Florida International University

FIU Digital Commons

FIU Electronic Theses and Dissertations

University Graduate School

$11-12-2009$

\title{
The Relationship between Classroom Interactions and Exclusionary Discipline as a Social Practice: A Critical Microethnography
}

Debra M. Pane PhD

Florida International University, debrapane@gmail.com

Follow this and additional works at: https://digitalcommons.fiu.edu/etd

Part of the Curriculum and Instruction Commons, Curriculum and Social Inquiry Commons, Inequality and Stratification Commons, Linguistic Anthropology Commons, Other Teacher Education and Professional Development Commons, Quantitative, Qualitative, Comparative, and Historical Methodologies Commons, and the Social and Cultural Anthropology Commons

\section{Recommended Citation}

Pane, Debra M. PhD, "The Relationship between Classroom Interactions and Exclusionary Discipline as a Social Practice: A Critical Microethnography" (2009). FIU Electronic Theses and Dissertations. 109. https://digitalcommons.fiu.edu/etd/109

This work is brought to you for free and open access by the University Graduate School at FIU Digital Commons. It has been accepted for inclusion in FIU Electronic Theses and Dissertations by an authorized administrator of FIU Digital Commons. For more information, please contact dcc@fiu.edu. 


\section{FLORIDA INTERNATIONAL UNIVERSITY}

Miami, Florida

THE RELATIONSHIP BETWEEN CLASSROOM INTERACTIONS

AND EXCLUSIONARY DISCIPLINE AS A SOCIAL PRACTICE:

A CRITICAL MICROETHNOGRAHY

A dissertation submitted in partial fulfillment of the

requirements for the degree of

DOCTOR OF PHILOSOPHY

in

CURRICULUM AND INSTRUCTION

by

Debra Mayes Pane

2009 
To: Interim Dean Marie McDemmond

College of Education

This dissertation, written by Debra Mayes Pane, and entitled The Relationship between Classroom Interactions and Exclusionary Discipline as a Social Practice: A Critical Microethnography, having been approved in respect to style and intellectual content, is referred to you for judgment.

We have read this dissertation and recommend that it be approved.

$\begin{array}{r}\text { Erskine Dottin } \\ \hline \text { Lynne D. Miller } \\ \hline \text { Angela Salmon } \\ \hline \text { Joan Wynne, Co-Major Professor } \\ \hline \text { Tonette S. Rocco, Co-Major Professor }\end{array}$

Date of Defense: November 12, 2009

The dissertation of Debra Mayes Pane is approved.

Interim Dean Marie McDemmond College of Education

Dean George Walker University Graduate School

Florida International University, 2009 
C Copyright 2009 by Debra Mayes Pane

All rights reserved. 


\section{DEDICATION}

To my husband, Robert Thomas Pane, for his love, dedication, patience, and encouragement throughout the entire process of writing this dissertation. 


\section{ACKNOWLEDGMENTS}

I wish to express my deepest appreciation to the members of my dissertation committee, Drs. Dottin, Miller, Rocco, Salmon, and Wynne for their guidance, support, and encouragement. Special gratitude is extended to my major professor, Dr. Tonette S. Rocco, whose guidance was crucial for developing my research method, dissertation writing and thinking skills, collegiate attitude, publication agenda, and professional fortitude. Her prompt responses, care for me as a student, dissertation peer review group and dissertation group meetings, and wittiness during the entire dissertation process were priceless. Another special thank you is extended to my major professor, Dr. Joan T. Wynne, whose kindness and solidarity supported and guided me in the direction of my final research topic and research design. I also wish to express my sincere appreciation to dissertation committee member Dr. Angela Salmon for her willingness to guide and work with me in research and publication endeavors. To Dr. Lynne Miller, thank you for introducing me to the love of literacy, writing, and research. To Dr. Erskine Dottin, thank you for your wisdom and concept of friendship between professors and students.

I also wish to thank faculty members in the College of Education who have been very supportive of my doctoral student endeavors over the years, especially Drs. Patricia Barbetta, Linda Bliss, Linda Spears-Bunton, Lisa Delpit, Mohammed Farouk, Gail Gregg, Lisbeth Dixon-Krauss, Joyce Fine, Adriana McEachern, and Isadore Newman. Special thanks are extended to Maria Tester, Office Manager, College of Education, Research and Grants; Maria Bovo, Office Assistant, College of Education, Department of Curriculum and Instruction; and Maria Macias, Secretary, College of Education, Department of Curriculum and Instruction, for their welcoming and professional 
assistance over the years. Research for this dissertation was supported in part through both a Florida International University Graduate School Doctoral Evidence Acquisition Fellowship and a Florida International University Graduate School Dissertation Year Fellowship.

I also wish to thank members of my peer review group and the dissertation group for their support. Special thanks to all the teachers and students who participated in this study for sharing their classrooms, experiences, and opinions with me. I am very grateful to the many alternative education students over the years who taught me about the conditions of life and education that I could not see prior to meeting them. To Jennifer Schuster, Esq., thank you for introducing me to the topic of my dissertation, supporting me over the years in the alternative education setting, and encouraging me to pursue my Ph.D.

Most importantly, I wish to thank my family for their love and prayers. To my mother Tommie June Mayes and daughter Heather Torenee Pane, thank you for the depth of listening, questioning, and faith in this study over the years. To my father Kenneth Orville Mayes, brother Kenneth Michael Mayes, brother David Leeke Mayes, son Justin Cory Pane, son Sean Christopher Pane, and sister-in-law Nancy Castle Mayes, thank you for your special encouragement and interest over the years. To my brother-in law Anthony Pane, Jr., sister-in-law Genevieve Mayes, daughter-in-law Erica Pane, and niece Laurie Pane, thank you for your words of support. To all of my other relatives, friends, and colleagues who have inspired and supported me during this process, thank you. Finally, I give God all credit for any good that may result from this dissertation. 
ABSTRACT OF THE DISSERTATION

THE RELATIONSHIP BETWEEN CLASSROOM INTERACTIONS

AND EXCLUSIONARY DISCIPLINE AS A SOCIAL PRACTICE:

A CRITICAL MICROETHNOGRAHY

by

Debra Mayes Pane

Florida International University, 2009

Miami, Florida

Professor Tonette S. Rocco, Co-Major Professor

Professor Joan Wynne, Co-Major Professor

Exclusionary school discipline results in students being removed from classrooms as a consequence of their disruptive behavior and may lead to subsequent suspension and/or expulsion. Literature documents that nondominant students, particularly Black males, are disproportionately impacted by exclusionary discipline, to the point that researchers from a variety of critical perspectives consider exclusionary school discipline an oppressive educational practice and condition. Little or no research examines specific teacher-student social interactions within classrooms that influence teachers' decisions to use or not use exclusionary discipline. Therefore, this study set forth the central research question: In relation to classroom interactions in alternative education settings, what accounts for teachers' use or non-use of exclusionary discipline with students? A critical social practice theory of learning served as the framework for exploring this question, and a critical microethnographic methodology informed the data collection and analysis. 
Criterion sampling was used to select four classrooms in the same alternative education school with two teachers who frequently and two who rarely used exclusionary discipline. Nine stages of data collection and reconstructive data analysis were conducted. Data collection involved video recorded classroom observations, digitally recorded interviews of teachers and students discussing selected video segments, and individual teacher interviews. Reconstructive data analysis procedures involved hermeneutic inferencing of possible underlying meanings, critical discourse analysis, interactive power analysis and role analysis, thematic analysis of the interactions in each classroom, and a final comparative analysis of the four classrooms.

Four predominant themes of social interaction (resistance, conformism, accommodation, and negotiation) emerged with terminology adapted from Giroux's (2001) theory of resistance in education and Third Space theory (Gutiérrez, 2008). Four types of power (normative, coercive, interactively established contracts, and charm), based on Carspecken's (1996) typology, were found in the interactions between teacher and students in varying degrees for different purposes.

This research contributes to the knowledge base on teacher-student classroom interactions, specifically in relation to exclusionary discipline. Understanding how the themes and varying power relations influence their decisions and actions may enable teachers to reduce use of exclusionary discipline and remain focused on positive teacherstudent academic interactions. 


\section{TABLE OF CONTENTS}

CHAPTER

PAGE

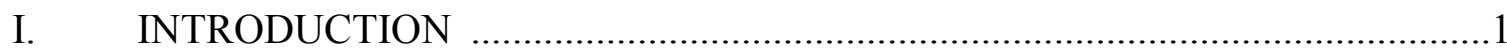

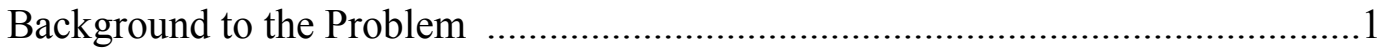

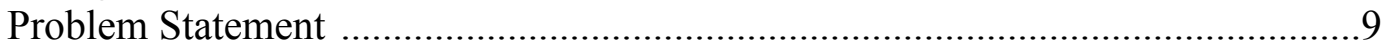

Purpose of the Study ................................................................................. 10

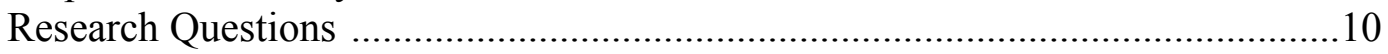

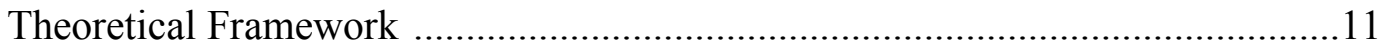

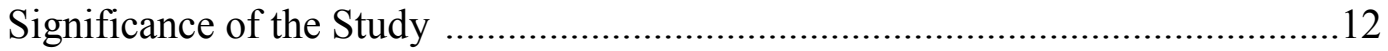

Assumptions of the Study ........................................................................13

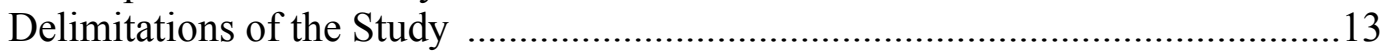

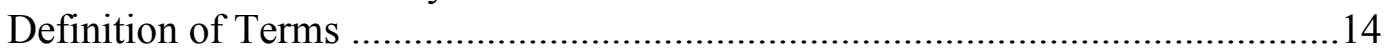

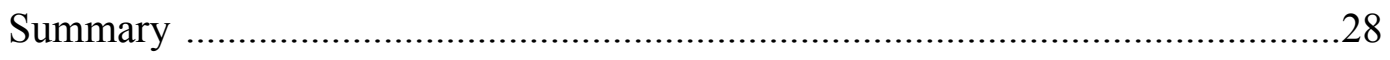

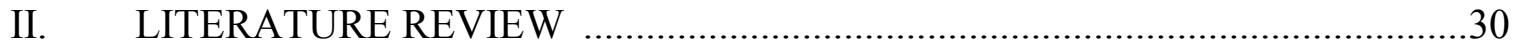

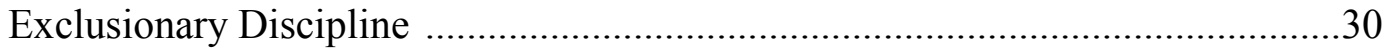

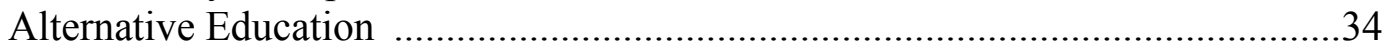

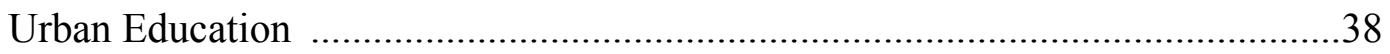

Views of Learning .......................................................................................63

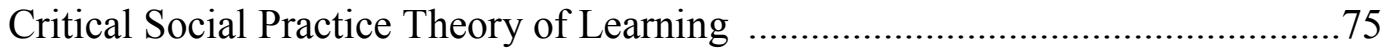

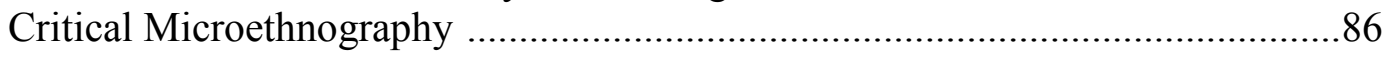

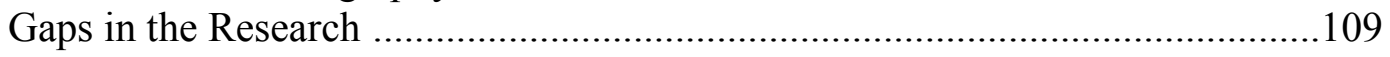

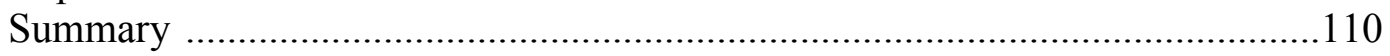

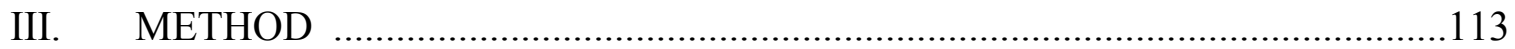

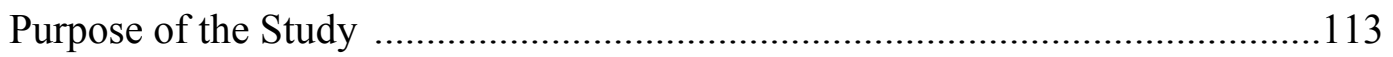

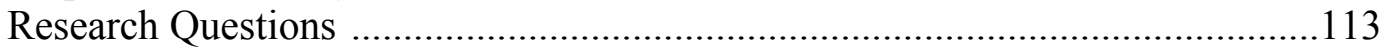

Critical Microethnographic Method .......................................................114

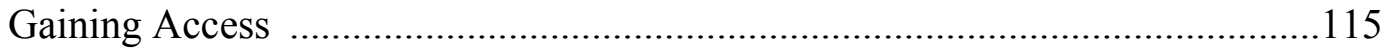

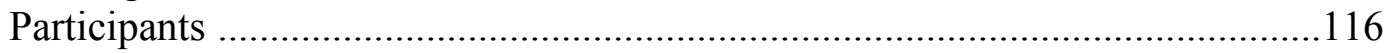

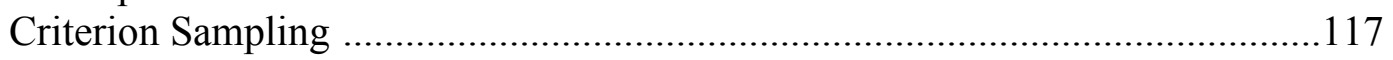

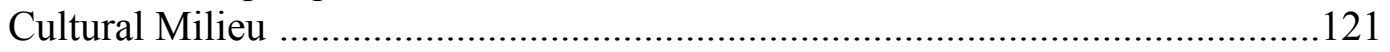

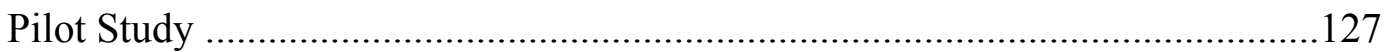

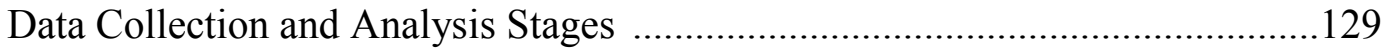

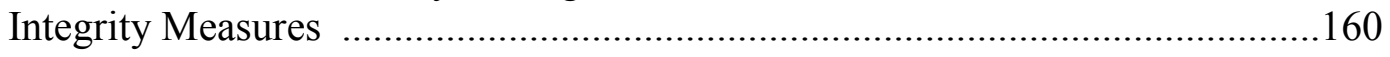

Validity Measures for Critical Social Research ....................................................161

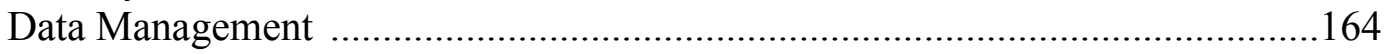

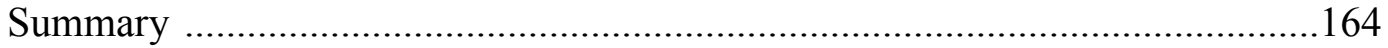


IV. DESCRIPTION OF CLASSROOM ONE FINDINGS _....................................166

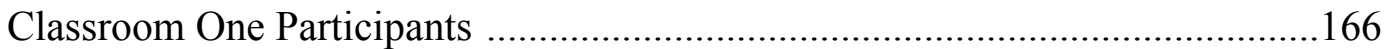

Findings from Interactive Power Analysis .......................................................167

Themes from Reconstructive Data Analysis ....................................................181

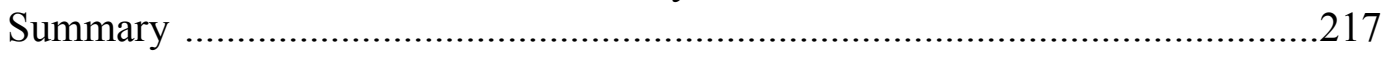

V. DESCRIPTION OF CLASSROOM TWO FINDINGS _.........................................

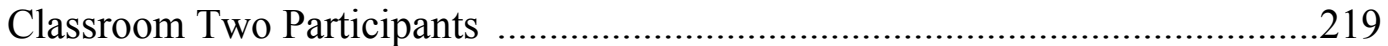

Findings from Interactive Power Analysis ......................................................222

Themes from Reconstructive Data Analysis ...................................................239

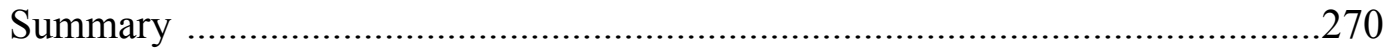

VI. DESCRIPTION OF CLASSROOM THREE FINDINGS _.............................272

Classroom Three Participants ....................................................................22

Findings from Interactive Power Analysis .....................................................274

Themes from Reconstructive Data Analysis ....................................................291

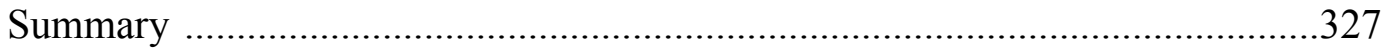

VII. DESCRIPTION OF CLASSROOM FOUR FINDINGS _....................................329

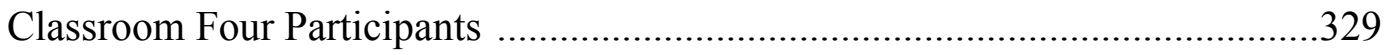

Findings from Interactive Power Analysis ....................................................331

Themes from Reconstructive Data Analysis ...................................................349

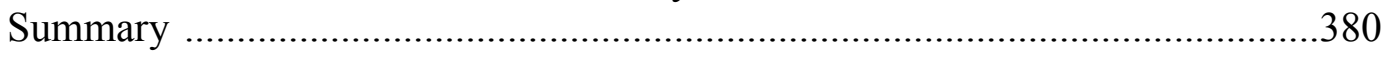

VIII. COMPARISON AND SYNTHESIS OF FINDINGS ........................................383

Classrooms Characterized by Frequent Exclusionary Discipline …………….....383

Classrooms Characterized by Rare Exclusionary Discipline.................................389

Classrooms Characterized by Frequent Versus Rare Exclusionary Discipline ..396

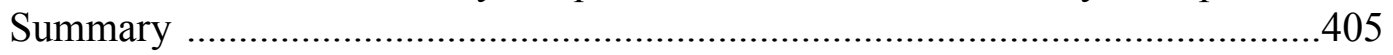

IX. SUMMARY, RESPONSES TO RESEARCH QUESTIONS, IMPLICATIONS,

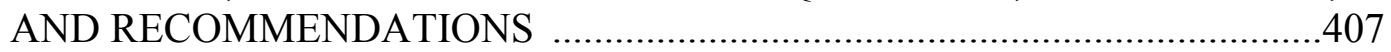

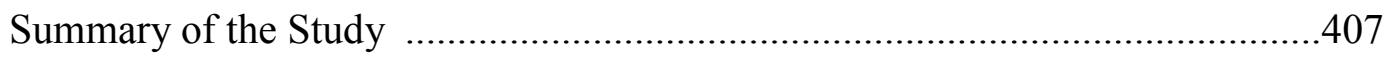

Responses to the Research Questions ...............................................................409

Implications for Teacher Education .............................................................421

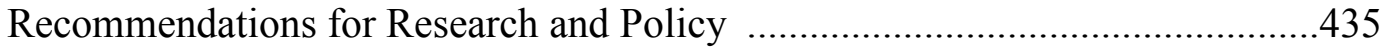

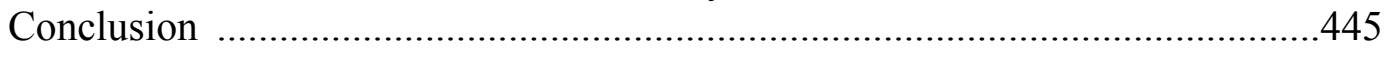

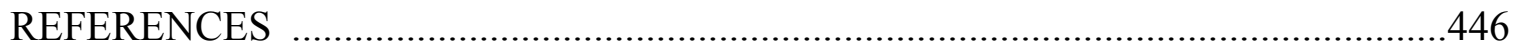

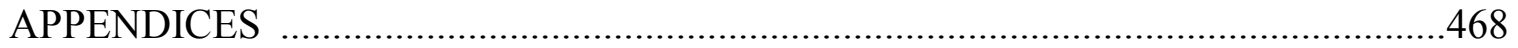

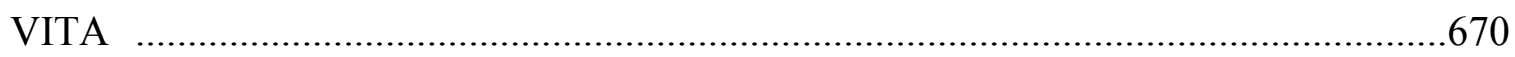




\section{LIST OF TABLES}

TABLE

PAGE

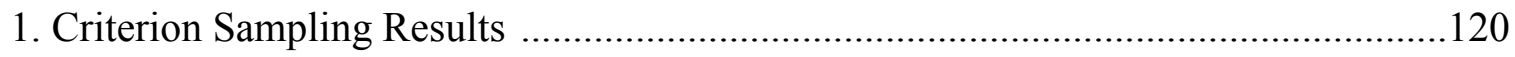

2. Student Classroom Observation Participants …………............................................131

3. Student Representation in Video Data .....................................................................136

4. Student Interview Participation .......................................................................138

5. Classroom One Student Participation ……………..................................................167

6. Types of Power Exhibited in Classroom One ..........................................................174

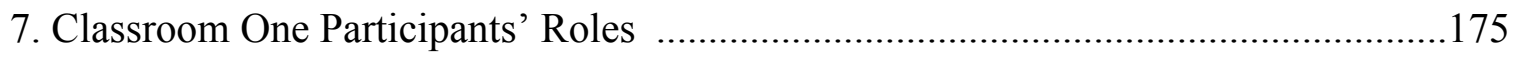

8. Classroom One Reconstructive Data Analysis .......................................................182

9. Classroom Two Student Participation ....................................................................22

10. Types of Power Exhibited in Classroom Two …………....................................232

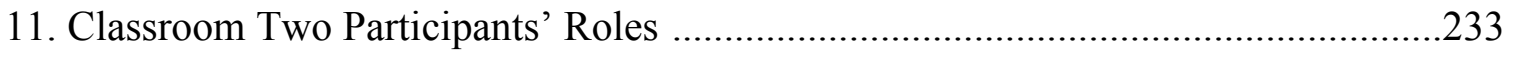

12. Classroom Two Reconstructive Data Analysis ........................................................240

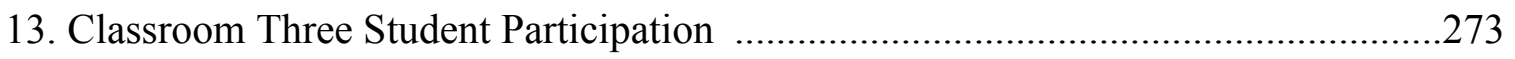

14. Types of Power Exhibited in Classroom Three ………...........................................28

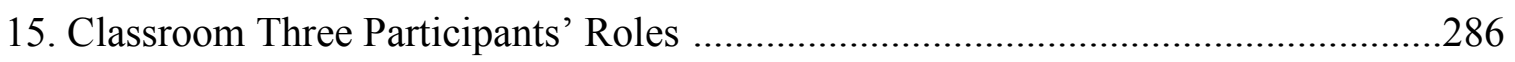

16. Classroom Three Reconstructive Data Analysis ......................................................22

17. Classroom Four Student Participation …………….............................................330

18. Types of Power Exhibited in Classroom Four …………...........................................341

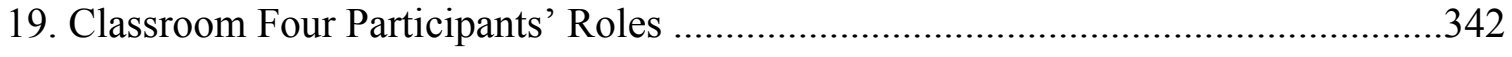

20. Classroom Four Reconstructive Data Analysis .....................................................350 
21. Comparison of Types of Power Exhibited in Classrooms Characterized by Frequent

Exclusionary Discipline .384

22. Comparison of Roles Exhibited in Classrooms Characterized by Frequent

Exclusionary Discipline

23. Comparison of Themes Exhibited in Classrooms Characterized by Frequent

Exclusionary Discipline .388

24. Comparison of Types of Power Exhibited in Classrooms Characterized by Rare

Exclusionary Discipline

25. Comparison of Types of Roles Exhibited in Classrooms Characterized by Rare Exclusionary Discipline

26. Comparison of Themes Exhibited in Classrooms Characterized by Rare

Exclusionary Discipline

27. Comparison of Types of Power in Classrooms Characterized by Frequent Versus

Rare Exclusionary Discipline

28. Comparison of Types of Roles Exhibited in Classrooms Characterized by Frequent

Versus Rare Exclusionary Discipline

29. Comparison of Themes Exhibited in Classrooms Characterized by Frequent Versus

Rare Exclusionary Discipline 


\section{LIST OF ACRONYMS}

$A R R A$. American Recovery and Reinvestment Act.

$A A V E$. African American Vernacular English.

AEP. Alternative education program.

$C D A$. Critical Discourse Analysis.

CHAT. Cultural Historical Activity Theory.

CRCM. Culturally responsive classroom management.

CRT. Critical Race Theory.

EAOP. Educational Alternative Outreach Program.

ESEA. Elementary and Secondary Education Act.

ETSPIC. Educational Testing Service Policy Information Center.

FDJJ. Florida Department of Juvenile Justice.

FDOE. Florida Department of Education.

FIU. Florida International University.

GED. General Education Diploma.

IDEA. Individuals with Disabilities Education Act.

$I-R-E / F$. Initiation-Response-Evaluation/Feedback.

JJDP. Juvenile Justice Dropout Prevention.

LPP. Legitimate peripheral participation.

M-DCPS. Miami-Dade County Public Schools.

NCES. National Center for Education Statistics.

NCLB. No Child Left Behind.

NLS. New Literacy Studies. 
NPTARS. National Partnership for Teaching in At-Risk Schools.

OC. Observer Comments.

OGS. Office of Graduate Studies.

OJJDP. Office of Juvenile Justice Dropout Prevention.

OJP. Office of Justice Programs.

PHA. Pragmatic horizon analysis.

$P R$. Primary record.

SES. Socioeconomic status.

SIZ. School Improvement Zone.

USDOE. United States Department of Education.

YLCI. Youth Literacies, Cultures, and Identities.

$Z P D$. Zone of proximal development. 


\section{CHAPTER I}

\section{INTRODUCTION}

The purpose of this critical microethnographic study was to explore the relationship between classroom interactions and exclusionary discipline as a social practice within and across classrooms in the same alternative education school. The background to the problem, problem statement, purpose of the study, research questions, theoretical framework, significance of the study, assumptions of the study, delimitations of the study, and definition of terms are presented in this chapter. A list of acronyms, list of tables, list of figures, list of appendices, and summary are also included.

\section{Background to the Problem}

In the United States, exclusionary school discipline is common. A large portion of the research deals with the African American population. Since the Children's Defense Fund (1975) research on school suspension, studies of school discipline have consistently documented the disproportionality of African American students, particularly males, in the administration of exclusionary school discipline (Skiba et al., 2000) and placement in disciplinary alternative education schools (Foley \& Pang, 2006; Van Acker, 2007) and the juvenile justice system (Office of Juvenile Justice Delinquency Prevention [OJJDP], 2009). Exclusionary school discipline includes removing students from the classroom by referrals to the office, suspensions, and expulsions. Exclusionary discipline consequences are more frequent, harsher, and less congruent to the incident for African American students, particularly males. Also, exclusionary discipline consequences increase for African American students, particularly males, in higher socioeconomic schools even 
though no evidence supports the claim that they are more disruptive than their peers (Skiba et al., 2000).

The disproportionality of African American students, particularly males, in exclusionary school discipline is termed the discipline gap (Monroe, 2005; Skiba et al., 2000). Disproportionality is determined by a $10 \%$ of the population standard, by considering a subpopulation "over- or under-represented if its proportion in the target classification (e.g., suspension) exceeds its representation in the population by $10 \%$ of that representation" (Skiba et al., 2000, p. 3). Since African Americans make up 16.9\% of the student population in our nation, $10 \%$ of the population standard for disproportionate suspension would be less than $15.3 \%$ or more than $18.7 \%$. The current $33.4 \%$ suspension rate of African American students is two to five times more than their White peers, which is well over $10 \%$ of the population standard, and confirms their disproportionate representation (Skiba et al., 2000).

As a result of being out of class so much, too many African American students get caught in the school failure, dropout, and juvenile justice system cycle, coined the schoolto-prison pipeline (Wald \& Losen, 2003). The school-to-prison pipeline theme, linking school discipline and juvenile justice data, emerged during a joint research conference cosponsored by the Civil Rights Project and Northeastern University's Institute on Race and Justice (Wald \& Losen, 2003). Results demonstrated an existing trajectory from the first time African Americans get in trouble at school to being (a) labeled a troublemaker and potentially dangerous; (b) referred to the office; (c) expelled to disciplinary alternative education schools; and (d) sent to jail or prison. 
Disproportionality increases as African American students move from referral to expulsion and, according to the disproportionate minority confinement data of the juvenile justice system, from detention to state prison (Florida Department of Juvenile Justice [FDJJ], 2007; Skiba et al., 2000). African Americans are twice as likely to be stopped and frisked, five times more apt to be detained, and up to ten times more prone to be incarcerated (Center on Crime and Juvenile Justice, 2008). Nationwide, African Americans comprise $15 \%$ of the juvenile population, $26 \%$ of juvenile arrests, $44 \%$ of juvenile detentions, $46 \%$ of youth sent to adult court, and $58 \%$ of youth sent to state prisons (Snyder \& Sickmund, 1999). In addition, African Americans comprise 27\% of all dropouts (National Center for Education Statistics, 2008), and 52\% of African American male dropouts have been incarcerated by age 30 (Day-Vines \& Day-Hairston, 2005). Disproportionate minority confinement has become the civil rights movement of our nation (Center on Crime and Juvenile Justice, 2008). However, explanations for the disproportionality in the discipline gap and school-to-prison pipeline are inconclusive.

Skiba and colleagues (2000) collected the disciplinary records of 50,000 middle school students in a large urban Midwestern public school district to explore possible explanations for "gender, race, and socioeconomic disparities in school discipline in sufficient detail to test alternative hypotheses concerning disproportionate school discipline" (p. 1). Results indicated that African American students represented 66.1\% of referrals to the office, $68.5 \%$ of suspensions, and $80.9 \%$ of expulsions, regardless of their being $56 \%$ of the student population. Males represented $63 \%, 67.2 \%$, and $83.7 \%$ of referrals, suspensions, and expulsions, respectively, and were $51.8 \%$ of the student population. African American students, particularly males, were also referred to the 
office, suspended, and expelled for more disruptive behavior compared to White students even though referrals were for less serious violations.

No evidence supported the hypothesis that African American students misbehaved more. Instead, African American students were referred for more subjective reasons such as disrespect or excessive noise while White students were referred for more serious and objective behaviors such as smoking and vandalism. Results also indicated that significant racial disproportionality existed after controlling for socioeconomic status regardless of analytical method used (Skiba et al., 2000). Racial and gender disparity appeared to originate at the classroom level as "systematic and racial discrimination" (p. 16). Nonetheless, empirical research conducted to explain racial and gender disparities in exclusionary school discipline is nonexistent (Skiba et al., 2000).

Broadly speaking, alternative education includes all educational programs and schools that fall outside traditional K-12 school systems (Cash, 2004). In the U.S., alternative education programs and schools differ in philosophy and organization (L. Delpit, personal communication, July 17, 2008). When referred to in this study, alternative education means programs and schools designed for students who are deemed disruptive and delinquent and suspended or expelled from their regular school or classroom. During the 1980s, the Office of Juvenile Justice Dropout Prevention (OJJDP) first promoted alternative education as a way to reduce youth crime. Since that time, traditional K-12 schools have been able to officially transfer youth deemed antisocial to alternative education programs (Wolford, 2000). Antisocial behavior is defined as the recurring violation of social norms and routines through disruptive, defiant, aggressive, or violent acts, such as fighting, substance abuse, truancy, and sexual deviance (Van Acker, 
2007). A $47 \%$ increase occurred in the number (from 2,606 to 3,850 ) of alternative education programs for antisocial youth between 1993 and 1998; today, approximately 11,000 alternative education programs exist (Foley \& Pang, 2006).

Thirty-nine percent of all public school districts in the U.S. have at least one alternative education program (NCES, 2003). There are approximately 11,000 alternative education programs (AEP) with a total of 612,000 students, $90 \%$ of which are for middle and high school youth (NCES, 2003). AEP can be situated anywhere from a separate facility where youth are transferred when they are suspended or expelled to a "schoolwithin-a-school" (Cash, 2004, p. 165). They range from alternative or charter schools for at-risk youth, district-operated community-based schools, or programs within juvenile detention centers (Foley \& Pang, 2006). Of all district-wide AEP, (a) 66\% are in urban areas; (b) $80 \%$ are in the southeast; (c) $95 \%$ have over 10,000 students; (d) $62 \%$ have more than half minority enrollment; and (e) $45 \%$ encompass student populations living in poverty. Urban, suburban, and rural public school districts across the 50 states define AEP differently, but approximately half of the youth referred to AEP are unsuccessful in the mainstream school setting and persistently involved in drug or alcohol abuse, fighting, truancy, academic failure, possession or use of a weapon, and disruptive verbal behavior. Twenty-five percent are referred for teen pregnancy or mental health issues (NCES, 2003).

This study took place in an alternative education school in a large urban multicultural southeastern area of the United States, Miami-Dade County Public Schools (M-DCPS), Florida, the fourth largest public school district in the country. An alternative education school setting was selected because of the likelihood of discipline issues and 
verbal and nonverbal interactions related to discipline, which is the focus of this study. M-DCPS has 418 schools, over 390,000 public school students, and 23,566 teachers (Berkshire Advisors, Inc., 2007). Miami-Dade County, Florida, population is approximately 57.3\% Latino/a, 20.3\% African American, and 20.7\% White of nonLatino origin (U.S. Census, 2008). However, in Miami-Dade County Public School (MDCPS), the percentage of African American students is higher at $30 \%$ and of Whites is lower at $10.6 \%$ with Latino students the same at $57.2 \%$. White, Black, and Latino teachers are distributed fairly equally across the district, but tend to more closely reflect the student racial profile of Black and Latino in the urban and alternative education schools (M-DCPS, 2007).

Students who populate M-DCPS alternative education schools have typically been suspended or expelled from their regular urban middle or high school (M-DCPS, 2007). Students who attend M-DCPS urban schools are characterized by higher than district average in-door and out-door suspension rates, higher truancy or absence rates, and lower achievement on standardized tests in reading comprehension (M-DCPS, 2007). Regulated by accountability and reporting auspices of NCLB (2002), M-DCPS 2005-2006 District and School Profiles (M-DCPS, 2007) indicate widespread academic failure characterized by very low standardized test scores, high suspension rates, and high absenteeism in the urban School Improvement Zone (SIZ). The 2004 M-DCPS SIZ proposal (now defunct) directed by the Superintendent and his staff identified a total of 39 elementary, middle, and high schools with 44,000 Black (67\%), Latino (30\%), and Haitian (3\%) students (no further breakdown in student demographics available), who have struggled academically for years (M-DCPS, 2004, 2007). During its tenure, the SIZ initiative aimed to promote 
high achievement and eliminate low performance in the identified schools, as measured by letter grades provided to schools based upon high-stakes test scores.

M-DCPS students who have continual and/or extreme behavior or academic problems in their regular schools, mostly Black and Latino students from the same feeder pattern, are referred to one of 16 M-DCPS alternative education schools (M-DCPS, 2007). Students released from detention or residential programs must attend an alternative education school successfully before returning to their mainstream school. If they are unsuccessful in alternative education schools and/or involved in crime, youth may be referred to one of the M-DCPS educational alternative outreach programs, small "last chance" centers for youth deemed extremely antisocial (M-DCPS EAOP, 2007). When school discipline and achievement data are analyzed, students who do better in school have fewer disciplinary consequences and also pass the high-stakes tests (MDCPS 2005-2006 District and School Profiles, 2007). Coupled with literature about alternative education programs and students, an implied link between school discipline, achievement, culture, and teaching practices exists but is not explicitly studied in educational literature.

Students who attend alternative education programs are vaguely characterized in the literature as (a) being "at-risk" of academic failure and dropping out of school, and (b) having been referred from traditional K-12 schools for disruptive and/or antisocial behavior (Foley \& Pang, 2006). However, a more recent and complete understanding of the characteristics of students who attend alternative education programs is gained from the literature about nondominant youth. Nondominant youth are defined as Black and Latino young people under the age of 18 who have been historically racialized (classified 
by race), marginalized, silenced, and segregated in schools and society (Fine, 1990, 1991; Fine \& Weiss, 2005; Gutiérrez, 2008; Nasir \& Hand, 2006; Oakes et al., 2006). Nondominant students are continuously failed and penalized by conventional attempts to reduce the achievement gap, the discipline gap, and the school-to-prison pipeline (Blanchett, 2006; Monroe, 2005). As reported in the literature, multiple risk factors disproportionately impact the vulnerability of nondominant youth for academic failure and dropping out of school. For example, nondominant youth are (a) three times more likely to live in poverty (Cash, 2004); (b) disproportionately diagnosed with behavioral, learning, and reading disabilities (Blanchett, 2006); and (c) disproportionately suspended or expelled from school—56\% Black, 38\% Latino, versus $28 \%$ White-for exhibiting disruptive and/or antisocial behavior (Foley \& Pang, 2006; Smink, 2004).

Nondominant youth with disabilities are also reported in the literature to attend alternative education programs in much higher proportions than nondisabled peers (Van Acker, 2007). Those who display antisocial behavior also have a significantly greater chance of being referred to alternative education programs and are at serious risk of relationship problems, dropping out of school, and increased rates of arrest, incarceration, and recidivism, which means a relapse into crime. Overall, $13 \%$ of Black and $27.8 \%$ of Latino youth drop out permanently compared to 3.8\% Whites (Snyder \& Sickmund, 2006). Relatedly, national youth recidivism rates hover between $60 \%$ and $84 \%$, and $57 \%$ of our country's inmates 16 and over are high school dropouts with only basic levels of literacy (Greenberg, Dunleavy, \& Kutner, 2007; Snyder \& Sickmund, 2006). Basic levels of literacy are the skills and knowledge needed from a conventional view of learning that 
enables one to use print information well enough to function in society and to achieve one's goals and potential.

Since Skiba and colleagues' (2000) study, Vavrus and Cole (2002) examined the "sociocultural factors that influence a teacher's decision to remove a student from the classroom" (p. 87). They studied how disciplinary moments, or "patterns of classroom interaction that often precede a suspension" (p. 89), are co-constructed or negotiated as social practice among teachers and students in moment-by-moment interactions. Results indicated that disciplinary moments vary by the sociocultural context of particular classrooms rather than occur as a series of events strictly defined in school discipline policy and presumably linked to violence. However, few studies have examined the social aspects of classroom interactions to understand how and why particular disciplinary actions occur (Monroe, 2006) even though misbehavior and discipline problems remain one of the main reasons teachers leave the profession (Evertson \& Weinstein, 2006; Public Agenda, 2004).

\section{Problem Statement}

Exclusionary school discipline is common in public schools. Nondominant students, mostly African American males, are disproportionately impacted by exclusionary discipline and end up in alternative education school settings. In alternative education school settings, nondominant students continue to be disproportionately involved in exclusionary discipline. Little or no research exists that focuses on the relationship between classroom interactions and exclusionary discipline as a social practice. An alternative education school setting was selected for this study because of the likelihood of discipline issues and verbal and nonverbal interactions related to 
discipline, which is the focus of this study. A critical social practice, or communities of practice perspective, guided the study for the purpose of finding potential spaces for transformation of oppressive conditions that perpetuate the disproportionality of nondominant students in exclusionary discipline consequences.

\section{Purpose of the Study}

The purpose of this critical microethnographic study was to explore the relationship between classroom interactions and exclusionary discipline as a social practice within and across classrooms in the same alternative education school.

\section{Research Questions}

The central research question was: In relation to classroom interactions in alternative education settings, what accounts for teachers' use or non-use of exclusionary discipline with students? Secondary questions were:

1. What verbal and nonverbal interactions typify the learning processes and exclusionary discipline practices within the selected classrooms?

2. What cycles of social and academic identity reproduction occur within the selected classrooms?

3. How does each teacher explain what happens within his or her classroom regarding classroom interactions, learning processes, and exclusionary discipline practices?

4. How do students explain what happens within their classroom regarding classroom interactions, learning processes, and exclusionary discipline practices?

5. What is the relationship between classroom interactions, learning processes, and exclusionary discipline practices across the selected classrooms? 


\section{Theoretical Framework}

While an expanded presentation of the theoretical framework is discussed in the literature review, key aspects of the framework include the following. A critical social practice theory of learning (Lave \& Wenger, 1991), or communities of practice perspective, is the framework that guided this study. A critical social practice theory of learning views learning, language, literacy, culture, and discipline, specifically school suspension, as integral social practices that are actively co-constructed in specific situations and simultaneously shaped by and shape societal processes. A critical social practice theory of learning is defined by the process of legitimate peripheral participation (LPP). LPP is a phrase coined by Lave and Wenger (1991) to describe how people engage in social practice and learn through various aspects of community membership.

\section{LPP}

provides a framework for bringing together theories of situated activity and theories about the production and reproduction of the social order .... a common ground for exploring their integral, constitutive relations, their entailments, and effects in a framework of social practice theory, in which production, transformation, and change in the identities of persons, knowledgeable skill in practice, and communities of practice are realized in the lived-in world of engagement in everyday activity. (Lave \& Wenger, 1991, p. 47)

In legitimate peripheral participation (LPP), learning is viewed as situated activity in communities of practice; that is, learners engage in learning to become full participants in the sociocultural practices of a particular community. Viewing each classroom as a community of practice in which the teacher and his or her nondominant students participate with each other based on previous experiences and how they understand the current situation enables the researcher to understand how and why disciplinary (or potential disciplinary) action may have occurred in particular alternative education (or 
other) classrooms. Legitimate peripheral participation (LPP) provides a framework for finding potential spaces for transformation of oppressive conditions that perpetuate the disproportionality of nondominant students in exclusionary discipline consequences.

\section{Significance of the Study}

This study lays a foundation for the study of interactions in alternative education classrooms to understand how and why particular disciplinary actions occur in order to reduce the need for exclusionary discipline. This study provides cases for discussion of interactions in alternative education classrooms with insight into potential spaces for transforming oppressive learning processes and cycles of social and academic identity reproduction. This study has implications for teachers who are competent teaching content and building on students' academic skills but rely on exclusionary discipline rather than preventing discipline problems in alternative education schools (Public Agenda, 2004). This study has implications for teacher education programs to include courses, field experiences, and units of study to help teachers transform their awareness of (a) teacher-student interaction patterns; (b) teacher responsibility/agency in creating positive interactions with all students to promote learning; and (c) social and academic consequences of classroom interaction patterns.

This study has implications for future research that builds on potential spaces for transformation of oppressive conditions in alternative education classrooms and schools like those presented in the findings of this study. This study has the potential of initiating “cross-cultural, global, and social justice conversations towards a theory of ongoing transformation in classrooms and schools" (Pane, in press, p. 1) and of contributing to the overall task of the anthropology of education as "the anthropology of cultural 
transformation" (Varenne, 2008, p. 356). Finally, this study has implications for policy changes that warrant and fund research and teacher education that transforms educational practices and conditions in alternative education classrooms and schools.

Assumptions of the Study

An assumption of this study is that classrooms are sociocultural contexts in which social practices (e.g., learning, language, literacy, culture, exclusionary discipline) are negotiated among all participants. Social practices in situated learning imply transformation and change in and of communities of practice. Another assumption of this study aligns with Lee's (2005) conclusions that problems teachers have in the classroom are derived from the paradigm from which intelligence and learning are viewed. A third assumption aligns with findings from the literature that teachers' decisions may be related to district or state high-stakes testing mandates and fear of the loss of control in the classroom (Noguera, 2003). Even though disciplinary moments are sociocultural negotiations between teachers and students and are bound by system constraints, teachers ultimately have the power to decide on exclusionary discipline consequences (Vavrus \& Cole, 2002).

\section{Delimitations of the Study}

The classrooms for this study were selected from a typical alternative education school in Miami, Florida. Delimitations exist by specifying teachers and students in alternative education classrooms as the population of this study. The results of this study do not necessarily generalize to other populations from other alternative schools in other school districts. Alternative education schools in Miami, Florida, are situated and defined in this study according to the definition of urban contexts in the K-12 education literature. 
Urban contexts in the K-12 education literature are represented as populated with students of color in lower income settings with meager resources and imposing bureaucracies. In light of these definitions, this study focused on what happens in classrooms with students who have been suspended or expelled from their regular urban school for disruptive or juvenile delinquent behavior, disproportionately African American and Latino, or nondominant, students.

\section{Definition of Terms}

African American. African American is the term used in this study to denote a racial category that differentiates between African American and European American students and/or teachers without comparing populations within each category (Nieto, 2002). The terms Black and White will be used interchangeably with African American and European American, respectively, by their use in cited studies. Further differentiating populations (e.g., Haitian) goes beyond the scope of this study.

Agency. Agency is "strategic making and remaking of selves" (Lewis, Enciso, \& Moje, 2007, p. 40). Spaces for agency may be promoted or limited by teacher, student, and/or institutional cultural models which continually frame and reframe what matters or not and what will be valued or not in literacy practices.

Antisocial behavior. Antisocial behavior is the recurring violation of social norms and routines through disruptive, defiant, aggressive, or violent acts, such as fighting, substance abuse, truancy, and sexual deviance (Van Acker, 2007).

Backgrounded. Backgrounded means where the idea originated (i.e., background experience or understanding). 
Caring and responsive curricula. Caring and responsive curricula arise from caring relationships among teachers and students. Caring relationships are defined as a "reciprocal and multidimensional process involving action, effort, achievement, accountability, respect, determination for self, community, and others, and responsiveness" (Bloome et al., 2005, p. 165).

Classroom management. "Classroom management refers to actions taken to create and maintain a learning environment conducive to successful instruction (arranging the physical environment, establishing rules and procedures, maintaining students' attention to lessons and engagement in activities"; Evertson \& Weinstein, 2006, p. 17).

Additionally, from the perspective of establishing an orderly learning environment in order to reduce misbehavior and increase opportunities for learning, classroom management is defined as

the ability of teachers and students to agree upon and carry forward a common framework for social and academic interactions, by creating an ethos of effort within a social fabric that is built over time, and ultimately leads to student selfdiscipline. (Freiberg \& Lapointe, 2006, p. 737)

Critical ethnography. Critical ethnography describes, interprets, and explains meanings people give to their own practices and experiences through an epistemological lens that links power, knowledge, and truth (Carspecken, 1996).

Critical microethnography. Critical microethnography merges ethnography, critical social research, and discourse analysis to study daily classroom life with attention to broader societal structures, learning processes, and social and academic identity reproduction (Bloome et al., 2005; Vavrus \& Cole, 2002). 
Critical sociocultural research methods. Critical sociocultural research methods

acknowledge and critique unequal power relations related to broader contexts of learning (Moje \& Lewis, 2007).

Critical sociocultural theory. Critical sociocultural theory accounts for issues of identity, agency, and power in the production of knowledge that are central to understanding literacy as a social and cultural practice .... as they shape and are shaped by individuals in particular cultural contexts .... [and] focuses on the central role of language and discourse in literacy events and practices. (Lewis et al., 2007, p. xi)

Cultural capital. Cultural capital is the ability to navigate and participate successfully in the culture of power (Delpit, 1995).

Cultural deficit orientation. Cultural deficit orientations have a history of explaining racial differences on IQ tests and school achievement as biological (e.g., inherited intelligence) and cultural deficits (e.g., poverty, social disorganization). More recently, scholars argue that students of color are culturally different rather than socially and cognitively deficient (Nasir \& Hand, 2006). In this view, culture is thought of as a system of meanings and practices, consistent over time, carried from place to place.

Cultural practices. A cultural practice is an agreed upon concept, or cultural model, that is performed in a particular set of events (Bloome et al., 2005). From a sociocultural perspective, culture is conceptualized as activities that are produced and reproduced as people live culturally. In other words, people create and recreate culture in social interactions as they participate in and reconstruct cultural practices (Gutiérrez \& Rogoff, 2003; Nasir \& Hand, 2006). Culture is

understood as the patterns of beliefs, values, and practices that we both inherit and transform over time...cultural practices are open or responsive to an everchanging environment...educational practices must be understood in terms of the 
activities that constitute them and in relation to the institutional contexts they constitute. (Gutiérrez, 2002, p. 3)

Cultural synchronization. "The concept of cultural synchronization is based on anthropological and historical research that advances the finding that black Americans have a distinct culture founded on identifiable norms, language, behaviors, and attitudes from Africa" (Irvine, 1990, p. 23). Cultural synchronization is what occurs in schools and classrooms when teachers' and students' cultures are similar. The White teacher and White student are the ones who usually understand the dominant "unstated rules and subtleties of verbal and nonverbal communicative processes ... [thus] interaction and learning with the teacher are productive and enjoyable" (Irvine, 1990, p. 23).

Culturally diverse. Stemming from Irvine's (1990) concept of cultural synchronization, culturally diverse will refer to the representation of more than one culture in this study. For example, a culturally diverse classroom has a teacher and students from more than one culture. The phrase culturally diverse students means that the group of students being referred to represent more than one culture.

Culturally responsive classroom management. Culturally responsive classroom management includes "five essential components derived from the literature on culturally responsive pedagogy, multicultural counseling, and caring" (Weinstein, TomlinsonClarke, \& Curran, 2004, p. 28):

(a) recognition of one's own ethnocentrism; (b) knowledge of students' cultural backgrounds; (c) understanding of the broader social, economic, and political context; (d) ability and willingness to use culturally appropriate management strategies; and (e) commitment to building caring classrooms. (Weinstein et al., 2004, p. 25) 
Culturally responsive pedagogy. Culturally responsive pedagogy is teaching that merges effective teaching standards with a focus on culture (Ware, 2006). It is characterized as validating, comprehensive, multidimensional, empowering, transformative, and emancipatory (Gay, 2000). Additionally, culturally responsive teachers understand the concepts of power, caring, communication, and cultural congruity to support culturally and ethnically diverse students' achievement in the classroom. Culturally responsive teachers appear to be more cognizant of students' cultural behaviors that are often perceived to be disruptive but not necessarily intended to be disruptive or disrespectful. For example, some teachers perceive hair platting in class to be disruptive when it is a deeply ingrained cultural behavior in Black communities.

Culture. Culture is defined as the "integrated pattern of human behaviors that include thoughts, communication, action, customs, beliefs, values, and instructions of a racial, ethnic, religious, or social group" (Day-Vines \& Day-Hairston, 2005, p. 237).

Cycles of social and academic identity reproduction. Cycles involve contradictory transformation and change of identities and social practices in synergistic relationships of participation, implicating the status quo. On the other hand, conventional apprenticeship and views of learning involve assimilation or transfer of learning in traditional teacherstudent relationships.

Dialogical data. Dialogical data are conversational data, such as data from interviews.

Discipline. Discipline, in the classroom context, "may be viewed as an action against as in disciplining or punishing someone .... In the context of behavior, discipline refers to control and with self-discipline to self-control" (Freiberg \& Lapointe, 2006, p. 
738). If a student is lacking self-discipline, teachers generally impose more external control equating classroom management with the "traditional unidimensional view of punishments and rewards, obedience and control” (Freiberg \& Lapointe, 2006, p. 738).

From a social justice perspective, Watts and Erevelles (2004) found that sometimes student behavior may be a healthy reaction to an unjust classroom learning environment. In socially just classrooms, teachers know how to model tolerance and respect for students (Landorf, Rocco, \& Nevin, 2007). “The essence of a [culturally] competent inclusive global educator is to go beyond tolerance to teach respect and social justice from the inside out" (p. 51). At the core of disproportionate "disciplinary referrals and inconsistencies between teacher-school expectations and urban student behavior are [limited] access and opportunity to learn" (Milner, 2006, p. 499).

Disciplinary moments. Disciplinary moments are "patterns of classroom interaction that often precede a suspension" (Vavrus \& Cole, 2002, p. 89). Disciplinary moments are co-constructed or negotiated as social practice among teachers and students in moment-by-moment interactions. Therefore, disciplinary moments are a result of the sociocultural context of particular classrooms. Disciplinary moments differ from the strict definition of discipline (a series of events linked to or resulting in violence) found in school discipline policy books.

discourse. Discourse with a "little $\mathrm{d}$ [means] language-in-use....how language is used 'on site' to enact activities and identities....[which are] rarely ever enacted through language alone" (Gee, 2006, p. 7).

Discourse practices. Discourse practices $(\mathrm{Gee}, 2006)$ are linguistic and semiotic (i.e., sign systems and knowledge for communicating). Thus, Discourse practices are 
the ways in which people position their bodies, the arrangement of furniture to support their social interactions, as well as their context-sensitive language behaviors all play a role in how people, through their daily interactions, establish social roles, power relationships, or, conversely, how they resist and subvert the status quo...a power issue as well. (Berkenkotter \& Thein, 2005, p. 179)

Discourses. Language-in-use, including verbal and nonverbal behaviors to enact specific activities and identities, is referred to as Discourse with a capital D (Gee, 1996, 2006). Discourses with a capital D can be thought of as shared ways of knowing (Heath, 1983), “thinking, believing, acting, and communicating” (Moje \& Lewis, 2007, p. 16) that are present in and out of school and influence how people teach and learn in school. Discourses with a capital D are

ways of being in the world...which integrate words, acts, values, beliefs, attitudes, and social identities...gestures, glances, body positions, and clothes. A Discourse is a sort of identity kit which comes complete with the appropriate costume and instructions on how to act, talk, and often write, so as to take on a particular social role that others will recognize. (Gee, 1996, p. 127)

Disproportionate. Disproportionate means a statistical overrepresentation of a particular group of people in a particular category (Foley \& Pang, 2006).

Disproportionality occurs when the "subpopulation may be considered over- or underrepresented if its proportion in the target classification (e.g., suspension) exceeds its representation in the population by $10 \%$ of that representation" (Skiba et al., 2000, p. 3). For example, in the United States, $60 \%$ of all youth are White, $20 \%$ are Latino/a, and 16.4\% are Black; however, those who attend alternative schools for disruptive and/or antisocial behavior are 5\% White, 41\% Latino/a, 50\% Black.

Exclusionary discipline practices. Exclusionary discipline practices occur when teachers or administrators remove students from the classroom for discipline reasons. 
Exclusionary discipline consequences (e.g., suspension, expulsion) are the "primary medium used once students are sent from the classroom” (Fenning \& Rose, 2007, p. 536).

Field journal notes. Field journal notes are written memories or other informal notes from conversations or other observations about the research experience. They are not transcribed and are used to augment and compare transcribed data analyses with additional contextual information for the researcher's interpretation (Wengraf, 2004).

Field journal notebook. The field journal notebook is where the researcher writes memories or other informal notes from conversations or other observations about the research experience (Wengraf, 2004).

Foregrounded. Foregrounded means the idea in focus (i.e., foreground object).

Initial meaning reconstruction. Initial meaning reconstruction is conducted to gain a holistic picture of routines or unusual events (i.e., continuity in settings and/or change or shifts in settings) in everyday life (Carspecken, 1996). Initial meaning reconstruction includes low-level coding, pragmatic horizon analysis, and validity reconstructions.

Interactive power. Power accompanies all actions and makes a difference in the subsequent events, "no matter how large or small" (Carspecken, 1996, p. 128). Interactive power analysis involves determining "who has what kind of power and why" (p. 129) and who has the most say in defining and determining the end result of interactions. However, in theory, even in the most constraining or coercive situations, participants have the choice to disobey or not comply.

Intersubjectivity. Intersubjectivity is "understanding the Self to understand the Other" (Roth, 2005, p. 15). In this study, hermeneutic inferencing requires the ability to 
take positions (i.e., intersubjectivity) and to recognize, reflect on, and explicitly examine and differentiate between personality and cultural typifications (i.e., intricacies in settings) of participants and others such as the researcher (Carspecken, 1996).

Interactions. Interactions include all verbal and nonverbal language between people in social settings (Carspecken, 1996).

Language. Language is the primary cultural tool and mediator of actions in activity systems, intertwined in macrostructures of power and ideology (Lewis et al., 2007). One's use of language to communicate impacts one's identity and is informed by one's cultural ways of knowing and making sense of an experience (Heath, 1983).

Latino. Latino is the term used in this study to denote students and/or teachers with an Hispanic background without differentiating populations within this category, which is beyond the scope of this study.

Lightly-structured interviews. Lightly-structured interviews allow more participation from the interviewee through the use of fewer and more open-ended questions about a general topic (e.g., a typical day at school).

Linguistic behaviors. Linguistic behaviors include all verbal and nonverbal interactions, such as speech acts, body movements, and postures (Carspecken, 1996).

Literacy. In this study, literacy is not defined as "some decontextualized ability to read and write, but the social practices into which people are apprenticed as part of a social group, whether as students in school, letter writers in a local community, or members of a religious group" (Gee, 1996, p. 57). Literacy will have no meaning outside of the cultural contexts in which it is used. 
Literacy event. A literacy event is any event in which literacy plays an important role. An event is a "bounded series of actions and reactions [in which] people act and react to each other" (Bloome et al., 2005, p. 6).

Literacy practices. Literacy practices are cultural practices involving written language (Bloome et al., 2005). A classroom literacy practice is a cultural practice that involves written language while "doing classroom life" (p. 50).

Low-level coding. Low-level coding is the process of making a list of short descriptions of objective features, or what anyone in the room who was listening would probably agree to have happened.

Marginalized. Marginalized youth are "outside of the mainstream of the regular school programs" (Miami-Dade County Public Schools [M-DCPS] Educational Alternative Outreach Program [EAOP], 2007, p.1). That is, they are unsuccessful in the mainstream school setting of traditionally expected cognitive skills and competencies (Pardoe, 2000). In this study, marginalized youth are students who have been involved in exclusionary discipline and/or in the juvenile justice system, and as a result, have been suspended or expelled to alternative education schools, which are located outside of the regular school programs and campuses.

Meaningful utterances. An utterance is meaningful only if and when it communicates who (i.e., not just a person but an identity being built) is doing what.

Mediator. Mediator, in this study, means a teacher who acts as a broker by supporting students' cultural backgrounds and needs in order to maximize opportunities for learning (Lee \& Ball, 2005). Based on practice or an activity system as the unit of analysis, mediation or mediated action occurs in a community in which the goals are 
emergent, negotiated, constrained, or made possible by the interactions among available tools used as artifacts, rules, ideas, and roles of participants as problems are solved. Roles of participants are considered tools since roles can interact with each other. For example, the role of teacher can be played by any or several participants in the classroom. The "teachers" will interact by negotiating, changing, or constraining each other's roles and ultimately each other's goals. Implications for identity reproduction or production apply.

Microethnography. Microethnography focuses on how people use language and other communication forms with attention to social, cultural, and political processes (Bloome et al., 2005).

Nondominant youth. Nondominant youth is a descriptor in literature pertinent to this study for young people under the age of 18 who have been historically marginalized, silenced, and segregated in U.S. schools and society (Fine, 1990, 1991; Fine \& Weiss, 2005; King, 2005; Lam, 2006; Nasir \& Hand, 2006; Oakes, Rogers, \& Lipton, 2006). In this study, nondominant youth are students in the alternative education classrooms and schools who are mostly African American and Latino and have been segregated, silenced, and marginalized by virtue of their expulsion or suspension from regular school programs and campuses. Nondominant will be the term of choice in this study to indicate African American and Latino students in alternative education classrooms and school as a group. If discussing a particular student or teacher, African American and Latino/a will be used. Normative-evaluative truth claims. Normative-evaluative truth claims are all perspectives on what is right or wrong about what is happening (i.e., right, good, or appropriate activities that others should agree on but usually argue about).

Objective truth claims. Objective truth claims are observable objects and events. 
Oppressive educational conditions and practices. Oppressive educational conditions and practices perpetuate dominant educational myths through systematic methods of indoctrination, measurement, testing, and rote learning (Goodlad, 1984). Oppressive education maintains the status quo, reproducing a common culture and not addressing the political nature of literacy and schooling (Wynne, 2002).

Passive observations. Passive observations are conducted by an uninvolved (i.e., passive) observer, who documents the observations with detailed notes, observer's comments, audio recordings, and/or videorecordings.

Pragmatic horizon analysis. Pragmatic horizon analysis the process of scrutinizing how all participants make sense of a situation together through the ongoing manipulation of physical objects, voice, body language, participation, cultural practices, social identities, and power relations. Pragmatic horizon analysis contrasts the idea in focus (i.e., foreground object) with where the idea originated (i.e., background experience or understanding).

Primary record. A primary record is a built by (a) videorecording and writing detailed notes (in a primary record notebook) of people interacting; (b) a first and second viewing of the videorecording to select segments representative of interaction patterns; (c) a third viewing of the videorecording to transcribe verbatim what people say into meaningful utterances (i.e., speech acts determined to be meaningful by what comes before and after); (d) a fourth viewing of the videorecording segments to explain the meaningful utterances and linguistic behavior observed (i.e., speech acts, body movements, and postures) on the transcripts; (e) and a fifth viewing of the representative 
videorecording segments to analyze and articulate possible objective validity claims (i.e., possible meanings) about observed linguistic behaviors on the transcripts.

Primary record notebook. The primary record notebook is the notebook in which the researcher writes detailed notes during each passive observation.

Raw codes. Raw codes are the list of multiple, overlapping general categories and many different codes under each category produced during high-level coding procedures and are reorganized to focus the final analysis of the study.

Recidivism. Recidivism is a relapse into crime (OJJDP, 2009).

Reconceptualize. Reconceptualize means to view a taken-for-granted concept from a different perspective. For example, the traditional concept of literacy (i.e., emphases on functional, or basic, literacy requirements) can be reconceptualized from a critical sociocultural perspective (i.e., emphases on the social and cultural aspects of literacy learning in which students are viewed as real people who resist and produce language and literacy together with others through interactive power).

Reconstructive data analysis. Reconstructive data analysis is the inductive (i.e., meanings not predetermined) process of interpreting implicit (i.e., unspoken) meanings of interactions in the primary record into explicit words.

Reflexivity. Reflexivity refers to how relationships, concepts, and/or meanings are simultaneously influenced by and/or influence each other (Patton, 2002).

Social theory of literacy. Practices, events, and texts "provide the first proposition of a social theory of literacy, that: literacy is best understood as a set of social practices; these are observable in events which are mediated by written texts" (Barton \& Hamilton, 2000, p. 9). 
Subjective truth claims. Subjective truth claims are one's personal thoughts and feelings.

Truth claims. Truth claims are explicit or implicit statements that can be judged to be true, false, right and/or wrong (see objective, subjective, normative-evaluative).

Urban. Urban (as opposed to suburban or rural) contexts are defined in K-12 education literature as "heavily populated with students of color ... large number of students from lower socioeconomic status, high attrition of teachers, heavy institutional and systemic barriers, and meager resources .... grossly underfunded, larger in size [than suburban or rural contexts], and infiltrated with administrative [local to federal] bureaucracy" (Milner, 2006, p. 493).

Validity claims. Validity claims are all the reasons one could give for subjective, objective, and/or normative-evaluative truth claims. Validity claims range from highly to remotely backgrounded or foregrounded.

Validity reconstructions. Validity reconstructions are an attempt to get at the meanings of what is happening that are often taken for granted (i.e., implicit) by producing a list of all possible reasons that a person could use to defend an action and/or to clear up a misunderstanding.

Youth. Youth are young people between the ages of 12 and 18 (Moje, 2007). Zone of proximal development. Three interpretations of Vygotsky's (1986) zone of proximal development are: (a) "the discrepancy between a child's actual mental age and the level he reaches in solving problems with assistance" (Vygotsky, 1986, p. 187); (b) the distance between one's cultural knowledge and everyday knowledge; and (c) taken from Engeström's (1987) activity theory and Lave and Wenger's (1991) LPP, 
focusing on the processes of social transformation that occur when people participate together in continuous, evolving communities of practice.

\section{Summary}

Exclusionary school discipline is common in the United States. A disproportionate number of African American students, particularly males, are referred out of the classroom and ultimately suspended and expelled from school. Exclusionary disciplinary practices lead to the disproportional involvement of African American and increasingly Latino (nondominant) students in school failure, alternative education, dropping out of school, and the juvenile justice system. The reasons for inequitable exclusionary discipline outcomes remain unclear, but exclusionary school discipline consequences appear to originate as racial and gender disparity in disciplinary actions at the classroom level (Skiba et al., 2000). Little research has been conducted in classrooms to examine specific teacher-student social interactions within classrooms that influence teachers' decisions to use or not use exclusionary discipline (Vavrus \& Cole, 2002). The central research question was: In relation to classroom interactions in alternative education settings, what accounts for teachers' use or non-use of exclusionary discipline with students? A critical social practice theory of learning served as the framework for exploring this question, and a critical micorethnographic methodology informed the data collection and analysis.

The background to the problem, problem statement, purpose of the study, research questions, theoretical framework, significance of the study, assumptions of the study, delimitations of the study, definition of terms, and a summary were presented in this chapter. Literature related to this study is reviewed in chapter 2 . The critical 
microethnographic method is explained in chapter 3 . In chapters 4-7, the findings from each classroom are presented. In chapter 8, a comparison and synthesis of the findings is provided. Chapter 9 includes a summary of the study, responses to research questions, implications for teacher education, and recommendations for research and policy. 


\section{CHAPTER II}

\section{LITERATURE REVIEW}

If we are to successfully educate all of our children, we must work to remove the blinders built of stereotypes, monocultural instructional methodologies, ignorance, social distance, biased research, and racism. We must work to destroy those blinders so that it is possible to really see, to really know the students we must teach .... I pray for all of us the strength to teach our children what they must learn, and humility and wisdom to learn from them so that we might better teach. (Delpit, 1995, p. 182)

The purpose of this critical microethnographic study was to explore the relationship between classroom interactions and exclusionary discipline as a social practice within and across classrooms in the same alternative education school. The literature review is organized into seven sections: (a) exclusionary discipline, (b) alternative education, (c) urban education, (d) views of learning, (e) critical social practice theory of learning, (f) critical microethnography; (g) and gaps in the research. A summary concludes the chapter.

\section{Exclusionary Discipline}

This section discusses how criminalization of African American males, race and class privilege, and zero tolerance policies impact exclusionary discipline practices. Exclusionary discipline practices, which include the referral of problem students out of the classroom and their subsequent suspension and expulsion to an alternative education school, disrupt the learning processes in thousands of U.S. urban classrooms (Aronson, 1995; Educational Testing Service Policy Information Center [ETSPIC], 2005; Fine, 1990, 1991; Wolford, 2000). School discipline data suggest that the dropout problem may stem from favoritism or deep-seated prejudice in school discipline practices beginning with teachers' beliefs and actions in the classroom (Skiba et al., 2000). A synopsis of 
research findings on the discipline gap and school-to-prison pipeline in which three conditions were identified that contribute to systematic bias at the classroom level is provided: (a) criminalization of African American males; (b) race and class privilege; and (c) zero tolerance policies (Monroe, 2005).

\section{Criminalization of African American Males}

Since Rist's (1970) study about the relationship between teacher expectations and student achievement, research has consistently documented teachers' perceptions and expectations of students as a key factor in decision making in the classroom (Noguera, 2003). If teachers harbor negative perceptions of African American students from the media, for example, they may implicitly and disproportionately distribute disciplinary consequences to keep control in the classroom. Teachers may not explicitly connect diversity awareness education, stereotypical perceptions, and discipline in the classroom.

African American behaviors are more often misconstrued as misbehavior and contribute to disciplinary action and the discipline gap (Monroe, 2006). Teachers who misunderstand students' cultural goals will refer Black boys more often to the office for misbehavior. "In the area of the discipline gap, teachers are subsequently encouraged to shift their thinking from 'Why can't Black boys behave themselves?' to 'How can my teaching and classroom ecology support Black male success?"” (p. 105)

For example, in an ethnographic study examining the discipline policies of two schools and a prison, Casella (2003) observed and interviewed school and prison staff, parents, and students. Results suggest that breaking of school rules was used as reasons for labeling and removing students. Students reported in interviews that it was very 
difficult to return to school once they had been removed. Suspension and expulsion occurred, and reinforced the school-to-prison pipeline.

\section{Race and Class Privilege}

Race and class privilege are intertwined with the administration of disciplinary consequences distributed by middle-class teachers and school leaders who lack experience with diversity in their professional and personal circles (Monroe, 2005). Disrespect, disobedience, disorderly conduct, and fighting are the most commonly reported reasons that teachers write referrals to the office (Skiba, 2001). However, studies comparing cultural interaction styles show African American behaviors, regularly referred as misbehaviors, were not intended as such (Weinstein et al., 2003, 2004). For example, cultural humor, play fighting, and overlapping speech were viewed as disrespectful and/or disorderly. African American cultural humor and discourse "play with" or "play on" words by using repetition, dramatic flair, "creative use of word patterns, and an overall playfulness in language usage" (Irvine, 1990, p. 101). Results imply that narrow views of community-based field experiences for teacher education students and African American students will perpetuate culturally irrelevant approaches to teaching, learning, and ultimately discipline in the classroom.

Within the context of the school-to-prison pipeline theme, school discipline data and juvenile justice data document the disproportional representation of nondominant students. Ethnographic studies and interviews as well as content analysis of disciplinary practices and procedures have been used to examine the impact of disproportionate punitive exclusionary discipline on students of color in low-income communities (Fenning \& Rose, 2007). For example, Fenning and Rose (2007) reviewed the literature 
to examine possible explanations for school discipline policies and for how and why teachers handle what they perceive to be discipline problems. Results indicated that disproportion of subgroups seen as problematic, particularly African American students, may be explained by the perception of teachers that these students do not fit the school norm because of "race, academic problems, and SES" (p. 537). Further, they found that this belief, augmented by federal mandates for all children to achieve specific academic standards and the fear of losing control in the classroom, leads to the notion that certain students are troublemakers and dangerous. Rose and Fenning (2007) conclude that the focus of alternative hypotheses based on internal factors (i.e., students' characteristics) resulting from teachers' beliefs need to shift to exploration of school and classroom level discipline policies and procedures.

\section{Zero Tolerance Policies}

Zero tolerance policies have been adopted by $94 \%$ of U.S. public schools even though not one of the five panels of experts on school violence recommended zero tolerance or school security as a best practice in reducing violence (Skiba \& Leone, 2001). In Skiba and Leone's (2001) report analyzing current education policies, procedures, and practices related to inequities resulting from student racial profiling, disparities are a greater concern. The report suggests that less attention is paid to the effectiveness of working with families and communities than trying to address discipline in the classroom and not issuing referrals to the office based on preconceived notions of (mis)behavior. Related ethnographic research suggests that teachers' fear of loss of control, heightened by recent zero-tolerance policies and power relations in the classroom, contribute to the increase of classroom clashes and suspensions. For example, 
Vavrus and Cole (2002) explored interactions in a classroom from the framework of suspension as a social practice. Data of interactions in two freshman high school science classrooms related to suspension events were documented with videorecorded observations, interviews of all stakeholders, and researcher field notes. The school was characterized by its large minority enrollment and its zero-tolerance of violence policies. Results revealed that suspensions were often issued by the teacher for unwritten or unspoken violations of classroom conduct codes after multiple disciplinary moments. Teachers often singled out particular students for exclusionary discipline based on their perception of loss of control rather than the occurrence of actual violent behavior (Noguera, 2003). Research is needed at the classroom level to examine the connection between teachers' perceptions of their sense of agency in making disciplinary decisions that may significantly reduce suspensions and expulsions and which contribute to the discipline gap and school-to-prison pipeline cycle (Monroe, 2005).

Alternative Education

This section covers two aspects of alternative education discussed in the literature: (a) legislative history, and (b) program characteristics and effectiveness.

\section{Legislative History}

The legislation of separate alternative education facilities occurred with The Delinquency Prevention through Alternative Education Initiative of 1974 through the enactment of the Juvenile Justice and Delinquency Prevention (JJDP) Act (JJDP, 1974, 2002; Office of Justice Programs, 2008). Subsequent legislative enactments, requirements, and partnerships have maintained, redefined, and contributed to alternative education program and school growth over the years. 
Current legislative requirements for reducing antisocial behavior and increasing low academic achievement are intertwined in The No Child Left Behind Act (NCLB) of 2001 (NCLB, 2002). NCLB is a federal effort toward accountability and universal student achievement of state-established standards. For example, The Individuals with Disabilities Education Act (IDEA) of 1997, reauthorized in 2004 (IDEA, 2004; USDOE, 2006) includes regulations related to discipline. NCLB also reauthorized and amended The Safe and Drug-Free Schools and Communities Act of 2004 as Part A of Title IV of $21^{\text {st }}$ Century Schools, the

federal government's primary initiative to prevent drug abuse and violence in and around schools. Through the program, state educational agencies, local educational agencies, and outlying areas are awarded grants ... to create programs deterring drug abuse and violence in and around schools (Cooper, 2005).

Partnerships between the U. S. Department of Justice and alternative education programs have become numerous with the recent rise in school safety concerns and legislative requirements for all schools to increase low academic achievement (NCLB, 2002; OJP, 2007). Partnerships attempt to ensure academic progress and the reintegration of youth into the community to prevent future antisocial behavior (Van Acker, 2007).

In an effort to meet federal accountability, universal student academic achievement, youths' social needs, and discipline requirements, education and juvenile justice education programs increasingly collaborate to provide comprehensive services authorized by legislation (OJJDP, 2009). Comprehensive services often provide the removal of youth from ineffective influences and their transfer to a safe alternative education setting that addresses academic, behavioral, and disciplinary needs (Van Acker, 2007). However, research indicates that youth who spend much of their school 
time in alternative education programs may develop failure-oriented and juvenile delinquent identities (Aronson, 1995; Foucault, 1977; Watts \& Erevelles, 2004). Moreover, segregating youth who are at risk of academic failure and dropping out of school results in their alienation (Blanchett, 2006; King, 2005; Nasir \& Hand, 2006).

Nonetheless, students in U.S. schools are widely sorted (i.e., tracked) according to their social and academic (usually reading or literacy) achievement (Fine, 1990, 1991; Oakes, 1985). A recently proposed reauthorization, Building On Results: A Blueprint for Strengthening the No Child Left Behind Act, promises funding, rewards, and tools to schools and teachers to ensure America's students read at grade level by 2014 (U. S. Department of Education [USDOE], 2007). Students in the successful track are lauded for making good grades, graduating from high school, attending college, and landing better jobs (Oakes et al., 2006). Penalties, such as failure to be promoted to the next grade or to graduate from high school are issued to academically unsuccessful students (Blanchett, 2006; Nasir \& Hand, 2006). This divisive phenomenon known as "the achievement gap" has become broadly accepted in our society (Ladson-Billings, 2005). Additionally, students on the failing track who are deemed antisocial are further penalized by being separated from their regular classrooms and schools into alternative education settings and the juvenile justice educational system (OJJDP, 2009).

A paradigm shift from a disciplinary to a more balanced approach to prevention and intervention is suggested in recent alternative education and juvenile justice education literature (Platt, Casey, \& Faessel, 2006; OJP, 2007). However, rather than getting at the root causes of antisocial behavior, the research, which is minimal in scope, 
largely describes program characteristics and effectiveness (OJJDP, 2009; Smink \& Schargel, 2004; Snyder \& Sickmund, 2006).

\section{Program Characteristics and Effectiveness}

Characteristics and effectiveness of alternative education programs for at-risk and disruptive students were developed in Raywid's (1994) study, resulting in three commonly cited descriptive categories: (a) Type I—schools of choice; (b) Type II—last option before expulsion programs; and (c) Type III—rehabilitation/ remediation programs for possible return to the traditional school. To date, characteristics and effectiveness of alternative education programs remain unclear, but most are site-based, have limited academic enhancement supports, use general and vocational education curriculum, and offer community activities (Foley \& Pang, 2006; Van Acker, 2007). For example, the Florida Department of Education Bureau of Family and Community Outreach provides seven types of dropout prevention programs for its 1.8 million youth of which 55\% are White, 20\% Latino, and 22\% Black (OJJDP, 2009). Programs include: (a) educational alternative; (b) teenage parent; (c) disciplinary; (d) truancy; (e) GED Exit Option; (f) mentoring; and (g) educational services. Within the Florida Department of Juvenile Justice (FDJJ) programs operate under the Bureau of Exceptional Education and Student Services (Florida Department of Education [FDOE], 2006, 2007). FDJJ also provides multiple prevention, intervention, detention, and treatment programs (FDJJ, 2007; OJJDP, 2009).

The effectiveness of alternative education and juvenile justice education programs is determined by their use of best practices and instructional approaches (OJJDP, 2009). Best practices rely on expert opinion and generalizations drawn from what works in 
successful alternative education and juvenile justice settings. What works and best practices are often referred to as effective dropout prevention strategies (OJJDP, 2009). Frequently cited best practices are (a) small, student-centered learning environments with career-training opportunities; (b) cooperative, engaging curricula; (c) caring, supportive teachers who provide a sense of community in the classroom; (d) involvement with community members; and (e) site-based management with strong teacher contribution (Smink \& Schargel, 2004). Evidence-based programs are developed to offer a continuum of youth services to assist juvenile justice practitioners, administrators, and researchers in offering effective instructional approaches, enhancing accountability and public safety, and reducing recidivism (OJJDP, 2009).

Effective instructional approaches for delinquent and antisocial youth are described in the literature as nontraditional, motivational, immediately responsive, and understanding of social factors related to dropping out (Blomberg \& Waldo, 2001; OJJDP, 2009). Examples include active learning strategies which incorporate students' learning styles and multiple intelligences into cooperative, positive interdependence, and project-based learning (Foster \& Shirley, 2004). However, few research studies explain how these results were reached or how the practices were accomplished. Moreover, statistics pertaining to delinquent and antisocial students continually report poor social and academic achievement (especially in reading), segregated classrooms and schools, and disproportionate dropout rates (NCES, 2008).

\section{Urban Education}

This section covers urban education literature related to (a) school, teacher, and student characteristics; (b) cultural synchronization, (c) culturally responsive pedagogy, 
and (d) culturally responsive classroom management. Urban education literature is useful for studying the implied link between school discipline, achievement, culture, teaching practices and the focus on reducing classroom conflicts and disproportional representation of African American students in the achievement gap (Delpit, 1995).

\section{School, Teacher, and Student Characteristics}

In urban education literature, urban (as opposed to suburban or rural) contexts are defined as

heavily populated with students of color . . large number of students from lower socioeconomic status, high attrition of teachers, heavy institutional and systemic barriers, and meager resources . ... grossly underfunded, larger in size, and infiltrated with administrative [local to federal] bureaucracy. (Milner, 2006, p. 493)

Urban schools are disproportionately populated with students of color and mostly White, middle class, and female teachers (Milner, 2006). Twenty-five percent of the entire nation's population of 72.8 million people is youth, young people between the ages of 13 and 18 (OJJDP, 2009). Approximately 60\% of all youth are White, 20\% are Latino/a, and $16.4 \%$ are Black. From the total population of public school teachers in the United States, 664,025 out of 772,460 are White, middle class, and female (NCES, 2005; National Partnership for Teaching in At-Risk Schools [NPTARS], 2005).

Most of the total population of public school teachers have had limited or no prior experiences with students from culturally diverse backgrounds (Hollins \& Guzman, 2005). In urban contexts, teachers' heightened concern with disruptive behavior often takes precedence over learning. Even though some researchers challenge the idea that people with "common ethnic and socioeconomic backgrounds share culturally shared perceptions of the world" (Fránquiz \& Salazar, 2004, p. 39), urban education literature 
(Gutiérrez, 2002, 2008; Lee, 2005; Milner, 2006; Monroe \& Obidah, 2004; Noguera, 2003; Skiba, 2001; Vavrus \& Cole, 2002) concludes that teachers in urban schools often struggle to manage their classrooms because of mismatches in cultural backgrounds and misunderstandings or conflicting expectations about appropriate classroom behavior, or lack of cultural synchronization (i.e., cultural incongruence).

\section{Cultural Synchronization}

This section includes literature related to cultural synchronization (a) theory (Gay, 2000; Irvine, 2000) and (b) research, which provides a base for understanding and reducing cultural incongruence in the classroom.

\section{Theory}

In an anthropological classroom theory of cultural synchronization, the role of culture in improving student academic success is recognized (Irvine, 1990). Cultural synchronization theory is rooted in Afrocentricity, which recognizes the central role and explanatory value of African culture in African American life and behavior (Asante, 1988; Hilliard, 1983, 1995; Irvine, 1990, 2003). In cultural synchronization theory, culture is defined as the "integrated pattern of human behaviors that include thoughts, communication, action, customs, beliefs, values, and instructions of a racial, ethnic, religious, or social group" (Day-Vines \& Day-Hairston, 2005, p. 237). Hall (1966) states that culture "obviously affects attitudes (p. 58) .... because people cannot act or interact in any meaningful way except through the medium of culture" (p. 188). In the various contexts of daily life, human beings respond to problems based on expectations from the dominant cultural group in society. Conflicting responses between cultural groups may cause miscommunication and misunderstanding based on cultural incongruence. 
Cultural synchronization theory is grounded in "anthropological and historical research that advances the finding that black Americans have a distinct culture founded on identifiable norms, language, behaviors, and attitudes from Africa" (Irvine, 1990, p. 23). This distinct African culture is incongruous and contradictory to European culture (Hilliard, 1983). Acknowledging the retention of a distinct rhythmic African culture in America helps to alleviate the myth that Blacks have no culture and are culturally, genetically, and psychologically deficient in respect to European culture and, thus, inferior to Whites.

Research (Hilliard, 1995; Larson \& Ovando, 2001) confirms the retention of identifiable and distinct African culture in America, especially in "lower-class Black communities where racial isolation persists and assimilation into the majority culture is minimal" (Irvine, 1990, p. 24). Many African Americans must respond to the triple quandary of living in the African-based, mainstream Eurocentric, and oppressed minority cultures at once (Boykin, 1986). In a comparison of African and European cultures, Boykin (1986) characterized African culture as rhythmic with highly charged style, vernacular, and feeling. African culture prioritizes spiritualism, harmony with nature, organic metaphors, expressive movement, interconnectedness, affect, events, oral communication, expressive individualism, and uniqueness. On the other hand, European culture, characterized by a linear and reserved style, values materialism, mastery over nature, mechanistic metaphors, impulse control, separateness, reason, time, print communication, possessive individualism, and sameness (Boykin, 1986).

Cultural synchronization in the classroom is evident when the teacher and student communicate with and understand each other's cultural orientations in a way that the 
classroom runs smoothly (Irvine, 1990). Lack of cultural synchronization in the classroom is apparent when a mismatch between the teacher's and students' cultures results in misunderstandings and miscommunications (Delpit, 1995). Students who lack cultural capital, or knowledge and initiative to assimilate into the classroom culture, have a greater chance of failing, dropping out, and involvement in the juvenile justice system.

Cultural synchronization in the classroom depends on culturally congruent expectations and responses among teachers and students (Irvine, 1990). School culture often collides with minority students' cultural traits. Culturally incongruent responses or behavior in the classroom may be interpreted as misbehavior by teachers. African American students' culture is different and often devalued or ignored in American schools and classrooms (Hilliard, 2003; King, 2005), typically grounded in Eurocentric paradigms (Howard, 1999) and taught by mostly White teachers (NPTARS, 2005). As a result, African American or other nondominant students and their mostly White teachers experience cultural incongruence, or lack of cultural synchronization, in the classroom. When teacher-student expectations and responses are out of cultural sync, they clash and conflict with each other (Delpit, 1995). Two seminal studies provide a link between teachers' expectations and cultural synchronization with academic performance (Rist, 1970; Rosenthal \& Jacobson, 1968).

Research

An experimental study that popularized the self-fulfilling prophecy concept is important for linking teachers' expectations and academic performance. The study was conducted in an elementary school of lower socioeconomic status (Rosenthal \& Jacobson, 1968). A sample of 18 first- to sixth-grade students was given a test of general 
ability. Then $20 \%$ of this sample of 18 students were randomly selected. Teachers were told that the randomly selected group of students was identified as academic late bloomers who would make the largest academic gains during the school year. The posttest results indicated large gains in reading for the academic late bloomers.

Additionally, teachers' perceptions and expectations of the academic late bloomers were more positive than for the control group. Results indicated that teachers' expectations and thus performance of the children were raised by the treatment suggestion that some students were academic late bloomers.

Rist (1970) conducted a longitudinal observational study of one group of Black children from kindergarten to second grade. By the eighth day of school in kindergarten, the teacher had already divided the children into three ability groups and permanent seating assignments. Children placed at the table closest to the teacher were well-dressed, clean, verbally interactive, and believed to be smarter by the teacher. Children placed at tables further away from the teacher were darker skinned, poorly dressed, less clean, and treated disrespectfully by the teacher and eventually by each other. Group assignments were repeated the following 2 years of school perpetuating what Rist (1970) referred to as a caste system of students' academic achievement based on the teacher's expectations. Irvine (1990) and Delpit (1995) provide a research base for both reducing the negative effects of a lack of cultural synchronization and increasing synchronicity. Irvine (1990) conducted an experimental study to examine the effects of student race, gender, and grade level on teacher-student communication interactions. That study addressed inconclusive results in teacher expectation research regarding how teacher verbal feedback varied by student race and gender. Three trained observers collected data from 
63 K-5 classrooms in 10 schools in four Southeastern U.S. public schools. Teachers' verbal feedback and student initiating behaviors and opportunities to respond were extensively noted on an instrument that provided quantitative and qualitative data.

The statistically analyzed data indicated that race and gender were complicated influencing factors in teacher-student communication interactions. The significance of the findings was that teachers formed achievement expectations within the first 2 weeks of school that were unchanging and often incorrect, especially about Black males. “Teachers' expectations for black males' achievement are more influenced by their stereotypes of black males as potential disruptors than by their academic ability" (Irvine, 1990, p. 77). Conclusions indicated that successful teachers proactively extended students' home lives into the classroom. Also, the lack of cultural synchronization was evident in "instructional situations in which teachers misinterpret, denigrate, and dismiss black students' language, nonverbal cues, physical movements, learning styles, cognitive approaches, and worldview" (Irvine, 1990, p. xix).

In Other People's Children: Cultural Conflict in the Classroom, Delpit (1995) incorporates conclusions from seminal research pertaining to cultural synchronization. Research was conducted in Papua New Guinea about how teaching in the mother tongue influenced "school-community relations, academic success, cultural change, and literacy development” (p. 78). Results from ethnographic interviews with parents and teachers demonstrated the academic and social value of prioritizing students' home language and cultural values in school. Delpit also recounts the results of interviews with teacher education students she instructed in Alaska. Results indicated the importance of not only 
validating students' experiences, contexts, and cultural values, but of teaching students how to learn in the dominant culture.

Delpit (1995) concludes that teachers need to explicitly teach other people's children how to participate in the culture of power in order for them to be successful in school. "If you are not already a participant in the culture of power, being told explicitly the rules of that culture makes acquiring power easier" (p. 25). Since we live in the "culture of power" (p. 25), "issues of power are enacted in the classroom ... through the power of the teacher over the student" (p. 24). Using teaching strategies that ignore students' linguistic and cultural strengths does not teach children what they need to know or build on what they already know. Rules for participating in power "relate to linguistic forms, communicative strategies, and presentation of self; that is, ways of talking, ways of writing, ways of dressing, and ways of interacting” (p. 25). Delpit's (1995) research has sparked multiple classroom interventions linking teachers' expectations, culturally responsive pedagogy, and academic achievement for culturally diverse students (Ballenger, 1999; Banks et al., 2005; Cochran-Smith \& Zeichner, 2005; Gay, 2000; Hollins \& Guzman, 2005; Ware, 2006).

\section{Culturally Responsive Pedagogy}

This section covers anthropological classroom literature related to culturally responsive pedagogy (a) theory and (b) research, which provides a base for understanding and reducing cultural incongruence (Delpit, 1995; Ladson-Billings, 1994).

\section{Theory}

In culturally responsive pedagogy theory, retaining students' cultural integrity during teaching and learning to achieve academic success is supported (Gay, 2000; 
Howard, 2001). Culturally responsive pedagogy theory is cited as a successful framework on which effective teachers of African American students base their classroom practices (Banks, 2001; Gay, 2000; Monroe \& Obidah, 2004). Teachers who practice culturally responsive pedagogy become familiar with their students' cultures, discern their students' strengths, and build on the strengths that students bring to school (Cochran-Smith \& Zeichner, 2005; Darling-Hammond \& Bransford, 2005). Culturally responsive teachers arbitrate on their students' behalf by working to prevent cultural conflicts in the classroom. Fewer conflicts in the classroom decrease students' chances of being removed from class and ultimately marginalized in the system due to lack of cultural capital (Delpit, 1995). Cultural capital is the ability to successfully navigate expected cultural values including those of the powerful in society (Anyon, 1981, 2005; Delpit, 1995).

Theoretical underpinnings of culturally responsive pedagogy (Ladson-Billings, 1994) are comprised of the (a) belief that all students are capable of academic success, (b) development of a community of learners, and (c) notion that knowledge is about doing. Additionally, the effect of students' cultural heritages on their "dispositions, attitudes, and approaches to learning" (Gay, 2000, p. 29) is acknowledged. Culturally responsive pedagogy occurs when the teacher is purposefully responsive to students' cultures, learning styles, knowledge, and histories (Ware, 2006). Culturally responsive teachers warmly demand student engagement and participation in academic learning to increase their social and political awareness inside and outside of the classroom (Milner, 2006). Research

The overall context of the culturally responsive education experience includes "ways of communicating, conceptions of knowledge, and methods of learning" (Gay, 
2000, p. 136) that support students' cultural backgrounds and needs. Culturally responsive teachers routinely (a) use multicultural content in all curricula, subjects, and skills taught; (b) build meaningful bridges between home and school experiences; (c) use multiple instructional strategies for different learning styles; and (d) teach students to know and value their own and others' cultural heritages (Gay, 2000). Research provides a link between teachers' beliefs and cultural responsive pedagogy with academic achievement (Gay, 2000; Ladson-Billings, 1994).

To investigate what teachers need to know to address cultural conflicts, researchers have examined the influence of teachers' backgrounds, beliefs, and experiences upon their teaching (Cochran-Smith \& Zeichner, 2005; Darling-Hammond \& Bransford, 2005). Conclusions reached are that teachers must address cultural conflicts by aligning students' home discourses with school discourses to maintain a respectful, orderly environment and achieve successful academic achievement (Delpit, 1995). However, how teachers develop the mindset to become culturally responsive educators has been overlooked in the literature (Cochran-Smith, 2005; Hollins \& Guzman, 2005). In a seminal ethnographic study, Ladson-Billings (1994) documented the "practice of highly effective teachers of African American students" (p. 145). The community nominated effective teachers who were asked to agree to participate. Data were collected through interviews, classroom observations and videorecordings. Data analysis consisted of collective interpretations among the teachers and researcher of transcribed interviews and videorecordings. Conceptualized within Afrocentric feminist epistemology, findings showed that culturally responsive teachers see color and culture and view (a) themselves and others with high regard; (b) all students as people who can 
succeed; (c) it as their duty to help students connect between themselves, their community, and national/world identities; (d) teaching as gathering knowledge from their students in a community of learners; (e) the teacher-student relationship as equitable and a way to connect with their students; (f) knowledge as something to be passionately, critically "re-created, re-cycled, and shared" (p. 81) rather than told; (g) it a requirement to help students develop necessary skills; and (h) excellence as a complex standard that takes cultural diversity and individual learning styles into account.

In Culturally Responsive Teaching, Gay (2000) "incorporates research, theory, and practice about culturally contextualized or mediated teaching for marginalized" (p. xix) culturally and ethnically diverse students from national data and well-known research over the past three decades. Conclusions from her extensive review of the research indicate that culturally responsive teaching is validating, comprehensive, multidimensional, empowering, transformative, and emancipatory. Also, culturally responsive teachers understand concepts of power, caring, communication, and cultural congruity to support culturally and ethnically diverse students' classroom achievement.

Culturally responsive educators have also been referred to as warm demanders (Monroe, 2006) who conceive of caring as accepting nothing less than high academic achievement rather than gently nurturing African American students to learn at their own pace. These three views of culturally responsive educators suggest that a particular frame of mind is necessary for teaching all students effectively. The consensus is that a culturally responsive frame of mind empowers the educator to intuitively enact a classroom culture based upon judgments that foster student resilience (Bondy, Ross, Gallingane, \& Hambacher, 2007). 
By developing caring relationships (Noddings, 2007), setting clear high academic and behavioral expectations, and providing meaningful learning opportunities, culturally responsive educators become "critical protective factors for fostering resilience" (p. 330). Moreover, culturally responsive educators also feel it is their duty to provide an equitable, socially just classroom culture as a microcosm of an equitable, socially just global society. Even though insinuated, the relationships among cultural synchronization, culturally responsive pedagogy, and discipline are rarely explicitly linked in the research.

\section{Culturally Responsive Classroom Management}

This section covers (a) effective classroom management; (b) culturally responsive classroom management research; (c) elements of culturally responsive management; and (d) culturally responsive classroom managers. Culturally responsive classroom management (CRCM) literature implicitly links cultural synchronization, culturally responsive pedagogy, and discipline.

\section{Effective Classroom Management}

How teachers establish expectations for behavior and what classroom management principles guide their teaching were first examined by Emmer, Evertson, and Anderson (1980). Based on extensive observations conducted during the first week of school, 27 new and experienced teachers from middle- to low-socioeconomic status schools were divided into effective and less effective classroom managers. Narrative and descriptive data were collected by multiple observers trained to gather several types of information during the first 3 weeks and used to compare the two groups of teachers. Data were quantitatively and qualitatively analyzed. 
Results from frequency counts, ratings, and narrative descriptions showed significant differences in initial behavior in "classroom rules and procedures, monitoring of pupils, and delivery of consequences" (Emmer et al., 1980, p. 224). Effective classroom managers initially integrated, discussed, practiced, and helped students learn classroom rules and procedures more than less effective managers. Maximum contact for the first day was planned and monitored through interesting activities for the whole group and continued throughout most of the first week. Establishing themselves as the classroom leader was accomplished early on by effective managers who purposely provided well-planned procedures for students to learn how to socialize in their particular classroom set the tone for the rest of the year.

In addition to facilitating effective behavioral management, effective classroom managers also exhibited better instructional management; purposefully accommodated student concerns; more effectively planned how to deal with constraints in room arrangement; and portrayed warm, listening, and predictable personal characteristics when interacting with their students (Emmer et al., 1980). Causal relationships were not drawn from the results, but certain conclusions were reached. Effective managers were more proactive and cognizant of students in ways that promoted cooperation and prevention of discipline problems in the first place. Emmer and colleague's (1980) study brought to researchers' attention the importance of studying classroom management principles as well as teachers' beginning of the year activities related to organization, communication, monitoring, and management. Effective classroom management literature concludes that teachers who are effective classroom managers can engage their students in learning (Evertson \& Weinstein, 2006). 


\section{Culturally Responsive Classroom Management Research}

Research on culturally responsive classroom management is derived from what researchers know about effective classroom management principles (Evertson \& Weinstein, 2006) and the art and the science of using culturally responsive pedagogy (Bondy et al., 2007). Culturally responsive pedagogy merges effective teaching standards with a focus on culture (Ware, 2006). Drawing from the large body of culturally responsive pedagogy research, some researchers have studied culturally responsive classroom organization and management tasks related to discipline (Weinstein et al., 2003). Results indicate that culturally responsive classroom management (CRCM) requires a different type of teacher. Teachers who are culturally responsive classroom managers (a) have a culturally responsive frame of mind; (b) practice culturally responsive classroom strategies; (c) organize and manage their classroom differently than generally effective classroom managers; and (d) develop different knowledge, dispositions, and skills than those found in the general classroom management literature. Monroe and Obidah (2004) used a case study approach to determine how and to what extent cultural synchronization influenced an African American teacher's responses to disruptive behavior. Interactions between a self-reported effective African American teacher and her students were examined. Data consisted of interviews, observations, and researcher field notes. Disciplinary actions were coded based on teacher-espoused practices and patterns that emerged from the data. Results indicated that the teacher used culturally responsive classroom management practices of cultural humor, and affect and emotion. 
Unique patterns of cultural humor used by students were reflected by the teacher to regulate student behavior. For example, prior to a pep rally, the teacher asked her students to announce beforehand that they were planning to "act the fool at the pep rally" (Monroe \& Obidah, 2004, p. 263) so she could go ahead and send them wherever they needed to go. In response, the class laughed and began to call out various comments as the teacher discussed her expectations about how to behave at the pep rally—continually promoting and affirming solidarity with the students.

Affect and emotion in the form of warm demands were displayed by the teacher when disciplining students on a rare occasion. For instance, when the teacher noticed one student intimidating or physically threatening another, she stood up and said loudly, "SHOW YOURSELF NOW! Dana." (Monroe \& Obidah, 2004, p. 264). The class got quiet. The teacher told Dana to wait for her on the sofa. After completing what she had been doing with a small group of students, the teacher took Dana into the hallway to reprimand her. The reprimand included a parent-like reminder of behavioral expectations and a request for Dana to go home and tell her parents how she acted like a fool.

Even though effective classroom management and discipline are top teacher concerns, they are not addressed in professional development and teacher education within the context of culture (Monroe \& Obidah, 2004). The researchers recommend that given the clear connection in the literature between "ethnicity, gender, and school discipline...culturally responsive disciplinary practices may be an effective way to manage African American students' behavior" (p. 266). They also suggest expanding on teachers' and including students' perceptions of effective classroom management for culturally responsive classrooms. 
Ware (2006) conducted a comparative case study to explore whether the practices and beliefs of two African American urban teachers reflected the elements of culturally responsive teachers in the literature. Descriptive data were collected through interviews and classroom observations. Codes from existing literature as well as categories that emerged from the data were used to analyze data. Codes depicting common traits of exemplary African American teachers from the culturally responsive pedagogy literature were (a) ethic of caring; (b) beliefs about students, teachers, parents, and community; and (c) instructional practices (Ware, 2006). These categories collapsed in the emerging codes that described teachers as warm demanders, a term illustrative of culturally responsive teacher traits, beliefs, and practices. Results indicated that as warm demanders, the teachers interacted with African American males (a) as authority figures and disciplinarians; (b) with special nurturing; (c) as caregivers; (d) with dedication to students' needs; (e) with other-mothering (not discussed in relation to fathering); (f) with culturally responsive pedagogy; and (g) with high expectations and beliefs about students no matter their limited literacy proficiency. Overall, a culture of achievement was created by the teachers for their African American students.

In another study, Brown $(2003,2004)$ interviewed 13 urban teachers to determine whether their self-professed effective classroom management strategies with culturally diverse students reflected culturally responsive pedagogy. Analysis of the data revealed five themes for culturally responsive classroom management. First, teachers developed personal relationships and mutual respect through individualized attention. Teachers created caring learning communities. They established business-like learning environments. They established congruent communication processes. Finally, teachers 
were assertive and clear about their expectations. Overall, these effective urban teachers acted as coaches who assumed responsibility for their students' academic achievement rather than custodians who watched over their students (Ladson-Billings, 1994).

Brown's $(2003,2004)$ study explicitly examined the connection between teachers' professed practices and elements of culturally responsive pedagogy, an important first step in defining the characteristics of CRCM (Bondy et al., 2007). However, observations, which would have provided a more thorough explanation of how teachers' professed practices related to their actual practices, were not conducted. Also, students' perspectives would have added another dimension to the interpretations.

\section{Elements of Culturally Responsive Classroom Management}

From an extensive review of the literature, Weinstein et al., (2004) propose a conception of CRCM which includes five essential elements:

(a) recognition of one's own ethnocentrism; (b) knowledge of students' cultural backgrounds; (c) understanding of the broader social, economic, and political context; (d) ability and willingness to use culturally appropriate management strategies; and (e) commitment to building caring classrooms. (p. 25)

Recognition of one's own ethnocentrism. Recognizing one's own ethnocentrism entails exploring the concept of Whiteness and privilege in an historical context (McIntosh, 1997). The examination of "taken-for-granted assumptions of a western, White, middle-class worldview, such as an emphasis on individual achievement, independence, and efficiency" (p. 29) is the focal point of culturally responsive teaching and missing in teacher preparation (Ballenger, 1999; Howard, 1999). Teachers who are culturally responsive classroom managers recognize and interrogate how their own ethnocentrism, or biases and values, relates to their interactions with students (Sleeter, 
2005). For instance, they may ask themselves whether they expect African Americans to be more disruptive than students from other cultures.

Based on recognition of their own ethnocentrism, teachers who practice CRCM strategies understand that appropriate classroom behaviors are culturally defined. This often means the "troublemaker" is looked on differently by the teacher, and discipline problems are proactively and effectively dealt with in the classroom. Teachers who practice CRCM purposely become knowledgeable about their students' cultures and communities, and they value the ways of speaking and interacting of their students. They also develop knowledge of their students' cultural backgrounds and needs and build caring classroom communities through positive social relations (Weinstein at al., 2004). Teachers who practice CRCM also realize that schools norms are based in White middleclass values through their dominance in society rather than an innate superiority. These teachers explicitly teach students how to succeed in the White middle-class "culture of power" (Delpit, 1995, p. 25).

The importance of "using culturally responsive curricular materials and instructional processes" (Brown, 2004, p. 273) has been documented somewhat less from students' perspectives. From available data, students report that the teachers' ability to manage students' behavior in an appropriate learning environment occurs through their caring attitude (Howard, 2001; Ladson-Billings, 1994), assertiveness and authority (Delpit, 1995), congruent communication processes (Gay, 2000), and demand of effort from their students. For example, students' perspectives and interpretations on culturally relevant teaching strategies conducted by four teachers in four urban elementary schools were examined (Howard, 2001). Data were collected through observations and 
interviews. Results showed that students preferred teachers who enacted caring attitudes, established community- and family-type classroom environments, and made learning fun (Howard, 2001).

Knowledge of students' cultural backgrounds. Developing knowledge of students' cultural backgrounds is a prerequisite for being able to develop cross-cultural interaction skills and achieve cultural synchronization in the classroom. Consulting with family and community members; making home visits; reading books about students' perspectives on school; and asking “questions about students' backgrounds, educational experiences, and cultural norms and values" (Weinstein et al., 2004, p. 30) are suggested ways of developing the cultural knowledge and, thus, the cross-cultural interaction skills needed to manage culturally diverse classrooms effectively.

Understanding of the broader social, economic, and political context. Awareness of the broader social, economic, and political context of teaching and learning accompanies awareness of how discriminatory practices and prejudice are normalized in our institutions through power (Freire \& Macedo, 1987). This awareness has implications for classroom management in how African American males are disciplined, profiled as troublemakers, and issued consequences unequal to the behavior (Wald \& Losen, 2003). Caring teachers will purposely listen to students, critically reflect, and provide spaces for students' voices and agency in the classroom to prevent discipline problems (Noddings, 2007).

Ability and willingness to use culturally appropriate management strategies. Ability and willingness to use culturally appropriate management strategies is part of the ongoing process of viewing behavior through the lens of cultural diversity (Weinstein et 
al., 2004). Culturally responsive teachers enact a social justice philosophy with the primary goal of providing all students the opportunity to learn rather than maintaining control over the students (Weinstein et al., 2003). Subsequently, culturally responsive classroom managers incorporate culturally responsive pedagogical philosophy into their classroom organization and management plans and procedures by

(a) creating a physical setting that supports academic and social goals; (b) establishing expectations for behavior; (c) communicating with students in culturally consistent ways; (d) developing a caring classroom environment; (e) working with families; and (f) using appropriate interventions to assist students with behavior problems. (Weinstein et al., 2003, p. 270)

Three challenges for teachers to undertake CRCM are provided in the literature (Weinstein et al., 2003). One challenge is for teachers to monitor their own behavior in terms of how they treat students, such as recommending expulsion for some students and in-school suspension for others for the same behavior. A second challenge is questioning taken-for-granted best practices for handling discipline in the classroom and being cognizant of mismatches between traditional and culturally responsive classroom management (Ballenger, 1999). A third challenge is when to accept and build on the students' cultural backgrounds and when to provide cultural knowledge for students to be able to succeed in the culture of power is another challenge (Delpit, 1995).

Commitment to building caring classrooms. Being committed to building a caring classroom community can be observed in teachers' classroom management strategies by how they facilitate cooperation, listening, reciprocity, and high expectations (Morris \& Morris, 2002). Teacher education research has begun to acknowledge caring, but the emphasis remains on "linking the subject matter competence and pedagogical knowledge of teachers to student performance on standardized tests" (p. 121). 
Morris and Morris $(2000,2002)$ conducted a case study in a small town African American school community in Alabama to identify the factors that the community perceived as making the school a quality segregated school. Alumni, teacher, administrators, parents, and community members associated with public schools in the community when it was segregated from 1909 and 1980 responded to a questionnaire and interviews (Morris, 2002, p. 120). All data were inductively analyzed for emerging themes. In addition, primary and secondary historical documents during the time the school was open were collected to confirm emerging themes followed by triangulation of the data for the final analysis. Results identified three themes: (a) Teachers were caring, competent, and committed; (b) the school offered a range of activities and programs; and (c) parents and community members were involved and supportive.

In another study, Bondy and colleagues (2007) examined the practices of three effective novice teachers during the first 2 hours of the first day and during the first weeks of school in three urban classrooms. Data were collected using videorecorded observations and interviews, collaboratively analyzed using an inductive approach, and triangulated for themes. Results indicated the significance of the beginning minutes of the first day. Even though the teachers varied in their race and cultural styles in the classrooms, they used CRCM strategies to "establish a positive psychological environment that supported student resilience and achievement" (p. 324). Results demonstrated that the teachers developed relationships through a personal approach to teaching and creating a safe and caring place to learn; insisted on clear expectations; used a culturally responsive communication style including cultural humor and discourse; and established a caring, respectful business-like community of learners. 
The teachers created a classroom environment that would lead to success and resilience for students who typically struggle in school (Bondy et al., 2007). High expectations for success were established by teaching rules and procedures. Insistence and proactive strategies were used to hold students accountable for meeting the high expectations in the classroom by respectfully repeating requests and "calmly delivering consequences to ensure appropriate behavior" (p. 341). Culturally responsive communication styles included humor; terms of endearment; Discourse style with familiar words and expressions, popular culture, and call-and-response interaction patterns; and assertive, explicit instructions. Bondy et al. (2007) conclude:

Early classroom management research demonstrated the link between orderly environments and student learning .... Emerging literature on CRCM suggests that effective teachers must also be culturally knowledgeable, able to analyze the role of culture in their perceptions of student behavior, and able to use culture to create classroom contexts that support, nurture, and respect students. (p. 344)

CRCM literature links teachers' effectiveness and students' learning with their being able to create and manage culturally responsive classrooms.

\section{Culturally Responsive Classroom Managers}

Culturally responsive classroom managers have a particular mindset about students' cultural behaviors that appears to be helpful in minimizing discipline problems and other classroom conflicts. Research concerning "cultural considerations in interpreting student behavior" (Milner, 2006, p. 498) concludes that teachers who understand their own ethnocentrism, and their students' cultures, are less likely to misinterpret cultural behaviors for misbehavior (Delpit, 1995; Gay, 2000; Howard, 2001; Irvine, 1990). They use culturally responsive classroom management (CRCM) strategies, grounded in their students' cultural backgrounds and needs, to proactively handle 
potential discipline problems within the classroom (Weinstein, Tomlinson-Clarke, \& Curran, 2004). As a result, culturally responsive classroom managers appear to be less likely to struggle with disciplinary moments, which are a series of classroom conflicts leading to exclusionary discipline practices (Vavrus \& Cole, 2002; Wald \& Losen, 2003).

Promise for reducing exclusionary discipline practices resides in the mindset of culturally responsive classroom managers who first understand themselves, others, and the context in order to recognize their own and others' biases, differences, and "ways that schools reflect and perpetuate discriminatory practices of the larger society" (Weinstein et al., 2003, p. 270). However, few studies explore teachers' conceptions of effective classroom management (Weinstein et al., 2004) or their underlying beliefs about discipline (Monroe, 2005).

Teacher education research alludes to teachers' mindsets in the following way. The research indicates that U.S. teachers are predominantly White middle class females who intuitively draw upon years of personal backgrounds, beliefs, and experiences in school to make decisions for practice in their classrooms (Cochran-Smith \& Zeichner, 2005). Most teachers have developed negative perceptions of the backgrounds and abilities of students of color, operating from the mindset of needing to fix their struggling students' deficits (Darling-Hammond \& Bransford, 2005; Lee, 2005). Teachers generally express a lack of competence in developing good relationships and responsive curricula for students who are different from themselves (Cochran-Smith \& Fries, 2005).

Teacher education research also indicates that knowledge of technical teaching skills, pedagogy, and subject matter are necessary but insufficient for teachers to address conflicts in the classroom with cultural sensitivity (Banks et al., 2005). Teachers must 
also possess dispositions and knowledge of how to incorporate "the cultures, experiences, and needs of their students into their teaching" (p. 243) to provide opportunities for success in school. However, how to gain the necessary dispositions and knowledge are not specified in the research. "We know a great deal about effective teaching practices for diverse students, but not much about how teachers who are effective with diverse students acquired the skills, knowledge, and dispositions needed" (Hollins \& Guzman, 2005, p. 512). Exploring classroom interactions through critical microethnographic methods may fill this gap. Critical microethnography integrates what happens during classroom interactions with why it happens from the researcher's, the teacher's, and students' viewpoints.

The influence of cultural incongruence on teachers' abilities to work successfully with students who are caught up in disproportionate school disciplinary consequences is agreed upon (Monroe \& Obidah, 2004; Weinstein et al., 2003, 2004). The correlation between discipline and academic achievement deems it important to engage students in the classroom with quality instruction (Darling-Hammond \& Bransford, 2005). Research attributes overrepresentation of minorities in academic failure and disciplinary consequences to cultural incongruence between teachers and students (Day-Vines \& DayHairston, 2005). However, "few researchers have explored the concept in relation to the discipline gap" (Monroe, 2006, p. 108) or explicitly connected culture, discipline, and teaching practices (Monroe \& Obidah, 2004).

The cultural and linguistic diversity of our nation's students, and the predominance of White, middle-class, and English-speaking females in our teacher workforce and teacher education student population has been well documented (Cochran- 
Smith, 2006; Ladson-Billings, 2006; NCES, 2008). According to this research, since behavioral expectations are culturally influenced, and students and teachers typically come from different backgrounds, classroom conflicts are probable. Lack of multicultural competence will complicate teachers' classroom management. Even so, general research on classroom management rarely addresses issues of cultural diversity (Weinstein et al., 2004). Additionally, teacher education and multicultural education research focus on teaching and learning outcomes and culturally responsive pedagogy (Banks, 2001; Gay, 2000; Hollins \& Guzman, 2005; Irvine, 1990; Ladson-Billings, 1994) to the exclusion of classroom management (Evertson \& Weinstein, 2006).

Villegas and Lucas (2002) suggest the importance of preparing teachers to be culturally responsive in organizing and integrating curriculum and classroom management. Culturally responsive managers provide a classroom that prevents disorder where students behave because they feel responsible and part of the learning community (Weinstein et al., 2004). The overarching goal of culturally responsive classroom management is for students to want to cooperate and learn.

The connection among organizing the physical environment, clarifying teaching expectations for behavior, preventing conflicts and escalation of conflicts, and communicating with families has not been explored (Weinstein et al., 2003, 2004). Even though effective classroom management is a greater influence on students' academic achievement than intelligence, home environment, motivation, or socioeconomic status and has been demonstrated to prevent misbehavior (Gettinger \& Kohler, 2006), few have examined the link between culturally responsive classroom management, teachers' beliefs, and discipline. In order to study what happens and why in classrooms, the 
conventional view of learning that predominantly guides educational research can be replaced with a critical social practice view of learning.

\section{Views of Learning}

This section compares and contrasts a (a) conventional view and (b) critical social practice view of learning pertinent to this study.

\section{Conventional View}

A conventional view of learning is the perspective from which alternative education programs were initiated and are maintained. Based on a conventional perspective, alternative education programs are structured to be isolated and segregated places where nondominant students who have been historically and continuously failed academically by the mainstream educational system are sent to be disciplined or cured (Carpenter et al., 2006; Cassidy \& Bates, 2005; Delpit, 1995; King, 2005; LadsonBillings, 2006; Moje \& Lewis, 2007). Conventional views of learning purport transmission-style teaching, which gained hold in colonial times and prevails in U.S. schools today. Transmission-style teaching is characterized by lecturing, authoritarianism, competition, and passivity (Giroux, 1991, 1994, 2001; McLaren, 2003). Transmission-style teaching is referred to as "banking education" (Friere, 1985, p. 21) by its opponents. Banking education occurs when knowledge is deposited into and withdrawn from the learners' heads by the authority, or teacher (Freire, 2000; Freire \& Macedo, 1987). Scripted lessons are intended to deliver knowledge that the authority has decided that everyone needs to learn. Students are expected to listen, learn, and regurgitate the information. Students who do not achieve the predetermined guidelines for success are declared to be failures. According to its opponents, banking education is 
oppressive education (Freire, 2000; Kozol, 2005). Oppressive education perpetuates dominant educational myths through systematic indoctrination, measurement, testing, and rote learning (Goodlad, 1984).

Oppressive education upholds the status quo of mainstream schools by reproducing the dominant culture and not addressing the political nature of literacy and schooling (Wynne, 2002). For example, alternative education schools that focus on remediating and controlling students provide status quo education, structured as separate facilities for students who are at-risk of academic failure and dropping out of school and have been expelled from mainstream K-12 schools for antisocial behavior (Cash, 2004; Foley \& Pang, 2006; Van Acker, 2007). Students remain in these alternative schools until their behavior changes to the point they can be returned to their regular school.

During their time in alternative education programs, students are taught reading from a conventional view of learning that focuses on remediation (M-DCPS). The teaching of reading has long been grounded in psycholinguistics and conventional methods of instruction, such as teaching students how to decode and encode text (Lankshear \& Knobel, 2006). Prior to 1970 in the United States, teaching someone to read was typically defined as a formal, autonomous activity separate from political, economic, social, or cultural practices and unrelated to power and ideology. Literacy programs were typically confined to nonformal educational settings for adults whose illiteracy was thought to be directly related to social conditions such as unemployment, drug abuse, teenage pregnancy, or incarceration (Greenberg et al., 2007). The term functional literacy was first used in formal educational contexts in the 1970s to publicly announce a widespread literacy crisis among adults who were unprepared for the 
emerging postindustrial labor market in the United States. Thus, illiteracy was initiated as something negative about individuals that needs fixing (Hamilton, 2000; Jimenez, 2004; Lee, 2005; Strickland \& Alvermann, 2004) making it difficult to construct alternative positive images of teaching reading with students who do not meet traditionally expected literacy standards (Pardoe, 2000). As a result of this deficit and functional logic that blames students for their failures, teachers are typically trained to practice banking education, or education that focuses on changing students to meet the required standards (Carpenter, Ramirez, \& Severn, 2006).

When teaching students to read or when students are declared in need of reading remediation, banking education is used to cure illiteracy, the disease (Freire, 2000). Students who are privileged typically learn to read well and benefit from banking education because scripted lessons originate in their social and cultural realities. In contrast, students who are not privileged are more likely to do poorly in school, not learn to read well, and be blamed for their failure. Since dominant scripted lessons do not originate in most alternative education students' social and cultural realities, failure to regurgitate information gives the impression that they cannot learn. Teachers who are conventionally trained to use transmission-style teaching typically accept banking education and the use of scripted lessons as the norm. Many students are compliant and regurgitate the information passively and successfully. However, disproportionate numbers of urban (and ultimately alternative education) students resist by disengaging, disrupting, and dropping out (Giroux, 2005). Teachers often utilize oppressive teaching practices such as removing disengaged and disruptive students from the classroom. Eventually, teachers tend to construct negative (and stagnant) images of students who do 
not meet traditionally expected behavioral and academic standards. A critical social practice view of learning provides a more positive and vibrant way of looking at what happens and why in classrooms with the goal of transforming oppressive conditions.

\section{Critical Social Practice View}

This section provides a brief rationale, followed by a synoposis of literature from a critical social practice view of learning related to (a) classroom relationships, (b) teaching practices, (c) critical views of literacy, and (d) critical sociocultural views of literacy. Critical social practice views of learning are useful for focusing on how and why teachers and students connect meanings to particular interactions and behaviors (Monroe, 2006). However, few researchers frame their studies within a critical social practice view of learning or explore the logic behind interactions and behaviors to understand why disciplinary consequences for nondominant students, particularly African American males, emerge at the classroom level. Studies of effective teachers of African American students (Ladson-Billings, 1994) have provided culturally responsive pedagogical interventions, but unless the logic behind nondominant students' behaviors are also understood, well-intended interventions will be unsuccessful (Noguera, 2001; Sleeter, 2005).

\section{Classroom Relationships}

Classroom relationships from a critical social practice view of learning are continually transformed through literacy, culture, and language as social practices that produce and reproduce conditions for learning with nondominant students in marginalized settings (Moje \& Lewis, 2007). Conditions for learning are not deemed stagnant; rather, learning is the interactional transformation of conditions, identities, or 
forms of membership that occurs by negotiations among community members (Gutiérrez, 2008). Looking at learning from a critical social practice perspective brings with it the assumption that (a) knowledge is politically, socially, and historically constructed (Giroux, 2001); (b) power operates through language (Friere, 2000); and (c) people have agency and will co-construct varied forms of community membership (Gutiérrez, 2008).

Taken-for-granted unjust conditions and classroom relationships can be transformed when literacy, language, and culture are viewed from a critical social practice perspective (Freire, 1997). From this perspective, classrooms are created to provide a culture in which students actively participate in public discourse, teaching, and learning (Apple, 1996; Giroux, 2005; McLaren, 2003). Students who are conventionally unsuccessful in school and ultimately labeled, segregated, and failed by structural and institutional functions in society are instead viewed as citizens in a vital democracy with the right and duty to question existing mechanisms and ideologies. The public space of the classroom is revitalized through educative politics (Dewey, 1916, 1939). Educative politics encourages members of a democratic community to participate in joint activity, producing “"economic literacy”" (Oakes et al., 2006, p. 39). Economic literacy is gained when people work together on everyday life problems in a public space of civic participation, engaging both academic texts and the participants' practical knowledge.

In order to transform the classroom, teachers challenge the status quo of conventional views of learning. Teachers who challenge the status quo disrupt the cultural norms and politics of schooling (Oakes et al., 2006) and view "schools as democratic public spheres and ... teachers as transformative intellectuals" (Giroux, 1988, p. xxxii). To feel comfortable challenging the status quo, teachers must view 
themselves as transformative intellectuals, replacing the narrow view of traditional teaching (Shor, 1992). As a result, teachers who are transformative intellectuals are not agents of oppression. They recognize the inequitable conditions of each teaching situation from a wider cultural political perspective and seek to transform these conditions for the benefit of society. "Transformative intellectual knowledge" (Sleeter, 2005, p. 83) enables teachers to combine critical "reflection and action in the interest of empowering students with the skills and knowledge needed to address injustices" (Giroux, 1988, p. xxxiv). To teach nondominant students successfully, practical aspects of education cannot be "divorced from historical, ethical, and political understanding without losing something in the process" (Apple, 1996, p. 100).

\section{Teaching Practices}

This section covers teaching practices that incorporate (a) experiential education for freedom (Dewey, 1910) and (b) dialogic problem posing pedagogy for conscientization (Freire, 1998, 2000, 2003). Teaching practices from a critical social practice view of learning are continually transformed through literacy, culture, and language as social practices that produce and reproduce conditions for learning with nondominant students in marginalized settings (Moje \& Lewis, 2007). Transformed teaching practices continuously focus on changing status quo educational practices and expectations embedded in the classroom (Geelan, 2005; Giroux, 2001, 2005; hooks, 1994; Kincheloe, 1991).

Experiential education for freedom. Teaching practices that incorporate experiential education for freedom (Dewey, 1910) can be used to transform teaching situations with nondominant students in alternative education programs. Experiential education for 
freedom cannot develop from predetermined knowledge or knowledge poured into students' heads by banking education (Dewey, 1938). Dangers of banking education reside in transmission of volumes of information, denying time for reflective thinking, thus miseducating and distorting the growth of future experience by producing insensitivity and lack of responsiveness (Dewey, 1938; Kincheloe, 1993). Transformative teachers educate nondominant students to participate in democracy by critically reflecting on culture and inquiring about phenomena which have become normative, or taken for granted, by most members of society. Transformative teachers choose educational experiences that help to create the kind of society we will ultimately have (Dottin, 2005; Westheimer, \& Kahne, 2004). If ideas are learned through drill and repetition, learners lose their capacity to act intelligently in new situations. If learning is segregated from experience, attitudes being formed at the time of learning that determine what actions will be used in future situations are not considered.

Transformative teachers plan meaningful experiences for nondominant students by using a "backward course design process .... [wherein the educator] starts at the 'end' of the learning process and works 'back' toward the beginning" (Dottin, 2007, personal communication, January 4, 2007). For most students, waste of time in school comes from the inability to connect experiences from outside of school with what is being learning within school. School should provide organic, natural connections between schools and the everyday lives of students. For instance, students' outside lives can be integrated with what is learned in literature, art, and history classes by producing cultural artifacts for a school-based museum (Dewey, 1915). Cultural artifacts include 
poems or lyrics about life, paintings of their neighborhood, or stories about the tribulations of family members.

Dialogic problem posing pedagogy for conscientization. Teaching practices that incorporate dialogic problem posing pedagogy for conscientization (Freire, 1998, 2000, 2003) can be used to transform teaching situations with nondominant students in alternative education programs. Transformative teaching is based on dialogic problem posing pedagogy for conscientization (Friere, 2000). Problem posing originates with students' knowledge, and it is based on dialogue among the students and teacher as equals. Dialogic problem posing pedagogy involves learners and teachers in critical reflection of their lived experiences for the purpose of "transform[ing] the world with their work and creating their own world" (Freire, 1985, p. 14). It merges nondominant students' everyday lives with what they are learning in order to expose the myths of their historical condition and reality so they can begin to transform those myths (Greene, 1988). For example, teachers and students can openly discuss and problematize the myth that alternative education students and their families do not care about school and begin to transform that myth in the classroom (Pane, 2005).

Transforming nondominant students' silence about their histories is a first step in changing oppressive conditions. The continual interplay between critical reflection and practice, or praxis, leads to conscientization, a person's realization that he or she is a creator of culture along with other humans (Shor \& Freire, 1987). Transformative teachers are cultural researchers or workers in Freireian-inspired transformative educational practices (Delpit, 1995; Freire, 2000; Roth-Lee, 2007). Transformative teachers are empowered and, in turn, empower their students to name and reflect upon 
their world and to then remake their culture and to read the word and the world - critical literacy (Freire \& Macedo, 1994). Teachers who believe and act on the notion that alternative education students, for example, can be successful in school will then empower students to act on that same notion.

When unequal power exists, however, as it does in conventionally reproduced classrooms and schools, transformative conditions are not easily constructed by interested educators, students, or researchers. Unless views on learning and teaching reading in particular are reconceptualized, spaces for transformation are not easily seen in many situations, especially in the most constrained alternative education programs. Critical literacy provides a potential space for transforming oppressive educational conditions.

\section{Critical View of Literacy}

The emergence of a critical view of literacy can be traced to periods of social and political unrest. In the 1960 s, skepticism of science and functionalist education demanded ways to change hegemonic, or dominant, practices, in schooling that deny human consciousness and perpetuate reproduction of dominant cultural knowledge as legitimate. In order to transform educational practices, illiteracy was thought of an historical production of a society structured to produce inequality (Freire, 2000). From its inception, reading was considered a political act; critical pedagogy began to be used for the purpose of social vision and liberation (Gore, 1993; Siegel \& Fernandez, 2001). During the 1970s, literacy campaigns promoted social and economic development in Third World countries and initiated epistemological shifts about literacy in developed countries. Freire's (2000) literacy campaigns in Brazil and Chile were most prominent in pushing literacy to the forefront of formal educational discourse. Freirean literacy 
education deemed illiteracy a consequence of unjust social processes and merged decoding and encoding text with reading one's world to gain empowerment to make changes.

Critical literacy highlights language as a tool to read the word and the world for social and political reconstruction rather than as a set of neutral, psychological skills for blaming students (Freire \& Macedo, 1987). Integrating nondominant students' own language with critical literacy emphasizes the "political character of education" (Freire, 1985, p. 171). Through critical literacy, nondominant students are given the chance to experience meaningful literacy learning and critically reflect on how "illiteracy is one of the concrete expressions of an unjust society" (p. 10) merely concerned with techniques and methods of transmitting knowledge (Giroux, 1987, 2006).

In meaningful critical literacy, students produce, transform, and reproduce meaning from their daily lived experiences (Bowles \& Gintis, 1976) rather than produce knowledge for purposes of social control. In critical literacy, students can name their world through their primary Discourse $(\mathrm{Gee}, 1996)$ and begin a dialectical relationship with the dominant Discourse $(\mathrm{Gee}, 1996)$ to transform their silence from years of isolation and segregation (Giroux, 1994; Kincheloe, 2005). Critical literacy engages people in collective action to challenge, rewrite, and transform taken-for-granted explanations and discourses of dominant practices and policies within societal structures. Critical Sociocultural View of Literacy

A critical sociocultural view of literacy challenges traditional reading instruction approaches and growing emphases on functional, or basic, literacy requirements for nondominant students. Critical literacy challenges the political nature of reading 
education. A critical sociocultural view of literacy developed from three seminal studies that introduced a social practice view of learning by (a) destabilizing conventional assumptions about literacy learning; (b) initiating the concept of situated literacy; and (c) introducing the notion of literacy (as social) practices. These studies provided the impetus for a growing "argument for a paradigm shift in what counts as learning and literacy for youth" (Gutiérrez, 2008, p. 148).

Destabilizing conventional assumptions about literacy learning resulted from Scribner and Cole's (1981) research. Through cross-cultural psychological tests, interviews, and observations, the influences of Western school-based literacy practices on problem solving abilities of schooled and nonschooled Vai people in Liberia were studied (Scribner \& Cole, 1981). Results showed that people learned English, Vai, and Arabic in formal and informal contexts depending on the purpose of the script with equally capable problem-solving abilities in all three scripts. Thereafter, researchers began focusing on the role of interaction and external factors in knowledge construction and problem solving, which destabilized conventional assumptions about literacy learning. From this perspective and relative to this study, knowledge and problem-solving skills of nondominant students from environments that stress daily survival are valued.

Initiating the concept of situated literacy was an important finding in Street's (1989) social anthropological study, which compared traditional and commercial literacies in the lives of Islamic villagers in Iran. Results revealed that traditional and commercial literacies differed in ideological and autonomous approaches. Everyday literacies were learned through ideological approaches which define literacy according to the situation. Commerical literacies were acquired by autonomous, or dominant, 
approaches which define literacy outside of the social context, or situation. Researchers began studying in-school and out-of-school literacy development as situated literacy. The concept of situated literacy has evolved to value multiple literacies, multiple contexts and communities of practice, various interpretations of the zone of proximal development, and the interactional value of learning processes and cycles of social reproduction including identity development and forms of community membership (Lave \& Wenger, 1991; Gutiérrez, 2008).

Introducing the notion of literacy (as social) practices was a significance of Heath's (1983) ethnographic and sociolinguistic study in which mismatches between reading and writing practices in three Appalachian communities and homes versus the school were described. The term "literacy events" was coined to mean what people do with reading and writing in their everyday lives rather than individual traits. Literacy events emphasizes how texts are mediated through dialogue and social interaction in particular practices and settings. People's literacy practices were seen as culturally relevant ways (e.g., talk, attitudes, feelings, social relationships) of how people make sense of literacy. Researchers began looking at how literacy events and practices acquired in different communities influence how and what is learned in and out of school. Through this notion, nondominant students' literacy practices in this study are viewed as culturally relevant ways of making sense of their world.

Destabilization and reconceptualization of conventional assumptions about literacy and learning (Vygotsky, 1978, 1986) links psychology, culture, and learning with activity through language and thought rather than focusing on individual cognition and behavior (Cole, 1996; Rogoff, 1990). A critical sociocultural view of literacy provides a 
different perspective on thinking, that is was no longer thought of as a private matter. Thinking is now understood to be mediated by cultural artifacts or languages that have been historically, socially, and culturally shaped for certain purposes by people who can transform them for new uses in new settings. Transformative teaching practices guided by critical sociocultural views of literacy provide insights into literacy, cultural, and language practices of nondominant youth (Lewis et al., 2007). Transformative teaching practices can mesh "intersection of social, cultural, historical, mental, physical, and political aspects of people's sense-making, interaction, and learning around texts" (p. 2).

Critical Social Practice Theory of Learning

The theoretical framework of this study, a critical social practice theory of learning (Lave \& Wenger, 1991), is presented in depth in this section. A critical social practice theory of learning, or communities of practice perspective, is defined by the process of legitimate peripheral participation (LPP). LPP describes how people engage in social practice and learn through various aspects of community membership within situated activity. Abstract knowledge in and of itself has no power, but generalization of "any form of knowledge always lies in the power to renegotiate the meaning of the past and future in constructing the meaning of present circumstances" (p. 34). Legitimate peripheral participation indicates a shift away from theories of situated activity in which learning is a cognitive process inside one's head or abstract activity that can be deposited into an activity or situation, devoid of participation in the social world.

For example, students who have been suspended multiple times over the years and are caught in the discipline gap expect to enter an unfamiliar classroom as a newcomer and be required to assimilate to conventional forms of teaching and learning 
(e.g., doing silent individual seatwork). However in communities of practice, the teacher and students co-construct different identities by engaging in jointly negotiated sociocultural practices to transform themselves and their conditions (Nasir \& Cooks, 2009). For instance, after discussing issues of significance for the students, a school newspaper that raises awareness and documents students' daily cultural experiences could be co-created, published, and distributed in their school, community, and homes.

In a theory of social practice with situated learning as legitimate peripheral participation, learning is understood as an integral aspect of all activity that can be analyzed by exploring the interconnections of its concrete relations in "historical terms, through time and across cultures" (Lave \& Wenger, 1991, p. 39). Learning takes place anywhere at any time and is not necessarily caused by intentional instruction. In other words, people learn both official (Apple, 2000) and incidental knowledge and practices from the social organization of the community of practice as much or more than from instructional material or techniques. Based upon previous experience with exclusionary discipline from classrooms and multiple suspensions from schools, students can quickly assess classroom situations without explicit instruction from the teacher. They can judge what types of interactions result in exclusionary discipline, what it takes to become a full participant in the classroom, the likelihood of either, and whether or how to proceed based on this knowledge in order to preserve their identity.

The notion of legitimate peripheral participation (LPP) helps one understand the social organization of classrooms or schools as communities of practice with assorted forms of membership. LPP provides a context for exploring what people learn or do not learn and do so with what meanings for identity production or reproduction. Learning 
occurs through the transformative potential of negotiated, often uncomfortable, interactions among members of specific communities of practice (Fránquiz \& Salazar, 2004). Social and academic identity transformation, and consequently academic resiliency, may develop from certain types of resistance to constraints in classrooms or schooling structures. By openly identifying him/herself as a troublemaker, the new student may be resisting constraining classroom structures with which he/she has been unsuccessful with in the past. From a communities of practice perspective, student resistance is recognized as a potential space for readjusting the classroom structure to allow the student to negotiate success. For example, the teacher could invite the newcomer to experience success immediately by helping members of the newspaper team deliver the most recent issue of the co-created school newspaper.

In order for readers to understand the phrase holistically, segments of LPP will now be analyzed. Legitimate participation is about ways of belonging to, or forms of membership in, communities of practice. For instance, all students belong to different communities of practice with diverse forms of membership both inside and outside of the school. Subsequently, each newspaper team member brings an assortment of experiences, ideas, and expertise to the table when engaged in social practice to develop the newspaper. The final product is co-constructed through multiple, dialogic, conflicting negotiations.

Peripheral participation suggests non-central ways of being located in the social world, referring to how "changing locations and perspectives are part of actors' learning trajectories, developing identities, and forms of membership" (Lave \& Wenger, 1991, p. 36). For example, as negotiations occur and are worked out, participants learn different 
perspectives and methods of interacting with various people to create new cultural forms of social and societal activity. In this example, a continually evolving newspaper emerges from the negotiations and struggles embedded in the ongoing interactions among the participants.

Legitimate peripherality, coined by Lave and Wenger (1991), is the practice of positioning community members through relations of power, implicating broader social structures. For example, each classroom community member who jointly develops the newspaper is positioned in various roles, such as editor, artist, or typist, to complete the monthly issue. Getting permission to deliver the newspapers, determining where the newspapers would be delivered and who would read them include a wide variety of communities of practice inside and outside of the classroom. Legitimate peripherality could also be exemplified by involving juvenile judges who work with alternative education students outside of the classroom. They could be invited to read the newspapers and later write special columns for the monthly issues. Involvement of judges is an example of legitimate peripherality from a societal perspective that also encourages teachers' and students' agency. Legitimate peripherality, albeit positioned from a different societal perspective, also involves parents who receive each issue of the newspaper and commend their child for participating on the team.

Full analysis of situated learning as legitimate peripheral participation (LPP) in communities of practice means connecting peripheral participation to the legitimacy of and control over the social organization's resources. Peripheral participation may lead to full participation, the rightful integration of evolving forms of community membership. Partial participation is not necessarily a disconnection from the practice, but a possible 
opening for gaining access to fuller participation. A communities of practice perspective may be useful for transforming oppressive classroom conditions that typically rely on exclusionary discipline for nondominant students (Gutiérrez, 2006). For example, teachers who prefer not writing referrals for disruptive behavior may try ventures similar to the newspaper team, encouraging students who partially participate to become full participants in the classroom community.

In contrast to a critical social practice theory of learning as defined by legitimate peripheral participation (LPP), conventional views of learning assume the easy absorption of given information in a process whereby individuals internalize knowledge by discovery, transmission, or interaction with others. A conventional view of learning supports the belief that all students will and should easily fit in and learn what is being taught in the classroom. To compare LPP theory of learning with conventional views of learning, three interpretations of Vygotsky's $(1978,1986)$ zone of proximal development and the social nature of learning will be undertaken here. The first two interpretations are compatible with conventional views of learning, the third with LPP theory of learning.

In the first interpretation, the zone of proximal development is interpreted as the "distance between the problem-solving abilities of a learner working alone and that learner's problem-solving abilities when assisted by or collaborating with more experienced people" (Lave \& Wenger, 1991, p. 48). Using this interpretation, teaching is thought of as scaffolding, or slowly relinquishing initial explicit support given for performance of a task until the learner can perform the task independently. An example of scaffolding is that of teaching students to write a five-paragraph essay by writing one 
together the first time, writing all but the last paragraph the second time, and so on until the students write the whole essay alone.

The second interpretation is a cultural understanding of the zone of proximal development as the distance between the cultural (understood) knowledge provided by the sociohistorical context (instruction) and the active knowledge owned by the individual. "This interpretation is based on Vygotsky's distinction between scientific and everyday concepts, and on his argument that a mature concept is achieved when the scientific and everyday versions have merged" (Lave \& Wenger, 1991, p. 48). An example is that of the teacher who bases her instruction of the scientific concept of precipitation on what students already know from their own cultural backgrounds about rain. In both interpretations, the social nature of learning is minimized to the internalization of the cultural given by individual acquisition.

A third and more recent interpretation of the zone of proximal development from activity theory (Engeström, 1987; Engeström, Miettinen, \& Punamaki, 1999) is compatible with Lave and Wenger's LPP and the theoretical framework of this study since it focuses on the processes of social transformation. In this interpretation, the zone of proximal development is defined as the "distance between the everyday actions of individuals and the historically new form of the societal activity that can be collectively generated" (Engeström, 1987, p. 174) from the solution to conflicts embedded in everyday actions. Learning is not the overt individual results of instruction based on given cultural information, but a "relational understanding of person, world, and activity" (Lave \& Wenger, 1991, p. 51) viewed as sociocultural transformations in terms of continuous, evolving, holistic participation in communities of practice. This third 
interpretation is exemplified when a student with multiple exclusionary discipline and suspension experiences enters an unfamiliar classroom in which the teacher focuses on social transformation. In the example of the classroom community of practice who creates a newspaper, the students and teacher both use agency to resist and participate in joint activities, continually negotiating and transforming their identities:

Understanding and experience are in constant interaction —indeed, are mutually constitutive. The notion of participation thus dissolves dichotomies between cerebral and embodied activity, between contemplation and involvement, between abstraction and experience: persons, actions, and the world are implicated in all thought, speech, knowing, and learning. (Lave \& Wenger, 1991, p. 52)

This subjective and objective focus on participation in relational social practice implicates people in the world as members of sociocultural communities as well as "a view of knowing as activity by specific people in specific circumstances" (Lave \& Wenger, 1991, p. 52).

Continuing the example of the newspaper team, learning involves the whole person in relationship to the specific continual activities of improving the newspaper. Members learn to interact with various social communities inside and outside of the school perimeter, such as the school director, staff members, and newspaper publishing agency. Members learn to plan, sell advertisements, take photographs, and interview people for articles. Each person provides his/her own computer expertise and learns to meet the deadline each month in multiple ways. The social nature of learning implies becoming a full participant through evolving transformed forms of community membership and ways of belonging in situated activities, which in this case could be a newspaper team based at an urban alternative education school for students who are expelled from their regular schools and are involved in the juvenile justice system. 
Learning is more than just involvement in new activities, performing new tasks, and mastering new understandings since these entities do not "exist in isolation but are a part of broader systems of relations in which they have meaning" (Lave \& Wenger, 1991, p. 53). Learning implies becoming a different person and necessarily involves the construction of identities, or living relationships among people participating in communities of practice, with possibilities facilitated by societal systems of relations. A relational view of the person and learning constructs whole persons with agency who define themselves in practice. For example, the newspaper team members are involved both in creating the monthly newspaper and in learning that transforms their social identities in the context of practice and trajectories of negotiated participation.

Since participation is the fundamental form of learning in the social practice theory of learning as legitimate peripheral participation (LPP), the situated nature of learning goes beyond the immediate sociocultural context. Theorizing LPP interrogates how societal forces both shape and are shaped by immediate contextual relationships, both the reproduction and transformation of social identities as well as communities of practice, depending on paths, relationships, and practices claimed by membership. In other words, what happens in classrooms mimics what happens in society and what happens in society influences classroom practice. The relational emphasis between changing identities and membership in communities of practice makes it possible to think of continuous learning, official or unofficial, as a basic characteristic of communities of practice. Studying and possibly changing classroom interactions, learning processes, and exclusionary discipline practices within classrooms will influence what happens outside of the classroom. 
Specific forms of legitimate peripheral participation (LPP) can be interpreted by exploring issues surrounding the sociocultural organization of space into places of activity and distribution of knowledgeable skill via ongoing historically constructed, conflicting, synergistic relations among participants and processes of community production and reproduction (Gutiérrez, 2008). In effect, a Third Space (Lefebvre, 1991; Moje et al., 2004; Muth, 2005; Wilson, 2003) is being created through the newspaper team. The teacher and students work together to solve academic and social issues for the good of the classroom learning community within and outside of the school. New students enter the space where they see themselves belonging and continually negotiating new forms of membership in the community of practice. A social practice theory of learning defined as LPP provides a way to analyze

changing forms of participation and identity of persons who engage in sustained participation in a community of practice: from entrance to newcomer, through becoming an old-timer with respect to new newcomers, to a point when those newcomers themselves become old-timers. (Lave \& Wenger, 1991, p. 56)

Rather than examining teacher-student relationships based on conventional apprenticeship and views of learning, synergistic relationships of participation within and across various cycles of learning can be studied. "These cycles emerge in the contradiction and struggle inherent in social practice and the formation of identities" ( $p$. 57), such as in the complexity of disruptions and interactions that lead to exclusionary discipline practices (Vavrus \& Cole, 2002). Sinc LPP implies that learning is never transfer or assimilation but rather problematic, contradictory transformation and change implicated in each other—-status quo and change both need to be explained. 
Because learning processes are part of the collective, generative working out of contradictions in communities of practice, social cycles of production and reproduction of the future of particular communities implies spaces, even momentarily, of agreement (Engeström, 1987). From personal experience, students with a history of exclusionary discipline from classrooms and suspension from schools may explain that they typically feel invisible and shunned at school even with friends from the neighborhood. However, these same students explain that being a member of generative classrooms where conflicts are welcomed as learning spaces makes them feel alive and visible to others around them. They desire and enjoy the chance to develop productive identities and help change things for the better in such ventures as a newspaper team. As their teacher, I also enjoyed, learned, and transformed within co-constructed generative learning spaces (Pane \& Rocco, 2009). Learning seems more palatable and available in generative spaces (Engeström, 1987). These potential spaces of transformation leave an historical residue of collectible physical, linguistic, and symbolic artifacts that are constructed and reconstructed in practice over time. These spaces can be experienced and studied anthropologically (Varenne, 2008) by teachers, students, and researchers if viewed as negotiable social practices in communities of practice.

A critical theory of social practice emphasizes the "relational interdependency of agent and world, activity, meaning, cognition, learning, and knowing" (Lave \& Wenger, 1991, p. 50). Meanings and communication are socially negotiated by people situated in the historical development of ongoing activity with others, which often includes discipline issues and practices in the classroom. Further, this theory is a critical social theory because it analyzes the social scientist's practice 
in the same historical, situated terms as any other practice under investigation. One way to think of learning is as the historical production, transformation, and change of persons. Or to put it the other way around, in a thoroughly historical theory of social practice, the historicizing of the production of persons should lead to a focus on processes of learning. (p. 51)

Therefore, the critical social practice theory of learning defined as legitimate peripheral participation (LPP), or communities of practice perspective, is a significant framework for this study based on its basic premise of engaging and including all members of society who have been marginalized. Viewing disciplinary (or potential disciplinary) actions as negotiable social practices among the teacher and nondominant students in a particular alternative education classroom shifts our perspective away from the common "reporting how many times something happened" mode. Instead, this view enables the researcher to discuss and analyze how and why something happened from participants' perspectives. Also, viewing learning, language, literacy, culture, and discipline, specifically school suspension, as negotiable social practices rather than reporting them as test scores or demographic descriptors of nondominant students who have been labeled unsuccessful in school and society enables the researcher to discuss and analyze the social and academic aspects of what is being learned.

Critical microethnography integrates research methods from a critical social practice theory of learning, the theoretical framework of this study to examine and interpret teacher-student social interactions within classrooms that influence teachers' decisions to use or not use exclusionary discipline in order to find potential spaces for transformation of oppressive educational practices and conditions. 


\section{Critical Microethnography}

This section covers reconceptualized (a) theoretical tools for analysis, (b) research contexts, and (c) research methods for critical microethnography, useful for studying classroom interactions related to exclusionary discipline for transforming stagnant, oppressive educational practices and conditions.

\section{Theoretical Tools for Analysis}

This section covers five theoretical tools for analysis (Bloome et al., 2005): (a) contextualization cues; (b) boundary-making; (c) turn-taking patterns; (d) thematic coherence; and (e) intertextuality (Fairclough, 2003). These tools are useful for viewing a moment-by-moment analysis of how language, literacy, culture, and discipline are produced or reproduced in teacher-student social interactions.

\section{Contextualization Cues}

Contextualization cues are the ways people act and react to each other depending on their understandings of what has happened, is happening now, and expect to happen in similar situations. Contextualization cues are both verbal and nonverbal behaviors. To understand how contextualization cues influence what goes on in the classroom, the ongoing social interaction and dialogue on a moment-to-moment basis, changing over time, needs to be analyzed. One of the major ways of analyzing dialogue is by understanding how people acquire Discourses $(\mathrm{Gee}, 1996)$ as tools for participation with others. Discourse with a capital D (Gee, 2006) is a person's overall presentation or persona that guarantees membership in particular social situations. Understanding students' Discourses provide a basis for understanding how culture unfolds and youth identities shift at multiple levels of development intertwined in activity among 
marginalized youth (Strickland \& Alvermann, 2004; Vadeboncoeur \& Stevens, 2005). The theory of Discourse (Gee, 1992, 2000) and literacy provides a framework for understanding that Discourse $(\mathrm{Gee}, 1996)$ is like having an identity kit with instructions on what to wear, how to act, and how to talk in particular roles in order to be recognized. Nondominant students' Discourses often exclude them from dominant societal structures and institutions because dominant members of society do not accept them due to the way they speak and act. Discourses marginalize or include people in power and hierarchical structures in society; thus, having control of the dominant Discourse of society may make it easier to succeed.

Primary Discourses are learned in homes and communities through acquisition (Gee, 1992). Acquisition is the process whereby one gains control of a primary Discourse in a natural, meaningful setting by trial and error. The primary Discourse being acquired is needed and wanted in order to succeed in a particular role (e.g., family member, lawyer). Primary Discourses make up students' prior knowledge, understandings, and cultural practices, or everyday funds of knowledge (Moll, 1992). Youth bring everyday funds of knowledge and Discourse to school with them, drawing on these funds and Discourses to make sense or not make sense of classroom texts. Secondary Discourses are learned, rather than acquired, through explicit gaining or teaching of knowledge and meta-knowledge of a discourse through explanations and analysis (Gee, 1992). Classroom, or academic, texts are secondary Discourses encountered in social institutions away from home. Since schools are based on middle and upper class Discourses, students from middle and upper classes typically grow up acquiring a primary Discourse congruent with the school's secondary academic Discourse (Gee, 1996). 
When students' primary Discourse from their home culture differs significantly from school Discourse, as in the case of nondominant students, a clash occurs (Delpit, 1995). Teachers may “misread students' aptitudes, intent, or abilities ... . [or] utilize styles of instruction and/or discipline that are at odds with community norms” (p. 167). When a clash occurs, teacher-student relationships suffer; teachers blame students for lack of effort or discipline; and students disengage, disrupt, or resist academic achievement (Lee, 2005). Knowledge of technical teaching skills, pedagogy, and subject matter are necessary but insufficient for addressing classroom clashes. Teachers must also possess dispositions and knowledge of how to incorporate "the cultures, experiences, and needs of their students into their teaching" (Banks et al., 2005, p. 243) so students have more opportunities to achieve literacy success in school.

\section{Boundary-making}

Boundaries are interactional behaviors that occur among teachers and students in a classroom. Boundaries are a result of how each person in the group identifies, interprets, and reacts toward other people's verbal and nonverbal behaviors, or contextualization cues (Bloome et al., 2005). When teachers do not understand the languages, Discourses, and cultures of nondominant students, boundaries based on the culture of power become rigid and oppressive when clashes occur. Teaching nondominant students well by understanding the culture of power concept has sparked classroom interventions which build on students' everyday languages, Discourses, and cultures (Delpit, 1995). To become transformative teachers, dominant teachers need to understand how to merge skills, critical and creative thinking, and progressive learning in meaningful contexts beginning with students' voices (Wynne, 2002). Students' cultural 
backgrounds must be valued and respected while awareness of dominant codes and Discourses of society are provided for success in and out of the classroom (Anyon, 2005).

Since we live in the "culture of power" (Delpit, 1995, p. 25), "issues of power are enacted in the classroom ... through the power of the teacher over the student" (p. 24). Broader institutional levels of power reinforce teacher-student power relationships. Teachers need to explicitly teach other people's children how to participate in the culture of power in order for them to be successful in school (Delpit, 1995). "If you are not already a participant in the culture of power, being told explicitly the rules of that culture makes acquiring power easier" (p. 25). Using generic skills and monocultural strategies which are decontextualized from students' lives ignores linguistic and cultural strengths of students and does not teach them what they need to know or build on what they already know.

Rules for participating in power "relate to linguistic forms, communicative strategies, and presentation of self; that is, ways of talking, ways of writing, ways of dressing, and ways of interacting" (Delpit, 1995, p. 25). The culture of power in our nation requires middle- and upper-class rules for successful participation in society and in school to determine who is literate (Foucault, 1977). Students who grow up in middleand upper-class homes come to school with these rules instilled in their ways of knowing. Middle- and upper-class students' particular shared ways of knowing (Heath, 1983), "thinking, believing, acting, and communicating" (Moje \& Lewis, 2007, p. 3) are congruent with most teachers' experiences and school cultures.

Nondominant students who do not grow up in middle- or upper-class homes come to school without already knowing the rules for successful participation. These students 
share particular ways of knowing that work well in their own community and home cultures but may clash with their teachers' experiences and expectations and school culture (NPTARS, 2005). Teachers can avoid the clash between home and school cultures by getting to know the nondominant students they teach, discovering the strengths students bring to school, and building on those strengths in order to support students' literacy learning (Cochran-Smith \& Zeichner, 2005; Darling-Hammond \& Bransford, 2005; Delpit, 1995). All students can be successful if teachers do not use the same instructional methods for all of them and if teachers share expertise in the classroom by learning from and about the students, their families and communities, and their intellectual legacies (Freire, 2000; Shor, 1992).

\section{Turn-taking Patterns}

Turn-taking occurs based on how contextualization cues are interpreted and boundaries are constructed by the group of people in a particular situation in a particular institution (Bloome et al., 2005). By viewing turn-taking as activity based on cultural practices, potential transformative spaces become apparent.

Cultural historical activity theory (CHAT) focuses on activity, or cultural practices, as the unit of analysis of human behavior (Cole, 1996). Cultural differences among groups are legitimated as valid cultural practices which depart from institutional expectations (Lam, 2006; Roth \& Lee, 2007). The zone of proximal development (ZPD) is a dynamic, rather than traditional, view of apprenticeship that privileges students' cultural funds of knowledge for learning. Traditional apprenticeships provide shadowing, experiential opportunities for persons to learn a trade within and outside of school. Dynamic apprenticeships focus on intersubjectivity_- "understanding the Self to 
understand the Other" (Roth, 2005, p. 15)—guided by dialogic (Bakhtin, 1986) relationships between school-based and out-of-school literacies.

An activity system involves dynamic interactions studied through the tool of mediated action among (a) a group of people who act as individuals and members of the community of practice over time; (b) available artifacts used as tools to solve problems; (c) rules that guide behavior; (d) division of labor to do the activity; and (e) emerging, negotiated, and possible goals of the activity (Engenström, Miettinen, \& Punamaki, 1999). Culture is broadly understood in CHAT as individual patterns of beliefs, values, and practices acquired and transformed over time through participation and engagement with multiple, heterogenous communities (Gutiérrez, 2002, 2008; Lam, 2006). Literacy practices in an activity system involve participation with others, even others who are not present. Dialogic theory (Bakhtin, 1986) is used to give voice or importance to each person's meaning and uses of language. For instance, in some areas of the U.S., dominant youth engage in African American Vernacular English (AAVE) speech genres (Lee \& Ball, 2005), an example of primary Discourse (Gee, 1996) dialect spoken by many African Americans.

Culturally responsive pedagogy (Ladson-Billings, 1995; Moje, 2004; Moll, 1992) values minority students' voices. Culturally relevant pedagogy is a well-documented yet underutilized responsive approach for successfully developing nondominant students' literacy learning (Banks, 2001; Ladson-Billings, 1994, 1999; Lee, 2005). Culturally responsive literacy teachers understand what people do with literacy as their literacy practices (Barton \& Hamilton, 2000; Maybin, 2000). Culturally responsive teachers are aware of, place value on, and build on the everyday "funds of knowledge and Discourse 
that shape and inform literate practice ..." (Moje \& Hinchman, 2004, p. 322) to challenge and reshape the academic-content knowledge and literacy practices of the curriculum.

Teachers can become a culturally responsive teacher by studying students' community or everyday funds of knowledge (Moll, 1992) and Discourse to understand how language and literacy practices are constructed outside of school (Moje et al., 2004). Studying everyday funds is the first step toward integrating often conflicting academic and everyday funds of knowledge and Discourses and increasing opportunities for literacy learning. Knowing how to integrate competing funds is essential at a time when more teachers and their students draw from different backgrounds and experiences to understand content area texts and concepts. Teachers who view education as transformation, grounded in culturally responsive pedagogy, will be able to teach other people's children how to participate in the culture of power to be successful in school (Ladson-Billings, 1994, 1999).

Cultural Modeling (Lee, 1993) begins with minority students' voices. Using a CHAT framework, Cultural Modeling is a way to design culturally responsive learning environments that link tacit, or taken-for-granted, knowledge students bring from home with disciplinary knowledge (Lee, 1993, 1995, 2000). For example, Lee and Ball (2005) found that AAVE speakers' tacit understanding of figurative language in rap lyrics can be merged with academic knowledge to generate possible meanings from figurative language in academic texts. However, Cultural Modeling necessitates an understanding of cultural models, cultural scripts, and an alternative view of Vygotsky's (1986) zone of proximal development. Cultural models (Lee \& Ball, 2005) are schemas shared within a cultural community through which people interpret experiences and base their actions. 
Cultural scripts are similar to Discourses (Gee, 1996) which are schemas for appropriate membership in an activity. Using ethnographic methods to uncover cultural models and scripts that are used in various communities by students in routine practice by teachers as researchers (Kincheloe, 1991) can privilege students' cultural funds of knowledge and negotiate goals together (Lee \& Ball, 2005).

\section{Thematic Coherence}

Thematic coherence takes place when teachers and students determine and agree on how meanings, ideas, and interactions during a literacy event have been organized and/or negotiated (Bloome et al., 2005). To account for negotiations that take place in activity systems and to reduce antisocial behaviors and disengagement, cultural practices in urban learning environments are viewed as spaces that can be analyzed for thematic coherence to discern opportunities and constraints for literacy learning (Gutiérrez, 1992; Gutiérrez, Bequedano-Lopez, \& Tejeda, 2003).

Within CHAT, cultural differences among nondominant groups are legitimated as valid cultural practices which depart from institutional expectations (Lam, 2006). Also, through Hybridity theory (Bhabha, 1994; Soja, 1996) the difficulties of examining people's different spaces (Gee, 1996) and multiple literacies (Moje et al., 2004) are acknowledged. An assumption of hybridity theory is that people in any community have access to and draw from multiple funds, or resources, to make sense of the world. Bringing various cultural funds of knowledge together can both support and constrain the development of identity, and literate, social, and cultural practices. Being in-between various funds of knowledge and Discourses can promote and limit identity development and literate, social, and cultural practices (McLaren, 1999). 
Third space theory is closely interrelated to CHAT and Hybridity theory. In one interpretation, construction of third space "merges the 'first space' of people's home, community, and peer networks with the 'second space' of the Discourses they encounter in more formalized institutions such as ... school ...” (Moje et al., 2004, p. 41). First space is equivalent to primary Discourses and second space to secondary Discourses. Similar to hybridity theory, third space theory (Lefebvre, 1991) invites the concept of first, second, and third spaces, the reconceptualization of binary spaces, either-or ways of thinking about literacy learning and success in school. Third spaces are in-between, or hybrid spaces (Muth, 2005; Wilson, 2003). Seemingly oppositional first and second spaces work together to generate new third space knowledges, Discourses, and literacy forms.

Third space is generated when people socialize together in and through language, mixing everyday and academic knowledge. Third space is used to explore literacy learning as a bridge or scaffold to move students through their zones of proximal development (ZPD) from marginalized (e.g., everyday) to privileged (e.g., dominant) content academic knowledge and Discourses. Third space is a navigational tool for guiding students across the boundaries of various privileged content texts through their everyday funds of knowledge and Discourse. Third space is also a space where everyday knowledge and Discourses are used in ways to "challenge, destabilize, and expand literacy practices that are typically valued in school” (Moje et al., 2004, p. 44).

Collective Third Space (Gutiérrez, 2008) can be viewed as an interactional interpretation of the zone of proximal development, incorporating a sociocritical literacy 
that historicizes everyday and institutional literacy practices and texts and reframes them as powerful tools oriented toward critical social thought .... in which [conventional] conceptions of academic literacy and instruction for students from nondominant communities are contested and replaced with forms of literacy that privilege and are contingent upon students' sociohistorical lives, both proximally and distally .... hybrid language practices; the conscious use of social theory, play, and imagination; and historicizing literacy practices link the past, the present, and an imagined future. (p. 148)

Outside of third space thinking, first space of students' out-of-school everyday knowledge and second space of in-school, academic knowledge are separate categories, read as everyday versus academic knowledge, implying one way to be literate (Moje, 2004). From a conventional view of learning, no third space is available for nondominant students since they are presumed to be illiterate or low-ability; they are labeled as failures. Third space thinking challenges fixed notions and dominant views of culture and language. Struggling to merge competing knowledges and Discourses can be fruitful if students are not defined according to dominant Discourse.

\section{Intertextuality}

Intertextuality occurs when two or more written, verbal, nonverbal, or electronic texts share a feature, refer to one another, or lead to another text (Bloome et al., 2005). The New Literacy Studies (NLS) challenge taken-for-granted deficit accounts of literacy in mass media, political discourse, and research (Pardoe, 2000). NLS stem from sociohistorical psychology, situated cognition, activity theory, cultural models theory, poststructuralist, and other work reacting against individualism and cognitivism (Gee, 2000). In NLS, "reading, writing, and meaning [are] always situated within specific social practices within specific Discourses” (p. 189). Reading and writing make sense only in the context of social, cultural, historical, political, and economic practices rather 
than of individual cognitive tasks. Knowledge and meaning are viewed as emerging from social practices in which "people, environments, tools, technologies, objects, words, acts, and symbols are all linked to ('networked' with) each other and dynamically interact with and on each other" (p. 184).

Classrooms are viewed as the local place where situated and multiple literacies develop: "the site at which people — in tandem with words, deeds, objects, tools, symbols, settings, times, and ways of being, doing, thinking, and valuing - work out their projects, as well as work on and rework the projects" (Gee, 2000, p. 194). Literacy is part of the way people keep up with their lives. Literacy practices are purposefully embedded in people's life goals and always entrenched in power relations (Street, 1989; Tusting \& Barton, 2005). Literacy practices in nondominant students' everyday lives, often referred to as out-of-school literacy, are viewed as dynamically and continually negotiated in everyday social, cultural, linguistic, and community contexts (Lewis et al., 2007; Strickland \& Alvermann, 2004).

All students — privileged and disadvantaged, dominant and nondominantstruggle to maintain their position in society, proactively engaging in multiple literacies with social networks in particular situations for authentic reasons. For example, electronic media play a large part in the development of youths' multiple everyday literacies outside of school (e.g., spoken-word performance, My Space, text messaging, instant messaging). Rarely are these everyday literacy practices thought of as particular funds of knowledge and Discourse which might be connected to, informed, and even integrated with knowledges and Discourses, valued, privileged, and taught in school (Moje, 2004, p. 41). 
Youth Literacies, Cultures, and Identities (YLCI) studies focus on "what and why texts matter to youth and on how youth texts and literacy practices might inform academic literacy development" (Lewis et al., 2007, p. 1) so that success can occur in the classroom. Literacy learning for nondominant students is reframed by reconsidering the notion of the so-called struggling secondary reader and writer and how to teach them. The assumption in YLCI studies is that all students have rich funds of knowledge, cultural practices, and Discourse that they bring to school. YLCI studies begin with what youth already and want to know, do, read, and write rather than what they struggle with in academic literacy learning. By doing so, YLCI studies are transformative by attending to the social, political, and economic importance of developing in-school literacy.

\section{Research Contexts}

This section provides a brief introduction, followed by a discussion of two aspects of reconceptualized research contexts: (a) culture as social practices and (b) classrooms as social sites. Research contexts are reconceptualized in critical microethnography to focus on how uses of language support or constrain opportunities for literacy learning in alternative education classrooms. Focusing on the critical social practice view of how language, literacy, culture, and discipline are produced among nondominant students and their teacher in an alternative education classroom necessitates a reconceptualization of the research context- "bringing together theories of situated activity and theories about the production and reproduction of the social order" (Lave \& Wenger, 1991, p. 47). The research context can no longer be viewed as an artificial container with controllable variables. By merging activity theory (Cole, Engeström, \& Vasquez, 1997; Engeström, 1987 ) with sociocultural theory, a static, context-free view of research reconceptualizes 
culture into seeing "how individuals participate in particular activities, and how they draw on artifacts, tools, and social others to solve local problems" (Nasir \& Hand, 2006, p. 450).

\section{Culture as Social Practices}

Viewing culture as social practices situated within the context of a hybrid, or problematic, learning environment provide reconceptualized understanding about the relationships among verbal and nonverbal interactions and learning processes (Gutiérrez, 2002). It addresses the "shifting attitudes, representations, and desires of this new generation of youth being produced within the current historical, economic, and cultural juncture" (Giroux, 2005, p. 21). In a seminal study about "working class cultural patterns of "failure"" (Willis, 1977, p. 1), insights about nondominant students are provided. Ethnographic methods were used to study how working class lads make choices, develop, transform, and reproduce cultural aspects of the society at large to maintain their place in society. Persistent failure of societal institutions to improve the life chances of working class lads was also explored. More social than intellectual, "education is one culture embracing another" (Rose, 1989, p. 225) with certain cultures expected to fail and others to succeed, depending on who is doing the embracing.

In research contexts that view culture as social practices, schools are understood as democratic public spheres where teachers and students actively participate in the construction of history, social change, and culture. Teaching is viewed as a cultural practice concerned with where and how knowledge should materialize. Curriculum is interrogated as to whether it is developed around interdisciplinary issues with questions about multiculturalism, race, identity, power, knowledge, ethics, and work. 
Transformative language and ways to think about the purpose of school, classroom relationships, and living in a globalized, high tech, racially diverse world need to be discovered (Giroux, 2005). Transformative language and thinking interrogates changing contexts and conditions as well as critical relationships among knowledge, culture, and power in our $21^{\text {st }}$ century postmodern democratic society.

Participants typically viewed as delinquent and disruptive nondominant students are instead viewed as real people who can articulate and rewrite their lived experiences within their own history through critical pedagogy. Critical pedagogy is a "central aspect of cultural studies and cultural work as a pedagogical practice" (Giroux, 2003, p. 3) for addressing social justice, democracy, and the struggle for equality. In critical pedagogy, people work to understand the "relationship between knowledge and power, language and experience, ethics and authority, student agency and transformative politics, and teacher location and student formations" (p. 6). Teachers and nondominant students may be viewed as community members interacting among culture, power, and transformation of social identities in ways that currently address the complexities of living in a postmodern age. Teachers and nondominant students may be viewed as asking new questions, developing new models, and producing counter-hegemonic learning communities that purposely build collective, visible identities of nondominant youth as literate and achieving people who participate in our democratic society (Hilliard, 2003; Perry, 2003; Steele, 2003).

Reconceptualized views of research contexts challenge the dominant discourse of reason, objectivity, and lack of students' and teachers' agency. Concerns with equality, social justice, and freedom through emancipatory cultural politics which offers 
possibilities of social reconstruction are embraced (hooks, 1994). With the onset and growth of critical and radical pedagogy after Freire's (2000) landmark work, transformative educators insist on eradicating all types of oppression as interlocking systems of domination. Knowledge is analyzed by how it is produced, refusing exclusions, hierarchies, and inequality (hooks, 1989). Mundane, conventional, pedagogy may be understood to be based on the fear of losing power needed to solidify racist, gender, religious, or classist stereotypes in the educational world. Excitement can co-exist with intellectual and academic engagement and conflicting negotiations if people's differences are recognized and affirmed in conjunction with change, turmoil, and critical reflections on one's self and actions. Moving beyond traditional boundaries toward freedom in education, a freedom many teachers and students fear to live, challenges the status quo of existing educational realities (Giroux, 2005; McLaren, 2003).

Ongoing issues of equity and justice are supported in reconceptualized approaches with the following premises: (a) Equitable, culturally appropriate education is a fundamental human right for civilization and freedom; (b) shared cultural continuities of African descent people are "sources of strength and resilience, which can be used to transform educational research and to design learning environments that meet the needs of African students at all levels" (King, 2005, p. xxiii); (c) Black education has been overstudied from mainstream epistemological perspectives and understudied from the perspective that focuses on culture as an asset for learning; and (d) formal education is organized to miseducate and dominate groups not in the dominant culture (Anyon, 2005; King, 2005; Perry, 2003; Woodson, 1933). 
An alternative vision for schooling and socialization of teachers and learners is necessary to see the potentials for transforming oppressive educational practices (Wynter, 2005). The state of Black education in the United States is abysmal (King, 2005). In order to produce "knowledge and understanding people need to rehumanize the world by dismantling hegemonic structures that impede such knowledge" (p. 5) transformation of oppressive educational practices must occur. Even though the general state of education may be considered abysmal, inequitable educational outcomes disproportionately fall on nondominant students. Teachers who successfully teach nondominant students may be able to teach all students more successfully (Cochran-Smith \& Zeichner, 2005; DarlingHammond \& Bransford, 2005; Delpit, 1995; Hollins \& Guzman, 2005; Ladson-Billings \& Tate, 2004; Lee, 2005).

The fundamental root of the abysmal state of education lies in the crisis in thinking and of knowledge (Lee, 2005), or an epistemological crisis. The "normal science paradigm" (King, 2005, p. 7) which perpetuates the cultural deficit orientation of learning conceptualizes Black students as at risk or disadvantaged. Transformative education replaces the normal science paradigm with the "science of learning" (Lee, 2005, p. 57) paradigm which uses the construct of culture as an asset when designing learning environments. The science of learning views intelligence as dynamic, prioritizing prior experiences and the influence of social contexts of learning. Equitable transformative education does not categorize people to track them through school and, ultimately, into society. Instead, collective consciousness and identity are nurtured for social transformation (Nembhard, 2005). 


\section{Classrooms as Social Sites}

Viewing classrooms as social sites reconceptualizes the research context. A "social site .... [is a region] within society in which routine activities, or interactions, take place .... [as face-to-face] coordinated activities between people at certain places and times" (Carpspecken, 1996, p. 34). Shifting rules and shared understandings when the teacher and students interact to negotiate or potentially negotiate consent (i.e., setting shifts) within the classroom are the focus of this study. This focus led to insights on learning processes and cycles of social reproduction, such as exclusionary discipline. Setting shifts, not directly observable but tacitly understood, make interactions possible. They are agreed upon, negotiated, or challenged via interactive power statuses and roles to introduce a new setting and continue interactions for social integration (see Figure 1).

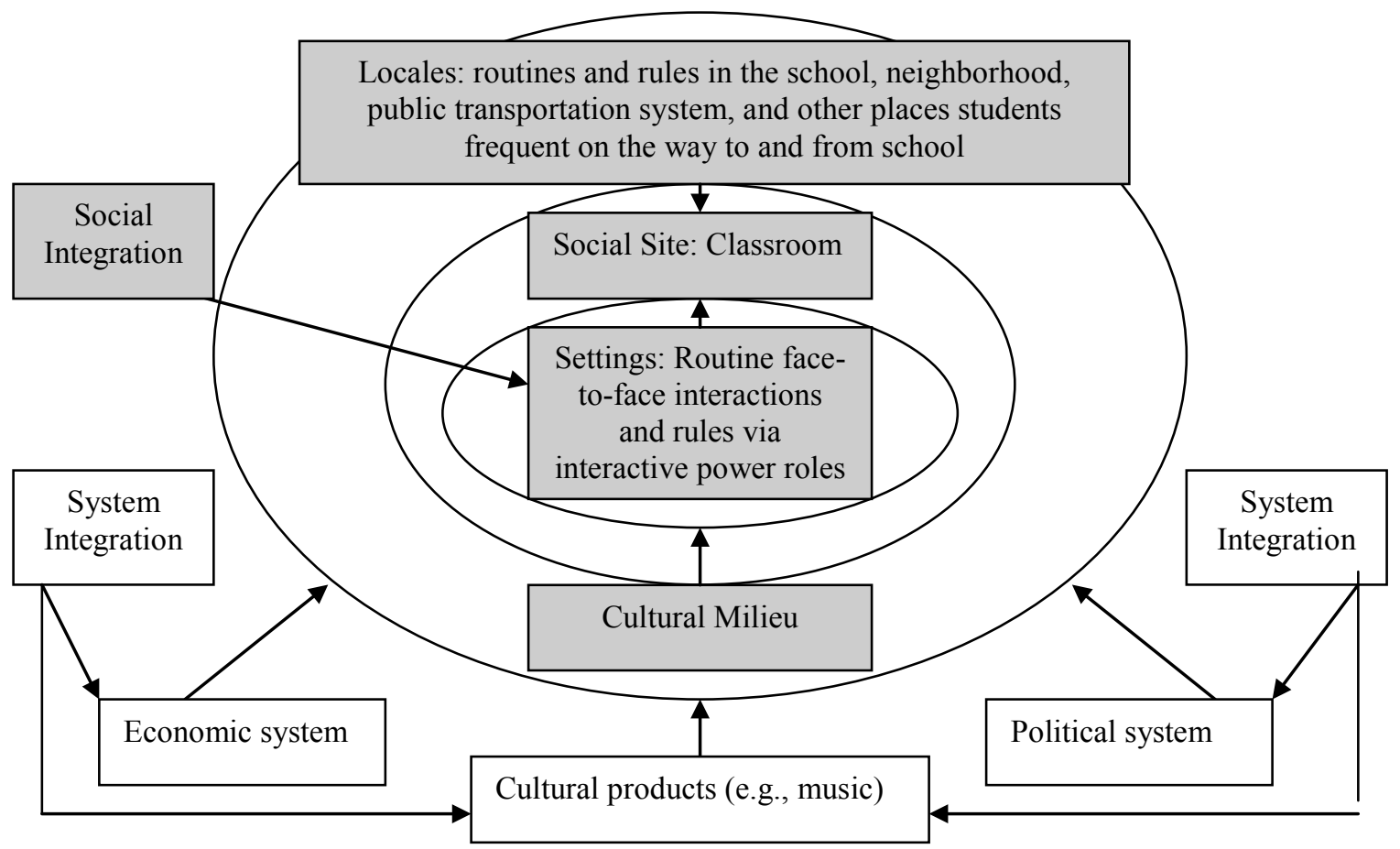

Figure 1. Social systems within social integration and system integration (adapted from Carspecken 1996, p. 39). 
In Figure 1, the social site of the classroom has predictable settings (i.e., routine face-to-face interactions) co-constructed by participants. Routines within classrooms include but not limited to decisions teachers and students make about behavior during classroom lessons (e.g., exclusionary discipline). Routines within classrooms are influenced by locales, "geographically wider areas [or conditions] surrounding the classroom" (Carspecken, 1996, p. 35) and the social integration of multiple face-to-face interactions. Routines can be explained by (a) institutional (i.e., school and classroom) rules; (b) compulsory district and state mandates; and (c) broad societal (i.e., economic, cultural, political) conditions.

Societal system patterns are widely distributed conditions that do not originate in one locale, but influence "activities throughout a large number of sites: a 'society" (Carspecken, 1996). For example, economic and political systems and cultural products that result from coordinated actions between groups of people separated by space and time in a society is referred to as system integration. Participants are either influenced or constrained by conditions in various settings. Influencing conditions "operate internally" (Carspecken, 1996, p. 37) and uphold participants' social identity, values, and beliefs allowing them to draw on and build upon familiar cultural themes when negotiating consent in particular situations. Constraining conditions operate externally forcing participants to cope with the particular system regardless of identity, values, or beliefs. Reproduction occurs when participants conform to the broad societal economic, political, and cultural conditions resulting from power relations (i.e., interactive power) during negotiations. However, in critical epistemology, participants have agency and may challenge, resist, and/or transform their conditions as they negotiate consent. 


\section{Research Methods}

This section covers (a) critical sociocultural theory; (b) Critical Race Theory (CRT) in education; (c) Critical Discourse (Theory and) Analysis (CDA); (d) critical social theory of literacy; and (e) informed by "interactive power analysis" (Carspecken, 1996, p. 130). These five critical theories and methods intertwine to inform critical microethnographic research methods in a reconceptualized research context.

\section{Critical Sociocultural Theory}

In order to understand historically and continuously marginalized, isolated, segregated, and nondominant "social practices and identities at work in society" (Gee, 2005 , p. 294) and to theorize transformative educational practices, a "critical sociocultural theory" (Lewis et al., 2007, p. 2) was needed for this study. Sociocultural researchers have focused on local situated literacies and cultural practices without attending to relationships among schooling, ideology, identity, power, and race. Taking on a critical sociocultural stance suggests literacy is found in multiple contexts and forms, makes sense to those involved as "part of other social, cultural, and political practices, and is therefore, never autonomous or decontextualized" (Gee, 2005, p. 5). A critical sociocultural viewpoint offers tools for studying local situated literacies and for distinguishing between culture and race. However, CRT in education and CDA were needed to more fully understand the uses of language among nondominant students and teachers in an alternative education classroom.

\section{Critical Race Theory in Education}

Microethnography can be used to explain within-group differences and individual identity development and agency by focusing on the daily life of classrooms (Bloome et 
al., 2005). Language is at the forefront, involving issues of social identity, power relations, and macroprocesses. However, since the microethnographic tradition rarely addresses how race informs the production of cultural practices, CRT in education (Ladson-Billings \& Tate, 1995) is needed. CRT provides additional tools for studying how micropractices of culture, learning, and power in classrooms with nondominant youth are intertwined in power and racism of societal structures.

CRT in education (Ladson-Billings \& Tate, 1995) consists of the following tenets: (a) counterstorytelling, told to challenge discourse that perpetuates racial stereotypes; (b) racism, recognized as permanent in society; (c) Whiteness as a form of property, used to challenge inequities in education reserved for White students; (d) interest convergence, used to question whose interests are at stake (e.g., Brown); and (e) critique of liberalism, used to challenge the proponents of "colorblindness, the neutrality of the law, and incremental change" (DeCuir \& Dixson, 2004, p. 29).

CRT in education enables a critical sociocultural perspective to get beyond a simple distinction between race and culture to discuss how race, racism, and power are deeply ingrained in American schools (Ladson-Billings \& Tate, 2005). In one study, literacy events such as reading and discussing books written from nondominant perspectives, or counternarratives, with characters the students can relate to were incorporated into pedagogy in traditional classroom settings (Perry, 2003; Rogers \& Fuller, 2007). Collective narratives, or classroom discussions, about how new types of literacy events help nondominant students pass the GED or other mandated tests were used when clashes occurred between student expectations and classroom practices. In this 
study, CRT tools were available to analyze how race informs the production of exclusionary discipline as a social practice.

CRT in education provides a forum for addressing "race, power, language, gender, identity, class, and social structure in relation to the opportunities and legal rights of individuals and groups" (Nasir \& Hand, 2006, p. 455). However, CRT alone tends to characterize racial or social class groups rather than explain within-group differences and individual identity development and agency.

\section{Critical Discourse Analysis in Education}

CDA in education is a theory and a method used to study the relationship between language and society through a critical social theory lens (Rogers, 2004). Discourse theory provides the tools for analyzing language in action (Rogers, 2005). Critical Discourse (Fairclough, 1995; Gee, 1996, 2005) and dialogic theory (Bakhtin, 1986) were needed to discuss how identities are produced and continually shift through language. In critical Discourse theory, identities are performed moment-by-moment in the classroom through constantly shifting power relations among teachers, students, and other participants. Language is the primary cultural tool and mediator of actions in activity systems, intertwined in macrostructures of power and ideology (Lewis et al., 2007).

CDA is used to theorize the uses of language and literacy learning and is a part of the event being analyzed. CDA in literacy research is "part of the study of social practices and identities at work in society" (Gee, 2005, p. 294). Unlike other discourse analysis methods which offer only a description and interpretation of discourse in context (Rogers, 2004), CDA includes an "explanation of why and how discourses work" (p. 2). The discourse part of CDA is the analysis of contexts to understand language in use 
assumed to be "constructing and constructed by social, cultural, political, and economic contexts" (p. 10). The critical part extends the analysis to learning processes and cycles of social reproduction. Educational micro- and macro-contexts include teacher-student conversations and interactions as well as larger sociocultural institutional and societal contexts. The analytic procedures systematically and reflexively move between a microand macro-analysis of texts and contexts to describe, interpret, and explain what is happening and what is not happening. CDA in education discerns how uses of language support or constrain opportunities for literacy learning in an alternative education classroom through potential spaces for transformation of oppressive educational practices.

\section{Critical Social Theory of Literacy}

Critical social theory of literacy (Freire, 1970/2000) supports CDA. Depending on how language is used, participants accept, resist, and/or transform educational practices. The underlying assumption is that all students are people who resist and produce language and literacy together with others (Moje \& Lewis, 2007). Although not assumed, unequal power relations related to historical, sociological, and political contexts of literacy learning should be actively acknowledged and critiqued (Barton \& Hamilton, 2000; Berkenkotter \& Thein, 2005; Tusting \& Barton, 2005). For example, who and what people are and how they interact (i.e., identities) can be critiqued. Who dominates or cares for others in a situation (i.e., power relations) can be interrogated. How people purposefully recreate themselves (i.e., agency) within shifting power relations can also be questioned. 
Critical social theory of literacy (Freire, 2000) supports the notion that all knowledge is produced within discourses of power/knowledge (Foucault, 1977). A social theory of literacy reconceptualizes literacy as a fundamental component of social events and practices rather than what students do or do not have (Hamilton, 2000; Maybin, 2000). Focus is on how people's oral language mediates their interactions around texts, how meaning and use of texts is dialectically and culturally shaped at the local level and influenced by broader discourses. Literacy practices are culturally constructed, historically situated, and represent people's social identities. Literacy practices are produced in and shaped by power relations that maintain the status quo of social institutions. Thus, some literacy practices are more dominant, valued, and influential than others.

Through critical social theory of literacy, schools are viewed as democratic public spheres (Dewey, 1916, 1939; Giroux, 2001; hooks, 1994). Critical literacy is a means of addressing social justice issues (Freire \& Macedo, 1987). Critical literacy also leads to shifts in agency and thinking and valuing of alternate Discourses (Gee, 1996) and perspectives. Discourses (Gee, 1996) with a capital "D" are identity kits by which we recognize others displayed in their attitudes, values, ways of acting, looking, interacting, and talking.

Interactive Power Analysis

Interactive power analysis involves determining "who has what kind of power and why" (Carspecken, 1996, p. 129) and who has the most say in defining and determining the end result of interactions. Power accompanies all actions and makes a difference in the subsequent events, "no matter how large or small" (p. 128). Even in the most 
constraining or coercive situations, participants have the choice to disobey or not comply in theory. Cultural power is noticed when tacit claims are distorted, recognized, and reflected by unequal power relations among participants whether unconsciously or semiconsciously. To spot the distortions, the claim to universality made by a participant assumes that all agree to the validity of the claim. When the norms and values claimed do not represent mutual interest of everyone involved and are false unreal choices, cultural power is at play. In reality, power and action are intertwined but the success of one's actions is dependent on how powerful the actions are - that is, how far removed the actions are from coercion. Interactive power is at its height when verbal and nonverbal inputs and interactions are most unequal among participants.

Gaps in the Research

Scant research examines communication patterns and interactions, which are fundamental elements in all social situations. Many quantitative studies from conventional views on learning and qualitative studies from sociocultural viewpoints have been conducted to examine educational problems and to suggest solutions for unsuccessful classrooms, students, schools, or teachers. A majority of these studies have taken place in urban K-12 contexts and informal educational settings, but rarely involved alternative education schools with nondominant students who have been suspended or expelled from their regular school for disciplinary reasons (Milner, 2006). Although relevant, classroom discourse studies rarely extend beyond sentence level analyses (Gee, 2006). Educational ethnographies rarely integrate CDA. Critical ethnographies rarely integrate classroom level interactions. Microethnographic studies integrate ethnographic methods, informed by discourse analysis (Bloome et al., 2005). However, few 
microethnographic studies have been conducted (a) from a critical social practice view on learning, language, literacy, and culture; (b) to establish and analyze interactive power relations involved in classroom situations; and (c) to describe, interpret, and explain classroom interactions related to exclusionary discipline in order to transform conditions.

\section{Summary}

The literature review was organized into seven sections: (a) exclusionary discipline, (b) alternative education, (c) urban education, (d) views of learning, (e) critical social practice theory of learning, (f) critical microethnography; and (g) gaps in the research. This summary concludes the chapter. Exclusionary discipline appears to originate at the classroom level as disproportionate systematic bias for nondominant students, particularly African American males. Research is needed to examine teachersstudent interactions preceding exclusionary discipline and sociocultural factors that influence teachers to use or not use exclusionary discipline. Alternative education was initiated under juvenile justice delinquency prevention and intervention legislation. Recent literature suggests a paradigm shift from a disciplinary to a more balanced approach to prevention and intervention. However, rather than getting at the root causes of antisocial behavior, the scant research that exists is largely descriptive. Urban education literature is useful for studying the implied link between classroom interactions and discipline. It focuses on reducing classroom conflicts and disproportional representation of African American students in the achievement gap. It acknowledges the importance of teachers' mindsets that lead to culturally responsive pedagogy and/or classroom management. However, few studies have explicitly examined the link between culturally responsive classroom management, teachers' beliefs, and discipline. 
A conventional view of learning initiated and still maintains alternative education today as isolated, segregated places where nondominant students are sent to be disciplined or cured of their academic and behavior problems. In contrast, a critical social practice view of learning provides a more positive and vibrant way of looking at what happens and why in classrooms with the goal of transforming oppressive educational practices and conditions, especially exclusionary discipline. Critical social theory of learning provides a framework for studying oppressive educational practices and conditions. However, few researchers frame their studies within a critical social practice theory of learning to explore the logic behind classroom interactions leading to the use or non-use of exclusionary discipline in order to transform the disproportional practice. Critical microethnography, informed by Critical Discourse Analysis and interactive power analysis, is useful for studying classroom interactions related to exclusionary discipline for transforming this stagnant, oppressive educational practice and condition.

Overall, this literature review covers a broader discourse about reconceptualizing teaching and learning, language, literacy, culture, classroom management, discipline, and research (reconceptualizations) necessarily supports and is supported by the theoretical framework of this study. Reconceptualizations are essential for understanding a critical social practice theory of learning defined within legitimate peripheral participation (LPP). As discussed in this chapter, the LPP theoretical framework focuses on processes of social transformation that shape and are shaped by how we think and participate individually and as a society. According to LPP, how and why interactions occur in a particular situation influence and are influenced by social practices simultaneously at micro- and macro-levels over time. No one person is in and of him or herself. No one 
situation is in and of itself. No one action is in and of itself. No theory is in and of itself and so on.

The key to understanding the synthesis of the LPP framework of this study and the suggested reconceptualizations presented in this literature review is to think of both as part and parcel to transformative studies of human activity and participation. As argued in the literature review, reconceptualizations are visualized as movement, transformation, or construction of knowledge among humans in social situations. To conjure up one term, action, or idea immediately elicits another for just long enough to change the situation for the moment and the discourse about the situation for a lifetime. Both changes depend on who is participating with whom. Reconceptualizations and the LPP framework, overall, provide a chance for anyone to transform or be transformed in the situation at hand or at large, an exciting prospect when working with nondominant students who have been labeled failures or in oppressive educational situations or marginalized segments of society.

The critical microethnographic method is explained in chapter 3. In chapters 4-7, the findings from each classroom are presented. In chapter 8, a comparison and synthesis of the findings is provided. Chapter 9 includes a summary of the study, responses to research questions, implications for teacher education, and recommendations for research and policy. 


\section{CHAPTER III}

\section{METHOD}

This chapter begins with the purpose of the study and research questions repeated from chapter 1 , followed by the critical microethnographic method, gaining access, participants, criterion sampling, cultural milieu, pilot study, and data collection and analysis stages. Integrity measures, validity measures for critical social research, data management, and a summary conclude the chapter.

\section{Purpose of the Study}

The purpose of this critical microethnographic study was to explore the relationship between classroom interactions and exclusionary discipline as a social practice within and across classrooms in the same alternative education school.

\section{Research Questions}

The central research question was: In relation to classroom interactions in alternative education settings, what accounts for teachers' use or non-use of exclusionary discipline with students? Secondary questions were:

1. What verbal and nonverbal interactions typify the learning processes and exclusionary discipline practices within the selected classrooms?

2. What cycles of social and academic identity reproduction occur within the selected classrooms?

3. How does each teacher explain what happens within his or her classroom regarding classroom interactions, learning processes, and exclusionary discipline practices? 
4. How do students explain what happens within their classroom regarding classroom interactions, learning processes, and exclusionary discipline practices?

5. What is the relationship between classroom interactions, learning processes, and exclusionary discipline practices across the selected classrooms?

\section{Critical Microethnographic Method}

A critical microethnographic method (Bloome et al., 2005) was used to answer the questions of this study. Critical microethnography originates in cultural anthropology and sociolinguistics and is "informed by discourse analysis to study the social practices of schooling" (Vavrus \& Cole, 2002, p. 90). After being explored for 30 years by linguistic anthropologists, it is now widely accepted that the role of language can be more fully understood by studying both the relationship between the verbal and nonverbal forms of language and the cultural practices that they help represent (Duranti, 2004). To study this relationship, "a microethnographic approach to discourse analysis allows the researcher to attend to how people use language and other systems of communication in constructing language and literacy events in classrooms with attention to social, cultural, and political processes" (Bloome et al., 2005, p. xv).

Critical microethnography also draws from critical ethnographic methods to focus on learning processes and cycles of social reproduction (Carspecken, 1996; Lave \& Wenger, 1991). Critical ethnographic methods are used to describe, interpret, and explain the meanings people give to their own cultural practices and experiences through a critical lens that links power, knowledge, and truth (Kamberelis \& Dimitriadis, 2005). Critical microethnographic methods provide qualitative, observational, cross-cultural, and ethnographic data, giving educational researchers the potential to (a) examine schools, 
teachers, and students within and across their community contexts (Cazden, 2001; Rist, 1970; Spindler \& Spindler, 1994), explicitly addressing class, power, and cultural structures of that community (Bowles \& Gintis, 1976; McLaren, 2003; Willis, 1977); and (b) explain disproportional educational achievement among subgroups, particularly the disadvantaged (Carlson \& Apple, 1999; Goetz \& LeCompte, 1984).

For this study, the critical microethnographic method accomplished three goals. First, the critical part of critical microethnography involved studying (a) what was being learned, (b) how and why that something was being learned, and (c) what was being socially reproduced as a result of the power relations that occurred during the learning processes. Second, the micro- part of critical microethnography involved using aspects of Critical Discourse Analysis (Gee, 2005; Rogers, 2004; Rogers, Malancharuvil-Berkes, Mosley, Hui, \& Joseph, 2005) to analyze how (a) people used verbal and nonverbal language; and (b) language was being used in the context of cultural, social, and political processes. Third, the ethnographic (Creswell, 1998; Goetz \& LeCompte, 1984; Spradley, 1979) part of critical microethnography involved (a) observing participants' cultural behaviors over time, (b) interviewing participants about what happened over time, and (c) collecting relevant documents and artifacts that were produced over time. The following section describes the procedures for gaining access to conduct research.

\section{Gaining Access}

Gaining access took place at the institutional and school level. For institutionallevel access, the M-DCPS Office of Research Services was contacted following proper protocol with FIU Institutional Review Board regulations. Approval was given to conduct research in M-DCPS. For school-level access, the researcher gained approval to conduct 
research from the principal of one of the 16 M-DCPS alternative education secondary $\left(7^{\text {th }}-12^{\text {th }}\right.$ grade) schools, henceforth referred to as The School. At their first meeting, the researcher explained the study to the principal, Dr. Jones, a White male, who welcomed the prospects of the study. He expressed an interest in the potential connection between the dissertation research topic and the Positive Behavior Support (PBS) program he had initiated two years earlier when he became The School's new principal.

The School was one of two single-gender male alternative education schools in M-DCPS. Student population was a mix of African Americans and Latinos. After determining a start date for the research study that did not conflict with high-stakes (FCAT) testing, Dr. Jones assigned his assistant principal, Ms. Garcia, a Latina, to serve as key informant. For classroom-level access, Ms. Garcia initiated an email to all teachers in The School, with the screening questionnaire (Appendix A) attached, requesting participants for the study. Ms. Garcia requested that teachers respond to the researcher who would be their contact person from that point forward. After her second request, six teachers who were willing to participate in the study responded to the researcher. Ms. Garcia ranked the six classrooms (cases) by each teacher's frequent or rare use of exclusionary discipline. Referrals were the required documentation for exclusionary discipline in The School per school district mandates.

\section{Participants}

Participants were chosen because they were information-rich in the coconstruction and negotiation of social practices and discipline consequences for their particular classroom as shaped by supportive or constraining classroom, school, and societal structures (Patton, 2002). For this study, cases that met the following criteria 
were selected: (a) 4 classrooms in the same M-DCPS alternative education school; (b) 2 classrooms with teachers who frequently used exclusionary discipline; and (c) 2 classrooms with teachers who rarely used exclusionary discipline.

\section{Criterion Sampling}

Criterion sampling occurred in two phases for (a) classroom observations and teacher interviews and (b) student observations and interviews. Criterion sampling was used to determine all cases that met the criteria needed to provide "a great deal [of information] about matters of importance and therefore worthy of in-depth study" (Patton, 2002, p. 242) in order to illuminate the questions of the study.

\section{Classroom Observations and Teacher Interviews}

The first sample selected for classroom (case) observations and interviews were teachers from 4 different classrooms. From the rankings provided by Ms. Garcia, the researcher selected 4 teachers ( 2 teachers who often and 2 teachers who rarely used exclusionary discipline in their classroom) who fit the criteria for the study (Patton, 2002). The 2 teachers who often used exclusionary discipline taught middle school Language Arts, one White male and one Latina; the 2 teachers who rarely used exclusionary discipline taught Culinary Arts, a White male, and Science, a White male from Sweden. Noting his national origin is significant because of the relationship between his accent and ensuing classroom interactions.

At the suggestion of Ms. Garcia, a group meeting was held with researcher and the 4 classroom teacher participants around a table in the Latina language arts teacher's classroom on a teacher workday. First, the researcher explained and answered questions the teachers had about the study. Second, the researcher and teachers developed a 
tentative data collection schedule for the classroom observations. Third, each teacher gave the researcher a copy of his/her official M-DCPS class list of students, and a date and time was agreed upon for the researcher to come to each class and discuss the study with the students. Teachers expressed concern about the timely collection of the necessary signed parental consent/teen assent form (see Appendix B) based on past unsuccessful attempts to collect signed permission forms for field trips and service learning projects. Therefore to jumpstart the collection, each teacher agreed to distribute the forms in class immediately, and the researcher committed to calling the students' guardians or parents to discuss the study and consent forms. Fourth, teachers signed a consent form (see Appendix C). Finally, the researcher visited each classroom to determine placement of the camcorder during observations. The researcher spent a few minutes in three of the classrooms; however, the researcher spend more than half an hour with the Culinary Arts teacher as he talked about how he ran his classroom.

\section{Student Observations and Interviews}

The second sample selected for student observations and interviews were students from each of the 4 selected classrooms. Prior to the researcher's entrance into each classroom to introduce herself, discuss the study, and collect signed consent/assent forms from the students, the researcher made several calls to each phone number on the class lists provided her at the group meeting held with the teachers. The purpose of the phone call was to inform the parents/guardians of the study and alert them to the consent/assent forms that their child would be bringing home for their signature to participate in the study. From 33 students listed, the researcher successfully contacted 8 parents/guardians; 
left 13 messages; and reached 10 incorrect or disconnected phone numbers. Ultimately, 16 students, 4 from each class, returned their signed consent/assent form.

Upon entrance into each classroom (named in the order each initial classroom observation occurred) to meet the students for the first time, the researcher was introduced by each teacher. In Classroom One, the White male Culinary Arts teacher sat at a table and introduced the researcher to the students, asked her to discuss the study thoroughly, encouraged questions from the students, and also asked the researcher questions. The atmosphere resembled an informal conversation around a kitchen table. In Classroom 2, the Latina Language Arts teacher stood to tell students that the researcher would introduce herself and tell them why she was there. She and the students, seated at desks in a circle, listened quietly while the researcher, also seated at one of the desks in the circle, talked about the study; then, students asked a few procedural questions. In Classroom 3, the White male Science teacher stood and did not introduce or solicit explanations about the study from the researcher, who also stood; the students, seated at tables, did not ask any questions. In Classroom 4, the White male Language Arts teacher introduced the researcher, discussed the students' participation in detail, asked the researcher to explain the study, and then asked for questions from the students. A few students asked questions.

In order to meet the start date requested by the principal and the criteria for student participants, the researcher told each teacher that she would begin data collection as soon as 4 students returned the signed form. Each teacher from Classrooms 1, 2, and 3 collected signed consent/assent forms from 4 students in the pre-selected class periods before the study was scheduled to begin. Classroom One consisted of 2 Latinos and 2 
African Americans. Classroom 2 consisted of one Latino and 3 African Americans.

Classroom 3 consisted of 4 Latinos. The teacher from Classroom 4 did not receive any signed forms from students in his originally selected class period; as a result, he selected another class period that did not conflict with the data collection schedule and successfully collected signed consent/assent forms from 4 students. Classroom 4 consisted of one Latino and 3 African Americans. Table 1 provides a summary of criterion sampling results.

Table 1

Criterion Sampling Results

\begin{tabular}{|c|c|c|c|c|}
\hline $\begin{array}{l}\text { Classroom } \\
\text { Subject Area } \\
\text { Grade Level }\end{array}$ & $\begin{array}{l}\text { Teacher } \\
\text { Pseudonym, } \\
\text { Ethnicity }\end{array}$ & $\begin{array}{l}\text { Student } \\
\text { Pseudonym }\end{array}$ & $\begin{array}{l}\text { Student } \\
\text { Ethnicity }\end{array}$ & $\begin{array}{l}\text { Student Age or } \\
\text { Grade Level }\end{array}$ \\
\hline $\begin{array}{l}\# 1 \\
\text { Culinary Arts } \\
6^{\text {th }}-12^{\text {th }}\end{array}$ & $\begin{array}{l}\text { Mr. Jenkins } \\
\text { (White) }\end{array}$ & $\begin{array}{l}\text { Green } \\
\text { Hector } \\
\text { Patt } \\
\text { Raul }\end{array}$ & $\begin{array}{l}\text { African American } \\
\text { Latino } \\
\text { African American } \\
\text { Latino }\end{array}$ & $\begin{array}{l}7^{\text {th }} \\
18 \text { y.o. } \\
7^{\text {th }} \\
\text { Unavailable }\end{array}$ \\
\hline $\begin{array}{l}\# 2 \\
\text { Language } \\
\text { Arts } 6^{\text {th }}-8^{\text {th }}\end{array}$ & $\begin{array}{l}\text { Ms. Gomez } \\
\text { (Latina) }\end{array}$ & $\begin{array}{l}\text { Alberto } \\
\text { Dann } \\
\text { Jonathan } \\
\text { Stanley }\end{array}$ & $\begin{array}{l}\text { Latino } \\
\text { African American } \\
\text { African American } \\
\text { African American }\end{array}$ & $\begin{array}{l}8^{\text {th }} \\
8^{\text {th }} \\
8^{\text {th }} \\
8^{\text {th }}\end{array}$ \\
\hline $\begin{array}{l}\# 3 \\
\text { Science } \\
6^{\text {th }}-8^{\text {th }}\end{array}$ & $\begin{array}{l}\text { Mr. } \\
\text { Frederek } \\
\text { (Swedish) }\end{array}$ & $\begin{array}{l}\text { Antonio } \\
\text { Carlos } \\
\text { Jack } \\
\text { Julio }\end{array}$ & $\begin{array}{l}\text { Latino } \\
\text { Latino } \\
\text { Latino } \\
\text { Latino }\end{array}$ & $\begin{array}{l}8^{\text {th }} \\
8^{\text {th }} \\
8^{\text {th }} \\
\text { Unavailable }\end{array}$ \\
\hline $\begin{array}{l}\# 4 \\
\text { Language } \\
\text { Arts } 6^{\text {th }}-8^{\text {th }}\end{array}$ & $\begin{array}{l}\text { Mr. Glass } \\
\text { (White) }\end{array}$ & $\begin{array}{l}\text { Derrick } \\
\text { Green } \\
\text { Juan } \\
\text { Roberto }\end{array}$ & $\begin{array}{l}\text { African American } \\
\text { African American } \\
\text { Latino } \\
\text { African American }\end{array}$ & $\begin{array}{l}7^{\text {th }} \\
7^{\text {th }} \\
7^{\text {th }} \\
7^{\text {th }}\end{array}$ \\
\hline
\end{tabular}


If more than 4 student consent/assent forms had been collected in any of the classrooms, a criterion sample of 4 or fewer students from each class who were most highly involved in disciplinary (or potential disciplinary) moments during observations would have been selected for the videorecording feedback interviews. However, since only 4 students from each classroom returned signed consent/assent forms before the start deadline and were involved in observations, all students who attended school the day of the scheduled student interview session participated in it.

\section{Cultural Milieu}

This section describes the cultural milieu of The School, Classroom One, Classroom Two, Classroom Three, and Classroom Four. A description of the cultural milieu provides common features of the research site for greater understanding of power and how it is distributed (Carspecken, 1996). Quotes are cited by transcript name (e.g., T6B), line number (e.g., L271), and participant pseudonyms when necessary (e.g., Mr. Jenkins).

\section{The School}

The School was in its third year as a single-gender male alternative education school. It had been temporarily relocated for the year from its previous site on the adjacent lot to an old vocational school building that had been used for administration

offices. The structure that previously housed The School had been razed in order to build a contemporary two-story "green" magnet school, which was in progress during the study. The busy daily hum of construction could be heard from the new green magnet school's towering building next door. The School was scheduled for relocation again the 
following year to an uninhabited elementary school much further south to make room for the new green magnet school's parking lot.

Resembling traditional vocational school building architecture, The School consisted of several freestanding buildings and portables. The main office area was located in the front of The School and housed administrative, secretarial, and support personnel offices (e.g., school psychologist, guidance counselor), as well as the media center. Classrooms, some of which were housed in portables, were located behind the office area with walkways or hallways leading to each one. Specific areas set up for vocational electives such as auto mechanics, culinary arts, or horticulture also fanned out behind the main office. Vocational electives were offered to students who had passed the FCAT. The cafeteria was across the street from the main office. After being transported to school in district school buses, students assembled in the cafeteria for a supply check before going to their first class. The cafeteria was also used to serve students lunch in several phases and as an auditorium for programs.

Visitors signed in at a designated table in front of the office, which was attended by a personable African American woman. Behind the sign-in table hung a PBS poster, promoting The School's philosophy. It was one of four schools in M-DCPS that had recently been recognized as a Florida PBS Model School. PBS offered a school-wide point system to assess students' behavior. Students who achieved sufficient points attended a weekly reward activity. PBS charts were exhibited in each classroom (but not referred to or used during classroom observations) to designate the location of the timeout bench, points earned by each student, and 10 rules students must follow in order to earn their points. The 10 rules are as follows: 
1. arriving for class on time punctually, not one second after the passing bell

2. attired in the proper school uniform

3. bringing one's own supplies: paper, pencil or pen

4. electronic devices, including ear-phone plug-ins, cell phones, i-pods, and mp-3 players are turned off and out of plain view

5. keeping one's hands and feet off of any other person in the classroom

6. using appropriate language without profanity

7. fully participating in all class activities

8. not creating or causing disruptions through one's language or behavior

9. giving maximum effort by using one's capabilities to the fullest extent

10. not leaving one's seat at the closure bell, waiting, instead, for the teacher to dismiss

The cultural milieu was pervaded with the 10 Positive Behavior Support rules for rewarding student behavior. Casual conversation and comments insinuated the following norms: (a) students must be coerced into behaving, (b) students behave badly so they need 10 rules to follow, (c) teachers must always be on the lookout for bad behavior, (d) students give some teachers a hard time, (e) teachers must help students do the academic material since the students have learning challenges, and (f) bad students are bad learners.

\section{Classroom One}

Classroom One was a Culinary Arts classroom/kitchen located across the outside hallway from the main office area in the front of The School. Colorful culinary arts posters and PBS charts lined the walls. The front wall had a board on which the daily vocabulary words were written. Writing tables were situated by the front door. The teacher/computer desk was located near the writing tables on the center of the side wall between the back door and the freezer. A long culinary work table stretched across the center of the room, separating the writing tables and the kitchen area. Movable cooling/ rising racks filled the side of the room opposite the teacher desk. The smell of freshly baked cookies and bread was common. Large kitchen appliances were located in the 
center of the kitchen area; storage shelves and cabinets covered the back periphery. To conduct classroom observations, the researcher sat near the front door at a table that openly stored students' journals and glossaries for vocabulary work. During the observations, Mr. Jenkins occasionally made a comment to the researcher or asked her opinion. Students casually talked to the researcher before but not during observations.

\section{Classroom Two}

Classroom Two was a language arts classroom located on the opposite side of the school from Classroom One in a separate wing that stretched out perpendicular to the main office area. From the office, an L-shaped covered walkway led to the double doors of the wing. Upon entering the building, Classroom Two was the first classroom on the right in a single hallway with six classrooms, three on each side. The sun shone brightly into the rectangular-shaped room. Colorful language arts posters and PBS charts lined the walls and front of a cabinet. Student desks were situated in a circle near the front door. To the right of the front door, in the corner, was a tall bookshelf that held language arts textbooks and FCAT workbooks. A white board, the teacher's bicycle, and the teacher/computer desk lined the adjacent wall. To the right of the teacher desk was a table that housed neatly organized trade books followed by a computer station. Separated by a back door, the rest of the computer station lined the back wall. In the center of the room near computer station was a kidney-shaped table with chairs, not used during the study.

To the left of the front door was another white board behind which was a table in the back of the room that blocked the cabinet door. To conduct classroom observations, the researcher sat next to the back table. Ms. Gomez did not acknowledge the researcher during classroom observations, and she expected the same from students. For example, 
when a student asked about the camera or the researcher's presence, Gomez reminded him to pretend that the camera was not there. She answered, "Why are we doing the [silent pause] that's not here? We already talked about that when she was here before" (T4A, L67). Ms. Gomez also eased the student's concerns of "other students watching us" (T4A, L72, Dann) by confirming that no other students would watch the videos.

\section{Classroom Three}

Classroom Three was a science classroom located at the back of The School in one of two portable buildings on site. From the office, Classroom Three was reached by exiting the L-shaped covered walkway that led to Classroom One and Two and walking straight past the neglected horticulture garden. Classroom Three was the third of three classrooms, next door to CSI (indoor suspension), in the portable on the left. Facing this portable building, divided by a concrete slab, was an identical portable. A parking lot for staff member or visitor use was behind Classroom Three's portable. Classroom Three was rectangular in shape; at one end were a student restroom, sink, folder storage table, and window overlooking the parking lot. The researcher sat at this end of the classroom during observations near several haphazardly located science supply boxes.

A teacher desk/computer, audio-visual equipment, and another window were at the other end of the classroom near the front door. Six student tables were spaced out in the middle of the classroom in three rows, facing the white board on the wall to the left of the front door. Frederek used one long table that stood near the white board to organize materials needed to teach each class. A tall cabinet, low bookshelf, and another long table lined the opposite wall behind the student tables and held science supplies and textbooks. Colorful science posters, maps, and PBS charts covered the walls. Mr. Frederek and the 
students did not talk to the researcher during observations; however, students glanced toward the camcorder periodically. Before class on the first day of observations, one student walked past the camcorder, blurted a surprised expletive, and flexed his muscle.

\section{Classroom Four}

Classroom Four was a language arts classroom located next door to Classroom Two. The sun shone brightly into the rectangular-shaped room. Just inside the front door of Classroom Four to the right stood a tall bookshelf filled with literature reference books and textbooks, and some dictionaries. Eight small cut-outs illustrating literature genres were affixed to the wall above the white board adjacent to the bookshelf. To the left of the front door was a small table where the researcher sat for observations. The wall behind the table was lined with PBS posters and charts and another white board. On the same side of the room were the teacher's computer/desk, overhead projector, and tall storage cabinet in the back corner. Beside the cabinet along the back wall was a bulletin board reserved for exemplary work and a table with folders organized by class. Student desks were in rows and faced different directions depending on the day's lesson plan. For example, desks faced the television, which was hanging from the front corner, if students watched an English literature play that day. On these days, some of the window shades were pulled down for easier viewing. Desks faced the wall opposite the windows if $\mathrm{Mr}$. Glass lectured with the overhead projector that day. Mr. Glass discussed all topics openly with the researcher before, during, and after the classroom observations. Students were cordial beforehand but did not talk to the researcher during the classroom observations. 


\section{Pilot Study}

As required by my dissertation committee and the Office of Graduate Studies (OGS) to indicate my experience with the complex nature of the proposed data analysis method before the research study began, I conducted a pilot study. The pilot study was based on a videorecorded 2-hour classroom observation and a videorecording feedback interview with two students involved in the classroom observations. Carspecken's (1996) critical ethnographic data collection and analysis research stages suggested for dissertation research and Bloome and colleagues' (2005) microethnographic research methods provided the model for this study. Both were adapted to the goals of this study as a result of the pilot study.

Three glitches that occurred during the data collection process were corrected in the data collection stages for this study. First, I videorecorded the classroom observations with a mini-DVD camcorder. Each mini-DVD provided less than an hour of recording time; it was disruptive when I had to take out the mini-DVD and replace it with a new one during the classroom observations. For an uninterrupted videorecording of each classroom observation, a digital camcorder with an internal hard drive and/or a flash memory was used, providing from 4 to 21 hours of videorecording at a time. Second, in order to reduce the amount of undecipherable talk when viewing and transcribing the videorecording, a digital recorder could be placed near the teacher or in a central location among participants while the camcorder runs during classroom observations. Clip-on microphones could also be used on each participant. However, neither adaptation was appropriate, due to the intimate context of each classroom setting, or essential due to the small number of participants. Third, the voice-activated mechanism on the digital audio 
recorder tended to delete the first syllable or word spoken by participants during the interview session; therefore, it was turned off during the study.

The critical microethnographic research stages were also focused, clarified, and expanded from the original five to the current nine stages for replicability and manageability, using Carspecken's (1996) terminology for critical ethnographic research: (a) Stage One - data collection for the primary record; (b) Stage Two-reconstructive data analysis; (c) Stage Three_- dialogical data generation; and (d) Stage Four-highlevel coding; and (f) Stage Five-final reconstructive data analysis. In addition to the adaptations resulting from the pilot study, the order of Carspecken's (1996) five stages were adapted in this study to take into consideration typically high attrition rate of the student population of this study. Walking through the transcription and analyses steps allowed me to determine an appropriate and meaningful length of the videorecorded segment to be analyzed from each classroom. Twenty-minute segments provided ample time to determine patterns in classroom interactions. As I transcribed and conducted the videorecorded observation and feedback interview data, I timed each stage. Then, I merged, revised, clarified, and detailed the steps within each stage and projected the time that would be necessary for each stage of data collection and analysis for this dissertation study (see Appendix D for Projected Data Collection and Analysis Schedule). I also created a 6-month timeline to be able to visualize the research schedule (see Appendix E for Tasks for Study Timeline). Then, I met with one of my dissertation committee cochairs and two doctoral peers to discuss the results of the pilot study as well as the reliability of the analyses procedures and the transcripts. Based on their feedback, the 
pilot study transcripts were enhanced for clarity by including a key prior to the analyses for each stage. Pilot study transcripts are included in Appendix F.

\section{Data Collection and Analysis Stages}

This section delineates the data collection and analysis stages for this critical microethnographic study (see Appendix G for Summary of Data Collection and Analysis Stages) as follows: (a) Stage One_-data collection for the primary record; (b) Stage Two - building a primary record from classroom observations; (c) Stage Threedialogical data generation; (d) Stage Four-building a primary record from representative classroom observations; (e) Stage Five-reconstructive data analysis of representative classroom observations; (f) Stage Six-building a primary record from interviews; (g) Stage Seven—reconstructive data analysis of interviews; (h) Stage Eight—high-level coding; and (i) Stage Nine-final reconstructive data analysis. Data collection and analysis in critical microethnographic research are recursive, or self-repeating, until a specific point is reached (Carspecken, 1996). For this study, the goal was to determine the pattern of how disciplinary (or potential disciplinary) moments were negotiated in each classroom in order to find potential spaces for transformation of oppressive conditions (exclusionary discipline). A sample transcript from Classroom Three data collection and analyses is used to illustrate each stage when applicable.

\section{Stage One-Data Collection for the Primary Record}

This section covers Stage One-data collection for the primary record, consisting of (a) passive observations, documented with (b) videorecordings, (c) primary record notes, and (d) field journal notes (Carspecken, 1996). 


\section{Passive Observations}

Three passive observations for a total of 5 hours were conducted in each classroom to document interactions among participants during classroom lessons. Passive observations are conducted by an uninvolved passive observer who builds up a primary record "through notetaking and...if desired, videotaping" (Carspecken, 1996, p. 41). Each passive observation was one hour and 40 minutes long, extending 5 minutes before and after each $1 \frac{1}{2}$ hour block schedule period. Block scheduling at this school consisted of class periods scheduled either on even (Monday and Wednesday) or odd (Tuesday and Thursday) days, rotating every other Friday.

In this study, classroom interactions were defined as linguistic behavior (i.e., verbal and nonverbal interactions). Verbal and nonverbal interactions can be thought of as any language activity going on anywhere, not the traditional notion of a language activity going on only in a language arts class but rather how the teacher and students use (verbal and nonverbal) language during interactions with each other during any classroom lesson (Lewis et al., 2007). Any classroom lesson in any subject area can be observed because language is an integral part of all classroom activity, interactions, and lessons. All students in the class and the teacher were observed during the classroom lessons. The unit of analysis was verbal and nonverbal interactions taking place in classroom activity between and among students, the teacher, and texts or documents (i.e., material culture) used or produced during classroom lessons. Table 2 summarizes the student classroom observation participants and total number of observations per student. 
Table 2

Student Classroom Observation Participants

\begin{tabular}{|c|c|c|}
\hline Participant & Classroom & Observations \\
\hline Green & One & 2 \\
\hline Hector & One & 3 \\
\hline Patt & One & $1 \frac{1 / 2}{2}$ \\
\hline Raul & One & 3 \\
\hline Jorge & Two & 1 \\
\hline Dann & Two & 3 \\
\hline Jonathan & Two & 3 \\
\hline Stanley & Two & 2 \\
\hline Antonio & Three & 2 \\
\hline Carlos & Three & 2 \\
\hline Jack & Three & 3 \\
\hline Julio & Three & 3 \\
\hline Derrick & Four & 3 \\
\hline Green & Four & 1 \\
\hline Juan & Four & 1 \\
\hline Roberto & Four & 3 \\
\hline
\end{tabular}




\section{Videorecordings}

A digital camcorder was used to document the 5 hours of passive observations in each classroom. For all classroom observations, the researcher placed the camcorder on a tripod in the predetermined section of the classroom and turned it on before the students began coming into the room. All classroom observations were used for data even though participants spent some time (and in various ways) getting used to the presence of the researcher and losing interest in the camcorder (Berkenkotter \& Thein, 2005; Bogdan \& Biklen, 2003). For example in Classroom One, Green verbalized how he did not feel like being filmed even though he signed the consent form to participate. Other participants rarely acknowledged the presence of the researcher and camcorder. In Classroom Two, when Dann asked Ms. Gomez about the researcher and camcorder, she talked about the researcher in third person reminding students that they were to act like it (the camera) was not there. In Classroom Three, while Mr. Frederek was standing at the door before class, Carlos shouted an explicative of surprise when he saw the camcorder. Otherwise, Mr. Frederek ignored the camcorder, and the students briefly glanced at it every now and then. In Classroom Four, Mr. Glass openly acknowledged the camera and introduced the researcher again to the students at the beginning of the study, detailed where the camera would be located, and talked directly to the researcher about setting up for the study. Throughout the study, Mr. Glass talked about what day of videorecording it was, when and how the interviews would occur in the future, and how and what the students were expected do during each part of the study. Three students barely acknowledged the camcorder or researcher. Green, however, who was also in Classroom One, negotiated 
with Mr. Glass during the opening minutes of classroom observations about the possibility of not being filmed and going to CSI instead.

\section{Primary Record Notes}

To augment data from the videorecordings which cannot capture everything that happens, the researcher wrote detailed primary record notes during each passive observation in a separate legal pad for each classroom (Carspecken, 1996). Primary record notes include (a) "highly detailed accounts of observed activity that include . . speech acts in verbatim form and many observations of body movements and postures" (p. 53); (b) the date, with time noted frequently, (c) classroom contextual information (e.g., posters on the wall or other material culture); (d) diagrams of how the classroom is set-up; and (e) observer comments [OC], bracketed to speculate meaning of interactions (Carspecken, 1996).

The following is a sample of primary record notes from Classroom Three, using Carspecken's (1996) format for primary record notes; spoken words are italicized.

Classroom Three, Monday, February 2, 2009

1:30 pm (Posters: science fair, chemistry, Positive Behavior Support)

Carlos: (headphones on, hoodie up)

Teacher: STOP IT! (to students laughing)

Carlos: Does it say sluts? (looking at word faults on board)

Teacher: (goes out door to get security) [OC: threat, loud voice]

Carlos: So it's faults? (gets ready, rips off shirt)

Security: (takes Carlos out, student head down, students looking quiet) Jack: Why did you kick him out?

Teacher: You're next. (immediately into the topic of faults and earthquakes) [OC: Why on earth does an earthquake matter at this point?]

In this sample, Classroom Three science teacher, Mr. Frederek, left the room to call the security guard after a student made a vulgar interpretation of the word faults, one of the words being copied from the board that day. The researcher's observer comment 
speculates that Mr. Frederek was threatening the student by using a loud voice and searching for the security guard. Then, one of the remaining students asked him why Carlos was kicked out to which Mr. Frederek replied that he may be next and immediately began lecturing on earthquakes. The researcher's observer comment emits a similar concern about Mr. Frederek's reaction to Carlos's misinterpretation of a word; observer comments and concerns are addressed during the interview sessions.

\section{Field Journal Notes}

After each passive observation, 15 to 20 minutes was set aside to self-debrief (Wengraf, 2004) about the experience in field journal notes (Carspecken, 1996). Selfdebriefing is "free associative flow" (Wengraf, 2004, p. 143) writing or "theoretical memos" (Wengraf, 2004, p. 210), which are notes about the experience, based on ideas and memories, feelings, or content intended to provoke the researcher to produce initial interpretations. The researcher wrote field journal notes as separate emails to herself with the classroom number and day number in the subject line and saved in a folder entitled The School. The field journal notes were reviewed and then a contact summary sheet (see Appendix H for both) was filled out (Miles \& Huberman, 1994). The contact summary sheet included questions that were helpful in summarizing information received from the observations, and new or remaining questions and concerns to guide fieldwork. Field journal notes and contact summary sheets were used to augment and compare transcribed data analyses with additional contextual information for the researcher's interpretation. As noted in the researcher's initial interpretations found in the samples provided in Appendix $\mathrm{H}$, concerns centered around what appeared to be a disconnection between the teacher and students. For example, the teacher ignored students' continual use of foul 
language but removed a student for a vulgar interpretation of a word on the board, and the teacher lectured to students who appeared to be tuning him out.

\section{Stage Two-Building a Primary Record from Classroom Observations}

This section covers Stage Two_- building a primary record from classroom observations, which consisted of viewing the videorecordings to determine a representative segment of interaction patterns for each classroom. "Building a primary record prioritizes the objective validity claim: claims open to multiple access ... . [it] is a sort of massive claim to represent what took place in a manner any observer or participant would report under ideal conditions" (Carspecken, p. 87). A primary record was built for each classroom based on Carspecken's (1996) format for building a primary record. To build a primary record, the videorecordings of each passive observation were viewed two times with notes taken by the researcher. The first viewing and notetaking session was conducted to determine a 1-hour segment with the disciplinary (or potential disciplinary) moments most representative of the classroom interactions patterns. The second viewing and theoretical notetaking session was conducted to determine a 20 minute segment with the disciplinary (or potential disciplinary) moments most representative of the classroom interactions patterns. Each representative 20-minute segment was burned onto a DVD and used as video data in the videorecording feedback interviews. As reflected in Table 3, two students were not represented in the video data for their class. Jorge had been invited to listen to the mayor speak at The School one day; Juan had gone home after being involved in a fight one day. 
Table 3

Student Representation in Video Data

\begin{tabular}{|c|c|c|}
\hline Participant & Classroom & Representation \\
\hline Green & One & Yes \\
\hline Hector & One & Yes \\
\hline Patt & One & Yes \\
\hline Raul & One & Yes \\
\hline Jorge & Two & No \\
\hline Dann & Two & Yes \\
\hline Jonathan & Two & Yes \\
\hline Stanley & Two & Yes \\
\hline Antonio & Three & Yes \\
\hline Carlos & Three & Yes \\
\hline Jack & Three & Yes \\
\hline Julio & Three & Yes \\
\hline Derrick & Four & Yes \\
\hline Green & Four & Yes \\
\hline Juan & Four & No \\
\hline Roberto & Four & Yes \\
\hline
\end{tabular}




\section{Stage Three-Dialogical Data Generation}

Stage Three — dialogical data generation consisted of two subsessions: (a) Stage Three A—interviews with students and (b) Stage Three B — interviews with teachers. Dialogical (i.e., conversational) data are generated during research interviews to democratize the research process (Freire, 2000) by soliciting and prioritizing participants' perspectives, or subjective and normative-evaluative truth claims (Carspecken, 1996). Participants' perspectives were solicited to augment the researcher's preliminary and reconstructive data analysis. The researcher should be cognizant of the "histories and social roles of those involved" (Wengraf, 2004, p. 42), which strongly affect the power relations and anxiety levels during research interviews.

\section{Stage Three A-Interviews with Students}

This section covers Stage Three A—interviews with students, consisting of (a) group videorecording feedback interviews, facilitated by an (b) interview protocol and documented with a (c) digital recorder and (d) field journal notes.

Group videorecording feedback interviews. Group videorecording feedback interviews with each sample of students were conducted to gain their perspectives on what happened and why during the representative 20-minute segment of videorecorded observations from their classroom. When a discourse analysis is not (as in this study) part of a larger ethnographic study, dialogical data can be generated with videorecording feedback interviews (Bloome et al., 2005; Carspecken, 1996). Videorecording feedback interviews are conducted by sharing video data and preliminary interpretations of it with one or more of the participants who are also asked "about contextual knowledge that might better inform the interpretations" (Bloome et al., 2005, p. 184). 
Videorecording feedback interviews were scheduled separately for each sample of students and were conducted in a quiet space in the school during the same classroom period as the observations. For Classroom One and Three student interviews, the media center specialist found empty classrooms to which the researcher took the student participants. For Classroom Two interview, the researcher used the media center which was empty for the first half of the interview, but a staff member came in to use a computer towards the end. Classroom Three teacher offered to take any students that were not part of the study to the media center while the student interview was conducted in his classroom. All participants in attendance the day of the interviews completed the interviews with the researcher. Table 4 summarizes student interview participation. As illustrated, Classroom One had one missing participant due to suspension; Classrooms Two, Three, and Four had two missing participants each due to suspension or absence.

Table 4

Student Interview Participation

\begin{tabular}{lll} 
Student Participant & Classroom & Participation \\
\hline Green & One & Suspended \\
Hector & One & Yes \\
Patt & One & Yes \\
Raul & One & Yes \\
Jorge & Two & Suspended \\
Dann & Two & Absent \\
Jonathan & Two & Yes
\end{tabular}




\begin{tabular}{lll}
\hline Student Participant & Classroom & Participation \\
\hline Stanley & Two & Yes \\
Antonio & Three & Yes \\
Carlos & Three & Suspended \\
Jack & Three & Yes \\
Julio & Three & Absent \\
Derrick & Four & Yes \\
Juan & Four & Yes \\
Green & Four & Absent \\
Roberto & Four & Absent \\
\hline
\end{tabular}

Interview protocol. The researcher constructed a lightly-structured videorecording feedback interview protocol (see Appendix I), which was read to the participants to introduce the videorecording feedback interviews. The interviewee(s) were told that they would view a 20-minute segment of the videorecordings from recent classroom observations. The interviewee(s) were invited to stop the videorecording whenever they wanted to discuss what was happening and why during the lessons. The videorecording feedback interview process consisted of playing the 20 -minute video data segments, selected for Stage Two — reconstructive data analysis, "back to the [participants] and allowing them to comment on any portion of the events they choose to . . . elicit 
articulations of tacit cultural material as well as for stimulating the expressions of subjective material" (Carspecken, 1996, p. 163).

The purpose of the videorecording feedback interview was to discuss both the researcher's and interviewees' interpretations of what was happening and why so that the interviewees' viewpoints were clearly understood by the researcher to incorporate in the analysis. Classroom One and Three students expressed their interpretations openly. If no (as was the case for Classroom Two) or few (as the case for Classroom Four) student interpretations were offered, the researcher asked the students questions about the video data to provoke conversation and comparison among interpretations. Nonetheless, Classroom Two students did not express any viewpoints; Classroom Four students expressed only a few viewpoints.

Digital recorder. To begin each interview session, the format was explained to the interviewees so that they felt comfortable with the situation. The presence of a digital recorder was discussed and then placed between the researcher and interviewees.

Field journal notes. Immediately after each interview session, 15 to 20 minutes was set aside to self-debrief about the experience in the field journal notes (Wengraf, 2004). Field journal notes were then reviewed, and a contact summary sheet (see Appendix H for both) was filled out to summarize the interview (Miles \& Huberman, 1994). The following is a sample of the field journal notes and contact summary from Classroom Three student video data feedback interview:

Field journal notes: The students were into the DVD and talked about what was happening and why very explicitly. They were in tune to Mr. Frederek's every move, other students' jaw movements and what they meant. They thought the teacher was so patient and that made him a good teacher because he doesn't get 
rid of kids easily. They also thought he was a good teacher in that he knew his content.

Contact summary sheet: The students were talkative about how the teacher is patient and they try him but know his and other students' moves and why down to the jaw muscles in someone's face. Students felt part of the room and wanted to talk about what goes on and compare it to other rooms. The students talked about what and why the teacher does things. They think a good teacher is one who is patient and wants to deal w/ you. Other teachers are not so patient so kids try them past the limit so they can laugh when they get mad. It is exciting - they want excitement in the classroom. The students were very involved and had much to say and knew a lot about Science.

After the student interviews, the researcher's initial interpretations of classroom interactions between the teacher and students began changing. Students viewed Mr. Frederek as patient and a good teacher, and they explained why and how they came to these conclusions. Also, they discussed intricacies about the topic of Science that they had learned in his class. The students' explanations and comments illuminated an understanding of the teacher that observations alone did not portray.

Stage Three B-Interviews with Teachers

This section covers Stage Three B-interviews with teachers, consisting of (a) individual lightly-structured depth (Wengraf, 2004) interviews and (b) individual videorecording feedback interviews, both facilitated by (c) interview protocols and documented with a (d) digital recorder and (e) field journal notes.

Individual lightly-structured depth interviews. Individual lightly-structured interviews were conducted to gain teachers' perspectives on school in general. Lightlystructured depth interviews (Wengraf, 2004) stimulate in-depth exploration of a topic by providing a space for each participant's voice and using participants' vocabulary (Schensul, Schensul, \& LeCompte, 1999). Each of the 4 teachers was interviewed 
separately. The interviews took place in each teacher's classroom during his or her planning period. All teachers completed the interview with the researcher and provided their interpretations of the video data.

Individual videorecording feedback interviews. Individual videorecording feedback interviews were conducted immediately after the individual lightly-structured interviews with each teacher. They were conducted to gain each teacher's perspective on what happened and why during the representative 20-minute segment of videorecorded observations from his/her classroom.

Interview protocols. The researcher constructed a lightly-structured depth interview protocol (see Appendix J) to facilitate the individual interviews. Three peer debriefers provided feedback on the protocol prior to the study. Peer debriefers were members of a small group of four doctoral students who provided feedback to each other on their dissertation writing before it was presented to a larger dissertation group of 25 doctoral students who met every 6 weeks to review each other's work. Lightly-structured interview protocols allow "maximum flexibility during the interview process" (Carspecken, 1996, p. 156) and draw out implicit theories comprising people's actions, rather than asking interviewees to talk about particular theories. Interview protocols also organize thoughts into headings and give "information about starting the interview, concluding ideas, information on ending the interview, and thanking the respondents" (Creswell, 1998, p. 126).

The lightly-structured interview protocol included open questions and possible follow-up questions in an effort to anticipate potential directions for more elaborate responses during the interview (Carspecken, 1996; Wengraf, 2004). The open questions 
pertained to concrete events (Gee, 2006) about a typical teaching day and a typical day for students in The School. Possible follow-up questions from the domains of life and society (Gee, 2006) were included in an effort to anticipate potential directions for more elaborate responses during the interview (Carspecken, 1996; Wengraf, 2004). Life questions pertained to each teacher's everyday classroom life in general. Society questions solicited more academic explanations and opinions about how and why things are used in class and by whom (Gee, 2006). The researcher developed classroom-specific follow-up society questions related to literacy according to observations prior to the interviews (see Appendix J). Depending on the dialogue level, active follow-up questions and probes were also improvised during the interviews (Wengraf, 2004).

The researcher then facilitated the videorecording feedback interviews in the same manner as conducted in Subsession One. Afterwards, the researcher took digital photos of the material culture of each classroom; she also collected documents that three of the teachers offered: one copy of a referral sheet from Ms. Gomez; two earthquake worksheets from Mr. Frederek; one Siddharta handout, one Merchant of Venice handout, and one suspension list from Mr. Glass.

Digital recorder. The digital recorder for Subsession Two interviews was treated in similar fashion to the Subsession One interviews.

Field journal notes. Field journal notes procedures were conducted in the same manner as the Subsession One interviews. The following is a sample of the field journal notes and contact summary from Classroom Three teacher interview:

Field journal notes: This teacher is caring about his students to the point that he tries hard not to kick out the students (like classroom 2). He weighs what is worth kicking kids out for before taking the step because he said that the referrals go on 
their record (like classroom 2) and besides that the referrals are political in that "they" (the administration) decides what to do about them. The teachers are isolated and do not find out about what happens once kids leave their room. He talked about they, they, versus us, us. He tries to do multiple things so as not to lose the kids' attention.

Contact summary sheet: The main concern of this contact was the gap between us and them (the administration). He said that he tries to keep the students from irritating each other, that he tries to do many different types of teaching, and that he tries not to refer them outside the class because he doesn't know what happens and it goes on their permanent record. He said that they come in and act good and then something happens that they change. He explained quite a bit about what happens and why he does what he does, he really cares about them and is patient picking his battles. He feels isolated from other teachers. He knows a lot about what is happening but ignores a lot. He was interested in seeing himself on the video and thought it was a good idea to do this for teachers.

After the teacher interview, the researcher's intial interpretations changed even more and comparisons between other classrooms entered her understandings. She saw another side of the teacher that coincided with what the students knew of him. He was patient and did not write referrals but let students go out for a breather instead. He understood the political agenda surrounding education as a gap between the teachers and administration. As a result, he tried to solve problems in class so that students would not get lost in the system through the written referral. Gaining an understanding of the teachers' perspectives illuminated another view and greater understanding of the classroom interactions that observations did not portray. After Stage Three procedures, the process of building a primary record from classroom observations began.

\section{Stage Four-Building a Primary Record from Representative Classroom Observations}

This section covers Stage Four-building a primary record from representative classroom observations, conducted in three subsessions: (a) Stage Four A-transcribe meaningful utterances, (b) Stage Four B - analyze meaningful utterances and linguistic 
behavior observed, and (c) Stage Four C — analyze possible objective validity claims. See Appendix K for an example of Stage Four procedures.

Stage Four A-Transcribe Meaningful Utterances

Stage Four - transcribe meaningful utterances involved the third viewing of representative 20-minute video segments from each class to transcribe meaningful utterances. Meaningful utterances were consecutively numbered throughout each transcript.

Stage Four B-Analyze Meaningful Utterances and Linguistic Behavior Observed

Stage Four B - analyze meaningful utterances and linguistic behavior observed involved the fourth viewing of the representative 20-minute video segments from each class to analyze the meaningful utterances for linguistic behaviors (e.g., speech acts, body movements, postures) observed. Verbatim transcriptions based on meaningful utterances go beyond traditional conversational analysis (see Appendix L, Transcription Key; Appendix M, Conversational Functions; Appendix N, Argument Elements) of turns at talk (Gee, 2005). Turns at talk are defined as changes in who talks and although important to acknowledge "do not provide a sufficiently integral unit of conversation to use as a basic unit of analysis for the purpose of understanding the social construction of conversational meaning and action" (Bloome et al., 2005, p. 22). Meaningful utterances, on the other hand, are part of a series of actions and reactions that are tentatively determined by what went on before and after each moment in the event. Meaningful utterances are often defined in terms of breath or other pauses from the perspective of what the speaker wants to accomplish (i.e., illocutionary perspective). 
Linguistic behaviors observed denote verbal and nonverbal interactions by describing how words are spoken with the use of the phrase as if. Each utterance is meaningful only if and when it communicates a "who-doing-what" (Gee, 2006, p. 23). A who does not necessarily mean people but involves identity-building and can be multiple and overlapping (i.e., heteroglossic). Making who-doing-what visible requires more than tabulating the turns at talk and analyzing the utterances made in language. It requires a study of how who-doing-what is recognized through language and interaction with others in particular situations and locations (i.e., linguistic behaviors observed).

\section{Stage Four C-Analyze Possible Objective Validity Claims}

Stage Four C-analyze possible objective validity claims involved reading the transcript to analyze possible objective validity claims (i.e., possible meanings) about the observed linguistic behaviors. Truth claims (i.e., assertions) are interpreted by considering the related validity conditions. Validity conditions or claims are based on what needs to be done or said to gain the consensus or understanding of any cultural group. Consensus involves negotiating, agreeing to, challenging, and/or transforming what is happening in a setting. Per critical epistemology, truth claims will never be final (Carspecken, 1996), but all possible claims suggested by an act or interaction were articulated. All participants have open access (i.e., or multiple access which means anyone in the room who was listening or looking could hear or see the interaction or object) to objects, spoken words, and observed linguistic behaviors. However, reaching agreement on observable truth claims, objects, and/or events depends on the cultural themes participants use to defend (i.e., negotiate) each claim and/or argument. For this reason, the researcher's interpretations and analyses of objective data were compared 
with subjective data (i.e., participants' personal perspectives with privileged access) subsequently collected in Stage Three during participant interviews.

After the primary record was built, primary record notes, field journal notes, and contact summary sheets were reread and compared. Linguistic behaviors and/or validity claims that augment the primary record were included.

\section{Stage Five-Reconstructive Data Analysis of Representative Classroom Observations}

This section covers Stage Five - reconstructive data analysis of representative classroom observations, conducted in three subsessions: (a) Stage Five A-initial meaning reconstruction, (b) Stage Five B_-“pragmatic horizon analysis” (Carspecken, 1996, p. 103), and (c) Stage Five C_-validity reconstruction. Stage Five took place immediately after the primary record was built. Reconstructive data analysis is the 3-step inductive process of interpreting tacit cultural and subjective material, that is, "meanings of interactions in the primary record" (p. 93), into explicit discourse.

\section{Stage Five A-Initial Meaning Reconstruction}

This section covers Stage Five A-initial meaning reconstruction, which involved (a) low-level coding and (b) explicit meaning reconstruction of the representative 20minute video segment for each class. Initial meaning reconstruction was conducted to gain a holistic picture of routines or irregular events (i.e., continuity in settings and/or change or shifts in settings) in everyday life (Carspecken, 1996). Initial meaning reconstructions provide illustrations and tentative explanations about what is happening in the setting (i.e., interactions) based on observations. Explanations in initial meaning reconstructions were derived holistically by inferring possible meanings of what was observed — and would probably be agreed upon by all participants — into words. 
Low-level coding. Low-level coding involved two steps. First, the primary record was read to holistically picture possible underlying meanings. Next, retaining the original, a copy of the primary record was coded with low-level coding procedures to construct categories that named types of interactions. Low-level coding references "mainly objective features of the primary record" (Carspecken, 1996, p. 147) that are open to multiple access which means anyone in the room who was listening or looking could hear or see the interaction or object (see Appendix O an example of low-level coding, noting objectivity levels of codes and categories).

Explicit initial meaning reconstruction. After low-level coding was completed, explicit initial meaning reconstructions, or possible underlying meanings of the interactions, were inferred hermeneutically and articulated in brackets line by line. Hermeneutic inferences are not gained by multiple access, but by noting possible intentions of actions, how actions are monitored by the actor, and how others understand the act. Hermeneutic inferencing requires the ability to take positions (i.e., intersubjectivity) and to recognize, reflect on, and explicitly examine and differentiate between personality and cultural typifications (i.e., intricacies in settings) of participants and others such as the researcher (Carspecken, 1996). See Appendix P for a sample transcription with inferences of possible underlying meanings.

Explicit initial meaning reconstructions were conducted with three peer debriefers on one of the 20-minute segments to check inference levels of the low-level codes and to support and/or challenge the researcher's articulated validity claims, biases, and cultural typifications. The researcher trained the peer debriefers briefly by going over the hermeneutic inferencing processes involved in the explicit initial meaning reconstructions 
completed on one of the 20 -minute segments. The peer debriefers then provided hermeneutic inferencing from other possible positions the researcher may have omitted. The peer debriefers also stressed the existence of unequal power relations between the teacher and students as well as possible implications arising from the fact that both classrooms with frequent suspension involved the subject of Language Arts.

Since initial meaning reconstructions are based only on observations and researcher-articulated possible validity claims, pragmatic horizon analysis and validity reconstructions are needed to analyze subjective and normative-evaluative claims. Subjective and normative-evaluative claims are of privileged access, existing in each person's individual opinions, understandings, beliefs, and/or consciousness. Stage Five B-Pragmatic Horizon Analysis

This section provides an (a) overview of pragmatic horizon analysis, followed by an explanation of (b) contextualization cues; (c) boundary-making; (d) turn-taking patterns; (e) thematic coherence; and (f) intertextuality (Fairclough, 2003), which are five theoretical tools for CDA that were adapted for pragmatic horizon analysis (Bloome et al., 2005). CDA is "a method of analyzing a wide variety of different types of spoken and written language within social practices" (Gee, 2005, p. 294). Analyzing specific communication structures that are used as tools to accomplish particular goals in interactions is an inductive process and can never fully determine the meanings expressed but can represent a range of potential meanings for a moment in time. Multiple products resulting from the CDA were used for subsequent validity reconstructions.

Overview of pragmatic horizon analysis. Pragmatic horizon analysis in this study involved the articulation of communication structures or linguistic behavior (i.e., 
pragmatic meaning units) within the interactive classroom context (i.e., semantic meaning units) of each of the four classrooms (Carspecken, 1996; Fairclough, 2003). Pragmatic horizon analyses of linguistic behaviors were conducted on each of the selected 20-minute representative video segments of the primary record. In pragmatic horizon analysis meaning reconstruction, actions — rather than perceptions —are the key to understanding experience (Habermas, 1981, 1987). A pragmatic horizon, or field of meaning, consists of temporal and paradigmatic axes. The temporal axis relates to shared awareness about past and future events. The paradigmatic axis relates to communication structures and "validity inferences at various levels of foregrounding" (Carspecken, 1996, p. 110).

Borrowed from phenomenology, a horizon of meaning encompasses how one perceives an entire experience, the equivalence of seeing a particular object in clear view with the background objects visible but out of focus. Pragmatic horizon analysis contrasts the idea in focus (i.e., foreground object) with where the idea originated (i.e., background experience or understanding). For example, Mr. Frederek, who after ignoring disruptive bothering each other behavior, suddenly used a very loud voice (i.e., foreground) to gain the attention of the class based on his experiences with students in this school (i.e., teachers who use loud voices gain attention of the students) even though he did not verbalize that understanding (i.e., background) at the time. The idea or action of using a very loud voice becomes understandable as a meaningful action when explicit and culturally shared linguistic behaviors are analyzed in reference to communication structures over time and space. 
Moreover, when Mr. Frederek used a loud voice followed by Carlos's sudden interests in copying a word off the board (i.e., foreground) is considered in reference to the possible consequences, Carlos's response can be further understood through the conjecture of possible underlying meanings (i.e., background). By changing his behavior, Carlos may be trying to make an attempt to not get kicked out of class because he is truly interested in learning but got carried away and/or does not want to entertain the possibility of getting suspended. For a moment in time, the power of his action could have been accepted by Mr. Frederek. However, Mr. Frederek decided to remove him for a breather. Even though this scenario ended in removal, the power relations of this moment could have shifted in multiple directions. The group will ultimately decide (coconstruct) by negotiating whose claim becomes legitimate (i.e., what will happen next).

Contextualization cues. Contextualization cues are ways people make their intentions known as they interact with others in the event (Bloome et al., 2005). People act and react to contextualization cues in a particular situation depending on past, present, and future understandings. Contextualization cues include (a) verbal (i.e., register and syntactical shifts); (b) nonverbal (i.e., gesture; facial expression and direction; eye movement, gaze, and contact; posture, postural configurations, and distancing; body movement and style of body movement); (c) prosodic signals (i.e., volume, tone, and rhythmic shifts; stress, stress patterns, and stress shifts; velocity shifts; pausing; intonation patterns and shifts); and (d) artifact or material culture (i.e., chairs, homework, books) manipulation. A "reflexive view of the relationship between language and context" (Gee, 2004, p. 29) was maintained during the process. In this context, reflexive 
means that utterances influence the meaning of the context as the context simultaneously influences the meaning of the utterance.

While simultaneously viewing each videorecording and reading the corresponding primary record segment to augment interpretations, contextualization cues were analyzed (see Appendix Q for a sample of a contextualization cues transcript). Transcription symbols were inserted into the transcripts to make contextualization cues visible (Bloome et al., 2005; Berkenkotter \& Thein, 2005). After contextualization cues were analyzed, boundary-making analyses of message unit boundaries, interactional unit boundaries, and phases of lessons were conducted.

Boundary-making. Boundaries are socially constructed interactional behaviors that are given meaning when participants propose, maintain, and/or resist them together (Bloome et al., 2005). The message unit, or "smallest unit of conversational meaning" (p. 19) were identified and interpreted by how participants acted and reacted to the contextualization cues. Message units are not necessarily turns at talk, meaningful utterances, or complete sentences. Rather, message units are defined as linguistic behaviors that hold shared meaning within the context of an event based on how the behavior impacts listeners. While reading each selected representative segment, message unit boundaries were identified and interpreted (see Appendix Q for a sample transcript identifying/interpreting message unit boundaries).

Larger segments of conversation, known as interactional units (Bloome et al., 2005), are tied by several message units. Interactional units are the "smallest units of joint social activity ... [ [involving] the actions and reactions of people toward each other" (p. 26). Interactional unit boundaries were graphically represented by a vertical line drawn 
across the page above and below the interactional unit and its number, written in caps to easily distinguish it from other words in the transcript (e.g., INTERACTIONAL UNIT 1). Closure (i.e., the end of the interactional unit) of interactional units depends on intonations and contextualization cues during the conversation. When closure was determined, a vertical line was recorded below this point. To augment interpretations, interactional units were identified while reading each selected representative segment (see Appendix Q for a sample transcript indicating interactional units).

Several interactional units comprise phases of lessons, and several phases of lessons constitute the entire event (Bloome et al., 2005). Phases may be explicitly introduced by the teacher; however, some phases are not planned for in advance (i.e., student interruptions); interactional units, phases of lessons, and entire lessons are culturally constructed prominent changes in interactional patterns signaled by linguistic behaviors and participation structures of group members. Phases were recorded numerically and by name (e.g., PHASE 17, TEACHER ATTEMPTS TO STOP STUDENT TALK WITH LOUD AND EMPHATIC DIRECTIVES AND THREAT OF KICKING STUDENT OUT OF CLASS) on the primary record with a double vertical line before the phase began and after the phase ended. If more than one lesson occurs in the transcript, each lesson is differentiated with a bold line across the transcript before and after each lesson. However, this did not occur in this study. While reading each selected representative segment, phases of lessons within the complete lesson were identified (see Appendix Q for a sample transcript indicating phases of lessons).

Turn-taking patterns. Turn-taking as simple tabulations of when and how often a person speaks is not interpretable unless defined within the social institution in which an 
event occurs and analyzed within the participation structure as socially constructed by the participants (Bloome et al., 2005). A common traditional classroom participation structure is the Initiation-Response-Evaluation/Feedback (I-R-E/F) sequence (Cazden, 2001). In I-R-E/F, the teacher initiates a question/statement, the student responds, and the teacher evaluates the response via explicit or implicit feedback about the correctness of the answer/repeating a response. Participation structures are used by participants to decide whether and how to interpret and participate or not in an event. I-R-E/F sequences usually limit students to short prerequisite answers and may limit generative, transformative thinking and learning. In addition, cross-cultural differences between students and the teacher may result in unshared expectations about how to participate in various phases of lessons/events (Delpit, 1995). While reading a new copy of each selected representative segment, I-R-E/F turn-taking patterns and/or anomalies were identified by noting whether the linguistic behavior was an initiation, response, evaluation, or feedback (see Appendix R for a sample transcript with I-R-E/F sequences indicated).

Thematic coherence. Thematic coherence is defined as how meanings are organized and/or negotiated during an event through ideas, interactions, and/or texts (Bloome et al., 2005). Participants decide whether an event has thematic coherence by asking what is going on in the event and what everyone is talking about. Not all events have thematic coherence, while some events may have multiple overlapping themes at multiple levels. Other events have little thematic coherence, noticeable when participants question and/or contest what is happening either through verbal or nonverbal interactions. Themes can be declared by any participant and agreed upon or not by the others involved. 
Themes are analyzed by determining what is and/or assumed to be forefronted (i.e., focused on) and what and how themes are shifted and negotiated during the lesson. How overlaps or interruptions are negotiated, confirmed and/or resisted with the other students and the teacher determines which theme will continue by agreement of the participants or whether thematic coherence is or is not achieved. For example, if interruptions become the focus of the class, thematic coherence of the lesson would be traditionally negotiated either by consensus (e.g., all students enter into the side conversation) or dissolution (e.g., teacher halts the lesson to call for the security guard or write a referral to the office). While reading each selected representative segment, thematic coherence was analyzed (see Appendix R for a sample transcription with thematic coherence indicated).

Intertextuality. Intertextuality occurs when two or more written, verbal, nonverbal, or electronic texts share a feature, refer to one another, or lead to another text (Bloome et al., 2005). A traditional view of intertextuality is that of students simultaneously reading from a text, writing notes, and conversing with others in the classroom. In his or her mind, the traditional teacher may isolate worksheets and/or textbooks, the traditional texts, from their production and consumption (Fairclough, 1995, 2003). For example, the teacher may not acknowledge publishers' ideological agendas. The teacher may not consider the notion that students have forgotten the lesson or have been talking to someone about what they did last night during the lesson. Also, students may not be able to connect previous knowledge to the worksheet and/or textbook.

As a theoretical tool for CDA, however, interactions among heterogeneous texts or material culture (i.e, television, math books, conversational text), or heteroglossia (Bakhtin, 1981), are acknowledged and recognized using Fairclough's (1995) three- 
dimensional framework for CDA of text, discourse practice, and sociocultural practice. Thus, intertextualities were defined as socially constructed interactions among texts, contexts (i.e., intercontextualities), and discourses (i.e., interdiscoursivities) with social consequence (Fairclough, 1995). Intertextualities, intercontextualities, and interdiscoursivities are proposed, acknowledged, recognized, agreed upon, or contested among those involved based on "bits and pieces of prior discourse that index social, cultural, and historical contexts" (Berkenkotter \& Thein, 2005, p. 203). While reading a copy of each selected representative segment, intertextualities were identified (see Appendix $\mathrm{S}$ for a sample transcription with intertextualities indicated). After pragmatic horizon analysis was accomplished through CDA, validity reconstruction was conducted.

\section{Stage Five C-Validity Reconstruction}

Validity reconstructions provide insights into a culture by attending to the "validity claims routinely employed in the construction of meaningful action" (Carspecken, 1996, p. 110). Validity reconstructions make the analysis of setting shifts and negotiations more precise (Carspecken, 1996, p. 117). Articulating validity claims entails reconstructing possible subjective, objective, and normative-evaluative claims (i.e, horizontal analysis) and simultaneously distinguishing between various levels (i.e., highly, immediate, less, remotely) of foregrounded and backgrounded claims (i.e., vertical analysis). Validity reconstructions get at the meaning of meaningful acts that are typically tacit, or backgrounded, in nature.

Validity reconstructions attempt to articulate the reasons a participant would use to defend an action or reaction and/or clear up a misunderstanding. These reasons, or validity claims, were differentiated by horizontal and vertical analysis for the final step of 
beginning analyses. While reading a copy of each selected representative segment, validity reconstructions (Carspecken, 1996) were conducted (see Appendix T for a sample transcription of validity reconstructions). At this point, validity reconstructions were conducted on multiple meaningful utterances, as themes are beginning to emerge more readily. After validity reconstructions were accomplished, Stage Five proceduresreconstructive data analyses of representative classroom observations were complete.

Stage Six—Building a Primary Record from Interviews

Stage Six — building a primary record from interviews was conducted in two subsessions: (a) Stage Six A-listen to and transcribe student interviews; and (b) Stage Six B - listen to and transcribe teacher interviews. The procedures were similar to those in Stage Four A for transcribing meaningful utterances, but using interview data.

\section{Stage Seven-Reconstructive Data Analysis of Interviews}

Stage Seven—reconstructive data analysis of interviews involved two sessions. Stage Seven A-initial meaning reconstruction, involved low-level coding of student interviews (See Appendix U), and Stage Seven B-initial meaning reconstruction, involved low-level coding of teacher interviews (See Appendix V). Stage Seven procedures adhered to the same logic of Stage Five A, Part 1, but using interview data. After all interviews were analyzed using Stage Seven procedures, Stage Eight—highlevel (i.e., abstract) coding was conducted.

\section{Stage Eight—High-level Coding}

Stage Eight—high-level coding procedures were based on data other than the representative segments from the primary record. High-level coding was supported by matches between interview statements and exemplary pragmatic horizon analyses. "High 
level codes are dependent on greater amounts of abstraction" (Carspecken, 1996, p. 148) than low-level codes which were used in initial meaning reconstruction and to select portions of the primary record for intensive analysis. Each high-level code "should be backed up with an exemplary horizon analysis .... [and] ideally ... match statements made by participants during the interviews ... of Stage Three" (Carspecken, 1996, p. 148). High-level codes are needed to "generalize findings that emerge from ... particularly meaning and validity reconstruction, horizon analysis, and the analysis of interactive power" (p. 148).

High-level coding produced general categories and many different codes under each category (i.e., raw codes). Multiple, detailed, redundant, and overlapping emergently developed raw heading categories and codes from each classroom existed at this point. The same three peer debriefers provided feedback on the high-level codes developed by reviewing the codes and categories and producing more of their own. See Appendix $\mathrm{W}$ for a sample of high-level coding, supported by matches between interview statements and exemplary pragmatic horizon analyses. After high-level codes were developed and matched, the raw heading categories and codes were then reorganized to focus the final analysis by conducting final reconstructive data analysis.

\section{Stage Nine - Final Reconstructive Data Analysis}

Stage Nine - final reconstructive data analysis was conducted in three subsessions: (a) Stage Nine A-interactive power analysis and role analysis, (b) Stage Nine B - thematic analysis, and (c) Stage Nine C—comparative analysis. 
Stage Nine A-Interactive Power Analysis and Role Analysis

Interactive power and roles were analyzed through the pragmatic horizon analysis and validity claims they entailed. First, the researcher deductively listed and analyzed the types of power that participants employed or claimed to gain consent and the right of power over others. Carspecken's (1996) typology of interactively established power was utilized, including (a) normative power, or by virtue of holding a higher position in the culture or society, (b) coercive power, or by forcing obedience after threatening punishment, (c) interactively established contracts, or by negotiating based on an implicit level of understanding between participants, and (d) charm, or by using one's personality.

Second, using Carspecken's typology, the researcher also determined the cultural milieu and cultural themes drawn upon by participants to answer why power was distributed. Interactive power is best found by utilizing the typology within the cultural milieu wherein participants' interactions occur and which the researcher has gained familiarity. In other words, power is distributed and consent is gained (or not) within cultural norms at play in each situation and the cultural themes negotiated among various participants in the roles they act out. See Appendix X for a sample of interactive power analysis. Finally, based on validity reconstructions and interactive power analysis, the researcher inductively determined and named participants' roles to provide a greater understanding of routine interactive patterns and sequences.

Stage Nine B-Thematic Analysis

Thematic analysis was conducted in several steps with the data from each classroom, augmented by the results of the interactive power analysis. First, "certain codes [were] grouped together into a few large categories" (Carspecken, 1996, p. 151) 
according to the questions of this research study. The resulting large categories and accompanying codes were organized thematically for patterns and meanings. Thematic analyses were reread and regrouped into many intermediate categories. The themes consisted of variations on teacher reproducing (conforming to) dominant ideology; student reproducing (conforming to) dominant ideology; teacher resisting dominant ideology; student resisting dominant ideology; and negotiation of classroom culture, norms, and values. Variations overlapped with student profiles and histories, PBS requirements, and authority figure in classrooms. At this point, the researcher went into the literature to find terms that would clearly summarize and capture at a glance the themes that had already emerged. Giroux's (2001) explanations of conformism, resistance, and accommodation in his theory of resistance in education and Third Space literature (Gutiérrez, 2008) concerning negotiation provided the terminology needed for clarity and differentiation of the nuances of the final themes. These use of these terms allowed explanations of the nuances that had already emerged from the data, but more concisely labeled. Finally, the overlapping intermediate categories were regrouped into final larger categories, or themes of social interactions, using the terms conformism, resistance, accommodation, and negotiation.

Stage Nine C-Comparative Analysis

A comparative analysis of the findings across the 4 classrooms was conducted in order to answer the questions of this study.

\section{Integrity Measures}

Integrity of qualitative research is determined by its trustworthiness, or value to the audience, through its credibility, transferability, dependability, and confirmability 
(Patton, 2002). Credibility of data collection was established through member-checking, which means to ask participants to confirm meanings developed by the researcher during initial inductive data analysis (Creswell, 2003). Data were triangulated from multiple observations and interviews to validate the findings of the study. Data were also triangulated through comparative analyses to address the research questions of the study.

Transferability of findings was augmented by maintaining a detailed researcher audit trail of the critical microethnographic method, enabling readers to determine if findings are relevant to their situation. Dependability was enhanced by my dissertation advisor and the Office of Graduate Studies (OGS) who provided feedback during the dissertation process. In addition, three doctoral students, who were in my dissertation peer group, provided feedback throughout the study. Confirmability was augmented by keeping objective, thorough, and organized records safely stored.

\section{Validity Measures for Critical Social Research}

This study was designed to maximize validity (Newman \& Benz, 1998) through the use of (a) transparent documentation of hermeneutically developed researcher judgments with evidence available to the public; (b) peer debriefer feedback and member checking to gain other perspectives and, if indicated, to incorporate into findings; and (c) triangulation of data for a more complete understanding of exclusionary discipline as a social practice. Strategies that provided internal validity to this study included (a) conducting extended interviews, (b) using participants' vocabulary during interviews, (c) accepting each statement made by participants as truth claims from their viewpoint, and (d) continually checking researcher subjectivity during analyses of data by recursive methods (Newman \& Benz, 1998). To enhance validity, interviews were facilitated by an 
interview guide that included questions pertinent to the purpose of the study (Newman \& Benz, 1998).

Measures were taken to meet validity, or trustworthiness, requirements for critical research throughout the study (Carspecken, 1996). Validation requirements in critical epistemology, or "“resistance"” (p. 15) postmodernism, are located in a nontraditional notion of signs and signifiers. Signs and signifiers are traditionally thought of as repeated language or actions that stand for the same thing and can be perceived through one's presence in experiences (Lee, 2005). In critical epistemology, however, visual perception is not used to validate claims, because "signs and signifiers do not relate to each other through perceptual experiences, but are 'mediated' through power relations" (Carspecken, 1996, p. 15). At the core of the validation procedures, critical epistemology gets at the holistic, internalized, taken-for-granted cultural expectations of routine social interactions. Communicative experiences are, thus, symbolized and communicated to others through communicative structures (Habermas, 1987).

\section{Construction of the Primary Record}

Validation procedures during construction of the primary record included the following suggested by Carspecken (1996). Passive observations helped reduce the effects of researcher presence (i.e., Hawthorn effect) on routine activities; however, participants' cultural schemas typically remain intact even when being observed. Triangulation of data through observational notes, theoretical notes, and videorecordings provided more than one viewpoint. Consistency checks for accuracy between written and video data were conducted when written notes were matched with signs and signifiers on video data. Low-inference vocabulary was used while taking notes during observations. 
Detailed low-inference notes (e.g., as if, appears to be) described interactions, speech acts, body movements, and body postures. Peer-debriefers checked notes, analyses, and codes during construction of the primary record.

\section{Generation of Dialogical Data}

Validation procedures during generation of dialogical data included the following (Carspecken, 1996). Peer debriefers provided feedback on the interview protocols. Dialogical data is "a sort of elaborate 'member check' .... [that] means sharing your notes with [participants] to see whether or not they agree with your record" (p. 89). Interviews were used as a consistency check to augment preliminary analyses of primary record data and as a form of member check from the participants' perspectives and vocabulary. For example, during videorecorded feedback interviews, the researcher shared preliminary analyses with participants and requested comparisons. Interview questions were open-ended to augment participants' perspectives. Rapport-building interview techniques used participants' vocabulary to reduce power relations and anxiety.

\section{Reduction of Power Relations}

Procedures for reducing assumptions of and distortions of power during the research process to result in objective claims in the primary record and during interviews (Carspecken, 1996) included the following. Lightly-structured interviews (Wengraf, 2004) provided the venue for developing supportive researcher-participant relationships and documenting conflicting accounts of an event. Importance was placed on rapport building, reporting, and analyses of verbal interactions by equalizing power relations during the interviews. Emphases were placed on minimizing the interviewer's voice and 
maximizing the "expression of the concerns, the systems of value and significance, the life-world, of the interviewee" (Wengraf, 2004, p. 69).

\section{Reconstructive Data Analysis}

Validation procedures for reconstructive data analysis overlapped those from the construction of primary record data, generation of dialogical data, and reduction of power relations (Carspecken, 1996). Data analysis was validated through (a) interviews that facilitated participants' reconstruction of their own meaning, (b) member checks on initial reconstructions to equalize power relations, (c) peer debriefers who checked researcher biases and/or omissions in analyses, (d) complementary and/or nested structures (Wengraf, 2004), and (e) researcher's familiarity with participants' cultures to both increase intersubjectivity in spotting cultural power in action and encourage participants' comfort and agency in making truthful claims. Through the use of validation procedures, alternative viewpoints were included in the conclusions of the study.

\section{Data Management}

Each participant was given a pseudonym for identity protection. All digital recordings, transcriptions, and analyzed data were collected and organized onto DVDs or CDs. A record of all analyses, self- and peer-debriefings, and additional member checks other than interviews were also kept. All data will be stored in a locked, fireproof file cabinet in my home office for 3 years from completion of the study (Florida International University Regulations for Thesis and Dissertation Preparation Manual, 2008).

\section{Summary}

Using critical microethnographic methods, this study explored the relationship between classroom interactions and exclusionary discipline as a social practice within 
and across classrooms in the same alternative education school. This chapter began with the purpose of the study and research questions repeated from chapter 1 , followed by the critical microethnographic method, gaining access, participants, criterion sampling, cultural milieu, pilot study, and data collection and analysis stages. Integrity measures, validity measures for critical social research, data management, and this summary conclude the chapter. In chapter 4-7, findings from Classrooms One, Two, Three, and Four are presented, respectively. In chapter 8, a comparison and synthesis of the findings is provided. Chapter 9 includes a summary of the study, responses to research questions, implications for teacher education, and recommendations for research and policy. 


\section{CHAPTER IV}

\section{DESCRIPTION OF CLASSROOM ONE FINDINGS}

This chapter presents a description of Classroom One findings in three sections based on the nine stages of data collection and analysis described in chapter 3 and illustrated in the Appendices: (a) Classroom One participants, (b) findings from interactive power analysis and role analysis, and (c) themes from reconstructive data analysis. A summary concludes the chapter. Quotes are cited by transcript name (e.g., T6B), line number (e.g., L271), and participant pseudonyms when necessary (e.g., Mr. Jenkins).

\section{Classroom One Participants}

Classroom One was a Culinary Arts course with a teacher who rarely used exclusionary discipline. When asked how he became a teacher, Mr. Jenkins, a White male, said that he "kinda stumbled into [teaching] actually" (T6B, L17). He had previously worked in kitchens before teaching part-time, attending a two-year cooking college, and becoming a full-time teacher. He was given a 2-year temporary certificate based on "basically having job experience, work experience, and the 2 years is to prove you know, that you can hold your own in a classroom" (T6B, L37). Meanwhile, he completed the required credits toward being a vocational teacher, noting that "a lot of people become vocational teachers out of- out of their industry and into vocational schools" (T6B, L41).

Mr. Jenkins did not know about alternative education when he applied for the job as Culinary Arts teacher at The School but said, "I tell you when I saw the kitchen that I first saw ... the kitchen really won me over. Yeah, we can really produce out of this 
kitchen!" (T6B, L45-47). After three years of teaching at The School, Mr. Jenkins maintained control of his classroom in order to prevent student suspension by merging system constraints, content requirements, and student behavior. He rarely relied on Positive Behavioral Support (PBS), a school-wide point system to assess and reward student behavior. Four students participated: Hector, Raul, Patt, and Green. Table 5 summarizes Classroom One student participation.

Table 5

Classroom One Student Participation

Name Observations Interview

$\begin{array}{llr}\text { Hector } & \text { x x x } & \text { X } \\ \text { Raul } & \text { x x x } & \text { X } \\ \text { Patt } & \text { x } 1 / 2 & \text { X } \\ \text { Green } & \text { x x } & \end{array}$

Hector and Raul, Latinos, participated in all 3 observations and the interview. Patt, an African American male, participated in the interview but only $1 \frac{1}{2}$ observations due to a parent conference and suspension. Green, an African American male, participated in 2 observations only due to suspension. Themes from interactive power analysis and reconstructive data analysis are discussed next, followed by a summary.

Findings from Interactive Power Analysis

This section provides an explanation of the findings from interactive power analysis, including (a) types of power, (b) roles, and (c) cultural power. Interactive power 
analysis determines who has what kind of power and why and whose power defines interaction outcomes.

\section{Types of Power}

The researcher deductively looked for four types of power based on Carspecken's (1996) categories of interactively established power: (a) normative power, (b) coercive power, (c) interactively established contracts, and (d) charm. This section defines and describes each type of power that existed.

Normative Power

Normative power is gained over others by virtue of one's position or status in the culture or society. Mr. Jenkins used his teacher status successfully to claim normative power over his students and define the outcomes of academic and culinary arts classroom interactions. He expected students both to stay in class and to complete the daily vocabulary assignment before working in the kitchen. In the end, Raul consented to the vocabulary after making several sarcastic remarks to maintain identity and agency as an entrepreneur. Hector feigned consent and maintained identity and agency by working on a personal drawing project and listening to headphones for as long as possible before doing the vocabulary. Green initially rejected Mr. Jenkins's normative claim by standing his ground upfront to maintain identity and agency with texting and excuses. However, hermeneutic inferencing based on reflecting on and examining the data provided evidence that Green knew that Mr. Jenkins would not give in until he agreed to stay in class and do his vocabulary, so Green finally consented. For example, Mr. Jenkins spent as much time as it took, whether it was one statement or 5 to 10 minutes, talking one-onone with whichever student needed motivated to get on board with the vocabulary 
assignment or cooking project for the day. Moreover, when a student walked out of the room, he went to get them. Students were used to the pattern of interactions Mr. Jenkins went through to get students on board.

Once finished with vocabulary and on board as culinary assistants, Mr. Jenkins made normative power claims by issuing directions about how to cook what he wanted, what to do with ingredients he decided to use, and doing the dishes in order to get products he wanted by the deadlines he had set. Mr. Jenkins dictated orders with redundant phrases that placed him in charge, such as I'm cooking, what I want to do, what I need done, what are the first two things you are supposed to do in the kitchen, do the dishes for me, read this recipe, or give this to [Green] for me. In the end, Raul consented as an assistant by doing the dishes, reading the recipe, doing the first two things he was supposed to in the kitchen, telling Mr. Jenkins what he knew or remembered, and working in the kitchen. Hector consented as an assistant by remembering part of the story about sausage for Mr. Jenkins and working in the kitchen. Green ultimately consented as an assistant by listening, reading the recipe, and cutting the celery. Patt was out of the room at a parent conference but upon return, consented to academic and culinary arts expectations.

Mr. Jenkins also made normative power claims as the teacher disciplinarian who puts out fires to keep students in the classroom. He maintained and redefined his authoritative status with a loud voice, active gestures, and brisk movements. He openly denounced students' poor choices made yet maintained solidarity by providing tacit reminders of the classroom norm-I help you stay in school, you help me cook products to sell. If necessary, he used explicit authoritative statements, such as send Patt back in 
room, go wash your hands, or you did not have to go see something. Patt consented to Mr. Jenkins's normative claims after finishing something more important to him first to maintain identity and agency. He was worried about his grandma, went to check on her, but returned to work in the kitchen.

\section{Coercive Power}

Coercive power is accomplished by forcing obedience after threatening punishment. Mr. Jenkins did not use coercive power to gain consent from his students. Interactively Established Contracts

Interactively established contracts are constructed by negotiations among participants, based on an implicit level of understanding, mutual consent, and equal communication input. Negotiations that lead to co-constructed interactively established contracts can be thought of as ongoing revisions on multiple drafts of a written paper passed back-and-forth between co-authors. It could go on indefinitely; co-authors may use varying amounts of charm, normative power, coercion, or a combination of techniques to sway the thinking for the final draft. However, the underlying ideas or meanings in the paper that are constantly being revised (albeit not necessarily face-toface) are what is viewed here as interactively established contracts.

In Classroom One, interactively established contracts with food as the motivation and reward pervaded classroom interactions. For example, when Mr. Jenkins was out of the classroom, students handled the kitchen with care and respected each other while waiting for his return so they could cook. When Mr. Jenkins needed to clarify Green's consent to do vocabulary and stop giving him a hard time, he negotiated an interactively established contract, based on the tacit claim of you give me no problem in class, and I'll 
give you no problem outside of class. Green reciprocated (clarified) and consented to the tacit contract, yet maintained identity and agency by tossing his notebook on the table and saying I gotta give you hard time. Vocabulary was something Mr. Jenkins had to say he did as a teacher (per student interviews) and that he needed to help him get situated (per Mr. Jenkins's interview), but once students did it and he got to check attendance, they could become assistants and he could become the chef.

\section{Charm}

Charm is the use of one's personality to gain consent from others. Mr. Jenkins's charming personality encouraged acceptable teacher and student identities, maintained his authority, and upheld tacit understandings of clearly defined outcomes in the classroom. Even though Mr. Jenkins made the decisions, students were involved as assistants. Mr. Jenkins conceptualized the situation as a kitchen in the workplace and by apprenticing assistants with charm, he won student consent. He used charm to motivate students, achieve desired cooking products, and provide encouragement. His charm was manifested vis-à-vis smiles, waves, nods, vernacular, humor, sarcasm, and stories. Mr. Jenkins told stories in a way that solidified his tacit claim of you help me cook food to sell and I'll help you have fun in class by using supplies and ingredients, learning to cook, and getting to eat. He told funny stories about previous cooking projects, such as repairing yeast rolls, salvaging sausage sandwiches, and working on a tortellini project, or future plans, such as doing a cooking show. Overall, Mr. Jenkins used charm to win the loyalty and trust of his students, based on the tacit claim of you do what I plan or am required to do from the administration and I will call on you to assist me in the kitchen and reap the rewards of eating. 
As exhibited in the pattern of interactions in this classroom, the students knew Mr. Jenkins would maintain solidarity with them and would not snitch on them. This conclusion was hermeneutically reached by reflecting on and differentiating among reoccurring intricacies in this classroom setting, combined with unsolicited student interview comments about Mr. Jenkins's positive influence and his willingness to talk with them and listen to them. For example, students saw and heard multiple opportunities for Mr. Jenkins to write referrals, such as when Patt walked out of the room unannounced or when students from other classrooms walked in without passes to buy food. When asked over the classroom phone during a parent conference about a student's recent behavior, Mr. Jenkins gave a positive answer about Patt's history of behavior, even though Patt had been a little bit of a clown lately, that all students could hear. Based on experience, they knew that he could be trusted to prevent their suspension; he would charmingly help them want to stay in class by telling stories or using sarcasm so they would get the point and if they forgot, he would quickly remind them that there were no other options. This was not considered coercive by the student participants or the researcher because Mr. Jenkins' ultimate goal was to prevent students' suspension, which they also desired. Students felt he was a positive influence and liked his equitable nononsense attitude.

Once in a while Mr. Jenkins combined charm with a normative power claim of no you don't have to, or an interactively established contractual reminder of you used to be kinda good in here or here is a story about how someone botched up a recipe. Rarely did students challenge Mr. Jenkins's definition of classroom norms, but they knew how to resist long enough to get their communication or other goals accomplished (e.g., drawing, 
texting, eating). As a result, even though he authoritatively walked around dishing out orders, he did it in a culturally congruent (charming) way with the students using vernacular, stories, smiles, catchy phrases to get them on board, and assuming that they knew how to do the task once they were ready to begin. Mr. Jenkins's stories also reminded students that even though he watched and discussed their behavior, he was human and supportive. For example, he told humorous stories about getting free tickets to a concert and noticing that the bus was on time but Green was late.

Positive Behavior Support rules were backgrounded in the classroom but only tacitly used as normative measures to get the students on board or to follow school rules. Instead, when students came by with a pass, Mr. Jenkins used charm and humorously called it a bootleg pass and examined it, all the while laughing that he was supposed to do this ain't I? or if a student got sucked out the door, he called him back and said no you don't have to go do something - end of story. As discussed in the interviews, students knew that Mr. Jenkins wanted them in class so they could accomplish their mission of having to be at The School for 18 weeks to return to regular school. They talked about how he differed from other teachers who called students names and got outwardly mad. Mr. Jenkins showed students that he liked them and also needed them to help him with his own deadlines. Hermeneutic inferencing based on the nuances in this classroom of students being liked and needed by their teacher led to the following conclusion. Students felt important, smart, and got to keep their identity as student, artist, or entrepreneur rather than as bad kids; therefore, they consented to the classroom culture definitions. All participants got to maintain identity and agency in this classroom. Table 6 summarizes types of power exhibited in Classroom One. 
Table 6

Types of Power Exhibited in Classroom One

Type of Power $\quad$ Exhibited

Normative $\quad x$

Coercive

Interactively Established Contracts $\mathrm{x}$

Charm $\quad x$

Normative power, interactively established contracts, and charm were exhibited in Classroom One; coercive power was not exhibited. The roles that participants exhibited are discussed next.

Roles

This section covers teacher roles and student roles. The researcher inductively found and named roles based on routine "interactive rhythms and sequences" (Carspecken, 1996, p. 137). Roles emerge as themes through complex ways of acting, which participants "recognize as having some unity" (Carspecken, 1996, p. 136). When participants recognize a role in play, they can predict, but not ensure, future actions. Table 7 provides a summary of Classroom One participants' roles, followed by a description of teacher roles and student roles. 
Table 7

Classroom One Participants' Roles

Participants Roles

Teacher - Mr. Jenkins The teacher who has a human side

The staunch student/culinary assistant supporter

The chef who solicits/needs culinary assistants in the kitchen

The multi-tasking product-oriented director

The disclosing comedic older peer/uncle

The neutral enforcer of administrative rules

The adamant classroom/kitchen rule enforcer

The no-nonsense traditional parent

Student- Raul The tit-for-tat team-player entrepreneur

Student - Hector The intense listening artist

Student - Green The socially networked peer/nephew

Student - Patt The clown/grandson assistant

\section{Teacher Roles}

The teacher exhibited eight roles. First, Mr. Jenkins was the teacher who has a human side. He needed time to get settled into work mode, relax from time to time, greet visitors, and make friends. He assigned vocabulary work to give him time to get settled. He was a teacher who intentionally sat behind the desk for social distance and authority position, surveyed culinary assistance prospects, and once assistants were confirmed, talked about his outside life with them to reduce social distance and gain loyalty and solidarity. Even when talking about students and their mishaps, he gained solidarity because he did it with charm, humor, and for the purpose of learning from mistakes which all of the stories he told portrayed. To administrative questions about Patt's 
behavior, he responded fairly by giving him the benefit of the doubt based on his history in the classroom thus far, that Patt had acted like a clown lately but did well most of the time. Students trusted him to look out for their best interests. Students compared the differences between him and other teachers who lost control, which gave the kids a laugh.

Second, as the staunch student/culinary assistant supporter, Mr. Jenkins talked to or about students, showed solidarity, and was consistent in his expressed desires to get them on board this school thing. He had high expectations and believed in students. For example, he told students they could probably do a task with their eyes closed, gave them directions, and then walked away knowing they could and would get the job done. He went to get students who should be in class, sent a student back to his own class so he would not get in trouble without a pass, never brought up coercive measures that would be taken if they did not do something, but expected they would consent to tasks in advance and that was that. He ignored something that did not matter at the moment. He reminded students gently about what was going on and needed to be changed, like done already? or what are the first two things?

Third, as the chef who solicits/needs culinary assistants in the kitchen, Mr. Jenkins told cooking stories of what students did and how he fixed the mistakes, using such phrases as I need you to do, help me cook, you cut celery and I'll do such and such. He showed students that he could not do this without them and being an assistant was very important. Tasks were important because of the results, such as selling and eating. This role lowered the importance of the teacher since he was busy doing things with the assistants for the purpose of getting products ready to sell. 
Fourth, as the multi-tasking product-oriented director, Mr. Jenkins could do many tasks at once, such as looking for salt, finding a missing student, helping someone do a kitchen task, and scooting intruding students out of the classroom, all with humor and authoritative demeanor and purpose. He could simultaneously solicit assistants, help the lady get ice, answer the phone, and move constantly. However, he could also sit and tell stories for the purpose of getting students on board, relax to reduce social distance between them, discuss cooking errors, and get prepped for the day's recipe. Mr. Jenkins was very busy being director, but included students all the while even if only for listening to his stories. He stayed physically at a distance except when he direly needed to get someone on board at which time he sat by them to reduce the social distance of directing.

Fifth, as the disclosing comedic older peer/uncle, Mr. Jenkins decreased social distance by using cultural humor, language, and movements as he told things about himself, what they could do together for service learning, what somebody did and should not have done. He always disclosed respectfully, and he got respect in return. Mr. Jenkins was there in all his roles to help students accomplish the PBS requirements of this school by having an alternative way of conceptualizing the situation. He used his chef role to direct, sway, humor, and produce his and the students' way to success. He was virtually successful. He was real in all of his roles. The students knew he was being real and thus did not resist outwardly in ways that ruined the teacher's definition of the setting. As a result, students also accomplished their own goals, even if only to voice momentary concern about a camera, or draw until the chef needed them, or sarcastically complain for a second but quickly get vocabulary done so they could cook, or go find something outside quickly and return ready to cook. 
Sixth, as the neutral enforcer of administrative rules, Mr. Jenkins got students both to stay in class and to not get suspended from other classes. His normative power was never in question, but he enforced it in order to support the students either in administrative rules or in becoming assistants in class, both of which retained students' identities. Students rarely resisted and usually consented quickly to classroom norms and definitions set up by Mr. Jenkins. For example, Green briefly resisted being filmed as an excuse for not doing vocabulary while texting. Mr. Jenkins's enforcement of the cell phone rule by interjecting that he did not accept vocabulary on a text showed Green that Mr. Jenkins was in tune to his desires, but that vocabulary still came first. Then Mr. Jenkins commented about Green having been good in class up to that point and why was he changing now, interjecting an interactively established contract. He solidified the comment with charm using a story about the bus being on time but Green being late, showing his support of Green not getting suspended for being tardy by neutrally enforcing a PBS rule. Mr. Jenkins got Green on board, although Green initially resisted and texted, retaining his identity of opposition to the camera and losing his journal as excuses up front.

Seventh, as the adamant classroom/kitchen rule enforcer, Mr. Jenkins increased social distance and implicitly claimed complete authority over kitchen rules and classroom rules to get students to stay in class. However, every now and then Mr. Jenkins had to state exactly and explicitly what students had to do right then so that health, safety, hunger reduction, and staying in school were accomplished. These goals were all in support of students' success. Supplies were not an issue in Classroom One; all students had writing utensils that they brought to class. 
Eighth, as the no-nonsense traditional parent, Mr. Jenkins stated adamantly and explicitly what needed to be done to make sure they did what should be done or to prevent someone from getting a written referral or getting suspended; for health purposes; to stop bucking a rule; and being a successful student. Mr. Jenkins infrequently used this role. He didn't appear to need this role; instead, he successfully negotiated consensual agreements with students. Real choices made the norms in this classroom work.

\section{Student Roles}

Raul, the tit-for-tat team-player entrepreneur, had several businesses outside of school such as a car wash service, and he knew the value of teamwork and getting something in return for doing something. He displayed entrepreneurial skills when he used confidence and agency to work on a recipe without telling Mr. Jenkins every step of the way.

Hector, the intense listening artist, knew when he was needed in the kitchen, and he worked on outside interests until that time was clear. His interests were his long-term self-researched art projects which he shared with the researcher before class. He quietly consented to classroom definitions, maintaining his identity and agency.

Green, the socially networked peer/nephew, achieved his communication goals and retained agency and identity, in the meantime, by texting, complaining about the camera, and saying he lost his notebook. However, he quickly and ultimately dropped the excuses, did the vocabulary, and worked in the kitchen.

Patt, the clown (as expressed by Mr. Jenkins on the phone and during the interview and Patt himself during the interview)/grandson assistant, achieved his communication goals by going outside to find out about his grandma, but he also came 
back in and worked in the kitchen. He thereby achieved agency and maintained identity through Mr. Jenkins's support of his values.

\section{Cultural Power}

Cultural power was discovered through the process of hermeneutic inferencing, or taking the position of participants in order to understand the basis of their claims. Cultural power exists when the norm or value claimed did not represent mutual interests of the entire group. In this classroom, Mr. Jenkins's normative-evaluative claims for doing what was right were real choices and he eventually gained consent from all students who realized their overall (universal) benefit. Not much cultural power was at play in this classroom, since each student could almost fully recognize himself as someone who could cook well, do vocabulary, use his own agency and healthily resist without punishment to a point, and participate in his own interests to a point. Mr. Jenkins's charm juxtaposed successfully with the students' resistant identities at this school. As experessed in the interview and exhibited in classroom interactions, students believed the teacher was fair and cared to support them in PBS and Culinary Arts goals, so they consented, agreeing to Mr. Jenkins's clearly defined classroom norms and values.

Mr. Jenkins and his students had little voice in The School and did not fully agree with rules and consequences related to PBS, referrals, and suspensions. Therefore, Mr. Jenkins tried to be flexible and enforced PBS to the benefit of students. In this classroom, students could eat and communicate while fulfilling the culinary purpose of cooking and selling food. For example, potential conflicts (the dislike of the camera) subsided quickly when the teacher juxtaposed charm, cooking, and his outside life to the student. The meaning of being on a team that produced a food product that was wanted inside and 
outside the class was universally agreed upon in this classroom. Vocabulary did not have universal meaning to all participants, but the students were willing to do it in order to gain the benefit of support, encouragement from the teacher and, more importantly, food to eat. The good feeling attained from completing a product and being needed on the cooking team was universally important in Classroom One.

Themes from Reconstructive Data Analysis

This section covers four themes that emerged from inductive reconstructive data analysis: (a) resistance, (b) accommodation, (c) conformism, and (d) negotiation. Because the emerging themes aligned with Giroux's (2001) theory of resistance in education and Third Space theory (Gutiérrez, 2008), terminology from both were used. Table 8 provides a summary of the findings from Classroom One reconstructive data analysis. 
Table 8

Classroom One Reconstructive Data Analysis

\begin{tabular}{|c|c|c|c|c|}
\hline Theme & Part. & Manifestation & & Purpose \\
\hline \multirow[t]{3}{*}{ Resistance } & $\mathrm{T}$ & Critique & $\mathrm{T}$ & Maintain control \\
\hline & $\mathrm{T}$ & Self-reflection & $\mathrm{T}$ & Prevent suspension \\
\hline & $\mathrm{T}$ & Struggles & $\mathrm{T}$ & Accomplish Culinary Arts \\
\hline \multirow[t]{6}{*}{ Accommodation } & $\mathrm{T}$ & Flexibility & $\mathrm{T}$ & Maintain control \\
\hline & & & $\mathrm{T}$ & Prevent suspension \\
\hline & & & $\mathrm{T}$ & Accomplish Culinary Arts \\
\hline & $\mathrm{S}$ & Flexibility & $\mathrm{S}$ & Maintain identity and agency \\
\hline & & & $\mathrm{S}$ & Prevent suspension \\
\hline & & & $\mathrm{S}$ & Accomplish communication \\
\hline \multirow[t]{4}{*}{ Conformism } & $\mathrm{T} \mathrm{S}$ & Vocational education & $\mathrm{T}$ & Maintain control \\
\hline & $\mathrm{T} \mathrm{S}$ & Banking education & $\mathrm{T} \mathrm{S}$ & Prevent suspension \\
\hline & $\mathrm{T} \mathrm{S}$ & Motivation/reward & $\mathrm{T}$ & Accomplish Culinary Arts \\
\hline & & & $\mathrm{S}$ & Reap reward \\
\hline \multirow[t]{5}{*}{ Negotiation } & $\mathrm{T} \mathrm{S}$ & Talk and humor & $\mathrm{T}$ & Maintain control \\
\hline & $\mathrm{T} \mathrm{S}$ & Social distance & $\mathrm{T} \mathrm{S}$ & Prevent suspension \\
\hline & & & $\mathrm{T}$ & Culinary Arts goals \\
\hline & & & $\mathrm{S}$ & Maintain identity and agency \\
\hline & & & $\mathrm{S}$ & Accomplish communication \\
\hline
\end{tabular}

Note $: \mathrm{T}=$ Teacher; $\mathrm{S}=$ Student; Part. $=$ Participant.

Table 8 denotes four themes that emerged from reconstructive data analysis. Resistance was manifested as subthemes teacher critique, self-reflection, and struggles.

Accommodation was manifested as subthemes teacher flexibility and student flexibility.

Conformism of teacher and students to the dominant ideology of Culinary Arts was manifested as subthemes vocational education, banking education, and motivation and reward. Negotiation was manifested as subthemes varying teacher and student talk, 
humor, and social distance. Teacher resistance, accommodation, conformism, and negotiation were manifested to maintain control, prevent suspension, and accomplish Culinary Arts goals. Student accommodation and negotiation were manifested to maintain identity and agency, prevent suspension, and accomplish communication goals. Student conformism was manifested to reap the rewards of food.

\section{Resistance}

This section covers Mr. Jenkins's resistance of the Positive Behavior Support system as manifested in three subthemes, highlighting Giroux's (2001) framework: (a) critique, (b) self-reflection, and (c) struggles. Resistance is a "form of refusal that highlights, either implicitly or explicitly, the need to struggle against the social nexus of domination and submission" (Giroux, 2001, p. 109).

\section{Critique}

Critique of domination manifested when Mr. Jenkins viewed the PBS system rules as a constraint to his goal of maintaining classroom control to prevent student suspension. When asked about PBS, Mr. Jenkins explained it as a program that provided incentives "to encourage the kids to, you know, to maintain good behavior" (T6B, L83). He felt that PBS broken down into a point system was a "good step in the right direction to clarify it for the teachers and for the students, you know, so that there is a consistent program around what's expected" (T6B, L85). Yet he added:

It'd be best, yeah, if it comes from uh, outside, you know, the committee, the administration, difficult to enforce equitably how it's gonna, how it's gonna be enforced, how it's gonna be made equitable, made consistent. Yeah, we've had a few, We've had a few, uh, some of the, some of the faculty meetings have been largely devoted to trying to make more clarification and I've pretty much said the same thing - that's why I'm not afraid to say - to repeat what I've said before. (T6B, L99-101) 
Mr. Jenkins critiqued the difficulty of enforcing the PBS point system equitably for the administration and for himself as an enforcer. He explained:

The way that it was explained to us was if you've got a 90 minute class, you can break it into about 20 minute segments for the 5 points. So that if a kid, because we don't want, if a kid has something that goes wrong in the beginning of class, for him to feel like he's blown it and he might want to try for the remainder of the class. And so the hope was that all right, well, you know, you've lost this point for this beginning or this middle segment of class, well, let's get back on track and get the points for the remainder of class. It's a nice theory except on that, based on that, um, that, uh, expectation, that guideline, what you are expecting is perfect behavior, even if it is only for 20 minutes to get that point. Therein lies some of the difficulty for me anyway as an enforcer for that, uh, uh. (T6B, L87-95)

He commented that PBS was a nice theory but further critiqued the notion of enforcing perfect behavior and trying to resolve the inconsistency between equitable enforcement and his own classroom goals. He critiqued the ambiguity of PBS rules:

All right, well, it's a little ambiguous, a little interesting that - basically, the school, the administrative stance, mantra is, don't give passes. The students are supposed to use the bathroom in between classes, there's really no reason why they should be going to another teacher unless it's some kind of an emergency. There, let's say they dropped the ball or the teacher, for some reason they didn't get their points, you know, like Jose did. Um, that was me, I dropped the ball on his points. I didn't make sure and get 'em well- [he was coming back] So he can get the points he's supposed to get, um, like I was telling you, you know, I have no way of knowing for sure whether he showed up at that class, you know there was a period of time that was not accounted for. Could be, like, you know, I have to use my gut whether or not to trust, you know, his story- and in this case, I did. Sometimes, I'll tell students no, I can't see you without a pass. (T6B, L145-153)

In general, Mr. Jenkins used his own insight to determine how to enforce PBS.

\section{Self-reflection}

Self-reflection occurred when Mr. Jenkins expressed the dilemma of enforcing

PBS rules for his classroom:

Once we came back from the winter break, um, I started seeing [Patt] a lot more out of his classes, and stopping by over here and seeing if I got something to feed 
him, and you know, even if he, even if he was looking to buy the cookies, that's that's one of the things that uh, is difficult for me and it's difficult for the principal is that, you know, the students are supposed to be in their class you know? And I'll tell 'em you know, you can't be here without a pass, but at the same time, you know, the principal has said you're not supposed to write students passes to go to Culinary Arts, you know, even for the bathroom, you're supposed to go in between classes- uh, you know when something is laid down as a- a zero tolerance like that, it's really tough to figure out the- what the right response is to it, and uh, you know, it's kind of like, the kid asks me, can I go to the bathroom? And I'll tell him no and see if I get away with it [laugh] (T6B, L337-343)

Mr. Jenkins reflected on the conflict between administrative and zero tolerance

expectations in the classroom. He explained his rationale:

And if they, if they don't push it, then I then you know that it wasn't that serious, but then sometimes you know, I gotta go, then I'll probably have a response more along these lines. I'll say, well, I'm not supposed to write you a pass, you know, but if you walk out that door, and you know, you go out and get in trouble, I'm not gonna back you up on that, because you walked out of my class, but if you go, you come back, you know, and you don't get, you know, in trouble, and you don't go off and waste your time, I'll let you back in. (T6B, L345)

Mr. Jenkins commented that he often left the choice with the student. He further reflected on the dilemma of enforcing PBS rules consistently in light of accomplishing his own Culinary Arts classroom goals:

The problem that, you know, can and does result from having different options is that there is going to be some inconsistencies there, you know, one day, you know, Joe had his headphones and I didn't say anything, and one day, Joe's got his headphones and I'm- we're having a confrontation. Um, that's a difficult and um, I got to admit I feel kind of bad personally that I do lack some consistency in how I address inappropriate, you know, negative behavior, specifically that's one of the big thing, those headphones, you know, um, for some of the teachers, their classes are very dependent on auditory, you know, input- (T6B, L85-87)

Overall, Mr. Jenkins was concerned with inconsistencies involved in his enforcement of PBS. 
Struggles

Struggles toward emancipation occurred when Mr. Jenkins released himself from

zero-tolerance of the PBS rules:

Yeah. Yeah, we have the standard referral form. They call it a SCAM. It's the acronym for something- students - I can't remember exactly what it stands for but uh, something administrative, um, however, since this is alternative ed, and they've wound up here as a result of a teacher exercising their authority- to remove a disruptive student from their class, which can only be done using the SCAM, and it becomes a part of their permanent record, there's I guess I'd say it's sort of unspoken hesitance on the part of most teachers to use a referral form for every infraction, you know, uh, when- I can't remember exactly what it was we received recently, something about, something within the positive behavior. I think it was a flow chart that uh, probably the school psychologist developed, you know, that when, you know, the next inappropriate behavior is observed, ok, which of these options are you going to choose? And one of them is to ignore the behavior, because sometimes you know, it is better to not make a big deal about it. And uh, you know, exactly how are you going to address it? (T6B, L175-183)

Mr. Jenkins ultimately chose to ignore behaviors that in other classrooms may be

considered disruptive for the purpose of keeping students in school. He explained further:

And there is some inconsistency, I mean, some teachers will obviously have a no tolerance policy. Don't have them on, don't have them visible, you know because that's basically what the parting line is, you know, no headphones, it's a- it's an offense, you know, uh, I think getting back to the whole thing about the referrals, since I've been out here, you know, there's, there can be, there can be a few problems with writing a kid a referral, uh, first and foremost, really, we are here as his last, you know, opportunity, really. If we're kicking him out of our school, or even out of our class, which is almost the same thing, where are we kicking him to, what are we accomplishing really? Yeah, outdoor suspension, they, you know, as far as we know, it's just any place but here. (T6B, L205-211)

Mr. Jenkins's concluded that zero tolerance provided limited opportunities for students.

Struggles toward emancipation occurred when Mr. Jenkins distanced himself

from bureaucratic suspension practices. For example, even though indoor suspension, or

CSI, was not encouraged, it was widely used in The School. However, he explained that

CSI was where disruptive students were seldom sent 
for more than a portion of a day. I-I've never really, I mean I- I don't have a chance to be much in the know but as far as I understand it, it's not gonna be too common that we're gonna tell a student, all right, well, you gotta come to school and stay over here in this room. (T6B, L213)

When asked about terms used for tracking student status in school, he "had no idea what" (T6B, L225) exclusion from school was but he said that

withdrawal, basically, is a you know, a just a bureaucratic term, that for example, if a kid gets locked up, then technically, a parent or a guardian is supposed to come down and withdraw them from this school so that they're not racking up absences and coming up with a truant status. (T6B, L219-221)

Mr. Jenkins's commented further about the bureaucracy of student status at The School.

Both Green and Patt had been added to the suspension list after the study began. Mr.

Jenkins thought it was "strange" (T6B, L231) that Green had been suspended for 10 days

but had returned early. On the absence list,

There's 24. Yeah, it's typical for there to be 20 to 25 names on there and 6 or 12 on the tardy list as well. Lately, it has been less so, but sometimes, I've seen them come in at 10:30 or 11 o'clock on the tardy list.

Mr. Jenkins noted more inconsistencies in the bureaucratic system related to suspension:

Yeah, you know, it's not like we're forcing them to be at home, we really can't do that and for a kid to have the choice to be at home or be at school, it doesn't seem to make a whole lot of sense that, yeah, most of 'em, they'll choose to be at home, uh, of course, they don't recognize that is has the longer term repercussion that they're gonna, you know, their grades are likely to suffer, especially if it's more than 10 days. You know, they might wind up spending more time in school as a result, trying to, if they fail the whole grade. (T6B, L257)

Mr. Jenkins's resistance of PBS system constraints both to maintain classroom control and to prevent student suspension provided the basis of his classroom culture, norms, and values. Students' uptake of Mr. Jenkins's classroom culture, norms, and values was interactively exhibited with him through accommodation, conformism, and negotiation. 


\section{Accommodation}

Accommodation manifested as two subthemes: (a) teacher flexibility and (b) student flexibility. Accommodation is perceived as simultaneously coping or accepting of the school's ideology while exhibiting some form of resistance to it (Giroux, 2001). The School's ideology consisted of scheduling, programming, and other interruptions during class and PBS rules, both of which impacted teachers' and students' daily and momentby-moment decisions.

\section{Teacher Flexibility}

Teacher flexibility was exhibited toward (a) multiple interruptions and (b) PBS rules to maintain classroom control and prevent suspension yet allow student expression and social contradiction (Giroux, 2001).

Multiple interruptions. Teacher flexibility was manifested when Mr. Jenkins accommodated multiple interruptions during a 90-minute period, including students leaving for field trips or programs, visitors knocking at the classroom door, and phone calls coming in from the office. When asked about an ordinary teaching day, Mr. Jenkins said, "Oh, um, uh, it's unpredictable .... I have to ... schedule it so that - how I can distribute it among the classes that I've got" (T6B, L55). He added, "You know, I can't assign something that's gonna exceed how much time I've got available" (T6B, L75). He explained:

Three blocks before lunch. Uh, it always entails a good deal of flexibility, you're never sure, how many of the students are going to be there, or if they're even gonna be available, or you know, willing to participate in the work. Sometimes you know, they're not available, they've got other things going on. Field trips, and just a lot of things. There tend to be a lot of, a lot of, um, adjusting of plan throughout the course of an ordinary day .... Yeah, yeah, I mean sometimes I've- I've pretty much just pulled the plug on one direction - one objective and 
plug in a different one instead. Just based on who's gonna be there for how much time. (T6B, L59, 73)

Teacher flexibility was exhibited in how Mr. Jenkins promptly handled interruptions. For example, the "first phone call out of 90 minutes" (T6B, L329) was the office calling about Patt. Mr. Jenkins answered:

This is Mr. Jenkins. Yes. Yes, I do. Yeah, yeah. Um-huh. Uh, generally he's good. But just the last maybe few weeks he's been a little bit more of a clown than usual. But general-, generally, he's uh, o-, on track. Alright? Alright. (T4A, L76)

Mr. Jenkins's flexibility and promptness in accommodating scheduling, programming, and other interruptions were often interwoven with multi-tasking and openly supporting students and achieving solidarity with them. He explained that the phone call was about

Patt, he was in a conference, a parent conference or something- And typically, 9 times out of 10, Patt's the first one finished, he's not skimpin' it, and he's up and uh, and wants to be on track. If I give him a project to do, um, he usually gives a good effort. I've had to, I've found I gotta, make sure to clarify and he understands what direction he's going in, you know, um, and he has, he's been real good, and then like I said, until just the last few weeks. (T6B, L333, 337)

When Patt was asked about what Mr. Jenkins said and why, Patt concurred, "[he said] You've been actin' like a clown, man. Cuz I always be clownin"” (T6A, L60, 62) . . . If I ain't doing nothin', I'll walk around the class [and] play" (T6A, L231, 244). Patt recognized Mr. Jenkins's flexibility when he walked around the classroom if he was not doing anything.

PBS rules. Teacher flexibility was also manifested when Mr. Jenkins accommodated PBS rules. For example, his daily requirement that a vocabulary assignment be completed by students during the first few minutes of class simultaneously gave students a class activity in which to participate, and it gave him needed preparation 
time in the beginning of class. Mr. Jenkins explained that he "tends to be kind of lax the first 10 to 15 minutes, because it's a 90-minute class, you gotta get this thing going" (T6B, L349). However, students had to complete the vocabulary assignment before they cooked. If someone asked how a student had been doing in his class, [he could say] "these guys, you know, they run it like you hope they will. They come in and get their vocabulary, do the vocabulary" (T6B, L335).

On another level, Mr. Jenkins accommodated PBS rules with flexibility by allowing students to listen to their headphones while they cooked or did their vocabulary assignment. This accommodation simultaneously allowed students to accomplish their own communication goals and allowed him to get things done. Mr. Jenkins explained:

You know, yes, sometimes I tell 'em put 'em away and sometimes I'll ignore it, um, you know, if they go right to their vocabulary book, they sit right down, they get right on writing it, they're not being disruptive - I'll ignore it -9 times out of 10. (T6B, L193-195)

During the interview, the students explained their understanding of the required vocabulary assignment. Raul said:

I guess he does it to say that he gives us some type of writing work, you know, it's so that-that's what I think. Pretty much we don't do any other besides, uh, the vocabulary except for when the midterms came around. Besides that, it's just cooking, we don't - we don't write nothing. (T6A, L46)

Hector agreed, "Yeah, because we don't usually do work" (T6A, L48). Students considered the vocabulary assignment to be busywork even though Raul added, "usually the words that he puts up there is related to the cooking that we do" (T6A, L50).

Mr. Jenkins also accommodated PBS rules with flexibility on a third level. He no longer stopped class every 20 minutes to discuss and record each student's points on the PBS Charts as suggested by administration in the beginning of the year. Instead, he 
exhibited extended and purposeful periods of silence and quick glances as a surveillance tool while sitting at his desk during class time. Surveillance allowed him the social distance to both determine who needed motivating to get on board and who needed reminding to get back on track. However, he questioned his own actions:

I guess I'm reaping some of the consequences of being lax on that, uh, yeah, yeah, I think if uh, if I was a little bit more, if I had the students a little bit more in tune, through the class that look, guys, I'm watching you here, I'm trying to give you points, and you know, and this is why somebody is getting 'em, and this is why somebody is not getting 'em. Something along those lines. Yeah, you know, over the course of 90 minutes, you know, our students tend to need reminders, where they are, on track or off track. (T6B, L123-129)

Mr. Jenkins thought he may be too lax, but on the whole, students understood Mr.

Jenkins's accommodations as equitable demonstrations of how he accomplished the goal of keeping them in class to prevent suspension. Student understandings of why Mr. Jenkins ran his class the way he did were derived from hermeneutic inferencing of the intricacies of the teacher's and students' interactions in class and the students' explanations about those interactions. For example, students explained that they liked how Mr. Jenkins was laid back and cool enough to let them be themselves and listen to them unless things got serious. On the other hand, he demanded respect and knew his stuff so they listened to him.

\section{Student Flexibility}

Student flexibility was manifested as (a) respect, (b) sarcasm, and/or (c) silence. Student flexibility was manifested when students accommodated Mr. Jenkins's classroom culture, norms, and values to get points and not get suspended yet maintain agency, identity, and communication goals. They knew Mr. Jenkins's limits and ultimately did what he wanted. 
Respect. Students exhibited respect even when Mr. Jenkins was not in the room. For example, Mr. Jenkins took a student who did not have his consent form to participate in the study to the coach even though he "wasn't supposed to, expecting them to go ahead and finish it up, finish up the vocabulary" (T6B, L271) while he was gone. Students were serious, careful, and responsible in the kitchen area. However, sometimes they forgot to do the vocabulary as happened on this day; they were each busy accomplishing their own goals—Raul looking for food, Green borrowing Raul's cell phone, and Hector drawing and listening to his headphones. However, upon Mr. Jenkins' reminders, they were usually respectful in carrying out his requests.

Sarcasm. Students exhibited sarcasm when Mr. Jenkins reminded them to do vocabulary. After hermeneutic inferencing of data, sarcasm usually indicated a sense of humor rather than a negative connotation. Upon Mr. Jenkins's return, he walked briskly through the back door, scanned the room, gestured actively and asked, "Done already" (T4B, L13)? With a slightly irritated tone, Green quietly said, "Man, dog" (T4B, L12). Hector continued to draw. Only Raul replied to Mr. Jenkins's question, "Doin' what" (T4A, L14)? When Mr. Jenkins said, "Vocabulary" (T4B, L15), Raul responded in a drawn out manner, "Oh, that's right" (T4B, L16) and began walking slowly in the direction of his journal. Mr. Jenkins directly and sarcastically reminded Raul while looking around at the other students, "Oh, gotta do that everyday. Just like you always do it every day" (T4B, L17)? Raul retorted with a smile, "Yes" (T4B, L18). Mr. Jenkins spoke again sarcastically, "Imagine that?" (T4B, L19) and Raul replied sarcastically under his breath, "Imagine" (T4B, L20). Raul only needed a humorous sarcastic reminder from Mr. Jenkins; he responded with similar sarcasm but began the vocabulary. 
Silence. Students also used silence to accommodate classroom norms. During the interview, Raul commented that "most of us forgot to do the journal, I'm guessing, because you [the researcher] were there" (T6A, L14). However, they voiced various excuses for forgetting. For example, when Raul heard Green say, "I don't wanna" (T4A, L43), he added, “Hmmm, I don't know, I guess that kid obviously doesn't want to be recorded" (T6A, L11). Hector clarified that Green's "not too interested, [he was] texting" (T6A, L12). Raul explained, "Which is my phone actually. I usually lend it to him every class. He texts his girlfriend" (T6A, L190-194). Hector continued, "I was just drawing, I was just waiting for him to say something before I would stop so I continue drawing” (T6A, L23). When asked if he was doing vocabulary yet later on in the class period, Hector replied, "I'm trying to look busy" (T6A, L183). Raul laughed, "No, he’s drawing" (T6A, L172)! Hector confirmed:

Yeah [I'm still drawing] .... the 100 themes. It's like this art challenge thing that um, is on the internet. And you get a hundred themes and you write a differentyou draw the themes. So to make it- improve my art skills, I got a hundred people to volunteer to model for me for each theme. So I think right now I'm working on the eyes. (T6A, L174-178)

The students were allowed to accomplish their own goals up to a point. They also realized and appreciated how Mr. Jenkins handled his classroom. Raul clarified, "Yeah, [he ignores certain things] unless it gets serious" (T6A, L198). Hector agreed, "Yeah, he's pretty laid back" (T6A, L199). Raul responded, "Yeah, he's a cool guy" (T6A, L200).

Students also manifested silence when they accommodated Mr. Jenkins's kitchen rules. For example during one class period, it appeared that Raul was following directions after Mr. Jenkins said: 
All right. Tell you what, generally when I have somebody measure it out especially if it is the first time, get everything measured, put in a little container of some kind, you know, the-uh, the . . . sunflower seeds you know you can just put them in a (undecipherable) mixing bowl, you know, salt in a little paper muffin cup, sesame seeds - in a little paper cup. Yeah, measure it out, put everything onto a sheet pan with separate containers, right? (T4A, L99-101)

However during the interview, Raul explained that once he realized Mr. Jenkins was making him do the multi-grain roll recipe on the card, he accommodated his directions:

There, he was telling me to put all the ingredients together, the first part, and then show it to him, which I didn't do. I put them together but I didn't show it to him . ... Because I was pretty sure I was doing it right. (T6A, L136-138)

Raul combined ingredients as directed in silence but made the decision not to show the first part to Mr. Jenkins, thus retaining his agency and identity. Another time, Mr. Jenkins told Raul to put away the dishes just as he was getting started on a recipe; Raul obliged silently but loudly clanged the dishes. When asked about the noisy clanging, Raul responded:

I was putting away the dishes. Pretty much, yeah. Actually, I got kind of mad cuz I was gonna start the recipe of the wheat bread or whatever. And he told me to go do the dishes and there was like lots of dishes. (T6A, L206-210)

Overall, students usually did what they were "supposed to" (T6A, L4-6, Raul) in Mr. Jenkins's class. Raul summarized: "Yeah, unless you do something bad, and then he'll like, he seems like the type of guy that when you really deserve a consequence, he'll give you one" (T6A, L405). The students used agency to test Mr. Jenkins's limits but were flexible and ultimately accommodated the classroom culture, norms, and values since they supported students' attempts to accomplish personal communication goals and maintain identity yet attain points, not get suspended, and return to regular school. 


\section{Conformism}

In this classroom, Mr. Jenkins and students exhibited conformism to the dominant ideology about Culinary Arts as three subthemes: (a) vocational education, (b) banking education, and (c) motivation and reward. Conformism, the opposite of resistance, is a form of behavior that simultaneously suppresses social contradictions and merges with the "logic of ideological domination" (Giroux, 2001, p. 109). Dominant ideological logic in education presumes the existence of an authority figure with the necessary knowledge to determine and issue decisions.

\section{Vocational Education}

Vocational education in Culinary Arts consists of (a) a chef in charge and (b) obedient assistants.

Chef in charge. As chef in charge, Mr. Jenkins was the authority figure in the kitchen that provided on-the-job training for his students by making all decisions and issuing directions, kitchen chores, and cooking tasks to his assistants, the students. Mr. Jenkins explained:

It does look like Hector is on task. I guess Green's on task with whatever recipe's got, Raul is back there, looks like he's doing things in the mixer, so I guess he's on task, yeah, see, and I love it, I have handed off the cards, they know what they're doing, you know, and- and pretty much I'm just, you know, ideally for me, you guys are here gaining the experience you know, the, what do they call it, muscle memory, you know, and- and- and using the scales and practicing with just you know, just clar- so that you know that when it says one and one half c on the paper, that translates into 12 ounces in the measuring cup, you know, in the tangible cup, um, Exactly! And then that way, you know, I'm just here for for clarification, for reference, you know, as long as they're keeping themselves motivated on track, in a reasonable timeline, great, you know, that that that's the goal really of vocational instruction, is to have them working as if it were a job. (T6B, L389-393) 
Mr. Jenkins was responsible for making sure that food was correctly produced in a timely manner. In order to accomplish these goals, Mr. Jenkins ensured that students knew that he made the decisions. To enforce the point that he made all the decisions, teacher talk was predominant and consisted of I-phrases such as "I was supposed to have those multigrain rolls" (T4A, L123), "I don't want to make the same old Chicken Noodle Soup" (T4A, L135), "What I'm doing with this sausage I got left over" (T4A, L140), or "This is what I'm after is to make these little circles" (T4A, L258). Other I-need or I-want-youphrases suggested that the chef needed assistants to get the job done. For example, Mr. Jenkins would say, "I need you to cut up the celery, and then do this sautee thing" (T4A, L170), "Green, I want you to look at that card, read the ingredients, and then read the steps it takes" (T4A, L95), or "I want you guys to actually be able to do a little cooking show thing" (T4A, L152).

Students conformed to Mr. Jenkins's position as authority figure in the kitchen. When asked who decided which recipe card would be used on a given day, Raul said, "Him, but supposedly the other day, the, um, he let the kids pick. But he- he says he doesn't usually do that cuz the kids pick something too extraordinary or something" (T6A, L113). Patt agreed, "He lets us pick recipes sometimes" (T6A, L119). Raul explained further about about Mr. Jenkins's scheduling decisions, "Yeah, unless we do cookies, and, cookies is the only thing we can make ourselves and eat ourselves. Yeah, everything else usually takes more than our class period to get it ready and all that stuff' (T6A, L124-126). Patt added, "Usually our class like our class does the .... getting ready for selling stuff “ (T6A, L127, 131). Raul concluded, "Yeah, cuz first period, they're the ones who get it ready kind of and we usually finish it .... [then they sell it] 
the next period, yeah. The period after ours" (T6A, L130, 132-134). Raul expressed confidence in Mr. Jenkins's decisions, “[Mr. Jenkins] usually knows his time, like his timing. So he knows when stuff should be done" (T6A, L237-239).

Obedient assistants. Obedient assistants, or apprentices, are necessary in Culinary Arts vocational education. Mr. Jenkins stated directives in subtle ways to gain obedience. For example, after concluding a story about a cooking mistake a student had made while making multi-grain rolls, Mr. Jenkins said to Raul, "what I ought to do is have somebody make another batch" (T4A, L90). He gave a recipe card to Raul to read. Then he gave Raul another card and said, "Hand that to Green for me" (T4A, L93). Mr. Jenkins explained:

Again, enlisting the peer to give that one to Green, you know, so that there is a little bit of a demonstration of obedience, you know, the teacher told me to do this and I'm doing it, you know, that's- that's a tool that I try to maximize in subtle little ways I mean, you know, it's me giving something to Raul and saying Raul, do this, and Raul does it- does it- and everybody gets the - exactly, it sets the example, yeah. (T6B, L365-373)

Raul provided insight on Mr. Jenkins's subtle ways of enlisting obedience:

He gave me a card and said to do the recipe. And he asked me if it made sense. I thought he was, at first I thought he was asking me to read it if it made sense, cuz relating to the kid with the yeast and the flour, whatever? Oh, oh, oh. But he was actually telling me to- to to do it to To do it. I thought he was telling me, because you know how the kid messed up? Uh-huh. He thought, I thought that he read it wrong, that's why the kid messed up. And that's why I'm reading it. To try to figure that out. Yeah. And then I realized that he was actually making me do that recipe. (T6A, L95-101)

Once Raul thought about it, he realized that Mr. Jenkins expected obedience. Mr. Jenkins also issued many spur-of-the-moment errands or busywork tasks to assistants to ensure obedience. For instance, he "told Raul to put away the clean dishes" (T6B, L461) in answer to Raul's comment about a bowl, "Mister, I'm about to throw this out 'cuz there's 
a crack in it" (T4A, L138). When asked what they mostly do in class, Hector commented, "He just tells me to get things to put them in to measure" (T6A, L223). Patt answered, "I don't even do that much. Sometimes. [I mostly do] errands" (T6A, L225-231). Overall, the students respected Mr. Jenkins's authority and expectations of obedience.

Banking Education

Banking education assumes the existence of (a) authoritative knowledge and (b) predictable knowledge (Freire, 2000). Authoritative knowledge is predetermined, unquestioned, and assigned, or delivered, as individual tasks to passive subjects. Predictable knowledge is best learned by being broken down into steps, or basic skills. Authoritative knowledge. Authoritative knowledge resided in Mr. Jenkins's prerequisite culinary knowledge. He predetermined the basic skills to be taught and did not encourage decision making input or creativity from his assistants in order to gain the desired food product. He expected correct answers; thus, students often feigned understanding.

In banking education, students or assistants are supposed to listen, regurgitate correct answers, and do tasks that are distributed to them. Teachers or chefs in charge lecture, ask questions with correct answers, and provide individual assignments for students to complete silently. Mr. Jenkins provided Culinary Arts experiences to his alternative education students through these banking education principles. Mr. Jenkins handed out recipe cards he had created, reminded students of the proper procedures, asked questions with correct answers, or conducted mini-lectures in order to get individual assignments or projects under way. Once cooking projects were under way, student participation was usually silent, "it's more of a hands on. Once they've got their 
instructions which commonly are in writing anyway, and the task to be performed can be done with headphones on" (T6B, L189).

Mr. Jenkins often provided the answers he expected from students during the process. If students did not know the correct answer, they often guessed or pretended to know. For example, after Raul completed his vocabulary and entered the kitchen, Mr. Jenkins asked him, “Okay, what's the first two things?" (T4A, L106) to which he expected the correct answer. However, Raul was thinking about his assigned recipe and answered, "Water, and butter" (T4A, L107). Mr. Jenkins pursued with another question to get the correct answer, "First two things when you go in a kitchen" (T4A, L108)? Raul answered not so confidently, “Oh, wash your hands. [And] Umm. Apron?" (T4A, L109111). Mr. Jenkins answered, “That's correct” (T4A, L112). Almost immediately, Mr. Jenkins looked over to Green and said, "Green, what's your recipe card say" (T4A, L113)? Green responded quietly, "Chicken Noodle Soup” (T4A, L114). Then, Mr. Jenkins asked, "Think you can pull that off" (T4A, L115)? Green said, "No" (T4A, L116), but Mr. Jenkins quickly responded for him, "With your eyes closed probably" (T4A, L117).

When Raul asked Mr. Jenkins if it was okay to use a whole stick of butter, Mr. Jenkins told him how to figure out the answer, "They're four ounces. Four ounces and four ounces. You need seven so cut a ..." (T4A, L182). Mr. Jenkins explained:

And the same thing, sticks of butter, there's 4 in a pound, 4 ounces each, and uh, I give him the recipe and said how much butter do you need? It's 14 ounces, I said do you know how to figure that out without using the scale? And he kind of did the same thing, you know, and he wasn't in it for some reason, his little brain disconnected at that moment. And so I had to kind of like, you know, hand him one, how much do you got, 4 ounces, now how much do you got, 8 ounces, one more, 16? Oh, you man, but it took him a second before he finally, wait a minute, 
well, ok, I need- how much more do you need, half a stick, you got it. So I was like-you can do it without a scale. (T6B, L509-515)

Mr. Jenkins made all decisions and provided all answers in his classroom during the cooking projects rather than let students learn by experience or trial-and-error.

Predictable knowledge. Predictable knowledge was necessary to fulfill Mr.

Jenkins's ideology about how to teach alternative education students and how they

learned. Mr. Jenkins explained an ordinary day of teaching Culinary Arts to alternative education students:

Uh, you know, I guess, the easy way to answer that is uh, I have a, uh, an objective, uhh, it's usually some kind of a dish, an entrée that we are preparing, that we are baking, and uh, I have to break down all the tasks. (T6B, L55)

Mr. Jenkins confirmed that he decided on the objective for the day and how to break down all the tasks. When asked to explain an ordinary school day for his students, Mr. Jenkins added:

I'd have to say pretty much the same thing that you've gotta uh, expect that there's you know, some work that we want to get done, um, some basic skills that I want you to learn, and it's gonna take some flexibility on your part, you know, I'm, I might, I might assign three people to do a uh, one thing and then the fourth person is doing something entirely different. (T6B, L63)

Mr. Jenkins explained students' ordinary day from the perspective of what he wanted

them to learn. He elaborated on how he graded students:

Basically, um, it's mostly on their participation, it's like $80 \%$ you know, is based on being, participating in class whether it is hands on cooking, cutting, mixing, or if it's a discussion, if I'm discussing things, you know, they're, if they're in tune, they're participating, it's participation, and then a smaller degree is their daily vocabulary and uh, And then they have a midterm and a final exam. (T6B, L133135) 
Grading students on participation accentuates predictability. In banking education, predictability is desired and attained by making things easier, consistent, and more organized for the students. Mr. Jenkins noted:

To me it's a lot easier to like, again, you boil it down to the common word, consistency, you know, the more that uh, that I can give them something that's uh, familiar and it's uh, a standardized recipe, it's easier, it has predictable results, it's a win-win, it's easier for me, it's better for them. Typically, it's the whole recipe, I might take and break down parts of it, you know, and say, all right, you're doing this part, and whatever, um, and then, obviously, it's not too complex of a recipe if I can fit it on one card [laugh]. (T6B, L59-61)

Raul agreed, "It's actually the easiest way, the card" (T6A, L109). Mr. Jenkins also assigned tasks that involved copying such as the classroom map he gave students onto which they copied names of appliances and other items that were labeled for them. He explained:

And that way, they'd they'd, all they had to do was copy everything down. By doing that, hopefully they would learn some of the names of the things, where things belong, you know, and um, I'd like to say it set them up for success, because that way you know, I tell them to do it and it's easy for them to do it and they do it. They've accomplished it. Um, the other thing, you know, it's one more piece of consistency in their day, you know, if everything belongs in the same place, you know, and that way, they- it's easier for them to put it away, and keep things organized and find it when they need it. (T6B, L141)

Copying and keeping things organized kept things easier. Students ultimately copied their vocabulary and conformed to Mr. Jenkins's classroom organization, always replacing items specifically where they belonged or asking Mr. Jenkins if they forgot.

\section{Motivation and Reward}

Motivation and reward were both accomplished in the Culinary Arts classroom with food. For example, when talking about what they would be cooking one day, Mr. Jenkins excitedly brought up the sausage they worked on last time. He said: 
Did you guys see that sausage? Only before it was cooked, huh? You saw it right, when I was putting it on the pan oh, you know what, yeah, I remember right. I piped out the first two long pieces, and then I said let's take and taste a little piece of it, right? And it didn't have enough flavor. (T4A, L125-129)

Hector looked up from his drawing and answered, "Yeah, and then you wanted to put that chicken flavor" (T4A, L130). Mr. Jenkins concluded the conversation, "Once we put that in there, man, that stuff was outstanding” (T4A, L131)! Mr. Jenkins explained:

Umm, well obviously, I have the advantage of having food as a reward, that's kind of nice, and I do try to- uh, try to work that in- I get a little extra motivation out of that, and it can go pretty far sometimes, um, occasionally you'll hear a student comment on it but, more often, they accept it without any comment, it's that most any kind of a uh, um, Yeah, any kind of a reward is generally food out here. It tends to be the motivator of choice. (T6B, L239-251, Mr. Jenkins)

Mr. Jenkins commented that food was the motivation and reward in his classroom. Raul confirmed what he was doing during the DVD, "I'm over there trying the dough" (T6A, L390-392). He explained his motivation:

I try to do more cooking so we get to eat. If you finish, then usually - sometimes he give us like a treat, you know like today, he gave us a cookie for example. Or like a sample, so I usually try to get the cooking part done. (T6A, L233-237)

On the whole, students conformed to the dominant ideology about Culinary Arts exhibited in Mr. Jenkins's classroom. They came to class, obeyed the kitchen cooking rules, tried their best to act interested and guess the right answer, were respectful to $\mathrm{Mr}$. Jenkins and each other, and accepted and completed the tasks he gave them. Conformism allowed them to reap the benefits of Culinary Arts products while staying in school.

\section{Negotiation}

This section discusses Mr. Jenkins's and students' negotiation of classroom situations, which manifested as five subthemes: (a) vocabulary, (b) student intrusion, (c) cooking projects, (d) cell phones, and (e) skipping class. A summary of negotiations 
concludes this section. Negotiation occurs when participants make bids to alter the “normative infrastructure" (Carspecken, 1996, p. 116). A normative infrastructure develops when all participants help coordinate the culture, norms, and beliefs within an interactive setting. If negotiations are successful, a setting shift results in replacement of the previous normative infrastructure with a new one. Mr. Jenkins initiated negotiations when student resistance first emerged in order to maintain control, accomplish his Culinary Arts goals, and prevent student suspension. In turn, students negotiated with Mr. Jenkins to accomplish personal communication goals, maintain agency and identity, and prevent suspension. Negotiations were exhibited by varying talk, humor, and social distance according to the severity of the situation.

\section{Vocabulary}

Negotiation of vocabulary occurred at the beginning of class. Mr. Jenkins recognized the need to negotiate with Green when he was the only student busy texting instead of doing vocabulary. Exhibiting his sense of humor and maintaining social distance while seated at his desk, Mr. Jenkins whispered Green's name, gestured as if writing on a pad, and said, “Green, I don't accept vocabulary on a text, man, come on, pencil and paper" (T4B, L27-28). Green was more interested in texting that day, however, so he entered into the negotiations with his first excuse, "I 'onno, I 'onno where my journal at" (T4B, L29). Mr. Jenkins answered a little louder, "Pencil and paper, please" (T4B, L30), emphasizing please. Green retorted, "Huh” (T4B, L31), still texting. Mr. Jenkins replied very loudly, "VOCABULARY” (T4B, L32). The negotiations continued back and forth, elevating with each round. Green said, "I ain't got my journal" (T4B, L33). Mr. Jenkins replied, “Try saying that in English” (T4B, L34). Green repeated 
in a different tone, "I ain’t got my journal" (T4B, L35). Mr. Jenkins tried a question, "Your-your book's not over there" (T4B, L36)? Green answered quietly, "No" (T4B, L37). Mr. Jenkins asked a leading question to let Green know he was getting tired of this, “Or you just ain’t got it” (T4B, L38)? Green seriously answered, "My book’s not over there" (T4B, L39). Mr. Jenkins knew better and emphatically responded, "Go over and git it! Please" (T4B, L40-41).

Mr. Jenkins realized the situation had become more severe when Green continued texting. Mr. Jenkins changed his tone to a more sarcastic one and reminded Green of his behavior in this class up until that moment, "Green, you ain't been buckin' me all year, man, don't start out now. I used to think you was kinda-" (T4B, L43-44). Green got the message, knew that he had reached Mr. Jenkins's limit, and interrupted him, "I don't wanna (undecipherable) over and see if I can find it" (T4B, L45). Green walked slowly over to the journal table. Mr. Jenkins surveyed Green silently for 25 seconds as he shuffled journals. Green found his journal and announced in a surprised slightly sarcastic tone, "How'd my folder appear here and I just looked for it" (T4B, L47)? Satisfied that Green would begin his vocabulary, Mr. Jenkins immediately started talking about early release day.

When asked about varying his voice tone, Mr. Jenkins said, "I do that sometimes, once in a while, I was taught by ... somebody else that once in a while you just vary your tone, sometimes that actually by lowering your tone, (undecipherable) better" (T6B, L279). Mr. Jenkins also commented both about Green saying he couldn't find his journal and on the long period of silence, "He's telling me he doesn't have his vocabulary book. They live right here, they're supposed to be right there, it should be pretty much an 
automatic thing. Waiting for him to go ahead and get it” (T6B, L281-283). Mr. Jenkins patiently waited.

During their interview watching the DVD, students explained what they thought

Mr. Jenkins was doing over there behind the computer, Raul answered, "Chillin' [laugh]" (T6A, L67). Hector agreed, "Yeah. Might be pretending to do something" (T6A, L6, 70). Raul concluded, "Probably waiting for us" (T6A, L72). They laughed in unison when Mr. Jenkins said that he didn't accept vocabulary as a text, but they also knew when Mr. Jenkins's limit had been reached. Hector commented, “He's telling him to lose the phone. He's got to lose the phone" (T6A, L186-188). Students' negotiations illustrated both their desire to stay in this class and their respect for Mr. Jenkins's classroom culture, norms, and values.

\section{Student Intrusion}

Negotiation of student intrusion was exhibited by Mr. Jenkins's multi-tasking, vernacular, lively talk, sarcasm, and leading questions to remind students what was expected and required in his class. For instance, a student from another class walked in and asked, "Y'all selling anything” (T4A, L219)? Mr. Jenkins was multi-tasking, directing the current cooking project while talking on the phone trying to locate the missing salt that students in another class had used. He replied with a smile to the student

intruder, pointed and nodded to him, and spoke vernacular, "Gimme this bootleg pass and I'll" (T4A, L220). The student responded with a smile, "It ain't no bootleg pass" (T4A L221). The negotiations continued. Mr. Jenkins said, "See you later, man" (T4A L222). The student begged, "Why ya not sellin' nothin"” (T4A L223)? Still on the phone, Mr. Jenkins answered humorously, "Is this Culinary Selling Class or is this Culinary Arts 
class" (T4A, L224)? The student retorted, "No bread, no nuthin"” (T4A, L225)? With a melodic sarcastic voice tone, Mr. Jenkins said, "I'll see you later, have a nice day" (T4A, L226). Student replied in the same tone, "Gon' do me like that" (T4A, L227)? Mr. Jenkins continued the negotiations, “That's exactly the way I'm doin' you. I'm doin' you just the way I'm s'pposed to, ain't I" (T4A, L228)? The student relented, "Oh” (T4A, L229). To complete the negotiations, Mr. Jenkins confirmed that he and the student agreed, "Ain't I" (T4A, L230)? The student walked out saying, "Not really" (T4A, L231).

Immediately after confirming negotiations, Mr. Jenkins walked over to the work table, turned the phone off, pointed to the pasta students were making, and said, "This is s'pposed to stay covered with plastic" (T4A, L233). Simultaneously, another student peeked in and said Mr. Jenkins's name to which Mr. Jenkins looked at the door, smiled, and waved (T4A, L235). Mr. Jenkins explained his rationale for using vernacular:

Ain't I? you know, oh, I tend to uh, I tend to give in, uh, to ummm, trying to I don't know, instead of trying to, uhhh, should I say I give in to using the vernacular, you know the common language, you know. I guess part of it, I- I- I guess I rationalize it, that uh, in some way it's a comical thing, you know, here's this teacher talking like he's you know, a kid on the street, you know that, I don't expect to be taken seriously in that sense, but uh, I don't know, I- I- I'm a little hesitant to defend that uh, behavior [laugh], that's not necessarily the best way for me to to be addressing the students. It's comfortable. Well, I mean, you know, we should be trying to set a better example, you know, deal with them a little more professionally - I guess, you know what it is? It's uh, it's kind of showing that I uh, that I don't take- I don't take things too seriously, in some ways, that's good, in some ways it's not good, right? I mean, you know, he's he's not in his class, not supposed to you know, he's not supposed to be interrupting my class but I'm not making a big deal of it. (T6B, L416-426)

Mr. Jenkins rationalized vernacular as comedy to lighten a situation. Mr. Jenkins showed students both that he liked them and other people and that he wanted people in his 
Culinary Arts classroom to cook or buy food. He summarized, "People love- I'm never lonely in a kitchen" (T6B, L155). Some people regularly visited the kitchen/classroom for food, ice, or other items. Patt commented that one lady "always comes in for something" (T6A, L355). At the same time, Mr. Jenkins exhibited that he cared about preventing student suspension and would not relent in his negotiations until this goal was accomplished. Mr. Jenkins's negotiations gained students' favor, support, respect, and solidarity. Raul concurred with the other students about Mr. Jenkins's consistency on and off camera, "No, no, naw, he's cool, he's the same" (T6A, L276).

\section{Cooking Projects}

Mr. Jenkins negotiated cooking projects by telling stories with a humorous sarcastic tone and interjecting catchy phrases, such as "Think you can pull that off?" (T4A, L115), or "With your eyes closed probably" (T4A, 1123). Appealing stories were used to motivate students to get on board with cooking projects, drive in a point about poor decision making, maintain students' attention, or gain some down time for himself. For example when Green finally opened his journal to write, Hector looked busy, and Raul had just completed the vocabulary and stored his journal, Mr. Jenkins started telling a cooking story from his desk:

Now, this is interesting. Last time you guys were here, we were making sausage, right? And I had somebody making soft rolls. And I had Randall Waddell working on the uh multi-grain rolls, wasn't it? Wasn't it Randall? The guy I said I wanted to hit him because he messed up the yeast, instead of putting it in with the flour he- (T4B, L85, 87)

Raul said, "Yeah, I think" (T4A, L88), leaning on the long work table to listen. Mr. Jenkins added, "He put it in the thing. I mean, no, they say that all's well that ends well, 
ever heard that before" (T4B, L89)? Raul laughed softly and answered, "Um-huh" (T4B, L90). Gesturing, Mr. Jenkins continued as if he did not hear Raul:

That means that if it ended okay, the bread turned out ok, then all is well. That means it's okay for me to say that I want to hit him, I don't know, that's not quite okay. Anyway, um, the bread turned out okay. (T4B, L91)

Raul whispered while peering down into the trashcan, "Where'd he put the yeast" (T4B, L92)? Mr. Jenkins held his hand on his chin and seemed puzzled, "He was in second period though" (T4B, L93). Raul still wondered, "What'd he do" (T4B, L94)? Mr. Jenkins sighed, snapped his fingers, and completed the story:

That's right, he wasn't with you guys. You guys just had to shape that. He put the yeast in the mixing bowl with the warm water and the egg and the stuff that goes in there. You know, basically, you know what I ought to do is just have somebody make another batch. (T4B, L95-96).

Mr. Jenkins's motivational story ultimately pointed out a student's cooking mistake.

He pulled a card out of his pocket and gave it to Raul, "Check this out, it's got two steps, step one, step two, make sense? Tell me in a minute" (T4B, L96). He waited silently for 15 seconds and then told Raul to give a recipe card to Green. During the interview, Mr. Jenkins questioned his approach to pointing out a mistake. He explained:

Raul's doing his thing. I always feel bad when I - have a - whenever I have a- asuboptimal response to, uh, to, uh, a negative behavior - when I don't respond as well as I should have. I'm just talking - I'm I'm what I was doing was responding to my feeling bad, saying look - and I yelled at this kid and I told him that I wanted to hit him and that's not the way I should have been, you know? And I feel bad about, you know, just even saying that, you know, you never know exactly how does that affect a kid, for especially a teacher to say I feel like hitting you, that's pretty unkosher. I guess he laughed and took it as, he knew that I wasn't serious, and uh, in reality, you know, the mistake that he made, you know, uh, if I would have been a little more careful, it would have been avoided. I had him going a little more on automatic, you know, he wound up with an oversight. (T6B, L349-361) 
Raul misinterpreted some of Mr. Jenkins's story. When asked if he had heard the phrase “all's well that ends well” (TB4, L89) before, he said, "Naw, actually I haven't. I didn't get that- what he means by- all's well that ends well" (T6A, L82, 90). He asked Hector if he had heard it. Hector said, "No" (T6A, L83). When asked if he remembered about the student who "messed up the yeast" (TB4, L87), he said, "I think I said yeah, but I don't know who that was. Go back, I think I nodded" (T6A, L87). Students negotiated by listening and agreeing out of respect for Mr. Jenkins in order to reap the rewards of cooking more quickly.

\section{Cell Phones}

Mr. Jenkins often negotiated the use of cell phones and other signs of slipping behavior that could result in student suspension. Even though Mr. Jenkins's talk did not come across as professionally as he thought it should or as it may appear objectively, he was ultimately concerned about preventing student suspension, giving real choices, and being fair. Students were attuned to these practices and, thus, willing to negotiate. For example, after Green found his journal and began walking to a writing table, Mr. Jenkins immediately interjected two short stories using leading questions and sarcasm to pursue the point that Green was slipping in behavior inside and outside of the class. First, Mr. Jenkins asked, "Did you guys get what they were saying about the early release day" T4B, L48)? Green said, “Today” (T4B, L49). Mr. Jenkins rephrased the question, "Did you hear what they said about it" (T4B, L50)? Green answered, "No" (T4B, L51) as he gently tossed the journal onto the table. Mr. Jenkins drove the point home, "Yeah, they said if you give Mr. Jenkins a hard time you don’t get the early release” (T4B, L53). Green dropped the journal on the table once more and answered sarcastically, "Oh, gotta 
give you a hard time" (T4B, L54). Mr. Jenkins immediately told the students what was really said about early release.

A few minutes later, Mr. Jenkins looked up from his computer and the online electronic gradebook showing that day’s absences and tardies. Green had been marked "tardy in the beginning so [Mr. Jenkins] figured [he'd] go ahead would ask him about that" (T6B, L327, Mr. Jenkins). He asked, "Green, you don't take the bus to school" (T4A, L69)? Green, texting under the table, answered, "Yeah" (T4A, L70). Mr. Jenkins asked, "Bus was late today" (T4A, L71)? Green answered, "No" (T4A, L72), finally put up the cell phone, and moved the Glossary they copied definitions from closer to his journal. Mr. Jenkins egged Green on, "Bus was on time, but Green was late” (T4A, L73)? Green admitted, "Green was tardy" (T4A, L74), and he began working on his vocabulary. Skipping Class

Negotiation about skipping class was immediate and abrupt to prevent student suspension, but neither Mr. Jenkins nor the student held grudges. For example, at the same time Mr. Jenkins gave the lady a bag for some ice and was on the phone looking for the missing salt, Patt left the room unexpectedly. Mr. Jenkins reacted immediately. First, he told the person on the phone to "Hang on one second" (T4A, L215). Then he yelled to someone outside, "If you see Patt Glover out there, tell him he better get his butt back in here" (T4A, L215)! Then he returned to the phone call, "All right. Robert. Mr. Jenkins. Listen, when you guys were making rice this morning, where'd you get the salt" (T4A, L215)? During a silent 35 second pause, Green was cutting celery, Raul was tasting dough, Hector was squeezing dough, and Mr. Jenkins was still on the phone looking for 
the salt. Someone knocked on the door and Raul answered it since he was closest. Patt entered as if nothing happened.

Mr. Jenkins briskly walked over to him and said briefly, definitively, and with his arm up in the air, "Hold up. Hold up. What are you going [walking] out of my class" (T4B, L225)? Patt faced sideways and answered quietly, "Go findin', go see something" (T4B, L226). Mr. Jenkins replied "Go wash your hands. You did NOT have to go and see something" (T4B, L227)! Patt walked quickly to the work table and got back to work without washing his hands. Mr. Jenkins nodded and smiled to the other student who just entered to buy something. When asked about what happened and why, Mr. Jenkins laughed and explained:

Yeah, he just popped outside the door for something. I have a recollection of that. Like I was just saying, he was just here a second ago, where did he go? He disappeared. Yeah, what happened is he was at the door, you know, he opened the door for the lady to come in, I guess. And then all of a sudden, he got sucked out. What happened? That's the difficulty of being the laboratory instructor, I mean, I guess any teacher faces that, you know, kid gets sucked out the door - you can't go and chase after them, you have to deal with what's in here and so now, not only is it going to wind being a response to the thing, you know, what - I gotta respond to what happened. So, so first try was to just tell somebody that if they see him, to send him back in. (T6B, L396-404)

Mr. Jenkins commented on how he must quickly respond to what happens in his classroom. His sense of humor and persistent negotiations with students supported both his and their goals to communicate and prevent suspension. He explained his rationale:

Yeah, he said, I had to go and look at something or something like that. And I said no you did not have to. Yeah, basically, I mean, well. I mean and- and largely in a situation like that, it's gonna be a judgment call, you know, is this, has this become a regular thing? Or is this an unusual behavior that can be excused as an exception? And you know, for Patt, yeah, generally, like I said, he's always up on task, everything usually works good, and then now, just just once in a while he's getting a little more off track and so you know, I want to make sure that I at least address it somewhat but not make it a bigger deal than I have to. (T6B, L238-234) 
Mr. Jenkins made judgment calls based on the situation and student's behavior history. Students did not take offense but rather thought of Mr. Jenkins as supportive when he talked to (or even about) them. When asked why he went outside that day, Patt said, "I went to the front, I thought my grandmamma was in the front" (T6A, L368). That was the day he had just returned from a parent conference. Patt said that there had been a fight outside and "what happened was that [a] lady had busted her nose" (T6A, L74). He explained that "when the lady came in [the] room to get ice" (T6A, L79) for the lady who busted her nose, he walked out "to see my grandma, I thought she was out there but she was gone already" (T6A, L384). He concluded, "I was just going to ask her for some money" (T6A, L388). Mr. Jenkins negotiated abruptly with Patt but did not make a big deal of the situation based on his behavior history. Students evaluated Mr. Jenkins's negotiations with them and understood that abrupt negotiations were initiated for their own good.

\section{Summary of Negotiations}

Mr. Jenkins negotiated with students about their behavior by varying his talk, voice tone, nonverbal cues, and social distance depending on the situation and the severity of the student's individual behavior. Students judged Mr. Jenkins's negotiations and responded in like manner until it behooved them to succumb. His talk dominated the classroom in general; however, during negotiations it was energetic and interspersed with purposeful spurts of emphasis. He often gestured and waved his hands like he was directing traffic. He smiled, nodded, and shifted his movements from slow and methodical to quick and urgent depending on the situation. Mr. Jenkins continually 
assessed the situation with alternate periods of silence, efficient multi-tasking, and persistent negotiations until satisfied that he had achieved or resumed successful control of the classroom. As a rule, Mr. Jenkins let nothing get by that interfered with his ultimate goals yet always had a sense of humor. He summarized his philosophy for dealing (negotiating) with unwanted student behavior as follows:

Obviously, whenever people ask me where do you teach, and I say [The School], you know, uh, if you say [Main High School], or if you say, you know, some obscure name, most people haven't heard of it, you know, I don't know the names of $95 \%$ of the schools in our district. Obviously if you say [River School or Downtown School], at least I've got a geographic location- And maybe I've got an idea of the student body there. The point is, obviously, is when you mention [The School], people will have an idea of the student body here, a little more commonly. This one guy, he said, oh, [The School], he said, and so you're a black belt of what field of, you know, martial arts, and I said- uh, comedy. I said it works every time. Well, it works for almost every situation. It helps to diffuse the situation. (T6B, L293-303)

Mr. Jenkins relied on the use of comedy to help negotiate or diffuse almost every situation.

In order to accomplish what he wanted, Mr. Jenkins negotiated less severe situations and behaviors with more humor and more social distance. If a less severe situation gradually became more severe, Mr. Jenkins constantly changed his tactics and spent as much time as he needed to negotiate the response he wanted. More severe situations and behaviors, which increased the likelihood of student suspension, involved shorter periods of negotiation with increasingly less humor and social distance. Once satisfied, he abruptly changed topics.

Students negotiated with Mr. Jenkins by knowing when and how much sarcasm and vernacular to use with him, responding with silence, and listening to stories that ranged from service projects he wanted to conduct with them at the nearby adult day care 
center in the future because his "head's really not in it today" (T4A, L161) to getting free tickets to "his first hockey game" (T4A, L166) the night before. They trusted him as a person and realized that his ultimate goals meshed with their own goals. Raul summarized:

Yeah, he uh, I guess he tries to like influence us in a way so we like come to school and like we stay positive you know and maybe if he just ignored us and like oh, cook and cook, you know, probably wouldn't like the class as much. But he takes time to talk to us and get to know us. That's what I like about this school, most teachers are like that. (T6A, L266)

The students knew Mr. Jenkins wanted to influence them positively. They also knew that referrals were not an option in this classroom. Several times during the interview, students provided unsolicited comparisons of Mr. Jenkins's negotiations. Students concluded that Mr. Jenkins was unlike the other teachers who (a) did not listen, (b) called students derogatory names, and (c) got outwardly mad. As a result, students respected Mr. Jenkins.

Mr. Jenkins was unlike other teachers who did not listen to students. Hector agreed, "Yeah, Mr. Frederek is that type though" (T6A, L410). Raul said, "Oh, yeah, Mr. Frederek is like that. Mr. Frederek is so lenient" (T6A, L411, 414). Hector added that he "has got a lot of patience" (T6A, L416). Raul explained, "That guy has got some patience. That guy needs an award" (T6A, L417). Hector agreed, "Yeah, seriously. Honestly” (T6A, L419). Raul continued, "The kids are screaming in his class, you know, constantly, AHHH, Mr. Frederek! Like calling his name, you know. Like saying stuff to him, jokes to him, messing with him" (T6A, L420, 422). Hector explained:

Like one time, one time when it was like my first day, so I- I didn't even know much about him. But I remember he was doing something on the board and this kid was calling him and Mr. Frederek was kind of occupied. So then the kid starts 
like hey, stupid b- and Mr. Frederek turns around and he's like you see, he only responds to me when I call him b-. (T6A, L423)

Raul laughed and added, "Yeah, that's right. I remember that" (T6A, L424). Hector concluded, "When I start acting nice to him, yeah you remember that, when I try to be nice to him, he doesn't listen to me" (T6A, L425). This statement may align with findings in Classroom Three that show how Mr. Frederek is king of patience and seems aloof until someone is getting bothered too much.

Mr. Jenkins was not like other teachers who called students derogatory names.

Hector explained about another teacher [not in the study] at The School:

Dude, when I was reading in his class, I kept mispronouncing a word and he was like are you dyslexic? Because some words sound the same and I kind of read it before I actually read it. And he started calling me dyslexia. I would get him [mad] on purpose. (T6A, L282-284, 305)

Mr. Jenkins was also unlike other teachers who got outwardly mad. Raul explained that other students got this same teacher mad on purpose.

Yeah, that's what they did in my class, in my class they took like this little paper that had, for example this, and then they hid the paper, and the paper was worthless but he wanted it for his next class. And for the next 20 minutes he was saying literally the same phrase, where's my mark, where's the thing where's my mark, where's my mark, where's my mark, he wouldn't say nothing but where's my mark - The little, it was, it's Germany money after the war that wasn't worth nothing or nothing or something like that and he had one of those. (T6A, L308310)

Raul explained further about why students got this teacher mad:

Because when he gets mad, it's hilarious. And then he's chill, and he's chill, and then some kid told him he got jacked. What? You jacked me, you jacked me. What's jacked? You jacked me on my back, huh? Come here ... jack my pen. He got a pen and he tells some kid to take the pen and he was like take the pen, take the pen, take it in front of me and the kids were like laughing and I was [too] at that point, cuz he was screaming take the pen! Come on, jack me! And some kids was hiding it from him just to see him get [mad]. (T6A, L315-323) 
Hector remembered his first day of class when this teacher got mad:

Oh, that reminds me when it was my first day when I was in his class, there was um, Juan I think, Luis, Nathan, and Jon or something, whatever, and we were in the classroom and then I don't know what- they started arguing about something and then the fat security guard came in, and then, I don't know, Mr. [the other teacher] tried to get Luis in a head lock or something I don't know, it was funny, he was trying to fight 'em, he was like move out of my way before I get you(T6A, L323-326, 335-336)

Students made [the other teacher] mad because it was funny, but it may end in a fight between the teacher and the student. Patt concluded, "But just don't make [the other teacher] mad. Yeah, they just do it on purpose because it's funny" (T6A, L333). Raul explained why students do not respond disrespectfully to Mr. Jenkins:

But [Mr. Jenkins] is not like, like uh, he's not going to let you take over him either. He's not that type .... no, then like then he'll get mad, like if you that's the thing, you can't disrespect him, maybe if you're fooling around, he's probably used to that. Mr. Jenkins would be the type if you disrespect him, he would like write you up or something. (T6A, L407, L429)

Students knew what was expected of them in various classes, realized the difference between inequitable or unfair and equitable or fair classroom practices, and knew each teacher's limits. In Mr. Jenkins's class, students used their agency and maintained their identity by accommodating and negotiating rather than resisting classroom cultures, norms, and values which offered real choices and the opportunity to accomplish some of their own communication goals. On the other hand, students also conformed to Mr. Jenkins's conformism of the dominant ideology about education by being obedient assistants.

Overall, Mr. Jenkins and his students had similar classroom goals, held similar ideologies, conducted similar manifestations, and worked together to prevent student suspension. 


\section{Summary}

This chapter presented a description of Classroom One findings in three sections: (a) Classroom One participants, (b) findings from interactive power analysis, and (c) themes from reconstructive data analysis. Mr. Jenkins is a White Culinary Arts teacher who had no experience or knowledge of alternative education before becoming a teacher at The School. His Latino and African American students were familiar with alternative education. Three types of power exhibited in Classroom One were normative power, interactively established contracts, and charm; coercive power was not exhibited. With this combination of types of power, Mr. Jenkins's and the students' roles meshed successfully. The teacher had a human and humorous side that was used to direct and gain assistance from his students, and to enforce classroom rules. Not much cultural power was at play in this classroom, since each student was allowed to participate in cooking projects and vocabulary while healthily incorporating his own interests without punishment to a point. Mr. Jenkins's charm and lack of coercive power balanced out the high dose of normative power he exhibited. Students believed he knew what he was doing and that he was fair and cared to support them in PBS and Culinary Arts goals. Therefore, they consented to Mr. Jenkins's clearly defined classroom norms and values.

Four themes of social interaction (resistance, accommodation, conformism, and negotiation) emerged and combined in the following ways. Mr. Jenkins resisted dominant ideology, such as PBS rules, when necessary to support his students (i.e., prevent suspension). Therefore, students did not resist his classroom norms. He and his students both accommodated classroom disruptions and system constraints by being flexible. Additionally, he and his students conformed to similar dominant vocational, educational, 
and motivational ideology, such as students listen to teachers lecture. Both he and his students also negotiated in class using similar humor, talk, and social distancing. All of the themes of social interaction were exhibited and combined successfully for similar purposes or goals of maintaining control and/or identity/agency, preventing suspension, and accomplishing Culinary Arts in the classroom. Chapter 5, 6, and 7 present the findings from Classroom Two, Three, and Four, respectively. In chapter 8, a comparison and synthesis of the findings is provided. Chapter 9 includes a summary of the study, responses to research questions, implications for teacher education, and recommendations for research and policy. 


\section{CHAPTER V}

\section{DESCRIPTION OF CLASSROOM TWO FINDINGS}

This chapter presents a description of Classroom Two findings in three sections based on the nine stages of data collection and analysis described in Chapter 3 and illustrated in the Appendices: (a) Classroom Two participants, (b) findings from interactive power analysis and role analysis, and (c) themes from reconstructive data analysis. A summary concludes the chapter. Quotes are cited by transcript name (e.g., T6B), line number (e.g., L271), and participant pseudonyms when necessary (e.g., Ms. Gomez).

\section{Classroom Two Participants}

Classroom Two was a Language Arts course with a teacher who frequently used exclusionary discipline. When asked how she became a teacher, Ms. Gomez, a Latina, said that "it was almost overnight an epiphany" (T6B, L5). She had been a paralegal for 7 years, gotten her bachelor's degree in Psychology, and was not sure what she wanted to do. Even though others told her she would be a good teacher, "it was never something [she] considered an option" (T6B, L3). She just "woke up one day and said you know what let me just go for my certificate and see what happens" (T6B, L5). She got a temporary certificate in Social Sciences, but because she really wanted to teach Language Arts, she took the Language Arts subject area test. When asked how she became a Language Arts teacher at The School, Ms. Gomez said that she "was actually offered a position at all three schools [where she applied]. Ms. Gomez laughed as she elaborated on her interviews for a teaching position: 
the guy who was doing the interview [downtown] said are you sure you want to work in that school? Yeah, he said, you know, I can put you somewhere else. We can find a better suit for you. And I said I want this school! That's what I chose ..... And even when I came for the interview [here], actually . . . the first question was do you know what kind of school this is? And I said yes, and they said and you still came for the interview. And I said yes, I did. (T6B, L13-17)

Even though she was not encouraged during the interviews she said, "I decided on [The School] because I figured I could use my Psychology degree and you know, mix that with education" (T6B, L7, 9). When asked if she was using her psychology in the classroom as she had hoped, Ms. Gomez stated that psychology

hasn't been as effective as I thought it was going to be. I still have a lot to learn in that department, because it doesn't always, reverse psychology does work, it does it really does. When I tell them, I had a few kids my first year, well I'm not going to do that. They wouldn't do the first assignment and I said ok, when the next things that we move onto, I wouldn't even give them the assignment, and then, they're like well how come I didn't get a paper, and I said well you said you weren't going to do any of the work! Well, I wasn't going to do that other one, you don't know if I'm going to do this one, so all of a sudden they want to do this one cuz I wasn't giving it to them. So that- that's sometimes effective. But other times they actually catch onto that so it doesn't always work. (T6B, L31-41)

She concluded that psychology doesn't always work in practice. Instead of using the

mandated textbooks, she would sometimes

pull stories that I think are interesting from there, that I think might work for the class but usually I'll do my own things like from what I find online. I find that especially with my high school classes that I use a lot of psychology, I use psychology-related articles. Like last week for my high school class we did 10, the 10 most irrational, why people do dumb or irrational things, 10 social psychology experiments, well-known, and so we read through that and we talked about why why there were, what was good about them, what was wrong about them, and the kids get into that, they like that, and using that, we can do the other stuff, like find the main idea, determine author's purpose, and summarizing on stuff that they like so I like to get things that they are interested in and otherwise I lose them, very quickly (T6B, L165-171)

Besides using psychology-related articles, Ms. Gomez viewed problematic classroom interactions through a psychology lens. She explained: 
I really want to get rid of is that, the way that they antagonize each other. I understand that they do it because it makes them feel better about themselves but I'm trying to find ways to show them that there are other ways to feel better about without having to put somebody down .... [but] I am still trying to figure them out! (T6B, L427, 437)!

Ms. Gomez was still trying to figure the students out and get rid of antagonistic behavior.

Ms. Gomez was also in the process of taking graduate classes for her professional certificate since her 3-year temporary certificate was up at the end of this school year "because I definitely want to continue . . . to stay in education" (T6B, L19, 23). After three years of teaching at The School, Ms. Gomez sought to simultaneously (a) develop and control a classroom culture that provided "an environment where the kids feel safe to talk about anything" (T6B, L413) and (b) enforce system academic and behavioral rules and regulations as closely as possible.

Four students participated: Dann, Stanley, Jonathan, and Jorge. Table 9 summarizes Classroom Two student participation.

Table 9

Classroom Two Student Participation

Name Observations Interview

\begin{tabular}{lll} 
Dann & x x x \\
Stanley & x x \\
Jonathan & x x x \\
Jorge & $\mathrm{x}$ & $\mathrm{x}$ \\
\hline
\end{tabular}


Dann, an African American male, participated in all three observations but not the interview due to a doctor's appointment. Stanley, an African American male, participated in two observations only; he was absent for unknown reasons. Jonathan, an African American male, participated in all three observations and the interview. Jorge, a Latino, participated in one observation and the interview. He was invited to listen to the mayor, also a Latino, speak at The School during the first missed observation and was reported as skipping during the second. Themes from the interactive power analysis and reconstructive data analysis are discussed next, followed by a summary.

Findings from Interactive Power Analysis

This section provides an explanation of the findings from interactive power analysis, including (a) types of power, (b) roles, and (c) cultural power. Interactive power analysis determines who has what kind of power and why and whose power defines interaction outcomes.

\section{Types of Power}

The researcher deductively looked for four types of power based on Carspecken's (1996) categories of interactively established power: (a) normative power, (b) coercive power, (c) interactively established contracts, and (d) charm. The next section defines and describes each type of power that existed in the order they were used. Normative Power

Normative power is gained over others by virtue of one's position or status in the culture or society. Ms. Gomez used her teacher status to claim normative power over her students and attempt to enforce system academic and behavioral rules and regulations. Ms. Gomez expected students to sit down and do their bellwork as soon as the bell 
sounded. During the interview, Ms. Gomez said that she had learned about bellwork form a workshop; bellwork is assigned work for the students to do right as they walk in the classroom after the bell rings to get them settled down and to give the teacher time to get organized. For example, she announced authoritatively for students to go sit down and do your bellwork. However, Stanley ignored her normative claim and blurted out an expletive, maintaining identity and agency. Dann appeared to accept Ms. Gomez's claim by picking up his bookbag, but he also maintained agency and identity by rejecting the claim and conversing about Stanley's cursing instead. Stanley rejected the claim again by more cursing, texting, and sitting at a great social distance in the computer chair behind the circle of desks reserved for this portion of class. Jonathan partially rejected the claim by keeping his hood on and remaining silent, and he partially considered it by sitting up in the reserved circle of desks. Ms. Gomez also expected students to follow PBS rules with normative claims, such as Dann enough! or stop running your mouth and Stanley, put up the phone or you can't use the phone no matter who it is. However, students challenged Ms. Gomez's normative power claims with persistent verbal and nonverbal disruptive behavior. Subsequently, she attempted interactively established contracts to enforce academic and behavior rules and regulations.

\section{Interactively Established Contracts}

Interactively established contracts are constructed by negotiations among participants, based on an implicit level of understanding, mutual consent, and equal communication input. Negotiations that lead to co-constructed interactively established contracts can be thought of as ongoing revisions on multiple drafts of a written paper passed back-and-forth between co-authors. It could go on indefinitely; co-authors may 
use varying amounts of charm, normative power, coercion, or a combination of techniques to sway the thinking for the final draft. However, the underlying ideas or meanings in the paper that are constantly being revised (albeit not necessarily face-toface) are what is viewed here as interactively established contracts.

Ms. Gomez attempted interactively established contracts by encouraging student conversations about what happened outside of school to gain solidarity with classroom norms - if I listen to you, you should listen to me. Students who were willing to converse (i.e., those who were not silent) steered their conversations away from academic topics that offered no real choices and toward social interactions that did. Ongoing conversations, including play fighting, dominated the classroom culture and norms. For example, when Ms. Gomez gave students the assignment to write FCAT prompts for practice, Stanley appeared to consent when he mentioned football, but then slumped down and said ohhh and returned to texting. Jonathan initially rejected the claim with a legitimate concern of I don't know what to write and began writing after Ms. Gomez clarified what to write.

Dann rejected the claim with I don't want to talk about football and stretched his arms back with $u h h h$. He changed the FCAT subject repeatedly to familiar topics such as the student who was absent because he got beat up, someone who peed on the wall last year, and his own decision to miss school the rest of the year. Dann relentlessly provided many reasons for not doing the academic work and not consenting to the interactively established contract claims made by Ms. Gomez. For example, he challenged authority and voiced his opinion about why they were writing in Language Arts class, why he disliked writing, and why he preferred a different prompt. Ms. Gomez tried to reach an 
agreement with I'll let you do that one if you want to and those are old FCAT prompts, that's why you saw that one already. Dann rejected the claim, responding with you didn't even read it to us, I could do better than ..., and no I already did that one. However, he maintained identity and agency, returning to the conversation with Stanley.

Ms. Gomez pursued an interactively established contract with Stanley implying if I listen to you, you should work for me. Stanley appeared to accept Ms. Gomez's claims by saying twice that he would get to work after coming back from the bathroom, implying a different agreement of if you let me go, I'll work. Upon return, he moved closer to the circle of desks and requested her academic assistance intermittently but continued the ongoing conversation with Dann. Stanley simultaneously strived for a student identity of one who accepts the teacher's help and a community identity of one who does not turn his back on his peers, the topic of their conversation. Jonathan rejected Dann's claim that they should not be writing in Language Arts. He consented partially to Ms. Gomez's claims by opening his notebook but complaining oh, my God, I'm not gonna. When conversations got out of hand and interactively established contracts were rejected, Ms. Gomez introduced coercive power claims but never dismissed the possibility of successful interactively established contracts.

\section{Coercive Power}

This section covers coercive power claims, manifested as (a) threats of academic failure and (b) threats of written referral. Coercive power is accomplished by forcing obedience after threatening punishment. Ms. Gomez used coercive power claims to convince students to do the right thing rather than force obedience and to enforce academic and behavior rules or regulations. 
Threats of academic failure. Threats of academic failure implied an interactively established contract. Ms. Gomez subtly threatened academic failure by deferring her decisions to authority. For example, to convince students to begin working and stop talking and texting, Ms. Gomez announced the bellwork as something they were supposed to read today, what we're supposed to do in each class per tacit district requirements, and to listen up this is what we're doing today because we've got FCAT Writes on Tuesday. Stanley rejected the subtle threats with a request to go to the bathroom, then begging to go pee, threatening to go outside, opening the door, and reporting who was calling on his cell phone at that time. Dann rejected the claim with a comment about Stanley going to the bathroom. Jonathan rejecting the claim silently.

Ms. Gomez reminded students why they were in her intensive writing class with subtle coercive power claims. For instance, she told them that students who did not try their best failed the FCAT and that a district sanction required students who failed the FCAT to take intensive writing. In this way, she also neutrally enforced administrative sanctions by not claiming responsibility for the decision. She then told students to put their name and date on their paper to check their consent to begin work. Dann rejected the claim saying he was not there for the test and then changed the subject to something he saw in the newspaper back to egging Stanley on saying a Steelers' player looked like his uncle, retaining agency and identity. Then, Dann challenged the administrative decision to put him and Stanley in the same class. Stanley appeared to accept Ms. Gomez's claim saying he would work when he returned, but then changed the subject to ask about her bike parked in the room, and then retorted to Dann's uncle comment while 
leaving the room, retaining his agency and identity. Jonathan rejected the claim by noticing Ms. Gomez's ring and asking about marriage.

Ms. Gomez combined interactively established contract possibilities with subtle coercive power claims to gain students' solidarity and consent to enforce academic and behavior requirements simultaneously. For example, she implied stick with me and I'll help you complete the program or else I'll refer you if you cause trouble when she used statements such as this is not the topic we are supposed to be writing about to insinuate the threat of referrals for not doing work. She also implied paper and pen agreements such as if you show me you will work, I'll give you supplies. Stanley acknowledged briefly that he had paper but not pen, offering tentative consent. However, he and Dann continued to define the interactive setting by rejecting her claims with persistent conversation, play fighting, and requests for Ms. Gomez to referee their ongoing conflict.

Ms. Gomez also combined implicit coercive power claims and interactively established contracts with sanction reminders if students did not obey classroom rules. She commented why don't you do the bellwork? or you're supposed to be writing on paper, not desk. However, she also talked with Dann each time he changed the subject, which clarified that she was not to the point of writing a referral yet. Ms. Gomez accepted Dann's charm to gain his loyalty, but her ulterior motive was to get students to do the work. She believed that if they tried they may pass the test.

At the same time, Ms. Gomez encouraged disruptive talk by getting involved in students' conversations. For example, when Stanley announced who was calling on the cell phone, she asked him about the caller's suspension status. Ms. Gomez also interjected comments into the conversation about what Stanley could write that related to 
what they did outside of school. She asked did that happen personally to you and clarified two personal experiences, reminding students of the prompt parameters. Ms. Gomez let Dann and Stanley continue conversing and then said that it sounds like you have something to write about, implying a tacit claim of I let you talk, you write for me.

Ms. Gomez also combined implicit coercive power claims and interactively established contract claims with reminders of they could use these grades. This reminder insinuated do what I ask and you get grades in return and we will both be meeting district requirements. She also used statements such as where's your bellwork, stop wasting time, start writing, the more you write today the less you gotta write Thursday, can't put it off until tomorrow that's a different class to Dann, do before $2 \mathrm{pm}$, and good time to start now isn't it? These claims referenced threats of failure or referral if students did not consent. Dann rejected her claims with I'm not going to be here Thursday, will be on field trip and I have a D in my bellwork and I'll talk to you tomorrow, will work tomorrow, I wasn't expecting to write today, ain't doing none of my work in none of my classes today, too late anyway, and finally talking about body building and his muscles. Stanley consented momentarily with a question about his grades saying what do I got so far?

Ms. Gomez added more explicit coercive power claims to the interactively established contract when time was running out with 3 more minutes left, Dann, start writing please. Dann rejected the claim again by continuing the conversation conflict with Stanley talking about beating each other up or something that happened in the neighborhood after school, who's tough and who's not, one upmanship conflict, maintaining agency and identity with excuses about cameras as his witness so he won't beat him up. Stanley appeared to accept Ms. Gomez's claim by ripping paper out of 
Jonathan's notebook without permission, asking Ms. Gomez what they were supposed to write, and adding I could say this or that. However, he returned to the conversation with Dann maintaining identity and agency.

When faced with Dann's challenging talk and Stanley's persistent begging, Ms. Gomez interjected forcefully with I need you guys to practice for FCAT implying failure if they did not. She added implicit threats of referral such as if you wait, I'll let you go to the bathroom. She repeated you gotta wait to go the bathroom, why didn't you go between classes, gotta wait 10 minutes, I can't write you a pass for the bathroom until you wait 10 minutes, and let's take 10 minutes to write two things. When students ignored her implicit verbal coercive power claims, Ms. Gomez began quietly documenting behavior in a notebook for future reference. At the same time, she continued to remind them with two things, I'm asking you to write and you could write about that. Overall, the continuing conflict conversation between Dann and Stanley was the prevailing agenda that determined the setting. Dann rejected her claim, still egging Stanley on about what happened after school. Stanley appeared to accept her claim with Miss and looking at the notes she was writing. He read the directions off the board if you want to talk about writing and asked can I put about dirt bike riding. However, Dann and Stanley continued the conflict conversation to maintain agency and identity not yet noting threats of written referral.

Threats of written referral. Threats of written referral served as subtle coercive power claims to remind students that she must enforce PBS rules. However, when subtle threats failed, Ms. Gomez used explicit threats before ultimately writing referrals to remove disruptive students from the classroom. For example, when Dann asked for pencil 
and paper, she asked you are not prepared? Ms. Gomez used this subtle coercive power claim to threaten Dann with referral for not being prepared for class. Dann rejected the claim by changing subjects to ask why they were being filmed, then initiating a long conversation with Stanley using back-to-back cursing, yelling, play fighting, and profane language. Stanley rejected by announcing who called him on his cell phone, then cursing, yelling, play fighting, and using profane language with Dann. Jonathan was silent.

Ms. Gomez used other subtle coercive power claims such as appearing to ignore undesired behavior but holding a referral form in hand. The referral form served as a constant reminder of the sanction of being written up if students did not consent to be quiet and do your work. For example, when Stanley refused to put up his cell phone, Ms. Gomez ignored his challenge to her authority but started writing on a referral form. As she wrote, she repeated guys so they would hear and consent to the coercive claim. When Stanley continued texting, she asked what are you doing wrong? This question implicitly reminded him that if he quit misbehaving, she would not refer him.

When subtle claims were challenged, Ms. Gomez interjected explicit requests such as stop focusing on each other, start focusing on getting your grades in, take it out on your essay, Stanley you're instigating, why are you asking him how when you should be writing, I thought you said you would do you work when you return, should I play the tape and Stanley let it go. Stanley rejected the claims, texting again, conversing, and play fighting with Dann. Jonathan laughed. After continual rejection of her claims, Ms. Gomez was forced to make explicit threats of referral such as don't make me write this referral. Stanley appeared to accept the claim with okay but he ultimately continued the 
conflict conversation with Dann. Dann rejected the claim, defining the interactive setting with their ongoing conversation.

Once Ms. Gomez started writing a referral, Dann proceeded to tell her what to write on the referral form. She played along with Dann saying right, at least you understand what I could write you up for. Ultimately, Dann used his charm to regain her loyalty. Knowing that the end result on that day would not be a referral, Dann rejected her claim and returned to the conversation with Stanley. Stanley asked if Ms. Gomez was writing him up as if considering consent and told Dann that he was not in on the mock referral being concocted. However, he rejected her claim and returned to the ongoing conversation, maintaining identity and agency.

Ms. Gomez used more blatant coercive power claims as the deadline for finishing their work approached and disruptive conversation persisted with I'm losing my patience with both of you, are you going to write or do you want to go? She warned the sanction of CSI with what do you got to Stanley. If students consented to the coercive power claims of a referral and began to write, Ms. Gomez entered into interactively established contracts possibilities about their work. For example, Ms. Gomez moved closer to Stanley who accepted the blatant coercive power claim momentarily and told him what to write step by step. She talked to Jonathan once during the whole time because he was silent, but he hummed and accepted the claim. Dann rejected the claim by continuing the conflict with Stanley and not agreeing to write, saying he was sleepy.

Ms. Gomez pursued further with Dann, you can do it, why do I have to push you so much, what's going on, and I know you've been lazy lately. These claims tacitly suggested that we have an agreement in here, but you are not upholding your end, 
implying that I think if you try, you could do this but you don't try. Ms. Gomez did not buy Dann's excuse of being sleepy and not liking Stanley with you don't have to like each other, just do your work in here is all I ask. Dann pondered the claim silently and ultimately rejected it, implying that he did not accept her help to write.

Dann reaffirmed his opposition to authoritative decisions and resistance to classroom activities. He made it clear that he preferred a community identity as someone who was strong and brave and stood by his word over a student identity that dominated The School culture as someone who was not smart, not well behaved, and not able to successfully return to regular school. Ultimately, being true to one's self and community defined the topic of conversation between Stanley and Dann and the classroom norm.

\section{Charm}

Charm is the use of one's personality to gain consent from others. Ms. Gomez did not use charm to gain consent from her students. Table 10 summarizes types of power exhibited in Classroom Two.

Table 10

Types of Power Exhibited in Classroom Two

Type of Power Exhibited

Normative $\quad \mathrm{x}$

Coercive $\quad \mathrm{x}$

Interactively Established Contracts $\mathrm{x}$

Charm 
Normative power, coercive power, and interactively established contracts were exhibited in Classroom One; charm was not exhibited. The roles that participants exhibited are discussed next.

\section{Roles}

This section covers teacher roles and student roles. The researcher inductively found and named roles based on routine "interactive rhythms and sequences" (Carspecken, 1996, p. 137). Roles emerge as themes through complex ways of acting, which participants "recognize as having some unity" (Carspecken, 1996, p. 136). When participants recognize a role in play, they can predict, but not ensure, future actions. Table 11 provides a summary of Classroom Two participants' roles, followed by a description of teacher roles and student roles.

Table 11

Classroom Two Participants' Roles

Participants Roles

Teacher - Ms. Gomez The business like teacher with a psychologist side The disclosing empathetic older peer/aunt The listening counselor/psychologist/mother The reluctant neutral enforcer of administrative rules The teacher who helps students make their grades The proactive observer and recordkeeper

Student - Dann The stubborn charming debater

Student - Stanley The peer challenger who wants his grades

Student - Jonathan The quiet observing student 


\section{Teacher Roles}

The teacher exhibited six roles. First, Ms. Gomez was the business like teacher with a psychologist side. She began class immediately to meet predetermined academic deadlines. She urged, pleaded, tried to convince, ignored, listened, and coerced students during class until the deadline got close and then business like teacher returned.

Second, Ms. Gomez was the disclosing empathetic older peer/aunt most of the time. She exhibited this role with other roles to try to accomplish the goal of students doing the work for grades and points. This role got in the way of accomplishing her goals because disclosure or empathy usually covered most of class period. Topics disclosed were about outside of school interests of both teacher and students (i.e., bike information or the fights occurring outside of class in the neighborhood). This role enhanced social understanding, reduced social distance, and encouraged the tacit claim of reciprocal trust which was accomplished consistently.

Third, Ms. Gomez played the listening counselor/psychologist often enough that students knew she would stop whatever was forefronted in her plans to listen and give advice that they did not necessarily oblige or consent to but solidarity was accomplished by their willingness to talk about any topic in her presence. Advice often pertained to the academic assignment, but this role was combined with advice for behavior as well and backfired in her definition of the setting norm to get work done.

Fourth, as the reluctant neutral enforcer of administrative rules, Ms. Gomez simultaneously disassociated herself from school/classroom rules and their related punishments and claimed enforcement of them. She asked students why they did not do their work, and did not come prepared, emphasizing the students' activities and 
consequences for not choosing to do the right thing. This role challenged her classroom order, which tacitly claimed that classroom/school/district rules were valid requirements, and gave students reasons to determine a different valid classroom norm. Ms. Gomez made claims prefaced with we had to do this work, we have this test we have to prepare for next week, etc. She tried in various ways to enforce the rules with why don't you ..., stop that, or let's do this or we have FCAT next week we have to do, but always resorted to coercive "reverse psychology" of holding the referral in hand, writing on it as record keeper just in case she needed it when something gets out of hand. In other words, she claimed normative power but had to use coercive power in the long run.

She was in the position of having to enforce the rules, but made her decisions as to how to enforce the claims such as you have to wait 10 minutes to go to the bathroom. The role was fairly effective in keeping students in class but not in getting them to do work except through tacit coercion to make grades and points (backgrounded in everyone's mind even though not brought up explicitly very often). This role was not effective when students (especially Dann) openly contested or challenged the rule and its authority. In this case, the students' identities as powerful people in the community were asserted to show that something was wrong here.

Fifth, as the teacher who helps students make their grades, Ms. Gomez tacitly claimed to be the helper of students who decided to consent to doing the work assigned and thus the underlying point system, by giving them the answers after providing some assistance to get them going. This role occurred after someone who had been disruptive showed that they were consenting to doing the academic assignment. The end goal was to get a grade in the gradebook (i.e, that was what was being consented to). Students who 
sat quietly were ignored and "checked" once in a while but the ones who caused disruptions were listened to, conversed with, cajoled, and coerced. When they finally consented, Ms. Gomez helped them with work by sharing how she thought they could write something from their conversation and telling them how to do it, by giving the answers.

Sixth, as the proactive observer and recordkeeper, Ms. Gomez's attention was always on the students, which demoted her authority in the sense that teacher activities were not predominant and social distance was reduced. Even though this role was supposed to raise her authority, when mixed with the reduction of social distance, it backfired. It ultimately raised the students' claim to authority in conducting classroom activities, which both helped and backfired. This role helped in the sense that students felt they could challenge or resist everything without coercive power such as referrals given even though referrals were foregrounded as a possibility. It backfired because students often misjudged Ms. Gomez's limits and thus frequently got suspended. The role backfired in the sense that Ms. Gomez's authority was challenged and, from experience in this classroom, students thought of her as an observer/listener that would not necessarily act upon her findings until overt actions coerced her into writing referrals as the last resort. This role manifested as a peer/psychologist who was reluctant to enforce the rules that were placed on them all.

\section{Student Roles}

Dann was the stubborn charming debater who resisted all classroom rules and whose activities forefronted his outside image that must be upheld. Based on things he said and his demeanor during classroom observations, it was evident that he believed that 
if he played the school game he would not maintain the powerful image he had outside of school. So he challenged authoritative decisions that affected students negatively. He explicitly shared reasons for every challenge he made with excellent results. His interactions played a large part in determining the definition of the classroom setting. He knew how to stay there and stay on top of the interactive power claims.

Stanley was the peer challenger who wanted his grades and who assisted in the resistance that Dann initiated as long as he thought he would not get referred or lose the grade he needed. He asked Ms. Gomez to referee their argument to achieve this goal. He knew that he would eventually do the work and that Ms. Gomez would help him if asked. Jonathan, the quiet observing student, was quiet almost the entire time, but watched the interactions among the other participants. He interjected once in a while how he felt about what should happen in this class, but being new had not yet determined how he fit in; he remained quiet but tentatively consented in his demeanor.

\section{Cultural Power}

Cultural power was discovered through the process of hermeneutic inferencing, or taking the position of participants in order to understand the basis of their claims.

Cultural power exists when the norm or value claimed does not represent mutual interests of the entire group. In this classroom, Ms. Gomez was compelled to accomplish certain tasks as a teacher at The School, but she was not convinced of their importance and thus deferred authority. Her claims (gotta do bellwork, gotta do FCAT prep, gotta wait 10 minutes, gotta put up phone, gotta stop writing on desk, got to be prepared, got to stop that talk, got to make grades) were spotted as invalid choices. Most claims were invalid 
since they did not win universal recognition and interest of all students involved. Since claims of universality were lacking, cultural power was at play.

Students did not recognize themselves or their interests within the articulated norms and values. This conclusion was based on hermeneutic inferencing of the perpetual and intricate patterns of students' verbal and nonverbal attempts to change the academic and behavioral topics that Ms. Gomez pursued and bring their own interests into the classroom discussion. Ms. Gomez never gained their full consent because of the play of power noted in her use of referrals, grades, passes, and school sanctions. Ms. Gomez never got the students to agree that the norms and values she proposed were as important as their discussion about who did not treat the other one right in the community. For example, when she delivered the bellwork assignment, Stanley suggested football as a topic to write about. Ms. Gomez picked up on this and tried to go with the football topic and later with the bike suggestion. However, she did not pick up on Dann's suggestion to write about fighting Stanley. Dann and Stanley tried unsuccessfully to bring their argument topic into the writing.

Ms. Gomez also gave mixed messages by listening, holding or writing on the referral form as a threat, and requiring behavior standards but reneging on those requirements. She recognized herself as a counselor, but not as a teacher of writing, and her own interests of keeping behavior to a minimum got in the way of other claims since she always held behavior up to the students. For example, once Stanley finally gave partial momentary consent to do the work, Ms. Gomez told him how and what to write, suggesting that he could not do the work without her help. 
Power play occurred the whole time between these conflicting claims. The silent student, Jonathan, did not offer suggestions or interests but did show he thought they should be writing when it was challenged. He consented to the power play, even though he was new and not established at The School yet, while he was trying to recognize himself and his interests in the classroom. He needed more time. So the teacher ignored him since he was not causing an overt behavioral problem.

Themes from Reconstructive Data Analysis

This section covers three themes that emerged from inductive reconstructive data analysis: (a) conformism, (b) resistance, and (c) accommodation. Because the three emerging themes aligned with Giroux's (2001) theory of resistance in education, his terminology was used. Table 12 provides a summary of the findings from Classroom Two reconstructive data analysis. 
Table 12

Classroom Two Reconstructive Data Analysis

Theme Part. Manifestation Purpose

\begin{tabular}{|c|c|c|c|}
\hline Conformism & $\mathrm{T}$ & $\begin{array}{l}\text { Deference to academic authority } \\
\text { Deference to behavior authority }\end{array}$ & $\begin{array}{l}\text { Rule enforcement } \\
\text { Safe talk environment } \\
\text { Inevitable suspension }\end{array}$ \\
\hline Resistance & $\begin{array}{l}\mathrm{S} \\
\mathrm{S} \\
\mathrm{S}\end{array}$ & $\begin{array}{l}\text { Critique } \\
\text { Self-reflection } \\
\text { Struggles }\end{array}$ & $\begin{array}{l}\text { Communicate } \\
\text { Maintain identity } \\
\text { Maintain agency }\end{array}$ \\
\hline Accommodation & $\begin{array}{l}\mathrm{T} \\
\mathrm{T} \\
\mathrm{T} \\
\mathrm{T} \\
\mathrm{T} \\
\mathrm{T}\end{array}$ & $\begin{array}{l}\text { Ignoring } \\
\text { Bribing } \\
\text { Mothering } \\
\text { Reminding } \\
\text { Threatening } \\
\text { Reverse psychology }\end{array}$ & $\begin{array}{l}\text { Rule enforcement } \\
\text { Safe talk environment } \\
\text { Inevitable suspension }\end{array}$ \\
\hline
\end{tabular}

Note: $\mathrm{T}=$ Teacher; $\mathrm{S}=$ Student; Part. $=$ Participant.

Table 12 denotes three themes that emerged from reconstructive data analysis. Teacher conformism to the dominant ideology of Language Arts was manifested as subthemes deference to academic and behavior authority. Student resistance was manifested as subthemes critique, self-reflection, and struggles. Teacher accommodation was manifested as (various combinations of) subthemes ignoring, bribing, mothering, reminding, threatening, and reverse psychology. Teacher conformism and accommodation were manifested to provide rule enforcement, safe environment for talk, and inevitable suspension. Student resistance was manifested to accomplish communication goals and maintain identity and agency. 


\section{Conformism}

Conformism manifested as two subthemes: (a) deference to academic authority and (b) deference to behavior authority. Conformism, the opposite of resistance, is behavior that simultaneously silences social incongruities and maintains the dominant ideological logic in education (Giroux, 2001). Dominant ideological logic is based in banking education. Banking education presumes that knowledge is predetermined by an authority and delivered to passive students; teachers are distributors and enforcers of decisions made by an outside authority. Banking education in alternative education is more stringent as noted in public discourse (Applied Research Center, 2001; Blanchett, 2006). Public discourse sustains a cultural deficit orientation, the belief that alternative education students possess unalterable (and often distinguishable) cultural deficits in intelligence and behavior. Thus, authorities must monitor and punish unsuccessful or disruptive alternative education students.

\section{Deference to Academic Authority}

Deference to academic authority manifested as (a) teacher authority, (b) curricular authority, and (c) expert authority. Deference to academic authority occurs through layers of rules and regulations when social incongruities and low expectations clash (Stevens \& Bean, 2005). Ms. Gomez sought to develop Language Arts classroom culture, norms, and values that provided an environment of reduced hostility safe for talking about anything.

Teacher authority. Ms. Gomez exhibited teacher authority at the beginning of each class by her brisk demeanor and by having different classes' assignments already written "on the board for everybody" (T6B, L431). However during class, her verbal and nonverbal discourse exhibited frequent deference to an authority other than herself, 
reducing her importance as decision-maker. Students (Dann, Stanley, and Jonathan) picked up on these clues and displayed an escalating interest in their own topics of conversation and communication goals throughout the class periods. For example as the bell rang, Ms. Gomez ordered stragglers out of her class and onto "eighth period. Everyone eighth period" (T5B1, L3) or "Come see me after class" (T5B1, L10). She shut the door, turned around quickly, and said authoritatively to Dann, "Go sit down and do your bellwork" (T5B1, L10). Immediately, four instances of student side talk between Dann and Stanley (who was also texting) started as Ms. Gomez got a notebook from her teacher desk. Ms. Gomez stood facing Dann and Jonathan (who was silent) both sitting in the circle of desks and Stanley sitting in a computer chair behind the circle of desks. She announced, "I got a [bellwork assignment] that you're supposed to read today. It's just us three today, or you three" (T5B1, L15). Dann changed the subject and seven more instances of student side talk and two comments from Ms. Gomez ensued as she walked past the circle of desks, reached the white board, stopped, and said in an excited louder tone, “ALL RIGHT, guys here's the bellwork for today guess what's on Tuesday next week?" (T5B1, L25). She held her notebook poised to write during 14 more instances of student side talk and five teacher comments about unrelated topics or texting. Unsuccessful as the only authority in class, she deferred to curricular authority.

Curricular authority. Ms. Gomez delivered the bellwork assignment with deference to curricular authority:

All right. Dann, listen up. This is what we're doing today. Because we've got the FCAT Writes on Tuesday, I need you guys to practice today. First for the bellwork, this is what I got. If you were given the topic of writing about personal experience, name two things you could possibly write about. And number two is 
pretty much the same thing if you were given the topic of writing about sports. Name two things you could possibly write about. What do I mean by that? (T5B1, L45)

Ms. Gomez deferred to curricular authority by stating what the students are supposed to read and need to do because they have the FCAT Writes the next week. When students misunderstand or were unwilling to do the assignment, Ms. Gomez added:

The point about this exercise the point of this exercise is because you don't know what the prompt is going to be on Tuesday. You have no idea. So I want you guys to get used to generating ideas about what you might talk about about a bunch of different topics. So when it comes to personal experience, I might say well, maybe I'll talk about a time I rode a bike home and it started raining on me, you know, something like that. You don't want to go into detail, I'm just talking about general topics. So do two things for personal experience, something that personally happened to you, and then two things for sports. And let's take 10 minutes for that and then we're going to start the prompt . . . A All right, guys, I'm going to give you 10 minutes for that. (T5B1, L52, 61)

Ms. Gomez divided the lesson into segments, reiterated its usefulness, told students what to write, and repeated time limits. During the interview, she explained her reasoning:

Well, in my mind, I wanted them to understand that you know, when they get these prompts, they have no idea what to expect and I want them to start thinking about that, I want them to start thinking about what sort of things they could talk about you know, and I'd be giving them an upper hand by practicing that ahead of time. (T6B, L321)

She focused on how practicing FCAT Writes would benefit students in the future. Ms.

Gomez deferred to curricular authority for her assignment choices. She explained why she assigned bellwork:

I have taken a lot of professional development courses and stuff and and I had read somewhere you know how important it is to have something a like a routine that they you know everyday ... there's an assignment on the board that they've got to do. The idea is that they have something to do while I take attendance or while I get settled in. (T6B, L433) 
Bellwork was suggested as a necessary routine during professional development courses. Ms. Gomez also explained what students did at the computer station and why, deferring to curricular authority: "We're given the suggestion that we're supposed to be focusing on FCAT and like when we're done with the work in the class, I'll have them get on the computer and do that FCAT Explorer" (T6B, L187).

Ms. Gomez also carried a notebook around during class, writing student academic and behavioral anecdotes that she could defer to later if necessary. She explained:

I've always got a notebook with me during class and I keep notes, who's doing what, who's not doing what, and uh, it's just like a little a little mental note that I make so that I so that when I go in for the grades or when I go in for make calls to the parents I know what to tell them. (T6B, L59-61)

Ms. Gomez always kept a notebook with her during class as a grade and behavior reminder. She also explained her grading procedures by deferring to curricular authority:

I grade them, every every bellwork is graded and I like I tell my kids, that's an easy A, because it's most of the time, it's an opinion question, something that they really can't get wrong, just to get them thinking, get them writing um, and then depending on what we do, like I'll weigh I'll weigh things differently, like the essay's always get 2 grades at least and you know, basically, we put about 2 to 3 , I put 2 to 3 grades a week in each of my classes. They say you are required 2 a week but I don't think that's really an official requirement, I think that's just a suggestion. (T6B, L141-145)

Ms. Gomez was required to give grades, and bellwork was an easy A. When deference to curricular authority was murky, Ms. Gomez deferred to expert authority.

Expert authority. Ms. Gomez deferred to expert authority for testing and tracking students' reading progress. For example, when she thought Dann "might have a speech problem .... We had a had a pathologist come out here and everything and test him but apparently he's not, he's just mumbles a lot" (T6B, L477-479). Ms. Gomez conformed to the ideology of needing reading and grade levels to know students' capabilities. For 
example, she compared Dann to another student by saying, "Dann's reading level, he's in eighth grade and he's probably a second grade reading level" (T6B, L337). When a new student arrived, she waited for his reading test scores to know how to teach him:

I actually don't know what reading level [Jonathan] is on yet, I haven't gotten the scores back from the tests we just did last week, uh .... the reading coach [gives the reading test], and she does it for everyone in the school and she does, it's an oral reading fluency test and they read aloud and she yeah, checks off what words they misspell and what their rate is - that's how we determine what level they're on. That's how I know.

Students' reading skills were assessed by an expert when they arrived at The School, their reading levels were determined, they were divided into reading classes accordingly, and teachers were given the scores and district-wide curricula to keep track of their progress. Ms. Gomez deferred to expert authority about how to teach reading:

For my lower grade classes, it is FCAT Explorer, and for my intensive reading classes, we actually have computer components for the programs so like for my middle school, it's called Solo and it's a reading program online that goes along with the curriculum and with my high school, it's Reading Navigator. It's a Jamestown program and I can track their progress on the computer and see what they got right what they got wrong. It's really (undecipherable) No, actually some some of them are [multiple choice]. Most of the Jamestown one is uh is fill in the blank or respond to this, yeah, sometimes they record themselves also to make sure their pronunciation is correct. (T6B, L205-211)

Ms. Gomez could track student progress with the online reading programs provided to her. In The School, students were also divided into classes and given elective choices by their performance on the FCAT high-stakes reading test. Ms. Gomez explained:

Because they have intensive reading, intensive writing, those are two electives they could have, like Culinary Arts or Auto Mechanics, whatever they want instead of those classes, but it's because of how they perform on the FCAT. So for example, I have Dann in seventh period, which is intensive reading. Now, if he passes the FCAT when we have it next month, then next year instead of doing intensive reading again, he will get to choose an extra elective. (T6B, L379-381) 
Ms. Gomez upheld cultural deficit logic about passing tests, blaming students for their own lack of success: "They don't get it that if they just tried that one day, they would get all these extra electives, they don't get it . . . They don't think long term, they think I don't feel like doing this right now" (T6B, L379, 381). She concluded:

I would be very surprised if they gave a genuine $100 \%$ and didn't pass, because really if you look at the FCAT, it's not that it's hard, it's long and it's boring but it's not hard. So the problem is that they see these long boring passages and right away, they give up! They give up before they even start! It's like I don't feel like doing this today and they just Christmas tree. They'll just circle whatever they want, and then they don't realize how that's going to affect them later on yeah, but it does, it comes back to haunt them. (T6B, L383-385)

Overall, Ms. Gomez believed that her students gave up before they started.

She summarized her cultural deficit orientation logic about alternative education students when she explained why she could not use three different reading programs in the same class of mixed grade levels. She said this is not like a "regular school where you can leave one group working and go to another but you can't do that in this type of school, they wouldn't, nothing would get done besides trashing the classroom" (T6B, L159-163). She was resigned to how long it took "to get them to finish the [short essay] bellwork alone. Yes! It's supposed to be a 5 minute activity, but it takes far longer than that" (T6B, L367-369). Yet, she assigned bellwork because "if I give them anything that has multiple choice, forget it, they just circled anything" (T6B, L177).

Ms. Gomez also exhibited conformism as deference to behavior authority through cultural deficit orientation.

\section{Deference to Behavior Authority}

Deference to behavior authority manifested as (a) teacher authority, (b) PBS authority and (c) discipline authority. Deference to behavior authority was layered with 
district and system rules and regulations for handling alternative education students' disruptive behavior that ultimately resulted in their expulsion from school. Ms. Gomez and her students knew that the classroom culture, norms, and values encouraged student talk about anything in a safe environment but that the impending last resort for disruptive students when Ms. Gomez lost her patience was their removal from the classroom.

Teacher authority. Ms. Gomez deferred to her teacher authority for helping develop the PBS system of points for rewarding student behavior. She had "volunteered for uh, for summer sessions and they- the administration chose who was going to be a part of [the PBS team]" (T6B, L103) who developed the charts:

The idea behind these [PBS] charts was to drive instruction it was- we're going to have these charts appear and every kid's name's going to be up there and they have an opportunity to earn up to five points per class period and they need to meet all of those expectations which is being on time, making sure you have your supplies, pen and paper to class, keep- we have a PBS team meeting that we met during the summer. I'm the team leader for that. There's a group of us that are dedicated, yeah, there's about seven or eight of us, yeah, we got together during the summer and we we brainstormed to see what are the main things we want to see the kids these kids accomplish, so, so, being on time, being respectful, having your supplies ready, keeping your hands to yourself is a big thing with these kids, using appropriate language, you know, we came up with these ten expectations that we want them to meet. And so by meeting these expectations, they earn points. So they're basically getting rewarded for doing what should do anyway. And that would never happen in a regular school but- (T6B, L63-75)

Ms. Gomez clarified from a cultural deficit orientation (i.e., viewpoint) that instruction

can be driven by observing and rewarding good behavior with points. She elaborated:

It ... is supposed to be a positive tool for positive reinforcement . . . because the idea was that every 20 minutes stop instruction and congratulations, you earned your point, you earned your point but a lot of teachers were saying Oh, you just lost a point. Now, that's negative reinforcement. They're not losing anything, they have the opportunity to earn their points, but sometimes in the and I have a couple it's hard, you know, when the kids are acting up and you you want to say you know, you just lost that, but but the best way to do it is tell someone who is doing what he's supposed to be doing, oh, congratulations, you just earned that point and try to take 
attention away from the negative and on to the positive. Cuz a lot of these kids, they do what they do because they want attention. They don't care if it's positive or negative they just want that attention because they don't get it anywhere else. (T6B, L77-85)

Ms. Gomez explained the strict enforcement of PBS expectations for alternative education students who had uncaring or inattentive families, a cultural deficit orientation principle. Additionally, she gained teacher authority from the principal's support of PBS:

He's very big on positive behavior support and I think it's an important thing because our kids they they need something extra and that's why every week what they earn from their points they get to go to an activity so every Friday, there's a PBS activity and whoever made $80 \%$ of their total available points gets to go, and it's a bunch of different things. Sometimes we've got you know, uh, cookouts in the park where they're making bar-b-q's, sometimes it's popcorn and a movie, or ice cream sundaes. (T6B, L95-97)

Ms. Gomez upheld the PBS reward system for alternative education students' success.

PBS authority. Ms. Gomez deferred to PBS authority for expecting students to be

responsible for collecting their points:

that's how we do it, two days a week, so every Wednesday we collect for the odd days and every Thursday we collect for the even day and then Friday is the activity so we can have them all tallied up, Well, the morning teachers, you know like for my first period or second period whatever is first block that day, I'll hand out the points to the kids in that class and they take, yeah, each kid gets a sheet and each sheet has the total available points, sometimes it's 10, sometimes it's 15 depending on how that week ended up since we alternate and so I'll give my first block period, you know, the points for my class and then I'll- they'll take that and they're responsible, the idea is to make the students accountable for their own points so then they're supposed to take that to each class and get each teacher to give them the points and then at the end of the day, they turn them in by the bus. (T6B, L127-135)

Overall, Ms. Gomez deferred to the authority of the PBS organizational flow when she talked about "how we do it" (T6B, L127), how often, who is supposed to do what, and why. She supported and enforced PBS as closely as possible through dominant logic. 
Discipline authority. Ms. Gomez deferred to a discipline authority when she had lost her patience with disruptive behavior. She sustained cultural deficit orientation logic about managing disruptive behavior dilemmas with her reasoning, riddled with expressions of guilt, lack of choice, and blame. For example when asked about an ordinary teaching day at The School, she explained:

Um-uh, really, it's a battle, everyday is a battle .... So really, it's a fight, everyday I find it to be just a battle, just this morning I had four kids, within a half an hour, I had one left. I just couldn't deal with them anymore, I had to throw them out" (T6B, L43).

Ms. Gomez continued to explain that rather than fight the battle, many teachers

"especially some we had last year were very quick to you know, get the hell out" (T6B,

L505). She added that teachers

gotta keep that in mind, it's not easy, it's not easy on a day to day basis because they really try your patience, and it's just really tries your patience but I I I try to keep that in my mind that you don't know what they're going through and it's not personal, you know? (T6B, L513-515)

However, Ms. Gomez ultimately deferred disruptive behavior to the appropriate discipline authority in The School by writing a referral, removing students from class, and sending them to CSI, even though it was not a good option. She explained:

We need to fix CSI here, CSI is basically right now one of those portables back there and it's just a babysitting room and the kids get to go in there and sleep or you know, play with their cell phone or whatever it is they do over there but there's no real consequence. (T6B, L243)

Ms. Gomez thought CSI was no real consequence for bad behavior and hated using it.

She explained:

and I hate I hate doing that. They went to pick up trash around the school. That's what they their CSI is like they go and they pick up the trash. I wrote referrals and I sent them to CSI and a few minutes later I saw them picking up trash in the front yard, so that's one of the things that they have them do, uh, sometimes I don't see 
that as much of a punishment because they'd rather be out picking up garbage than, you know, getting bitched at here by me wanting them to do their work, so that that's for me a last resort I hate kicking them out but sometimes there's certain groups of kids that I know it's just not going nothing I do is gonna get to them so that particular class it's gotten to the point that I'm at my rope's end. (T6B, L45-51)

Ms. Gomez commented on how she finally loses her patience with some students.

Ultimately, she repeatedly deferred to a discipline authority when she ran out of options in the classroom and to support her decisions and actions for what should be appropriate punishment for disruptive students.

When asked about writing passes, she also adhered to the dominant logic about alternative education students and deferred to the proper discipline authority:

We're we're uh, we're not supposed to be giving passes very much. [The principal] likes us to hold them in class as much as possible and if they have to go to the bathroom which is the problem we have, it's like we've got to determine whether or not we believe it's an emergency, and it's really hard because these kids are very good at convincing you of anything, so sometimes they'll say it's an emergency, and it might not be but I'm afraid of a kid going in the room which has happened in the past, not to me but to other teachers in this school and I, yeah, uh, it's a, the passes, the regular district passes that they give out and I'll put where they're coming from, where they're going, and what time, the date. (T6B, L149-153)

Ms. Gomez abided by cultural deficit orientation logic that alternative education students are not trustworthy and teachers must determine whether or not to believe these students. Mr. Jenkins had decided not to use referrals at all. However, Ms. Gomez thought she did not use them often when she issued three in one day. When asked about writing referrals, Ms. Gomez explained:

I don't use [referrals] often, I used three today .... I don't like using them. I really don't because it goes on their permanent record and it might just be that they're having a bad day that day and a lot of them uh, they have to report to their probation officers, to the judge, and when they see these things, you know, they really get into a lot of trouble. So I try to leave this as a last resort, so basically 
you put the school number, the student name, student's information, the date that it happened, what period of the day, the time, and then you got to talk about if you've already spoken or made parent, guardian contact for this behavior or another behavior, whether you actually made the contact or not and then the teacher referring who are you referring it to which is now it's [Mr. Mannes], the administrator uh, they go to him, it used to be him and uh another one of our assistant principals, but we no longer have him, so now it's just [Mr. Mannes]. He usually calls home and makes a decision as to whether they are going to be suspended or what kind of uh, you know, consequence they are going to have. So here, we have to write a narrative, we have to say exactly why. (T6B, L217-227)

Even though she did not like using referrals and knowing what ultimately happened to the students in the system, Ms. Gomez used them as a last resort and deferred the decision as to what happened to students after they left her classroom to the discipline authority.

A similar dominant logic of deferring behavior problems to the discipline authority was exhibited in Ms. Gomez's discourse about phone usage rules at The School. As she watched the DVD during the interview, she remarked:

Oh, there's Stanley on his phone again! Ahh! Yeah, I had just mentioned that to him, that drives me crazy, absolutely crazy. No, the school board rule is that they can have it on them, but they can't use it during the school day is the idea and the rule here even though it's not really enforced the way it should be is that the idea is that ok, the first time they take it out during the school day, you know, you give them a warning or whatever if they take it out again, then you've got to call security or one of the PBS coaches to come in and take the phone and when they take the phone the first time they get it back at the end of the day. The second time a parent or guardian has to come and sign for the phone. (T6B, L441-448)

She gave warnings, but repeated the cultural deficit discourse of how nothing ever changed in alternative education and deferred to the discipline authority for dealing with disruptive students. In this case, PBS Coaches are staff members who are

assigned, I mean they have other functions, usually it's a paraprofessional, and one of job descriptions is to act as a PBS coach. So [Ms. Jammeson] is one example, she's the one that I'll call because she's assigned to me and a few other teachers, so if I have a real problem with a student, I'll call her and she'll come in, try to counsel them, take them out of the room, you know, take their phone, whatever the problem is .... when when it's something that's disrupting my class 
and I don't want to keep having it disrupt and I'll have her come in and deal with it. (T6B, L452-457)

When counseling did not work, she deferred to the proper discipline authority and The School requirements for removing students from the classroom:

If I send them out of the room at all, I have to do this [write a referral]. I'm not allowed- whether they pick up or they go to CSI? Coach Charles, he's in charge of discipline and stuff. He'll usually make that determination. Most of the time when they don't have their supplies, he has them cleaning up so they can earn their supplies and he'll give them paper, pen, whatever he needs, but he's the one that decides, you know if they're going to be in CSI or if they're going to be cleaning up, yeah, yeah, that's why they don't want us kicking them out, but at the same time, if we do, we have to document everything. (T6B, L245-249)

Coach Charles was in charge of discipline after they left her room. After providing the required documentation in the form of a written referral, Ms. Gomez left the decision of what to do with the students in Coach Charles' hands. When students were asked why Ms. Gomez carried a referral around during class, Jorge answered: "It's just normal, she doesn't care, she just has a referral"' (T6A, L51).

When Ms. Gomez was asked how student turnover and suspensions were tracked in the system, she explained with cultural deficit orientation logic, "We've got kids changing all the time, you know, they come and they go, so they have to update this, this is a class list" (T6B, L285). She clarified what teachers could gather from the list, "These kids have been added to your class in this period, these kids have been dropped from your class in this period" T6B, L289). She detailed how she "got a new kid" (T6B, L291) in one period and "might have lost a few, uh, yeah, see I know [Osmond] is still no longer here, [Jason] is no longer here, and [Clifton] is no longer here" (T6B, L293). When asked what happened to students who were no longer here, Ms. Gomez explained the nature of alternative education through cultural deficit orientation logic: 
No, no, they they I know that they were, for example, [Osmond] went back to his home school. [Jason] I have no idea what happened to him but he was withdrawn. Um, [Clifton], I believe was excluded from school. I believe he was kicked out. And [Govani] went back to his home school. So they get to earn their way they're here depending on what they did for a minimum of 18 weeks and if they don't mess up in those 18 weeks, if they don't get a referral, if they don't miss too many days of school. They can't get two [referrals]. I think they're allowed one, but if they get two referrals, that's it, that semester is out the window for them, they've got to start from scratch. So if they get two referrals or more, or if they miss too many days of school, or if they get a D or lower in any of their classes, they're not allowed to go back. So it's pretty hard for the kids to keep up with that, they don't get sent back very often, but we do that to make sure we don't get them right back here right after. (T6B, L297-301)

Overall, Ms. Gomez's discourse sustained cultural deficit orientation logic about alternative education as unchangeable and when her teacher authority was unsuccessful, she deferred to outside authorities for handling her students' lack of academic or behavioral success in school.

\section{Resistance}

This section covers students' resistance, integrating Giroux's (2001) framework of critique, self-reflection, and struggles throughout the five subthemes exhibited: (a) resistance to bellwork and PBS rules, (b) resistance to dominant reasoning and deference to authority, (c) resistance to dominant ideology about reading and writing, (d) resistance to authoritative decisions, and (e) resistance to classroom culture, norms, and values.

Resistance is a "form of refusal that highlights, either implicitly or explicitly, the need to struggle against the social nexus of domination and submission" (Giroux, 2001, p. 109). Even though Ms. Gomez's students came to class on time as expected, they immediately and continually exhibited implicit and explicit resistance, or refusal to comply, with classroom culture, norms, and values based on banking education and enforcing PBS. Students' resistance illustrated the use of agency to merge a critique of 
domination, self-reflection, and struggles to achieve self- and social-liberation. Students' resistance exhibited awareness both of Ms. Gomez's underlying goal of having an environment where students feel safe to talk about anything and of her limits pending ultimate referral.

\section{Resistance to Bellwork and PBS Rules}

Students exhibited resistance to the validity of bellwork and PBS rules about inappropriate language by explicitly partaking in personal communication goals and implicitly questioning Ms. Gomez's authority. For example, immediately after Ms. Gomez began class with the bellwork directive, Dann egged Stanley on instead, "I remember that day Stanley was shaking his ass on Tamaris" (T4B, L11). Stanley responded with a common vernacular phrase, "Fucked up" (T4B, L12). Ms. Gomez ignored the resistance and repeated the directive, stressing that it was something "that you're supposed to read ... just you three today" (T4B, L15). Stanley began texting on his cell phone. Dann ignored the deference to authority and the directive but knowingly reminded Ms. Gomez that "it's just us three every day" (T4B, L19) and the student who was absent "got beat up" (T4B, L23). Ms. Gomez said, "Really?" (T4B, L23). Stanley continued texting as he issued a common vernacular insult, "Puss ass nigga" (T4B, L24).

\section{Resistance to Dominant Reasoning and Deference to Authority}

The students resisted Ms. Gomez's dominant reasoning and deference to authority for doing the bellwork by feigning the role of interested student, asking to be excused, critiquing the value of the assignment, and pursuing another topic. Ms. Gomez ignored the previous comments again and asked the students if they knew what was going to happen soon that related to the bellwork. Jonathan whispered, "Halloween?" (T4B, L26). 
Dann said rotely, "What" (T4B, L27). However, Stanley said, "I gotta I gotta I gotta pee so bad, Miss" (T4B, L30). Stanley begged during the next 13 utterances to which Jonathan responded in disgust, "Oh, my God, I ain't gonna be like-" (T4B, L31). Dann stated, "I'm not comin' for the rest of the school year" (T4B, L32). Stanley finally threatened, "I'm gonna go outside" (T4B, L37). After Ms. Gomez tried convincing Stanley to wait, Dann interjected, "I remember last when Harry peed on the wall, that junk was funny" (T4B, L47).

Ms. Gomez redirected students' attention to the bellwork assignment. First, she answered her own question telling students that the FCAT Writes was coming up soon. Then, she asked them to name two things they could write about. Stanley said, "Football," (T4B, L45). Ms. Gomez elaborated on his answer but after several utterances Dann explicitly resisted, stopped the lesson, and said, "I don't wanna talk about it" (T4B, L50). Ms. Gomez continued lecturing, delivered more information about the point of the exercise, and issued a time limit for finishing the assignment. Stanley slumped down and moaned his dislike for the assignment. Dann stretched his hands back behind him and also moaned his disdain for the assignment. Jonathan expressed his misunderstanding, "I don't know what to write" (T4B, L54). Ms. Gomez responded, "You can write, you don't have to write the whole story, I'm just asking for the general topic" (T4B, L56). Jonathan thought she said "write the prompt" (T4B, L57) but Ms. Gomez clarified, "No, that's the next thing" (T4B, L58). Ms. Gomez laughed and explained what was going on in this situation from a dominant ideological perspective:

See, they don't make a connection, they just, they never make a connection to what we ask them to do, I feel like it's just like well, you just want to torture me, see the expressions on their faces and they're just bored, they're tired, they don't 
want to do anything and that's an everyday thing, it's so hard to get them to feel like this is something meaningful. (T6B, L317-319)

Ms. Gomez made dominant excuses for student resistance and for the meaningfulness of the assignment. During the interview, Jonathan said that they had bellwork "every day" (T6A, L17) but when asked what it was for, he said "I donno" (T6A, L19), confirming its lack of meaning for him. When asked why he never told Ms. Gomez what he was going to write about, he said, “Cuz, I won’t, I didn’t feel like talking. I was bored, I don't talk when I'm bored" (T6A, L11, 13).

Ms. Gomez restated the time limit, and students rephrased their resistance. For example, Dann responded, “Can I have a pencil and paper?” (T4B, L61), offering a statement of explicit resistance to the PBS rule about being prepared for class. During the interview, Ms. Gomez commented with dominant reasoning, "They couldn't care less, they just want to get it over with and get done and they don't want to think about anything. It's frustrating for me. Yeah, in that regard a lot. Yeah, they're very very unprepared" (T6B, L321-322). Stanley continued texting and said, "Hey, Miss, Billy callin' me!" (T4B, L76), explicitly resisting the validity of the PBS rule about phone usage. During the interview, Ms. Gomez explained Stanley’s resistant behavior from a dominant perspective, "Stanley is so smart and he just wastes, he's so intelligent and he really wastes his potential just horsing around in class and stuff like that" (T6B, L337).

Then, Stanley quietly made another derogatory statement to Dann, “Dann’s gay” (T4B, L80). Dann replied, “I'm gay? You sure you calling me gay? You talking to yourself, right?” (T4B, L83, 86). Ms. Gomez commented on the history of this situation:

These two want to fight. There's tension going between them. I don't know what happened but there's some tension between the two of them they had a conflict 
outside of school, yeah, and I don't know what it was but it's been getting progressively worse. Uh, well, I only have, I have him seventh and eighth period. So I have him every day but Stanley I only have for eighth period, but he has even told me Stanley uh, Dann has told me that in seventh period that he's that Stanley's getting on his nerves that he wants to fight him, so there's something going on there. (T6B, L327-331)

Ms. Gomez tried to figure the students out, but 20 utterances later, they were yelling at each other and asking Ms. Gomez to listen. For instance, they took turns saying, "Tell her how you ...” T4B, L105, 106). They stopped for a second when Stanley noticed Ms. Gomez writing on a referral form and asked, "You writin' me up?" (T4B, L110). However, Dann exhibited resistance by critiquing, reflecting, and maintaining his identity in the scenario. He seriously initiated his resistance by telling Ms. Gomez what to write on the form. For example, he said, "Put Daniel Grey, being disrespectful" (T4B, L113114) but eventually changed his tone to that of sarcasm with "and that he called Stanley gay" (T4B, L120).

After several more vernacular utterances and inappropriate language, Dann acknowledged that Ms. Gomez may have reached her limit with their resistance. He laughed and asked her, “Are you puttin' that for real? I was just playin' .... You're puttin' my name for practice? You practicin"” (T4B, L123)? Ms. Gomez said that she did not put his name yet and then reminded the students again about the bellwork assignment. Dann resisted again and changed the subject, "I don't feel it right now, cuz I got two cameras watchin' me" (T4B, L127). Stanley listened to several utterances from Dann about the camcorder in the corner of the room that they were supposed to ignore and the surveillance camera that Ms. Gomez had installed in the classroom. Then, Stanley repeated the previous insult to Dann, "He's gay!" (T4B, L134). Dann and Stanley 
bickered back and forth and eventually told the story of their disagreement to Ms. Gomez over the course of 100 utterances. From time to time, Ms. Gomez interjected suggestions for writing to no avail. The argument prevailed, resting on an underlying tension between them about which of the two "was sellin' out" (T4A, L164), a vernacular phrase for the concept of being a traitor or being afraid to stand up for one's friend. Dann summarized, "I told you to meet me at the park and you never came" (T4B, L235). Their personal communication goals were real choices in their lives and trumped the assignment delivered by Ms. Gomez.

\section{Resistance to Dominant Ideology about Reading and Writing}

Dann and Stanley exhibited resistance to the dominant ideology about reading and writing, specifically practicing for the FCAT Writes. Dann critiqued and self-reflected, "I don't get it, I'm a good writer but I don't like writin"” (T4B, L246). He continued, “This ain't even a writin' class and I gotta write" (T4B, L248). Jonathan and Dann disagreed about whether Language Arts was for reading only or for reading and writing. Stanley changed the subject and said, "Miss, I gotta pee so bad" (T4B, L259). Dann pursued his critique, “Are we s'posed to be writin'?" (T4B, L261). Ms. Gomez answered from dominant ideology and deferring to authority, "Yes, we are supposed to be writing, we're supposed to practicing for the FCAT Writes" (T4B, L262). Dann requested clarification, "In Language Arts?" (T4B, L263). Stanley resisted further and promised, "As soon as I come back in, I'll do my best to get done" (T4B, L264).

\section{Resistance to Authoritative Decisions}

Critique, self-reflection, and liberatory goals were continually issued by the students in order to show resistance to authoritative decisions and to accomplish their 
own (more important) communication goals. For example, on his way out the door, Stanley picked up a newspaper from the teacher's desk and commented about a football player pictured on the front page, made another promise, and then asked Ms. Gomez about the padded seat of her bicycle that was parked along the wall, "That guy's big. As soon as I get back in, mam, I'll get done. Does this warm your booty or som'in'?" (T4B, L268). Ms. Gomez laughed and answered, "No, it just makes it more comfortable" (T4B, L270, 275). Dann interjected another vernacular insult to Stanley, "Number 66, playing for uh, the Steelers. He look like Stanley uncle" (T4B, L273, 276). While Stanley was gone, Dann critiqued and self-reflected on The School's policy of sorting students into classes, "Miss, why they put me and Stanley in the same class and we don't get along?" (T4B, L288). Ms. Gomez replied deferring authority, “I don't know, I didn’t make the schedule" (T4B, L289). Dann responded with plans for retaining his identity, "I gotta talk to them. I'm gonna end up punching him in his mouth" (T4B, L290).

Ms. Gomez gave additional directions to the students and walked closer to check on Jonathan's paper, "Write your name and date on your essay. What are you doing?" (T4B, L291). Jonathan saw Ms. Gomez's ring and said, "You're married” (T6B, L292). Dann readily responded, “I know for a couple of months. And you just now realizin' she was married" (T4B, L296). Then, Dann announced with a smile that he was sleepy. However, when Stanley walked back in and said, “Aw, whuz up?” (T4B, L302), Dann suddenly woke up and replied, "I don't like you" (T4B, L303). They argued about not liking each other for awhile until Ms. Gomez stopped them and said to finish the essay “today in class. Before 2 pm” (T4B, L327). Dann explained his forethought, "I wasn’t expectin' to write today. I ain't done none of my work in none of my classes" (T4B, 
L329). Ms. Gomez suggested, "It's a good time to start then, isn't it" (T4B, L230)? However, Dann resisted, "We too close to goin' home" (T4B, L331). He promised to finish tomorrow when he finished all of his other work, and then looked at his muscles and asked Ms. Gomez, "I’m big, huh, Miss?” (T4B, L333).

Resistance to Classroom Culture, Norms, and Values

Dann continued exhibiting resistance to the classroom culture, norms, and values based on cultural deficit and banking education principles. He asked Ms. Gomez why she did not read them the other prompts on the board and said he would probably do better on one of the other ones because he had already done it in the other teacher's room. Ms. Gomez replied, "It comes from an old FCAT Writes" (T4B, L347), and Dann answered with apparent knowledge of the system, "I could tell" (T4B, L248). About that time, Stanley returned to texting and announced that it was Billy again. Dann responded with another insult to rekindle the ongoing argument. After 20 more utterances, Dann and Stanley noticed Ms. Gomez silently writing on the referral form again. However, they also knew that she had not reached her limit and continued arguing for another 100 utterances. Stanley finally relented when Ms. Gomez asked, “Are you gonna do your essay or do you want to go" (T4B, L437)? He quickly requested and received Ms. Gomez's help writing the essay. Meanwhile, Jonathan hummed, and Dann acted sleepy. Ms. Gomez asked to no avail, "Having trouble, Dann" (T4B, L455)? Overall, students continually exhibited resistance to classroom culture, norms, and values and system authority that provided them no valid or real choices while Ms. Gomez accommodated their resistance or until Ms. Gomez eventually ran out of patience and wrote a referral to remove disruptive students from the classroom. 


\section{Accommodation}

This section provides an overview of Ms. Gomez's accommodation, followed by an explanation of the accommodations that manifested as various combinations of tactics in six subthemes: (a) ignoring and bribery; (b) mothering and reminding; (c) threatening and reverse psychology; (d) reminding, begging, and pleading; (e) reminding and reverse psychology; and (f) threatening, mothering, and reverse psychology. Accommodation is perceived as simultaneously coping or accepting of the school's ideology while exhibiting some form of resistance to it (Giroux, 2001).

Ms. Gomez's accommodation of student resistance resulted from her triple quandary of supporting the dominant ideology about teaching Language Arts and enforcing PBS while providing an environment of reduced hostility where the kids feel safe to talk about anything. During the interview, Ms. Gomez commented after multiple instances of Dann's resistance to the classroom culture, norms, and values, "Dann likes to negotiate" (T6B, L397). She said that she "would rather have them talking . . . interacting, having conversation ... because when they're just looking at a paper, I don't know what's going through their minds" (T6B, L177). She elaborated:

I like to talk about that stuff, I like to know who they are, and really, if you listen to some of these kids' backgrounds, it's no wonder that they are the way that they are. I mean, I'd be pretty messed too if I dealt with half the things they've had to go through and yeah, that's probably what I like most about this is being able to get to know these kids and being able to offer them a different perspective on certain things that they go through so they do open up a lot a lot to me. Sometimes a little too much a little too much, but and I'm pretty open too, they know exactly where I'm coming from and I think that that's important too, because a lot of the teachers they feel like they don't care because you know, they're not being real with me and I hear that a lot from them and I don't want them to feel that way. (T6B, L403-405) 
Ms. Gomez listened to students to both gain an understanding of their lives and to

hopefully provide them a different perspective. Ms. Gomez explained further about the

environment she wanted to create:

I want to have an environment that my biggest challenge I think is what's going on between Dann and Stanley because there's this and it's the same in every class with different kids, there's this animosity and this hostility it's almost like they're the insecurity really shows because they're always trying to one up each other or insult each other and sometimes you know in back-handed ways and that's the biggest challenge I have, they're trying to put on a show for their classmates and in the process they're losing out on you know, on all the things that they could be learning or what they could be gaining from the situation, they don't see it that way, they are not mature enough, but I these guys are Dann is 13, Jonathan's 12, and Stanley is 13 or 14, they're eighth graders. And um, it's that hostility in the classroom that cuz that's what I want to get rid because I want to have an environment where the kids feel safe to talk about anything. They feel safe to talk about a lot of things that they do, but I don't like the way they antagonize each other. That's one thing that I still haven't been able to get rid of in my classes. And especially the whole, uh, because it's an all boy's school now, everything is faggot this and faggot that and you're gay and this is gay and that is gay. (T6B, L411-413)

Ms. Gomez accommodated student resistance of the assignments until she ran out of patience and "had to throw them out" (T6B, L43) in order to provide an environment for reducing hostility among students. Everyday she explained that she had

to come up with some way to trick these kids into learning something new that day because most of the time, you know, they just fight me on it or they want to sleep or they want to talk or they just don't want to do it so but you know, I've gone through in the three years that I've been teaching, I've tried pretty much everything. I've tried bribing them, I've tried threatening them, I've tried babying them, you know, anything that I can, sometimes with some kids certain tactics work, but with most of them, I still don't know how to get through to them. A lot of them. (T6B, L29)

Ms. Gomez struggled to provide a safe environment for talking within system ideology through multiple tactics and with negligible results. Ms. Gomez initiated incremental 
accommodations in response to the level of student resistance for as long as she had the patience or to prevent a fight. The six subthemes are explained next.

\section{Ignoring and Bribery}

When students first resisted her directives and dominant reasoning for doing bellwork, Ms. Gomez often combined ignoring those who posed the least threat to the accomplishment of her goals with bribing those who were most outwardly resistant. For example in the beginning of class, Ms. Gomez ignored initial off-the-topic vernacular insults and brief complaints about the bellwork. However, after Stanley persistently texted and begged to go to the bathroom for 13 utterances, Ms. Gomez tried bribery, "You gotta wait Stanley. Wait Stanley, why didn't you go between classes" (T4B, L3638)? Then she specified, "Stanley. You gotta wait you gotta wait at least 10 minutes. You have to wait at least 10 minutes before I can give you a pass" (T4B, L41).

\section{Mothering and Reminding}

When Stanley first told Ms. Gomez that Billy was calling him, Ms. Gomez tried mothering, including sternness and appeal to his conscience. For example, Ms. Gomez said, "You can't use the phone in here, you know that" (T4B, L77), and "I don't care who it is, you can't use the phone in here" (T4B, L79), or "What are you doing wrong right now" (T4B, L251)? Mothering tactics involved listening as the two boys stepped up their argument of rapid-fire increasingly louder, fouler vernacular insults to each other throughout the period combined with oral reminders such as "Paper and pen" (T4B, L103) or "No, you don't have anything to fight about" (T4B, L182). When Ms. Gomez thought things were getting out of hand, she exhibited the nonverbal reminder of writing in her notebook to tone students down so they could continue to talk things out in a safe 
nurturing environment. Ms. Gomez explained how she knew when things were getting out of hand:

I've been lucky enough to not yet, knock on wood, to have a fight in my room. But it's come close. Yeah, yeah, you can tell, I have a pretty good idea when they're really serious. So the teacher, and I for me, you can tell. You can tell when they are playing around or when it's going to get to that point. I don't want to let it I don't want to wait until it gets to the swings, by that time, I'll be calling security they're already on each other you know. I've been lucky enough to not have that happen yet but it's happened in several other classrooms you know, I know these kids well enough that I can tell when it's really going to get to that point. (T6B, L459-465)

Knowing the students well enough was helpful, but she explained how and why writing in a notebook also helped her accomplish her goals:

In the beginning of the year, I used [the PBS] system a lot. Every twen- because the idea was that every 20 minutes stop instruction and congratulations, you earned your point, you earned your point, I found and most of the teachers found that to be more of a distraction than anything else because when we would do that, then the kids that didn't earn their points well, how come I didn't earn my point? I did my, you know. Then it's a big argument. And it totally takes the class off course. That's why I went from doing to like kind of keeping it to myself, just going up there, it's up there, they can look at it if they want to but I don't make a big deal out of it right in front of the class. (T6B, L85-91)

Ms. Gomez found the PBS charts to be ineffective and sometimes used them to "keep track of what what student's doing what at the end of the class ... for [her] own benefit" (T6B, L59), but mainly relied on her notebook. The PBS bench was also ineffective for reducing hostility in the classroom. Ms. Gomez laughed and explained:

But that's what the PBS ideally is supposed to be, initially we also used the bench, see that bench thing over there? That was supposed to be, the idea was a time out kind of thing for it, the kids weren't really responding to it the way that we wanted them to cuz the idea was okay if you're not doing what you're supposed to be doing in class, then instead of automatically reprimanding you, I'm going to say okay, take a time out, sit over in the bench, think it over, and in about 5 minutes I'm going to come back to you and I'm going to see if you want to get back in the game doing your work or if we're going to take other other actions. It just, they wouldn't go to the bench. Well, yeah, make me, what do you 
do? When they won't even go to the bench? It's like how do you, yeah, no, it didn't work. So I'm still trying to figure out something we can do we can we are still trying to continually improve it. (T6B, L109-117)

Ms. Gomez preferred mothering and reminding over the PBS bench. Students knew Ms. Gomez's preferences and goals; thus, they invited her to listen in on their arguments from time to time with such comments as, "Miss, let me tell you what he said" (T4B, L102). Threatening and Reverse Psychology

Ms. Gomez also combined the tactic of threatening with reverse psychology. For example, the first time Stanley noticed Ms. Gomez writing something down in her notebook, he stopped exchanging insults with Dann for a second and asked, "Wha'dat? I do got paper, I just don’t got a pen” (T4B, L104). Ms. Gomez said, “Guys” (T4B, L109) as a warning while she walked over to her desk and picked up a referral form. Stanley says, "All right" (T4B, L110) and asked her if she was writing him up. Ms. Gomez responded, "I'm just holding onto referrals right now. What I do with it is up to you guys" (T4B, L111). At this point, Dann exhibited resistance by telling her what to write down for his behavior. Ms. Gomez played along with reverse psychology statements such as, "You want me to put that" (T4B, L113)? and "Right, at least you understand what it is I would write you up for" (T4B, L115), and "Just setting it up" (T4B, L124). Finally, they asked her so many questions about the sincerity of her referral writing that she issued a threat, "I didn't put your name yet. Why don't you focus on the two questions for the bellwork" (T4B, L126)? Dann immediately changed the subject, this time toward his dislike of the two cameras in the room. His comments stalled the referral threat for the moment; everyone knew that Ms. Gomez had not reached her limit yet. Ms. Gomez laughed and explained, "When stuff like that's going on, I keep writing down the details. 
See, he noticed. See, it'll get them back on task for like, a short period of time but then they forget that I'm writing a referral" (T6B, L371-373).

Ms. Gomez also combined threats and reverse psychology when cell phone usage got out of hand even though school board rules suggest more rigid guidelines. She explained:

That's the idea [of the school board guidelines], does it really happen that way? NO! it doesn't really happen that way so a lot of the times when this happens in my class, because it's one thing that really drives me nuts is that phone so a lot of the times even though they tell us we're not supposed to hold it, I'll say you know, give me that phone and if they don't want to, it's either give it to me or you give it to security and who's going to give it back to you sooner? Because with me, you get it back at the end of the period, with security who knows when you get it back? So usually, they will hand it over when I when I put it that way. But I don't like taking it and I give them a few chances but a lot of them will just keep on and keep on, it drives me nuts. (T6B, L449)

Besides threatening to lose their cell phone to security if they would not give it to her temporarily, Ms. Gomez used other threats in her classroom, such as the surveillance camera that Dann mentioned as one of his critiques of the system. She explained:

The camera, it's not recording now, I was told I can't use it. I bought it. I bought it, no no, I had an issue with uh, another class where a student came in and verbally threatened one of mine and uh, and then he denied it right after. When I turned him in to the security and the school police because he was, this kid is a bully, in a lot of different ways, and I have him, actually for a different class, but you know, he did it right in front of my face and then wants to try and say, like he's telling the security and the cop that look, I didn't say that, I didn't say that, until they had to pull me out of class to go into the security room and when I looked him in the eye, he was like, ok, maybe I said it but I didn't mean it. So that's when you know, I got fed up and I was like you know what, that's not going to happen again. So I had gone to Radio Shack and they were on sale, $\$ 30$, for a perfectly, you know, good camera that picks up sound and color picture. I couldn't resist, so I bought it! And I put it up thinking maybe it'll curb some of the behaviors, which it didn't really work that way, I mean the two weeks I was using it and the only thing it did was make them a little more aware of their behaviors but it didn't stop what was going on uh, and yeah, I was told by an administration that I gotta take it down, so they said that apparently I'm not allowed to do it. It doesn't make any sense, they are videotaping the hallway all 
the time, but yeah, there's cameras in every hallway. Yeah, they have recorded many fights. (T6B, L355-365)

Threats and reverse psychology did not always curb behaviors as desired.

\section{Reminding, Begging, and Pleading}

Ms. Gomez often resorted to repetitive reminding, begging, and pleading. For example, she would give constant reminders, "Dann, you're supposed to be, Dann, you're supposed to be writing on the paper, not the desk please" (T4B, L141) or "You don't have to worry about [cameras], you have to worry about getting done" (T4B, L128). At one point when the boys were screaming “I WAN'T FIXIN' GO TO THE GAME! I WAS GOIN' TO THE GAME TO FIND THAT CHICO” (T4B, L178), Ms. Gomez interjected an implicit reminder, "Stanley, sounds like you got something to write about, so write" (T4B, L179). She also repeated time-related reminders such as, "Okay, guys, come on, we only got three more minutes" (T4B, L185), and again, "Three more minutes" (T4B, L186).

Amidst multiple expletives, insults, and vernacular storytelling, Ms. Gomez often used begging, "Dann, start writing please" (T4B, L204), or "Stanley, you can say I was dirt bike I could write about the time I was dirt bike riding in the rain" (T4B, L226). Stanley intermittently requested help to write the essay. For example, he interjected while arguing with Dann, "Say it again, Miss" (T4B, L229). Ms. Gomez begged, "You want to use complete sentences, so I'm asking you- I'm asking you what you could possibly write about .... You could say I could possibly write about the time when I was riding dirt bikes in the rain" (T4B, L230-232, 236). 
Ms. Gomez also strongly pleaded for students to do their work. For example, after most of the period had passed and she had ignored the foul language, arguing, and resistance to the assignment, Ms. Gomez pleaded, "Guys, focus on this, and you haven't even started. Come on, Dann, you can do it! Why do I have to push you so much, I know you've been lazy for the past couple of weeks. What's going on" (T4B, L299)? Dann answered, "I'm sleepy" (T4B, L300) to which Ms. Gomez pleaded, "No, I don't buy that. You were doing really well. I won't see you go downhill” (T4B, L301). When Stanley returned from the bathroom, he and Dann returned to their argument. Ms. Gomez pleaded again, "You could use these grades. Two. You already got one in your bellwork, right?" (T4B, L310), and "Come on guys, stop wasting time, start writing" (T4B, L315). She often combined pleading with bribery, "The more you write today, the less you gotta write on Thursday. Remember that" (T4B, L317)? At that point, Dann explained why he was not going to work and where he would be on Thursday. Ms. Gomez pleaded again, "So will you please finish today" (T4B, L323)? However, Dann knew her limits and continued explaining his plans that did not include doing the work that day.

When students heard Ms. Gomez say something like “Dann, don't make me write this referral out" (T4B, L371), they would include things they could write about into their argument. By playing on words with Ms. Gomez, students could bide more time to talk about what they thought was important and to resist doing the work. They continued to talk about not liking each other or writing about fighting each other. For example, Dann stated, “If it were somethin' on my paper 'bout whippin' Stanley's mouth about how I was gon' do it I got a A++" (T4B, L386). Ms. Gomez continually reminded with such phrases as, "Guys, stop focusing on each other. Start focusing on getting your grades in" 
(T4B, L378) and "Take it out on the essay. You don't like that either, right" (T4B, L382)?

Threatening, Mothering, and Reverse Psychology

Ms. Gomez combined threatening with a mothering tone and reverse psychology when she was running out of patience, "Dann! Enough!” (T4B, L389) and "Stop running your mouth" (T4B, L392) or "Stanley, you're instigating” (T4B, L398). Stanley denied it, "I'm not instigating, I'm asking him how" (T4B, L401). Ms. Gomez responded with reverse psychology, "Why are you asking him how when you should be thinking of another reason to write about" (T4B, L402)? Almost to the end of her rope, Ms. Gomez tried another threat and a mothering plea to Stanley's conscience, "I thought you said . . . I'm going to the restroom, and when I come back my test is gonna be done. Isn't that what you said? Should I play back the tape" (T4B, L404, 405)?

Finally, when all else failed, Ms. Gomez said, “Stanley, I'm losing my patience with both of you" (T4B, L417) and "Are you gonna do your essay or do you want to go" (T4B, L437)? Stanley quickly asked Ms. Gomez to tell him how to start his essay, Jonathan hummed, and Dann acted sleepy and would not accept help. Ms. Gomez explained the ultimate ineffectiveness of threatening, mothering, and reverse psychology:

It's not really that effective because they see me and ok, ok, we'll do our work and they'll look at their paper for like a minute and then they forget about it and they start doing what they were doing again, so usually I'll write it ahead of time and then if they if they push me over the edge then it goes to the door and the security gets it. (T6B, L239)

Ms. Gomez accommodated student resistance with multiple tactics for as long as possible until students pushed her over the edge to stall the inevitable written referral or prevent a fight. Simultaneously, students knew Ms. Gomez's limits and resisted classroom culture, 
norms, and values based on dominant ideology for as long as possible until they saw that she had been pushed over the edge to accomplish their own communication goals, maintain their identity, and stall their removal from the classroom.

Overall, Ms. Gomez and her students had dissimilar classroom goals and exhibited few manifestations in order to stall the inevitable written referral and ultimate suspension.

\section{Summary}

This chapter presented a description of Classroom Two findings in three sections: (a) Classroom Two participants, (b) findings from interactive power analysis, and (c) themes from reconstructive data analysis. Ms. Gomez is a Latina Language Arts teacher who had no experience or knowledge of alternative education before becoming a teacher at The School. Her Latino and African American students were familiar with alternative education. Three types of power exhibited in Classroom Two were normative power, interactively established contracts, and coercive power; charm was not exhibited. These types of power combined in a way that her and her students' roles ultimately conflicted with each other. The teacher had a business side which was conducted as a combination of psychologist, empathetic aunt or mother, and record keeper, attempting students to do what the system expected of them (pass the FCAT). Cultural power was at play in Classroom Two, because the students were not convinced of Ms. Gomez's claims of the importance of FCAT preparation. As a result, time was spent on other things that she viewed as more important, such as figuring out how to get the students to get along. Ms. Gomez's lack of charm and encouragement of student initiated interactively established contracts (e.g., arguing about what happened in the neighborhood) counteracted with 
deference to authority (e.g., need to practice the FCAT) to stop the arguing reduced the validity of normative power claims (e.g., threats of referral) she exhibited. Student behaviors showed that they did not believe she would follow through with her threats. In turn, her threats sounded like begging and pleading due to their receipt on deaf ears.

Three themes of social interaction (resistance, accommodation, and conformism) emerged and combined in the following ways. Ms. Gomez conformed to dominant ideology, both PBS and academic rules and regulations, in order to provide a safe environment for talking but that ultimately supported student suspension. Therefore, students resisted her classroom norms by communicating with each other to maintain their identity and agency in the face of knowing they would ultimately receive a written referral. In turn, Ms. Gomez accommodated their resistance by ignoring, bribing, mothering, reminding, threatening, and using reverse psychology. The combination of these themes resulted in no room for negotiation between the teacher and students. The teacher's goals were different than the students' and were consistently exhibited; thus, conflicts rather than negotiation arose in social interaction and ultimately ended in students being removed from the room with a written referral. In this Language Arts classroom, Ms. Gomez encouraged talk about social incidents outside of school with few academic connections made during the process, which resulted in unsuccessful student outcomes when they were ultimately referred out of the classroom for disruptive behavior. Chapter 6 and 7 present the findings from Classroom Three and Four, respectively. In chapter 8 , a comparison and synthesis of the findings is provided. Chapter 9 includes a summary of the study, responses to research questions, implications for teacher education, and recommendations for research and policy. 


\section{CHAPTER VI}

\section{DESCRIPTION OF CLASSROOM THREE FINDINGS}

This chapter presents a description of Classroom Three findings in three sections based on the nine stages of data collection and analysis described in chapter 3 and illustrated in the Appendices: (a) Classroom Three participants, (b) findings from interactive power analysis and role analysis, and (c) themes from reconstructive data analysis. A summary concludes the chapter. Quotes are cited by transcript name (e.g., T6B), line number (e.g., L271), and participant pseudonyms when necessary (e.g., Mr. Frederek).

\section{Classroom Three Participants}

Classroom Three was a Science course with a teacher who rarely used exclusionary discipline. When asked how he became a teacher, Frederek, who was Swedish, said, "I always thought of becoming a teacher but it was not my first choice [when] I used to apply for different jobs" (T6B, L2). However, teaching was one of the first jobs he got in Sweden and has always

been convenient for me because I like to have the summers off so I can travel so especially in America when you only have a few weeks vacation if you have a regular job. Teaching is very good for me because it allows me to go back to Sweden for 2 to 3 months. (T6B, L8-12)

He had a bachelor's degree in Chemistry and took education courses for "three years to complete ... my certificate, professional certificate in education" (T6B, L22). He became a Science teacher at The School by going through the

Human Services downtown for the [public schools] and they gave me a list of different schools that had openings so that's how I applied for this job, I didn't know it was an alternative school it was just kind of random too. (T6B, L24) 
Getting the job was random, but an ordinary teaching day regularly involved getting up early, planning, and copying, and "teaching and behavior problems" (T6B, L26). He said that his students also got up early so they "are kind of grumpy in the morning" (T6B, L28). Students were dropped off by the buses at the school cafeteria before first period where "they talk to the students and they check to see if they have supplies and books, [and] they're supposed to eat breakfast here I think" (T6B, L30). He summarized an ordinary day for students in his class:

I think they have a pretty good time, not a good time but uh, you know, I think they learn somewhat and you know, I don't know, but ... I think they learn at least sometimes and I don't think they hate to come to my classes. (T6B, L36-38)

After three years of teaching at The School, Frederek maintained control of his classroom in order to teach Science content and prevent student suspension by balancing "good learning and something students like to do" (T6B, L346).

Four students participated: Julio, Antonio, Jack, and Carlos. Table 13 summarizes Classroom Three student participation.

Table 13

Classroom Three Student Participation

\begin{tabular}{lll} 
Name & Observations & Interview \\
\hline Julio & x x x & \\
Antonio & x x & x \\
Jack & x x x & x \\
Carlos & x x & \\
\hline
\end{tabular}


Julio, a Latino, participated in all three observations but was absent for the interview for unknown reasons. Antonio, a Latino, participated in two classroom observations and the interview, but was absent during one observation session for unknown reasons. Jack, a Latino, participated in all three observations and the interview. Carlos, also a Latino, only participated in two classroom observations, having been put on suspension during one observation and the interview. Findings from interactive power analysis and themes from reconstructive data analysis are discussed next, followed by a summary.

Findings from Interactive Power Analysis

This section provides an explanation of the findings from interactive power analysis, including (a) types of power, (b) roles, and (c) cultural power. Interactive power analysis determines who has what kind of power and why and whose power defines interaction outcomes.

\section{Types of Power}

The researcher deductively looked for four types of power based on Carspecken's (1996) categories of interactively established power: (a) normative power, (b) coercive power, (c) interactively established contracts, and (d) charm. The next section defines and describes each type of power that existed in the order they were used. Normative Power

Normative power is gained over others by virtue of one's position or status in the culture or society. Mr. Frederek used his teacher status to claim normative power over his students and gain their consent to classroom norms, including teacher teaches and protects so students can enjoy learning. Mr. Frederek stood with authoritative stature and demeanor at the door before class both directing his students sternly into class and 
protecting his classroom from intruders, using normative verbal claims, such as come on, in a loud deep voice. He used normative claims, such as $O K A Y$, to begin class and conduct lectures from the front of the room behind a long table that provided social distance. Mr. Frederek also used normative nonverbal claims during class by walking around continually among students to pass out supplies, provide directions for maps, handouts, and seatwork students had to do, and check their work, using little eye contact. He gave students many opportunities to consent to his normative claims to do their work and let him teach Science with virtual success. To provide an enjoyable classroom for teaching and learning Science, and to protect students from suspension, Mr. Frederek entered interactively established contracts.

\section{Interactively Established Contracts}

This section covers interactively established contracts, manifested as two subthemes: (a) to provide an enjoyable class and (b) to protect students. Interactively established contracts are constructed by negotiations among participants, based on an implicit level of understanding, mutual consent, and equal communication input. Negotiations that lead to co-constructed interactively established contracts can be thought of as ongoing revisions on multiple drafts of a written paper passed back-and-forth between co-authors. It could go on indefinitely; co-authors may use varying amounts of charm, normative power, coercion, or a combination of techniques to sway the thinking for the final draft. However, the underlying ideas or meanings in the paper that are constantly being revised (albeit not necessarily face-to-face) are what is viewed here as interactively established contracts. 
In Classroom Three, students were rewarded with candy to confirm successful interactively established contracts.

To provide an enjoyable class. An enjoyable class for teaching and learning Science to Mr. Frederek meant that the teacher teaches, and students have fun and learn something. First, Mr. Frederek got to teach Science because he provided supplies if needed, but did not deduct points from unprepared students' PBS point sheets, in order to prevent suspension. Mr. Frederek also provided Science information in pictorial ways to gain students' attention and make learning more enjoyable. He changed teaching approaches, such as drawing and using overhead transparencies, every 15 minutes or so. He answered questions if they asked, but did not initiate the questions to students. Then, when students had consented to letting him teach and doing their work, he passed out candy as a reward. Students consented to the teacher teaching but retained agency by voicing their opinions during class.

Having fun meant students could talk (or not), did not bother each other, and did not get suspended. Having fun manifested itself before or during class when students talked among themselves if desired. The tacit agreement was that Mr. Frederek would ignore them if they did not bother each other too much. For example, while Mr. Frederek directed students at the door before class, Julio and Antonio silently waited. Jack and Carlos were verbal and animated at this point, accepting classroom norms with some reservations and using agency to communicate with each other.

Students had fun constructing acceptable identities by talking about what they liked and letting Mr. Frederek teach at the same time. They also made fun of Mr. Frederek's accent, writing, teaching topics, and mannerisms. Mr. Frederek did not take 
critique as a challenge to his authority, he usually ignored them. However, when students became disruptive or bothered another student to the point that his definition of the classroom setting was in jeopardy, Mr. Frederek interspersed normative claims. For example, Mr. Frederek used such phrases as watch your language, turn off your IPOD, or Carlos, stop it to warn students that they were not holding up their end of the interactively established contract in motion.

Students often initiated interactively established contracts with Mr. Frederek or each other during class. For example, Jack asked to see his report card during a lecture about earthquakes. Jack argued that he will copy if Mr. Frederek will let him look at them quickly. Then, Jack asked Antonio about his bookbag, and then asked Mr. Frederek if he could go get it. Jack then began pencil tapping incessantly during the lecture, then asked what's in the box, went to the restroom, and returned to pencil tap while Antonio and Mr. Frederek talked. Mr. Frederek entered into the negotiations by talking about why Jack could not see his report card yet, letting Antonio go get the book bag, ignoring the incessant pencil tapping, confirming what was in the box, continuing the lecture, and manipulating the overhead.

Students learn something about Science and social interactions in Mr. Frederek's class as exhibited during their conversations (or lack of talk). Students regurgitated Science terminology to position themselves as intelligent multitaskers who negotiated interactively established contracts. For example, Jack talked about what he knew, questioned Frederek's Gulf of Mexico comment, then asked Carlos what the lyrics on his book bag meant, hummed them while he listened to Mr. Frederek's directions, then 
changed the subject to Starbursts while working on the handout, looked through a magazine, maintained agency and identity, and teased Antonio.

Carlos partially accepted Mr. Frederek's contractual claim to teach with his hand on his elbow holding up his head and appearing to listen. He asked questions, hummed while listening to his IPOD, accepted the normative claim to turn it off, told Jack what the lyrics meant, asked Mr. Frederek about directions, cursed the class because he did not get it, maintained agency and identity, threw paper on the floor, and said Mr. Frederek should pass out weed instead. Antonio accepted the claim to learn while still looking at and fiddling with the overhead, maintained a sleeping demeanor, traded Starbursts, maintained agency and identity, scrunched eyes at Jack for teasing, and put his head on the table again. Julio wrote silently to maintain agency and identity, accepting the claim to learn. Interactively established contracts interspersed with normative reminders usually resulted in Mr. Frederek teaching, students having fun and learning at least something about Science, and no one getting suspended. However, when interactively established contracts got unruly and students bothered each other too much, Mr. Frederek stepped in to protect them from each other and ultimately from suspension.

To protect students. To protect students from each other and from suspension, Mr. Frederek made disciplinary decisions based on what was happening at the moment rather than on strict enforcement of PBS rules. He wanted students to like the Science class, and he did not want to ruin their chances at school by writing referrals that removed students from class and usually led to suspension. He also did not want students to leave class in anger without permission, which could also lead to suspension. For example, Julio was silent during most of the time; Mr. Frederek talked quietly to him if he got bothered or 
lacked supplies. When students bothered Julio by trying to get him to talk, Mr. Frederek first reduced the social distance between himself and Julio to gain solidarity with him. $\mathrm{He}$ spoke quietly to Julio, reassuring him that it was safe to stay in class because he would protect him from the taunters. Then, he said to the taunters in a loud voice such phrases as leave him alone or be quiet. Julio consented to the interactively established claim and stayed in class. Through such interactively established contracts, Mr. Frederek also protected Julio from suspension.

Mr. Frederek allowed some claims to be made by students either by ignoring their references to cultural differences unless it bothered another student, or by letting them talk quietly about community processes, like fighting, while he passed out folders. When students bothered Antonio too much, however, Mr. Frederek had to initiate several interactively established contracts before Antonio agreed to stay in class. First, he ignored the conversation between Jack, Antonio, and Carlos, based on the interactively established contract of the accepted classroom norm to converse while waiting for the teacher to start. References to the counterculture of fighting and profane language finally got too loud and were bothering Antonio too much. Then, Mr. Frederek used normative claims such as leave each other alone now, don't talk to him, that's enough, don't look at him, and Jack's name. Antonio remained upset. Jack accepted the claim with all right, I'm ready, but looked around quietly. Carlos opened his notebook and accepted the claim momentarily. The conversation reignites bothering Antonio again. Jack rejected Mr. Frederek's claim with sarcasm to Antonio. Antonio rejected Mr. Frederek's claim with cursing to get the guys off his back, a sarcastic retort, and then got up and headed for the door threatening to leave. He challenged how the class was run implying that Mr. 
Frederek was not protecting him like he tacitly claimed to be able to do. Antonio used agency to initiate an interactively established contract, taking care of the situation by himself since Mr. Frederek did not appear to be holding up his end of the agreement.

Frederek accepted this interactively established contract from Antonio and tried to get him to stay with you have been in this class and walking by him as he headed toward the door. The tacit contract to Antonio was I'll protect you in here and not write referral if you will stay. He told Carlos you are the one who should be leaving, tacitly claiming that he had broken the classroom norms here. He interjected normative claims to Jack and Carlos with loud be quiet leave him alone and sit down to Antonio.

Antonio opened another interactively established contract with Mr. Frederek telling him to call the counselor to change the schedule. Jack accepted the claim to leave Antonio alone and didn't want to get into trouble. Carlos accepted the claim to leave Antonio alone and tried to get Antonio to stay fearing repercussions for the guys; then Carlos used his own normative power with Antonio dropping his rank in the peer group. This reference to the peer group from Latino culture is an attempt to identify themselves separately from school.

Mr. Frederek claimed don't worry about it to Antonio, interactively reestablishing the contract that he protects students, followed by a loud normative claim of leave him alone that's enough. Jack rejected the claim with sarcasm about how long they have been working on earthquakes, saying Frederek deserved an award for patience, laughing, saying the board is dirty, mouthing to Carlos, then laughed at what Carlos said, and then accepted Antonio's stare as straight, chillin', mack. Carlos rejected the claim by pulling up his hood and listening to his IPOD, turned it up loud, rejected the claim further by 
continuing to listen to his IPOD even though asked to put it up, and mouthed words to Antonio. Carlos appeared to accept the second normative claim to turn it off but with reservation to maintain identity and agency that he wanted to listen to it, told Jack that he was going to listen to Frederek, but immediately egged Antonio on. Antonio accepted the claim but also nodded to Jack, stared at both guys, and guys accepted him as straight, chillin, mack.

Students knew that Mr. Frederek would protect them if necessary and he wanted them to all stay in class, but if students bothered each too much, he would use the underlying CSI option for a few minutes, trying to not write referrals until absolutely necessary. Jack accepted the claim as he talked and waited. Carlos accepted the claim as he talked and waited. Antonio accepted the claim as he talked and waited, stood up once angry and sat back down. Julio accepted claim as he waited silently. Frederek ultimately gained consent from students and administered candy as a reward to uphold the tacit interactively established contract for not bothering anyone else and settling down into the classwork.

Even though trouble with the law got students into this school, family opinions were important to many students. Their parents did not want teachers to call home to bother them with school infractions, because cultural mores dictated that students were supposed to mind their teachers. Mimicry of Mr. Frederek's accent positioned the student peer group as Latino versus Swedish, referencing a counterculture that was interested in peer status. However, students often negotiated interactively established contracts with sarcastic remarks referencing cultural humor, music culture, and identities that got out of 
hand. If interactively established contracts got out of hand and were not being negotiated successfully, Mr. Frederek resorted to coercive power, albeit seldom.

\section{Coercive Power}

Coercive power is accomplished by forcing obedience after threatening punishment. Mr. Frederek used coercive power to maintain control of his classroom when his goal of teaching and learning Science in an enjoyable environment was not being achieved. Threats were issued to students based on their history of behavior in his class. Mr. Frederek used coercive power claims to call home if he knew from experience that the student would ultimately stop disrupting when he heard this. He believed that a good student with a good family took this threat seriously. For example, Mr. Frederek used coercive power by talking to the CSI staff about Antonio's desire to leave class. Antonio accepted the claim as a threat he was unwilling to pursue because it might involve a call home to his parents, but he came back into the classroom by his own choice with agency.

Rather than write referrals to kick students out of class, Mr. Frederek used coercive power with a threat to call home when good students bothered each other too much or questioned his disciplinary decisions. For example, he said be quiet or I'll call your parents to Jack who was bothering Antonio too much. Jack accepted the claim with all right all right. Mr. Frederek interjected you may be next and threatened a call home when Jack questioned why Carlos was kicked out. Jack accepted the threat to call home by waving his hands and saying naw naw naw. He immediately asked a question about earthquakes, and began copying words off the board, but also maintained identity and agency by questioning Mr. Frederek about why he broke a pencil to illustrate faults 
Antonio accepted the claim. Julio partially rejected the claim by silently texting and writing at the same time.

On the other hand, if a student showed Mr. Frederek that he did not seem to care by challenging his classroom goals $24-7$, he eventually kicked him out to CSI for a breather. From experience, Mr. Frederek believed that students who were not good and made bad choices and had no support at home would not stop disruption otherwise. Therefore, he used coercive power when the history of a situation had gone on too long and someone was getting hurt or demeaned even if not in the room. For example, Mr. Frederek had ignored much of Carlos's talk until he asked if the word on the board was sluts. This was the last straw for Mr. Frederek, and he finally stepped in and removed Carlos from class. The reason for the disciplinary action was not obvious to students because all they heard was the one word. However, they also knew that Mr. Frederek was patient but, like most people, had a limit. Students tried his limits until he reacted with you're out of here and getting the CSI staff for Carlos. Mr. Frederek never accepted students' challenges as a threat to his authority as he waited for security. He interjected normative claims, telling Carlos what the word was, that he caused the problems, to be quiet, to write it down even if he doesn't understand it, but be quiet! When Carlos continued to argue, Mr. Frederek said loudly, you're out from here. To security, he said authoritatively I need Carlos out.

Carlos appeared to accept the normative claim to leave Antonio alone but rejected the coercive claim to leave. He said what does that say about the word he was copying from the board, sluts? He rejected coercive power by reclaiming his position of wanting to copy but couldn't read. Saying sluts appeared to be an innocent mistake; he appeared 
to really think faults was sluts as he pursued with some cursing, more asking, disbelief, and questioning how he can copy if he doesn't know the word. He never accepted the normative claim to leave, but he obligingly accepted the coercive claim of CSI with agency and identity in tact by not giving in without a fuss, taking a while to write the word and put on his jacket. Jack rejected the claim with a question, why did you kick him out? Julio texted silently as he had been doing for most of the period. Antonio laughed, accepting the claim but with reservation.

Carlos's breather in CSI occurred as a result of an historically disruptive period of time in Mr. Frederek's class. Carlos returned from CSI after 15 minutes, the time limit allowed in there without a written referral. He appeared to accept Mr. Frederek's normative rule to listen to the lecture but with a quiet insult to Mr. Frederek to maintain identity and agency. He began writing and tapping his pencil, laying his head on his chin. Julio looked at the overhead, accepting the claim. Antonio looked at the floor, accepted the claim, and asked several lecture related questions which Mr. Frederek answers. Jack accepted the claim. Through coercive power threats, Mr. Frederek ultimately gained consent from students to not bother anyone else and settle down into the classwork.

\section{Charm}

Charm is the use of one's personality to gain consent from others. Mr. Frederek did not use charm to gain consent from his students. Table 14 summarizes types of power exhibited in Classroom Three. 
Table 14

Types of Power Exhibited in Classroom Three

Type of Power $\quad$ Exhibited

Normative $\quad \mathrm{x}$

Coercive $\quad x$

Interactively Established Contracts $\mathrm{x}$

Charm

Normative power, interactively established contracts, and coercive power were exhibited in Classroom Two; charm was not exhibited. The roles that participants exhibited are discussed next.

Roles

This section covers teacher roles and student roles. The researcher inductively found and named roles based on routine "interactive rhythms and sequences" (Carspecken, 1996, p. 137). Roles emerge as themes through complex ways of acting , which participants "recognize as having some unity" (Carspecken, 1996, p. 136). When participants recognize a role in play, they can predict, but not ensure, future actions. Table 15 provides a summary of Classroom Three participants' roles, followed by a description of teacher roles and student roles. 
Table 15

Classroom Three Participants' Roles

Participants Roles

Teacher - Frederek The patient quiet teacher with a disciplinarian/protector side

The teacher/lecturer who answered questions

The aloof patient teacher

The roving traditional disciplinarian parent

The quiet protector

Student - Jack The curious student with an uninhibited sense of humor

Student - Carlos The uninhibited leader and destroyer

Student - Antonio The perceptive team player

Student - Julio The silent/distrustful observer

\section{Teacher Roles}

The teacher exhibited six roles. First, Mr. Frederek was the patient quiet teacher with a disciplinarian/protector side. He exhibited this role when he passed out folders and other supplies, walking by each student, nonverbally forefronting his status as the teacher.

Second, Mr. Frederek exhibited the role of teacher/lecturer who answered questions after consent had been reached that he would lecture. Sometimes Mr. Frederek stopped lecturing if someone asked a valid question to answer their questions and carry on a conversation about what was asked. To him, this was how students best learned, but he did not initiate the questions. Students knew that they could ask him questions and get valid responses, thus validating their student identity. A tacit agreement existed that 
students were allowed to resist classroom activities by validating their own identity as a person and challenging his authority as a teacher to protect them as he had claimed he would when he said leave him alone. Once students felt protected or free to validate their student identity, they would venture forth to ask academic questions. The teacher has to have validated his own claim to be the protector in order for this to happen.

Third, Mr. Frederek exhibited the aloof patient teacher role quite often when his own authority was being challenged. He acted as if his authority was not being challenged by ignoring insults and humor about his accent, writing, and other details. The social distance created by his patience and ignoring tactics made space for him to take his time to assess the situation, let the students identify with the classroom space, and decide whether they would eventually consent to doing the Science and stop bothering each other. The students acknowledged his patience, tried his patience, and eventually consented to learning Science if he fulfilled his part of the agreement to protect them from others who bothered them in this classroom. Once the teacher had validated his part of the agreement, they usually consented and did the work.

Fourth, Mr. Frederek exhibited the role of roving traditional disciplinarian parent most often to prevent conflicts so that students could and would stay in class. To do so, Mr. Frederek walked around when necessary to survey or set up the situation to make sure students did the classwork and left each other alone. If someone bothered someone else, Mr. Frederek told them to leave each other alone. If students were not bothering anyone else, they could talk. If they bothered him, Mr. Frederek ignored it and went about his business of teaching Science in as many ways as he could think of - ways that included pictures, drawings, overhead, and so on but not to get students too active. If the 
student would not do as told after many demands, he got into trouble. If the student usually shaped up but got out of hand, Mr. Frederek would call home or threaten to call home to get him to stop. If someone was getting bothered or if someone disrupted inappropriately after a long history of the same, he would get kicked out but referrals were not used except in dire situations. Mr. Frederek wanted kids to stay, like class, and learn Science. With a little authority, they could learn.

Fifth, Mr. Frederek usually played the quiet protector role when students were still deciding whether to consent to learning Science or not, and students were challenging his authority to protect them from others who bothered them. Either he would go over and whisper what the student needed to know about the work being done or follow the student around to convince him to stay in class. He implied that he would protect the student from the others who would never consent to classroom norms of leaving each other alone. Usually, Mr. Frederek could convince students to stay, learn, do the work, and enjoy it in the meantime. In this classroom, rules were debatable and discussable; choices were valid. Eventually with normative power claims foregrounded, contractual agreements and coercive power claims backgrounded but foregrounded when necessary, protection guaranteed for feeling safe for learning Science or being sent out to CSI for a few minutes but allowed to return, the students consented to stay in class and to the work. This teacher upheld a façade of social distance with his quiet/authoritative demeanor but in the end, passed out candy, knew students' interests, knew their ethnicities, called home, and protected each students' identity claims so that they could like and learn some Science in the classroom. He was natural in each role he played. 
Because of his consistency and as expressed during the interviews, the students knew where he stood even though he was not talkative about his own life.

\section{Student Roles}

Jack played the role of the curious student with an uninhibited sense of humor throughout most of the class and got away with it, because he knew just when to discontinue the behavior. He did not want Mr. Frederek to call his home.

Carlos was the uninhibited leader and destroyer, named by his peers. He was uncontrollable when he got into a realm of behavior that would give him attention and provide him a facade to hide behind when he did not or did not want to understand the work being assigned in class. When talking to peers about music, he calmly taught them what he knew.

Antonio was the perceptive team player and could manipulate a situation in order to gain Mr. Frederek's assistance. Antonio knew how other peers worked for and against each other and would use this knowledge to gain an upper hand in the classroom. However, he knew when to stop because he did not want Mr. Frederek to call his home. Julio was the silent/distrustful observer $100 \%$ of the time. He did not want to get involved in Carlos's intrusions. His peers acknowledged this behavior and understood it because they had once been in his position as a new student in The School.

\section{Cultural Power}

Cultural power was discovered through the process of hermeneutic inferencing, or taking the position of participants in order to understand the basis of their claims. Cultural power exists when the norm or value claimed does not represent mutual interests of the entire group. In this classroom, Frederek's continual reference to the universal 
claim of leaving each other alone was only partially recognized by the students, noted in how they continued to bother each other. Interests of all participants were not fulfilled by this claim. Students wanted to bother the quiet guy since he did not talk to them; they wanted to bother Antonio because he was part of their crew and they always discussed rank and other interests to remain a part of the group. The interest of bothering Mr. Frederek by making fun of his language and writing was part of an interest they wanted to fulfill in solidarity with each other. Students knew they could bother Mr. Frederek without being punished, but he gave mixed messages by allowing them to bother him but not each other.

Based on hermeneutic inferencing through the recognition, reflection, and examination of students' interactions with each other and their explanations of how they assess and test teachers' patience limits, students recognized themselves as taunters and guys who could get teachers to react with no repercussions, up to a point, that is. For example, Carlos misjudged when he said sluts for faults and Mr. Frederek reacted uncharacteristically to the students; they were surprised. Mr. Frederek's interest to keep kids in class fell through when he felt he had to have Carlos leave. He fulfilled this same interest by telling Jack he would call home and Jack subsided and consented to stay and do the work. After students achieved their interests of bothering Antonio, they consented to do the work. They recognized themselves in the work somewhat. Antonio asked questions and had a conversation with the teacher; Jack interjected things he remembered even though he continued to bother Mr. Frederek or Antonio in the meantime. Carlos did not see himself in the work; he considered it but ultimately gave up. 
Mr. Frederek did not forefront, or openly threaten, the inevitable written referral, because he did not want to use it. So he surprised the students when he sent them out or when he called home. Cultural power was somewhat in play but worked to the interests of the students in most cases. Jorge got to be silent which he recognized himself doing and got to do work (with some texting which was ignored). Jack got to be humorous, creative and sarcastic with language to a point and remembered some of the work and interjected information he had retained. Antonio got to stay and not be bothered which was why he wanted to leave earlier but the teacher convinced him to stay, He also got his questions answered about earthquakes. Carlos got to be the rank master among his peers, a position he wanted, but he also got sent out of class because he would not consent and had a history of not consenting and took it too far. However, he also got to come back in to class and begin doing the work, but he did not really recognize himself in the work so he said he hated this class to save identity/agency. In the end, Mr. Frederek gave out candy which all students consented to, recognizing that the interactively established claim of rewarding work with candy was in their interests too.

Themes from Reconstructive Data Analysis

This section covers three themes that emerged from inductive reconstructive data analysis: (a) conformism, (b) accommodation, and (c) negotiation. Because the emerging themes aligned with Giroux's (2001) theory of resistance in education and Third Space theory (Gutiérrez, 2008), terminology from both were used. Table 16 provides a summary of the findings from Classroom Three reconstructive data analysis. 
Table 16

Classroom Three Reconstructive Data Analysis

\begin{tabular}{|c|c|c|c|}
\hline Theme & Part. & Manifestation & Purpose \\
\hline Conformism & $\mathrm{T}$ & Banking education & $\begin{array}{l}\text { Maintain control } \\
\text { Teach Science } \\
\text { Prevent suspension }\end{array}$ \\
\hline Conformism & $\mathrm{S}$ & Banking education & $\begin{array}{l}\text { Learn Science } \\
\text { Prevent suspension }\end{array}$ \\
\hline Accommodation & $\begin{array}{l}\mathrm{T} \\
\mathrm{T}\end{array}$ & $\begin{array}{l}\text { Teaching Science } \\
\text { Enforcing PBS }\end{array}$ & $\begin{array}{l}\text { Maintain control } \\
\text { Balance learning and needs } \\
\text { Prevent suspension }\end{array}$ \\
\hline Negotiation & $\begin{array}{l}\mathrm{T} \\
\mathrm{T}\end{array}$ & $\begin{array}{l}\text { Cognizance } \\
\text { Patience }\end{array}$ & $\begin{array}{l}\text { Maintain control } \\
\text { Teach Science } \\
\text { Student enjoyment } \\
\text { Students' needs } \\
\text { Prevent suspension }\end{array}$ \\
\hline Negotiation & $\begin{array}{l}\mathrm{S} \\
\mathrm{S} \\
\mathrm{S} \\
\mathrm{S}\end{array}$ & $\begin{array}{l}\text { Bothering each other } \\
\text { Giving teacher hard time } \\
\text { Inappropriate negotiations } \\
\text { Preventing boredom }\end{array}$ & $\begin{array}{l}\text { Communication goals } \\
\text { Maintain identity and agency } \\
\text { Enjoy class } \\
\text { Prevent suspension }\end{array}$ \\
\hline
\end{tabular}

Table 16 denotes three themes that emerged from reconstructive data analysis. Teacher and student conformism to the dominant ideology of Science education was manifested as banking education. Teacher accommodation manifested as teaching Science and enforcing PBS. Teacher negotiation manifested as patient reminders, threats to call home, call home, vary talk/distance, and 15-minute CSI breather. Student negotiation manifested as bothering each other, giving teacher a hard time, inappropriate negotiations, preventing boredom, and varying talk/distance. Teacher conformism was 
manifested to maintain control, teach Science, and prevent suspension. Student conformism was manifested to learn Science and prevent suspension. Teacher accommodation was manifested to maintain control, balance learning and needs, and prevent suspension. Teacher negotiation was manifested to maintain control, teach Science, provide student enjoyment, meet students' needs, and prevent suspension. Student negotiation was manifested to accomplish communication goals, maintain identity and agency, enjoy class, and prevent suspension.

\section{Conformism}

This section covers how both Mr. Frederek and his students exhibited conformism to dominant ideology about teaching Science to alternative education students from a banking education perspective. Conformism, the opposite of resistance, is a form of behavior that simultaneously suppresses social contradictions and merges with the "logic of ideological domination" (Giroux, 2001, p. 109). Dominant ideological logic in education is based in banking education. Banking education assumes that Science knowledge is classified, sorted, distributed, and assessed by an authority and that alternative education students possess academic and behavioral deficits that are classified, sorted, and assessed by an authority.

Frederek's classroom culture, norms, and values for teaching Science followed banking education principles. As the classroom authority figure, Frederek had the necessary Science knowledge to conduct science labs or lectures using textbook information and academic vocabulary. Jack and Antonio agreed, "Yeah, he knows his stuff" (T6A, L212). Sometimes, Frederek conducted science labs but "since we changed 
to this classroom, I haven't done so much labs because of the- it doesn't really have storage" T6B, L130). He explained from a dominant perspective:

I've used the goggles once or twice with my, the older kids that have my eighth period Tuesdays and Thursdays, they're older, they're easier to do stuff with because they can sit still, the younger kids, it's a lot of work to do something like that. I used the lab, goggles and aprons, I've done with these kids, uh, a lab will be hands-on, yeah [using goggles and putting on aprons]. (T6B, L130-136)

Frederek laughed and clarified that hands-on to his students "can also be trying things out, yeah, when I ask the kids kids say it is putting chemicals together" (T6B, L137-138).

Typically, Frederek stood behind the long table near the white board at the front of the class when he lectured, keeping his distance from the students. Students were supposed to (and typically did) stay seated at the tables during class. Frederek stored, distributed, and collected student folders, handouts, and other supplies as needed during class. During the interview watching the DVD, Antonio confirmed that Frederek was "just picking up the folders from the other class" (T6A, L10).

Mr. Frederek wrote academic information and made scientific drawings on the board daily for students to copy into their notebooks. He supplemented the lectures with audio-visual aids to get his students involved through different approaches. Mr. Frederek explained:

I think that the more stuff that you use, it's like the more, like movie or overhead they're both, especially the big things that catches their you know, attention and then changing stuff and do some work on there and on there. (T6B, L106-108)

Besides different approaches, Mr. Frederek used supplemental materials to simplify the academic information. Mr. Frederek explained:

This [computer] I use for software so I can explain on this like from the textbook, you know, power point slides and that sort of stuff, yeah, [overheads that come 
with textbook too], the chapters are divided, you know, simpler to show with pictures, so that's how. (T6B, L124-126)

Frederek sustained banking education principles by simplifying various approaches. Students waited quietly while Mr. Frederek changed approaches, walked to another position in the classroom, or wrote on the board.

Mr. Frederek did not ask students any questions and expected silence from them when he was lecturing. However, he would entertain student questions. For example during a lecture about earthquakes, Antonio raised his hand, leaned over to point to something on the transparency, and asked, "Let me see, what is this right here? Is this an earthquake" (T4A, L281)? Mr. Frederek pointed to the transparency and answered this and subsequent questions from Antonio during the next 20 utterances. During the interview, Mr. Frederek explained that "when someone asks me a question like that like a serious question, I have to go with that because that's when they learn most. So whatever it takes" (T6B, L344). Additionally, if students ever asked something he did not know, Mr. Frederek tried his best to find the answer. Jack said, “Only one time he didn't know what it was" (T6A, L220). Antonio laughed and explained:

Carlos asked him something that I don't remember, something about the planets. It was something about the planets and Carlos asked him, and Mr. Frederek just put to the face something like he didn't know it! And everybody was like oh, he doesn't know. And he starts searching in the book like crazy. (T6A, L221)

Jack concluded, "Yeah, he'll look for the answer" (T6A, L222). After Mr. Frederek lectured with simpler supplemental materials and students copied notes from the board, he passed out handouts for students to complete at their tables while he walked around to assist students as needed. He explained: 
the point of this is when you have a small class like this four to one, (undecipherable) to help them more easily than if you was to do it in front of the class it's not going to turn out so good. You can help each student out, you can do that here, because you only have four persons. That's nice, a small class like this. I've had classes last year I had a class that was always 20 people. They' $d$ sit everywhere. That was not in this classroom. This was before this classroom. Yeah, but this was for the older students they're easier to handle so with these when you have 20 kids of these you can't do that [walk around to help individual students]. [They're] eighth graders, but some of them are retaking it, so I don't know how old [they are]. (T6B, L356-361)

Mr. Frederek classified, sorted, and assessed his students from a banking education perspective that assumes alternative education students learn better individually and silently at their desks, and are easier to handle when older.

Talking about worksheets during the interview, he summarized his philosophy of

teaching alternative education students:

This is not really a good worksheet, but it's something they like to do so you have to make that balance between good learning and something they like to do as well. Mostly these yeah, this is a worksheet, yeah, yeah, it has to do with drawing or plotting, not writing, usually they like to do what is in a worksheet maybe it's not the best learning experience but uh, yeah, we write we try to write something everyday. Yeah, they take notes everyday or something not I wish I could do it more but I like they write today's date and then write something everyday, [not] like thoughts or decisions, uh, maybe the older kids, uh, they could do it much more, yes, it's definitely they can do much more, I do usually notes or drawings. (T6B, L346-354)

Mr. Frederek's low expectations were derived from students' dislike for writing and superseded encouragement of their thoughts or decisions in the classroom. Mr. Frederek elaborated on his alternative education philosophy as it related to grades and report cards:

Report cards are given out four- every quarter. Or the interim, there's also the interim. Uh, some kids care, some kids some kids care about their grades, some kids they can't you know, they got their report cards for the second quarter, you know, they are given out report cards three weeks after the second quarter and uh, they can't connect those grades that the got then with what happened three weeks ago. Same with the points, they need to have this worksheet everyday, everyday, so when then I usually give them out the last 10 minutes of the class period 
because some students are going to run around asking their teachers and stuff like that. They ask sometimes about their grades but uh, and their report cards should have grades and so. Yeah, yeah, for the students yes, I like grades, I mean I give at least two grades each day for each student, you know participation grades or for stuff they are doing in this class ... . I don't personally like grades but I think that more grades gives a better picture, you know. (T6B, L66-72)

Mr. Frederek portrayed low expectations and static behavioral and academic principles when he mentioned students who ran around wanting to look at their report cards and when he graded for participation.

Overall, both Mr. Frederek and his students sustained dominant logic in education. They presumed that Science content is predetermined knowledge that is classified, sorted, distributed, and assessed by an authority for students who have also been classified, sorted, distributed, and assessed by an authority.

\section{Accommodation}

This section covers accommodation, manifested as two subthemes: (a) teaching Science and (b) enforcing PBS. Accommodation is perceived as simultaneously coping or accepting of the school's ideology while exhibiting some form of resistance to it (Giroux, 2001). Mr. Frederek demonstrated accommodation by simultaneously coping with system rules and regulations about teaching Science and enforcing PBS while exhibiting some form of resistance to flaws in the system. He coped by distancing himself from the system, continually referring to we as teachers and they as the administration or district:

It's really in public teaching I think it's one of the things I've noticed, it's like them, it's like the administration and the teachers, they're so se-, it's like a hierarchy, you know, it's not working together, everybody does the thinking for someone else. (T6B, L118-122) 
By distancing himself from administrative decision-making flaws, he could accomplish his goal of balancing good learning and attending to students' needs based on their profiles and histories to prevent suspension.

\section{Teaching Science}

Mr. Frederek coped with public school system regulations about teaching Science but ultimately made his own decisions in order to accomplish his goals. For example, he covered the walls with various Science posters both according to what was being worked on in class and to accommodate how "they want [us] to have, they want [us] to have [something on the walls], the administration" T6B, L116). Mr. Frederek also used textbooks as a reference for his lectures because of his banking education philosophy but this practice simultaneously accommodated administrative discouragement of the use of textbooks as busywork. He explained:

I use textbooks somewhat, not that much, I should probably use them more but uh, some teachers use them too much, I think. It's kind of also something that they [the administration] discourage to use too much textbooks, I think. Yeah, [because] the teachers put up questions from the textbook and they sit down and let yeah, the textbook itself does not have a good stigma to it. (T6B, L88-94)

In addition to using textbooks only as a reference, Mr. Frederek explained that he used worksheets as a guide for students' learning, a practice that also accommodated administrative discouragement of worksheets as busywork:

If the worksheets are good, they're they're fine, if they're like busywork they don't like them so, the administration, yeah, they have a now they have a limit of 150 copies per month, that's nothing, but I think worksheets are good, especially with these kids because they give them a, you know, a roadmap you know. I likeI like worksheets. (T6B, L96-98, 104)

Mr. Frederek liked using textbooks and worksheets as a roadmap for his students while also accommodating the administration. 
Mr. Frederek's philosophy on testing also accommodated district mandated FCAT testing procedures. He said, "We have a have a curriculum that's ... right now in front of FCAT ... . review sheets for FCAT" T6B, L300) that students are

supposed to study weekly, so for the FCAT, these are the eighth graders are going to take the FCAT, it's a month from now, so this is usual science, it doesn't it doesn't count for the students, it only counts for the school, so that's the dilemma, [students] know they don't count for the students but it counts for the school. They don't really pass, uh, there's a few students that pass the science FCAT but most of them will not pass. (T6B, L302-314)

Knowing the dilemma between what counts for the school and how many students pass

the FCAT, he accommodated district standardized testing policies by focusing on

students' immediate needs in the classroom. He elaborated:

I think when they have these standardized tests in science, math, and uh, reading, English across, well, these four are, there's other ones from the district those uh, I'm gonna give some out this week but every quarter we have those from the district it only goes to the district so those that those are used to grade the students' grades as well, they have it on their report cards, yes. But uh, those yeah, those are the main tests I give. I don't really do so much tests myself. (T6B, L140-146)

Mr. Frederek rarely gave teacher-made tests and ultimately accommodated system requirements when teaching Science in order to accomplish his own classroom goals of balancing good learning with students' likes.

\section{Enforcing PBS}

Mr. Frederek also coped with system regulations about enforcing PBS but

ultimately made decisions in the classroom to prevent suspension. He explained:

I think that the idea of positive behavioral support is good uh, you know we have the chart there that we take we're supposed to give them points for uh good behavior but it doesn't work out so good the point system but I always give them points at the end of the class. You know, summarize how good that they did during the class. And then at the end of the week, they they get all these points together and they get to go to the point activity. But I think that it's a good idea 
but the actual giving out of the points sometimes in the middle of the class is not good. Yeah, every 20 minutes, they supposed, when they started they- we were supposed to give 20 minutes into us you know, in the announcement but you know that would probably be even worse, tell you, every 20 minutes ring the bell every 20 minutes right. No, it was too much [to keep up with]. (T6B, L40-48)

He thought the PBS system was a good idea but voiced how he thought the point collection system could be made better for students:

[Students] collect point sheets that they are going to give to the administration so that they can count their points and if they get enough points they get to do something fun on Fridays usually. But uh, they only collect points every once a week, the point sheets only once a week but I think it would be much better if they actually had a point sheet every day, you know. They collect for the week, yes, it's actually two or three days, right, except for if you have it on the board, but nobody really, teachers, you know, so it would work much better if students collected points every day every day because it would be more routine. I told them that too, but they haven't implemented it, [students] should have a big worksheet like that [one on the wall] so you can actually see you know, everything with points every day. (T6B, L60-64)

Even though his suggestions for a routine daily point collection sheet had not yet been implemented, he accommodated the enforcement of PBS by focusing on what was going on in his own classroom with his students before making decisions about how to deal with their behavior. When asked about an ordinary day for his students at The School, he explained:

I don't really know how they they how they have it you know, I see them in my class period but it's kind of- I stay in my room pretty much, you know, yeah, that's what I think is yeah, it's very anonymous, don't work together, it's- you know what is going on in your classroom. (T6B, L28-32)

From his perspective, teachers and administration do not work together. Mr. Frederek continually reasoned that he made decisions in the classroom about enforcing PBS based upon what happened and what is going on in his classroom to ultimately prevent student suspension. For example, during a lecture about "seismic energy, seismic waves" (T4B, 
L242, Mr. Frederek), Jack asked Antonio which class another student had that period. Antonio answered Jack, and Jack immediately asked the teacher, "Frederek, will you call her really fast? I need to get my book bag from her" (T4B, L250). Mr. Frederek stopped lecturing and asked, "From who?" (T4B, L251). Jack responded with the student's name and confirmed the location with Antonio. Mr. Frederek walked over to his desk and wrote Antonio a pass so he could go get the bookbag for Jack. Jack explained during the interview, "I lended it to him because he had a field trip and to get inside the bus you need a bookbag and he didn't bring one so I just lended it to him" (T6A, L230). Antonio commented while watching the DVD, "That was the part where I went [to] go get his bookbag" (T6A, L232). During the interview, Mr. Frederek laughed and said understandingly about getting asked to go get a book bag, "There's always something, always something" (T6B, L296).

Mr. Frederek accommodated the enforcement of PBS rules depending on students' immediate needs. He explained the administrative stance on writing passes:

Passes, well, passes are not encouraged, they don't like that us to send any students out for any reason very much so but sometimes you just give in and give passes anyway but [they go to the] bathroom, but they- there might be some other purpose for that trip. They have like, phew, 20 cameras here there's so much, yeah, as soon as you get out from this classroom, there's cameras so they can you know, if you went into the security room, they have like so many cam- there's like a camera for every- yeah, all the time, yeah, so it's like a Ft. Knox. Yeah, they'd be on the camera, sometimes they call down the kids because they have done something they have seen on the cameras. No, not in here, I don't know but think it's probably some union regulation or something like that. So passes are dis- they don't like us to use passes but uh, sometimes we do it anyway. (T6B, L74-86)

Mr. Frederek sometimes wrote passes depending on students' needs and profiles. He also rarely used written referrals in order to prevent student suspension, a practice that 
accommodated administrative discouragement of written referrals. He explained his philosophy based on his knowledge of school politics:

I use them [referrals] not so often. Maybe, I don't know, last week I think I gave about two to three but I that's kind of unusual. No, I give them to the uh, well the thing is that when you have there is a CSI next door, the CSI where they keep the students there to (undecipherable) but you can only keep the students in there if you give them referral, to have written them up. See, you can't just send kids in there because they are being painful. I think the referrals is too, you know, if you write the referral they can't go back to their home schools to their home school. No they cannot with referrals, so that is kind of a too harsh punishment, there should be something in between.

Mr. Frederek believed that referrals were unnecessary punishment for students; this belief aligns with his thoughts on how students only get worse the longer they stay at The School. He continued talking about the policital repercussions of referrals for The School when they are tabulated downtown:

Yeah but these referrals aren't, huh, they're all political the referrals too, they don't really like them to be given out, because you know, that information goes downtown and if it's a lot of referrals it looks good or bad on a school if there's a lot of behavior problems. Referrals are not encouraged either. It's all politics, I think. But I give, you know, if if but you you send them over there and then you write them up and then you send the referrals over there too and then you're supposed to get a copy too, but whatever happened to that referrals you don't know if it actually, yeah, sometimes it might not even be executed so you don't know if you don't. (T6B, L150-160)

Writing referrals looks bad on a school and is often too harsh of a punishment, but Mr.

Frederek explained another flaw in the system that deterred him from writing referrals.

He confirmed that part of the politics is that once referrals leave the teachers' hands, no one knows what or how it will be executed. He would rather solve the problems himself. For example, during the interview while watching the DVD, Mr. Frederek noticed Carlos returning to the classroom after being sent next door to CSI for 15 minutes. He said that he was 
going to try to put him in the detention here, yeah, but I he can probably be in there for 15 minutes [without a referral] yeah, but this problem I think I could have solved because I know them so well so I I at least I think I know them so well. (T6B, L252-256)

He elaborated on the conflict between solving problems in the classroom, writing referrals, and preventing suspension:

That's the flaw in the system. Once you send them away from here, right, maybe I didn't write the referral, that's the reason he came back, yeah, but the thing is that when you send a student uh, to to detention here and then you are going to sit down to write your referral, they might be hundreds others of issues in the classroom then you're going to sit and write, but they won't let as soon as he you send him away, you should write the referral but that could wait until the class is over or something. But sometimes you send them away with the referral you know and they come back and begging you to not you know send them. I don't know [why Carlos returned to class], yeah. It's probably because I didn't write the referral, I don't know. Or or he wants another chance, yeah, that shouldn't happen. (T6B, L320-326, 338)

Based on his views of the politics of schooling, Mr. Frederek accommodated PBS by making decisions and solving problems himself in the classroom except in very dire situations to prevent student suspension. To accomplish his goals, Mr. Frederek ignored some PBS infractions based on what else was going on in the classroom. For example, he explained his reasoning for ignoring [Carlos's] lack of proper uniform attire:

Sometimes I think if they forget one, they will get they have to borrow one but, no, they can have black, I don't know what he has, if he has, they're supposed to have a collar you know, but I don't know if that is a collar but I don't I don't pay attention to that stuff you know we're supposed to but that doesn't catch my attention so they can come in here pretty much naked, no, we're supposed to but I don't. (T6B, L218-222)

Mr. Frederek explained, "I'm probably too nice sometimes to them but you know, these kids are, I think I'm I'm I could probably enforce some rules more often but sometimes I think these kids you know they need a break sometimes too" (T6B, L34-36). Overall, Mr. Frederek accommodated PBS to ultimately prevent suspension both by knowing what 
was going on in his classroom and by balancing good learning with what students like to do. Thus, he and his students negotiated many potentially problematic situations successfully.

\section{Negotiation}

This section covers negotiations manifested as six subthemes: (a) teacher cognizance, (b) teacher patience, (c) students bothering each other, (d) students giving the teacher a hard time, (e) students exhibiting inappropriate negotiations, and (f) students preventing boredom. Negotiation occurs when participants make bids to alter the "normative infrastructure" (Carspecken, 1996, p. 116). A normative infrastructure develops when all participants help coordinate the culture, norms, and beliefs within an interactive setting. If negotiations are successful, a setting shift results in replacement of the previous normative infrastructure with a new one. An interactive setting, or normative infrastructure, occurred as Mr. Frederek and his students participated in continual negotiations of the classroom culture, norms, and values. Mr. Frederek's classroom culture, norms, and values simultaneously enabled him to maintain control of his classroom in order to teach Science content and prevent student suspension yet allow students to negotiate, enjoy the class, and learn something. Mr. Frederek was the authority figure in the classroom as exhibited in his stature and stoic appearance as well as in abrupt decision making when necessary to prevent exclusionary discipline.

\section{Teacher Cognizance}

Cognizance was exhibited in Mr. Frederek's negotiations as being in tune to students' profiles and histories, what was going on in class, and the degree to which his classroom culture, norms, and values were at risk of being jeopardized. He knew specific 
details about his students. For example during the interview, he elaborated about each student on the attendance/suspension list:

Pedro is gone, Juan is supposed to be here, I don't know where he is, he told me he was going to San Francisco for vacation on February 3, but then we have another new one that's no, they just sent him over to Mr. Wheyan last week, he was here once or twice .... Carlos is Venezuelan with Italian descent. He like talks about it. [Jack's] Cuban. Antonio is Cuban. Julio, I don't know where he is from. I think he's probably Cuban too but I'm not sure where he's from. He hasn't been here for a long time yet. [In my classes] it's probably $60 \%$ Hispanic, maybe 30 Black and maybe 10 White. ( T6B, L162-208)

Mr. Frederek was cognizant of students' backgrounds, histories, and idiosyncrasies and was willing to negotiate with them with extensive patience.

\section{Teacher Patience}

Patience was exhibited in how Mr. Frederek's negotiations often lasted so long and so rarely ended in a written referral that students referred to him as the "king of patience" (T4B, L130). Mr. Frederek responded, "Yeah, I have given too many breaks, [but] to send these students out all the time, it's not going to work, you have to find a balance" (T6B, L258-260). Mr. Frederek found a balance between maintaining control and exhibiting patience in his negotiations. His patient negotiations encouraged student negotiations, resulting in incremental setting shifts that momentarily appealed to both $\mathrm{Mr}$. Frederek and the students, thereby preventing student suspension in most cases. Yet, Mr. Frederek's patient negotiations also gave mixed messages to student negotiators who misjudged his limits. If a student negotiated setting shifts that could ultimately jeopardize Mr. Frederek's ultimate goals, Mr. Frederek patiently negotiated by varying his social distance and voice tone, which ranged from nearby quiet reminders to louder warnings and threats issued from a distance. In the event a student went too far with inappropriate 
negotiations, Mr. Frederek would threaten to call home, send him to CSI (detention) for a 15-minute breather, or sometimes call home unannounced. In this way, Mr. Frederek maintained control of the classroom so that he could accomplish his teaching goals and prevent student suspension.

Knowing Mr. Frederek's ultimate goals, students successfully initiated and negotiated the classroom culture, norms, and values for as long as possible, enjoyed class, and learned something in order to accomplish their own personal communication goals, maintain their image, but not get suspended. Both teacher and student negotiations were exhibited by varying talk and social distance. Mr. Frederek negotiated persistent studentinitiated negotiations of his classroom culture, norms, and values based on student profiles and histories in the following four situations.

\section{Students Bothering Each Other}

In the beginning of class one day, Mr. Frederek was thumbing through folders to distribute before his lecture. Huddled near one table, Carlos was quietly telling Jack and Antonio about a fight he had while removing his yellow uniform shirt to expose a plain black shirt. He said, "This shirt is too too bright" (T4B, L21). Antonio asked Carlos who he fought as Carlos made a punching move with his right arm. Jack argued, "You didn't fight nobody ... I was there" (T4B, L27). Mr. Frederek was busy helping Julio, silently sitting at a distant table, find his folder. Carlos hiked up his cheek and argued in return, "Yeah, I did, I scared the shit out of him, he went like, dude" (T4B, L32)! Jack turned around in his chair, pointed toward Antonio and asked, "Oh, it was him” (T4B, L33)? Antonio realized he was being insulted, stood up, and responded with a common vernacular phrase among students at The School, "Fuck you, nigga" (T4B, L34). They 
argued back and forth for 14 utterances until Antonio told Jack to turn around and shut up a couple of times. Jack repeated twice, "Say it again!" (T4B, L45, 47) to further agitate Antonio. Antonio refused loudly to which Mr. Frederek turned around from distributing supplies and said to Antonio, "Hey, watch your language" (T4B, L49)! Jack sided with Mr. Frederek and repeated, "Yeah, watch your language" (T4B, L50). Antonio thought Mr. Frederek had been unfair and yelled, "WHY ARE YOU TELLING ME WHEN HE'S THE ONE TURNING AROUND, BRO” (T4B, L51)? Mr. Frederek patiently stood in the middle of the tables with his hands in his pockets, looked at Antonio and said, "Yeah" (T4B, L52), as if pondering the situation.

In the meantime, Jack was impressed with Antonio and said, "Ohh, ohh, getting feisty, rough guy, tough guy" (T4B, L53) and Carlos agreed. Mr. Frederek warned, "Hey, hey, Jack" (T4B, L55) who quieted down. Appeased for the moment, Mr. Frederek gave Julio a folder he could use for the day and got out some pencils while the other three students continued talking about Antonio's prowess for 10 more utterances until Antonio realized they were again making fun of him. He yelled again, "YO, SHUT THE FUCK UP AND TURN AROUND” (T4B, L65)! Four utterances later, Mr. Frederek said, "Hey, leave each other alone" (T4B, L69), but Jack and Carlos continued bothering Antonio for six more utterances until Antonio walked toward the classroom door and threatened to leave the class, "Oh, naw, Frederek, I don't wanna be in this class, you call her and tell her to change my schedule. I don't want to be in this class anymore" (T4B, L76).

Carlos poked fun, "Haaah, haaah, he feels, he feels so baaad" (T4B, L77). Jack, afraid he would get in trouble, said, "Now he's gonna go to, now he's gonna go to the principal, maaan" (T4B, L78)! Carlos begged him to sit down. Mr. Frederek realized his 
goal of keeping students in a class they could enjoy and learn something was in jeopardy, walked over toward Antonio and said in a loud booming voice, "BE QUIET, BE QUIET, JUST LEAVE HIM ALONE" (T4B, L81)! Mr. Frederek tried convincing Antonio to stay but Antonio repeated, “No, man, no, dog, no, I don't want to stay no, I'm going home, I don't want to stay here" (T4B, L87). Carlos also tried to get Antonio to sit down, commenting, "Your rank your rank just went super down" (T4B, L91). Jack tried to get Antonio to sit down, adding, "I'mona! Get! In! Trouble” (T4B, L101)! Mr. Frederek called Jack's name to quiet him down and told Carlos, "Hey, hey. Carlos, you are the one that should be leaving this class" (T4B, L95). Mr. Frederek opened the classroom door and talked to the security outside, came back inside, and 20 utterances after Antonio first threatened to leave, he sat back down. Mr. Frederek followed him, touched his shoulder, and said, "OK!" T4B, L106). He then walked to the front of the room.

Mr. Frederek explained his negotiations based on the profiles and histories of the student and the situation. For example, he only talked to the security guard about Antonio, but did not send him next door to CSI because

I think I go outside to get some personnel and then he uh, things like this I don't notice. So what he did was, yeah, I think I did talk to somebody outside the door, and yeah, then I changed my mind. Because I wanted to have him in class, that issue was not big enough. I think the issue was not big enough. I think that it was not big enough for him to leave, I think that would cause more problems. (T6B, L264-288)

He weighed each issue and conducted his negotiations based on what was going on with the students at the time:

For some reason they're they're you know, that's, but they have had a fairly good relationship, you know before, the month before but there's always something coming up but I don't know what's been going on, I know that [Jack] was in boxing before and then he stopped I think you know, you get a lot of respect if 
you attend a boxing match, but if you stop you're like outside. Yeah, they have talked about it before. But uh, yeah, no I remember they came to me earlier in the day and there was some issue in another class. I don't know if you remember, I told you about this yeah, there was an issue because I moved I don't know but, I don't know what this issue is really, you know, they start, he has some friends too in school and there might be another class that he's with them and he's they're kind of picking on one another so and then another class group and he doesn't have anybody that you know. (T6B, L224-237)

Mr. Frederek was in tune to students' issues with each other and he negotiated accordingly. He added, "These students are so uh, they have this conflict one second you know and then the next minute it's completely different, like it never existed" (T6B, L250).

During the interview, Antonio remarked how they were just playing. He said:

We're talking about the fight that um, remember when he said he punched John, and then, Scorpion, John? It was not a fight, he was just making it up, you know, play, we was just playing. Carlos said he punched John, but he was playing. He didn't say it to like, he said it serious but he didn't do it. He was just making that up, and then I got up. [and Jack told him to] stop fucking around with him. Oh, you [Jack] said ghetto or something like that. And then Carlos was like, oohh. [Jack keeps saying, say it again] to me. (T6A, L49)

Jack added, "I'm just carrying on. And then you [Antonio] go oh, no, I don't want to be here no more. Ohohohhh" (T6A, L40, 43)! Antonio clarified, "But they got me mad, both of them" (T6A, L47). Jack added that he kept repeating, say it again, "for [Frederek] to get mad. We were playing around. He does get mad" (T6A, L55-59). Antonio agreed, "It's funny when he gets mad. Yeah, he like, his face is funny" (T6A, L60-62). Jack reiterated what he said when Antonio threatened to leave the class, "I thought he was going to go to the principal" (T6A, 67). Antonio clarified, "No, man, I wasn't" (T6A, L68). Jack added, "He was like, ohh, I want to go, all y'all are bothering me, yeah" (T6A, 
L70). Antonio explained, “But, I wasn't going to leave the class though, all I wanted to do was like get you guys to stop because I was getting mad already" (T6A, L72).

The students were in tune to Mr. Frederek's limits on their negotiations but sometimes misjudged his patience. Antonio remembered, "I went once to CSI . . . . for eating um, what's that thing called, oh sunflower seeds" (T6A, L152, 154). Jack also "went one time, I forgot for what" (T6A, L155, Jack). Antonio thought it was for bothering um, Duval .... because Duval got mad and then [Frederek] was like AHHH, you know how Duval gets mad and then he went back there and started knocking on the window and ... then Mr. Frederek. (T6A, L158)

Jack disagreed as to why Mr. Frederek got mad:

NO, he kicked me out because I had the book and then I dropped it and made it look I did it by accident and then he's like comeoncomeoncomeon don't do that again, and then he gives me a book and I go like that and I drop it again and he goes THAT'S IT, YOU'RE OUT! Then I got out, then I came back. (T6A, L159163)

Jack concluded by noting a flaw, or inconsistency, in the system that you return to class from CSI "when they want you to" (T6A, L166). Bothering each other often led to giving the teacher a hard time.

\section{Students Giving the Teacher a Hard Time}

The minute Antonio sat down, Carlos and Jack snickered but Mr. Frederek said very loudly, “HEY, JACK! LEAVE EACH OTHER ALONE, NOW! Don't-” (T4B, L109). Antonio glanced back and forth from Mr. Frederek to Jack who said, "I'm talking to him" (T4B, L111), pointing to Carlos. Mr. Frederek told him not to talk to Carlos either. Jack whispered, "Sweet man” (T4B, L113) under his breath. Mr. Frederek said, "That's enough" T4B, L114)! Jack repeated the comment to which Mr. Frederek yelled, "JACK! JACK” T4B, L116, 118)! Jack taunted Mr. Frederek again, “All right, let’s go. 
I'm ready" (T4B, L117)! Antonio thought he was being bothered again and told Jack to stop. Mr. Frederek said, “Don’t look at him, just be quiet, Jack, I will call your-" (T4B, L120). Jack looked left and right, laughed, chewed on a straw, and then conceded the negotiation as if he knew what Mr. Frederek was going to say and could not have the teacher call home or he would get into real trouble with, "All right right right" (T4B, L121). Mr. Frederek confirmed, "I will call your house" (T4B, L122). During the interview, Mr. Frederek explained that he threatened to call Jack's home based on previous, positive contact with them. He said, “[Jack's] okay, I think he knows what's wrong or right, I called his parents before and he- they are- and then he will stop. He's easier, much easier than Carlos" (T6B, L238).

Mr. Frederek began lecturing at the board and drawing about the topic of earthquakes. He said, “All right, okay, let's see, Now, we're working on earth- quakes” (T4B, L124). Carlos, who had opened up his notebook as if ready to copy, had his hood up maintaining the option to listen to his IPOD. He smirked as if he is making fun of Mr. Frederek's Swedish accent and choice of words, then laughed and mimicked quietly, "Okay, let's see" (T4B, L125). Jack tossed back his head and laughed. Then Carlos continued giving Mr. Frederek a hard time, "Hey, we been working on earthquakes since the beginning of the year. You don't know how to teach anything else" (T4B, L127)? Jack added, "Frederek, Frederek, Frederek, Frederek, Frederek" (T4B, L128). Mr. Frederek answered Jack, "Yes" (T4B, L129)? Jack said, "You're the king of patience. I give it to you" (T4B, L130).

Carlos put hands in his pockets, his hood still up, pulled out his IPOD player and tuned it to the song he preferred (T4B, L131). Mr. Frederek ignored Jack's comment and 
Carlos's tuning and began to lecture. Jack enunciated carefully and slowly, "What happened to the board? It's not erased properly" (T4B, L133). Mr. Frederek remarked that he knew it was dirty. Jack asked, "What" (T4B, L135)? Mr. Frederek lectured, "It's ok, ok, so we have these different fault lines, these plates, and when they intersect, we have fault lines" (T4B, L136). For the next 20 utterances, Mr. Frederek lectured, during which time he told Carlos to turn off the IPOD three times. Carlos denied twice that it was him, laid his head down to listen, and then said, "Damn, dog, I want my IPOD on" (T4B, L151), and "I wanna listen to him. Shit" (T4B, L155). He bothered Antonio again saying, "Come on, hoss. Come on" (T4B, L158, 160) when he got caught taking Antonio's notebook. Mr. Frederek told Antonio, “Don't worry about it” (T4B, L161). Jack and Carlos bothered Antonio again. Mr. Frederek said very loudly, "LEAVE HIM ALONE! THAT'S ENOUGH” (T4B, L165)!

Mr. Frederek patiently negotiated this scenario of students giving him a hard time. However, when they incessantly bothered Antonio, he responded with a very loud voice that he had had enough. Mr. Frederek explained that he patiently negotiated based on students' profiles and histories for several reasons. First, "Carlos, Carlos was picking oh him, Carlos picks on anybody, yeah. He's like a constant behavior problem, I called [his parents] and they didn't speak English. Yeah. I should probably call more" (T6B, L238242). Sometimes he tried

to think too when you see so many conflicts in the classroom they have to- they have to- you miss- you probably get some of these conflicts wrong, you probably support the wrong person you know, so I think that is the problem, you being in this classroom environment for day after day after day that's completely you know, and then you start to sometimes listen to these conflicts and you try to calm it down but if something like this kid here [Antonio]. I don't like them to to mess around with him because he doesn't do anything bad. So if when he might I went 
to talk to the administration about that issue so because, yeah, there was an issue before with him and Carlos in here, but he has caused some of these problems himself I'm sure so uh, so I'm not that, unless it's continuous, continuous, continuous then I'll take more notice of it but this is something that happens pretty much every class. Yeah, I try to talk to them but you know, it's interesting to see because it's something that you know, just it happens so frequently that you don't take too much notice of it. (T4B, L242-250)

Mr. Frederek tried to assess each situation accurately and protect the student who was getting harassed, but it was easy to become immune. He reflected further on students' profiles and histories at The School:

It's interesting that just one person can change the whole dynamics in the classroom and uh, the interactions. Antonio is not really doing anything right now. Antonio, he is the one that sits over there and he was really a good student when he first came here. He did everything. To be in this school for a long- for a longer period of time is not good. You shouldn't be, the purpose is to be sent back to your normal school. I think 18 weeks [time requirement] but it used to be 9 weeks which is much better, in 9 weeks you can keep up, you know, concentration, but after 18 weeks, it's uh, so many bad bad influences for a lot of kids. Sometimes I have to you know, try to encourage them by open up the textbook for them, and I can try something. (T6B, L290-294)

Mr. Frederek emphasized how one student can change the whole dynamics and after 18 weeks bad influences change students for the worse. He tries to encourage students.

During the interview watching the DVD, Antonio and Jack evaluated their negotiations related to giving the teacher a hard time. Jack commented, "I was whispering, 'Say it again, you're the king of patience.' It means he doesn't get mad. He doesn't get mad, he doesn't lose his temper” (T6A, L75-80). Antonio added, “To get him mad, you have to do something bad, really bad like" (T6A, L81). Jack interrupted, "Like make a hole in the wall" (T6A, L82). Antonio laughed and repeated, "Like making a hole in the wall" (T6A, L83). Jack clarified, "I didn't' do it, like. And then somebody like hit it too hard hit the wall and made a hole and he was like .... and then he walks away 
[shaking his head]" (T6A, L85-88). Antonio explained, "He's a good teacher, though, naw, he's patient. Yeah. All the other teachers wouldn't have handled it like that. They would have just kicked us out” (T6A, L91-94). Jack concluded, “After one little thing, you can't have fun" (T6A, L95).

The students were also very in tune to Mr. Frederek's nonverbal clues. For example, Antonio said during the interview:

He's [Frederek's] always scratching his ear .... Yeah, when he's teaching, everytime he's looking at the paper he goes sometimes he goes like this, when he can't take it no more, he just sits down and goes. Yeah, sometimes we start banging and the table just starts shaking. You [Jack], me, Carlos, and Israel. (T6A, L238-243, 282)

Jack clarified, "Yeah, we all [bang the table] at the same time. We all do it at the same time. We haven't done it [in Frederek's class]. We did it in Gomez's class" (T6A, L283290). Antonio and Jack laughed when Mr. Frederek got a loud voice on the DVD. Antonio said, "Oh, I like this part. And sometimes when ... he's talking, he sort of like randomly he just goes like WRWRWRWRAW, starts screaming" (T6A, L308). Jack added, "He raises his voice, he goes okay we're going to SEE THIS THING RIGHT HERE and I was like don't scream" (T6A, L309). Students were in tune to all teachers' limits when they gave them a hard time. They would usually judge and succumb to Mr. Frederek's patience limits successfully by his nonverbal behaviors. However, every now and then inappropriate negotiations occurred after Mr. Frederek lost his patience.

\section{Students Exhibiting Inappropriate Negotiations}

Immediately after Mr. Frederek had warned Carlos multiple times for various infractions of the classroom culture, norms, and values, Carlos smirked and looked at the board poised to take notes. He asked the following question in a serious tone as he copied 
the word faults, "Hey, what does it say there? Sluts? Like sluts" (T4B, L166)? Mr. Frederek had reached his limit with Carlos and responded, "You're out of here" (T4B, L167) and abruptly walked to the door to call security. Carlos held his left arm straight out walking toward the teacher and yelled, trying to stop Mr. Frederek from sending him out of the room. Then Carlos quickly took off his jacket, grabbed his yellow uniform shirt, and tried to put his shirt on quickly as if he would get into worse trouble with it off. Carlos yelled at Mr. Frederek as he struggled with his uniform shirt, YO, BUT WHAT DOES IT SAY? YOU DON'T KNOW HOW TO WRITE, DOG! HEY” (T4B, L164)! Then, he said in a lower tone, "I gotta put on my shirt" (T4B, L164). Finally, he repeated softly and then loudly, "What does it say there? WHAT DOES IT SAY THERE "(T4B, L164)?! As Mr. Frederek talked to security at the door, Carlos finished putting on his uniform shirt and used a common vernacular phrase, "What the fuck" (T4B, L166)? In the meantime, the other students faced forward in their chairs and remained silent.

Carlos relentlessly repeated, "Yo, what does it say there" (T4B, L169)? Mr. Frederek told him that the word was faults. Carlos held his pencil between both hands in front of him propped on the table, then pointed with one finger to the word on the board and replied, "That's fault? Are you serious? Faults" (T4B, L174)? Mr. Frederek responded, "Faults, you know, you are causing these problems, just be quiet ... If you don't understand what it says, write it down, but be quiet" (T4B, L175-177)! Carlos answered, "Oh, my God, how can I write it down if I don't know it says" (T4B, L179)? Mr. Frederek replied in a very loud voice, "YOU'RE OUT FROM HERE (T4B, L180)! Carlos held his head low, shook it, and said, “Go', dog, fuck” (T4B, L181). 
Mr. Frederek continued lecturing, "Yeah, you have these fault lines" (T4B, L182) just as the door opened again. Mr. Frederek said to the security guard, "I need Carlos out" (T4B, L183) and to Carlos, "Go now, go now" (T4B, L185). The security guard tried to hurry Carlos along, but Carlos kept writing and said, "Hold on, so it’s faults" (T4B, L188)? Then, he calmly and slowly stood up, pulled up his pants, fixed his shirt, got his jacket off the chair, fixed it, looked for arm holes, turned it right side out, and finally walked out with the security guard. The security guard told Carlos, "You can put it on outside, man, come on" (T4B, L190). Carlos responded courteously, "What's gonna be the difference" (T4B, L195)? After Carlos left the room, Jack asked Mr. Frederek seriously, "Why'd you kick him out" (T4B, L199)? Mr. Frederek answered, "Why? You know" (T4B, L200). Then Jack laughed, pointed once with his right arm, and said, "Why do you look at me with that face" (T4B, L201)? Antonio laughed and put his knees up against the table, and Julio silently texted. Mr. Frederek responded to Jack, "You too are the next" (T4B, L204). Jack wondered aloud, "Why" (T4B, L205)? Mr. Frederek answered, "Well, it's complication" (T4B, L206), and Jack waved his right hand, shook his head from side to side, smiled, and said, "I'll say it is" (T4B, L207). Mr. Frederek warned, "Jack, I will call your father" (T4B, L208) and then held up two textbooks to demonstrate faults. Jack said, "Naw, naw, naw, naw, naw, naw” (T4B, L209).

Mr. Frederek continued lecturing about fault lines, holding two textbooks to demonstrate, "So these fault lines, um, they are, they are, they- it's the plates touching each other, you know, the plates move past each other or crash into each other or move away from each other" (T4B, L210). He stacked the textbooks, then picked them up again and continued, "So they uh, they build up strength, and they moving, they moving 
about half an inch a year in some places so when they touch each other" (T4B, L210). Then he said, "Jack" (T4B, L210)? Jack looked up and said, "I didn’t say anything" (T4B, L211). Mr. Frederek continued lecturing and rubbing textbooks back and forth against each other, "Then they touch each other and then after a while the strength is building up and then they can't bend anymore and then they snap and you have this snap" (T4B, L212). Jack asked with feigned interest, "That's from an earthquake" (T4B, L213)?

During the interview, Jack said that at first he was just "giving him a hard time that's all" (T6A, L151) when he asked Mr. Frederek why he kicked Carlos out. He further explained why he asked a serious question after Mr. Frederek threatened to call his home, "I'm trying to look like I'm interested so he won't call my parents because he's sneaky, he does it without even telling you" (T6A, L193). He and Antonio explained why they did not want Mr. Frederek to call home and report problems in school. Watching the DVD, Jack confirmed, "[Frederek] said I'm calling your father again and I was like naw, naw, naw, naw [because my dad gets mad]" (T4B, L168). Antonio added:

I think it's worse if I go to CSI and then if I like I think it is worse if I I think it's worse that if Mr. Frederek calls my mom to like tell them, to like tell my mom that I'm behaving bad than like if I got CSI and then my mom knows about it. I think that it's worse when Mr. Frederek calls her, when the teachers call her. Yeah, cuz then, cuz they think like when when your parents like they're gonna be like naw uh, why's your teacher wasting his time when like calling me to tell like you're behaving bad when like you should be paying attention and he should be teaching. That's what my mom tells me. (T6A, L178-181)

Besides not wanting their parents called, Jack and Antonio further explained the negotiation between Mr. Frederek and Carlos from a student perspective. Antonio said, "The problem- the problem with Mr. Frederek is that you can't understand what he is 
saying or like his handwriting you can't understand” (T6A, L195). Jack agreed, “Oh-h, his handwriting is ba-a-a-d" (T6A, L196). Antonio added, "You can’t understand what he is saying like when he talks" (T6A, L197). Jack explained, "It gets me f-f-f-rustrated too, I'm trying to do the work and there's a word there that I can't copy I'm like what does that say and he takes long to tell you, and he goes, oh, oh, and I look at another word" (T6A, L199). Jack continued, “And I'm like oh, my God, what does that say” (T6A,

L201)? Antonio elaborated:

And he tells you like you- you ask him what is that word mean- not only what does it mean, but what does it say, but spell it for me and he tells you what it means and then he like spell it. (T6A, L203)

Jack continued, "No, oh, when he go, when you go oh, what does it say and he says a word and you can't understand him. Like he says plate and he says plaet or something like that" (T6A, L204-206). Antonio kept repeating, "Plaet. Plaet" (T6A, L207-209).

They both said, "Yeah, [he is from] Sweden" (T6A, L211).

Students insinuated a language issue during some unsuccessful negotiations. From

Mr. Frederek's perspective, however, Carlos is

a troublemaker 24-7 and he will try to get you know, he will try to create you know, I think there was something on the board that he said that he couldn't read it but then he translated it to something inappropriate, yeah, because it's just going to get worse and worse with him, it's there's not too much you can do when he gets started. Well, they are supposed to receive a pass. The watch the hallways and stuff like that. I thought that maybe there was something that that had led up to (undecipherable) asked him why. I remember it was something on the board and he translated it to something inappropriate and I knew that it was just going to continue and continue and continue and I think it was maybe, yeah, and also because he had just bothered him, but Carlos you know, he's a good student too but some days and this is the worst period of the day because it's after lunch and they are all [wound] up. (T6B, L264-288) 
Mr. Frederek believed at that point, the situation would only become worse and worse with Carlos based on his history with him. Mr. Frederek patiently negotiated students' inappropriate negotiations according to their profiles and histories and what was going on in class at the time. However, much of the time was spent negotiating students' interactions related to preventing boredom.

\section{Students Preventing Boredom}

Mr. Frederek lectured and provided worksheets for 224 more utterances, during which time students learned something but also initiated negotiations to prevent boredom. In turn, Mr. Frederek patiently negotiated. For example, Julio texted with his head bent while Mr. Frederek broke a pencil to demonstrate to the students "that when you have this, breaks and you have a release of energy that's what an earthquake is" (T4B, L220). Jack questioned the action, but then changed the subject and asked, "Frederek, are you going to give us the report cards" (T4B, L229)? Mr. Frederek answered that he would "in the last 10 minutes" (T4B, L232). Jack asked him if he could "see it really fast" (T4B, L233). Mr. Frederek honestly responded, "No, when I give it out, it's just going to be chaos" (T4B, L234). They argued back and forth and Jack promised that he would just look really fast, that he would copy, that he was already copying, and so on. However, Mr. Frederek held his ground and began lecturing again and drawing on the board about seismic waves.

Jack turned to Antonio and asked him about another student that had his bookbag. After Antonio got a pass to go get Jack's bookbag, Jack began tapping his pencil. Antonio returned with the bookbag, began listening to Mr. Frederek's lecture, and asked a serious question. For 16 utterances, Jack tapped his pencil and Mr. Frederek ignored 
him as he lectured. Then, Jack stood up, walked over to peer inside a box on the floor, and asked, "Frederek, what is that" (T4B, L270)? Mr. Frederek asked, "What" (T4B, L271)? Jack answered his own question, “That. Oh, they're just goggles? Oh” (T4B, L232). Mr. Frederek confirmed that they were goggles.

Jack sat back down and Carlos returned from CSI and said, “They didn't, uh, they sent me back over here" (T4B, L274). Mr. Frederek muttered that he must have not written a referral and told Carlos to sit down. Carlos immediately said, "Somethin' not right, dog" (T4B, L278). Mr. Frederek waited for a long pause as if to make sure Carlos was calmed down and then walked back to the overhead as if ready to talk again. $\mathrm{He}$ closed his hands together in front of himself, looked over to the screen, and walked back to board as if he needed to point as he talked for the students to understand. Then he went back over to the overhead, changed transparencies, and checked what it looked like on the screen. Then he turned out the light to make it easier to see the transparency and walked back to the overhead and began lecturing about the "reason we don't have too many of these earthquakes here in Florida" (T4B, L279). Students silently waited. Julio looked up, Antonio looked at the floor, Carlos laid his head on his chin and wrote, and Jack returned from the restroom.

Antonio then pointed to the overhead and asked if what he was pointing to was an earthquake. Mr. Frederek answered for 10 utterances at which point Jack started tapping his pencil again. Antonio asked questions about various continents and states for 10 more utterances, and Jack stopped tapping his pencil. Mr. Frederek then said, "Okay, this is a chart” (T4B, L309). Jack sarcastically interjected, “Ah! A chart” (T4B, L310)! Mr. Frederek ignored the comment and continued, "-showing you the major earthquakes 
that's happened in the United States" (T4B, L312). Jack looked at the chart and read that "over a hundred and fifty" (T4B, L316) happened in California. Mr. Frederek continued lecturing. Carlos announced, "You got one in Florida" (T4B, L318) to Jack's disbelief. Antonio and Julio were silent and looked disinterested. Mr. Frederek replied that the earthquake happened a year ago in the Gulf of Mexico. Jack repeated with another tone of disbelief, "Golf of Mexico in Florida? There's no Golf of Mexico in Florida" (T4B, L321, 326)! Mr. Frederek said it was on the other side of Florida and then passed out a map.

Mr. Frederek said, “Here's a map, of the United States, I'm going to give you, put out the number of earthquakes" (T4B, L330). Jack remained interested and conversed with Mr. Frederek for 13 more utterances until Mr. Frederek realized Carlos was singing and listening to his IPOD. He said loudly, "HEY! Hey, Carlos" (T4B, L339). Carlos pointed a finger at the teacher and nodded one time to the right as his finger swayed in the air as if he is on the same page as the teacher. Mr. Frederek called Carlos's name again and Carlos whispered, "Oh, my god. Yes. Yes" (T4B, L343), and he immediately started writing. Mr. Frederek reminded him, "Just calm down, mind your own business" (T4B, L344). Carlos repeated the directions. Mr. Frederek began looking for a better map in the textbook.

Meanwhile, Carlos changed his tone, "That's stupid, oh, yeah, I need that shit" (T4B, L347)! Jack turned sideways with his left arm on Carlos's table, leaned his head way down, then sat back up, held Carlos's backpack strap up as if reading some words and wanted to know what they meant. He asked, "What does that mean, on my way" (T4B, L348)? Carlos smiled at Jack and used his hand to talk as if he knew all about it 
and loved sharing this knowledge. He answered seriously, "On my way. If you listen to Iceberg, you'll see how it goes" (T4B, L349). Jack asked, "Oh, how does it go" (T4B, L350)? Carlos sang the words and nodded his head briefly. They discussed and sang the lyrics for 12 more utterances at which point Mr. Frederek came over to Jack's table to reiterate the directions. He talked while Jack sang the words and listened to the directions simultaneously. Mr. Frederek then discussed the directions with Carlos.

Jack began tapping his pencil again and asked courteously in slow elaborated syllables, "May I please have a Starburst" (T4B, L371, 373)? Mr. Frederek replied, "Yes, but get started" (T4B, L372). Carlos howled like a wolf and Jack turned around and dropped a wad of paper on Carlos's desk. They taunted each other with the wad of paper, tossed it back and forth, and sang blurbs of lyrics for 20 utterances. Flipping through a magazine, Jack repeated, “Mr. Frederek, may I please have a Starburst” (T4B, L394)? Mr. Frederek walked around and opened the textbook for each student, pointed, and said, "Here there's another map in this book that's actually better" (T4B, L397). Carlos complained with a common vernacular phrase, “Where the fuck's is Alabama? I don't like this stupid class, man" (T4B, L398). Ten utterances later, Mr. Frederek nonchalantly passed out the candy.

The students began bartering. Jack asked, "Can I have two" (T4B, L406)? Carlos asked, "Hey, anybody got pink" (T4B, L411)? As they bartered with each other, Carlos tried to get Julio to answer. He shook his head no, but Carlos argued, "Yeah, you do, I just saw it right now" (T4B, L419). Antonio preferred lollipops, and they argued about which was better. Mr. Frederek got a lollipop out of the drawer and gave it to Antonio. He said, "With Starbuust you get some ... energy" (T4B, L433, 435). Jack corrected Mr. 
Frederek's pronunciation of the word, "Starburst" (T4B, L434) and then told Mr.

Frederek what kind of candy he should get next time. Mr. Frederek stood in the front of the room with his hands in his pockets and looked at the students silently as if enjoying the peace and quiet while they ate their candy.

Mr. Frederek chose to ignore or minimize certain things that students did to prevent boredom, such as make fun of his accent and writing. He was casually resigned during the interview, "I don't know, they sometimes make fun of my accent. They talk about my writing all the time" (T6B, L262). Mr. Frederek also protected Julio and Antonio from students who bothered them too much out of boredom. For example, Julio silently texted during the majority of the class with no repercussions from Mr. Frederek. He minimized Julio's silence (and texting) by walking over to him if necessary. $\mathrm{He}$ commented, "When I walk up next to him he says something. He doesn't come forward and ask me something" (T4B, L298). Students commented about Julio's unyielding silence, “[Julio] wouldn't talk” (T4B, L97). Jack added, “He won’t ever say anything. He only says something once in a while to his other friends, but like. When he's with his other friends, he'll talk, but only a little bit" (T4B, L99-101). Antonio explained, "Yeah, but Carlos is bothering him too much. That's why he doesn't talk, cuz anything that he says-" (T4B, L103). Jack interrupted:

Ever since he came into the school, I don't know, somebody started bothering him so he's quiet because Carlos was talking to him so he can talk and be friendly but he don't want to and Carlos from there on said oh, I'm going to bother you and bother you and bother you until you start talking. (T4B, L104-106)

Antonio continued about how the same thing happened to him:

That happened to me, that's what Carlos did to me. Yeah, at the beginning of the year, I used to come in and try to do my work and then go to sleep. And they used 
to throw paper bombs at me and then one day I just got mad, threw one back and started cursing a lot and then whatever. (T6A, L108-112)

Preventing boredom commonly ended discord among students, but it also led to infractions of PBS rules and overstepping Mr. Frederek's patience limits even though students were cognizant of both. Jack repeated the PBS rule about phones, "You can't have them out, you can't have them out" (T4B, L121). Antonio added, "You can't use them in class" (T4B, L122). Jack elaborated, "You can have them in your pocket but not out, like the teacher can't see them. Yeah, he's [Julio] texting or whatever" (T6A, L123125). Jack explained further about preventing boredom. When asked why he went to look at the goggles, Jack said, "I was bored" (T6A, L245). When asked if they ever got to use the goggles, Antonio said, "Naw, [but] I would like that" (T4B, L247-249). Jack added that he would like to do "a project, yeah. Like open up a frog or something" (T4B, L250). Antonio elaborated, "Like when, like when it's like only like me, him, and the other kid, like the class is quiet. But it's boring, like" (T4B, L254).

Jack reflected on a larger scale, "But I understand why we don't have labs here, they can't trust us with them, yeah, most of us are like bad. We'd probably start throwing frogs at each other or something, somebody doing something stupid" T4B, L255-259). Antonio asked, "Like Carlos" (T4B, L262). Jack added, "He'll start slapping the thing or stomp them. Carlos is like a destroyer, he destroys everything" (T4B, L263-264)! Antonio agreed, "Yeah! I was, the other day I was at the computer in Ms. Gomez's class" (T4B, L266). Jack continued, “And he punches the screen” (T4B, L267)! Antonio said, "He just punches the screen for no reason! And then he just grabs the screen and goes like watch what is this" (T4B, L268)? Jack laughed, "Yeah, it was funny, it was funny 
though" (T4B, L270). Antonio continued, "He's an animal! He's an animal” (T4B, L271). Jack laughed, “He's like he's there looking, he's on My Space, he's looking and all of a sudden, Bong" (T4B, L272)! Antonio concluded, "I was like without him, this class would be bored. Without Carlos, this class would be bored" (T4B, L277).

The students talked about how they try to make Science less boring. Antonio said, "We tell [Mr. Frederek] to like we tell him to like take us outside one day you know, and do something and he like says no." (T4B, L293). Jack said, "Yeah, but you gotta understand that it's a science class, it's not PE" (T4B, L295). Antonio disagreed, "No, like we could test" (T4B, L297). Jack caught on, "We could see how hard we hit with the ball" (T4B, L298). Antonio added, "Yeah, like the speed of the ball, gravity, all of that. That's all science" (T4B, L300). Jack added, "Yeah, it's just so we could play, I'll tell him all of that. Tell him about velocity all of that" (T4B, L301-303). Antonio continued, "How long does the ball take to-" (T4B, L304). Jack said, "How far you can throw it (T4B, L305). Antonio concluded, "Yeah, that's all science" (T4B, L306).

Jack explained that he incessantly tapped his pencil because he "was bored" (T6A, L311). Mr. Frederek laughed when he explained why he ignored Jack's incessant pencil tapping during class, "Everything is relative you know, if there was five things, you know you can live with that [pencil tapping]. If it's completely quiet, then they start with that, then you notice it, but this much I can take" (T6B, L316-318). Other topics surfaced in the discussion of preventing boredom in class. Jack looked for things to do during class that interested him. He explained about looking back at Carlos's bookbag and thinking, "There's some things there's something on his bookbag that said on my way and I was like what does that mean? And Carlos was like it was a rap and I was like how 
does it go" (T4B, L311). Students also enjoyed when Jack used a play on words to give them a break during boring classes. He used the phrase say it again 12 times in a 20minute period of time to liven up the class. For example, he asked Mr. Frederek to "say it again" (T4B, L318) when he said Starbuust with a Swedish accent.

The students laughed. They also knew that they sometimes misjudged Mr.

Frederek's patient negotiations. Antonio remembered, "I was quiet for a little while, but then when I stop playing, I got mad like, like, I just forgot about everything and then I started talking again and then everything came back. We're talking about me leaving" (T4B, L127-131). Jack remembered the history of what happened when Carlos translated the word faults inappropriately:

Oh, we may be fucking ourselves. You're out, cuz we kept on, we were bothering him [Frederek] at the same time that he was using the phone and then there he got, that's it, he goes oh, is that sluts and then he got mad, see. And Carlos in previous classes before you did the camera, he would do moaning noises. (T4B, L132-142)

Mr. Frederek patiently negotiated but summarized how intense it is to have block scheduling of 90-minute classes with these students:

I think that the shorter periods are better but I think that maybe an hour every second day but even then I think it's every 40 minutes [everyday], yeah, I had that before, but not in this school, everyday for 45 minutes I think the block schedule's probably better and not meet every day, because it gets so it's so much we need a day you know away from each other because it's so intense with these kids, everyday see each other, I think it's better. (T6B, L370-374)

Mr. Frederek tried to end each day with the students on a positive note. He assessed the DVD, "I think we ended on a good note, that's what I think, but this is every day every class period. You're going to have some issues" (T6B, L388). 
Overall, Mr. Frederek and his students understood each other's goals in the classroom and negotiated successfully in most cases to prevent suspension.

\section{Summary}

This chapter presented a description of Classroom Three findings in three sections: (a) Classroom Three participants, (b) findings from interactive power analysis, and (c) themes from reconstructive data analysis. Mr. Frederek is a White Science teacher from Sweden who had no experience or knowledge of alternative education before becoming a teacher at The School. His Latino students were familiar with alternative education. Three types of power exhibited in Classroom Three were normative power, interactively established contracts, and coercive power; charm was not exhibited. Through a particular combination of these types of power, Mr. Frederek's and the students' roles meshed successfully. Mr. Frederek was a disciplinarian and aloof, yet he protected students from other students who bothered them too much and from system rules in order to prevent suspension and so that they could learn Science in as enjoyable manner as possible in this situation. Cultural power was somewhat in play but worked to the interests of the students in most cases, because students were allowed to resist classroom activities, validate their own identities, and challenge his authority as a teacher if they did not cross the line as far as bothering other students. Therefore, the tacitly understood interactively established contracts between Mr. Frederek and his students balanced out the normative and coercive power that were used to protect students' ultimate goals in class. Students believed he knew what he was teaching, patient, and supported them to ultimately learn something in Science and to prevent suspension. Therefore, they ultimately consented to Mr. Frederek's classroom norms and values. 
Three themes of social interaction (accommodation, conformism, and negotiation) emerged and combined in the following ways. Mr. Frederek and his students conformed to dominant ideology about teaching Science, such as teachers lecture to passive students, in order to maintain control of the classroom and prevent suspension. However, Mr. Frederek accommodated system constraints so that he could teach and students could learn, but he did not write referrals because of the political ramifications of losing the students in the system. In order to accomplish their similar goals, Mr. Frederek and the students negotiated with each other. Mr. Frederek was patient and cognizant of what was happening in classroom interactions even though he exhibited aloofness about them until someone was getting bothered too much. The students negotiated by bothering each other to a point (unless they misjudged), giving the teacher a hard time, and other antics to prevent boredom. Due to the high level of negotiations and accommodations on the teachers' part, students negotiated but rarely resisted Mr. Frederek's classroom norms. The tacit understanding of each other's goals in the classroom successfully accomplished negotiations and interactively established contracts in most cases to prevent suspension. Chapter 7 presents the findings from Classroom Four. In chapter 8, a comparison and synthesis of the findings is provided. Chapter 9 includes a summary of the study, responses to research questions, implications for teacher education, and recommendations for research and policy. 


\section{CHAPTER VII \\ DESCRIPTION OF CLASSROOM FOUR FINDINGS}

This chapter presents a description of Classroom Four findings in three sections based on the nine stages of data collection and analysis described in chapter 3 and illustrated in the Appendices: (a) Classroom Four participants, (b) findings from interactive power analysis and role analysis, and (c) themes from reconstructive data analysis. A summary concludes the chapter. Quotes are cited by transcript name (e.g., T6B), line number (e.g., L271), and participant pseudonyms when necessary (e.g., Mr. Glass).

\section{Classroom Four Participants}

Classroom Four was a Language Arts course with a teacher who frequently used exclusionary discipline. When asked how he became a teacher, Mr. Glass, a White male, said that he "wound up doing some teaching at the university level uh, long before I- I got into secondary education" (T6B, L2) after majoring in English literature but going into seminary and becoming a police officer first. After his police career ended, he retired and moved with his family to Ecuador. He elaborated:

The quickest job- the first job I- I obtained was as a teacher and it was with young people it was with $7^{\text {th }}$ and $8^{\text {th }}$ well actually it was with $7^{\text {th }}$ through $12^{\text {th }}$ graders and it was a horrible experience because I had no prior background in teaching children I had done previous teaching with adults, hah, and so it was kind of uh, baptism by fire. (T6B, L2)

Mr. Glass continued to teach children, attained a Masters of Arts, and

obviously got better and then when I began to understand pedagogy when I studied in educational courses, it made sense to me how to instruct young people, what to do in the classroom with with uh, children, with adolescents. (T6B, L4) 
He came to The School "by way of a sudden uh, transfer [after] happily teaching" (T6B, L6) in a public high school downtown for his first 5 years in the area. He explained:

I had a professional communications dispute with the principal and I had a decision to make on a moment's notice at the beginning of the 2006 school year whether I was going to stay or an opportunity with the then principal here who presented himself and said he needed an English teacher and I jumped, I took the offer to leave [the school downtown]. (T6B, L6)

After 3 years of teaching at The School, Mr. Glass maintained control of his classroom in order to teach literature in an "orderly climate" (T6B, L390) to students who "are forced to either adopt [his] expectations" (T6B, L12) or eventually get suspended from school.

Four students participated: Derrick, Roberto, Juan, and Green. Table 17 summarizes Classroom Four student participation.

Table 17

Classroom Four Student Participation

Name Observations Interview

Derrick $\quad$ x x x

Roberto $\quad \mathrm{x} x$

Juan $\quad \mathrm{x}$

Green $\quad$ X $\quad$ X

Derrick, an African American male, participated in all three observations and the interview. Roberto, a Latino, participated in the first two classroom observations only. He was absent for the last observation session and the interview for unknown reasons. Juan, a Latino, participated in the first classroom observation only; he was absent from school 
for the last two observations and the interview due to involvement in a fight. Green, an African American male, who also participated in Classroom One, participated in the last classroom observation and the interview, returning early from a 10-day suspension. Themes from the interactive power analysis and reconstructive data analysis are discussed next, followed by a summary.

\section{Findings from Interactive Power Analysis}

This section provides an explanation of the findings from interactive power analysis, including (a) types of power, (b) roles, and (c) cultural power. Interactive power analysis determines who has what kind of power and why and whose power defines interaction outcomes.

\section{Types of Power}

The researcher deductively looked for four types of power based on Carspecken's (1996) categories of interactively established power: (a) normative power, (b) coercive power, (c) interactively established contracts, and (d) charm. The next section defines and describes each type of power that existed in the order they were used.

\section{Normative Power}

This section covers normative power manifested as four subthemes: (a) announcements about skippers, (b) announcements about preparations, (c) details about testing, and (d) lecturing. Normative power is gained over others by virtue of one's position or status in the culture or society. Mr. Glass used his authoritative teacher status to claim normative power over his students in order to teach literature in an orderly climate to students who were forced to do as expected or eventually get suspended. Mr. Glass conducted normative power claims from behind the computer or pacing back and 
forth leisurely in front of the students, providing social distance. Mr. Glass's normative power was never in question since he used normative claims after students had already decided whether they would stay in class and comply with the classroom norms of waiting silently or quietly for Mr. Glass to conduct class. Normative power claims took up most of the class period.

Announcements about skippers. Announcements about skippers were used by Mr. Glass with normative claims describing in detail what he was doing to achieve his classroom goals and to solidify that he was in charge. For example, the cultural norm at this school was that teachers kept track of students and recorded their attendance properly on electronic sheets distributed daily and that students must be in class to get PBS points. Mr. Glass used normative power claims to announce that he was doing attendance first, making it clear that students must abide by PBS rules in his class. He announced that he was making phone calls to look for missing students with phrases such as I'm looking for skippers, I need them here, I'm going to find them.

Mr. Glass used normative power claims when he took attendance orally even though only two students were there, commenting sarcastically about one student who was in school but did not come to his class, naw, not here, got punched in the nose, and got in a fight. Mr. Glass blamed students for bad choices and implied that he could not make them come to class and that it was out of his hands. However, his normative comments insinuated that those who made good choices, such as coming to class and doing as expected once they got there were good students. Derrick and Roberto both consented to the claim and waited quietly for Mr. Glass. 
Finally, Mr. Glass also used normative power claims when he gave directives to students who could not come in the class since they did not get the consent form signed for the study. He directed the students knocking at the door with you cannot come in, before you go there, go next door and get ORF test done, tell her you're one of my students, she'll ORF you. Students consented to the claims and did as directed.

Announcements about preparations. Mr. Glass also made announcements about preparations for the lecture with gesturing, little eye contact, and normative claims, such as here's what we're going to do today, okay, today, we're going to start a new curriculum, this is how I do it, and this is what you do. He announced that he was passing out a play, setting up the overhead projector, and for students to look at it, let's go, and is that clear. Derrick consented, answering yeah. Roberto consented, moving his desk over to see better. Derrick confirmed that he accepted the normative claims by having his notebook and pencil out and ready on the desk. Roberto accepted the claim by sitting down, getting his point sheet out of his pocket.

Details about testing. Mr. Glass used normative power claims to give students details about testing that he conducts in his classroom. The cultural milieu was one of students who were poor students in the system so he would help them make a good grade and earn points to get back to regular school. He and students knew that this is not the typical way of taking a test. However, Derrick accepted the claim, fiddling with his pencil eraser. Roberto also accepted the claim with his chin on his hand. Mr. Glass announced authoritatively that students were not expected to do the test alone, that he would help. He stressed that he would do all he could to make it easy so they could make good grades. 
Students knew they would not make good grades the typical way teachers gave tests, so they played the normative power game to maintain identity, listening and following along. Mr. Glass announced in step-by-step fashion that he would give them a copy of the play and asked if they had something to write with and said you gotta write on it, and absolutely when students asked him if they were supposed to write on it. He announced that he was giving them copies so they had it in front of them with normative claims such as here's the whole play because the test is on whole play, I'll direct you toward where I think you can find the answers, I'll put the test up here, I'm going to use the overhead projector to help you guys take the test, and you shouldn't have problems since you took part of it as a quiz. Derrick consented by looking at the test and listening. Roberto consented by moving his jacket over and getting ready for the test.

Mr. Glass continued announcing I'm going to get my marker so that it helps us, and then asked normatively without expecting an answer, how do you want to do it, chronologically or getting a letter, either one that works for you, do you remember the answers to this stuff. Derrick consented with yeah, said chronologically but wasn't heard. Roberto consented with yeah but was not acknowledged. Mr. Glass also used normative power claims when he asked what the answer was and when students guessed the wrong letter replied with maybe it would help you if I said to you where in the act the event occurred. Derrick consented with guesses and agreement to maintain agency and identity. Roberto consented with guesses and agreement to maintain agency and identity.

Lecturing. Normative claims manifested as lecturing juxtaposed with continuous, repetitive, sing-song voice intonation and nonverbal gestures that did not come across as natural or believable, in context, to the researcher when compared to comments made 
during the interview about how these students could not learn the material he presented. For example, Mr. Glass interjected normative lecturing with such phrases as I'll give you a handout and I'll help you, or I was impressed with your knowledge on the test, pointing to the bulletin board showcasing the two tests on which he helped the students with the answers. He also interjected lecturing with directive normative statements such as the other kids who did not have the kind of preparation you did will take the test while you go with the researcher for the interview and now you're going to take notes, and Green, if you care to, will take notes and then we're going to work on a 10-question handout after lunch. His claims implied you listen to me, I'll help you later. Mr. Glass used lecturing to confirm that he was the helper of students who played the guessing game to get a grade once they had consented to stay in class. Mr. Glass used lecturing to confirm that students were silent guessers with a student identity of those who do not know much and have to have help to make good grades. Decisions were made by both teacher and student at the beginning of class as to whether they would stay or not. Once a student consented to stay, he also consented to being quiet, listening to the continuous rhythm of Mr. Glass's lecture voice, and playing the game of being helped by the teacher. Derrick consented. Green rejected with a last attempt to get out of class saying can I go get a pen from my uncle and then consented and accepted a pencil from Mr. Glass.

Interactively Established Contracts

Interactively established contracts are constructed by negotiations among participants, based on an implicit level of understanding, mutual consent, and equal communication input. Negotiations that lead to co-constructed interactively established contracts can be thought of as ongoing revisions on multiple drafts of a written paper 
passed back-and-forth between co-authors. It could go on indefinitely; co-authors may use varying amounts of charm, normative power, coercion, or a combination of techniques to sway the thinking for the final draft. However, the underlying ideas or meanings in the paper that are constantly being revised (albeit not necessarily face-toface) are what is viewed here as interactively established contracts.

In Classroom Four, Mr. Glass used interactively established contracts minimally with students in two situations: (a) checking attendance and (b) discussing points. While he checked attendance, Mr. Glass asked students in class if they had information about other students who were not there. If they responded with relevant information, he conversed with them to attain more details. For example, Derrick accepted the contract and said that he had seen one student earlier in another class. Roberto accepted the contract and gave Mr. Glass some information about the student who got into a fight. While making preparations for the lecture, Derrick said that it was point sheet day and he was going to collect his points. Mr. Glass conversed with Derrick about how he would help him.

Students also interactively established contracts with each other when Mr. Glass was busy checking attendance, consenting to the classroom norm. For example, at the same time that Derrick and Roberto were giving Mr. Glass information about skippers, they also talked quietly about the cops outside, referencing a counterculture that affirmed their opposition to school/cops with explicit talk about Black and White cops versus the students. Another day before class began and while Mr. Glass was on the phone checking for skippers, students talked quietly about big customers they sold to outside of school. 


\section{Coercive Power}

Coercive power is accomplished by forcing obedience after threatening punishment. Mr. Glass used coercive power to maintain an orderly teaching climate and force students to either adopt his expectations upon entrance into the classroom, which meant be quiet and do what he said, or get kicked out immediately. Students knew that Mr. Glass dictated the norms and defined the outcomes of their decisions. Therefore, they decided in advance whether to come into class ready to let him teach, come into class disruptively and get kicked out immediately, or skip class altogether. As a result, Mr. Glass often taught only one student. However, he wanted to make sure the four student participants were in class for the study, so he used coercive power claims to that end.

The cultural milieu during the study was that of a play with actors being rounded up and directed. The director, the teacher, promised the actors, the students, that if they stayed and played along, things would all go back to normal in a few days. The coercive power claims implied you stay for study and I'll not write you up. However, Green rejected the claims and decided to leave class before it began with the other students who did not belong. As Green got up to leave, Mr. Glass stated authoritatively do not go, just stay, sit! He continued with Green, come back in here and sit and goodbye to the other students. This verbal interaction was not natural, because Mr. Glass usually kicked troublemakers out before class officially started and did not beg them to stay. However, Mr. Glass was doing this for the study, and all students knew he would typically write Green a referral for this behavior. Mr. Glass acted atypical for the study and he openly stated the reasons in class and during the interview. 
Coercive power was exhibited when Mr. Glass directed disruptive students to leave or stay in his class. For example, when students knocked on the door to come into class unwarranted, Mr. Glass used a voice tone intended as a voice tone of charm but it came across as awkward sarcasm because the words implied punishment (i.e., goodbye). When Green wanted to leave class unwarranted, Mr. Glass used a voice tone intended as charm again but it came across as disconcerting sarcasm because the words implied punishment in a context of pleading (i.e, sit! stay! please). Green unwillingly consented.

Green said he did not want to stay and Mr. Glass argued with I really don't care what you want. Green rejected the claim again and argued that he did not want to be filmed, I don't want to stay here, and can I go to CSI. Students who did not belong in the class and were leaving but were Green's friends consented to Mr. Glass's claim by telling Green not to get suspended and to chill out and stay. When friends told him to stay, Green had reasons to do so because he was maintaining identity with them. Green tried to undermine the coercive agreement to stay one more time and then things would be back to normal. As Mr. Glass prepared for the lecture after Green finally consented to stay, Green rejected again with can I go to CSI, why not, and I don't want to be here. However, Mr. Glass would not allow him, saying no, I don't care what you want. By asking to go to CSI, Green resisted classroom norms and maintained his identity as a student who gets referred and suspended, he's comfortable there because he can be himself and not feel dumb in class. While Mr. Glass announced the test, he stated to Green, you will do work like Derrick and then Thursday it will be normal in here. This is the last videorecording session. Derrick consented, waited. 
Coercive sanctions of exclusionary discipline were always underlying but Mr. Glass did not want to kick anyone out this time because of the study; he needed bodies to do his part as a teacher participant. By laying the choice ultimately on Green, Mr. Glass could retain neutral enforcer through this interactive sequence and only by walking out the door would he write a referral and both knew that. With his friends' support, Green could maintain identity and stay one time. Green consented under duress and sat down; he asked to leave throwing the responsibility on Mr. Glass, who had the authority to let him leave. Green got mad when Mr. Glass did not take that authority for him. Green never conceded in getting Mr. Glass to agree to the interactively established contract of letting him go to CSI. Mr. Glass threw it back to Green with a different contract—stay once more and we'll be done with the study. Mr. Glass finally resorted to no, I don't care what you think, I'm the authority and you will stay or I will use the underlying coercive power I have to write you up.

The school imposed a tightly constrained student identity for the boys; they must meet 10 requirements on PBS, by each teacher's interpretation of it. Although not used explicitly, Mr. Glass would only recognize Green or any students in a validating way if Green played the school game of PBS. Green knew that Mr. Glass would treat him as the student he would help if he agreed to play the school/classroom game. Green also knew that trying to play the classroom/school game would give him the student social identity as an alternative education, behavioral problem student who was not able to learn the material like they do in regular schools. He would rather go to CSI where his social identity prospered, but agreed to stay because his friends suggested him to just stay here 
for today and not get suspended. He had little to defend himself with, so he complied just for that day.

Students come to The School because they were kicked out from regular school, and they have that held over them with the PBS system, referral, suspension route that is the ultimate end if they do not do what will keep them in class to which Mr. Glass upheld with his coercive power claims. In this case, mostly Black and Latino boys are here and they are requested to keep quiet in class and halls and to have their supplies (which they may not have money to buy), since most are from lower class and minority communities. Green may not interpret it in this way, but he only sees what he experiences, that he will not do well, so why try to play the game. Through his resistance, Green attached himself to the students who told him what to do at that point, stay so you don't get suspended, an interactively established contract between themselves. Staying meant being quiet in class. Derrick consented and waited silently. Green finally consented and was silent.

\section{Charm}

Charm is the use of one's personality to gain consent from others. Mr. Glass did not use charm to gain consent from his students. Table 18 summarizes types of power exhibited in Classroom Four. 
Table 18

Types of Power Exhibited in Classroom Four

Type of Power Exhibited

Normative

X

Coercive

$\mathrm{X}$

Interactively Established Contracts $\mathrm{x}$ (minimal)

Charm

Normative power, coercive power, and interactively established contracts were exhibited in Classroom Four; charm was not exhibited. The roles that participants exhibited are discussed next.

\section{Roles}

The researcher inductively found and named roles based on routine "interactive rhythms and sequences" (Carspecken, 1996, p. 137). Roles emerge as themes through complex ways of acting, which participants "recognize as having some unity" (Carspecken, 1996, p. 136). When participants recognize a role in play, they can predict, but not ensure, future actions. Table 19 provides a summary of Classroom Four participants' roles, followed by a description of teacher roles and student roles. 
Table 19

Classroom Four Participants’ Roles

Participants Roles

Teacher - Mr. Glass The teacher who is very busy with many things

The expert lecturer of great English literature

The teacher who helps his students get the right answer

The no-nonsense strict disciplinarian

The neutral enforcer of administrative rules

The Director in charge

Student - Derrick The quiet willing participant who wants his points

Student - Green The unwilling participant who is biding his time

Student - Roberto The very quiet participant who is not much trouble

\section{Teacher Roles}

The teacher exhibited six roles. First, Mr. Glass, the teacher with

helper/disciplinarian/expert side, let students know that if they agreed to stay in class at

the beginning, they would be helped through to make the grade and points since they

could not do it alone per the teacher's statements.

Second, Mr. Glass, the teacher who is very busy with many things, was often

combined with the Director role. This is not the same thing as multi-tasking like Mr.

Jenkins accomplished. While he directed, he was busy doing things one at a time (back-

to-back) such as taking attendance, setting up the overhead, finding supplies, talking on

the phone to find students or ask favors, and getting ready in general. Social distance was

accomplished during this role too by little eye contact or listening which discouraged

intimacy, including forms of disruptive behavior and challenges designed to gain 
teacher's attention or quick reactions. The teacher's importance was elevated while the students' importance was minimized in the entire situation from a tacit appeal to authority in this role. The important activities carried out by the teacher were not shared by the students, even though he did explain to them each step of what he was doing, even attendance. This provided greater control of the situation by the teacher in the classroom through this role.

Third, Mr. Glass played the role of the expert lecturer of great English literature during the director and busy roles. His expert knowledge distanced him from the students and lessened their role in the classroom, especially when combined with the helper role he used after lecture periods. He used this role to tacitly claim authority and tacitly claim their reciprocal agreement to sit, stay, and be quiet while he lectured. This was a very natural role because he was not interrupted and did not interrupt quiet students who looked like they were sleeping. The goal was quiet. When the phone rang or the door was knocked on, he could step away from the lecturer role with no interruptions and return immediately because of the tacit consent of the students.

Fourth, Mr. Glass, as the teacher who helps his students get the right answer, frequently helped his students out on tests and other written work. This role of helper was in direct contrast to the other roles he played and was combined with the underlying coercive power claim of not getting suspended that all in the room knew was the end result of both not being quiet and not staying in class. As a result of the students' tacit consent to Mr. Glass's requirement to be quiet and stay in class, he would help them to get a good grade since they could not do it alone, which he frequently told them. Students outwardly accepted the role as incompetent students who needed help when they stayed 
in the class. Most of the time, students would misbehave and get kicked out before class started. So those who chose to stay knew what they must do during the period. They played the school game in order to get their points in order to claim the Friday activity. They could go back to their regular school after 18 weeks of good behavior, documented by sufficient points, less than two written referrals, or not getting suspended during that time. Mr. Glass believed that the students could not learn the material but wanted to teach it because he was good at it, so he helped them with the answers so they could have at least heard of great authors.

Fifth, in the no-nonsense strict disciplinarian role, Mr. Glass elevated his authority to get to stay in class so he could help the researcher conduct the study with all the participants. Typically, he said he used this role to get the students out who were going to be problems during class. In using this role to make a student stay in class who did not want to stay, it was awkward. "Sit, stay" sounded awkward.

Sixth, when Mr. Glass took on the neutral enforcer of administrative rules role, he disassociated himself from the school rules for the classroom but claimed the role of enforcer for them. He tacitly claimed this role to emphasize the end result of choices students made about not coming to class, skipping, or getting into fights. The tacit claim to authority rested on the idea that the school PBS/suspension/referral rules were not debatable, necessary, and logical and that Mr. Glass as teacher must enforce them even though he could not make students make the right choice related to them. The fault lies in the students' poor choices.

Seventh, Mr. Glass, as the Director in charge role, explicitly explained the parts he and students would play or were playing during the particular section of the class at 
that time. When in this role, Mr. Glass already tacitly claimed and tacitly received the consent of the students to stay and sit in class and validated their consent by acknowledging their part during the process. During this role, Mr. Glass spoke to the students with little eye contact, providing social distance between them.

\section{Student Roles}

Derrick, as the quiet willing participant who wants his points, agreed tacitly to the teacher's claims by sitting down and waiting for the teacher to begin class and going through the motions to get the points and grades he needed to accomplish the goal of not getting suspended and returning to regular school. He gave the teacher information when asked about another student which elevated his importance in the classroom; during class lecture, though, students' importance was minimized. Participation consisted of guessing answers while Mr. Glass provided clues.

Green, as the unwilling participant who is biding his time, openly challenged $\mathrm{Mr}$. Glass's rules for coming in and playing the school game of being quiet and letting the teacher claim authority in the classroom. Green positioned himself with the students who get kicked out to CSI by asking to go there but Mr. Glass wanted him to stay as a participant instead. Mr. Glass made a contractual agreement and Green consented but backgrounded by his friends' support not to get suspended. He also asked to go get paper from his uncle who worked in the school, but Mr. Glass provided paper and pen for him.

Roberto, as the very quiet participant who is not much trouble, consented to Mr. Glass's rules by coming in, sitting down, being quiet, guessing when asked. 


\section{Cultural Power}

Cultural power was discovered through hermeneutic inferencing, or taking the position of participants in order to understand the basis of their claims. Cultural power exists when the norm or value claimed does not represent mutual interests of the entire group. In this classroom, cultural power was at play, recognizable when Mr. Glass's claims failed to win recognition or fulfill a universal interest to all participants in the classroom. For example, students would momentarily stop their quiet conversations among each other to provide information to Mr. Glass in his endeavor to find skippers while taking attendance. However, they did not appear to condone his implicit references to skippers as students who make bad choices. They did not seem to be listening and did not interject any words of agreement when Mr. Glass said sarcastically about the students who were skipping (sounding as if he is making fun of them, degrading them, or commenting on behavior he expected from them) that he's never here or he's never around. Instead, the students returned to their own quiet conversation (while Mr. Glass was busy doing other things) about whether the cops at The School were White or Black.

Cultural power was at play when Green wanted to leave the class and was not allowed to, which distorted the typical protocol in this classroom. Mr. Glass usually kicked students out immediately when they disrupted, but when he told Green to stay, verbal and nonverbal interactions did not appear or sound genuine. Mr. Glass did not want any disruptive student in class normally, but he tried to get Green to stay for the study. This was not a natural role for Mr. Glass. The students knew this, so some friends who were leaving this classroom to go their own class, told Green to stay so he would not get suspended. Green consented but explicitly let it be known that he did not want to be 
on camera and asked to go to CSI. Mr. Glass said no, and that he did not care what Green wanted at this point. He told him to sit, stay in a pretentious, or hollow, tone. Then Mr. Glass made the claim that this would all be back to normal after one more day so we would do this one more time. He built a coercive contract with them to agree to staying for the study with him. Normal meant he would kick students out or not beg them to stay in class in his natural roles as teacher at The School.

Once Green settled down, his demeanor showed that cultural power was at play, that the normative claims were a sham. He and other students were quiet and played the game of school according to the interests of the teacher. Mr. Glass claimed that we will do this and that and walked them through each step. They played the game. When he checked attendance, Derrick and Roberto talked about the cops that were on campus, what color (race) they were and so on. This was of interest to them, and they knew they could talk quietly at this point because the teacher was busy doing the attendance. The teacher recognized himself as a good record keeper, keeping care of student status for the administration, kicking them out when their behavior conflicted with his teaching. He recognized himself as an excellent English literature teacher and even though he said that the students could not really learn the material, he wanted to at least let them hear about these great writers. Since Mr. Glass knew that they could not learn the material, he helped them get answers on the quiz so they could pass the course.

The students did not recognize themselves as good students by interacting with the lecture. They recognized themselves as good students in this class by guessing and putting the right answer on the test. They recognized themselves as good students when they stayed in class. They recognized their own interests when they talked about the cops, 
when Green asked to go to CSI and when Green said he did not want to be on camera. When one cannot fully recognize one's self, one's own interests, within an articulated norm/value, then one can only consent to this norm/value because of the play of power.

Students consented to the norms/values of the teacher's setting because of the cultural power of The School enforced by the teacher. Once or twice students told Mr. Glass what they knew about other students' whereabouts. Otherwise, they said nothing at all except one word guesses to the test questions. Mr. Glass asked questions but did not listen to their answers. When he complimented their knowledge on the test stapled to the bulletin board, it was a sham; the students knew that he gave them the answers. They did not realize their papers were on the bulletin board even after he pointed to them. Mr. Glass universally claimed to students how he taught and how they would learn, with his help. Students played the role of students who needed help because of the play of power from the teacher.

Cultural power is the falseness of universal claims. Internal assessment of universalized claims was possible because of the researcher's familiarity with both cultural realms, the students' and the teacher's. Cultural power was at play by how the participants' cultural realms were incongruently juxtaposed. For example, the PBS rules were strictly enforced by Mr. Glass who recognized himself as an enforcer of the rules for the benefit of teaching literature. Typically, if the students did not consent immediately he usually kicked them out. However, this was not the case on the day Mr. Glass told Green, who was being disruptive, to stay for the study. He announced multiple times that participating in the study was the right thing to do for all students and how many times were left. He also gave out pencils during the study but usually wrote a 
referral or docked points if students were not prepared. Consent to the teacher's claims was not natural during the study, but a sham; students consented because of the cultural power in play of referral/suspension and not getting back to regular school. They were used to this pattern in the classroom, but during the study the typical pattern of kicking kids out was conducted for different reasons. The ones who did not bring back signed forms were the one who would be kicked out during the study.

The interests of the teacher were recognized as those of walking the researcher and guys through every step of the play so to speak. Students were actors and Mr. Glass was the director/main character. The interests of the teacher were to have a classroom with students in it that were going to follow along.

Themes from Reconstructive Data Analysis

This section covers three themes that emerged from inductive reconstructive data analysis: (a) conformism, (b) accommodation, and (c) resistance. Because the emerging themes aligned with Giroux's (2001) theory of resistance in education, his terminology was used. Table 20 provides a summary of the findings from Classroom Four reconstructive data analysis. 
Table 20

Classroom Four Reconstructive Data Analysis

Theme Part. Manifestation Purpose

$\begin{array}{llll}\text { Conformism } & \mathrm{T} & \begin{array}{l}\text { Banking education } \\ \text { Cultural deficit orientation }\end{array} & \begin{array}{l}\text { Maintain control } \\ \text { Teach in orderly climate } \\ \text { Enforce expectations } \\ \text { Inevitable suspension }\end{array} \\ \text { Accommodation } & \mathrm{T} & \text { Authority figure } & \begin{array}{l}\text { Maintain control } \\ \text { Teach in orderly climate } \\ \text { Enforce expectations } \\ \text { Inevitable suspension }\end{array} \\ \text { Accommodation } & \mathrm{S} & \text { Compliant subjects } & \begin{array}{l}\text { Follow classroom norms } \\ \text { Communication goals }\end{array} \\ & & \mathrm{S} & \begin{array}{l}\text { Critique } \\ \text { Self-reflection } \\ \text { Struggles }\end{array} \\ \text { Resistance } & & \begin{array}{l}\text { Communicate } \\ \text { Maintain identity } \\ \text { Maintain agency }\end{array}\end{array}$

Note: $\mathrm{T}=$ Teacher; $\mathrm{S}=$ Student; Part. $=$ Participant.

Table 20 denotes three themes that emerged from reconstructive data analysis. Teacher conformism to the dominant ideology of Language Arts was manifested as two subthemes: (a) banking education and (b) cultural deficit orientation. Accommodation was manifested as two subthemes: (a) teacher as authority figure and (b) students as compliant subjects. Student resistance was manifested as three subthemes: (a) critique, (b) self-reflection, and (c) struggles. Teacher conformism and accommodation were manifested to maintain control, teach in an orderly climate, and enforce expectations and inevitable suspension. Student accommodation was manifested to follow classroom 
norms and accomplish communication goals. Student resistance was manifested to communicate and maintain identity and agency.

\section{Conformism}

Based on his philosophy of schooling, Mr. Glass's conformism manifested in this classroom as two subthemes: (a) banking education and (b) a cultural deficit orientation. Conformism is the opposite of resistance, which is an emancipation-seeking behavior. Rather, conformism is behavior that simultaneously suppresses social contradictions and unites with dominant ideology (Giroux, 2001). Mr. Glass exhibited conformism to the dominant ideology in education pertaining to the structure of schooling and how it related to the cultural deficits of his alternative education students. For Mr. Glass and his students, schooling

is structured in a way that if you don't uh, conform to certain group rules, then you get put out of the class and you get put out of the class several times and you'll wind up with an out of school suspension and enough of those and you'll be withdrawn. (T6B, L12)

\section{Banking Education}

In dominant ideology, banking education relies on an authority figure that provides the requisite knowledge, predetermined curricula, and nonnegotiable conditions to compliant subjects who regurgitate the knowledge and actions expected in the preset conditions for learning. In this classroom, Mr. Glass was the authority figure who possessed the necessary knowledge to "bring the positive energy each day to the subject that I teach, I love to teach what I do which is literature" (T6B, L10). He elaborated:

You must be thoroughly comfortable with uh, an author like Shakespeare, my feeling is if I'm going to teach them what I am good at, which is literature . . . . I know enough writers of short stories, poetry, and and novels uh, uh, that uh, I I very rarely go into the $7^{\text {th }}$ grade or $9^{\text {th }}$ grade for and except for having enough 
books a classroom set, the $9^{\text {th }}$ grade text has the Odyssey by Homer so I'll use the textbook for that or a Shakespearean play where I don't have the ability to make copies. (T6B, L26)

Mr. Glass was thoroughly comfortable teaching literature, and was subsequently, the authority figure who predetermined the curricula for his alternative education students.

He explained:

I mean I teach, again, maybe strangely, the same writers or authors that I would teach an AP class of $11^{\text {th }}$ and $12^{\text {th }}$ graders and now we're talking about $7^{\text {th }}, 8^{\text {th }}$, and $9^{\text {th }}$ graders. The $7^{\text {th }}$ grade just finished a unit on Shakespeare's The Merchant of Venice. (T6B, L26)

In order to teach alternative education students the same authors as an AP class, Mr.

Glass was also the authority figure who set up the conditions for learning. He confirmed these conditions during the interview when he saw Derrick working on the assignment:

The conditions have been set to allow him to be successful and he is following up on that, he is not disengaged from what we are doing which is important to me as I say or I've said before I'm here in my own understanding of my purpose to help a student to increase the self-confidence um their their um the famous psychologist uh, from Stamford, uh, well self-regulated behavior is what I'm trying to get at that when a person feels capable they tend to believe that they're going to be successful in what they do. When they have doubts about their capabilities, uh, they tend not to perform well. So I'm I'm trying to get the kids to believe in themselves that they can do academic work. Bandura is the psychologist. (T6B, L78)

As the authority figure, Mr. Glass set the conditions to allow students to be successful.

He required engaged, self-regulated behavior before he began teaching his content.

However, Mr. Glass stressed that alternative education students only come to school to socialize, they come to hang out, they do not come with a mindset to study, or or to do um, um, um, conceptual academic work, they do not like it when um, ask them to think they love busywork or what I would call as um handouts that do that do not require them to do much more than fill in the blanks, copy material from the board, that they consider I did my work I should get an A or I should get a B uh, but it doesn't use their mind, it doesn't it doesn't exercise their thinking skills, uh, and so I don't do much of that kind of stuff and that throws them off too 
because a lot of teachers give in in subtle ways. Ah, this is my personal opinion, obviously, to this entitlement mentality, that that that the kid or the student can dictate to you what they will do and how they will do it and I don't accept that so they then are forced to either adopt my expectations or fight me on it and a lot of kids will choose to fight me on it and so we will wind up with kids going to detention or CSI because they are preventing other students from learning. So I I don't from the student vantage point, I see them coming here because they are required to go to school. Oftentimes I've said uh, in the 3 years I've been here, why don't you go to the movies, why don't you go to the beach, why do you come to school? You don't like to be here. You are uncomfortable about being here, but my parents make me go, or the law makes me go. (T6B, L12)

He had to force them to adopt his expectations. In order to determine which students were compliant to his expectations on a given day, Mr. Glass spent a great deal of time tracking students' whereabouts, progress, and attitudes. While watching the DVD during the interview, Mr. Glass noticed the amount of time he spent tracking down students:

As you can see, as we view this together, it takes me um, too much time, I'm selfcritiquing here, too much time to get them on point with what we're doing, first, we were trying to find out if he was coming, if you, and so Derrick is just sitting there and he's waiting for me because I haven't given him the test and I'm telling him that we're going to take the test, so I would be critiqued if an administrator were observing this tape as to well, Mr. Mr. Glass, all of this lost learning time has gone down before you actually get into what you're doing. (T6B, L56)

Mr. Glass critiqued himself on the lost learning time for Derrick, the only student in attendance at this point, who waited for him to start the academic portion of the class.

While evaluating student compliance, Mr. Glass verbalized his intentions to everyone in the classroom who waited for class to begin. For example while seated at his computer/desk at the beginning of class, Mr. Glass said from a distance:

I'm gonna um, take a few moments ... I'm going to try to find these two .... Okay I don't want them skipping, I need them in here but I don't know what they're doing, I don't where they are, but I'm going to FIND 'EM if I can, and that may take me a moment. (T4B, L2) 
While Mr. Glass searched, Derrick waited silently at his desk until Roberto arrived. Mr. Glass picked up the phone and called another classroom and then CSI to find the two missing students. He stated openly, "I'm told they're here in school, but uh, because of a study going on in my classroom, some videotaping, I need them in my classroom so I hope they are not skipping and that's why I'm trying to find them" (T4B, L10). Neither faculty member had seen the missing students. Mr. Glass said loudly after hanging up the phone, "Hokay" (T4B, L11) and then checked again on the computer. He announced, "I used to be able to pull their schedules up, and my electronic gradebook is not helping me do that" (T4B, L13). Roberto arrived, and Mr. Glass completed dictation of the attendance check. Then, Mr. Glass called Derrick and Roberto's attention to the assignment for that day. Derrick and Roberto had been talking quietly but stopped to listen to Mr. Glass give directions. They were his compliant students.

\section{Cultural Deficit Orientation}

Once Mr. Glass had compliant students identified and in class, he conducted his class as the authority figure, teaching Language Arts to alternative education students from a cultural deficit orientation. A cultural deficit orientation manifests when students are compared and taught according to their level of compliance with the dominant structure of schooling. In our society, school is structured largely and unwaveringly as a middle-class endeavor. As a result, when students do not comply with dominant middleclass ideology, their intelligence, behavior, and family/community are stereotypically blamed for being culturally deficient, and low expectations prevail. Mr. Glass explained from a cultural deficit orientation how regular education students differ from alternative education students: 
Teaching in alternative ed is different in many ways from regular schools. Uh, uh, first of all, the students in this setting have been uh removed from, expelled from their regular school, so they obviously are coming here uh, not in a normal situation. They have uh had uh fights or they have been involved in drugs, or they've had problems with truancy, so they are students who have certain either emotional dysfunctionalities or or learning differences, learning problems and they are not motivated to come to school in general so they are restless in the classroom. They are students of of uh to whatever degree of impulsivity. They will not acknowledge that they're part of a group so everything revolves around the individual kid and you have to teach here on any given day and every day with a uh much greater degree of patience than you have in the regular school setting. You're not going to have kids who will give you the benefit of the doubt in accepting normal classroom behavior standards. Um, they will push you, they will test you by horseplay, throwing paper balls at each other uhh, sleeping, umm, all sorts of disengaged and disruptive behaviors.

Mr. Glass explained that his students were not motivated to come to school and that their emotional dysfuntionalities and learning problems led to impulsivity and restlessness in class. He therefore believed, "There is a very close connection in my view between behavior modification and learning gains or roles in academic achievement" (T6B, L14).

From a cultural deficit orientation, he explained:

If the student has lots of anger management issues and a very low tolerance for conflict, then they are not going to do well academically because they are always going to get into some type of escalating uh, conflict situation, whereas if they learn that's not the way to handle pressure, it's better to deescalate it and uh, learn how to focus on what's my self interest to learn and to not to give in to the uh, cultural peer pressure amongst young men of that have not done well in school or reluctant readers, you know, several grade levels behind by the time they're adolescents and then they pull anybody down who stands out as somebody who wants to learn. And this negative peer pressure is rampant in the culture, it's not just in [The School], so students who do try to do their best are isolated, they are insulted, they are harassed, they are bullied um, um, and uh, just the opposite goes on in middle and upper class cultural environments where kids are pushed very hard towards academic achievement and so the norm is that everybody's trying to be the top student in the class as opposed to in an alternative ed situation like this where everybody is pulling down anybody who stands out as a nonconformist. And so you see, again, that's why it's very hard on students to to to actually get a decent education. 
Mr. Glass stressed that his students have anger management problems, come from a culture rampant with negative peer pressure, and are reluctant readers who have not done well in school. As a result, they do not push toward academic achievement like students do who are from middle and upper class environments.

During class, Mr. Glass commented loudly on his students' cultural deficits. For example while Mr. Glass was looking for skippers, Derrick suddenly remembered that Roberto had been in woodshop with him a few minutes ago. Roberto came in shortly thereafter and said that Juan had gotten into a fight. A security guard opened the door to report that Juan got punched in the nose and went home. Mr. Glass responded loudly with resigned sarcasm as he continued to check attendance, "He got into a fight . . . he got punched in the nose" (T4B, L33, 37). Then Mr. Glass announced to the two students in class, “All right, guys, here's what we're gonna do" (T4B, L39), prepped them not to fear the upcoming test since he would be helping them, and talked to Derrick about his point sheet before dictating aloud:

Let me take the attendance first . . . let me take the attendance and then we'll get started. Umm, okay, uh, Joseph's here, Paulo naw, he's not around. Exante's here, Derrick, Stephan not here, Green's never here. Elvis never comes to class, although I see him in Ms. Rubio's class in period seven. (T4B, L55)

Mr. Glass elaborated on who was not around, never here, and never comes to his class. Then he highlighted deficient decision-making with additional comments such as, "but he just decides to skip. And Juan got punched in the nose, so he won't be here" (T4B, L57).

Mr. Glass reasoned students' lack of involvement or engagement in class through cultural deficit orientation that blames students' backgrounds for their lack of success. He 
explained during the interview that even though he brings positive energy to the subject

he loves to teach, he rarely

get[s] a positive reaction from students because I'm always pushing them to do more work or to participate, read aloud, um, use their minds in analyzing and interpreting the literature through the writing activities I have, so I make students through the way I teach generally with their backgrounds uncomfortable and so I am naturally provoking the kinds of conversa confrontations I've just described.

He reasoned that students with their backgrounds were uncomfortable when pushed to do more work. He further explained that when they are reading "say Huckleberry Finn or a Shakespearean play, and these kids are restless, they lose track of the plot, they uh start having sidebar conversations, and then I have to stop and ask them to get reengaged" (T6B, L10). He referred to this type of teaching as a

herky jerky kind of teaching but again I'm used to it, I expect it, uh, on rare occasions and it does happen I will get 60,70, or even a whole period's worth of of cooperation out of the 5 or 6 or 4 however many students I have and the class will be a wow experience I mean where that would be the norm in regular school where kids could uh, because in the regular school there a lot more students who dream about going into higher education. These kids do not dream about going into higher education and I see a special role working over here in trying to um, um, get that candle lit in some students because all of our students are bright and I think that if they were able to develop more organized study habits and could understand um normal behaviors in a classroom, they would go on and do fine in higher education, but but they need practice in those types of behaviors and I try to model my class and and my expectations along those lines and so then there's this clash of you know, confrontation because kids are like I'm not going to college and I don't I I don't like the subject matter you teach, it's boring and all we do is the same thing everyday. (T6B, L10)

He believed that these kids did not dream of going into higher education. Therefore, he modeled his classroom expectations so students could practice the behaviors needed to go to college but conflicts ensued. He reasoned further:

We read and we write. Those are two language arts or English skills that are universal and need to be practiced. A lot of other things need to be practiced too but you're teaching on several levels here you're not just teaching content, you're 
teaching kids as I say, how to develop more self-confidence in themselves to be able to do academic work. And that takes a lot of psychology, a lot of strategies, to encourage kids but at the same time also get them to understand that they're not entitled, they don't have the right to prevent other kids from learning and so you uh work a lot on uh uh positive behavioral support rules! (T6B, L10)

A conflict existed in his mind about how to encourage kids but at the same time to get them to understand that they were not entitled. He concluded from a cultural deficit orientation:

most of the kids in the class couldn't tell you the plot of the play, or uh, even a part of it ... some of them may well find the experience of Shakespeare to be interesting to them, to be something unusual which most teachers of English don't want to work with because it is difficult. (T6B, L26)

Mr. Glass reasoned that even though his students could not remember the plot of a play as difficult as Shakespeare, just having the experience of Shakespeare may be interesting and worthwhile for them.

Mr. Glass explained other aspects of teaching alternative education students from a cultural deficit orientation. For example, he said that a class size as small as one student is the norm for this "special population" (T6B, L52) at The School. "The majority of [these students] maybe even a high percentage of them have probation officers, they have been in trouble in their neighborhoods uh, or with the school police and they have experienced the uh juvenile justice system here" (T6B, L40). Mr. Glass explained from a cultural deficit orientation how and why he lowered his grading expectations to combine participation, effort, and substantive content:

I used to weight grading when I was at [the regular high school downtown] and I would uh, pass out a syllabus to my classes uh, and in it would be the grading scale uh, uh, which basically uh, A's 4, B's 3, C's 2 , D's 1 or or on a percentage basis, A's 90-100, B's 80-89, C's 70-79, etc, um, and then I would weight certain categories, for example, tests would be worth $15 \%, 20 \%$, tests and quizzes, um, a project during the quarter might be worth $20 \%$, um, participation $10 \%$, homework 
$10 \%$ in other words, I would establish enough categories to come up with a $100 \%$ system. Um, here I don't weight grades at all, I don't give homework which is surprising I guess, but I've found trying to do that is a disaster, students just don't do it and so you're penalizing them more than they need to be penalized in my opinion so what I do is each class every day I will have writing associated with the lesson and the writing will be related to the literature and so I will give a grade every day in every class to every student who shows up and I will see how much effort and participation they put in I'm not so picky on did they get all the answers on the reading analysis correct.

He found that giving homework was a disaster, because students did not do it. $\mathrm{He}$ explained how he assesses participation and effort instead:

If they showed me they were trying as best they could to do the work, they'll probably get a B and if and if they get most of the work correct, they get an A, I am more interested in their participative attitude, their effort than I am in how uh, uh, intellectually capable they are because so many of them have uh, these different degrees of learning problems so it is very difficult for them to do the kinds of intellectual work with the great writers I teach. (T6B, L26-28)

After disastrous results, he had become less picky on providing a syllabus, weighting grades, grading tests, and assigning homework. Mr. Glass's philosophy of grading and assessment had graphically changed since he had experienced teaching with alternative education students. He used to have "that opening 5 minutes or uh, startup activity that engaged them with some writing on the board and to get them to think" (T6B, L60) at the regular high school. Some students took it seriously there but when he got to The School, he discontinued the practice.

As the authority figure in the classroom that sustained a cultural deficit orientation and kept his social distance, Mr. Glass also verbally dictated his intentions to students in a step-by-step manner and introduced academics as if he already knew students would do or feared they would do poorly. For example before taking attendance, Mr. Glass prepared students for the upcoming test by saying, "Now, I'm'onna help you so this is 
not something you need to be fearful of like- geez, you know, I'm gonna fail, or I'm not gonna get a good grade" (T4B, L49). He continued prepping them mentally for the test by first making a buzzer sound and then saying, "Aaahhhh, you're both gonna get good grades because you're gonna participate, and we're gonna do this not the way that we normally take tests which is, um, where, you know, the test is in front of you" (T4B, L52). He sat down to work on the computer as he talked, "Let me take the attendance first um, I uh, just expect you to do this all by yourself, no Mr. Mr. Glass is going to help, so let me take the attendance and then we'll get started" (T4B, L55). Roberto looked at the paper in front of him, flipped it over, and then laid his chin in his hands on the desk while he waited for Mr. Glass. Derrick looked at his pencil, wrote on the desk, looked up, and then pushed the pencil tip onto the end of the desk, erasing and tapping while he waited.

After taking attendance, Mr. Glass said, "Okay, you uh, I'm going to give you a copy of the play [meaning test]. You guys have something to write with" (T4B, 71)? Derrick wanted his pencil sharpened, which Mr. Glass did for him. During the interview watching the DVD, Mr. Glass commented:

It looks like Derrick because we did a quiz on Acts 1 through 3 that were the first part of the test, he's already working and it shows again that he has good recall of what has happened, he remembers events in the play, he has a generally good understanding of the plot, and this is a rare example, many students that I have in the ninth grade we did Dracula, over about 16 weeks or 15 weeks a long 400 page novel, uh, they could not carry that plot for any period of time it was just, it was all Greek to them I mean, because their concentration levels or periods is so short ... they work in 5 to 8 minutes spans of time and then their mind wanders off and they may be thinking about sex or about about drugs or why whatever they're thinking about but it's away from concentrating on the task at hand in the classroom whether it could be algebra, science, social studies, or English. (T6B, L76) 
Mr. Glass commented that it looked like Derrick was already working, showing that he had good recall and understanding of the plot, which was rare. From a cultural deficit orientation, he elaborated that many of his students exhibited short concentration levels and drifted off into thinking about sex, drugs, or anything other than academic topics.

Mr. Glass further opined about other student diversions from learning:

Another comment, this sharpening pencil business, I have this opinion that students who don't want to learn will use any trick they can come up with to divert the teacher from teaching and one of the diversions is this constant every period telling the teacher, please I need to sharpen my pencil whether it's a good student like Derrick saying that or a manipulator like another student who's not a good student saying that I am constantly besought upon by students in every period to sharpen pencils or worse, which I don't enable anymore, I used to enable, when they ask Mr. Mr. Glass, I don't have any pencil or pen can you give me something to write with, and the principal told the faculty in more than one occasion in our faculty meetings or or in community meetings we have every Monday mornings or the beginning of the week, uh, he does not want teachers to enable students who do not have supplies by giving them pencils so following his directive, his oral directive, I've told the students for some time now, no, I may well have pencils in the desk, but I say no I don't have anything, or no I will not give you anything and if you can get a pencil or a pen from a classmate, that's great but you'll see one of the Positive Behavior Support questions, PBS questions, did the student bring supplies so I said if you get something from me, I take a point off uh, from the 5, the max, you know, the maximum. (T6B, L70)

Also based on a cultural deficit orientation, Mr. Glass used the pencil sharpening issue as an example to explain how he believed that students try to manipulate the teacher away from teaching. In line with PBS rules about being prepared for class and the principal's request that teachers do not enable students by giving them pencils, Mr. Glass resolved supply issues by deducting a point if students get a supply from him or sharpening pencils himself to reduce the amount of time students spent at the pencil sharpener. 
After receiving his copy of the test during class, Roberto asked if they were going to write on it. Mr. Glass answered in an authoritative voice laying out his step-by-step intentions as he passed out papers and clipped plays together:

You gotta write on it, absolutely you gotta write on it. I'm going to sharpen your pencil, and that. Now, we're almost ready to go. Now, I'm going to give you copies of the play so we have it in front of us. (T4B, L77)

Expounding on inconsequentialities, Mr. Glass authoritatively and melodically answered Roberto's question about writing on the test and then sharpened Derrick's pencil. They discussed the date and then Mr. Glass directed again with especially melodic intonation:

Okay, here's the whole play because the test is over the whole play. Okay? So feel free to uh, I'll direct you to where I think you can find answers, now, let me put the test up here. Okay? So we're gonna, what, the way we're going to do this is I'm going to use the overhead projector to help you guys take this test ... part of it you also should not have any problems with because we took it as a quiz on Act 12 3. (T4B, L82, 85)

From a cultural deficit orientation, Mr. Glass introduced the test focusing on how he would direct them to where they could find answers. Then, he introduced the first part of the test again, exhibiting a cultural deficit orientation. He said in the same melodic voice:

SO! The first part of this test should go pretty quickly because we did it a couple of weeks ago (or a week ago) OK? So let's look at it, ok? Let's look at this. Let's get it so it's clear .... . SO you guys can put your name and today's date's the fifth of February and is that clear? And do you need to move closer? Can you see can you see this? (T4B, L88)

Mr. Glass reminded the students that they had done the first part of the test already and that it should go pretty quickly. Then, 15 utterances later, he repeated the introduction in a similar fashion:

Okay! So! The first part of the test is write the letter of each event in the order it occurred beside the corresponding number. And we've got ten events in the play. So gentlemen, what is the first? Well, let's look however you want to do this um, and I can get my marker out here so that it helps us, let me get my marker OK, so, 
do you wanna go with one by one? Getting a letter? Or do you want to do this chronologically. How do you want to do it? (T4B, L103, 106)

Mr. Glass focused on writing the letter of each event during this introduction to the first

part of the test. During the interview, he explained how and why the students are ill-

equipped and how he addresses this problem in class:

These students are not equipped because they won't go to the library and get a personal copy of whatever we are reading in class, they won't do homework, so if I were to use the same standard I held in the regular school setting, where when I gave a test, I gave an advance announcement that we were going to take a test whenever the date was and then I gave the test out and it might or might not be open book, I tend to give open book tests because I think in objective tests just being able to spew out facts is not demonstration to me of capability to do academic work but anyway, but I would not go to the length that I do here where I actually help the students get the answers to questions by walking them through them and probing with them to see if they know the answer and if they don't, then I come up with answer and again, their participation in this will ensure that both of them, I guess, got an A on the first part of the Merchant of Venice test, when in reality they didn't take a test that demonstrated their independent knowledge of the play, because I didn't test them that way, if I had done so, they both would have gotten an F!

He has lowered his standards with the students at The School by actually helping them get the answers and if they do not know it, he comes up with the answer for them. He explains what he means by testing students they way he does so they do not make an F:

By that I mean they would have not been able to do this because it does require some ability to recall in general at least the plot so that if you did have an open book that you could refer to um, you could check to see well, yeah, if it was part of the test required putting events in chronological order, as this test does have a part, they could check where certain events happened in which act, but as I say, I don't see that kind of of independent intellectual ability if one, a student is a reluctant reader so they hardly ever read or only read when they are forced to read, and uh, and their unfamiliarity with the elements of literature, so it it's just something that I don't put a lot of emphasis on here, were I back in a regular school setting, my procedures for testing would be different. It wasn't a test of knowledge of the play so much as their ability to follow the the test content and see if they could associate previous knowledge from watching the DVD and also studying over 8 or 9 weeks of text. (T6B, L72) 
Mr. Glass went into great detail to explain how he cannot set the same standards for alternative education students because they are not equipped, do not go to the library, and will not do homework. Therefore, he gave them the answers if they participated. From a cultural deficit orientation, Mr. Glass reduced his expectations and graded students according to how well they followed the test content.

Mr. Glass often stopped class for as long as it took to deal with interruptions. For example, each attempt to introduce the test was discontinued temporarily to handle interruptions concerning the whereabouts of students. With an authoritative and emphatic voice, Mr. Glass first answered a phone call while students waited quietly for him:

Mr. Mr. Glass. Yeah. No! Here's what I want you to do. CALL De Ramirez and the following kids should be sent to you by him for ORF. Ready to copy a few names? Jason Fabes, Exante Less, uh, Bale Contuse, that's it. Those three kids are with him and if you call him, one by one they can come and go and take that test. De Ramirez has those three students, they didn't sign parent permission forms so I have to send them to Ramirez for this period, uhh, I do but he's in fifth period, and he was absent yesterday. (T4B, L85)

During this first interruption, Mr. Glass stopped class to send students (who did not have signed parent permission forms for the study and were with another teacher at that moment) to take the ORF test. The second interruption between introductions of the first part of the play occurred just as Mr. Glass said to Derrick and Roberto, "Let's go through part one" (T4B, L95). A student knocked on the door and opened it. Mr. Glass replied quickly, "You can't come in" (T4B, L95). The student questioned why he could not come in again. Mr. Glass explained rapidly:

Talk to De Ramirez because you didn't sign the parent permission form and we're videotaping. Auto. Mechanics. You know where it is? Know where it is? Actually, before you go before you go go into that office and take the ORF test tell Ms. Jackson that you're here you just came you're one of my students sixth period Demetrius Lewis, ok. She'll she'll ORF you. (T4B, L97, 99) 
The second interruption occurred by a student who also could not participate in the study. Returning to the first part of the test, Mr. Glass asked for the answer to number one. Derrick guessed remotely, "H" (T4B, L113). Mr. Glass asked, "This is A" (T4B, L114)? Derrick replied, "I mean H. H" (T4B, L115). Mr. Glass responded as if giving a clue, "This is H" (T4B, L116)? Derrick answered remotely again, "Yeah, I think" (T4B, L117). Mr. Glass replied in a leading tone, "No" (T4B, L118)? Roberto interjected, "It's F” (T4B, L119). Mr. Glass said, “Okay” (T4B, L120). Roberto said, "No” (T4B, L121). Finally in the same melodic sympathetic voice:

Maybe it'll help you if I try to say to you where in the uh which act these events occur, ok? Jessica elopes with Lorenzo occurs in Act 1 . So it can't be $h$ because that's way way way too far away, ok? (T4B, L122)

Mr. Glass fed answers continually to his compliant alternative education students. During the interview, Derrick said that it was helpful when Mr. Glass gave them the act number but contradicted himself when he noted that he only knew what happened in all the acts, "so so" (T6A, L83). As a result of cultural deficit orientation and banking education perspective, low expectations offered students little opportunity and few real choices or meaningful options for academic success.

\section{Accommodation}

Accommodations of school ideology manifested as two subthemes: (a) teacher as authority figure and (b) students as compliant subjects, making accommodations to Mr. Glass's classroom culture, norms, and values. Accommodation occurs as the simultaneous coping or acceptance of school ideology and the display of some form of 
resistance to it (Giroux, 2001). The School's ideology required teachers and students to follow PBS rules and the prevailing educational philosophy.

\section{Teacher as Authority Figure}

Mr. Glass coped with PBS rules and school ideology about teaching content unless it prohibited him from maintaining control in the classroom in order to teach his content in an orderly climate. In order to retain an orderly climate for teaching what and how he loved to teach, he exhibited some resistance as the authority figure that made the final decision. When asked about The School's PBS rules and regulations, Mr. Glass explained from a cultural deficit orientation:

I thought before I knew anything about the Positive Behavior Support system that it was a gimmicky type of behavior modification program, I have changed my views um, considerably on it, I do think that it is an important part of education in an alternative education program and it is also an important behavior modification program in a regular school, it can work anywhere. What it does is focus on observable behavior of students so that you will see a series of 10 specific questions related to the system that students and teachers should review periodically however they do it, every teacher's different, uh, coming to class on time, being punctual, not being tardy, uh, the school has a uniform, uh, being attired in the school uniform, uh, bringing supplies to class, biggest problem I have with my students- they never have pencils, paper, or pen, they don't have anything to do the work with. They say they lose them on the bus, they lose them in the cafeteria, whatever so these are observable behaviors that you can get the student to understand that they either meet the criterion or they don't, keeping your hands to yourself, not engaging in horseplay, uh, participating in the lesson actively, not putting your head down saying I'm sick I don't feel good, I'm not going to do anything today, Mr. Mr. Glass. Uh, being respectful, not using profanity, again all of these are clear specific behaviors that you can practice with students to get them to understand there's normal routines that encourage learning and if you practice them and believe in them, you will learn! (T6B, L14)

Mr. Glass came to believe that PBS was an important behavior modification program that may encourage students to learn observable behaviors and normal routines at school. Mr.

Glass elaborated on the PBS system rules and regulations: 
The way it works um, in brief is that in each class a student can earn a maximum of five points if they do all of those 10 specific behaviors for the full period they're going to earn the maximum number of points or close, they might be off task, you know, for a little bit, get 4 instead of 5 points, but they won't get 3, 2, or 1. Just showing and doing nothing will get them a point. Zero points comes when they get thrown out of class. They get a referral, they go to CSI or detention, and then the system as it functions here is that each week, there is a reward activity for the top $30 \%$ of the student body point wise, so in any given week, maybe 25 or 30 out of a hundred or more, uh, have an ice cream sundae or a movie or they go bowling or they get a Chinese luncheon, uh, the incentive is to earn the points to be involved in the reward activity at the end of each week. (T6B, L16)

PBS rewarded students for complying with specific behaviors. Even though students were becoming more connected to PBS over the last two years and the system was probably working better each year, Mr. Glass explained that they had also experienced where let's say that $40 \%$ of the student body is trying to participate in the in the uh PBS program, and 50 or $60 \%$ give up and aren't concerned at all, don't care about the points, don't turn in their point sheets, just say I I don't really care about the rewards, I don't care about the program, just leave me alone, and I I don't want to earn the points, so turning around the attitude of the student to invest in the external rewards is a big challenge. Yes. (T6B, L18)

Mr. Glass noted that more than half of the students did not care about earning the points.

Therefore, when students' attitudes interfered with his ability to maintain control of his desired classroom culture, norms, and values and teach literature, Mr. Glass accommodated PBS rules and made the final decision. For example, with disruptive behavior he would

normally, uh, be patient and redirect a disruptive student or a student who is constantly bringing out their cell phone to text message or having their ears plugged in with ear plugs and listening to their IPOD. I will give that student an opportunity to put it away out of plain view and I will do that maybe twice. If the student ignores me beyond two redirects, two warnings, then I will call for staff assistance or security and I will write a referral and they will go out of class. Occasionally, if a student has a pattern or history of disruptiveness and we have a good number of those kinds of students across the student body, I might not be so patient. If the student comes into the class where I have 5 or 6 people or more, and they're automatically disruptive from the moment they hit the door, I won't 
wait for any redirects, the moment they turn the class from low-level chaos to high-level chaos, I will get rid of them and I have been noted to throw kids out even before the class period started because they came in here tussling and horseplaying with anther student.

He explains his exclusionary discipline policies further:

So I think I don't know you'd have to ask the administration or find out from the administration, I probably write more referrals than any teacher on the staff, at least that is what I have heard second hand, but I do write a lot of referrals and I write them because again, I need to have an orderly climate to teach, and if a kid is preventing anyone, one or more students from that learning experience, they got to go and I I don't hesitate about that, maybe another teacher has a softer approach or a more, but that doesn't mean that I don't have patience. I really will do everything I can to figure out how to keep a kid in class because I know it's not the the principal want students to be in the classroom and not outside in detention uh, and he was very strict about that in the beginning of the school year, he said we don't have CSI this year and there is no more detention and we started this PBS system with the idea, well you can see a poster up there called the bench, when a kid acted up for whatever reason you put them in your designated bench part of the classroom and they sat there for five minutes to cool out and then you went over and you asked them if they were ready to come back and participate and if they said no you called the staff person, well, by this time in the school year I would be shocked or greatly surprised if anybody [used the bench] (T6B, L30)

Even though the principal discouraged sending students to detention (CSI), Mr. Glass accommodated by giving students two warnings to put up cell phones or IPODs, but if they ignored him or if they were disruptive when they came into the classroom, he did not hesitate to throw kids out before the class period ever started. He interjected that he probably wrote more referrals than any teacher on the staff. He was the authority figure in his classroom.

Mr. Glass also accommodated PBS rules that restricted the use of passes in order to accomplish his own classroom goals. He explained that:

the first two quarters, the opening quarter of the year, that begins August through October and then the second quarter that covers Thanksgiving through the winter break holidays until we come back after the New Years, I'm very, me personally, I'm very strict with passes, I tell students that in those first two quarters that I will 
allow them to go to the restroom two times in nine weeks, hey, or ten weeks and that after that they better use the restroom during the passing because if they ask me to go to the restroom and they have exhausted their two uh, free passes, I won't let them go and then if they leave the room without permission that's a referral. Um, I have uh, eased up on that in this, we're in the third quarter uh, and I I no longer uh, take that approach, it it it worked, uh, it had a useful purpose for me but I don't find a lot of abuse now that we're so far into the school year so that if a student asks me to go to the bathroom, I'm not keeping track of of the number of passes they have had, you know, I have to keep a record of that just like with the points and it uh, uh, it uh, I I don't need to do that now for me. (T6B, L24)

Mr. Glass was the authority figure and decided how and when to write passes. Mr. Glass also acted as the authority figure and accommodated PBS rules regarding point sheets. For example when Mr. Glass had finished checking attendance and began introducing the first part of the test to Derrick and Roberto, Derrick said, "Yeah! It's our point sheet day. It's our point sheet day" (T4B, L41). Mr. Glass, who often referred to himself in third person, answered, "Yeah, you got point sheets for us?" Derrick reached onto his pockets and fished around, saying, "Ms. Lamb's got-" (T4B, L44). Mr. Glass replied, gesturing with his arm toward Derrick, "I got- I got one if you if you want the one from Lamb, I'll sign it later on if you want one from me I got an extra one, ok?" (T4B, L45). Derrick answered that he would "wait for Ms. Lamb" (T4B, L46). Mr. Glass replied, "Yeah, ok. And then you're going to come by and see me eighth period?" (T4B, L47) and turned around and walked the other way toward his desk. Derrick nodded his head and said, "Yes," and Mr. Glass responded:

Cuz I don't have any students eighth period. Ok, here's what we're going to do today, and you know, just like the other day ... um, you're going to take the test, with me, for the Merchant of Venice. (T4B, L48) 
During the interview, Mr. Glass clarified how he accommodated the PBS point sheet rules (that hold students responsible for keeping up with them) when he deemed necessary:

What that discussion was about was that he uh, he has a problem because this is not the first time where he doesn't carry, the student is responsible for carrying the point sheet from class to class and giving it to a staff person before they get on the bus Wednesday and Thursday, those are the collection days. So if this is a Thursday then he should, or a Wednesday, er or but it's a Thursday because it's an even class, then I'm saying to him, okay I've got an extra point sheet so you know, or maybe if we finish this in time he can go get his old point sheet and bring to this class. (T6B, L54)

Mr. Glass decided that he would give Derrick an extra point sheet or let him go get his old one if they finished their work in time.

Mr. Glass also explained that he taught literature to his students because he thought they had "the right to know about the best writers in the world, in world history" (T6B, L261). He believed that using mandated textbooks was watering down the curriculum. He preferred lecturing from his authoritative knowledge of literature. For example, Mr. Glass explained the new curriculum unit to compliant students in a melodic authoritative voice. As he talked, he slowly got the overhead set up, turned out the classroom lights, turned on the overhead light, walked over to desk to get something, read something on his desk, put the transparency on the overhead projector, fixed the focus, and moved the projector back a little bit. He always kept his social distance and had no eye contact with the students when he lectured, expecting they were watching and listening to him. He said:

Today we are going too start, a new, curriculum unit. And the way that I always start, when I introduce a play or novel (or a) new author is to begin with background context in other words to talk about the work and the uh, writer and so, we're gonna take a look at the writer of a book called Sidd-har-ta. And you're 
gonna to take some notes, and then after lunch, we're going to do a handout! Okay? Which I'll help you with. (T4B, L172)

Mr. Glass told the students how he always conducted a new curriculum unit and what they would be doing that day. He explained during the interview that he was not the type of teacher to water the curriculum down and give students "easier uh, work assignments or busywork type textbook-driven lessons" (T6B, L261). He lectured for long periods of time even though

kids do not like to sit for a long period of time, a 90 minute, 85 minute block uh, period that we have as opposed to a regular 50 minute period class, and um, if you want to be very successful, you will change the activities at least every 15 or 20 minutes. Now I don't do that and I often have uh uh educational philosophy differences with the administration because when they come in and observe me, they see that I might be spending 30 to 35 minutes on a textual passage. (T6B, L10)

As the authority figure in the classroom, Mr. Glass accommodated the administration's educational philosophy in his final decision of what and how to teach. He explained:

Quote unquote the administration expects that you will use the language and literature textbook that's assigned to the grade level you are teaching so this would be Language Arts II we would use a seventh grade McDougal Mattel uh, Anthology. I don't and no one has complained that I don't because they know again, from observing me that I can teach these great writers so they're happy to let me to it and I'm pleased that I have the freedom to do it and not to be shut down and say you must use the textbook, Mr. Mr. Glass. (T6B, L28)

Mr. Glass lectured, gestured, and dictated his actions and intentions for long periods of time throughout the class period if no interruptions occurred. For example to begin the unit on Siddharta, he said:

All right? So, let's get this in focus. Okay, that should be. I'll move it around because the bottom part of it, I need to bring up more, okay. Now, that's probably is as clear as I'm going to get his picture. Okay, getting this off of an internet website a little biography of Hermann Hesse. Now, this novel Siddharta is something different very very different from what we have finished. I mean we started the Merchant of Venice and Shakespeare in the second quarter. Umm, we 
basically finished the short stories with the Africans and with Tolstoy right at the break. It was a clean break, so that the new curriculum unit began as soon as we began the second quarter. Now the Merchant of Venice took us longer, than one quarter to do, which is okay! But we finished it with the test that you took and and Stephan took, both of your work products are over there, you know, you both did well on what we did the last period and I was very impressed you know, with your knowledge, so, umm. (T4B, L174)

As Mr. Glass talked, he walked over to cabinet, opened it up, got some papers, walked back over in front of the students and started moving his left hand flinging it back and forth, paced in front of the students, put his hand to his forehead and held it there, not looking at the students. With his left hand fingers together, Mr. Glass pointed toward a place on wall, stood sideways to the students looking off at the wall, rolled his wrist for awhile, put his hand to his chin, and sawed his hand up and down in the air as talked. His left hand, with fingers together again, moved to the right and pointed, he nodded, pointed once to Derrick, pointed and rolled his fingers toward the exemplary work bulletin board where two papers were stapled, nodded at Derrick once again, and then looked back at the overhead material, flashing the light on the white board in front of the students.

During lectures, Mr. Glass interjected information about students who had been suspended, taking notes, getting supplies out, and other things. However, students very rarely talked, moved around, or negotiated with Mr. Glass during this time. Mr. Glass also rarely asked for student input about classroom curriculum or school requirements. For example when asked during the interview if he saw his paper on the exemplary work bulletin board or if he knew one of the two papers were his, Derrick commented in the negative with, "Hmmp. Hmm" (T6A, L95, 98). When asked what the ORF test was that students were being sent to take during class, Derrick answered, "I donno" (T6A, L58). 
Negotiation attempts by students usually failed shortly after initiation. For example, after Mr. Glass had introduced the new curriculum unit on Siddharta and was seemingly anxious for students to take notes during his lecture, he said melodically to Green who had requested to go to CSI at the beginning of class that same day:

Okay. You're going to take some notes. And [Green], if you care to, you're gonna take some notes because uh, we're gonna work on a a 10-question handout after lunch that deals both with Hesse and with this nov with this novel. NOW! The name of the book we're going to read is right up here. S-I-D-D-H-A-R-T-A. (T4B, L178)

Green responded, “Can I have a pen?” (T4B, L179), sounding ready to take notes. Mr. Glass continued lecturing, saying the title of the novel with fondness, "Sidd-harta" (T4B, L180). Green asked again with an additional reason, "May I go get a pen from my uncle?" (T4B, L181). Mr. Glass responded as he walked over to Green, "What do you need, paper and pencil?" (T4B, L182). Green said he preferred a pen. Mr. Glass replied happily but not congruent to Green's request, "Okay, yeah, you need paper and pencil" (T4B, L184). Mr. Glass supplied Green with a pencil, accommodating a PBS supply rule, and continued lecturing authoritatively.

\section{Students as Compliant Subjects}

Students accommodated Mr. Glass's classroom culture, norms, and values that provided few options or real choices to successfully stay in class. They simultaneously coped as compliant subjects if they did not want to go to CSI that day but exhibited quiet resistance when Mr. Glass was busily conducting teaching tasks. For example the day Mr. Glass checked attendance aloud and looked for skippers, Green was not in class because he was "still out on [10-day] suspension" (T6B, L44, Mr. Glass). Derrick was the only compliant student at the time. He was leaning over the back of his chair with one 
arm hanging down and other arm resting on the desk as if bored. From time to time, he glanced out the window. During the interview, Mr. Glass explained:

I think what's going on, I could be wrong, but that since his, this is Derrick, and his demeanor is he's waiting for me to engage him, that I'm probably getting the overhead projector ready to start the new curriculum unit on Siddharta, and as soon as I'm set up, he'll bring his head up and we'll because he is a uh, good student uh, he has been so most of the year, there was a drop off during the second quarter, I think he got a $\mathrm{C}$ in the second quarter, but he now has a $\mathrm{B}$ and $\mathrm{I}$ think he is going to maintain that for this quarter, he comes to school prepared, he does have supplies, you can see he's got a notebook and uh, even though this scene doesn't look like he's doing anything, I think it's partly because I'm trying to start whatever I'm going to say about Hermann Hesse now let's find out. (T6B, L42)

Mr. Glass commented that Derrick's quiet compliant demeanor signaled his engagement even though it looked like he was not doing anything while he waited for Mr. Glass.

After Roberto arrived and sat down, both he and Derrick were compliant students during attendance check. They either provided information Mr. Glass requested about missing students or talked quietly about what had happened that morning. By doing so, they both complied with classroom norms and achieved their own communication goals. On this particular day, Derrick asked Roberto, "How many cops out there?" (T4B, L58). Roberto replied, “Cops? I don’t know. I saw one” (T4B, L59, 61). Derrick responded, “I saw a White one, a White cop, you saw a White cop?" (T4B, L62). Roberto answered, "I saw a Black cop" (T4B, L63). They discussed the two cops and how another student got slapped in the teeth. Suddenly, Mr. Glass announced that he was going to give them a copy of the play. The boys stopped talking. They knew it was time to listen.

Other times during class, compliant students were silent and waited for extended periods of time for Mr. Glass to begin or resume class. During the interview, Derrick said that he was silent because he was bored. If he talked which was not often (T6A, L74), he 
may make something up to accommodate the classroom norms of being compliant and willing to participate. For example, when Mr. Glass asked the students if they wanted to answer the questions by getting a letter or doing it chronologically, Derrick answered that he wanted to answer the questions chronologically. He explained during the interview, "I don't know what that meant I just made it up" (T6A, L76). Compliant students accommodated classroom culture, norms, and values in order to communicate with each other in the few moments they perceived that option. Complaint students also knew Mr. Glass's limits and ultimate goals of teaching in an orderly climate to which they accommodated in order not to get referred to CSI or suspended. In the event students were not willing to comply with the classroom culture, norms, and values on a particular day, they preferred going to CSI.

\section{Resistance}

Resistance is behavior that implicitly or explicitly exhibits refusal as well as a need to struggle between domination and compliance (Giroux, 2001). Resistance is manifested in three subthemes: (a) critique, (b) self-reflection, and (c) struggles (Giroux, 2001). This section discusses students' resistance of Glass's classroom culture, norms, and values, highlighting Giroux's framework.

\section{Critique}

Critique of domination manifested when students either skipped Mr. Glass's class altogether or disrupted in the beginning of class, knowing they would get sent to CSI. Mr. Glass defined skipping, "When they skip a class, they uh, duck, dodge, and hide on campus roaming however they can keep away from somebody intercepting them and asking them what they are doing" (T6B, L46). 
Having returned early from 10-day suspension, resistance was exhibited in Green's struggle between not getting suspended again and the desire to be emancipated from the constraints of classroom culture, norms, and values. Ultimately, though, Green accommodated. Before class began one day while Mr. Glass checked attendance aloud and talked to another staff member on the phone about students' whereabouts, Green and other students talked quietly at the student desks. Using common vernacular, one student said, “No, man, I'm stretching the money" (T4B, L131). Another student replied, "I've got a big ass customer" (T4B, L132). Another student asked, "You poppin' the click, boy?" (T4B, L133). One student replied, "What's up? Mr. Glass ignored them until the bell rang. Seven students and Green, the only one who belonged in the class, stood up and began filing out. From a distance, Mr. Glass suddenly said in a loud authoritative voice, “OK! Those people who take, Green you stay! GREEN! DO! NOT! GO!” (T4B, L140).

\section{Self-reflection}

Self-reflection occurred when Green and other students expressed the dilemma of staying in class with an excuse that he did not want to be in "front of the camera" (T4A, L146). The students laughed, but one student tried to calm his buddy down and pleaded, “Green, chill out! We told each other we can do it! Chill out! Chill out! Don’t get suspended!" (T4B, L142, 143, 145). Green critiqued the class again using self-reflection, saying that he did not want to stay. The student told him to stay. Still retaining his distance, Mr. Glass interjected in a stilted manner, "Just stay! SIT! Sit, okay? Thank you" (T4B, L148, 150). Green said, "Naw, naw" (T4B, L151). Mr. Glass demanded, "Green, come back in here and sit. [to the other students] Bye, good bye" (T4B, L153). Green sat 
down after stating again, I don’t wanna stay here, man” (T4B, L158). Mr. Glass

responded, "I r I really don't care, Green, I mean, that's neither-" (T4B, L159).

Struggles

Struggles toward emancipation occurred when Green stood up and asked a

different question, reflecting on past experiences of emancipation from class, "All right, then let me go to CSI or som'in?" (T4B, L160). But Mr. Glass denied the request, "You're going to do some work for us. Just like Derrick is" (T4B, L161). Green asked again if he could go to CSI, seeking emancipation. Mr. Glass again said no, confirming, "I'm just going to get the attendance made up and we're ready to go. Okay" (T4B, L170).

Finally, Green succumbed and laid his head on the desk. Shortly afterward, Green asked Mr. Glass if he could go get a pen from his uncle who worked at The School.

During the interview, Mr. Glass commented:

Okay, so here's an example of where, not because it's Green, but any student, a typical student that doesn't have the supply materials, how can they do anything academic achievement wise, okay it's virtually impossible because they're not prepared mentally for the school experience. For them, school is just being here, just showing up and not having supplies is that that attitude, an example of that attitude. (T6B, L82)

Mr. Glass viewed students' resistance from a cultural deficit orientation of not being mentally prepared for the school experience. He compared Derrick and Green from the same cultural deficit orientation:

Green is someone who I've come to learn more about his family background through some of the other staff people around here, he's got a lot of issues that uh, that make him the kind of clown type student that he projects, he's not initially when he came in here at the beginning of the school year I was so impressed with his purposefulness, he like Derrick showed me a desire to want to do well in the classroom you know within the first two weeks of the school year and I was I was pleased and then after that, he just unraveled. His overall demeanor is one of being a jokester, acting like a fool, and he's uh earned very poor grades because 
he doesn't care, he doesn't try, uh, except to copy at the off somebody's paper but he does have a native intelligence that he could do much better if he uh was motivated to be a uh a student and I believe that his situation at home has caused a lot of his attitude problems, he is not a good student because he does not have the support that he could have or might have from a more stable home situation which is demonstrated here in his desire not to stay ... [Even his uncle said] that the family situation has gotten to the point to where Jim has just washed his hands, yeah, he may be blood related to me but I don't I don't care about him anymore. He's his parents' responsibility not mine and if he messes up then send him to detention or CSI and he has gotten suspended for his behavior (T6B, L80)

Mr. Glass elaborated about the connection between Green's deficient family background, native intelligence, and overall jokester demeanor in the classroom. On the other hand, Derrick has showed him a desire to do well in the classroom over a longer period of time. From a cultural deficit orientation, student resistance is often blamed on lack of desire at the fault of the family background.

Green confirmed during the interview that he was asking to "go to CSI" (T6A, L94). He added, "I didn't want to stay in there. They were saying don't get suspended" (T6A, L86-88). Students knew the PBS rules and the rewards. Green clarified, "You gotta get points from every teacher, if you make your points, there's an event on Fridays. Every Friday" (T6A, L35). However, students also weighed the validity of the choices provided them in classroom culture, norms, and values with the consequences. Derrick explained, "It's fun in CSI" (T6A, L12). Green clarified that CSI was "detention." (T6A, L14). Derrick elaborated about what they did in CSI, "You usually sit around if you don't know the work, but they don't give you work" (T6A, L18). Green added, "If you get kicked out of class and don't go, you get suspended. It's like indoor [suspension] . . . I guess [they send you to CSI first before suspending you] to give you a chance" (T6A, L20-27). 
Green noted that there was no hard fast rule about getting suspended. He said:

I got suspended 2 weeks ago, I just came back this week. When you get suspended, you can't go back to class you go to CSI. Yeah, I went to CSI for the rest of that day and then can't come back. You stay home. (T6A, L42-47)

When asked if it helped to stay home, he said that it did not help and that he did not know why they suspend students except maybe for "punishment, I guess, from the school" (T6A, L51). Derrick said we "need to ask them that" (T6A, L52). Students also knew the teacher's expectations and limits provided in the classroom culture, norms, and values. Green commented on what ultimately happened in class that day, "We're fixing to read a new story about Jesse Hess" (TA, L104). Derrick corrected him and laughed, "It's Hermann Hess" (T6A, L105). Green laughed and said, “Oh, Hermann” (T6A, L106). Derrick told him, "A writer" (T6A, L110). Green explained what he was doing with his head down, "Laying there. I was listening. (T6A, L116).

Mr. Glass viewed student resistance from the perspective of what he encountered daily. He commented on how he viewed teaching at The School:

You need to try to help [students] develop more self-confidence that they can do classroom work of any subject and if you and if you are frustrated by the uh type of situations you encounter in maybe every class or certainly at least once a day in one class with with students disrupting your lesson, throwing you off your rhythm uh, then you will be very frustrated person in alternative ed. Most of the staff here has lots and lots of experience with these kinds of students so even though they do lose patience, we all lose patience, we are more able to handle the stresses here than teachers who have never had this kind of experience, teaching experience. (T6B, L8)

Mr. Glass wanted to help students develop more self-confidence with academic material in the classroom, but concluded that teaching in alternative education consisted of the daily frustration of disruptive behavior that threw off a teacher's rhythm. Students resisted his classroom culture, norms, and values by either skipping Mr. Glass's class or 
by being disruptive in the beginning of class and critiquing the constraint at hand so that they could get sent to CSI where it was more fun.

Overall, in order to teach his content, Mr. Glass maintained control of his classroom by requiring an orderly climate and compliant students. Students knew their options and the teacher's limits and were forced to decide whether to be compliant with no opportunity for negotiations or be resistant with the consequence of ultimate referral and suspension.

\section{Summary}

This chapter presented a description of Classroom Four findings in three sections: (a) Classroom Four participants, (b) findings from interactive power analysis, and (c) themes from reconstructive data analysis. Mr. Glass is a White male Language Arts teacher who had no experience or knowledge of alternative education before becoming a teacher at The School. His Latino and African American students were familiar with alternative education. Three types of power exhibited in Classroom Four were normative power, (minimal) interactively established contracts, and coercive power; charm was not exhibited. These types of power combined in a way that Mr. Glass and his students' roles conflicted. He was very busy with many things, while lecturing, providing clues to answers, disciplining, enforcing rules, and directing the classroom minute-by-minute. Cultural power was at play in Classroom Four, because Mr. Glass did not gain universal interest of his classroom goals and norms from his students. According to their demeanor and/or their participation in class, students consented to the norms/values of the teacher's setting because of the cultural power of The School which was enforced by the teacher. Mr. Glass's lack of charm and rarely exhibited interactively established contracts (only 
when it involved talking about other students' whereabouts) increased the importance of normative and coercive power in this classroom. Student demeanors abided accordingly. Three themes of social interaction (resistance, accommodation, and conformism) emerged and combined in the following ways. Mr. Glass rigidly conformed to dominant ideology, both PBS and academic rules and regulations, in order to maintain control, teach in an orderly climate, and enforce expectations by using written and supporting student suspension. Therefore, students resisted his classroom norms by either not coming to class, trying to get removed from class upon arrival and not being in the mood, or being silent and rotely following directions. Mr. Glass accommodated the dominant ideology as well by being the authority figure who decided how and when to accomplish his classroom norms and system rules. In turn, students also accommodated his classroom norms by acting as compliant subjects but talking quietly if he was busy doing other things and did not notice. The combination of these themes resulted in no room for negotiation between the teacher and students. The teacher's goals were different than the students' and since they were inconsistently exhibited according to how he exhibited authority as normative and coercive power, conflicts arose at two extremes of the spectrum. For example, at one moment conflicts may be demonstrated by students with very loud disrespectful talking, while the next moment conflicts may be illustrated by students' silent, limp demeanor. In this Language Arts classroom, Mr. Glass did the majority of talking as lecturing or walking students through expected actions to take socially or academically, which resulted in unsuccessful student outcomes either way. If they did comply with the lecture, they were told the answers to questions being asked; if they did not comply with instructions, they were referred out of the classroom for 
disruptive behavior. In chapter 8, a comparison and synthesis of the findings is provided. Chapter 9 includes a summary of the study, responses to research questions, implications for teacher education, and recommendations for research and policy. 
CHAPTER VIII

COMPARISON AND SYNTHESIS OF FINDINGS

This chapter provides a comparison and synthesis of findings and is divided into three sections: (a) classrooms characterized by frequent exclusionary discipline, (b) classrooms characterized by rare exclusionary discipline, and (c) classrooms characterized by frequent versus rare exclusionary discipline. A summary concludes the chapter.

Classrooms Characterized by Frequent Exclusionary Discipline

This section compares and synthesizes findings in classrooms characterized by frequent exclusionary discipline $(\mathrm{C} 2, \mathrm{C} 4)$ in three sections: (a) power, (b) roles, and (c) themes.

Power

Power is compared and synthesized in this section. Normative power was exhibited by both classroom teachers in varying degrees - Classroom Two, sporadically; Classroom Four, continually. Coercive power was exhibited by both teachers yet differed in execution-Classroom Two teacher, implicitly and gradually; Classroom Four teacher, explicitly and immediately. Interactively established contracts were initiated by Classroom Two students continually and encouraged by Classroom Two teacher continually so that she could figure out ways to rehabilitate students. Classroom Four teacher exhibited interactively established contracts rarely so that he could document student whereabouts. Neither teacher exhibited charm. Table 21 illustrates the comparison of types of power exhibited, followed by a discussion related to cultural power. 
Table 21

Comparison of Types of Power Exhibited in Classrooms Characterized by Frequent Exclusionary Discipline

Types of Power Classroom Two Classroom Four

Normative power

Coercive power

Interactively established contracts

Charm

$$
\text { Sporadically }
$$

Implicitly and gradually

Student initiated continually and teacher encouraged continually for student rehabilitation
Continually

Explicitly and immediately

Rarely for student documentation
Not exhibited
Not exhibited

Cultural power was at play in each classroom, because both teachers defined and determined the end result of academic and social interactions through exclusionary discipline rather than through charm. However, disciplinary actions preceding exclusionary discipline in Classroom Two were more successful than in Classroom Four, because they were further removed from coercion (Carspecken, 1996). For example, students in Classroom Two were encouraged to talk during class (until the teacher lost her patience from the incessant student talk and wrote a referral) which provided both a space for their interests and for the teacher to figure out ways to reduce student hostility. On the other hand, students in Classroom Four who exhibited disruptive or talkative behavior at the beginning of class were typically issued referrals and removed from the classroom immediately. This exclusionary discipline practice set up rigid boundaries between the teacher and students who remained in class and provided little space to 
incorporate students' interests through talk other than quietly with each other when the teacher was busy or briefly answering a direct question from the teacher. Academic interactions in both classrooms were minimized as a result of the focus on exclusionary discipline to maintain control in the classroom. For example, assignments to write prompts and an essay for FCAT practice were not completed in Classroom Two. Answers for a test on "The Merchant of Venice" were provided with assistance from the teacher in Classroom Four.

\section{Roles}

Teachers' and students' roles are compared and synthesized in this section. Teachers' roles were similar in their surveillance of students yet differed in executionClassroom Two teacher through empathetic practice; Classroom Four teacher through rigid expertise. Students' roles in Classroom Two and Four exhibited agency to maintain identity. Table 22 summarizes roles exhibited, followed by a discussion.

Table 22

Comparison of Roles Exhibited in Classrooms Characterized by Frequent Exclusionary Discipline
Roles
Classroom Two
Classroom Four

Teacher Roles

Empathetic practice for surveillance

Agency to maintain identity
Rigid expertise for surveillance

Agency to maintain identity

Both teachers monitored students' academic and social behaviors closely through the various Discourses and roles they had acquired in their educational and professional lives 
(Cochran-Smith \& Zeichner, 2005; Darling-Hammond \& Bransford, 2005; Gee, 1992; Hollins \& Guzman, 2005). However (as supported in interview data), based on the scope of teachers' experiences and dominant conceptualization of alternative education students as poorly behaved and academically challenged, their teacher roles clashed with students' roles and Discourses, providing few spaces for students' cultural and literacy practices to emerge and develop over time (Delpit, 1995; Gutiérrez, 2008; Lee, 2005; Moll, 1992). For example, Classroom Two teacher took anecdotal notes on students' behavior, and filled out referral forms regularly while trying to get students to practice for the FCAT, in anticipation of either using her psychology knowledge to rehabilitate students or issuing exclusionary discipline when she ran out of options. Classroom Four teacher lectured to students about the lives and works of "great" authors and helped them take tests after ridding the classroom of disruptive students who he felt did not care about learning.

Students resisted both teachers' academic and disciplinary practices through roles and Discourses they had acquired in their lives to attempt to maintain acceptable identities in alternative education classrooms and schools. In Classroom Two, students came to class knowing they would be allowed to produce vernacular and cultural humor during class while the teacher made suggestions about their academic work. In Classroom Four, students chose either to skip class, act disruptive to get sent to CSI, or remain compliantly in class to get PBS points and a grade for that period.

\section{Themes}

Themes are compared and synthesized in this section. Teacher resistance was not exhibited in either classroom. Student resistance was exhibited in both classrooms to communicate and maintain identity and agency yet was executed differently. In 
Classroom Two, students expressed resistance continually during class, both verbally and nonverbally to change the topic away from Language Arts. In Classroom Four, students expressed resistance continually either by skipping class, being disruptive in order to get removed from class, or silently complying during class. Teacher accommodation was exhibited in both classrooms, pending ultimate exclusionary discipline, but was executed differently and for different interim purposes. Classroom Two teacher executed accommodation by using various tactics to enforce rules and maintain an environment safe for talking. Classroom Four teacher executed accommodation as an authority figure to maintain control, teach in an orderly climate, and enforce expectations. Student accommodation was not exhibited in Classroom Two. However, student accommodation of classroom norms and minimal communication opportunities in Classroom Four was exhibited continually as compliant subjects. Teacher conformism was exhibited in both classrooms continually but executed differently—Classroom Two through deference to academic and behavior authority and Classroom Four through banking education and cultural deficit orientation. Table 23 summarizes themes exhibited, followed by a discussion. 
Table 23

Comparison of Themes Exhibited in Classrooms Characterized by Frequent Exclusionary Discipline

Themes Classroom Two Classroom Four

Teacher resistance

Student resistance

Teacher accommodation

Student accommodation

Teacher Conformism

Student Conformism

Teacher Negotiation

Student Negotiation
Not exhibited

Continual to change topic away from academics during class

Varying tactics to enforce rules, maintain environment safe for talk, pending exclusionary discipline

Not exhibited

Continually through deference to academic and behavior authority, pending exclusionary discipline

Not exhibited

Not exhibited

Not exhibited
Not exhibited

Continual to skip class, disruptive removal from class, or silence during class

Authority figure to maintain control, teach in orderly climate, enforce expectation, pending exclusionary discipline

Continually as compliant subjects

Continually through banking education, cultural deficit orientation, pending exclusionary discipline

Not exhibited

Not exhibited

Not exhibited

As supported by interview data, both teachers strictly abided by conventional views of learning that sustained mainstream educational ideology about alternative education students as those who need to be disciplined or cured of their social and academic deficiencies (Carpenter et al., 2006; Cassidy \& Bates, 2005; Delpit, 1995; King, 2005; 
Ladson-Billings, 2006; Moje \& Lewis, 2007). Neither teacher's classroom practices provided room for student input or negotiation about what was being learned academically or socially, and how that learning was accomplished. As a result, students resisted teachers' classroom practices, teachers and students were on different wavelengths about academic and social issues, and teachers strived continually for more control, ultimately using exclusionary discipline to stop conflicts and discord in their classrooms.

Each teacher differed in how and why he/she strived for control in both the academic and behavioral areas. For example, Classroom Two teacher used Language Arts requirements issued from outside authority (e.g., FCAT, computer reading programs) as a backdrop for listening to students talk in order to figure out ways to reduce hostility among her students. Classroom Four teacher used his expertise in lecturing about great authors and their works of literature as a backdrop for keeping his students compliant and quiet. Even though each classroom sounded (ie., loud versus quiet) different, both teachers upheld conventional Language Arts teaching and alternative education learning practices that required passive students and used exclusionary discipline to control student behavior.

\section{Classrooms Characterized by Rare Exclusionary Discipline}

This section compares and synthesizes findings in classrooms characterized by frequent exclusionary discipline (C1, C3) three sections: (a) power, (b) roles, and (c) themes. 
Power is compared and synthesized in this section. Normative power was exhibited by both classroom teachers consistently. Classroom One teacher did not exhibit coercive power; Classroom Three teacher exhibited coercive power rarely. Interactively established contracts were exhibited by both classroom teachers consistently. Classroom One teacher exhibited charm constantly, whereas Classroom Three teacher did not exhibit charm. Table 24 illustrates the comparison of types of power exhibited, followed by a discussion related to cultural power.

Table 24

Comparison of Types of Power Exhibited in Classrooms Characterized by Rare Exclusionary Discipline

Types of Power Classroom One Classroom Three

$\begin{array}{lll}\text { Normative power } & \text { Consistently } & \text { Consistently } \\ \text { Coercive power } & \text { Not exhibited } & \text { Rarely } \\ \begin{array}{l}\text { Interactively established } \\ \text { contracts }\end{array} & \text { Consistently } & \text { Consistently } \\ \text { Charm } & \text { Constantly } & \text { Not exhibited }\end{array}$

Cultural power was rarely at play in either classroom since both teachers defined and determined the end result of academic and social interactions to prevent exclusionary discipline, a goal agreed upon by students. Disciplinary actions to prevent exclusionary discipline were more successful in Classroom One since the teacher did not use coercion but did incorporate charm which provided a space for students' voices in the classroom. 
For example, Classroom One teacher never mentioned writing referrals, but used vernacular and other types of charm both to stop unwelcome behaviors abruptly and to get students involved in the daily cooking project immediately. Not using the option of written referrals produced a fluid atmosphere of mutual interest and negotiation between the teacher and students.

On the other hand (supported by observational data), Classroom Three teacher very rarely used coercion and did not exhibit charm, but he provided a space for students' voices during class. For example, Classroom Three teacher mentioned calling home or sending a student out of the room for a breather only when he thought students needed a reminder that he did not allow their talk to bother others too much or veer too far from the Science topic. Providing behavior and academic reminders that did not lead to referrals produced a negotiable atmosphere that acknowledged students' families (Weinstein et al., 2004). Academic interactions increased in both classes by not focusing on exclusionary discipline, allowing students to talk within limits, and by providing motivational rewards during class. For example, the motivation and reward of food was a natural consequence of doing the vocabulary and following the recipes in Classroom One. The prospect of getting candy was motivation for completing the daily Science work in Classroom Three.

\section{Roles}

Teachers' and students' roles are compared and synthesized in this section. Teachers' roles were similar in their support of students, yet differed in executionClassroom One teacher through humor and friendliness; Classroom Three teacher through seriousness and aloofness. Students' roles in Classrooms One and Three 
exhibited agency to maintain identity. Table 25 summarizes the roles exhibited, followed by a discussion.

Table 25

Comparison of Types of Roles Exhibited in Classrooms Characterized by Rare Exclusionary Discipline
Roles
Classroom One
Classroom Three

Teacher Roles

Humor and friendliness for

Seriousness and aloofness support of students for support of students

Student Roles

Agency to maintain identity

Agency to maintain identity

Both teachers supported students' academic and social behaviors through the various

Discourses and roles they had acquired in their educational and professional lives (Cochran-Smith \& Zeichner, 2005; Darling-Hammond \& Bransford, 2005; Gee, 1992;

Hollins \& Guzman, 2005). Based on the scope of these two teachers' experiences and conceptualization of alternative education students as people who deserve the chance to stay in school, teachers' roles provided spaces for students' roles and Discourses to emerge by prioritizing tolerance, respect, and learning academic material over exclusionary discipline in the classroom (Landorf, Rocco, \& Nevin, 2007; Noguera, 2003). Students negotiated both teachers' attempts to produce socially just practices and opportunities to learn through agency, roles, and Discourses (Gutiérrez, 1992; Gutiérrez, Bequedano-Lopez, \& Tejeda, 2003; Milner, 2006) that were indicative of their lives and acceptable identities outside of school. As a result, they ultimately accepted the opportunity to learn the academic material that each teacher provided them. 


\section{Themes}

Themes are compared and synthesized in this section. Teacher resistance was exhibited in Classroom One to maintain control, teach Culinary Arts, and prevent exclusionary discipline successfully; teacher resistance was not exhibited in Classroom Three. Student resistance was not exhibited in either Classroom One or Three. Teacher accommodation was exhibited in Classroom One through flexibility to maintain control, teach Culinary Arts, and prevent exclusionary discipline. Student accommodation was also exhibited in Classroom One through flexibility to maintain identity and agency, communicate, and prevent exclusionary discipline. Classroom Three teacher exhibited accommodation by teaching Science and enforcing PBS to maintain control, balance learning and needs, and prevent exclusionary discipline. Classroom Three students did not exhibit accommodation. Classroom One teacher and students exhibited conformism to vocational education, banking education, and Culinary Arts as motivation and reward - the teacher to maintain control, teach Culinary Arts, and prevent exclusionary discipline; the students to prevent exclusionary discipline and reap rewards. Classroom Three teacher and students exhibited conformism to banking education- the teacher to maintain control, teach Science, and prevent exclusionary discipline; the students to prevent exclusionary discipline and learn Science.

Classroom One teacher and students exhibited negotiation - the teacher to maintain control, teach their content, and prevent exclusionary discipline in an enjoyable environment by varying talk, humor, and social distance; the students to prevent exclusionary discipline, maintain identity and agency also by varying talk, humor, and social distance. Classroom Three teacher and students exhibited negotiation- the teacher 
to maintain control, teach Science, and prevent exclusionary discipline in an enjoyable environment that balances students' needs through cognizance and patience; students for the purpose of preventing exclusionary discipline and learning Science in an enjoyable manner as well as achieving agency, identity, and personal communication goals. Table 26 summarizes the themes exhibited, followed by a discussion.

Table 26

Comparison of Themes Exhibited in Classrooms Characterized by Rare Exclusionary Discipline

Themes

Classroom One

Classroom Three

Teacher resistance

Student resistance

Teacher accommodation

Student accommodation

Teacher Conformism
To maintain control, teach content, prevent exclusionary discipline

Not exhibited

To maintain control, teach Culinary Arts, and prevent exclusionary discipline through flexibility

To maintain identity and agency, communicate, and prevent exclusionary discipline through flexibility

To vocational education, banking education, and Culinary Arts as motivation and reward to prevent exclusionary discipline, teach Culinary Arts, and maintain control
Not exhibited

Not exhibited

To maintain control, balance learning and needs, and prevent exclusionary discipline by teaching Science and enforcing PBS

Not exhibited

To banking education to maintain control, teach Science, and prevent exclusionary discipline the students to prevent exclusionary discipline and learn Science 


\begin{tabular}{|c|c|c|}
\hline Themes & Classroom One & Classroom Three \\
\hline Student Conformism & $\begin{array}{l}\text { To vocational education, } \\
\text { banking education, and } \\
\text { Culinary Arts as motivation } \\
\text { and reward to prevent } \\
\text { exclusionary discipline and } \\
\text { reap reward }\end{array}$ & $\begin{array}{l}\text { To banking education to } \\
\text { prevent exclusionary } \\
\text { discipline and learn Science }\end{array}$ \\
\hline Teacher Negotiation & $\begin{array}{l}\text { To maintain control, teach } \\
\text { Culinary Arts, and prevent } \\
\text { exclusionary discipline in } \\
\text { an enjoyable environment } \\
\text { by varying talk, humor, and } \\
\text { social distance }\end{array}$ & $\begin{array}{l}\text { To maintain control, teach } \\
\text { Science, and prevent } \\
\text { exclusionary discipline in } \\
\text { an enjoyable environment } \\
\text { that balances students' } \\
\text { needs through cognizance } \\
\text { and patience }\end{array}$ \\
\hline Student Negotiation & $\begin{array}{l}\text { To prevent exclusionary } \\
\text { discipline, maintain identity } \\
\text { and agency by varying talk, } \\
\text { humor, and social distance }\end{array}$ & $\begin{array}{l}\text { To prevent exclusionary } \\
\text { discipline, enjoy class, } \\
\text { agency, identity, personal } \\
\text { communication goals }\end{array}$ \\
\hline
\end{tabular}

Both teachers upheld conventional views of learning in mainstream educational ideology about transmission-style teaching by how they were prone to lecturing, authoritarianism, competition, and passivity (Giroux, 1991, 1994, 2001; McLaren, 2003). However, their classroom practices provided some room for student input and negotiations about what was being learned academically and socially and how that learning was accomplished. As a result, students negotiated and participated in their own way, teachers and students were on similar wavelengths about academic and social issues, and teachers accomplished most of their academic and behavioral classroom goals. Even though both teachers were successful in achieving an enjoyable atmosphere in their classroom, Classroom One 
teacher was most successful in reducing oppressive conditions, because he resisted school PBS expectations by never mentioning or using exclusionary discipline in his classroom.

Classrooms Characterized by Frequent Versus Rare Exclusionary Discipline

This section compares and synthesizes findings in classrooms characterized by frequent exclusionary discipline $(\mathrm{C} 2, \mathrm{C} 4)$ versus rare exclusionary discipline $(\mathrm{C} 1, \mathrm{C} 3)$ in three sections: (a) power, (b) roles, and (c) themes.

Power

Power is compared and synthesized in this section. Classrooms are designated by classroom number (e.g., C1, C2, C3, C4). Normative power was exhibited by all classroom teachers in an attempt to maintain control, albeit in various ways, to varying degrees, and for different purposes_-classrooms with rare exclusionary discipline exhibited normative power consistently; classrooms with frequent exclusionary discipline exhibited it continually (C4) and sporadically (C2). Coercive power was rarely (C3) or not (C1) exhibited in classrooms with rare exclusionary discipline; coercive power was exhibited regularly but in different ways in classrooms with frequent exclusionary discipline_explicitly and immediately (C4) or implicitly and gradually (C2).

Interactively established contracts were exhibited consistently in classrooms characterized by rare exclusionary discipline $(\mathrm{C} 1, \mathrm{C} 3)$. However, classrooms characterized by frequent exclusionary discipline exhibited interactively established contracts in varying degrees and in different ways. In C2, interactively established contracts were initiated by students continually and defined the classroom norms. In C4, interactively established contracts were exhibited rarely in order to document student whereabouts and behavior (e.g., point sheets, attendance). Charm was exhibited only in 
$\mathrm{C} 1$, characterized by rare exclusionary discipline. Charm was not exhibited in $\mathrm{C} 3$, characterized by rare exclusionary discipline, or $\mathrm{C} 2$ and $\mathrm{C} 4$, both characterized by frequent exclusionary discipline. Table 27 summarizes the types of power exhibited, followed by a discussion related to cultural power.

Table 27

Comparison of Types of Power in Classrooms Characterized by Frequent Versus Rare Exclusionary Discipline

\begin{tabular}{lll} 
Types of Power & $\begin{array}{l}\text { Classroom Two; } \\
\text { Classroom Four } \\
\text { (Frequent) }\end{array}$ & $\begin{array}{l}\text { Classroom One; } \\
\text { Classroom Thre } \\
\text { (Rare) }\end{array}$ \\
\hline Normative Power & $\begin{array}{l}\text { Sporadically; } \\
\text { Continually }\end{array}$ & $\begin{array}{l}\text { Consistently; } \\
\text { Consistently }\end{array}$ \\
Coercive Power & $\begin{array}{l}\text { Implicitly and gradually; } \\
\text { Explicitly and immediately }\end{array}$ & $\begin{array}{l}\text { Not exhibited; } \\
\text { Rarely }\end{array}$ \\
Interactively established & $\begin{array}{l}\text { Student initiated } \\
\text { continually, teacher } \\
\text { encouraged continually for } \\
\text { student rehabilitation; } \\
\text { Rarely for student } \\
\text { documentation }\end{array}$ & $\begin{array}{l}\text { Consistently; } \\
\text { Consistently }\end{array}$ \\
& $\begin{array}{l}\text { Not exhibited, } \\
\text { Not exhibited }\end{array}$ & $\begin{array}{l}\text { Constantly; } \\
\text { Not exhibited }\end{array}$
\end{tabular}

Cultural power was more at play in classrooms with frequent (versus rare) exclusionary discipline because teachers determined in advance that they would use referrals, which was incongruent with students' ultimate goals of not getting suspended. Disciplinary actions preceding exclusionary discipline were less successful in classrooms characterized by frequent (versus rare) exclusionary discipline because teachers used 
more coercion and less charm that conflicted with students' cultural behaviors and humor. Even though C3 teacher did not use charm, his consistent exhibition of other types of power outweighed the use of cultural power. As a result of heightened cultural power, coercion, and use of written referrals (from high to low- 4 , $\mathrm{C} 2, \mathrm{C} 3, \mathrm{C} 1$ ), academic interactions that included students' voices were reduced, respectively (from most to least $-\mathrm{C} 4, \mathrm{C} 2, \mathrm{C} 3, \mathrm{C} 1)$.

\section{Roles}

Teachers' and students' roles are compared and synthesized in this section. Teachers' roles in classrooms characterized by frequent exclusionary discipline were similar in their surveillance of students but were exhibited in different ways - empathetic practice $(\mathrm{C} 2)$ and rigid expertise $(\mathrm{C} 4)$. On the other hand, classrooms characterized by rare exclusionary discipline teachers' roles were similar in their support of students but were exhibited in different ways-with humor and friendliness (C1) and with seriousness and aloofness (C3). Students' roles across classrooms exhibited agency to maintain identity. Table 28 summarizes the roles exhibited, followed by a discussion. 
Table 28

Comparison of Types of Roles Exhibited in Classrooms Characterized by Frequent Versus Rare Exclusionary Discipline

\begin{tabular}{lll} 
Roles & $\begin{array}{l}\text { Classroom Two; } \\
\text { Classroom Four } \\
\text { (Frequent) }\end{array}$ & $\begin{array}{l}\text { Classroom One; } \\
\text { Classroom Three } \\
\text { (Rare) }\end{array}$ \\
\hline Teacher Roles & $\begin{array}{l}\text { Empathetic practice for } \\
\text { surveillance; } \\
\text { Rigid expertise for } \\
\text { surveillance }\end{array}$ & $\begin{array}{l}\text { Humor and friendliness for } \\
\text { support of students; } \\
\text { Seriousness and aloofness } \\
\text { for support of students }\end{array}$
\end{tabular}

Student Roles

Agency to maintain identity

Agency to maintain identity

Teachers' roles in classrooms with rare (versus frequent) exclusionary discipline more successfully accomplished both teacher and student classroom goals related to (a) exclusionary discipline, (b) teaching and learning content area material, and (c) teacherstudent relationships. Students' roles in both types of classrooms were based on their desire and agency to maintain what they envisioned to be a good academic or social image; however, students' academic and social identity development was more successful in classrooms with rare (versus frequent) exclusionary discipline (from more to less successful- $\mathrm{C} 1, \mathrm{C} 3, \mathrm{C} 2, \mathrm{C} 4)$.

\section{Themes}

Themes are compared and synthesized in this section. Teacher resistance was not exhibited in classrooms characterized by frequent exclusionary discipline $(\mathrm{C} 2, \mathrm{C} 4)$ or in C3, characterized by rare exclusionary discipline. However, teacher resistance was exhibited by $\mathrm{C} 1$, characterized by rare exclusionary discipline to maintain control, teach 
Culinary Arts, and prevent exclusionary discipline successfully. Student resistance was not exhibited in classrooms characterized by rare exclusionary discipline $(\mathrm{C} 1, \mathrm{C} 3)$. However, student resistance was exhibited continually in classrooms characterized by frequent exclusionary discipline - to steer the topic away from academics during class (C2); to skip class, for disruptive removal from class, or to comply silently with teacher expectations (i.e., classroom culture, norms, and values) during class (C4).

Teacher accommodation was exhibited in all classrooms, albeit in various ways, for different purposes. In classrooms with frequent exclusionary discipline, teacher accommodation was exhibited by varying surveillance tactics to enforce rules and maintain an environment safe for talk, pending exclusionary discipline (C2) or by acting as an authority figure to maintain control, teach in an orderly climate, and enforce expectations, pending exclusionary discipline (C4). In classrooms with rare exclusionary discipline, teacher accommodation was exhibited through flexibility to maintain control, teach Culinary Arts, and prevent exclusionary discipline (C1) or to maintain control, balance learning and needs, and prevent exclusionary discipline by teaching Science and enforcing PBS (C3). Student accommodation was exhibited differently across classrooms. In classrooms with frequent exclusionary discipline, student accommodation was not exhibited in $\mathrm{C} 2$, but exhibited continually as compliant subjects in $\mathrm{C} 4$. In classrooms with rare exclusionary discipline, student accommodation was not exhibited in $\mathrm{C} 3$, but was exhibited in $\mathrm{C} 1$ through flexibility to maintain identity and agency, communicate, and prevent exclusionary discipline.

Teacher conformism to dominant ideology in education was exhibited across all classrooms, albeit in different ways and for different purposes. In classrooms with 
frequent exclusionary discipline, teacher conformism was exhibited continually through deference to academic and behavior authority, pending exclusionary discipline (C2) or through banking education and cultural deficit orientation, also pending exclusionary discipline (C4). In classrooms with rare exclusionary discipline, teacher conformism was exhibited to prevent exclusionary discipline, teach Culinary Arts, and maintain control (C1) and to prevent exclusionary discipline, teach Science, and maintain control (C3). Student accommodation was not exhibited in classrooms with frequent exclusionary discipline $(\mathrm{C} 2, \mathrm{C} 4)$. However, in classrooms with rare exclusionary discipline $(\mathrm{C} 1, \mathrm{C} 3)$, student accommodation was exhibited to prevent exclusionary discipline, reap rewards, and learn content.

Teacher negotiation was not exhibited in classrooms with frequent exclusionary discipline. However, in classrooms with rare exclusionary discipline, teacher negotiation was exhibited by varying talk, humor, and social distance to maintain control, teach Culinary Arts, and prevent exclusionary discipline in an enjoyable environment (C1) and through cognizance and patience to maintain control, teach Science, and prevent exclusionary discipline in an enjoyable environment that balances students' needs (C3). Student negotiation was not exhibited in classrooms with frequent exclusionary discipline. However, in classrooms with rare exclusionary discipline, student negotiation was exhibited by varying talk, humor, and social distance to prevent exclusionary discipline, maintain identity and agency $(\mathrm{C} 1)$ and to prevent exclusionary discipline, enjoy class, and achieve agency, identity, and personal communication goals (C3). Table 29 summarizes the themes exhibited, followed by a discussion. 
Table 29

Comparison of Themes Exhibited in Classrooms Characterized by Frequent Versus Rare Exclusionary Discipline

Themes

Classroom Two;

Classroom Four

(Frequent)
Classroom One;

Classroom Three

(Rare)
Teacher Resistance

Student Resistance

Teacher Accommodation

Student Accommodation
Not exhibited;

Not exhibited

Continually to change topic away from academics during class;

Continually to skip class, disruptive removal from class, or silence during class

Varying tactics to enforce rules, maintain environment safe for talk, pending exclusionary discipline; Authority figure to maintain control, teach in orderly climate, enforce expectation, pending exclusionary discipline

Not exhibited; Continually as compliant subjects
To maintain control, teach content, prevent exclusionary discipline; Not exhibited

Not exhibited;

Not exhibited

To maintain control, teach Culinary Arts, and prevent exclusionary discipline through flexibility; To maintain control, balance learning and needs, and prevent exclusionary discipline by teaching Science and enforcing PBS

To maintain identity and agency, communicate, and prevent exclusionary discipline through flexibility; Not exhibited 


\begin{tabular}{ll} 
Themes & $\begin{array}{l}\text { Classroom Two; } \\
\text { Classroom Four } \\
\text { (Frequent) }\end{array}$ \\
\hline Teacher Conformism & $\begin{array}{l}\text { Continually through } \\
\text { deference to academic and } \\
\text { behavior authority, pending } \\
\text { exclusionary discipline; } \\
\text { Continually through } \\
\text { banking education and } \\
\text { cultural deficit orientation, } \\
\text { pending exclusionary } \\
\text { discipline }\end{array}$
\end{tabular}
Classroom One; Classroom Three (Rare)

To vocational education, banking education, and Culinary Arts as motivation and reward to prevent exclusionary discipline, teach Culinary Arts, and maintain control; To banking education to maintain control, teach Science, and prevent exclusionary discipline

Student Conformism

Not exhibited; Not exhibited

To vocational education, banking education, and Culinary Arts as motivation and reward to prevent exclusionary discipline and reap reward; To banking education to prevent exclusionary discipline and learn Science

Teacher Negotiation Not exhibited;

To maintain control, teach Not exhibited Culinary Arts, and prevent exclusionary discipline in an enjoyable environment by varying talk, humor, and social distance; To maintain control, teach Science, and prevent exclusionary discipline in an enjoyable environment that balances students' needs through cognizance and patience 


\begin{tabular}{lll} 
Themes & $\begin{array}{l}\text { Classroom Two; } \\
\text { Classroom Four } \\
\text { (Frequent) }\end{array}$ & $\begin{array}{l}\text { Classroom One; } \\
\text { Classroom Three } \\
\text { (Rare) }\end{array}$ \\
\hline
\end{tabular}

Student Negotiation

Not exhibited;

Not exhibited
To prevent exclusionary discipline, maintain identity and agency by varying talk, humor, and social distance; To prevent exclusionary discipline, enjoy class, agency, identity, personal communication goals

Teachers in classrooms characterized by frequent (versus rare) exclusionary discipline abided more closely to conventional views about fixing the academic (especially reading and writing) and behavioral deficits of alternative education students (Carpenter, Ramirez, \& Severn, 2006; Hamilton, 2000; Jimenez, 2004; Lee, 2005; Strickland \& Alvermann, 2004). This finding is relevant to the fact that Classrooms Two and Four were language arts classrooms which are monitored more closely and held accountable for FCAT scores. As a result, students in classrooms characterized by frequent (versus rare) exclusionary discipline resisted teachers' expectations more, exhibited less learning of academic material, and experienced more exclusionary discipline. Overall, classrooms characterized by rare (versus frequent) exclusionary discipline had teachers who were more successful in achieving negotiable, flexible, and academic student identities (from more to less successful $-\mathrm{C} 1, \mathrm{C} 3, \mathrm{C} 2, \mathrm{C} 4)$ since they were more flexible in challenging or resisting to some extent the constraints that they decided they could not live with each day at school. 


\section{Summary}

This chapter presented a comparison and synthesis of findings from classrooms characterized by frequent, rare, and frequent versus rare exclusionary discipline. Cultural power was at play in each classroom characterized by frequent exclusionary discipline, because both teachers defined and determined the end result of academic and social interactions through exclusionary discipline rather than through charm. Roles exhibited showed that both teachers monitored students' academic and social behaviors closely, and students resisted both teachers' academic and disciplinary practices. Both teachers strictly abided by conventional views of learning that sustained mainstream educational ideology about alternative education students as those who needed fixing, leaving little room for student input or negotiation about what was being learned.

In both classrooms characterized by rare exclusionary discipline, cultural power was rarely at play since both teachers defined and determined the end result of academic and social interactions to prevent exclusionary discipline, a goal agreed upon by students. Both teachers supported students' academic and social behaviors through their roles that provided room for students to give input and/or negotiate what was being learned. Both teachers upheld conventional views of learning in mainstream educational ideology about transmission-style teaching by how they were prone to lecturing, authoritarianism, competition, and passivity, but these views were also held by students.

Overall, cultural power was more at play; teachers' and students' roles were less successfully accomplished; and students resisted teachers' expectations more and exhibited less learning of academic material in classrooms with frequent (versus rare) exclusionary discipline because teachers determined in advance that they would use 
referrals, which was incongruent with students' ultimate goals of not getting suspended. This finding is relevant to the fact the classrooms with frequent exclusionary discipline were language arts classrooms which are monitored more closely and held accountable for FCAT scores. Additionally, classrooms characterized by rare (versus frequent) exclusionary discipline had teachers who were more successful in achieving negotiable, flexible, and academic student identities since they were also more flexible in challenging or resisting system constraints. Chapter 9 provides a summary of the study, responses to the research questions, implications for teacher education, and recommendations for research and policy. 


\section{CHAPTER IX}

\section{SUMMARY, RESPONSES TO RESEARCH QUESTIONS, IMPLICATIONS, AND RECOMMENDATIONS}

This chapter consists of five sections: (a) summary of the study, (b) responses to research questions; and (c) implications for teacher education, (d) recommendations for research and policy, and (e) conclusion.

\section{Summary of the Study}

This study examined specific teacher-student social interactions within classrooms that influence teachers' decisions to use or not use exclusionary discipline. Four themes of social interaction emerged: (a) resistance, (b) accommodation, (c) conformism, and (d) negotiation with terminology adapted from Giroux's (2001) theory of resistance in education and Third Space theory (Gutierrez, 2008). Four types of power, derived from Carspecken's (1996) typology of interactive power, were analyzed: (a) normative, (b) coercive, (c) interactively established contracts, and (d) charm. Themes and power were exhibited and integrated in different ways, to varying degrees, and for different purposes to influence each teacher's use or non-use of exclusionary discipline. Based on the integration of themes of social interaction and types of power, teachers' and students' roles were found and named and cultural power was discovered. Teachers' decision making and actions were guided by the constraints of dominant alternative education ideology, The School ideology, and incongruent student-teacher goals. Overall, findings showed that more exclusionary discipline and fewer academic interactions occurred in classrooms with more cultural power at play (see Figure 2). 


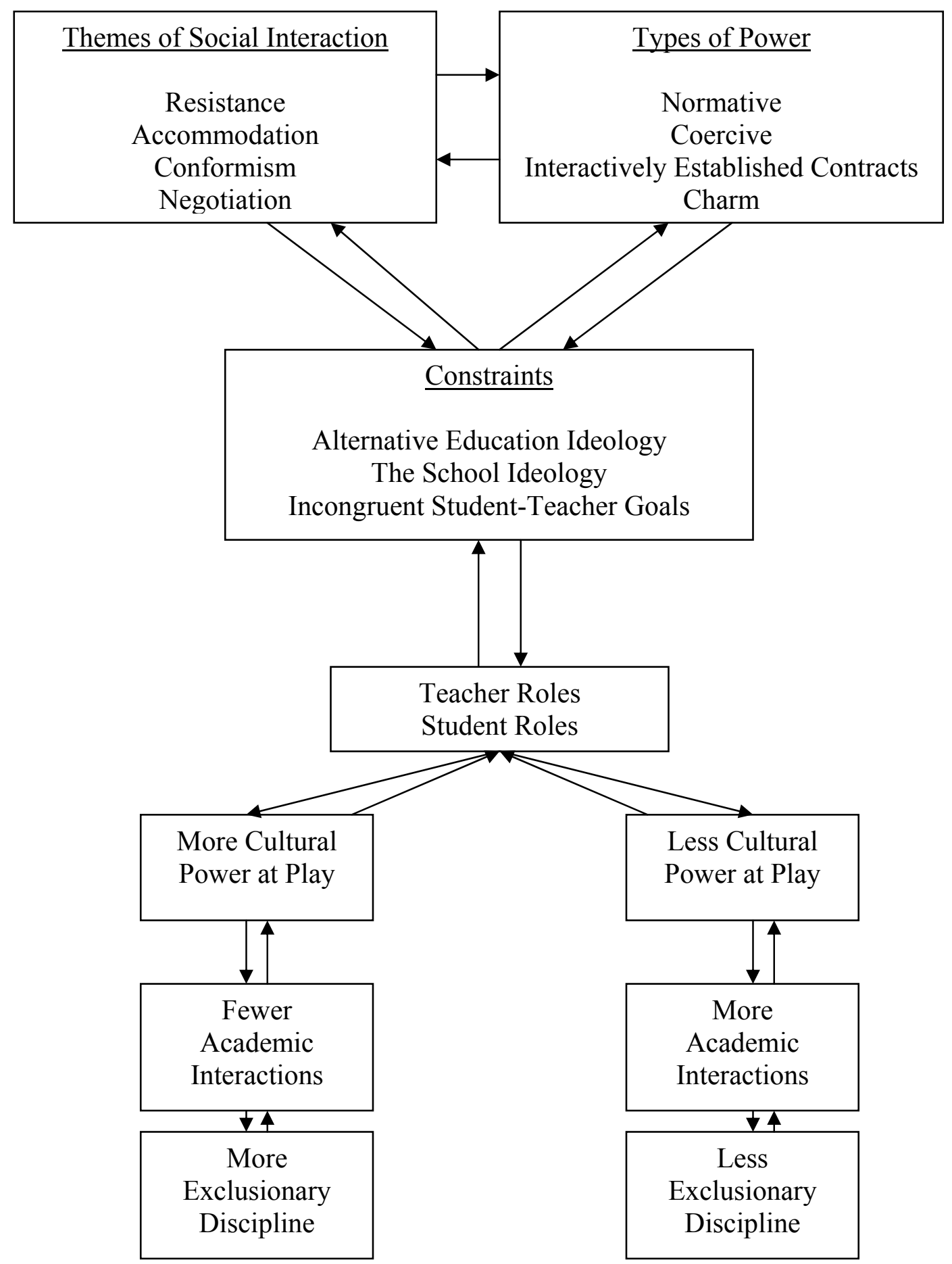

Figure 2. Interaction of themes of social interaction, types of power, and constraints that result in the use or non-use of exclusionary discipline. 


\section{Responses to the Research Questions}

Responses to the research questions are provided in this section. Discussion begins with responses to the secondary research questions to gain a clearer understanding of potential spaces of transformation within and across classrooms followed by a response to the central research question to gain a more holistic understanding of what happens in alternative education classrooms characterized by frequent and rare exclusionary discipline.

\section{Secondary Research Question 1:}

What verbal and nonverbal interactions typify the learning processes and exclusionary discipline practices within the selected classrooms?

Learning processes in all classrooms consisted primarily of transmission-style teaching including lecturing, authoritarianism, competition, and passivity (Giroux, 1991, 1994, 2001; McLaren, 2003). However, when teachers' classroom practices provided some room for student input, negotiations, and enjoyment about what was being learned, more congruent teacher-student relationships and accomplishment of academic goals resulted. When teachers' classroom practices provided no room for student input about

what was being learned, incongruent teacher-student relationships, student resistance, and fewer academic goals were accomplished.

Disciplinary actions preceding exclusionary discipline were less successful in classrooms characterized by frequent (versus rare) exclusionary discipline since teachers used more coercion and less charm, which conflicted with students' cultural behaviors and humor. Therefore, disciplinary actions were more successful and reduced the need 
for exclusionary discipline when further removed from coercion and were most successful when they also incorporated charm, as found with Mr. Jenkins.

Secondary Research Question 2: What cycles of social and academic identity reproduction occur within the selected classrooms?

As a result of heightened cultural power, coercion, and predetermined use of referrals by teachers, academic interactions were reduced, and resistant student identities were increased in classrooms characterized by frequent (versus rare) exclusionary discipline. On the other hand, as a result of more teacher flexibility in challenging or resisting to some extent the school and system constraints, negotiable, flexible, and academic student identities were more successfully achieved in classrooms characterized by rare (versus frequent) exclusionary discipline.

Secondary Research Question 3:

How does each teacher explain what happens in his or her classroom regarding learning processes, classroom interactions, and exclusionary discipline practices?

All teachers' explanations were founded in dominant ideology, based in conventional views of teaching and learning and cultural deficit views of behavior and intelligence about alternative education students, and enacted in their roles. Teachers explained how and why they interacted with students in different situations, based on how they had learned to handle situations in their previous classroom experiences.

Teachers' educational and vocational backgrounds pervaded their decision making in the classroom about academic and behavior issues. For example, the teacher who majored in psychology used counseling techniques in her classroom. The teacher who had a background in Culinary Arts set up his classroom to resemble a professional 
kitchen with a chef and assistants. The teacher who majored in Science conducted (or desired to conduct) labs and building projects when (if) supplies were available in his classroom. The teacher who was an ex-police officer and English literature expert combined his experiences to develop a tightly run classroom environment. Since all teachers had no previous knowledge or experience of alternative education before working at The School, their explanations of student behavior or academic identities were constructed from dominant ideology about alternative education students as socially and academically unsuccessful, bad, or unable to change. However, Mr. Jenkins showed some resistance to dominant ideology by expecting students to change their behavior in his classroom with successful results as noted in his non-use of exclusionary discipline.

\section{Secondary Research Question 4:}

How do students explain what happens in their classrooms regarding learning processes, classroom interactions, and exclusionary discipline practices?

Students explained what happens in their classrooms through the lens of their personal, educational, and cultural experiences. All students' explanations were founded in dominant ideology, based in conventional views of teaching and learning in classrooms, and enacted in their roles. Students explained why they interacted with the teacher and each other in different situations, based on how they had learned to handle situations in their previous classroom experiences. Students' decision making emerged from years of experiences in nondominant communities and in alternative education. Even though explanations were often demonstrated silently or with few words, students were quick to assess whether or not educational practices supported their academic and social goals and acted accordingly to protect their self-images. 


\section{Secondary Research Question 5:}

What is the relationship between classroom interactions, learning processes, and exclusionary discipline practices across the selected classrooms?

The relationship between classroom interactions, learning processes, and exclusionary discipline practices across classrooms was guided by the mix of teachers' and students' background experiences, classroom goals, and definition of how to accomplish them. Before teaching at The School, all teachers had preconceived classroom goals and implicit theories of how to accomplish them, based on their educational degrees, cultural and vocational backgrounds, and individual personalities. However, previous educational, vocational, and cultural experiences did not prepare them to teach alternative education students in alternative education schools. Therefore, all teachers at The School were forced to learn how to refine their classroom goals in order to maintain control of their classrooms within system and school constraints while teaching their content.

Teachers who decided beforehand that exclusionary discipline was not an optional classroom goal for them tended to overlook what other teachers would define as disruptive behavior and found ways of supporting students. For example, Mr. Frederek voiced the political ramifications of using written referrals that he learned after becoming a teacher at The School. Therefore, he decided that he would not use referrals unless someone was getting hurt or bothered extensively. By solving problems himself in the classroom, he could support his students. Mr. Frederek and Mr. Jenkins developed opportunities for merging successful teacher-student classroom interactions and as a result, more academic teaching and learning was accomplished and less cultural power 
was at play. On the other hand, teachers who decided beforehand that exclusionary discipline was the ultimate option for disruptive behavior in their classroom used various surveillance tactics. For example, Mr. Glass discussed how students would eventually get suspended if they did not immediately cooperate with his expectations upon entrance to the classroom. Overall, fewer opportunities were available in Mr. Glass's and Ms. Gomez's classrooms for merging successful teacher-student classroom interactions, less academic teaching and learning was accomplished, and more cultural power was at play. During the process and with the options available in teachers' minds, they all learned and expressed in their interviews that successful teaching and learning of their content was not guaranteed and often disappointing.

Before entering The School, students were already experienced with alternative education (having been sent to alternative educations schools more than once as an exclusionary discipline outcome) and the alternative education student population, most of whom were African American or Latino just like them. Even though every classroom in this study involved various nuances of teacher-student interactions, students already knew from experience how system constraints worked to rotate them between schools, how to be a student by being quiet or how to get suspended by being disruptive, how to assess the fairness and intricacies of teachers' classroom goals and limits, and when and how to push teachers' goals and limits to liven up the classroom if they deemed it boring. These implicit theories were acknowledged in the interviews and/or in classroom interactions. For example, Dann told Ms. Gomez outright that he did not like the decisions that administration made about student placement in classes. He believed he was going to get in trouble in this classroom and get suspended. Throughout classroom 
observations in Classroom Two, student talk took for granted that written referrals and suspension were par for the course and the only option in alternative education for dealing with disruptive behavior. They knew from experience that exclusionary discipline was the underlying theme of the alternative education system.

The students also knew of the prevalent belief that African American or Latino students who were sent to alternative education were bad and could not or would not change. For example, students in Classroom Three discussed how people thought they were bad and that was the reason they could not liven up the Science classroom with projects such as the dissection of frogs. However, they brainstormed multiple other suggestions for learning more Science in active and fun ways. Students also knew how to weigh the options of whether to work within the system or not by how it damaged or supported their self-image in the classroom situation. During Classroom One interview, students explicitly compared and contrasted how Mr. Frederek (who they had in another classroom period) was a good, patient teacher who made them feel smart versus how another teacher who was not in the study called students names and made them feel dumb. Therefore, they acted differently in classrooms depending on the nuances of the classroom interactions.

Students also knew that being silent and compliant signaled to most teachers that they were working within the system constraints. Being disruptive or loud may signal they were not working within the system constraints depending on the situation and often resulted in exclusionary discipline, especially if they misjudged the teachers' limits. Therefore, when students came to The School, they were quick to assess as claimed in the interviews and/or during classroom observations each teacher's expectations or 
classroom culture, norms, and values related to exclusionary discipline and each teacher's limits. If a teacher's classroom goals and limits seemed fair and prevented exclusionary discipline, they felt supported and were more willing to work with them. Students learned that in classrooms with teachers who rarely used exclusionary discipline, they were given more opportunities to promote successful social and academic identities, learn something new, and be rewarded in the classroom. For example, Classroom Three students interjected multiple Science terms and background knowledge during classroom discussions and the interview. Classroom One students conversed in Culinary Arts lingo and worked on cooking projects for periods of time independently. Students had learned something related to the academic topic in both of these classrooms.

On the other hand, students learned that in classrooms with teachers who frequently used exclusionary discipline, which was more typical in alternative education, they had few opportunities for successful identity development or enjoyment in the classroom. For example, Dann stated during the observations that he had already written about the FCAT prompt on the board in Classroom Two. He pursued disruptive behavior, never doing the assigned work. Students also learned various tactics for resistance, including skipping class, being disruptive in class so they would be removed, giving teachers a hard time so they could get a humorous reaction, or being silent so they could get rewarded with points and grades long enough to go back to their regular school in Classroom Four. Students' voices in both Classroom Two and Four were either silent or unrelated to the topic of Language Arts.

A fine line exists between resistance and accommodation when students exhibit silence. Being silent in class, in this case, is more than accommodation, because the 
students are using their silence to "voice" critique, self-reflection, and struggle against the classroom norms rather than just coping with them. If they were exhibiting accommodation, or coping with slight resistance, students would show evidence of implicitly sharing some of Mr. Glass's classroom goals. However, hermeneutic inferencing of students' demeanors, expressions, and explanations did not reveal that they shared the goals of students being kicked out of class or being given answers to a test.

The findings showed that the two classrooms with the highest levels of referrals were both classes that were subject to FCAT testing. In contrast, the classroom with the lowest level of referrals was a vocational class rather than an academic one. This is a relevant point and carries several implcations. First, teachers and students in Language Arts classrooms hold certain assumptions about the course. Teachers are expected to teach so that students can pass the FCAT; students are supposed to learn the information and pass the FCAT. However, in alternative education settings, students are not only disproportionately caught in the exclusionary discipline cycle, but are also involved in the school failure cycle. This puts teachers on alert. Dominant ideology about passing highstakes tests provides no windows of opportunity for those who do not comply. Thus, the need of Language Arts teachers to maintain control in their classroom so that they can teach their class to pass the FCAT is heightened in alternative education.

On the other hand, the vocational classroom teacher is not expected to teach his students to pass the FCAT, and thus has more leeway in how he runs his class. Mr. Jenkins did include journal writing of glossary definitions related to Culinary Arts each day, but this was done to allow him time to get organized and to get the students settled in before the cooking projects began for the day. Dominant ideological expectations were 
entirely different in Culinary Arts versus Language Arts classrooms. Future studies may address this limitation by selecting only Language Arts classrooms for research in order to focus on the implications of high-stakes testing in regards to exclusionary discipline.

\section{Central Research Question:}

In relation to classroom interactions in alternative education settings, what accounts for teachers' use or non-use of exclusionary discipline with students?

In relation to classroom interactions in alternative education settings, teachers' and students' decision making and subsequent actions about teaching and learning were guided and differentiated by (a) dominant alternative education ideology, (b) The School ideology, and (c) students' and teachers' background experiences.

\section{Dominant Alternative Education Ideology}

Dominant ideology guides decision making about teaching and learning across all classrooms through its pervasive hegemonic qualities. Current dominant ideology sustains an educational ecology resulting in a "tight-tight culture" (Gutierrez, 2006, p. 1) of accountability in which low-performing schools with low high-stakes standardized test scores are more heavily monitored than high-performing schools through policies that mandate "unprecedented surveillance and regulation of student and teacher behavior and practices" (p. 1). Tight-tight school cultures of accountability disguised as educational reform "create an ethic of sanctions and failure that is difficult to overcome" (p. 1).

Dominant ideology also sustains an unnamed, unproblematized, oppressive educational ecology of alternative education which emerges from the findings of this study as a tight-tight-tight culture. The FCAT is highly monitored in Language Arts classrooms in general. When taking into consideration that the two highest levels of 
referrals, or exclusionary discipline, in this study were in the Language Arts classrooms, accountability is more tightly monitored on a third level that includes behavior. This triple (tight school culture) bind in alternative education is reminiscent of the triple quandary many African Americans have been forced to respond to historically, that of living in the African-based, mainstream Eurocentric, and oppressed minority cultures at once (Boykin, 1986). The tight-tight-tight culture in alternative education schools and classrooms makes visible the national debt that has accumulated in our educational caste system found as oppressive conditions in alternative education for mostly African American and more recently Latino students and their teachers. The suggested triple bind from the findings of this study is not reflected in Gutiérrez, Asato, Zavala, Pacheco, and Olson's (2004) original (A New Institutional Architecture) model of tight-tight culture of accountability. The shaded portions of Figure 3 depict suggested additions to Gutiérrez and colleague's (2004) original model.

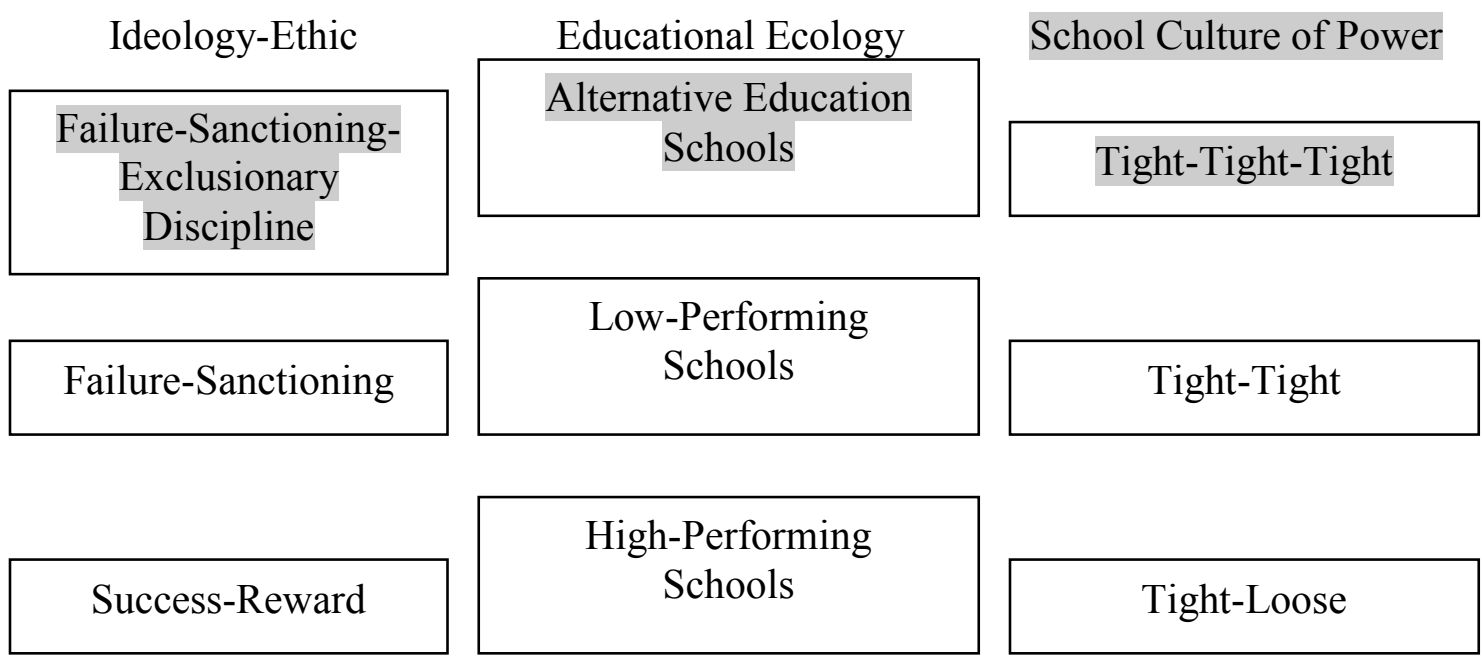

Figure 3. Triple bind of oppressive conditions in alternative education school culture

The oppressive conditions in the tight-tight-tight alternative education culture is experienced by nondominant alternative education students "whose identities fall outside 
of the norms established by standardization" (Au, 2009, p. 68) pursued by both highstakes testing and juvenile justice system norms. As a result, alternative education students are monitored severely in isolated settings to the demise of academic, especially literacy, opportunities. Ultimately, both alternative education students and their teachers lose control of classroom decision making and critical, student-centered pedagogies from the pressures of high-stakes testing (Au, 2007) and juvenile justice mandates (OJJDP, 2009). This is especially true in Language Arts classrooms that must be accountable to the FCAT. Ultimately, dominant alternative education ideology sustains banking education, cultural deficit orientation, surveillance, silencing, and exclusionary discipline of nondominant students. Teachers are isolated from quality critically reflexive teacher education that is closely aligned to alternative education students' cultural, social, and literacy practices (Stevens \& Bean, 2005). A tight-tight-tight culture denotes the triple bind of being ousted from low-performing schools to alternative education where behavioral constraints trump those of academics and both are monitored severely.

\section{The School Ideology}

The School ideology was bound by dominant public school district and juvenile justice regulations and ideology about alternative education even with administrative attempts to create camaraderie and discussion among faculty members and students, a potential space for transformation. The School ideology provided remedial academic (reading) classes for students who had failed the high-stakes tests; electives were offered as a reward to students who had already passed the tests. However, minimal opportunities for teachers or students to develop critical mindsets or participate in curricula invalidated the success of academic outcomes. For example, Mr. Frederek discussed that the topic of 
his next workshop would be how to build a rocket, which did not address his main concerns in the interview data. The School ideology also provided opportunities for students to buy into PBS, the point system for rewarding student behavior, and for faculty teamwork to refine the point system for classrooms. However, as noted in the non-use of PBS Charts for recordkeeping and the Bench for cooling off, inconsistencies in student and faculty beliefs about the equitability of the PBS system invalidated how it was carried out in classroom practice.

\section{Students' and Teachers' Background Experiences}

Students' and teachers' background experiences have been unaddressed in the literature or in practice except to note the differences between the mostly White teaching force and the increasingly diverse student population, especially in urban settings. Students' extensive alternative education and juvenile justice backgrounds were incongruent with their own nondominant cultural experiences and goals and provided few valid choices for their successful academic and social identity production. Teachers' lack of alternative education and juvenile justice experiences or teacher education prior to teaching at The School, were incongruent with their own educational and cultural backgrounds and resulted in few valid options in their minds for successful teaching practice. As a result of incongruencies, exclusionary discipline was more frequent in classrooms with more cultural power at play due to teachers' heightened needs to maintain control of their alternative education classroom while trying to successfully teach their content area (i.e., Language Arts) with no prior experiences to guide them.

This section provided responses to the secondary research questions followed by a response to the central research question for a clearer and more holistic understanding of 
what happens in alternative education classrooms characterized by frequent and rare exclusionary discipline. The next section provides implications for teacher education. Implications for Teacher Education

This section provides a discussion of implications for teacher education from a critical analysis of potential spaces for transformation of oppressive conditions from the findings of this study for (a) classrooms characterized by frequent exclusionary discipline, (b) classroom characterized by rare exclusionary discipline, and (c) classroom characterized by frequent versus rare exclusionary discipline.

\section{Classrooms Characterized by Frequent Exclusionary Discipline}

For classrooms characterized by frequent exclusionary discipline, implications for teacher education lie in transforming power relations, teachers' and students' roles, and themes of social interaction. First, the inverse relationship noted between cultural power and students' voices (i.e., input) related to academic interactions needs to be transformed. To reduce cultural power and increase students' voices, teachers can be encouraged to develop their own version of what I suggest be termed "cultural charm." Cultural charm differs from the charm exhibited by Mr. Jenkins in Classroom One. Mr. Jenkins's charm was convincing, because he consistently merged his verbal and nonverbal behavior in class with his implicit theories about what he wanted to accomplish as far as preventing students' suspension. His version of charm was also congruent with the students' cultural behaviors and humor. Because his and his students' classroom goals aligned (e.g., prevent suspension), his sarcasm was also viewed in a positive light as part of his charm. An implicit understanding existed between Mr. Jenkins and his students. His sarcasm and constantly pointing out (in both charming and normative ways) of what students could 
and could not do were tempered with students' trust in him to prevent their suspension if at all possible. Through hermeneutic inferencing of the nuances involved in Classroom One interactions, Mr. Jenkins's consistency as far as why he conducted himself as he did was viewed in a positive light because of its equitable outcomes for students.

In contrast, Ms. Gomez did not consistently merge her verbal and nonverbal behavior in class with her implicit theories about inevitable suspension. Her lack of charm, verbal and nonverbal behaviors, and classroom goals also conflicted with students' goals, cultural behaviors, and humor. For example, Ms. Gomez continually pointed out what students could and could not do. However, as exhibited by students' reoccurrences of a deaf ear to her requests and sudden interest when she filled out a referral form, students did not trust that the outcomes to what she requested from them would be advantageous for them. At one point during the classroom observations, Ms. Gomez and students discussed their poor and/or failing grades. Discussion also involved why they were in this class; they had all previously failed the FCAT. They had learned from experience that she would help them write the prompt which did not give them the impression that she believed they could do it alone. They had also learned from experience that Ms. Gomez would inevitably write a referral when she ran out of patience with students' behaviors that she previously encouraged. Therefore, because of the inconsistency between her words and actions, her words came across as begging and pleading before she finally wrote the referral. Through hermeneutic inferencing of the nuances involved in Classroom Two interactions, Ms. Gomez's inconsistency, as far as why she conducted herself as she did, was viewed in a negative light because of the inequitability of outcomes for students. 
Similarly, Mr. Glass did not consistently merge his verbal and nonverbal behaviors with his implicit theories about teaching AP English Literature to his students in alternative education and enforcement of his expectations about disruptive behavior with immediate exclusion from class. He voiced during the interview that he usually threw students out of class in the beginning so that he could teach to students who wanted to learn and would listen. As a result, his attempts to use a charming voice tone during disruptive situations observed came across as superficial or pretentious. Additionally, through hermeneutic inferencing of the nuances invoved in Classroom Four interactions, Mr. Glass's attempts at humor came across as misplaced sarcasm and, based on the lack of discernment or no response from students, his humor was incongruent with students' cultural behaviors and humor. Therefore, Mr. Glass's inconsistency, as far as why he conducted himself as he did, was viewed in a negative light because of the inequitability of outcomes for students.

Nonetheless, charm as exhibited by Mr. Jenkins was not what I term cultural charm. Even though his charm was congruent with students' cultural behaviors and humor, he did not build on students' cultural behaviors and backgrounds to develop curricula. Therefore, to distinguish between charm and cultural charm, I define cultural charm as a component of culturally responsive classroom management (Weinstein et al., 2003), resulting from understanding, valuing, and building on students' cultural backgrounds. Teachers who develop their own versions of cultural charm, thus, are already, or purposely become, aware of their own ethnocentrism and of their students' cultural behaviors and backgrounds. Then, coupled with their own personality and charm, teachers can consciously build on students' cultural backgrounds and behaviors to 
develop curricula through their own versions of cultural charm. As a result, teachers may also feel more competent encouraging student input in the co-construction of knowledge and daily curricula the minute they walk in the classroom door and, thus, reduce the need for exclusionary discipline (Shor, 1992; Wynne, 2002).

Implications for teacher education related to teachers' and students' roles lie in teachers' surveillance of student behavior, teachers' knowledge of their content (in this case, Language Arts and English literature), and students' agency and aspiration to preserve their identities. Potential spaces for transformation begin with raising teachers' awareness of their own ethnocentrism and strengths of students' cultural and literacy practices to increase literacy learning and identity development opportunities (Lewis et al., 2007; Strickland \& Alvermann, 2004), and simultaneously reduce the need for punitive classroom management, especially in Language Arts classrooms (Sleeter, 2005; Weinstein et al., 2004).

Implications for teacher education related to themes of social interaction lie in providing reconceptualized views of teaching and learning Language Arts that rely less on transmitting information to passive, compliant students and more on critical, sociocultural, and multicultural interactional negotiation and transformation of classroom conditions, academic and social identities, and forms of community membership (Gutiérrez, 2008). Potential spaces for transformation begin with how teachers use or do not use talk in the classroom to provide a classroom culture of teachers and students who experience empowerment by actively participating in decision making and critically reflecting about what is being learned and how learning is accomplished (Giroux, 2005; McLaren, 2003, Oakes et al., 2006). For example, potential spaces for transformation lie 
in Classroom Two teacher's willingness to learn more about her content area (Language Arts) and accommodate student cultural behaviors, albeit for rehabilitative purposes, and Classroom Four teacher's deep content area (English literature) knowledge and profound desire to teach it to students in all walks of life. Potential spaces for transformation occur in viewing students' perceptions and resistance of inequitable classroom goals as cultural and literacy practices on which to build.

\section{Classrooms Characterized by Rare Exclusionary Discipline}

For classrooms characterized by rare exclusionary discipline, implications for teacher education lie in potential spaces for transformation by building on the (a) positive relationship between the use of charm and congruence between teacher and student cultural and linguistic backgrounds (Monroe \& Obidah, 2004), and (b) negotiable atmosphere that could also include students' voices in the co-construction of daily curricula (Freire \& Macedo, 1987). First, teacher education that includes culturally responsive pedagogical courses on students' linguistic and vernacular nuances would be helpful for teachers who are unfamiliar with both. For example, the teacher in Classroom Three may have had other options for handling the interpretation of the word faults if students' linguistic and vernacular nuances had been previously studied. Second, teacher education that includes courses on culturally responsive classroom management would be helpful for teachers to study unique patterns of cultural humor used by students that can be reflected on by teachers to regulate student behavior (Monroe, 2004).

Implications for teacher education related to teachers' and students' roles lie in Classroom One and Three teachers' positive mindsets toward students' cultural behaviors, their desire for students to learn their content deeply, and students' cognizance 
of the value of meaningful classroom opportunities. Potential spaces for transformation could build on teachers' mindsets ripe for developing culturally responsive classroom management and critical literacy, both of which value and search for social justice and respect in the alternative education classroom (Evertson \& Weinstein, 2006; Freire \& Macedo, 1987; Pane, 2005; Stevens \& Bean, 2005).

Implications for teacher education related to themes of social interaction lie in the study of teaching practices that incorporate experiential education for freedom (Dewey, 1910), dialogic problem posing pedagogy for conscientization (Freire, 1998, 2000, 2003), and critical literacy and pedagogy (McLaren, 2003). This can be accomplished by "engaging teachers in conversations ... and the critique of practice grounded in their classroom experiences" (Stevens \& Bean, 2005, p. 46) that introduce and provide teachers experience with critical literacy practices. Potential spaces for transformation begin with dialogue possibilities that already existed in the classroom, both teachers' love and deep knowledge of their content area, willingness to negotiate with students and accommodate them to prevent exclusionary discipline, and the inverse relationship between increased academic learning processes and minimized the need to use exclusionary discipline. Mr. Jenkins's usage of journals to write definitions of Culinary Arts terms to begin each classroom period could also include students' literacy practices in the cooking projects. For example, students could summarize in their journals the steps they took to achieve the product of the day for starters. Implications and potential spaces for transformation also lie in students' perceptions of common classroom goals and determined willingness to negotiate them, acknowledge teachers' limits, achieve 
communication goals, maintain an acceptable academic and social identity, and learn academic material.

\section{Classrooms Characterized by Frequent Versus Rare Exclusionary Discipline}

In classrooms characterized by frequent versus rare exclusionary discipline, implications for teacher education lie in potential spaces for transformation to increase teachers' understanding (in both types of classrooms) of (a) students' cultural behaviors and humor in order to value student input in the curricula, and increase academic interactions (Ware, 2006) and (b) how to disrupt education discourses that silence student (and their own) voices with "prescribed, precanned curriculum packages” (Stevens \& Bean, 2005, p. 43) through critical literacy practices.

Implications for teacher education and field experiences related to teachers' and students' roles lie in the positive relationship between less cultural power, supportive teacher roles, and successful student academic and social identity development. Potential spaces for transformation to increase teachers' positive experiences and understanding of a critical sociocultural view of learning, language, literacy, and culture as social and cultural practices (Freire, 2000; Gutiérrez, 2008; Lewis, Enciso, \& Moje, 2007) may increase their desire and ability to critique and reduce unequal power relations which deter students' academic and social identity development.

Implications for teacher education related to themes of social interaction build on the potential spaces for transformation that lie in the mindsets of teachers and students by providing experiences with critical literacy classroom practices. Potential spaces for transformation may be noted from critiquing the omissions related to empowering teachers and students in co-constructing their own daily curricula for the purpose of 
producing respectful, democratic citizens in our schools and society. For example, findings indicate that teachers rarely if ever mentioned the ethnic identity of their students during the study. Findings also indicate that students were not asked to and did not learn how to work together in classrooms with their teachers to transform their conditions. During the study, African Americans were not discussed or pictured in curricula materials or practices in the classrooms. Documents collected did not represent students' literacy practices, interests, or experiences.

Findings indicate and concur with other research that the teachers' mindset about teaching students reproduce what they are comfortable with and have experienced (Banks et al., 2005; Pane, 2006). Even when teachers, who are mostly middle-class and White, work at schools similar to their educational and background experiences, academic and behavior problems arise. However, when teachers work at schools that are out of their cognitive, educational, and cultural comfort zone, problems seem insurmountable much of the time, as noted in the findings. This is especially true in alternative education schools, which house nondominant students in disproportionate numbers in marginalized physical settings that are lodged in the boundaries of people's imaginations and ideologies. Mr. Frederek, as well as the other teachers, mentioned in various ways that it was very hard to teach at The School and that they had not known about alternative education before teaching at The School. Specifically voiced by Mr. Frederek in his we versus they explanation of teachers versus administration, when coupled with the isolation they felt from not being specifically prepared before teaching at The School, teachers (and students) were left to their own devices. Rather than being left to their own devices, teacher education opportunities should include experiences for teachers to 
actively participate in a democratic critical, sociocultural, multicultural communities of practice in order to develop multiple perspective and options for praxis in their classroom practices. In order to be effective, praxis cannot exist without the combination of theory, practice, and critical reflection or interrogation of each other for the purpose of equitable or socially just educational practices (Freire, 2000; Stevens \& Bean, 2005).

Findings also indicate that teachers rarely if ever mentioned the ethnic identity of their students in thinking about, planning, or conducting their classroom practices. Since all students at The School were nondominant males, either Black or Latino, the most imposing omission is that of not acknowledging their ethnic and cultural histories and backgrounds, and their disproportional exclusion into schools and societies, in order to transform oppressive educational conditions (Banks et al., 2005; King, 2005). Potential spaces for transformation of teacher education should begin with teachers learning to interrogate their own ethnocentricities. Then, teachers should become reflexive teacher researchers (Freire; 2000; Kincheloe, 2005) who realize the importance of knowing and empowering their students to know their own backgrounds and cultural strengths and from whence both are empowered to build on the notion that knowledge is politically, socially, and historically constructed (Giroux, 2001).

Implications for teacher education also lie in the striking silence of students' and teachers' ideas in the classrooms, especially about literacy, and the distance between teachers and students even in the most successful of the four classrooms in this study at The School. Learning how to construct (critical literacy, sociocultural, multicultural) communities of practice would benefit the teachers of nondominant alternative education students who have been isolated from the mainstream schools in oppressive conditions 
most of their educational experiences (Applied Research Center, 2001; Kardos \& Johnson, 2007; Pasque, Bowman, Small, \& Lewis, 2009; Stevens \& Bean, 2005). Findings indicate that students were not asked and did not learn how to work together in classrooms with their teachers to co-construct curricula. In other words, students were not asked what they wanted to learn that day for the next unit. They did not learn how to talk together about academic material in ways that empowered them to desire and continue further learning. If and when alternative education students are offered this opportunity, they may be more likely to learn how to work within society at large to transform their conditions.

Potential spaces for transformation reside in teachers' desires for a successful and enjoyable classroom and students' keen assessments and agency in resisting inequitable classroom goals. Building on the potential spaces of teachers' desires to provide an enjoyable place for learning for their students, albeit within different time frames of patience, is a window of opportunity for constructing conscientization (Freire, 2000) that leads to critical literacy practices (Alvermann, Moon, \& Hagood, 1999; Stevens \& Bean, 2005 ) in communities of practice. Constructing conscientization involves learning to communicate (Pasque et al., 2009) and critique one's situation with others involved to empower academic and social identities to participate in a democracy to transform one's conditions. Conscientization could be produced during the learning processes at The School in communities of practice by building on the predominance of teacher-student interactively established contracts and negotiations. Windows of opportunity for developing teacher-researcher (Kincheloe, 2005) communities of practice that work 
through academic and behavior issues together are also potential spaces of transformation when based on teachers' desires to solve common classroom problems among them.

Providing professional development as praxis at The School or teacher education programs based in a critical sociocultural theory of learning could involve teachers as communities of practice that continually interrogate ways of constructing successful academic and behavior identities for their students through critical literacy practices. Critical sociocultural theory could provide teachers with new ways of thinking about their marginalized students as people with agency who make choices within the context of their situation. With a foundation in critical sociocultural theory, teachers could learn how to provide critical pedagogies and critical literacy experiences in their classrooms (McLaren, 2003; Stevens \& Bean, 2007). Additionally, teacher education courses based in Critical Race Theory (practice and reflexivity) in education (Ladson-Billings \& Tate, 1995, 2005) may provide teachers with new ways of viewing and teaching nondominant alternative education students. For example, at the risk of oversimplifying the findings, students arriving on campus at The School with teachers who have gained a Critical Race Theory grounding would find positive images, posters, books, media, conversations, and thinking about themselves and their histories and cultural backgrounds and experiences in every aspect of the curriculum and participation structures (Zinn, 2005).

During the study, African Americans were not discussed or pictured in curricula materials or practices in the classrooms. Documents collected did not represent students' literacy practices, interests, or experiences by providing them "with opportunities to examine the discourses of the subject-area texts in relation to the discourses of everyday life" (Moje, Overby, Tysvaer, \& Morris, 2008, p. 4). Potential spaces for transformation 
found in student-initiated conversations could be seriously taken up by teachers who are educated through critical multicultural sociocultural education to simultaneously provide motivation through students' social networks and honor their students' ethnic histories and cultural backgrounds through critical literacy practices (Alvermann et al., 1999; Stevens \& Bean, 2005). Thinking together critically in the classroom would consist of problem posing (Freire, 2000) and problem solving for positive outcomes that validate real lives and real issues. For example, teachers and students could research recipes to cook or literature to study or perform from their own cultures. Critical decisions made together in communities of practice that honor each other's racial and cultural backgrounds would produce empowered teacher and student academic and social identities in a critical multicultural alternative education.

Building on the potential spaces illustrated in the findings, communities of practice can be constructed to empower teachers to be researchers of the strengths of their own and their student's backgrounds and begin to transform oppressive classroom conditions. Teachers and students would then explain what happens in alternative education classrooms differently. For example, learning processes would be based on students' literacy and cultural practices and spoken of as meaningful, worthwhile, and exciting (hooks, 1994; Stevens \& Bean, 2007). Classroom interactions would be spoken of as respectful, caring, character building, community building, and fulfilling (Noddings, 2007). Exclusionary discipline practices would not dominate since time would be spent creating racially visible, transformative outcomes for the school within each classroom. Teachers would be educated through praxis (Freire, 2000; Stevens \& Bean, 2005) for alternative education before they are placed in The School; students would enter and 
be surprised at first that they felt wanted, needed, smart, and beautiful. Findings implied that students in the Culinary Arts classroom felt needed and smart; Mr. Jenkins needed them to work on the cooking projects, and he dished out directions but then let them to independently work on their part of the project—giving them the idea they they were smart. With an understanding of communities of practice that honors racial, cultural, and literacy backgrounds, freedom would be given to participants in teacher education field experiences, workshops, or professional development courses to brainstorm a different picture of what alternative education could look like through a communities of practice perspective (Pane, in press). For example, the communities of practice teacher education groups may devise and experience arts-based critical literacy curricula that build on students' interest in music and dance in order to develop critical literacy and, in turn, be able to do the same with their future students. Content area teachers would learn how to devise a Third Space (Gutiérrez, Baquedano-López, \& Tejeda, 2003; Gutiérrez, 2006; Pane, 2009) for critical literacy by learning how to blend literacy and cultural practices into their subject area in order to be prepared to work with their future students.

Ongoing collaborative workshops, teacher education, and professional development for social justice should energize, educate, and support teachers and staff for alternative education (McDonald, 2007). Alternative education schools should have an abundance of good news and exemplars of co-constructed curricula to share. They should be visited by researchers, student interns, and others interested in understanding a communities of practice perspective that honors alternative education students who work alongside teachers, staff, and administration to transform conditions. 
Findings suggest that teacher education programs need to incorporate research findings from this and future studies related to transforming alternative education classroom conditions into their courses and requirements. Teacher education programs have introduced critical, multicultural, sociocultural, and special inclusive courses and pedagogies, but not specifically for teachers in alternative education. Teacher education programs also have not combined critical multicultural sociocultural practice pedagogies into the coursework and field experiences of future teachers specifically for alternative education classrooms. This is a great omission when dealing with oppressive conditions in alternative education that overpower students' cultural communities and claim "white innocence" (Gutierrez, 2006, p. 1) at what happens in classrooms characterized by exclusionary discipline.

According to the findings of this study, teachers would benefit from learning how to work together both with each other and with their alternative education students via race-conscious, equity-oriented humanist approaches before being placed in the specific context of The School. If teachers are afforded the opportunity to learn to work together in such critical communities of practice (Freire, 1998, 2000, 2003; Lave \& Wenger, 1996; McDonald, 2007) before coming to The School, and ongoing while teaching at The School, they would be more prepared to offer empowering opportunities to their students. For example, Mr. Frederek's workshop would address this suggestion if it involved a group of teachers learning how to build a rocket while simultaneously learning how to involve students in critical pedagogy related to rockets. Critical pedagogy related to rockets may critique the monetary benefits of blasting rockets into space for the students involved in the discussion. In critical communities of practice, people create praxis and 
socially just curriculum together. People learn from each other by listening, negotiating, critically reflecting, and developing positive, congruent norms and outcomes for their specific situation. Teachers who learn to participate with one another about problems that face them will also learn how to offer this opportunity to their students. Classrooms would be exciting rather than mundane (Freire \& Macedo, 1987; hooks, 1994).

Findings showed that the relationship between classroom interactions, learning processes, and exclusionary discipline practices across alternative education classrooms are shaped and also shape the power relations within the context of cultural, social, and political processes presently mired in the cradle-to-prison pipeline (Children's Defense Fund, 2007). Steeped in conformism to dominant academic and behavior ideology about alternative education, teachers at The School strived to survive the illiterate (Freire, 2000) and prison atmosphere (Foucault, 1977); students bided time until transferred, suspended, or put in jail. Implications for teacher education are urgent if we are to gain momentum for reconceptualizing alternative education and repay the debt we have accumulated related to nondominant students in our country's public classrooms and schools (LadsonBillings, 2007).

\section{Recommendations for Research and Policy}

This section provides recommendations for research and policy through a critical analysis of potential spaces for transformation of oppressive educational practices and conditions in the findings.

\section{Research}

Future research in alternative education settings can build on potential spaces for transformation highlighted in the findings of this study. Research studies that have been 
conducted in alternative education classrooms using a critical social practice framework and critical microethnographic method to transform oppressive educational conditions for nondominant students have not been found to date (Gutiérrez, 2006; Pane \& Rocco, 2009). Potential spaces for transformation of conditions for nondominant students in classrooms characterized by rare and frequent exclusionary discipline are deeply lodged in reconceptualizing dominant ideology, school ideology, and educational experiences of students and teachers in alternative education (Gutiérrez, 2006; Ladson-Billings, 2006; Moje, Overby, Tysvaer, \& Morris, 2008; McLaren, 2003; Pasque, Bowman, Small, \& Lewis, 2009). Recommendations for research are made next.

First, teachers' commitment to teaching their content area provides a foundation for building on the intersection of critical sociocultural theory of learning, practice, and praxis (Ax \& Ponte, 2008; Kincheloe, 2005) and content area literacy (Moje et al., 2008; Stevens \& Bean, 2007), critical pedagogy (McLaren, 2003), and transformation of verbal and nonverbal interactions focusing primarily on exclusionary discipline in alternative education settings (Gutiérrez, 2006). A suggested future research question is: What happens in alternative education classrooms when critical sociocultural theory of learning, practice and praxis guides content area literacy and critical pedagogy in classroom practices?

Second, teachers' willingness to negotiate and accommodate student behavior is a good foundation for building on the intersection of critical communities of practice (Lave $\&$ Wenger, 1996; Pane, in press), culturally responsive pedagogy, culturally responsive classroom management, and transformation of individualistic teaching and learning practices. A suggested future research question is: What happens in alternative education 
classrooms when classroom practices resemble culturally responsive classroom management?

Third, teachers' low-key resistance to dominant behavior ideology to prevent exclusionary discipline in their classrooms is an important mind-set for building on the intersection of Critical Race Theory (Ladson-Billings \& Tate, 2004) and anti-racist, antioppressive, academic learning processes (Freire, 2000; Goodlad, 1984, Pane \& Rocco, 2009; Wynne, 2002). One of the most significant omissions in the findings was the lack of acknowledgement of teacher ethnocentricities or students' cultural backgrounds and experiences in the juvenile justice system in combination with critical literacy development opportunities. A suggested future research question is: What happens in alternative education classrooms when anti-racist, anti-oppressive communities of practice are pursued in combination with critical literacy development opportunities?

Fourth, future research can pursue these omissions by intersecting the critical framework and method of this study with participatory and longitudinal critical research methods in teacher education and other alternative education classrooms (Carspecken, 1996; McLaren, 2003; Freire, 2000; Ladson-Billings \& Tate, 2004). A suggested future research question is: What happens over time in alternative education classrooms characterized by exclusionary discipline? Making banking education learning processes and race visible through critical communities of practice may reduce the need for exclusionary discipline practices in the classroom. When non-dominant students are viewed as capable people with agency for decision making, teachers are less likely to conform to banking education from a cultural deficit orientation, which thrives on rehabilitative purposes. Teachers are more likely to incorporate and honor students' 
cultural practices, literacy practices, and racial backgrounds into their expertise through these frameworks. Through critical frameworks and research settings, teachers are free to inquire to create exciting meaningful critical pedagogies and curricula with their students (Freire, 2000; McLaren, 2003) even in this high-stakes testing era. A suggested future research question is: What happens in alternative education classrooms when teachers provide critical pedagogies and curricula with their students that they have experienced in teacher education?

In the meantime, the need for exclusionary discipline is also minimized because participation in critical communities of practice is more equitable and produces more congruent outcomes. According to the findings, students wanted to learn and appreciated teachers who made them do the work, albeit via banking education learning processes. However, when students come to a school (or when every school students enter) thrives on how students behave or misbehave to the neglect of academic (especially literacy) learning, students know they are receiving inequitable schooling. Instead, if students enter a school that thrives on respectful verbal and nonverbal interactions led by faculty and administration who honor students' backgrounds and put academic learning in which students are viewed as inquirers at the forefront, behavior is no longer the controlling issue. In the end, all members of the school feel they are doing something important and worthwhile when conditions are being continually transformed and they are an integral part (Lankshear \& Knobel, 2006; Nieto, 2002; Oakes et al., 2006; Pane \& Rocco, 2009).

Overall, this study has implications for researchers to "rethink the ways we theorize individuals from non-dominant groups, their practices, and their communities" (Gutiérrez, 2006, p. 1). There is an urgency for researchers to develop a "new educational 
discourse and analytical framework that makes visible the persistence of inequity and supremacy in educational policy, practice, and empirical work" (p. 1). Researchers must address the debt our nation has accumulated (Ladson-Billings, 2006) by pursuing raceconscious, equity-oriented humanist approaches when studying cultural communities that "account for the sociohistorical, political and intellectual context" (Gutiérrez, 2006, p. 2) of our work. To do so, future research may utilize "white innocence" (p. 1) to build analytical frameworks and methodologies for rethinking educational discourse and inquiry rather than sustaining dominant analytical frameworks and methodologies that constrain non-dominant students' opportunities for successful education. Recommendations for research from a white innocence perspective are suggested in order to make visible, name, and construct critical sociocultural, multicultural, and empowering communities of practice for alternative education students and their teachers. Implications for policy are discussed next.

\section{Policy}

Implications for policy are significant within the context of current dominant education ideology, advocacy, and legislation. Policy within dominant ideology, according to Partnership for $21^{\text {st }}$ Century Skills (P21), formed in 2002 through the collaboration of the U.S. Department of Education and partnerships among education, business, and community leaders, "emerges from a complex mingling of needs, opportunities, resources, personalities, and political will ... [and] regulates the activities, distributes the resources, and sets the priorities that determine, in large measure, the role of education within society" (Partnership for $21^{\text {st }}$ Century Skills, 2009, p. 26). Even though couched within dominant conventional ideology, educational 
advocacy and legislation provide potential spaces for transformation of oppressive conditions in alternative education suggested in the findings of this study through a critical analysis of policy. Critical discourse analysis (Fairclough, 1995, 2003) of policy within dominant ideology provides opportunities to interrogate sociocultural values, language, and potentialities for transformation represented in the policy for particular communities of practice.

Since No Child Left Behind Act of 2001 (NCLB, 2002) was signed into law, P21 has been a leading advocacy organization for infusing $21^{\text {st }}$ century learning into U.S. K12 education. Even though alternative education is not explicitly mentioned, suggestions for how to create and sustain $21^{\text {st }}$ century communities for learning have policy implications for alternative education. Partnership for $21^{\text {st }}$ Century Skills (2009) suggest that learning should take place in connected communities rather than in isolated entities where classrooms are "isolated from other classrooms, teachers isolated from other teachers, [and] schools isolated from the outside world" (Partnership for $21^{\text {st }}$ Century Skills, 2009, p. 22). As suggested in the findings of this study, however, isolation is a persistent oppressive condition in alternative education that reduces the opportunities for communities of learning within classrooms as well. Critical analysis of policy conducted in connected critical communities of learning within teacher education, research, and alternative education classrooms and schools would help transform their isolated conditions. They decide together who benefits from the policy at hand, "which aspects of being a student, a teacher, a literate person were authenticated, silenced, marginalized, and subverted within these policy spaces" (Stevens \& Bean, 2005, p. 98), and what possibilities exist for students, teachers, and opportunities in literacy learning. 
Since its implementation in 2002, the language of NCLB remains closely bound to accountability for achievement, success, and progress. For example, stronger accountability was recently announced as a pilot by the U.S. Secretary of Education for schools declared to be lowest-performing (USDOE, 2008). From 17 state applications, 6 (including Florida) were accepted for the pilot. Differentiated accountability

will allow states to vary the intensity and type of interventions to match the academic reasons that lead to a school's identification. In addition, some states and districts have a large percentage of their schools identified, impacting capacity to provide meaningful, intensive reforms. Differentiated accountability will assist those states by targeting resources and interventions to those schools most in need of intensive interventions and significant reform. (p. 1)

Even though not mentioned in this report, the differentiated accountability reform suggests a tight-tight school culture as mentioned in the findings. Differentiating, or separating schools or curricula for accountability provides little room for learning that thrives on communities of practice. Moreover, it negates P21 suggestions for reduction of isolation in learning. According to the Children's Defense Fund (2009a)

states spend about 2.8 times as much money per prisoner as per public school pupil ... [and] unless we focus our efforts on early intervention and prevention, rather than punishment, we are robbing thousands of youths each year of their futures and our country of vital human resources. (p. 1)

Based on the findings of this study and the suggestion of the tight-tight-tight alternative education school culture, omissions of empowering literacy education in Office of Juvenile Justice Delinquency Prevention (OJJDP, 2009) model programs are significant. OJJDP Model programs thrive on unequal power relations, do not incorporate students' literacy and cultural practices, and are not integrated into alternative education schools. Potentialities under current policy lack research methods that get beyond best practices and functionalist assumptions about alternative education schools and programs 
as places to isolate, measure, and punish unacceptable behavioral skills. Policy and language about alternative education in OJJDP provides no room to acknowledge the "ideological, historical, social, and political nature" (Stevens \& Bean, 2005, p. 99) of what works in OJJDP model programs. Power relations are heightened into a triple bind of oppressive educational conditions (Foucault, 1977). Critical analysis of OJJDP policy to discover what is missing, who benefits, and what possible spaces for transformation lay dormant in the language could take place in critical communities of practice in teacher education, research, and alternative education settings. In turn, the cradle-toprison pipeline could be replaced with critical communities of practice where alternative education students are no longer looked on as deficient and undeserving of empowerment but as inquirers of the world around them.

An analysis conducted by Children's Defense Fund (2009b) of the FY2010 federal budget request submitted by the Obama Administration, named the American Recovery and Reinvestment Act (ARRA, 2009), noted new and expanded alternative school options for reducing the cradle-to-prison pipeline (e.g., Promise Neighborhoods program, Charter Schools Grants program). For example, the Promise Neighborhoods program awards competitive grants to non-profits to open education programs similar to Harlem Children's Zone (Tough, 2008) in New York City. Miami Children's Initiative is a similar effort for Liberty City, Miami, funded by Florida legislation (Ounce of Prevention Fund of Florida, Florida Statute 409.147). Even though alternative education is not explicitly mentioned in these initiatives, the addition would represent a potential space for transformation of oppressive conditions as suggested in the findings of this study. An alternative education school guided by the (critical sociocultural multicultural) 
communities of practice perspective of this study incorporated into the Miami Children's Initiative, for example, could interrogate the omission. This implication for policy may be very significant since unequal power relations and exclusionary discipline were at their height in the Language Arts courses, which are most tightly bound by NCLB mandates and accountability.

According to the language of the Reading First policy (2002) within NCLB and subsequent funding opportunities for schools, the cultural model of a good reader in the U.S. is a student who is able to decode 120 words per minute by the end of third grade. Tests used at The School such as the Oral Reading Fluency (ORF) are examples of how student readers should be measured and tracked through Reading First policy discourse prioritizing code breaking, or decoding, behaviors. Critical analysis of the language and cultural model of a reader that is promoted notes the omission of the "image of a complex reader" (Stevens \& Bean, 2005, p. 101) and non-standard, nondominant fluencies. Cognitive, psycholinguistic, sociolinguistic, and ethnographic researchers investigate comprehension as the ability to make meaning and become a critical reader over time (Freire \& Macedo, 1987; Gee, 1996; Heath, 1983; Lankshear \& Knobel, 2006; Luke \& Freebody, 1999). Models that incorporate the historical, political, and sociocultural nature of reading texts wherein readers engage "with different types of literacy events, for different purposes, and with different results" (Stevens \& Bean, 2005, p. 101), such as the Four Resources model (Freebody \& Luke, 1990) of critical reading, consciously acknowledge the complexities of literacy rather than universality of end-results of reading. 
Building on teachers' and students' opinions noted in the findings of what was wrong or missing in their classrooms provides a foundation for rethinking alternative education in critical communities of practice. Instead of thinking of alternative education as another place to hold students who are disruptive, delinquent, multi-disabled, and bad in general, conditions could be transformed by reconceptualizing alternative education across contexts and disciplines. Policy makers, curriculum writers, administrators, teacher educators, teachers of all content areas, and support staff can interrogate policy by thinking transformatively about how to include critical communities of practice in the context of alternative education and student populations. Long-term transformative critical literacy pedagogies (McLaren, 2007) and communities of practice would replace the revolving door of punishment based on time spent in alternative education settings that reinforces the cradle-to-prison pipeline (Children's Defense Fund, 2009a).

Research, and policy implications and recommendations are based on the conclusion that alternative education classroom and school conditions for nondominant students need to be transformed. However, the status of alternative education must first be declared oppressive before transformation of conditions can be undertaken. This declaration is difficult because it deals with reconceptualizations of people's mindsets. Nonetheless, once alternative education conditions are declared oppressive by policy makers, policy changes can be written. Only after policy changes are written may reconceptualized research and teacher education programs related to alternative education be funded and undertaken. 


\section{Conclusion}

The purpose of this critical microethnographic study was to explore the relationship between classroom interactions and exclusionary discipline as a social practice within and across classrooms in the same alternative education school. Overall, various forms of Discourse that interacted within layers of system constraints accounted for teachers' use or non-use of exclusionary discipline with students. Specifically, classroom interactions, learning processes, and exclusionary discipline practices were cyclically and rigidly intertwined in dominant educational Discourse. Dominant educational Discourse, based in systematic and systemic prejudices, racism, and power relations, implicitly shaped the school and classroom Discourses which, in turn, shaped and solidified the dominant Discourse, repeating the cycles of prejudice, racism and power relations. School Discourses included surveillance, reward, and punishment. Classroom verbal and nonverbal Discourses included patterns of teacher authoritarianism, student disruptive behavior, and teacher threats to maintain control. Learning processes involved cyclic Discourses of teacher lecturing unrelated to students' backgrounds, student passivity, and power relations rather than social, cultural, racial, and academic empowerment and development. As a result, teachers and students were trapped in the dominant educational Discourse even though they used agency to superficially retain their identities and achieve some of their goals. Through teacher education programs based in Critical Race Theory and other critical stances, teachers could begin to learn to interrogate their own ethnocentricities and those of their students, represent and build on students' backgrounds and inquiry in the curricula, and initiate a break in the systemic cycle of educational oppression for nondominant students and their teachers. 


\section{REFERENCES}

Alvermann, E. E., Moon, J. S., \& Hagood, M. C. (1999). Popular culture in the classroom Teaching and researching critical media literacy. Newark, DE: International Reading Association.

American Recovery and Reinvestment Act of 2009. (2009). Pub. L. No. 111-5. Retrieved August 14, 2009, from http://frwebgate.access.gpo.gov/cgibin/getdoc.cgi?dbname=111_cong_bills\&docid=f:h1enr.pdf

Anyon, J. (1981). Social class and school knowledge, Curriculum Inquiry, 11(1), 3-42.

Anyon, J. (2005). Radical possibilities: Public policy, urban education, and a new social movement. New York: Routledge.

Apple, M. W. (1996). Cultural politics \& education. New York: Teachers College Press.

Apple, M. W. (2000). Official knowledge: Democratic education in a conservative age. New York: Routledge.

Applied Research Center. (2001). Zero Tolerance and School Security Measures: A Failed Experiment. In T. Johnson, J. E. Boydon, \& W. J. Pittz (Eds.), Racial Profiling and Punishment in U.S. Public Schools: How Zero Tolerance Policies and High Stakes Testing Subvert Academic Excellence and Racial Equity. Oakland, CA: Skiba, R. J., \& Leone, P. E.

Aronson, S. R. (1995). Examining effective alternative programs. Insights on education policy, practice, and research, 6 . Retrieved June 5, 2005, from http://www.sedl.org/policy/insights/

Asante, M. K. (1988). Afrocentricity. Trenton, NJ: Africa World Press.

Ax, J., \& Ponte, P. (2008). Praxis: Analysis of theory and practice. In J. Ax, \& P. Ponte (Eds.), Critiquing praxis (pp. 1-20). Rotterdam, The Netherlands: Sense.

Bakhtin, M. M. (1981). The dialogic imagination. Austin: University of Texas Press.

Bakhtin, M. M. (1986). Speech genres and other late essays. Austin: University of Texas Press.

Ballenger, C. (1999). Teaching other people's children: Literacy and learning in a bilingual classroom. New York: Teachers College Press. 
Banks, J. A. (2001) Approaches to multicultural curriculum reform. In J. A. Banks, \& C. A. Banks, (Eds.), Multicultural education: Issues and perspectives ( $4^{\text {th }} \mathrm{ed}$.) (pp. 225-246). New York: John Wiley \& Sons.

Banks, J., Cochran-Smith, L., Moll, L., Richer, A., Zeichner, K., LePage, P., DarlingHammond, L., Duffy, H., \& McDonald, M. (2005). Teaching diverse learners. In . Darling-Hammond \& J. Bransford (Eds.), Preparing teachers for a changing world: What teachers should learn and be able to do (pp. 232-274). San Francisco: Jossey-Bass.

Barton, D., \& Hamilton, M. (2000). Literacy practices. In D. Barton, M. Hamilton, \& R. Ivanič (Eds.), Situated literacies: Reading and writing in context (pp. 7-15). New York: Routledge.

Beach, R., \& Kalnin, J. (2005). Studying values stances in institutional settings. In R. Beach, J. Green, M. Kamil, \& T. Shanahan (Eds.), Multidisciplinary perspectives in literacy research ( $2^{\text {nd }}$ ed., pp. 209-242). Cresskill, NJ: Hampton.

Berkentotter, C., \& Thein, A. (2005). Settings, speech genres, and the institutional organization of practices. In R. Beach, J. Green, M. Kamil, \& T. Shanahan (Eds.), Multidisciplinary perspectives in literacy research ( $2^{\text {nd }}$ ed., pp. 179-208). Cresskill, NJ: Hampton.

Berkshire Advisors, Inc. (2007). Best financial practices review (Ch. 2). Available from http://www.oppaga.state.fl.us/reports/pdf/dadechp1_2002.pdf

Bhabha, H. (1994). The location of culture. New York: Routledge.

Blanchett, W. J. (2006). Disproportionate representation of African American students in special education: Acknowledging the role of White privilege and racism, Educational Researcher, 35(6), 24-28.

Blomberg, T. G., \& Waldo, G. P. (2001). Implementing research-based best practices in juvenile justice education. Corrections Today, 63(7), 144-148. Retrieved May 29, 2004, from http://vnweb.hwwilsonweb.com.ezproxy.fiu.edu/hww

Bloome, D., Carter, S. P., Christian, B. M., Otto, S., \& Shuart-Faris, N. (2005). Discourse analysis and the study of classroom language and literacy events: A microethnographic perspective. Mahwah, NJ: Lawrence Erlbaum.

Bogdan, R. C., \& Biklen, S. K. (2003). Qualitative research for education: An introduction to theories and methods ( $4^{\text {th }}$ ed.). New York: Allyn \& Bacon. 
Bondy, E., Ross, D. D., Gallingane, D., \& Hambacher, E. (2007). Creating environments of success and resilience: Culturally responsive classroom management and more. Urban Education, 42(4), 326-348.

Bowles, S., \& Gintis, H. (1977). Schooling in capitalist America: Educational reform and the contradictions of economic life. New York: Basic.

Boykin, A. W. (1986). The triple quandary and the schooling of Afro-American children. In U. Neisser (Ed.), The school achievement of minority children (pp. 57-92). Hillsdale, NJ: Lawrence Erlbaum.

Brown, D. F. (2003). Urban teachers' use of culturally responsive management strategies. Theory into Practice, 42(4), 277-282.

Brown, D. F. (2004). Urban teachers' professed classroom management strategies: Reflections of culturally responsive teaching. Urban Education, 39(3), 266-289.

Carlson, D., \& Apple, M. W. (Eds.). (1999). Power/knowledge/pedagogy: The meaning of democratic education in unsettling times. Boulder, CO: Westview.

Carpenter, D. M., Ramirez, A., \& Severn, L. (2006). Gap or gaps: Challenging the singular definition of the achievement gap [Electronic version]. Education and Urban Society, 39(1), 113-127.

Carspecken, P. F. (1996). Critical ethnography in educational research: A theoretical and practical guide. New York: Routledge.

Casella, R. (2003). Punishing dangerousness through preventive detention: Illustrating the institutional link between school and prison. New Directions for Youth Development: Deconstructing the School-to-prison Pipeline, 99, 55-70.

Cash, T. (2004). Alternative schooling. In J. Smink, \& F. P. Schargel (Eds.), Helping students graduate: A strategic approach to dropout prevention (pp. 165-176). Larchmont, NY: Eye on Education.

Cassidy, W., \& Bates, A. (2005). "Drop-Outs" and "Push-Outs": Finding hope at a school that actualizes the ethic of care [Electronic version]. American Journal of Education, 111(1), 66-103.

Cazden, C. (2001). Classroom discourse: The language of teaching and learning. Portsmouth, NH: Heinemann.

Center on Crime and Juvenile Justice. (2008, March). Race and the juvenile justice system. San Francisco: Butts, J. 
Children's Defense Fund. (1975). School suspensions: Are they helping children? Cambridge. MA: Washington Research Project.

Children's Defense Fund. (2009a). Children's Defense Fund Cradle to Prison Fact Sheet Florida. Washington, DC, Retrieved August 12, 2009, from http://www.childrensdefense.org/child-research-data-publications/data/state-datarepository/cradle-to-prison-pipeline/cradle-prison-pipeline-florida-2009-factsheet.pdf

Children's Defense Fund. (2009b). A new commitment to our nation's future.

Washington, DC, Retrieved August 12, 2009, from

http://www.childrensdefense.org/helping-americas-children/ending-childpoverty/president-obama-fy2010-budget-analysis.html

Chubbuck, S. M. (2004). Whiteness enacted, whiteness disrupted: The complexity of personal congruence. American Educational Research Journal 41(2), 301-333.

Cochran-Smith, M. (2005). The new teacher education: For better or for worse? Educational Researcher, 34(7), 3-17.

Cochran-Smith, M., \& Fries, K. (2005). Researching teacher education in changing times: Politics and paradigms. In M. Cochran-Smith, \& K. M. Zeichner, (Eds.), Studying teacher education: The report of the AERA panel on research and teacher education (pp. 69-110). Mahwah, NJ: Lawrence Erlbaum.

Cochran-Smith, M., \& Zeichner, K. M. (Eds.). (2005). Studying teacher education: The report of the AERA panel on research and teacher education. Mahwah, NJ: Lawrence Erlbaum.

Cole, M. (1996). Cultural psychology: A once and future discipline. Cambridge. MA:The Belknap Press of Harvard University Press.

Cole, M., Engeström, Y.,\& Vasquez, O. (1997) Introduction. In M. Cole, Y. Engeström, $\&$ O. Vasquez (Eds.) Mind, culture and activity: Seminal papers from the laboratory of comparative human cognition. New York: Cambridge University Press.

Cole, M., \& Scribner, S. (1974). Culture and thought. San Francisco: John Wiley \& Sons.

Cooper, E. F. (2005). The safe and drug-free schools and communities act: reauthorization and appropriations (CRS Report for Congress Received through the CRS Web, Order Code RS20532). Retrieved August 1, 2008, from http://www.law.umaryland.edu/marshall/crsreports/crsdocuments/RS2053202152 005.pdf 
Creswell, J. W. (1998). Qualitative inquiry and research design: Choosing among five traditions. Thousand Oaks, CA: Sage.

Creswell, J. W. (2003). Research design: Qualitative, quantitative, and mixed methods approaches $\left(2^{\text {nd }}\right.$ ed.). Thousand Oaks, CA: Sage.

Darling-Hammond, L. \& Bransford, J. (Eds.) (2005). Preparing teachers for a changing world: What teachers should learn and be able to do. San Francisco: Jossey-Bass.

Day-Vines, N. L., \& Day-Hairston, B. O. (February, 2005). Culturally congruent strategies for addressing the behavioral needs of urban, African American male adolescents. Professional School Counseling, 236-243.

DeCuir, J. T., \& Dixson, A. D. (2004). "So when it comes out, they aren't that surprised that it is there": Using critical race theory as a tool of analysis of race and racism in education. Educational Researcher, 33(5), 26-31.

Delpit, L. (1995). Other people's children: Cultural conflict in the classroom. New York: The New Press.

Dewey, J. (1910). How we think. Mineola, NY: Dover.

Dewey, J. (1915). The school and society. New York: Dover.

Dewey, J. (1916). Democracy and Education. New York: Macmillan.

Dewey, J. (1938). Experience and education. New York: Simon \& Schuster.

Dewey, J. (1939). Freedom and culture. New York: Capricorn.

Dixon, D., \& Green, J. Studying the discursive construction of texts in classrooms through interactional ethnography. In In R. Beach, J. Green, M. Kamil, \& T. Shanahan (Eds.), Multidisciplinary perspectives in literacy research $\left(2^{\text {nd }} \mathrm{ed} ., \mathrm{pp}\right.$. 349-390). Cresskill, NJ: Hampton.

Dottin, E. S. (2005). Creating a professional community through means-ends connections to facilitate the acquisition of moral dispositions: Developing, living, and evaluating a conceptual framework in teacher education. New York: University Press of America.

Duranti, A. (Ed.). (2004). A companion to linguistic anthropology. Malden, MA: Blackwell. 
Educational Testing Service Policy Information Center. (2005, February). One-third of a nation: Rising dropout rates and declining opportunities. Princeton, NJ: Barton, P. E.

Elementary and Secondary Education Act. (2002). Title I, Part B, Subpart 1 - Reading First. Retrieved August 12, 2009, from U.S. Department of Education website http://www.ed.gov/programs/readingfirst/legislation.html

Emmer, E. T., Evertson, C. M., \& Anderson, L. M. (1980). Effective classroom management at the beginning of the school year. The Elementary School Journal, 80(5), 219-231.

Engeström, Y. (1987). Learning by expanding: An activity-theoretical approach to developmental research. Human Development, 31, 137-151.

Engeström, Y., Miettinen, R., \& Punamaki, R. (Eds.). (1999). Perspectives on activity theory. New York: Cambridge University Press.

Evertson, C. M., \& Weinstein, C. S. (Eds.). (2006). Handbook of classroom management: Research, practice, and contemporary issues. Mahwah, NJ: Lawrence Erlbaum.

Fairclough, N. (1995). Critical discourse analysis: The critical study of language. Essex, England: Longman Group.

Fairclough, N. (2003). Analyzing discourse: Textual analysis for social research. New York: Routledge.

Fenning, P., \& Rose, J. (2007). Overrepresentation of African American students in exclusionary discipline: The role of school policy. Urban Education, 42(6), 536559.

Finders, M. (2005). "Gotta be worse": Literacy, schooling, and adolescent youth offenders. In J. A. Vadeboncoeur, \& L. P. Stevens (Eds.) Re/constructing "the adolescent": Sign, symbol, and body (pp. 97-122). New York: Peter Lang.

Fine, M. (1990). Making controversy: Who's "at-risk". Journal of Urban and Cultural Studies, 1, 55-68.

Fine, M. (1991). Framing dropouts: Notes on the politics of an urban high school. Albany: State University of New York Press.

Fine, M., \& Weiss, L. (Eds.). (2005). Beyond silenced voices: Class, race, and gender in United States schools (Rev. ed.). Albany, NY: State University of New York Press. 
Florida Department of Education, Bureau of Family, Community Outreach. (2007).

Dropout prevention programs and strategies. Retrieved November 24, 2007, from http://www.fldoe.org/family

Florida Department of Education, Division of Public Schools Bureau of School Improvement Program Guide. (2006). Available from FLDOE website http://data.fldoe.org/programguide

Florida Department of Juvenile Justice. (2007). Learn more about the Florida Department of Juvenile Justice. Available from FLDJJ website http://www.djj.state.fl.us/AboutDJJ/index.html

Florida International University Regulations for Thesis/Dissertation Preparation Manual. Retrieved November 30, 2008, from http://gradschool.fiu.edu/thesis.html

Foley, R. M., \& Pang, L. (2006). Alternative education programs: Program and student characteristics, The High School Journal, 89(3), 10-21.

Foster, D. H., \& Shirley, L. J. (2004). Active learning. In J. Smink, \& F. P. Schargel (Eds.), Helping students graduate: A strategic approach to dropout prevention (pp. 197-210). Larchmont, NY: Eye on Education.

Foucault, M. (1977). Discipline and punish: The birth of the prison. New York: Vintage.

Fránquiz, M. E., \& Salazar, M. C. (2004). The transforming potential of humanizing pedagogy: Addressing the diverse needs of Chicano/Mexicano students. The High School Journal 87(4), 36-53.

Freebody, P., \& Luke, A. (1990). Literacies programs: Debates and demands in cultural context. Prospect: Australian Journal of TESOL, 5(7), 7-16.

Freiberg, H. J., \& Lapointe, J. M. (2006). Research-based programs for preventing and solving discipline problems. In C. M. Evertson, \& C. S. Weinstein (Eds.). Handbook of classroom management: Research, practice, and contemporary issues (pp. 735-786). Mahwah, NJ: Lawrence Erlbaum.

Friere, P. (1985). The politics of education: Culture, power, and liberation. New York: Bergin \& Garvey.

Freire, P. (1997). Pedagogy of hope. New York: Continuum.

Freire, P. (1998). Teachers as cultural workers: Letters to those who dare to teach. Boulder, CO: Westview Press.

Freire, P. (2000). Pedagogy of the oppressed. New York: Continuum. 
Friere, P. (2003). Education for critical consciousness. New York: Continuum.

Freire, P., \& Macedo, D. (1987). Literacy: Reading the word and the world (pp. 29-36). Westport, CT: Bergin \& Garvey.

Gay, G. (2000). Culturally responsive teaching: Theory, research, \& practice. New York: Teachers College Press.

Gee, J. P. (1992). What is literacy? In P. Shannon (Ed.), Becoming political: Readings and writings in the politics of literacy education (pp. 21-28). Portsmouth, NH: Heinemann.

Gee, J. P. (1996). Social linguistics and literacies: Ideology in discourses (2 ${ }^{\text {nd }}$ ed.). Bristol, PA: Taylor \& Francis.

Gee, J. P. (2000). Discourse and sociocultural studies in reading. In M. L. Kamil, P. B. Mosenthal, P. D. Pearson, \& R. Barr (Eds.), Handbook of reading research: Volume III (pp. 195-207). Mahwah, NJ: Lawrence Erlbaum.

Gee, J. P. (2004). Discourse analysis: What makes it critical? In R. Rogers (Ed.), An introduction to critical discourse analysis in education (pp. 19-50. Mahwah, NJ: Erlbaum.

Gee, J. P. (2005). Critical discourse analysis. In R. Beach, J. Green, M. Kamil, \& T. Shanahan (Eds.), Multidisciplinary perspectives in literacy research $\left(2^{\text {nd }} \mathrm{ed} ., \mathrm{pp}\right.$. 293-318). Cresskill, NJ: Hampton.

Gee, J. P. (2006). An introduction to discourse analysis: Theory and method $\left(2^{\text {nd }}\right.$ ed.), NY: Routledge.

Geelan, D. R. (2005). Weaving narrative nets to capture school science classrooms. In W.-M. Roth (Ed.). Auto/biography and auto/ethnography: Praxis of research method (pp. 227-242). Rotterdam, The Netherlands: Sense.

Gettinger, M., \& Kohler, K. M. (2006). In C. M. Evertson, \& C. S. Weinstein (Eds.). (2006). Handbook of classroom management: Research, practice, and contemporary issues (pp. 73-96). Mahwah, NJ: Lawrence Erlbaum.

Giroux, H. A. (1987). Literacy and the pedagogy of political empowerment. In P. Freire and D. Macedo, Literacy: Reading the word and the world (pp. 1-25). Westport, CT: Bergin \& Garvey.

Giroux, H. A. (1988). Teachers as intellectuals: Toward a critical pedagogy of learning. Westport, CT: Bergin \& Garvey. 
Giroux, H. A. (Ed.). (1991). Postmodernism, feminism, and cultural politics: Redrawing educational boundaries. Albany, NY: State University of New York Press.

Giroux, H. A. (1994). Living dangerously: Identity politics and the new cultural racism. In H. A. Giroux \& P. McLaren (Eds.), Between borders: Pedagogy and the politics of cultural studies (pp. 29-55). New York: Routledge.

Giroux, H. A. (2001). Theory and resistance in education: Towards a pedagogy for the opposition. Westport, CT: Bergin \& Garvey.

Giroux, H. A. (2003). Youth, higher education, and the crisis of public time: Educated hope and the possibility of a democratic future. Social Identities, 9, 141-168.

Giroux, H. A. (2005). Doing cultural studies: Youth and the challenge of pedagogy. Available from http:/www.gseis.ucla.edu/courses/ed253a/Giroux/Giroux1.html

Goodlad, J. (1984). A place called school: Prospects for the future. New York: McGraw Hill.

Gore, J. M. (1998). Disciplining bodies: On the continuity of power relations in pedagogy. In T. S. Popewitz, \& M. Brennan (Eds.), Foucault's challenge: Discourse, knowledge, and power in education (pp. 231-251). New York: Teachers College Press.

Greene, M. (1988). The dialectic of freedom. New York: Teachers College Press.

Greenberg, E., Dunleavy, E., \& Kutner, M. (2007). Literacy behind bars: Results from the 2003 national assessment of adult literacy prison survey (NCES 2007-473). U. S. Department of Education. Washington, DC: National Center for Education Statistics. Retrieved August 26, 2007, from http://nces.ed.gov/pubsearch/pubsinfo.asp?pubid=2007473

Gutiérrez, K. (1992). A comparison of instructional contexts in writing process classrooms with Latino children. Education and Urban Society, 24, 224-262.

Gutiérrez, K. (2002). Studying cultural practices in urban learning communities. Human Development, 45, 312-321.

Gutiérrez, K. (2006). White innocence: A framework and methodology for rethinking educational discourse. International Journal of Learning, 12, 1-11. Available at http://www.gseis.ucla.edu/faculty/files/gutierrez/gutierrez_whiteInnocence.pdf

Gutiérrez, K. D. (2008). Developing a sociocritical literacy in the Third Space. Reading Research Quarterly, 43(2), 148-164. 
Gutiérrez, K., Baquedano-López, P., \& Tejeda, C. (2003). Rethinking diversity: Hybridity and hybrid language practices in the Third Space. In S. Goodman, T. Lillis, J. Maybin, \& N. Mercer (Eds.), Language, literacy and education: A reader (pp. 171-188). Trent, UK: Trentham.

Gutiérrez, K., \& Rogoff, B. (2003). Cultural ways of learning: Individual traits or repertoires of practice. Educational Researcher, 32(5), 19-25.

Habermas, J. (1981). The theory of communicative action, volume one: Reason and the rationalization of society. Boston: Beacon.

Habermas, J. (1987). The theory of communicative action, volume two: Lifeworld and system, a critique of functionalist reason. Boston: Beacon.

Hall, E. T. (1966). The hidden dimension. New York: Anchor.

Hamilton, M. (2000). Expanding the new literacy studies: using photographs to explore literacy as social practice. In D. Barton, M. Hamilton, \& R. Ivanič (Eds.), Situated literacies: Reading and writing in context (pp. 16-34). New York: Routledge.

Heath, S. B. (1983). Way with words: Language, life and work in communities and classrooms. New York: Cambridge University Press.

Hilliard, A. G. (1983). Psychological factors associated with language in the education of the African American child. The Journal of Negro Education, 52(1), 24-34.

Hilliard, A. G. (1995). The maroon within us: Selected essays on African American community socialization. Baltimore: Black Classic Press.

Hilliard, A. G., III (2003). No mystery: Closing the achievement gap between Africans and excellence. In T. Perry, C. Steele, \& A. Hilliard (Eds.), Young, gifted, and black:Promoting high achievement among African American students (pp. 131166). Boston: Beacon.

Hollins, E. R., \& Guzman, M. T. (2005). Research on preparing teachers for diverse populations. In M. Cochran-Smith \& K. M. Zeichner (Eds.), Studying teacher education: The report of the AERA panel on research and teacher education (pp. 477-548). Mahwah, NJ: Erlbaum.

hooks, b. (1989). Thinking feminist: Thinking black. Boston, MA: South End Press.

hooks, b. (1994). Teaching to transgress: Education as the practice of freedom. New York: Routledge. 
Howard, G. R. (1999). We can't teach what we don't know: White teachers, multiracial schools $\left(2^{\text {nd }}\right.$ ed. $)$. New York: Teachers College Press.

Howard, G. R. (2001). Telling their side of the story: African American students' perceptions of culturally relevant teaching. The Urban Review, 33(2), 131-149.

Individuals with Disabilities Education Act of 2004, Pub. L. No. 108-446, 118 Stat. 2647 (2004). Retrieved August 27, 2007, from U. S. Department of Education site http://www.ed.gov/about/offices/list/osers/osep/index.html? src=mr

Irvine, J. J. (1990). Black students and school failure: Policies, practices, and prescriptions. Westport, CT: Praeger.

Irvine, J. J. (2003). Educating teachers for diversity: Seeing with a cultural eye. New York: Teachers College Press.

Jimenez, R. T. (2004). Reconceptualizing the literacy learning of Latino students. In D. S. Strickland \& D. E. Alvermann (Eds.) Bridging the literacy achievement gap (pp. 17-29). New York: Teachers College Press.

Juvenile Justice and Delinquency Prevention Act of 1974, 42 U.S.C. (1974). Retrieved October 8, 2008, from http://www.ncjrs.gov/html/ojjdp/juris_tap_report/ch2_03.html

Kamberelis, G., \& Dimitriadis, G., (2005). Focus groups: Strategic articulations of pedagogy, politics, and inquiry. In N. K. Denzin \& Y. S. Lincoln (Eds.), The Sage handbook of qualitative research ( $3^{\text {rd }} \mathrm{ed}$, pp.887-907). London: Sage.

Kardos, S. M., \& Johnson, S. M. (2007). On their own and presumed expert: New teachers' experience with their colleagues. Teachers College Record, 109(9), 2083-2106. Retrieved August 4, 2009, from http://www.tcrecord.org

Kincheloe, J. (1991). Teachers as researchers: Qualitative inquiry as a path to empowerment. London, England: Falmer.

Kincheloe, J. (1993). Toward a critical politics of teacher thinking: Mapping the postmodern. Westport, CT: Bergin \& Garvey.

Kincheloe, J. (2005). Critical ontology and auto/biography: Being a teacher, developing a reflective teacher persona. In W.-M. Roth (Ed.). Auto/biography and auto/ethnography: Praxis of research method (pp. 155-176). Rotterdam, The Netherlands: Sense. 
King, J. E. (2005). A transformative vision of Black education for human freedom. In J.E. King (Ed.), Black education: A transformative research and action agenda for the new century (pp. 3-18). Mahwah, NJ: Erlbaum.

Kozol, J. (2005). The shame of the nation: The restoration of apartheid schooling in America. New York: Crown.

Ladson-Billings, G. (1994). The dreamkeepers: Successful teachers of African American children. San Francisco: Jossey Bass.

Ladson-Billings, G. (1995). Multicultural teacher education: Research, practice, and policy. In J. A. Banks \& C. A. M. Banks (Eds.), Handbook of research on multicultural education (pp. 747-759). New York: Macmillan.

Ladson-Billings, G. (1999). Just what is critical race theory and what's it doing in a nice field like education? In L. Parker, D. Deyhle, \& S. Villenas (Eds.) Race is ...race isn't: Critical race theory and qualitative studies in education (pp. 7-30). Boulder, CO: Westview.

Ladson-Billings, G. (2005). Beyond the big house: African American educators on teacher education. New York: Teachers College Press.

Ladson-Billings, G. (2006). From the achievement gap to the education debt: Understanding achievement in U. S. schools. Educational Researcher, 35(7), 312.

Ladson-Billings, G., \& Tate, W. (1995). Toward a critical race theory of education. Teachers College Record, 97(1), 47-68.

Ladson-Billings, G., \& Tate, W. F. (2004). Toward a critical race theory. Teachers College Record, 97(1), 45-66.

Lam, W. S. E. (2006). Culture and learning in the context of globalization: Research directions, Review of Research in Education, 30(1), 213-237.

Landorf, H., Rocco, T. S., \& Nevin, A. (2007). Creating permeable boundaries: Teaching and learning for social justice in a global society. Teacher Education Quarterly, 34, 41-56.

Lankshear, C., \& Knobel, M. (2006). New literacies: Everyday practices \& classroom learning $\left(2^{\text {nd }}\right.$ ed.). New York: McGraw-Hill.

Larson, C. L. \& Ovando, C. J. (2001). The color of bureaucracy: The politics of equity in multicultural school communities. Belmont, CA: Wadsworth. 
Lave, J., \& Wenger, E. (1991). Situated learning: Legitimate peripheral participation. New York: Cambridge University Press.

Lee, C. D. (1993). Signifying as a scaffold for literary interpretation: The pedagogical implicationsof an African American discourse genre. Urbana, IL: National Council of Teachers of English.

Lee, C. D. (1995). A culturally based cognitive apprenticeship: Teaching African American high school students' skills in literary interpretation. Reading Research Quarterly, 30(4), 608-631.

Lee, C. D. (2000). Signifying in the zone of proximal development. In C. D. Lee \& P. Smagorinsky (Eds.), Vygotskian perspectives on literacy research: Constructing meaning through collaborative inquiry (191-225). New York: Cambridge University Press.

Lee, C. D. (2005). The state of knowledge about the education of African Americans. In J. E. King (Ed.), Black education: A transformative research and action agenda for the new century (pp. 45-72). Mahwah, NJ: Lawrence Erlbaum.

Lee, C. D., \& Ball, A. (2005). All that glitters ain't gold: CHAT as a design and analytical tool in literacy research. In R. Beach, J. Green, M. Kamil, \& T. Shanahan (Eds.), Multidisciplinary perspectives on literacy research $\left(2^{\text {nd }}\right.$ ed., $p p$. 101-132). Cresskill, NJ: Hampton.

Lefebvre, H. (1991). The production of space. (D. Nicholson-Smith, Trans.) Malden, MA: Blackwell. (Original work published 1984)

Lewis, C., Enciso, P., \& Moje, E. B. (Eds.). (2007). Reframing sociocultural research on literacy: Identity, agency, and power (pp. 15-48). Mahwah, NJ: Lawrence Erlbaum.

Luke, A., \& Freebody, P. (1999). Further notes on the four resources model. Reading Online. Retrieved August 15, 2008, from www.readingonline.org

Maybin, J. (Ed.). (2000). Language and literacy in social practice. Philadelphia: The Open University.

McIntosh, P. (1997). White privilege and male privilege: A personal account of coming to see correspondences through work in women's studies. In E. Delgago \& J. Stefancic (Eds.) Critical white studies: Looking behind the mirror (pp. 291-299).

McLaren, P. (1999). Schooling as a ritual performance: Toward a political economy of educational symbols and gestures $\left(3^{\text {rd }}\right.$ ed.), New York: Rowman \& Littlefield. 
McLaren, P. (2003). Life in schools: An introduction to critical pedagogy in the foundations of education. ( $4^{\text {th }}$ ed.). New York: Allyn \& Bacon.

Miami-Dade County Public Schools. (2004). Article XXVIII - School improvement zone. Retrieved on August 3, 2008, from

http://www2.dadeschools.net/employees/labor_union/UTD/pdf05/Art_XXVIII.pdf

Miami-Dade County Public Schools. (2007). Miami-Dade County Public Schools 20052006 District and School Profiles (2007) [Data file]. Available from http://oada.dadeschools.net/dsprofiles/ds_profiles.asp

Miami-Dade County Public Schools Educational Alternative Outreach Program. (2007). Program description. Retrieved on November 21, 2007, from http://outreach.dadeschools.net

Miles, M. B., \& Huberman, A. M. (1994). An expanded sourcebook: Qualitative data analysis $\left(2^{\text {nd }}\right.$ ed.). Thousand Oaks, CA: Sage.

Milner, H. R. (2006). Classroom management in urban classrooms. In C. M. Evertson, \& C. S. Weinstein (Eds.). (2006). Handbook of classroom management: Research, practice, and contemporary issues (pp. 491-522). Mahwah, NJ: Lawrence Erlbaum.

Moje, E. B. (2004). Powerful spaces: Tracing the out-of-school literacy spaces of Latino/a youth. In K. Leander and M. Sheehy (Eds.), Spatializing Literacy Research and Practice (pp. 15-38). New York: Peter Lang.

Moje, E. B., Ciechanowski, K. M., Kramer,K., Ellis, L., Carrillo,R., \& Collazo, T. (2004). Working toward third space in content area litearcy: An examination of everyday funds of knowledge and Discourse. Reading Research Quarterly, 39(1), 38-70.

Moje, E. B., \& Hinchman, K. (2004). Culturally responsive practices for youth literacy learning. In J. Dole \& T. Jetton (Eds.), Adolescent literacy research and practice (pp. 331-350). New York: Guilford Press.

Moje, E. B., \& Lewis, C. (2007). Examining opportunities to learn literacy: The role of critical sociocultural literacy research. In C. Lewis, P. Enciso, \& E. B. Moje (Eds.), Reframing sociocultural research on literacy: Identity, agency, and power (pp. 15-48). Mahwah, NJ: Lawrence Erlbaum.

Moje, E. B., Overby, M., Tysvaer, N., \& Morris, K. (2008). The complex world of adolescent literacy: Myths, motivations, and mysteries. Harvard Educational Review, 78, 1-35. Retrieved August 1, 2009, from http://www.edreview.org/harvard08/2008/sp08/p08moje.htm 
Moll, L. (1992). Literacy research in community and classrooms: A sociocultural approach. In R. Beach, J. L. Green, M. L. Kamil, \& T. Shanalas (Eds.), Multiciplinary perspectives on literacy research (pp. 211-244). Urbana, IL: National Council of Teachers of English.

Monroe, C. M. (2005). The cultural context of "disruptive behavior": An overview of research considerations for school educators. Improving Schools, 8(2), 153-159.

Monroe, C. R. (Winter, 2006). African American boys and the discipline gap: Balancing educators' uneven hand. Educational HORIZONS, 102-111.

Monroe, C. R., \& Obidah, J. E. (2004). The influence of cultural synchronization on a teacher's perceptions of disruption: A case study of an African American middleschool classroom. Journal of Teacher Education, 55(3), 256-268.

Morris, V. G., \& Morris, C. L. (2000). Creating caring and nurturing education environments for African American children. Westport, CT: Bergin \& Garvey.

Morris, V. G., \& Morris, C. L. (2002). Caring - the missing C in teacher education. Journal of Teacher Education, 53(2), 120-123.

Muth, W. R. (2005). Literacy learners in prison: Finding purpose in the "second space". In P. E. Linder, M. B. Sampson, J. R. Dugan, \& B. A. Brancato (Eds.), Building bridges to literacy: The $27^{\text {th }}$ yearbook of the College Reading Association (pp. 1934). Bryan, TX: Texas A \& M University-Commerce.

Nasir, N. S., \& Hand, V. M. (2006). Exploring sociocultural perspectives on race, culture, and learning. Review of Educational Research, 76(4), 449-475.

National Center for Education Statistics. (2003). Public alternative schools for at-risk students. In The condition of education 2000-2007 (sect. 4). U. S. Department of Education. Retrieved October 2, 2009, from http://nces.ed.gov/programs/coe/2003/section4/indicator27.asp

National Center for Education Statistics (2005). Characteristics of schools, districts, teachers, principals, and school libraries in the United States: 2003-04 schools and staffing survey. U.S. Department of Education. Retrieved August 16, 2006, from NCES 2006-313

National Center for Education Statistics. (2008). Numbers and rates of public high school dropouts: School year 2004-05. In Common core of data: Selected findings. U.S. Department of Education. Retrieved February 14, 2008, from http://nces.ed.gov/pubs2008/hsdropouts/findings.asp 
National Partnership for Teaching in At-Risk Schools (2005). Qualified teachers for atrisk schools: A national imperative. (Inaugural report). Washington, DC: Warner.

Nembhard, J. G. (2005). On the road to democratic economic participation: Educating African American youth in the postindustrial global economy. In J. E. King (Ed.) Black education: A transformative research and action agenda for the new century (pp. 225-240). Mahwah, NJ: Lawrence Erlbaum.

Newman, I., \& Benz, C. R. (1998). Qualitative-quantitative research methodology: Exploring the interactive continuum. Carbondale, IL: Southern Illinois University Press.

Nieto, S. (2002). Language, culture, and teaching: Critical perspectives for a new century. Mahwah, NJ: Lawrence Erlbaum.

No Child Left Behind Act of 2001, Pub. L. No. 107-110, 115 Stat. 1425 (2002). Retrieved August 27, 2007, from U.S. Department of Education website www.ed.gov/legislation/ESEA02

Noddings, N. (2007). Philosophy of education ( $2^{\text {nd }}$ ed.). Cambridge, MA: Westview.

Noguera, P. A. (2001). Finding safety where we least expect it: The role of social capital in preventing school violence. In W. Ayers, B. Dohrn, \& R. Ayers (Eds.), Zero tolerance: Resisting the drive for punishment in our schools (pp. 202-218). New York: The New Press.

Noguera, P. A. (2003). The trouble with black boys: The role and influence of environmental and cultural factors on the academic performance of African American males. Urban Education, 38(4), 431-459.

Oakes, J. (1985). Keeping track: How schools structure inequality. New Haven: Yale University Press.

Oakes, J., Rogers, J., \& Lipton, M. (2006). Learning power: Organizing for education and justice. New York: Teachers College Press.

Office of Justice Programs (2008). Legislation. Retrieved August 1, 2008, from http://ojjdp.ncjrs.org/about/legislation.html

Office of Juvenile Justice Delinquency Prevention (2009). The Office of Juvenile Justice Delinquency Prevention's Model Programs Guide. Retrieved August 12, 2009, from http://www.dsgonline.com/mpg2.5/mpg_index.htm 
Pane, D. M. (in press). Viewing classroom discipline as negotiable social interaction: A communities of practice perspective. Teaching and Teacher Education: An International Journal of Research and Studies (2009), doi:10.1016/j.tate.2009.05.002

Pane, D. M. (2009). Third space: Blended teaching and learning. Journal of the Research Center for Educational Technology [Special Issue], 5, Art. 8, http://www.rcetj.org

Pane, D. M., \& Rocco, T. S. (2009). Critical microethnography: The search for emancipatory methods. Forum: Qualitative Social Research (FQS), 10(2), Art. 7, http://www.qualitative-research.net/index.php/fqs/issue/view/31

Pane, D. (2006). Pre-service teacher education for diversity. In A. Heron-Hruby (Ed.), American Reading Forum Online Yearbook, 26, http://www.americanreadingforum.org/06_yearbook/html/arf_06_pane.htm

Pane, D. (2005). Shifting our gaze to the strengths of alternative education students. In The proceedings of the fourth annual College of Education research conference, Florida International University, Miami, FL, USA, http://coeweb.fiu.edu/research_conference, 93-98.

Pardoe, S. (2000). Respect and the pursuit of "symmetry" in researching literacy and student writing. In D. Barton, M. Hamilton, \& R. Ivanič (Eds.), Situated literacies: Reading and writing in context (pp. 149-166). New York: Routledge.

Pasque, P. A., Bowman, N. A., Small, J. L., \& Lewis R. (2009). Student-created curricular and co-curricular pathways toward participation in a diverse democracy. Multicultural Perspectives, 11(2), 80-89.

Partnership for $21^{\text {st }}$ Century Skills. (2009). $21^{\text {st }}$ century learning environments white paper. Tucson, AZ. Retrieved August 12, 2009, from http://www.21stcenturyskills.org/index.php?option=com_content\&task=view\&id $=600 \&$ Itemid $=185$

Patton, M. Q. (2002). Qualitative research \& evaluation methods ( $3^{\text {rd }}$ ed.). Thousand Oaks: Sage.

Perry, T. (2003). Freedom for literacy and literacy for freedom. In T. Perry, C. Steele, \& A. Hilliard (Eds.), Young, gifted, and black: Promoting high achievement among African American students (pp. 11-51). Boston: Beacon.

Public Agenda. (2004). Teaching interrupted: Do discipline policies in today's public schools foster the common good? Available at http://www.publicagenda.org

Raywid, M. A. (1994). Alternative schools: The state of the art. Educational Leadership, 52(1), 26-31. 
Rist, R. C. (1970). Student social class and teacher expectations: The self-fulfilling prophecy and ghetto education. Harvard Educational Review, 40, 411-51.

Rogers, R. (Ed.). (2004). An introduction to critical discourse analysis in education. Mahwah, NJ: Erlbaum.

Rogers, R. \& Fuller, C. (2007). “As if you heard it from your momma”: Redesigning histories of participation with literacy education in an adult education class. In C. Lewis, P. Enciso, \& E. B. Moje (Eds.), Reframing sociocultural research on literacy: Identity, agency, and power (pp. 75-114). Mahwah, NJ: Lawrence Erlbaum.

Rogers, R., Malancharuvil-Berkes, E., Mosley, M., Hui, D., \& Joseph, G. O. (2005). Critical discourse analysis in education: A review of the literature. Review of Educational Research, 75(3), 365-416.

Rogoff, B. (1990). Apprenticeship in thinking: Cognitive development in social context. New York: Oxford Unviersity Press.

Roscoria, T. (2009, June 10). Teaching for the $21^{\text {st }}$ century. Converge. Retrieved August 14, 2009, from http://www.convergemag.com/workforce/Teaching-for-the-21stCentury.html

Rose, M. (1989). Lives on the boundary: A moving account of the struggles and achievements of America's educationally underprepared. New York: Penguin.

Rosenthal, R., \& Jacobson, L. (1968). Pygmalion in the classroom: Teacher expectation and pupils' intellectual development. New York: Holt, Rinehart, and Winston.

Roth, W.-M. (2005). Auto/biography and auto/ethnography: Praxis of research method, Rotterdam, The Netherlands: Sense.

Roth, W., \& Lee, Y. (2007). "Vygotsky's neglected legacy": Cultural-historical activity theory. Review of educational research, 77(2), 186-232.

Schensul, S. L., Schensul, J. J., \& LeCompte, M. D. (1999). Essential ethnographic methods: Observations, interviews, and questionnaires. New York: Rowman \& Littlefield.

Scribner, S., \& Cole, M. (1981). The psychology of literacy. Cambridge, MA: Harvard University Press.

Siegel, M., \& Fernandez, S. L. (2000). Critical approaches. In M. L. Kamil, P. B. Mosenthal, P. D. Pearson, \& R. Barr (Eds.), Handbook of reading research: Volume III (pp. 141-151). Mahwah, NJ: Lawrence Erlbaum. 
Shor, I. (1992). Empowering education: Critical teaching for social change. Chicago: The University of Chicago Press.

Shor, I., \& Freire, P. (1987). A pedagogy for liberation: Dialogues on transforming. Westport, CT: Bergin \& Garvey.

Shor, I. \& Freire, P. (2003). What are the fears and risks of transformation? In A. Darder, M. Baltodano, \& R. D. Torres (Eds.) The critical pedagogy reader (pp. 479-496). New York: Routledge Falmer.

Skiba, R. (2001). When is disproportionality discrimination? The overrepresentation of black students in school suspension. In W. Ayers, B. Dohrn, \& R. Ayers (Eds.), Zero tolerance: Resisting the drive for punishment in our schools (pp. 176-187). New York: The New Press.

Skiba, R. J., \& Leone, P. E. (2001). Zero tolerance and school security measures: A failed experiment. In T. Johnson, J. E. Boydon, \& W. J. Pittz (Eds.), Racial Profiling and Punishment in U.S. Public Schools: How Zero Tolerance Policies and High Stakes Testing Subvert Academic Excellence and Racial Equity. (Research Report). Applied Research Center, Oakland, CA.

Skiba, R. J., Michael, R. S., Nardo, A. C., \& Peterson, R. (2000). The color of discipline: Sources of racial and gender disproportionality in school punishment (Policy Research Report \#SRS1). Indiana University: Indiana Education Policy Center.

Sleeter, C. E. (2005). Un-standardizing curriculum: Multicultural teaching in the standards-based classroom. New York: Teachers College Press.

Smink, J., \& Schargel, F. P. (2004). Helping students graduate: A strategic approach to dropout prevention. Larchmont, NY: Eye on Education.

Snyder, H. N. \& Sickmund, M. (2006). Juvenile population characteristics. In Juvenile offenders and victims: 2006 national report (chap. 1). Washington, DC: U. S. Department of Justice, Office of Justice Programs, Office of Juvenile Justice and Dropout Prevention. Retrieved August 26, 2007, from http://www.ojjdp.ncjrs.gov/ojstatbb/nr2006/downloads/NR2006.pdf

Soja, E. W. (1996). Thirdspace: Journeys to Los Angeles and other real-and-imagined places. Malden, MA: Blackwell.

Spindler, G., \& Spindler, L. (1994). What is cultural therapy? In G. Spindler, \& L. Spindler (Eds.), Pathways to cultural awareness: Cultural therapy with teachers and students (pp. 1-33). Thousand Oaks, CA: Corwin.

Spradley, J. P. (1979). The ethnographic interview. Belmont, CA: Wadsworth. 
Steele, C. (2003). Stereotype threat and African American student achievement. In T. Perry, C. Steele, \& A. G. Hilliard, III (Eds.), Young, gifted, and black: Promoting high achievement among African American students (pp. 109-130). Boston: Beacon.

Stevens, L. P., \& Bean, T. W. (2007). Critical literacy: Context, research, and practice in the K-12 classroom. Thousand Oaks, CA: Sage.

Street, B. V. (1989). Literacy in theory and practice. New York: Cambridge University Press.

Strickland, D. S., \& Alvermann, D. E. (2004). Learning and teaching literacy in grades 412: Issues and challenges. In D. S. Strickland \& D. E. Alvermann (Eds.) Bridging the literacy achievement gap (pp. 1-14). New York: Teachers College Press.

Tough, P. (2008). Whatever it takes: Geoffrey Canada's quest to change Harlem and America. Boston: Houghton Mifflin.

Tusting, K., \& Barton, D. (2005). Community-based local literacies research. In R. Beach, J. Green, M. Kamil, \& T. Shanahan (Eds.), Multidisciplinary perspectives in literacy research $\left(2^{\text {nd }}\right.$ ed., pp. 179-208). Cresskill, NJ: Hampton.

U.S. Census Bureau. (2008). The 2008 statistical abstract [Data file]. Available from U.S. Census Bureau website http://www.census.gov/compendia/statab

U.S. Department of Education, Secretary Spellings approves 6 states' differentiated accountability proposals, Washington, D.C., 2008, Retrieved August 12, 2009, from http://www.ed.gov/nclb/accountability/differentiated/factsheet03.html

U.S. Department of Education, Building on results: A blueprint for strengthening the No Child Left Behind Act, Washington, D.C., 2007. Retrieved July 24, 2008, from http://www.ed.gov/policy/elsec/leg/nclb/buildingonresults.pdf

U.S. Department of Education, Office of Special Education Programs, Building the legacy: IDEA 2004, Discipline, Washington, DC, 2006, Retrieved August 1, 2008, from USDOE, OSEP website http://idea.ed.gov/explore/home

Vadeboncoeur, J. A., \& Stevens, L. P. (2005). (Eds.) Re/constructing "the adolescent": Sign, symbol, and body. New York: Peter Lang.

Van Acker, R. (2007). Antisocial, aggressive, and violent behavior in children and adolescents within alternative education settings: Prevention and intervention, Preventing School Failure, 51(2), 5-12. 
Varenne, H. (2008). Culture, education, and anthropology. Anthropology \& Education Quarterly, 39(4), 356-368.

Vavrus, F., \& Cole, K. (2002). "I didn't do nothin"”: The discursive construction of school suspension. The Urban Review, 3(2), 87-111.

Villegas, A. M., \& Lucas, T. (2002). Preparing culturally responsive teachers. Journal of Teacher Education, 53(1), 20-32.

Vygotsky, L. S. (1978). Mind in society: The development of higher psychological processes. Cambridge, MA: Harvard University Press.

Vygotsky, L. S. (1986). Thought and language. Cambridge, MA: MIT Press.

Wald, J., \& Losen, D. J. (Eds.). (2003). New directions for youth development: Deconstructing the school-to-prison pipeline: No. 99. San Francisco: Jossey-Bass.

Ware, F. (2006). Warm demander pedagogy: culturally responsive teaching that supports a culture of achievement for African American students. Urban Education, 41(4), 427-456.

Watts, I. E., \& Erevelles, N. (2004). These deadly times: Reconceptualizing school violence by using critical race theory and disability studies. American Educational Research Journal, 41(2), 271-299.

Weinstein, C. M., Curran, M., \& Tomlinson-Clarke, S. (2003). Culturally responsive classroom management: Awareness into action. Theory into Practice, 42(4), 270276.

Weinstein, C. M., Tomlinson-Clarke, S., \& Curran, M. (2004). Toward a conception of culturally responsive classroom management. Journal of Teacher Education, 55(25), 25-38.

Wengraf, T. (2004). Qualitative research interviewing: Biographic narrative and semistructured methods. Thousand Oaks, CA: Sage.

Westheimer, J., \& Kahne, J. (2004). What kind of citizen? The politics of educating for democracy. American Educational Research Journal, 41(2), p. 237-269.

White House. (2009). Education issues. Retrieved August 14, 2009, from http://www.whitehouse.gov/issues/education/

Willis, P. (1977). Learning to labor: How working class kids get working class jobs. New York: Columbia University Press. 
Wilson, A. (2003). Researching in the third space: Locating, claiming and valuing the research domain. In S. Goodman, T. Lillis, J. Maybin, \& N. Mercer (Eds.), Language, literacy and education: A reader (pp. 293-307). Trent, UK: Trentham.

Wolford, B. I. (2000). Youth education in the juvenile justice system. Corrections Today,62(5), 128. Retrieved May 29, 2004, from http://vnweb.hwwilsonweb.com.ezproxy.fiu.edu/hww

Woodson, C. G. (1933). The mid-education of the negro. Trenton, NJ: African World Press.

Wynne, J. (2002). “We don't talk right. You ask him.” In L. Delpit \& J. K. Dowdy (Eds.) The skin that we speak: Thoughts on language and culture in the classroom (pp.203-220). New York: The New Press.

Wynter, S. (2005). Race and our biocentric belief system: An interview with Sylvia Wynter. In J. E. King (Ed.), Black education: A transformative research and action agenda for the new century (pp. 361-366). Washington, DC: American Educational Research Association.

Zinn, H. (2005). A people's history of the United States: 1492-present. New York: Harper Collins. 


\section{APPENDIX A}

\section{Screening Questionnaire}

Hello, I am Debra Pane, a doctoral student from FIU. As a partial requirement for the $\mathrm{PhD}$ degree in Curriculum \& Instruction, I will complete a dissertation on the topic of classroom interactions in an alternative education school. I chose this topic after teaching in a M-DCPS educational alternative outreach classroom for nine years. M-DCPS has given me the official approval to conduct research in the classroom. The principal of this school has given me official approval to distribute this questionnaire to the teachers.

This questionnaire allows me to determine which teachers would be willing for me to conduct research in their classrooms. I understand the challenges of teaching in alternative education classrooms and will keep the time and disruption to a minimum.

If you answer "yes" to the following questions, I will contact you and set up a 15-minute meeting to discuss the possibility of your participation in this study. If you agree to participate, we will confirm a schedule and I will ask you to sign a consent form at that time. My contact information is debra.pane@fiu.edu or 786-371-6938.

Thank you for your interest, time and effort.

1. Are you willing for the investigator, Debra Pane, to observe in one of your classroom periods for five days?

2. Are you willing for the investigator, Debra Pane, to videorecording the classroom observations?

(yes) (no)

3. Are you willing to participate with the investigator, Debra Pane, in a one- to twohour digitally recorded interview to discuss the classroom observations?

(yes) (no)

4. Are you willing for a small group of your students to participate with the investigator, Debra Pane, in a digitally recorded interview to discuss the classroom observations?

(yes) (no) 


\section{APPENDIX B}

\section{Parent or Guardian Consent / Teen Assent Form To Participate in a Research Study Title: The Relationship between Classroom Interactions and Suspension as a Social Practice: A Critical Microethnography}

This form is to request your consent and your teen's agreement to be in a research study. The investigator of this study is Debra Pane, a student at Florida International University. The purpose of this study is to describe verbal and nonverbal interactions during classroom lessons. The study will begin on November 1, 2008, and end on December 15, 2008. Your teen will participate between 5 and 7 total hours. First, the investigator will observe and videorecording what happens during five 1-hour classroom lessons. Then, your teen may be asked to watch a segment from some of the videorecordingd observations to discuss what happened and why during the lessons. Discussion of the videorecordings will take about an hour and will be digitally recorded.

No harm is expected to your teen during this study. There will be no greater risk than what happens during a regular school day. Your teen may refuse to provide information if desired. Your teen's participation will, however, assist the investigator gain information and knowledge in this area. There are no direct benefits and no cost to your teen for participating. Your teen may withdraw from the study at any time with no negative consequences. All information that pertains to this study will be locked in an office. Files will be identifiable to the investigator, but final reports will not use your teen's name in any way. If you would like more information about this research study, you can contact Debra Pane by email at debra.pane@fiu.edu, by phone at 305-233-8645, or by mail at 9235 SW 144 St., Miami, FL 33176. If you would like to talk with someone about your rights of being in this study, you may contact Dr. Patricia Price, the Chairperson of the FIU Institutional Review Board at 305-348-2618 or 305-348-2494. Your signature below indicates that all of your questions have been answered and that you agree to help in the study. A space for you to refuse permission for your teen to help in this study is also available below. Check if you require a copy of this consent form.

\begin{tabular}{|c|c|c|}
\hline Parent/Guardian Signature of Permission & Printed Name & Date \\
\hline Parent/Guardian Signature of Refusal & Printed Name & Date \\
\hline Teen's Signature & Printed Name & Date \\
\hline
\end{tabular}

I have explained the research procedure, subject rights and answered questions asked by the parent and/or participant. I have offered them a copy of this informed consent form.

Investigator




\section{APPENDIX C \\ Teacher Consent Form \\ Title: The Relationship between Classroom Interactions and Suspension as a Social Practice: A Critical Microethnography}

You are being asked to participate in a research study. The investigator of this study is Debra Pane, a student at Florida International University. The purpose of this study is to describe verbal and nonverbal interactions during classroom lessons in one of the classes you teach. Your participation will include being part of five 1-hour classroom videorecordingd observations and a 2-hour digitally recorded individual interview. The interview will consist of asking for your perspective on school in general and then watching a 20-minute segment of the videorecordings to get your opinion about what happened and why during the classroom observations. Before the study begins, the investigator will gather contact information for each student in your class. Using the information provided, the investigator will contact the students' parent or guardian to gain permission and signatures for their teen to participate in the study. This form requests your permission as the teacher for the investigator to observe and videorecording in your classroom and your approval to be interviewed.

No harm is expected to you or the students during this study. There will be no greater risk than what you do during a typical workday. You may refuse to provide information if you choose. Your participation will, however, assist the investigator gain information and permission needed to begin study in this area. There are no direct benefits and no cost to you for your participation. You may withdraw your participation at any time with no negative consequences. All information that pertains to this study will be locked in an office. Files will be identifiable to the investigator, but final reports will not use your name in any way. If you would like more information about this research study, you can contact Debra Pane by email at debra.pane@fiu.edu. If you would like to talk with someone about your rights of being in this study, you may contact Dr. Patricia Price, the Chairperson of the FIU Institutional Review Board at 305-348-2618 or 305-348-2494. Your signature below indicates that all of your questions have been answered and that you agree to help in the study. Check if you require a copy of this informed consent form.

Participant's Signature

Printed Name

Date

I have explained the research procedure, subject rights and answered questions asked by the participant. I have offered him/her a copy of this informed consent form. 


\section{APPENDIX D}

\begin{tabular}{|c|c|c|c|c|}
\hline \multicolumn{5}{|c|}{ Projected Data Collection and Analysis Schedule } \\
\hline Monday & Tuesday & Wednesday & Thursday & Friday \\
\hline \multicolumn{5}{|c|}{ Stage One-Data Collection for the Primary Record (20 hours) } \\
\hline \multicolumn{5}{|c|}{ Week 1-Classroom Observations (10 hours) } \\
\hline $\begin{array}{l}\text { Class } 1 \\
\text { 1-hour passive } \\
\text { observation in } \\
\text { classroom }\end{array}$ & $\begin{array}{l}\text { 1-hour passive } \\
\text { observation in } \\
\text { classroom }\end{array}$ & $\begin{array}{l}\text { 1-hour passive } \\
\text { observation in } \\
\text { classroom }\end{array}$ & $\begin{array}{l}\text { 1-hour passive } \\
\text { observation in } \\
\text { classroom }\end{array}$ & $\begin{array}{l}\text { 1-hour passive } \\
\text { observation in } \\
\text { classroom }\end{array}$ \\
\hline $\begin{array}{l}\text { Class } 2 \\
\text { 1-hour passive } \\
\text { observation in } \\
\text { classroom }\end{array}$ & $\begin{array}{l}\text { 1-hour passive } \\
\text { observation in } \\
\text { classroom }\end{array}$ & $\begin{array}{l}\text { 1-hour passive } \\
\text { observation in } \\
\text { classroom }\end{array}$ & $\begin{array}{l}\text { 1-hour passive } \\
\text { observation in } \\
\text { classroom }\end{array}$ & $\begin{array}{l}\text { 1-hour passive } \\
\text { observation in } \\
\text { classroom }\end{array}$ \\
\hline \multicolumn{5}{|c|}{ Week 2-Classroom Observations (10 hours) } \\
\hline $\begin{array}{l}\text { Class } 3 \\
\text { 1-hour passive } \\
\text { observation in } \\
\text { classroom }\end{array}$ & $\begin{array}{l}\text { 1-hour passive } \\
\text { observation in } \\
\text { classroom }\end{array}$ & $\begin{array}{l}\text { 1-hour passive } \\
\text { observation in } \\
\text { classroom }\end{array}$ & $\begin{array}{l}\text { 1-hour passive } \\
\text { observation in } \\
\text { classroom }\end{array}$ & $\begin{array}{l}\text { 1-hour passive } \\
\text { observation in } \\
\text { classroom }\end{array}$ \\
\hline $\begin{array}{l}\text { Class } 4 \\
\text { 1-hour passive } \\
\text { observation in } \\
\text { classroom }\end{array}$ & $\begin{array}{l}\text { 1-hour passive } \\
\text { observation in } \\
\text { classroom }\end{array}$ & $\begin{array}{l}\text { 1-hour passive } \\
\text { observation in } \\
\text { classroom }\end{array}$ & $\begin{array}{l}\text { 1-hour passive } \\
\text { observation in } \\
\text { classroom }\end{array}$ & $\begin{array}{l}\text { 1-hour passive } \\
\text { observation in } \\
\text { classroom }\end{array}$ \\
\hline \multicolumn{5}{|c|}{ Stage Two-Building Primary Record from Classroom Observations (24-32 hours) } \\
\hline \multicolumn{5}{|c|}{ Week 3-View Videorecordingd Classroom Observations } \\
\hline $\begin{array}{l}1^{\text {st }} \text { View of } 5 \\
\text { hours of video- } \\
\text { Select } 1-\text { hour } \\
\text { segment } \\
\text { representative of } \\
\text { Class } 1 \\
\text { interaction } \\
\text { patterns } \\
\text { (3-4 hours) }\end{array}$ & $\begin{array}{l}1^{\text {st }} \text { View of } 5 \\
\text { hours of video- } \\
\text { Select } 1-\text { hour } \\
\text { segment } \\
\text { representative of } \\
\text { Class } 2 \\
\text { interaction } \\
\text { patterns } \\
\text { (3-4 hours) }\end{array}$ & $\begin{array}{l}1^{\text {st }} \text { View of } 5 \\
\text { hours of video- } \\
\text { Select } 1 \text { - hour } \\
\text { segment } \\
\text { representative of } \\
\text { Class } 3 \\
\text { interaction } \\
\text { patterns } \\
(3-4 \text { hours })\end{array}$ & $\begin{array}{l}1^{\text {st }} \text { View of } 5 \\
\text { hours of video- } \\
\text { Select } 1 \text { - hour } \\
\text { segment } \\
\text { representative of } \\
\text { Class } 4 \\
\text { interaction } \\
\text { patterns } \\
\text { (3-4 hours) }\end{array}$ & \\
\hline $\begin{array}{l}2^{\text {nd }} \text { View of } 1- \\
\text { hour segment- } \\
\text { Select } 20 \text {-minute } \\
\text { segment most } \\
\text { representative of } \\
\text { Class } 1 \\
\text { interaction } \\
\text { patterns } \\
\text { (3-4 hours) }\end{array}$ & $\begin{array}{l}2^{\text {nd }} \text { View of } 1- \\
\text { hour segment- } \\
\text { Select } 20 \text {-minute } \\
\text { segment most } \\
\text { representative of } \\
\text { Class } 2 \\
\text { interaction } \\
\text { patterns } \\
\text { (3-4 hours) }\end{array}$ & $\begin{array}{l}2^{\text {nd }} \text { View of } 1- \\
\text { hour segment- } \\
\text { Select } 20 \text {-minute } \\
\text { segment most } \\
\text { representative of } \\
\text { Class } 3 \\
\text { interaction } \\
\text { patterns } \\
(3-4 \text { hours })\end{array}$ & $\begin{array}{l}2^{\text {nd }} \text { View of } 1- \\
\text { hour segment- } \\
\text { Select } 20 \text {-minute } \\
\text { segment most } \\
\text { representative of } \\
\text { Class } 4 \\
\text { interaction } \\
\text { patterns } \\
(3-4 \text { hours })\end{array}$ & \\
\hline
\end{tabular}




\begin{tabular}{|c|c|c|c|c|}
\hline \multicolumn{5}{|c|}{ Stage Three A-Dialogical Data Generation (4 hours) } \\
\hline \multicolumn{5}{|c|}{ Week 4 -Interviews with Students } \\
\hline $\begin{array}{l}\text { Class } 1 \\
\text { Group } \\
\text { Videorecording } \\
\text { Feedback } \\
\text { Interview } \\
\text { (1 hour) }\end{array}$ & $\begin{array}{l}\text { Class } 3 \\
\text { Group } \\
\text { Videorecording } \\
\text { Feedback } \\
\text { Interview } \\
\text { (1 hour) }\end{array}$ & & & \\
\hline $\begin{array}{l}\text { Class } 2 \\
\text { Group } \\
\text { Videorecording } \\
\text { Feedback } \\
\text { Interview } \\
\text { (1 hour) }\end{array}$ & $\begin{array}{l}\text { Class } 4 \\
\text { Group } \\
\text { Videorecording } \\
\text { Feedback } \\
\text { Interview } \\
\text { (1 hour) }\end{array}$ & & & \\
\hline \multicolumn{5}{|c|}{ Stage Three B-Dialogical Data Generation ( 8 hours) } \\
\hline \multicolumn{5}{|c|}{ Week 5-Interviews with Teachers } \\
\hline $\begin{array}{l}\text { Class } 1 \\
\text { Teacher } \\
\text { Individual } \\
\text { Interview and } \\
\text { Videorecording } \\
\text { Feedback } \\
\text { Interview } \\
\text { (1-2 hours) }\end{array}$ & $\begin{array}{l}\text { Class } 2 \\
\text { Teacher } \\
\text { Individual } \\
\text { Interview and } \\
\text { Videorecording } \\
\text { Feedback } \\
\text { Interview } \\
\text { (1-2 hours) }\end{array}$ & $\begin{array}{l}\text { Class } 3 \\
\text { Teacher } \\
\text { Individual } \\
\text { Interview and } \\
\text { Videorecording } \\
\text { Feedback } \\
\text { Interview } \\
\text { (1-2 hours) }\end{array}$ & \begin{tabular}{|l} 
Class 4 \\
Teacher \\
Individual \\
Interview and \\
Videorecording \\
Feedback \\
Interview \\
(1-2 hours)
\end{tabular} & \\
\hline \multicolumn{5}{|c|}{$\begin{array}{c}\text { Stage Four A-Building Primary Record from Representative Classroom Observations } \\
(16-36 \text { hours })\end{array}$} \\
\hline \multicolumn{5}{|c|}{ Week 6-View 20-minute Segments } \\
\hline $\begin{array}{l}3^{\text {rd }} \text { view-- } \\
\text { Transcribe } \\
\text { meaningful } \\
\text { utterances for } \\
\text { Class } 1 \\
\text { (4-9 hours) }\end{array}$ & $\begin{array}{l}3^{\text {rd }} \text { view- } \\
\text { Transcribe } \\
\text { meaningful } \\
\text { utterances for } \\
\text { Class } 2 \\
\text { (4-9 hours) }\end{array}$ & $\begin{array}{l}3^{\text {rd }} \text { view- } \\
\text { Transcribe } \\
\text { meaningful } \\
\text { utterances for } \\
\text { Class } 3 \\
\text { (4-9 hours) }\end{array}$ & $\begin{array}{l}3^{\text {rd }} \text { view- } \\
\text { Transcribe } \\
\text { meaningful } \\
\text { utterances for } \\
\text { Class } 4 \\
\text { (4-9 hours) }\end{array}$ & $\begin{array}{l}\text { (See Pilot Study } \\
\text { Transcripts: } \\
\text { Stage 4A) }\end{array}$ \\
\hline \multicolumn{5}{|c|}{$\begin{array}{c}\text { Stage Four B-Building Primary Record from Representative Classroom Observations } \\
(16-20 \text { hours })\end{array}$} \\
\hline \multicolumn{5}{|c|}{ Week 7-View 20-minute Segments } \\
\hline $\begin{array}{l}\text { 4th view- } \\
\text { Explain } \\
\text { meaningful } \\
\text { utterances and } \\
\text { linguistic } \\
\text { behavior } \\
\text { observed for } \\
\text { Class } 1 \\
\text { (4-5 hours) }\end{array}$ & $\begin{array}{l}\text { 4th view- } \\
\text { Explain } \\
\text { meaningful } \\
\text { utterances and } \\
\text { linguistic } \\
\text { behavior } \\
\text { observed for } \\
\text { Class } 2 \\
\text { (4-5 hours) }\end{array}$ & $\begin{array}{l}\text { 4th view- } \\
\text { Explain } \\
\text { meaningful } \\
\text { utterances and } \\
\text { linguistic } \\
\text { behavior } \\
\text { observed for } \\
\text { Class } 3 \\
\text { (4-5 hours) }\end{array}$ & $\begin{array}{l}\text { 4th view- } \\
\text { Explain } \\
\text { meaningful } \\
\text { utterances and } \\
\text { linguistic } \\
\text { behavior } \\
\text { observed for } \\
\text { Class } 4 \\
(4-5 \text { hours })\end{array}$ & $\begin{array}{l}\text { (See Pilot Study } \\
\text { Transcripts: } \\
\text { Stage 4B and } \\
\text { 4C) }\end{array}$ \\
\hline
\end{tabular}




\begin{tabular}{|c|c|c|c|c|}
\hline \multicolumn{5}{|c|}{$\begin{array}{c}\text { Stage Four C-Building Primary Record from Representative Classroom Observations } \\
(8-12 \text { hours })\end{array}$} \\
\hline \multicolumn{5}{|c|}{ Week 8-Analyze 20-minute Segments } \\
\hline $\begin{array}{l}5^{\text {th }} \text { view- } \\
\text { Articulate } \\
\text { possible objective } \\
\text { validity claims } \\
\text { about linguistic } \\
\text { behavior } \\
\text { observed for } \\
\text { Class } 1 \\
(2-3 \text { hours })\end{array}$ & $\begin{array}{l}5^{\text {th }} \text { view- } \\
\text { Articulate } \\
\text { possible objective } \\
\text { validity claims } \\
\text { about linguistic } \\
\text { behavior } \\
\text { observed for } \\
\text { Class } 2 \\
\text { (2-3 hours) }\end{array}$ & $\begin{array}{l}5^{\text {th }} \text { view- } \\
\text { Articulate } \\
\text { possible objective } \\
\text { validity claims } \\
\text { about linguistic } \\
\text { behavior } \\
\text { observed for } \\
\text { Class } 3 \\
(2-3 \text { hours })\end{array}$ & $\begin{array}{l}5^{\text {th }} \text { view- } \\
\text { Articulate } \\
\text { possible objective } \\
\text { validity claims } \\
\text { about linguistic } \\
\text { behavior } \\
\text { observed for } \\
\text { Class } 4 \\
(2-3 \text { hours })\end{array}$ & $\begin{array}{l}\text { (See Pilot Study } \\
\text { Transcripts: } \\
\text { Stage 4B and } \\
\text { 4C) }\end{array}$ \\
\hline \multicolumn{5}{|c|}{$\begin{array}{c}\text { Stage Five A-Reconstructive Data Analysis of Representative Classroom Observations } \\
(20 \text { hours })\end{array}$} \\
\hline \multicolumn{5}{|c|}{ Week 9-Initial Meaning Reconstruction of 20-minute Segments (12 hours) } \\
\hline $\begin{array}{l}\text { Low-level } \\
\text { Coding } \\
\text { (categories to } \\
\text { name } \\
\text { interactions) for } \\
\text { Class } 1 \\
\text { ( } 3 \text { hours) }\end{array}$ & $\begin{array}{l}\text { Low-level } \\
\text { Coding } \\
\text { (categories to } \\
\text { name } \\
\text { interactions) for } \\
\text { Class } 2 \\
\text { (3 hours) }\end{array}$ & $\begin{array}{l}\text { Low-level } \\
\text { Coding } \\
\text { (categories to } \\
\text { name } \\
\text { interactions) for } \\
\text { Class } 3 \\
\text { (3 hours) }\end{array}$ & $\begin{array}{l}\text { Low-level } \\
\text { Coding } \\
\text { (categories to } \\
\text { name } \\
\text { interactions) for } \\
\text { Class } 4 \\
\text { ( } 3 \text { hours) }\end{array}$ & $\begin{array}{l}\text { (See Pilot Study } \\
\text { Transcripts: } \\
\text { Low Level } \\
\text { Stage 5A, Part } 1 \\
\text { and 2) }\end{array}$ \\
\hline \multicolumn{5}{|c|}{ Week 10-Initial Meaning Reconstruction of 20-minute Segments ( 8 hours) } \\
\hline $\begin{array}{l}\text { Explicit meaning } \\
\text { reconstruction } \\
\text { (possible } \\
\text { underlying } \\
\text { meanings) for } \\
\text { Class } 1\end{array}$ & $\begin{array}{l}\text { Explicit meaning } \\
\text { reconstruction } \\
\text { (possible } \\
\text { underlying } \\
\text { meanings) for } \\
\text { Class 2 }\end{array}$ & $\begin{array}{l}\text { Explicit meaning } \\
\text { reconstruction } \\
\text { (possible } \\
\text { underlying } \\
\text { meanings) for } \\
\text { Class } 3\end{array}$ & $\begin{array}{l}\text { Explicit meaning } \\
\text { reconstruction } \\
\text { (possible } \\
\text { underlying } \\
\text { meanings) for } \\
\text { Class } 4\end{array}$ & $\begin{array}{l}\text { (See Pilot Study } \\
\text { Transcripts: } \\
\text { Stage 5A, Part } 1 \\
\text { and 2) }\end{array}$ \\
\hline \multicolumn{5}{|c|}{$\begin{array}{c}\text { Stage Five B-Reconstructive Data Analysis of Representative Classroom Observations } \\
\text { (34 hours) }\end{array}$} \\
\hline \multicolumn{5}{|c|}{ Week 11-Pragmatic Horizon Analysis of 20-minute Segments (22 hours) } \\
\hline $\begin{array}{l}\text { Boundary- } \\
\text { making- } \\
\text { Contextualization } \\
\text { cues ( } 4.5 \text { hours) } \\
\text { for Class } 1\end{array}$ & $\begin{array}{l}\text { Boundary- } \\
\text { making- } \\
\text { Contextualization } \\
\text { cues ( } 4.5 \text { hours) } \\
\text { for Class } 2 \\
\end{array}$ & $\begin{array}{l}\text { Boundary- } \\
\text { making- } \\
\text { Contextualization } \\
\text { cues ( } 4.5 \text { hours) } \\
\text { for Class } 3\end{array}$ & $\begin{array}{l}\text { Boundary- } \\
\text { making- } \\
\text { Contextualization } \\
\text { cues ( } 4.5 \text { hours) } \\
\text { for Class } 4\end{array}$ & $\begin{array}{l}\text { (See Pilot Study } \\
\text { Transcripts: } \\
\text { Stage 5B, Part } \\
\text { 1) }\end{array}$ \\
\hline $\begin{array}{l}\text { Boundary- } \\
\text { making- } \\
\text { Interactional } \\
\text { units ( } 1 / 2 \text { hour) } \\
\text { for Class } 1\end{array}$ & $\begin{array}{l}\text { Boundary- } \\
\text { making- } \\
\text { Interactional } \\
\text { units ( } 1 / 2 \text { hour) } \\
\text { for Class } 2 \\
\end{array}$ & $\begin{array}{l}\text { Boundary- } \\
\text { making- } \\
\text { Interactional } \\
\text { units ( } 1 / 2 \text { hour) } \\
\text { for Class } 3\end{array}$ & $\begin{array}{l}\text { Boundary- } \\
\text { making-- } \\
\text { Interactional } \\
\text { units ( } 1 / 2 \text { hour) } \\
\text { for Class } 4\end{array}$ & $\begin{array}{l}\text { (See Pilot Study } \\
\text { Transcripts: } \\
\text { Stage 5B, Part } \\
\text { 1) }\end{array}$ \\
\hline $\begin{array}{l}\text { Boundary- } \\
\text { making-Phases } \\
\text { ( } 1 / 2 \text { hour) for }\end{array}$ & $\begin{array}{l}\text { Boundary- } \\
\text { making-Phases } \\
(1 / 2 \text { hour }) \text { for }\end{array}$ & $\begin{array}{l}\text { Boundary- } \\
\text { making-Phases } \\
(1 / 2 \text { hour }) \text { for }\end{array}$ & $\begin{array}{l}\text { Boundary- } \\
\text { making-Phases } \\
(1 / 2 \text { hour }) \text { for }\end{array}$ & \\
\hline
\end{tabular}




\begin{tabular}{|c|c|c|c|c|}
\hline Class 1 & Class 2 & Class 3 & Class 4 & \\
\hline \multicolumn{5}{|c|}{ Week 12-Pragmatic Horizon Analysis of 20-minute Segments (12 hours) } \\
\hline $\begin{array}{l}\text { Turn-taking for } \\
\text { Class } 1(1 / 2 \text { hour })\end{array}$ & $\begin{array}{l}\text { Turn-taking for } \\
\text { Class } 2(1 / 2 \text { hour })\end{array}$ & $\begin{array}{l}\text { Turn-taking for } \\
\text { Class } 3(1 / 2 \text { hour })\end{array}$ & $\begin{array}{l}\text { Turn-taking for } \\
\text { Class } 4(1 / 2 \text { hour })\end{array}$ & $\begin{array}{l}\text { (See Pilot Study } \\
\text { Transcripts: } \\
\text { Stage 5B, Part } \\
\text { 2a/b) }\end{array}$ \\
\hline $\begin{array}{l}\text { Thematic } \\
\text { Coherence for } \\
\text { Class } 1 \\
(1.5 \text { hours })\end{array}$ & $\begin{array}{l}\text { Thematic } \\
\text { Coherence for } \\
\text { Class } 2 \\
\text { (1.5 hours) }\end{array}$ & $\begin{array}{l}\text { Thematic } \\
\text { Coherence for } \\
\text { Class } 3 \\
(1.5 \text { hours })\end{array}$ & $\begin{array}{l}\text { Thematic } \\
\text { Coherence for } \\
\text { Class } 4 \\
(1.5 \text { hours })\end{array}$ & $\begin{array}{l}\text { (See Pilot Study } \\
\text { Transcripts: } \\
\text { Stage 5B, Part } \\
\text { 2a/b) }\end{array}$ \\
\hline $\begin{array}{l}\text { Intertextuality for } \\
\text { Class } 1 \text { ( } 1 \text { hour) }\end{array}$ & $\begin{array}{l}\text { Intertextuality for } \\
\text { Class } 2 \text { (1 hour) }\end{array}$ & $\begin{array}{l}\text { Intertextuality for } \\
\text { Class } 3 \text { (1 hour) }\end{array}$ & $\begin{array}{l}\text { Intertextuality for } \\
\text { Class } 4 \text { (1 hour) }\end{array}$ & $\begin{array}{l}\text { (See Pilot Study } \\
\text { Transcripts: } \\
\text { Stage 5B, Part } \\
\text { 2c) }\end{array}$ \\
\hline \multicolumn{5}{|c|}{$\begin{array}{c}\text { Stage Five C-Reconstructive Data Analysis of Representative Classroom Observations } \\
\text { (12 hours) }\end{array}$} \\
\hline \multicolumn{5}{|c|}{ Week 13-Validity Reconstruction of 20-minute Segments } \\
\hline $\begin{array}{l}\text { Validity } \\
\text { Reconstruction } \\
\text { for Class } 1 \\
\text { (3 hours) }\end{array}$ & \begin{tabular}{|l|} 
Validity \\
Reconstruction \\
for Class 2 \\
(3 hours) \\
\end{tabular} & \begin{tabular}{|l|} 
Validity \\
Reconstruction \\
for Class $\mathbf{3}$ \\
(3 hours) \\
\end{tabular} & \begin{tabular}{|l|} 
Validity \\
Reconstruction \\
for Class 4 \\
(3 hours) \\
\end{tabular} & $\begin{array}{l}\text { (See Pilot Study } \\
\text { Transcripts: } \\
\text { Stage 5C) }\end{array}$ \\
\hline \multicolumn{5}{|c|}{ Stage Six A-Building Primary Record from Interviews (28 hours) } \\
\hline \multicolumn{5}{|c|}{ Week 14—Listen to Student Interviews } \\
\hline $\begin{array}{l}\text { Transcribe } \\
\text { meaningful } \\
\text { utterances for } \\
\text { Class } 1 \\
\text { (7 hours) }\end{array}$ & $\begin{array}{l}\text { Transcribe } \\
\text { meaningful } \\
\text { utterances for } \\
\text { Class } 2 \\
\text { (7 hours) }\end{array}$ & \begin{tabular}{|l|} 
Transcribe \\
meaningful \\
utterances for \\
Class 3 \\
(7 hours) \\
\end{tabular} & \begin{tabular}{|l|} 
Transcribe \\
meaningful \\
utterances for \\
Class 4 \\
(7 hours) \\
\end{tabular} & $\begin{array}{l}\text { See Pilot Study } \\
\text { Transcript: } \\
\text { Stage 6A) }\end{array}$ \\
\hline \multicolumn{5}{|c|}{ Stage Six B-Building Primary Record from Interviews (28 hours) } \\
\hline \multicolumn{5}{|c|}{ Week 15-Listen to Teacher Interviews } \\
\hline $\begin{array}{l}\text { Transcribe } \\
\text { meaningful } \\
\text { utterances for } \\
\text { Class } 1 \text { Teacher } \\
\text { (7 hours) }\end{array}$ & $\begin{array}{l}\text { Transcribe } \\
\text { meaningful } \\
\text { utterances for } \\
\text { Class } 2 \text { Teacher } \\
\text { (7 hours) } \\
\end{array}$ & $\begin{array}{l}\text { Transcribe } \\
\text { meaningful } \\
\text { utterances for } \\
\text { Class } 3 \text { Teacher } \\
\text { (7 hours) }\end{array}$ & $\begin{array}{l}\text { Transcribe } \\
\text { meaningful } \\
\text { utterances for } \\
\text { Class } 4 \text { Teacher } \\
\text { ( } 7 \text { hours) } \\
\end{array}$ & \\
\hline \multicolumn{5}{|c|}{ Stage Seven A-Reconstructive Data Analysis of Interviews (18 hours) } \\
\hline \multicolumn{5}{|c|}{ Week 16-Initial Meaning Reconstruction of Student Interviews (9 hours) } \\
\hline $\begin{array}{l}\text { Low-level } \\
\text { Coding for Class } \\
\mathbf{1}(2.25 \text { hours) }\end{array}$ & $\begin{array}{l}\text { Low-level } \\
\text { Coding for Class } \\
\mathbf{2}(2.25 \text { hours }) \\
\end{array}$ & \begin{tabular}{|l|} 
Low-level \\
Coding for Class \\
$\mathbf{3}$ (2.25 hours) \\
\end{tabular} & \begin{tabular}{|l|} 
Low-level \\
Coding for Class \\
$\mathbf{4}(2.25$ hours $)$ \\
\end{tabular} & $\begin{array}{l}\text { See Pilot Study } \\
\text { Transcript: } \\
\text { (Stage 7A) }\end{array}$ \\
\hline
\end{tabular}




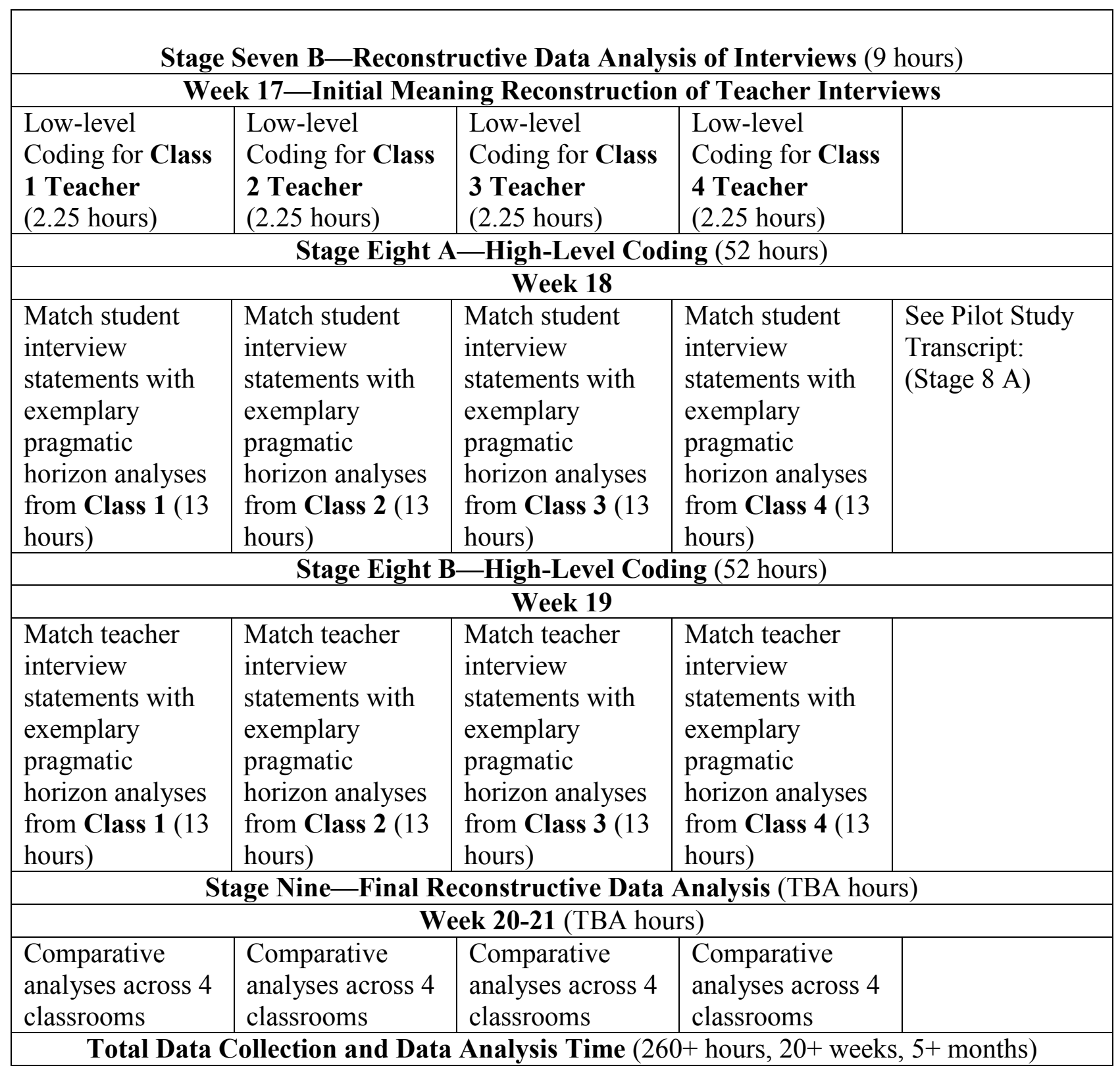


APPENDIX E

Tasks for Study Timeline

\begin{tabular}{|c|c|c|c|c|c|c|c|c|c|c|c|c|c|c|c|c|c|c|c|c|c|c|c|c|c|c|c|c|}
\hline \multirow{2}{*}{$\begin{array}{l}\text { Tasks for Study } \\
\text { Weeks }\end{array}$} & \multicolumn{4}{|c|}{ Month 1} & \multicolumn{4}{|c|}{ Month 2} & \multicolumn{4}{|c|}{ Month 3} & \multicolumn{4}{|c|}{ Month 4} & \multicolumn{4}{|c|}{ Month 5} & \multicolumn{4}{|c|}{ Month 6} & \multicolumn{4}{|c|}{ Month 7} \\
\hline & 1 & 2 & 3 & 4 & 5 & 6 & 7 & 8 & 9 & $\begin{array}{l}\mathbf{1} \\
\mathbf{0}\end{array}$ & $\begin{array}{l}1 \\
1\end{array}$ & $\begin{array}{l}1 \\
\mathbf{2}\end{array}$ & $\begin{array}{l}1 \\
\mathbf{3}\end{array}$ & $\begin{array}{l}1 \\
4\end{array}$ & $\begin{array}{l}1 \\
5\end{array}$ & $\begin{array}{l}1 \\
6\end{array}$ & $\begin{array}{l}1 \\
7\end{array}$ & $\begin{array}{l}1 \\
8\end{array}$ & $\begin{array}{l}1 \\
9\end{array}$ & $\begin{array}{l}2 \\
0\end{array}$ & $\begin{array}{l}2 \\
1\end{array}$ & $\begin{array}{l}2 \\
2\end{array}$ & $\begin{array}{l}2 \\
3\end{array}$ & $\begin{array}{l}2 \\
4\end{array}$ & $\begin{array}{l}2 \\
5\end{array}$ & $\begin{array}{l}2 \\
6\end{array}$ & $\begin{array}{l}2 \\
7\end{array}$ & $\begin{array}{l}2 \\
8\end{array}$ \\
\hline Gaining Access & $\mathbf{x}$ & $\mathbf{x}$ & & & & & & & & & & & & & & & & & & & & & & & & & & \\
\hline Criterion Sampling/Consent Forms & & & $\mathbf{x}$ & $\mathbf{x}$ & & & & & & & & & & & & & & & & & & & & & & & & \\
\hline Classroom Observations & & & & & $\mathbf{x}$ & $\mathbf{x}$ & & & & & & & & & & & & & & & & & & & & & & \\
\hline $\begin{array}{l}\text { Building Primary Record } \\
1^{\text {st }} / 2^{\text {nd }} V \text { iew/Criterion Sampling } \\
\text { for Student Interviews }\end{array}$ & & & & & & & $\mathbf{x}$ & & & & & & & & & & & & & & & & & & & & & \\
\hline Student Group Interviews & & & & & & & & $\mathbf{x}$ & & & & & & & & & & & & & & & & & & & & \\
\hline Teacher Individual Interviews & & & & & & & & & $\mathbf{x}$ & & & & & & & & & & & & & & & & & & & \\
\hline Building PR $3^{\text {rd }}$ Viewing & & & & & & & & & & $\mathbf{X}$ & & & & & & & & & & & & & & & & & & \\
\hline Building PR $4^{\text {th }}$ Viewing & & & & & & & & & & & $\mathbf{x}$ & & & & & & & & & & & & & & & & & \\
\hline Building PR $5^{\text {th }}$ Session & & & & & & & & & & & & $\mathbf{x}$ & & & & & & & & & & & & & & & & \\
\hline $\begin{array}{l}\text { Initial Meaning Reconstruction } \\
\text { (low-level coding/explicit meaning } \\
\text { reconstruction) of PR }\end{array}$ & & & & & & & & & & & & & $\mathbf{x}$ & $\mathbf{x}$ & & & & & & & & & & & & & & \\
\hline $\begin{array}{l}\text { Training/Peer Debriefing of } \\
\text { Explicit Meaning Reconstruction }\end{array}$ & & & & & & & & & & & & & & & $\mathbf{x}$ & & & & & & & & & & & & & \\
\hline $\begin{array}{l}\text { Pragmatic Horizon Analysis } \\
\text { of PR }\end{array}$ & & & & & & & & & & & & & & & & $\mathbf{x}$ & $\mathbf{x}$ & & & & & & & & & & & \\
\hline $\begin{array}{l}\text { Validity Reconstruction } \\
\text { of PR }\end{array}$ & & & & & & & & & & & & & & & & & & $\mathbf{x}$ & & & & & & & & & & \\
\hline Building PR of Interviews & & & & & & & & & & & & & & & & & & & $\mathbf{x}$ & $\mathbf{x}$ & & & & & & & & \\
\hline $\begin{array}{l}\text { IMR, PHA, VR } \\
\text { of Interviews }\end{array}$ & & & & & & & & & & & & & & & & & & & & & $\mathbf{x}$ & $\mathbf{x}$ & & & & & & \\
\hline High-level Coding & & & & & & & & & & & & & & & & & & & & & & & $\mathbf{x}$ & $\mathbf{x}$ & & & & \\
\hline $\begin{array}{l}\text { Peer Debriefing } \\
\text { of High-level Coding }\end{array}$ & & & & & & & & & & & & & & & & & & & & & & & & & $\mathbf{x}$ & & & \\
\hline Final Comparative Analyses & & & & & & & & & & & & & & & & & & & & & & & & & & $\mathbf{x}$ & $\mathbf{x}$ & \\
\hline
\end{tabular}




\section{APPENDIX F}

Pilot Study Transcripts

Stage Four A-Building Primary Record from Representative Classroom Observations (Transcribe Meaningful Utterances)

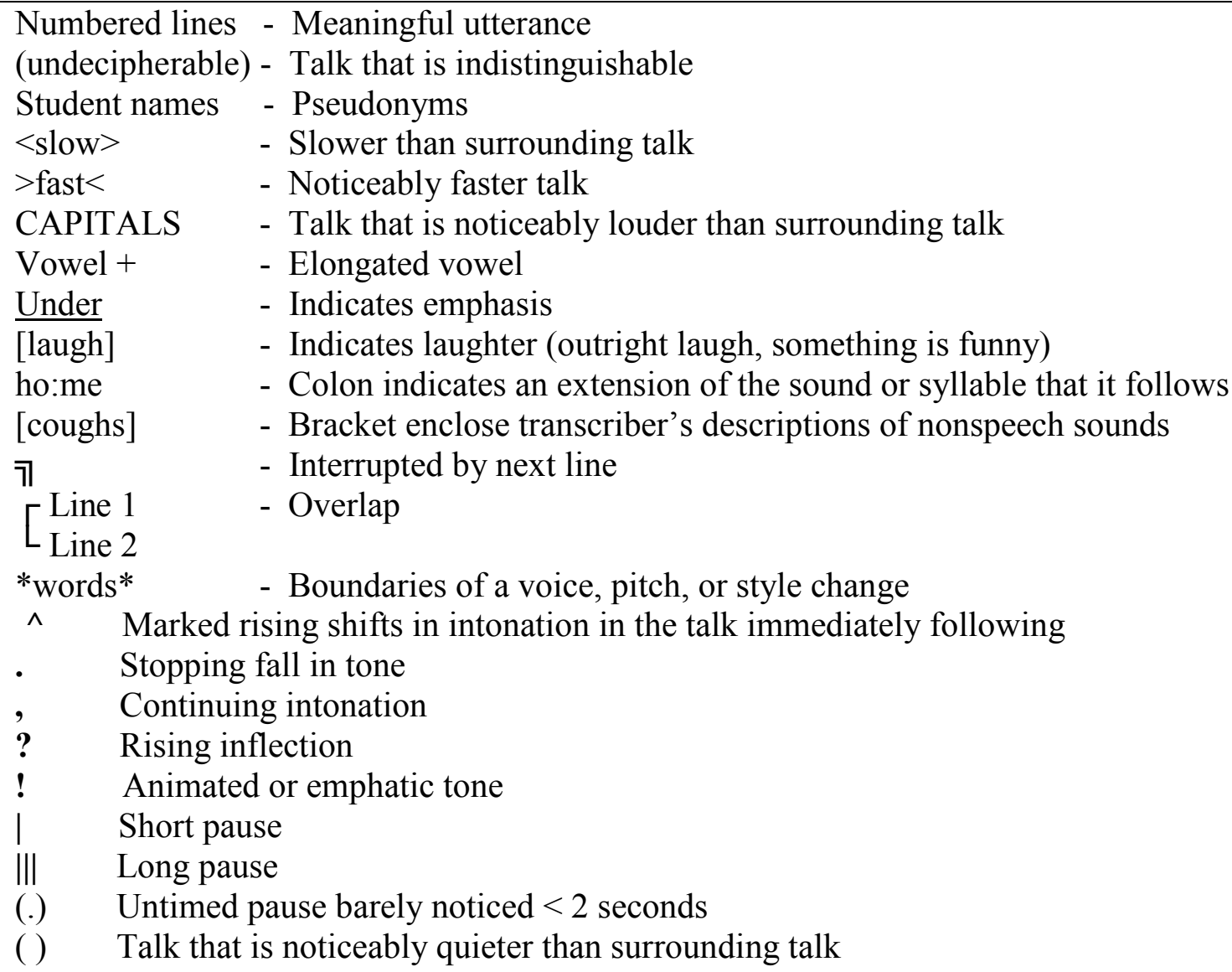

1. Students: [laugh]

2. Students: (undecipherable)

3. Professor: Uh. Who did? You know I left my notebook. Where's Jim?

4. Students: [laugh]

5. Professor: Didn't he just? Who are you?

6. Students: [laugh]

7. Professor: O:h, forget it. He's not here yet. It was me.

8. Students: [laugh]

9. Professor: This is the hardest thing I do all year. Doris, were you here last time?

10. Doris: Yes

11. Professor: Lola Lesser, does anybody know if she was here last two weeks ago?

12. Professor: All right. Jose Ruiz?

13. Professor: All right. Marissa Sanders? 
14. Professor: Johnson I know was, ()

15. Professor: How about Natalia? [laugh]

16. Professor: All right. Niara?

17. Niara: Yes

18. Professor: Were you last?

19. Niara: Yes

20. Student: [cough]

21. Professor: Christy, you were too, Acacia was not because they lied to her, Alicia was not ${ }^{\wedge}$ Ginny was ${ }^{\wedge}$ Rachel was, you're Rachel right, Emily.

22. Emily: I was here

23. Professor: You were here then. Are you here now?

24. Professor: I think you were here when I called your name!

25. Students: (undecipherable)

26. Professor: $O k, o k \mid M i-a h$ ?

27. Mia: Me-ah.

28. Professor: $M e-a h$.

29. Mia. Yeah.

30. Professor: I knew who you were ^ [laugh]!

31. Mia: Yeah. That's a plus ${ }^{\wedge}$

32. Professor: That's a plus! [laugh] I didn't have to ask ^ you. Ok, you were here last time too.

33. Professor: $O K, I$ know June was, all right. NOW, is there somebody in the room right now that was here two weeks ago and here tonight that's not on my list ${ }^{\wedge}$ ? III

34. Professor: Ok, how many of you received the things that I sent you today? | All right, uh, you will find that I-ah frequently will send you things the day of, actually, there is no rhyme or reason to my sending things.

35. Students: [laugh]

36. Professor: To tell you the truth ${ }^{\wedge}$,

37. Professor: If one of you asks me a question, now the answer you ^ get from me may aggravate the shit out of me, and you notice that in my response.

38. Professor: But then ^ but then ^ I will decide it's not that dumb of a question [laugh] after all [laugh], it was my husband ${ }^{\wedge}$ that was really aggravating me, and I will try ${ }^{\wedge}$ to respond in a fuller message to the class because I think that it is something in fact that you don't understand as a group, ok^?

39. Professor: <So you've got a message > about problem statements from me, right? And I took, I think I took two of my own papers?

40. Students: (undecipherable)

41. Professor: What?

42. Professor: Yeah, and uh, I showed you what a problem statement with a purpose looked like from, I know from my CRT one and I don't remember what the other one was,

43. Student: [cough]

44. Professor: But I think it was two of them, 
45. Professor: At any rate, $u$ h | all right, so, somebody asked me something and that's what I did,

46. Professor: Now, today ^ I was preparing for class a little later than I would have liked because I distracted myself and did something else, and uh, and anyhow, so I sent these things late, >Now they're in your email ^ so you can go back and get them if you don't have them but it occurred to me that it might be useful for somebody to have ${ }^{\wedge}$ these handouts so you can print them out and write on them $<$, ok?

47. Professor: So, that's why I sent them, you know, it's not required, it's just I was trying to be helpful.

48. Professor: All right, um ||

49. Professor: Now, I'm going to talk to you tonight about writing a research paper and, umm, I going to spend probably what some of you will consider an inordinate amount of time, trying to teach you how to write research papers. I do this for a variety of reasons. One, I believe very strongly about the notion that students and professionals have a voice, and the only reason we don't know what you know is because you don't know how to access the world of publication.

50. Professor: Ok?

51. Professor: What do you think, Johnny?

52. Johnny: You are perfectly, perfectly right.

53. Professor: [laugh] I like Johnny. [laugh]

54. Professor: Anyhow, and uh, I think this because when I was a grad student, uh, in the 90s, look at my vitae, I'm very young, um, all four of my degrees come from the 1990s. Anyhow, um, when I was a grad student, I knew when I went to get my doctorate that I wanted to be a professor, that was what I was going to do, um, I figured it out but so, I didn't know what you needed to do as far as career ^ ${ }^{\wedge}$ path to become a professor, so I started asking faculty^. You know, what does a student need to do as a student to become a faculty member later? And they said you need to publish.

55. Professor: $\mathrm{OKa}+y$, [laugh], how do you do that ${ }^{\wedge}$ ? Well, no faculty members really tell you how to do that except for a few rare ones, like me ${ }^{\wedge}$, and um, because most faculty assume that you go on and get a doctorate and somehow this magic of writing this dissertation which is really unlike any other kinds of writing, will magically make you understand the publication process. And it doesn 't. It doesn't prepare you at all, uh, others ${ }^{\wedge}$ think that students have nothing more () to say, therefore, they shouldn't be encouraged |

56. Professor: [laugh]

57. Professor: It's getting more and more crowded, there's two seats up here. There's one here, this one's mine ||| Nobody wants to sit by me, Johnny.

58. Johnny: [laugh]

59. Student: You're so ()

60. Professor: ||| All right, you know what, we are expecting some more. So, you're going to have to sit there, I hope you're()

61. Student: (undecipherable) [laugh]

62. Professor: And ||| 
63. Professor: Actually, Miguel ${ }^{\wedge}$ was going to put the desks this way, but I thought this would be a better way. I think you might have a little more room sort of. If there's a fire drill though

64. Students: [laugh]

65. Professor: I think this row needs to go out this door so that that group can go out that door. Oh, yeah, the restrooms down this door.

66. Professor: () Where was I in my sad tale?

67. Professor: Oh! The rest of you don't know anything worth getting published, therefore, nobody should teach you how. Now that's one reason. Another ${ }^{\wedge}$ reason is that if you come to graduate school, the assumption is you have a

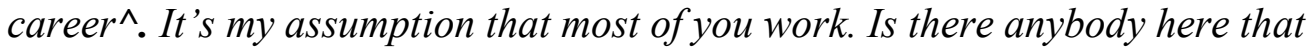
doesn't have a regular job?

68. Professor: Me, [laugh].

69. Students: (undecipherable]

70. Professor: All right, but you're in the minority [cough] () but you are, and especially true in the fields that I represent, Adult Education and Human Resource Development.

71. Professor: SO, if you have a job, how many of you never write a thing at work?

72. Yeah, most of you write at work. Now, how people perceive you at work has a lot to do with how you communicate. Not ${ }^{\wedge}$ the nature of your ideas $\mid$ how you look, how you sound, whether they can pick up an accent in your writing.

73. Professor: I learned this in Columbus, Ohio, so it has nothing to do with a Spanish accent that I'm talking about. It has everything to do with a Black accent, or a Cleveland accent, ok, or West Virginia. Because even from different places in this country^, we write differently according to where we were brought up and how we talk. And generally^ one thing I can guarantee all of you, I write a whole hell of a lot better than I talk.

74. Professor: [laugh], yeah, and in fact, you can probably read me and not think it was me because it is so good.

75. Students: [laugh]

76. Professor: No cussing nothing ^ [laugh]. The sentences are in the right order [laugh], uh, it's focused, organized, all of the things I'm not in person, [laugh].

77. Professor: All right, SO, these are the reasons that I do this, I think that you've got something important to say. I think that you represent different groups, whether it be graduate students masters' level, graduate students at the doctoral level, different ethnic groups, different other kinds of groups, women, African American, Haitians, Jamaican, uh,

78. Student: [cough]

79. Professor: Other groups that in this country have been disenfranchised, and not [cough] allowed in.

80. Professor: OK? So, those are some of the reasons that I'm doing this.

81. Professor: Now, my process is probably one most of you are not used to, and actually the nurses from summer from spring you didn't get a taste of this because we went on that giant escapade.

82. Students: [laugh] [laugh] 
83. Student: You had to bring that up, didn't you?

84. Professor: [laugh]

85. Professor: I'm still living with it. We're still trying to do it.

86. Student: It really $<\underline{\text { honed }}$ my lit search skills $>$.

87. Professor: It's ^ an important skill if you go on to get a doctorate. [laugh]

88. Professor: All right, so, I don't, I, one thing I learned, I'm never going to do that in that class again and it wasn't, it just wasn't the right place, but at any rate, because I probably will do such a stupid thing again, I know. [laugh]

89. Professor: All right, So, what I will do, is you'll send me drafts, and given my parameters timewise, I'll look at them and I'll give you feedback. I use track changes and I use comments. And I use short (.) to the point (.) sentences. And I have never learned Spanish, I don't know come from a polite culture. Those of you who haven't noticed that yet. I'm Italian, I'm the oldest, I have four brothers $^{\wedge}$, I was raised in a very male neighborhood^ ${ }^{\wedge}$ in Cleveland, we're just like New Yorkers and Chicagoans, which means we're not very polite. [laugh]

90. Professor: Nonetheless, Miguel is here to keep me in check, right? [laugh]

91. Miguel: Yeah.

92. Professor: Right? [laugh]. [laugh]. Good luck to him^, huh^? [laugh]

93. Professor: So, I just tell you this in way of explanation, because our cultures differ, and there are a lot of you in the room and there's only one of me,

94. Professor: Cuz he can't give you feedback on your writing. No offense, [laugh].

95. Professor: Well, he can and he can't. I want to take that back, because Miguel has come a hell of a long way since I've met him. When ^did I meet ^you? Three years ago?

96. Miguel: 2006.

97. Professor: Only two years ago? It seems like I've known you forever.

98. Professor: Anyhow, [laugh], all right, but Miguel has come a long way, because in the interview, the dissertation, the interview to get in to be a doc student, I didn't understand half of what he said. All I understood was a few references to Habermas, who else? Foucault, uh, [laugh].

99. Professor: Anyhow, so, well, you had only been here, what, a year at that point?

100. Miguel: Yeah.

101. Professor: And he did what I think is common for a lot of people that come here from another country to take-go to school and that is they sit silently in classes because they don't want to look stupid. And so you don't speak and you don't learn to speak it. He's forced to talk to me.

102. Professor: So, anyhow, you've got the idea? I'm going to give you feedback within my time parameter. Now some of you may need to make appointments with me, may need to come in during my office hours. If you want help on your papers, though, don't come to me with nothing, come to me with a work plan, come to me with an outline, come to me with a draft of some sort, because I can't work from nothing^ and if you give me something concrete, I can give you something concrete back.

103. Professor: Ok? Now, another thing that is going to be unusual is that I believe writing is process, and I believe it takes as long as it takes to get it right. 
Richard Smith here is embarking on a paper that I think has the potential to be an article. Not just a class paper, not just a conference paper. And it actually, probably $^{\wedge}$ has the potential if he was in another field maybe this one to be a dissertation topic.

104. Professor: And we'll put this up and you'll see.

105. Professor: All right, so I believe writing is a process and all things that are a process, you learn by practicing those skills. Now any of you athletic? Basketball, soccer, soccer, like I know shit about soccer. Anyhow^, when you are little, most of us are older than death, and don't want to play soccer or basketball anymore, but when we are little, and we used to play these things, we practiced! And all athletes know that they have to practice these skills, and we drill it into ${ }^{\wedge}$ them. But we don't do the same things when it comes to writing, when it comes to reading. We talk about it other ways, we don't talk about these thing in terms of building skills.

106. Professor: Now, I don't think for the most part, the books you have to read, this term, for these classes are going to be difficult reads. In years past, I've used the Handbook of Adult Education and it was a difficult read, because the last version took a postmodern stance. And postmodernists believe that the only good writing is dense circuitous writing, on purpose! OK.

107. Professor: So, and, but it takes practice sometimes, well it does, it takes practice to read academic literature, it takes practice to develop your reading skills, it's just practice, same with writing, so.

108. Professor: I also think some of you should get into peer groups possibly, join forces, send drafts back and forth, though I'm not going to organize that because you're always better off getting outside ()

109. Professor: All right, we're going to start off talking about the components of a paper. I'm gonna walk through what I think are the components of a position paper which is what most of you will be writing in here from the way that I saw your purposes. Then I'm gonna go through some samples, I'm going to walk you through Lisa's paper, I'm gonna show you Miguel's paper from two years ago in this class, and uh, and then I'm gonna go through your purpose statements that you sent me, okay?

110. Professor: Now, the other thing. I generally don't give breaks because I don't remember to give em, and if you really need a break, you've got to consult with Miguel, he's gonna have to work it out and he's gonna have to tell me to stop. Now, otherwise, I'm just going to go (.) until I'm done, [laugh], and then we go home ${ }^{\wedge}$. If you need to get up and go to the bathroom, they're down the hall. Or you can take the elevator or the stairs all the way down^ and go on the first floor. I don't know where they are on the second floor. I don't think I've been on the second floor, um.

111. Students: (undecipherable)

112. Professor: Yeah, you can go out that door and down this hall, yeah, you're really concerned about the bathrooms?

113. Students: [laugh]

114. Student: I'm a nurse. You're worried about if we don't find them. 
115. Professor: So, <papers have parts >. Papers have parts, and the papers that you're going to do for me will have three parts. It will have an introduction^, a discussion section, and implications. The introduction ${ }^{\wedge}$ section contains some parts $^{\wedge}$.

116. Professor: Are you looking for a chair? We've got two over here. | And there are no other rooms in this place.

117. Professor: But she better be on time next time. [laugh], or she'll be sitting in the hall, [laugh], [laugh].

118. Professor: All right, now (.) a problem ^ statement. I sent you that article, or the chapter in the book from Merriam and Simpson, where they walk through a problem statement. Now, you're going to hear terms kind of bantied about, you might have already in your travels as students. Some people will talk about a thesis, ok? When you took uh, English, they probably talked about a thesis, um, what other ways do they talk about it? Well, that's good enough, we know that one.

119. Professor: But the thesis^ and the problem statement is the beginning of a paper that grounds your paper in something. Ok? Some one of you wants to do something on obesity, ok? And, uh, I had a student last year that did something on obesity, I don't exactly remember how it went but it was on the educational ${ }^{\wedge}$ value of obesity programs that we have. 
Pilot Study Transcripts

Stage Four B-Building Primary Record from Representative Classroom Observations

(Explain Meaningful Utterances and Linguistic Behavior Observed)

Stage Four C-Building Primary Record from Representative Classroom Observations

(Articulate Possible Objective Validity Claims)

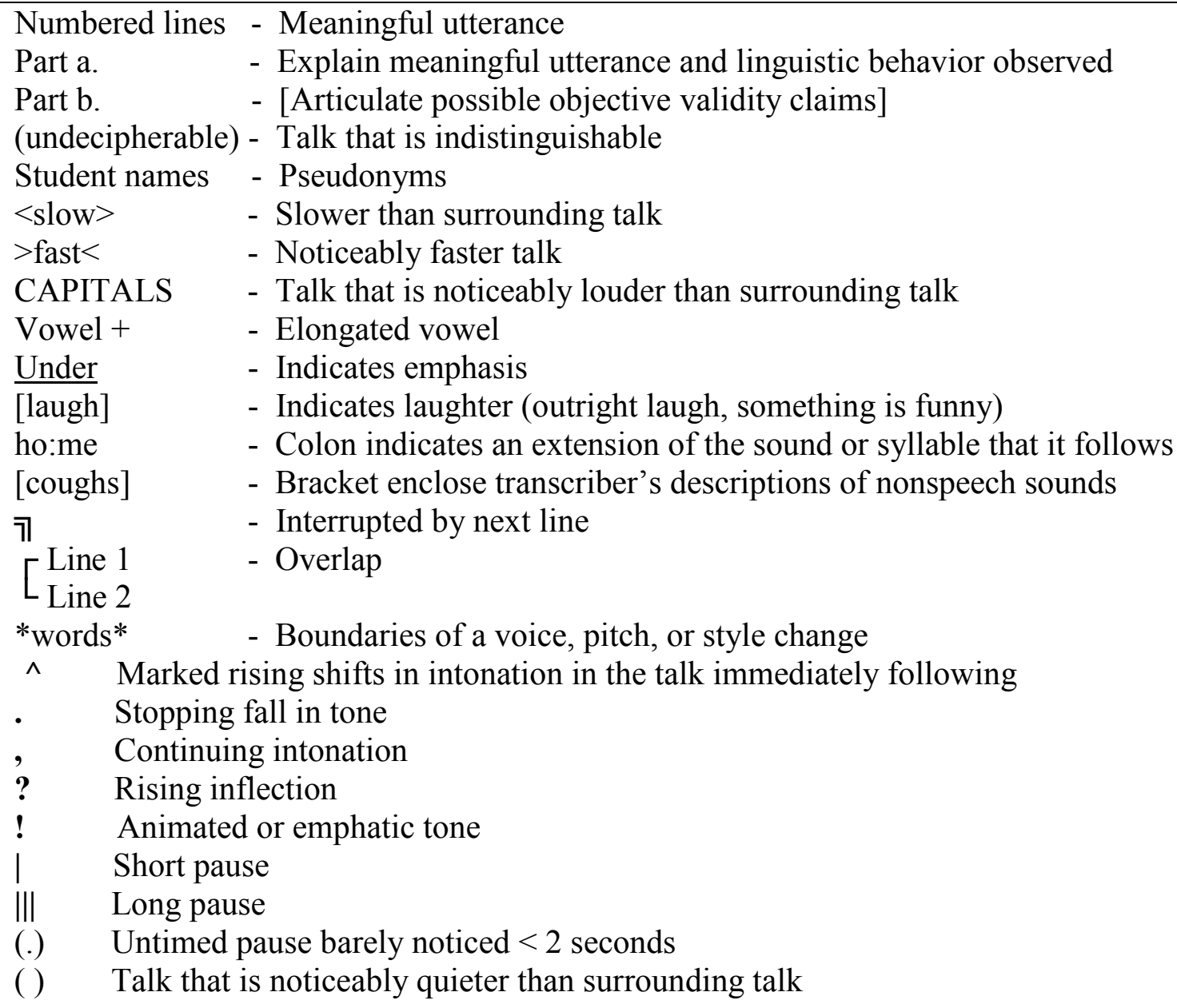

1. Students: [laugh]

a. Students are coming in and casually and quietly talking and laughing to whoever is near them as professor fiddles with the laptop at the front of the class. A Power Point slide is showing on the screen. The long tables are situated in a rectangular shape so the students can sit on the outside and look towards each other. Several students have laptops. Most have notebooks to write in.

b. [We're having fun talking together in a classroom with other students before class officially starts AND/OR I need to find a seat before class starts so I don't get called on and potentially embarrassed.]

2. Students: (undecipherable) 
a. Around 20 students are here now and either quiet or buzzing quietly to the person next to them. It is obvious who the professor is by the placement of the tables. The professor is setting up at the front table, the one right in front of the screen.

b. [It is acceptable to talk together quietly before the professor gets here AND/OR begins class.]

3. Professor: Uh. Who did? You know I left my notebook. Where's Jim?

a. Sitting down in front of laptop and looking around as if lost somebody or something.

b. [I always forget something AND/OR I can always ask Jim what I did with my notebook since he usually remembers what I do with stuff better than I do.]

4. Students: [laugh]

a. Students all laughing at once as if someone just told a joke.

b. [This feels good when the professor asks for students to help AND/OR This professor did the same thing last time and I think that is funny AND/OR The professor loses things and it reminds me of myself AND/OR The professor does not match what I thought a professor does.]

5. Professor: Didn't he just? Who are you?

a. Point to a person as if that might be Jim.

b. [I thought I just saw Jim. To save face, I will ask someone if they are Jim to ease the embarrassment level. I usually have good luck with that tactic.]

6. Students: [laugh]

a. Students laughing at once again as if someone told another joke.

b. [This may be a fun class since the professor cracks jokes AND/OR has a different sense of humor AND/OR I hope she does not point to me since I don't like to be called on even at this level.]

7. Professor: Ohh, forget it. He's not here yet. It was me.

a. Throws up one hand in the air as if tossing something out.

b. [Oh, yeah, I remember where it is. I'll just admit my preoccupation out loud and get a laugh out of the students. AND/OR I like to make them feel at ease. They have had too many insensitive professors.]

8. Students: [laugh]

a. Students all laugh together again as if someone told another joke.

b. [She's human, thank heavens. This may not be so hard after all. Being at the master's level may be something I can do after all. AND/OR Oh, no, she's a basket case. Can I endure this?]

9. Professor: This is the hardest thing I do all year. Doris, were you here last time?

a. Checking things on the laptop as if it is required AND/OR being in charge of taking roll.

b. [I like to say things that students have to reconsider, like pretending something mundane is really hard. AND/OR I need to put this task forced upon me by the university into perspective. I hope the students caught that innuendo.]

10. Doris: Yes 
a. Students are listening now as if they know the rules of school are the same as rules of university, that is, that when the teacher or professor begins checking roll, class begins and you become quiet. The class includes students from various cultures from looking around or listening to them answer the professor. Some are Black, some are Latino/a, a few are White with most appearing to be Latino/a and Black.

b. [I have been in school for so long that roll call is familiar to me.] 11. Professor: Lola Lesser, does anybody know if she was here last two weeks ago?

a. Looks around as if she would know or see the person being called $\mathrm{AND} / \mathrm{OR}$ as if she knows the person is not there or would raise hand and be acknowledged.

b. [Where is she? I was hoping she would come to class. AND/OR I purposely look around and get people involved even in what seems like the smallest ways. This sets the tone for the class.]

12. Professor: All right. Jose Ruiz?

a. Looks around briefly as if knows the person is there.

b. [This is boring AND/OR Why does the university require such mundane things from me? AND/OR Why don't I just let someone else do this task? I follow this rule to the $t$ in every class. I sure wouldn't do this if not required.]

13. Professor: All right. Marissa Sanders?

a. Says all right as if to get attention of whole class. Looks up at student as if remembers her and she was there.

b. [I want to make sure I have the attention of everyone from the getgo.]

14. Professor: Johnson I know was, ()

a. Did not look up as if she knows Johnson.

b. [I like knowing students so I can get this task over with AND/OR to let students see how I interact closely with students even at mundane levels.]

15. Professor: How about Natalia? [laugh]

a. Looks up briefly as if knows Natalia is not there but checking anyway and then giggles as if something is funny.

b. [Sometimes I tickle myself AND/OR I want to make the students feel like they can be comfortable.]

16. Professor: All right. Niara?

a. Looks up briefly as if looking for student.

b. [I may remember him if I just scan the room.]

17. Niara: Yes

a. [I know how roll call goes.]

18. Professor: Were you last?

a. Talks directly to the student as if to verify last week's attendance.

b. [It feels good looking at students' faces when I talk to them.]

19. Niara: Yes

a. Niara answers as if she knew the professor wanted to know about last week's attendance.

b. [Don't you remember? AND/OR Why don't you know me yet?] 
20. Student: [cough]

21. Professor: Christy, you were too, Acacia was not because they lied to her, Alicia was not ${ }^{\wedge}$ Ginny was ${ }^{\wedge}$ Rachel was, you're Rachel right, Emily.

a. Speaks to Christy as if she remembers her. Then looks up as talks about Theorela at same time pushes a button on the laptop and brings that finger up into the air as she speaks. Each time makes big strokes as if to make sure the students see her take attendance. She looks at who she thinks is Rachel to confirm and looks up to find Emily.

b. [I am getting tired of this so I feel like moving faster and acting out what I do so they know I am still taking attendance AND/OR I need to speed this up before I lose people over something unimportant in the scheme of things.]

22. Emily: I was here

a. Answers as if she wants to clarify her attendance.

b. [I must make sure she knows I was here last time so I will use something other than a simple yes just to make sure.]

23. Professor: You were here then. Are you here now?

a. Talks as if making connections between this and last week's attendance.

b. [It feels good when I can pull in historical construction even when taking roll.

24. Professor: I think you were here when I called your name!

a. Converses with Emily as if her name is a very important topic.

b. [I like making people feel important. AND/OR She is taking over the power here so I will dominate the conversation.]

25. Students: (undecipherable)

a. Students mumble together.

b. [I see she really gets to know people AND/OR I wonder what she will say to me in the future? I better keep on my toes.]

26. Professor: $O k$, ok $\mid M i-a h$ ?

a. Stops and asks the student her name as if asking for correct pronunciation.

b. [What culture does this name come from? Better check pronunciation.]

27. Mia: Me-ah.

a. Student says name as if to respectfully correct mispronunciation of name.

b. [It feels good when teachers ask me how to pronounce my name. It makes me feel important. AND/OR It is getting old having to say my name in every class year after year. I feel like an outcast.]

28. Professor: $M e-a h$.

a. The professor repeats as if trying to say the name correctly.

b. [I need to repeat so I can remember. Years of this has shown me how important names are to people.]

29. Mia: Yeah.

a. Mia answers as if verifying the correct pronunciation.

b. [I'll just muffle my answer so we can just get off this topic.]

30. Professor: I knew who you were ${ }^{\wedge}$ [ [laugh] 
a. With both hands as if directing an orchestra for a second AND/OR as if very happy she knew which student she was talking to.

b. [This feels good when I remember someone after only one class.]

31. Mia: Yeah. That's a plus ^

a. Student agrees as if it was important.

b. [I guess she really means well, so I will go along.]

32. Professor: That's a plus! [laugh] I didn't have to ask ^ you. Ok, you were here last time too.

a. Reiterates the point as if this is an important part of the agenda for all to know.

b. [I love bringing in culture and community feel even during mundane tasks like taking roll.]

33. Professor: $O K$, I know June was, all right. NOW, is there somebody in the room right now that was here two weeks ago and here tonight that's not on my list ${ }^{\wedge}$ ? III

a. Points with one finger down for each time she said here as if to confirm she means right here in this room. Then spends a while finishing something on the laptop as students watch her.

b. [I'm ready to get done here so let me finalize the task right here!]

34. Professor: Ok, how many of you received the things that I sent you today? | All right, uh, you will find that I-ah frequently will send you things the day of, actually, there is no rhyme or reason to my sending things.

a. She looks up as she asks the question, still sitting. Then she reconsiders what she asked as if the process of sending needed to be explained.

b. [I don't know if that came out right, what did I say? Let me rephrase that so students will think it was a funny comment AND/OR so that students will listen better thinking that I may be an oddball by letting out my thoughts. It feels good having students think that I am an oddball because most professors are so boring and closed.]

35. Students: [laugh]

a. Muffled laugh as if smiling slightly out loud

b. [I may enjoy listening to you tell us how you think and do things, since most professors are so straight-laced and never let on that they really might think like you are about to expound on. I like this. AND/OR Oh, no, what have I gotten myself into? Surely, she has a rhyme or reason. I want to make an $\mathrm{A}$ in this class. Come on!]

36. Professor: To tell you the truth ${ }^{\wedge}$,

a. Reconsidering again as she decided another way to explain her process of sending papers AND/OR as if reconsidering how to explain her process.

b. [I like coming at things from a different angle than anyone would expect. I want them to think about things so I'll just bring in how I do it.]

37. Professor: If one of you asks me a question, now the answer you ${ }^{\wedge}$ get from me may aggravate the shit out of me, and you notice that in my response.

a. She tells her process as if trying to get at the possible students' perspective when they receive a response from her. 
b. [If I act like I think about them when I am reading their stuff and having a life at home, they will get the idea that I am not an oddball, but a person who they can talk to, relate to, and construct knowledge with.]

38. Professor: But then ^ but then ^ I will decide it's not that dumb of a question [laugh] after all [laugh], it was my husband ${ }^{\wedge}$ that was really aggravating me, and I will try ${ }^{\wedge}$ to respond in a fuller message to the class because I think that it is something in fact that you don't understand as a group, ok^?

a. Stands up and starts using hands as if to reiterate the importance of the new way of explaining the process AND/OR showing students she rethinks her first response and reaction, acknowledging she realizes how students may feel when they get that first response and decides they all might need help, thus providing help on something they all might not understand.

b. [I can get my point across when I show them how I actually learn something by reconsidering what seems stupid when taken at face value, but upon rethinking it seems like a good question from someone else's perspective. I feel good when I show students that it is hard to be a critical thinker but I expect them to do the same thing sooner or later.]

39. Professor: <So you've got a message > about problem statements from me, right? And I took, I think I took two of my own papers?

a. Two hands going up in the air as she asks the first question, looking down at laptop as if to answer the second question she asks.

b. [I do lots of work here, did you at least retrieve the messages? AND/OR I like to ask them questions I already know, but sometimes I get sidetracked and maybe didn't even send what I meant to send so I'll put it in their court.]

40. Students: (undecipherable)

a. Quiet buzzing of students' voices as if they don't want to be heard.

b. [I know the answer but am afraid to speak really loud.]

41. Professor: What?

a. Looks at who asked the question as if to hear the question again.

b. [I wonder what you said but am not going to press the matter since I don't think it matters right now AND/OR since she looks not sure of herself.]

42. Professor: Yeah, and uh, I showed you what a problem statement with a purpose looked like from, I know from my CRT one and I don't remember what the other one was,

a. Quickly registers the question and begins using hands two up touching a point in the air and then lowers them a little to touch another point in the air to answer the question and then telling the students what was sent with one hand under chin as if she feels she has their attention now.

b. I'll just answer my own question since I think I remember what I sent, and it feels good to talk about my CRT paper. I like to banty terms around that I want them to hear for future reference anyway.]

43. Student: [cough]

44. Professor: But I think it was two of them, 
a. Says this as if it were an afterthought.

b. [The second paper is not a priority right now in my mind.]

45. Professor: At any rate, uh $\mid$ all right, so, somebody asked me something and that's what I did,

a. Uses this phrase to change the topic some and using hands as if directing an orchestra in an up, down, and around fashion.

b. [I need to get back to the topic of this class or at least the topic of how I respond when someone asks me a question just in case I have had a busy day AND/OR in case someone doesn't understand my cultural behaviors.]

46. Professor: Now, today ^ I was preparing for class a little later than I would have liked because I distracted myself and did something else, and uh, and anyhow, so I sent these things late, $>$ Now they're in your email ${ }^{\wedge}$ so you can go back and get them if you don't have them but it occurred to me that it might be useful for somebody to have ${ }^{\wedge}$ these handouts so you can print them out and write on them $<$, ok?

a. Using hands as if to direct an orchestra and talking more quickly as she goes along as if knowing what is coming next in the information being given.

b. [I have a life and want you to know that even so I will still do my work and will still provide you with all sorts of models for your own writing. I like to throw hints about writing on handouts and other things that I've learned along the way about the writing process AND/OR and other things that other professors could care less about sharing.]

47. Professor: So, that's why I sent them, you know, it's not required, it's just I was trying to be helpful.

a. Two hand up together with the first word and then pointing to places in the air as if to make sure students see she is concluding the explanation.

b. [See, I really do care because I go beyond requirements of the university system. I want to help you become better writers.]

48. Professor: All right, um ||

a. Starts to sit back down again, looks at laptop as if deciding where to go next.

b. [I need a second to remind myself what I want to talk about next.]

49. Professor: Now, I'm going to talk to you tonight about writing a research paper and, umm, I going to spend probably what some of you will consider an inordinate amount of time, trying to teach you how to write research papers. I do this for a variety of reasons. One, I believe very strongly about the notion that students and professionals have a voice, and the only reason we don't know what you know is because you don't know how to access the world of publication.

a. Stands back up, hands together at first, and casually walks into the middle of the oblong table arrangement with all students facing inward as if she is confident enough about what she is saying that there is no need to point to places in the air for emphasis AND/OR begins making two hand motions from elbow level in and out many times as she is talking as if she is sure of the meaning of what she is saying to the students (OC: Does she point 
in the air when she is searching for what comes next versus move hands at elbow level in and out when she deeply believes and knows what she is saying or going to say next?)

b. [I better preface how long I intend to talk about writing research papers because I know that students are not used to this and they need to know how important this is. It feels good to tell them what I believe about their potential because I have seen how students (including myself as a doc student) are treated and I am determined not to do have any student leave my class thinking they cannot write well or thinking that writing is not a process!]

50. Professor: $O k$ ?

a. End of discussion.

b. [I feel like I made my point.]

51. Professor: What do you think, Johnny?

a. Looks at Jimmy, hands elbow level together and still.

b. [I better lighten things up and bring in a student's remarks, a student I know well right now.]

52. Johnny: You are perfectly, perfectly right.

a. Sounds as if he halfway agrees but with tongue in cheek.

b. [I understand the professor and it is fun to be able to tease her and vice versa. My history with school, work, and society has been a disaster. She gives me hope. It's the least I can do at this point.]

53. Professor: [laugh] I like Johnny. [laugh]

a. Laughing, pointing with one hand toward Johnny.

b. [I know Jimmy has had a hard time so I feel good when I let him show what he knows even if it is about me. Aren't I funny? Sometimes I tickle myself. I'm so glad I became a professor.]

54. Professor: Anyhow, and uh, I think this because when I was a grad student, uh, in the 90s, look at my vitae, I'm very young, um, all four of my degrees come from the 1990s. Anyhow, um, when I was a grad student, I knew when I went to get my doctorate that I wanted to be a professor, that was what I was going to do, um, I figured it out but so, I didn't know what you needed to do as far as career ^ path to become a professor, so I started asking faculty^. You know, what does a student need to do as a student to become a faculty member later? And they said you need to publish.

a. Still standing, little bit closer to laptop, hands elbow level, slightly in and out as if truly believes and knows what she is saying, one hand up to confirm importance of four degrees, finger shaking at figured it out, point to three places on a ladder at career path, hands still low.

b. [I like bringing in my own history because I know how past, present, and future connect and maybe by doing so my students will begin to look at the world differently, at what they can do differently.]

55. Professor: $\mathrm{OKa}+y$, [laugh], how do you do that ${ }^{\wedge}$ ? Well, no faculty members really tell you how to do that except for a few rare ones, like me^, and um, because most faculty assume that you go on and get a doctorate and somehow this magic of 
writing this dissertation which is really unlike any other kinds of writing, will magically make you understand the publication process. And it doesn't. It doesn't prepare you at all, uh, others ${ }^{\wedge}$ think that students have nothing more () to say, therefore, they shouldn't be encouraged |

a. Continues the same stance every now and then backing up a step and looking at laptop as if checking to see if she is getting closer to it, two hands sway left and right at somehow magically, then two hands pointing to places in front of her as if to stress unlike other kinds of writing AND/OR as if to stress that she disagrees with what other faculty think about writing and their students relationship to the magical process AND/OR hands higher than elbow level and pointing for emphasis when she emphasizes certain words when talking as if showing where she disagrees with other faculty.

b. [I learned that I needed telling but no one would do it and from that point in time I made a commitment to tell students how to do something like publishing papers explicitly. At this point, I can say I am a rare case at the cost of appearing a braggart because of how I have handled the class up to this point. They must see that I am trying to share what I know and have learned yet don't think myself a know it all.]

56. Professor: [laugh]

a. Smile on face and hands in front of her swaying with upper body is as if she just got away with opening up a previously secret bit of information.

b. [I think I got away with that. That's funny to me. But I made my point.]

57. Professor: It's getting more and more crowded, there's two seats up here. There's one here, this one's mine ||| Nobody wants to sit by me, Johnny.

a. Points to places to sit and then tells Johnny no one wants to sit by her as if to ease student unfamiliarity with her and others $\mathrm{AND} / \mathrm{OR}$ as if trying to get on the same level with students AND/OR trying to get students to enter into the conversation as people who know something and don't have to steer clear of the professor.

b. [I like to make people laugh and feel comfortable AND/OR like they can be humorous especially in a forced closed in setting like the university classroom.]

58. Johnny [laugh]

a. Johnny laughs as if he thinks something is humorous.

b. [They will learn that sitting by you is okay as soon as they get to know you.]

59. Student: You're so ()

60. Professor: ||| All right, you know what, we are expecting some more. So, you're going to have to sit there, I hope you're()

a. Stops to let students sit down and lets them take their time by talking to Miguel while they get comfortable.

b. [I really don't like when students come in late but don't want to belabor the point. Humor needed here.]

61. Student: (undecipherable) [laugh] 
a. Students laugh as a group as if to show that they feel comfortable and get the humor she presents.

b. [So that's we get if we come late?]

62. Professor: And |||

a. Says as if biding for time.

b. [I need to get back on topic, but how?]

63. Professor: Actually, Miguel ${ }^{\wedge}$ was going to put the desks this way, but I thought this would be a better way. I think you might have a little more room sort of. If there's a fire drill though

a. Brings Miguel into the conversation as if to make students feel comfortable AND/OR as if to show she got the last say in arranging tables and then adds some humor as if to bring back the comfort level again for students.

b. [It works well to bring in a student and something they did in context to gain attention sometimes. I'll try it here.]

64. Students: [laugh]

a. Students laugh as if to show they understood the humor of a crowded room and fire drill.

b. [It feels great when I can get students to laugh and regain attention at the same time.]

65. Professor: I think this row needs to go out this door so that that group can go out that door. Oh, yeah, the restrooms down this door. I

a. Pointing with two hands as if directing traffic, she continues the humor of the fire drill as if letting students know she heard their laughter AND/OR as if she wants to elevate ownership and comfort in the classroom and as if making a final input for student comfort she adds more about the restrooms and points to the door she is referencing (OC: does she point often because she thinks students are or should be looking at her or because of her cultural background of Italian or to elevate comfort level?)

b. [I like telling the whole story of what could happen. It's sort of like rethinking what is given at face value and coming up with something out of the norm.]

66. Professor: () Where was I in my sad tale?

a. Almost said as if an aside to get back to the topic of the class.

b. [I'm ready to begin talking about writing again.]

67. Professor: Oh! The rest of you don't know anything worth getting published, therefore, nobody should teach you how. Now that's one reason. Another ${ }^{\wedge}$ reason is that if you come to graduate school, the assumption is you have a career $^{\wedge}$. It's my assumption that most of you work. Is there anybody here that doesn't have a regular job?

a. She starts in as if the new students knew what she was talking AND/OR didn't think about how they just got there (OC: I wonder if the new students thought SHE meant that they knew nothing and nobody should teach them. I wonder what they thought the reasons were that she is listing. I wonder if the students who had been there remembered where 
she left off from here.) Then she asks the class about their jobs as if to bring everyone into the conversation.

b. [I was talking about how professors don't think students know anything, so that's where I'll start. But I think differently, everyone has careers and that must mean they know something.]

68. Professor: $M e$, [laugh].

a. She answers her own question as she points with one hand in the air and laughs at her answer as if being a professor was not a regular job AND/OR as if trying to let students realize that any job could be considered not regular $\mathrm{AND} / \mathrm{OR}$ as if trying to get students to think outside the box about their own job.

b. [What did I mean by regular, I'll bring myself into the conversation again and say that my job is not regular. It feels right when I laugh about myself in order to build the students' confidence.]

69. Student: (undecipherable)

70. Professor: All right, but you're in the minority [cough] () but you are, and especially true in the fields that I represent, Adult Education and Human Resource Development.

a. Looks at the student who answered the question and then critiques the students' answer quickly as if to bring everyone back to the topic of the class papers, that is $\mathrm{AE} / \mathrm{HRD} \mathrm{AND} / \mathrm{OR}$ as if to introduce the topic of minorities.

b. [Was that an insult? I'd better clarify about minority here. I know I would bring it up sooner or later anyway.]

71. Professor: SO, if you have a job, how many of you never write a thing at work?

a. Immediately emphasizes the word SO as if to not pursue the students' answer AND/OR as if to bring the topic back to writing. Then looks around for a second as if for nods or some response.

b. [It is important to relate this class to what students do in the real world, that is, work. I know the answer to this question already and so do they.]

72. Professor: Yeah, most of you write at work. Now, how people perceive you at work has a lot to do with how you communicate. Not ${ }^{\wedge}$ the nature of your ideas how you look, how you sound, whether they can pick up an accent in your writing.

a. Still standing at front of room near laptop. Sees she got a response and continues talking with one hand out at a little above elbow level on the topic of writing as if to introduce identity and communication and writing with work AND/OR as if trying to connect what she is saying to students through their work.

b. [It is also important to relate writing to culture and identity development.]

73. Professor: I learned this in Columbus, Ohio, so it has nothing to do with a Spanish accent that I'm talking about. It has everything to do with a Black accent, or a Cleveland accent, ok, or West Virginia. Because even from different places in this country^, we write differently according to where we were brought up and how we talk. And generally^ one thing I can guarantee all of you, I write a whole $h$ - of a lot better than I talk. 
a. Holds one hand still by her neck, then begins explaining how different places write differently moving her hands up and down and then talks about her own writing keeping hands elbow level and still. Connects other cultures to her own as if bringing in connections so students can begin to understand writing as part of bigger picture AND/OR as if making connections to culture in general and writing in particular.

b. [It seems like the time to bring in the connection between culture and writing and language. I need to show them these are all related and that we are going to discuss them in this class.]

74. Professor: [laugh], yeah, and in fact, you can probably read me and not think it was me because it is so good.

a. Brings in humor by talking about herself in ways that most people shy away from.

b. [It works well when I can insult myself in good humor. Students like that. It makes them feel comfortable, which I want in my classes.]

75. Students: [laugh]

a. Some students laugh as if to say they got the humor and understand.

b. [I see, I can be my own person in here. AND/OR I see, writing is more than just words on the paper. AND/OR What is she talking about? I'm lost, I thought this was a research writing class.]

76. Professor: No cussing nothing ^ [laugh]. The sentences are in the right order [laugh], uh, it's focused, organized, all of the things I'm not in person, [laugh].

a. Continues explaining the disconnect between her own writing and talking in person as if to show the importance for the class in their own thinking about writing AND/OR as if sharing with class that they can all learn to write well even if they don't talk well.

b. [I wonder if anyone gets it yet, the new students, that writing is a process connected to identity development and history and culture and that's why I talk about why I do stuff the way I do too.]

77. Professor: All right, SO, these are the reasons that I do this, I think that you've got something important to say. I think that you represent different groups, whether it be graduate students masters' level, graduate students at the doctoral level, different ethnic groups, different other kinds of groups, women, African American, Haitians, Jamaican, uh,

a. She discusses more about the connections between culture and writing and identity as if to say that everyone one in the class has all the pieces necessary for becoming a good writer AND/OR as if to take the spotlight off of her to the students AND/OR as if to share her mission of bringing in representation of disenfranchised groups even at this level.

b. [I think you are all important and I think we need to express out loud who and what we represent. I give you permission.]

78. Student: [cough]

79. Professor: Other groups that in this country have been disenfranchised, and not [cough] allowed in. 
a. She says the word disenfranchised in the context of writing as if to connect the two meanings for the students (OC: I wonder if anyone in the room has heard this word or knows its meaning?) AND/OR as if she assumes everyone knows this word and its meaning AND/OR as if she wants to share her mission with the students and invite them to join her on the mission and is doing this in the context of this class.

b. [I am ready to make my big plunge into racism. It feels good to say the word disenfranchised in this context when no one would expect it. Isn't this what it is all about? Accepting everyone?]

80. Professor: OK? So, those are some of the reasons that I'm doing this.

a. Concludes her reasons as if everyone remembers what reasons she is discussing even the ones who came in late (I wonder if they are lost?) $\mathrm{AND} / \mathrm{OR}$ as if she has already talked about the reasons in a previous class (I may have missed this in previous class. I know what she means because I have been her doctoral student for years. I wonder how long it took me to understand her mission like I do now as I listen to this class.)

b. [I hope they got the connection that writing and the reasons I conduct this class the way I do is all about inclusion and just practices.]

81. Professor: Now, my process is probably one most of you are not used to, and actually the nurses from summer from spring you didn't get a taste of this because we went on that giant escapade.

a. Hands mostly elbow level, but come out to reinforce a few words as she speaks. She refers back to the process she keeps talking about as if she realizes maybe some students are lost AND/OR as if she thinks maybe she should reiterate how most students are unfamiliar with her process $\mathrm{AND} / \mathrm{OR}$ as if she is bringing in her realization of the various ways people historically construct knowledge. Then she reminds nurses in the class who had her before of a project that got them waylaid from the process she is referring to here.

b. [I feel like reminding the class again that I know they are not used to my process and bringing in what happened in the past with some of the students in this class right now will reinforce context, history, and learning from what happens to us.]

82. Students: [laugh] [laugh]

a. Nurses laugh as if they remember.

b. [That was funny to think that the professor thought of our last class as an escapade. We are a community.]

83. Student: You had to bring that up, didn't you?

a. Student uses a bit of humor reminiscent of PROFESSOR's as if to connect to what PROFESSOR is saying here.

b. [I feel like I can be sarcastic in here.]

84. Professor: [laugh]

a. Laughs as if to show she is enjoying the conversation and memories.

b. [I like students to feel like they can be sarcastic and reminisce with me and others in the class. I think it builds community.] 
85. Professor: I'm still living with it. We're still trying to do it.

a. Reminds students that there is much to get involved in in here and there is a history between her and her students.

b. [I'm trying to show you that I don't give up easily.]

86. Student: It really $<\underline{\text { honed }}$ my lit search skills $>$.

a. The student spoke as if wanting others to know the importance of what they did in the class being referred to AND/OR as if to let others know to beware.

b. [I feel like I learned something at least and it was hard work.]

87. Professor: It's ^ an important skill if you go on to get a doctorate. [laugh]

a. Says by framing face and smiling with two hands directly in front of her face.

b. [I believe in you so nothing we do is in vain.]

88. Professor: All right, so, I don't, I, one thing I learned, I'm never going to do that in that class again and it wasn't, it just wasn't the right place, but at any rate, because I probably will do such a stupid thing again, I know. [laugh]

a. Says all right and so as if to bring students back to the topic AND/OR continues on discussing the topic with the nurses anyway as if to historicize what happened in the context of this class referring to what was learned and how it will affect future actions.

b. [I probably should have learned to not venture out and go with the flow, but I know myself and I will do the same thing again if called for in context.]

89. Professor: All right, So, what I will do, is you'll send me drafts, and given my parameters timewise, I'll look at them and I'll give you feedback. I use track changes and I use comments. And I use short (.) to the point (.) sentences. And I have never learned Spanish, I don't know come from a polite culture. Those of you who haven't noticed that yet. I'm Italian, I'm the oldest, I have four brothers $^{\wedge}$, I was raised in a very male neighborhood ${ }^{\wedge}$, in Cleveland, we're just like New Yorkers and Chicagoans, which means we're not very polite. [laugh]

a. Holding hands together in front elbow level, periodically throwing out a hand slightly, going over the process of feedback integrating with her culture as if to provide reasons for how she gives feedback tied to her culture. Closes with a slight laugh looking quickly from one side to the other as if to get approval AND/OR as if to make everyone at ease.

b. [I'm trying to share with you how we do things in here, but don't expect me to be a typical professor that never lets you in on my own life. My cultural behaviors are learned from my own history and even though I am organized and work hard, I will still have cultural norms come out that you may not like or understand or be used to. So don't get offended is what I am trying to say. I hope you hear me.]

90. Professor: Nonetheless, Miguel is here to keep me in check, right? [laugh]

a. Points to Miguel as if to remind students they are included in this class AND/OR to ease the comfort again between instructor/student power 
levels. And then laughs for a minute as if something is funny AND/OR as if it might be ironic that she must be kept in check.

b. [I am trying to show you how students become close to me over time and take over when I just don't get it. I give students permission.]

91. Miguel: Yeah.

a. Answers in affirmative as if he knows what is expected AND/OR as if he wants to go along with the Professor's process or humor. (OC: Miguel has known the professor for a couple of years and understands where she is coming from.)

b. [I appreciate what she has done for me and how she gives me permission to think outside of the box.]

92. Professor: Right? [laugh] [laugh] Good luck to him ${ }^{\wedge}, h^{\wedge} h^{\wedge}$ ? [laugh]

a. Laughs as if tickled at the prospects of what she just said.

b. [I hope they got the humor in thinking Miguel can take over and tell me to do something. This is a good example of sharing the community of learners.]

93. Professor: So, I just tell you this in way of explanation, because our cultures differ, and there are a lot of you in the room and there's only one of me,

a. Hand movements criss cross at differ and then open out and then hold up one finger to reinforce one of me as if students need reinforcement as they listen to her speak AND/OR as if students are still new to process and way of thinking the Professor has about writing and cultures.

b. [I want you to understand that we are all included here AND/OR I need you to know that I am cognizant of power level structures in university classroom settings.]

94. Professor: Cuz he can't give you feedback on your writing. No offense, [laugh].

a. Points to Miguel and reminds students who cannot give feedback as if to reinforce her status AND/OR as if to reinforce Miguel's status.

b. [But I also want to remind you that I am leading the pack so to speak, I am the one teaching you how to write research papers even though some students have learned a lot.]

95. Professor: Well, he can and he can't. I want to take that back, because Miguel has come a hell of a long way since I've met him. When ^did I meet ^you? Three years ago?

a. Takes back what she said as if rethinking how to say what she wants to say to the students AND/OR as if trying to take back an insult. Then turns directly full body to Miguel and asks him when they met.

b. [Sometimes what I say sound like an insult so I need to clarify. And I will at the expense of sounding different. I just want you to know what I am thinking and how I believe in students.]

96. Miguel: 2006.

a. Miguel knew exactly when they met as if it coincided with a special event like when he began his degree.

b. [I remember the day we met for sure.]

97. Professor: Only two years ago? It seems like I've known you forever. 
a. Starts turning back to face the class while states knowing him forever as if it were a bad or good thing AND/OR as if leading students to another thought.

b. [I want to share with you how will I come to know my students.]

98. Professor: Anyhow, [laugh], all right, but Miguel has come a long way, because in the interview, the dissertation, the interview to get in to be a doc student, I didn't understand half of what he said. All I understood was a few references to Habermas, who else? Foucault, uh, [laugh].

a. Now she fills the class in on what she meant as if they need to understand the connection between language usage and culture and knowledge AND/OR as if students knew Habermas and Foucault (OC: Do AE/HRD masters students have knowedge of Habermas, Foucault?) AND/OR to try to take back an insult with an compliment on how smart Miguel is since he knows about Habermas and Foucault enough to talk about them in conversation.

b. [I want you to connect intelligence with what people know not how they speak English.]

99. Professor: Anyhow, so, well, you had only been here, what, a year at that point?

a. Faces Miguel again to ask him this question as if to reiterate how smart he is or the connection between language and culture and knowledge.

b. [I think he spoke pretty good English for only a year in the US.]

100. Miguel: Yeah.

101. Professor: And he did what I think is common for a lot of people that come here from another country to take-go to school and that is they sit silently in classes because they don't want to look stupid. And so you don't speak and you don't learn to speak it. He's forced to talk to me.

a. Hands held together elbow level, she talks about common behavior that she has seen for people who come here knowing another language better than English as if taking back the insult again to reiterate his intelligence AND/OR as if to share he cognizance of how students sit back and don't learn to speak English AND/OR as if to share how she relates to what people need to learn English better and also provides it for them, that is talking with them.

b. [I have seen lots of students in the same situation in a regular classroom, but with me I make people talk sooner or later by working closely with them on writing and other projects.]

102. Professor: So, anyhow, you've got the idea? I'm going to give you feedback within my time parameter. Now some of you may need to make appointments with me, may need to come in during my office hours. If you want help on your papers, though, don't come to me with nothing, come to me with a work plan, come to me with an outline, come to me with a draft of some sort, because I can't work from nothing^ and if you give me something concrete, I can give you something concrete back.

a. Still standing in front of class, hands mostly in front still elbow level and asking if they have the idea (OC: Idea of what? I wonder if students are 
staying with her?). She continues explaining the idea as if it is about talking to her so they can learn things AND/OR explaining the idea as if it is about the process of getting feedback in general AND/OR as if the idea is how she needs concrete evidence of their work before she can provide feedback. Hands up once to reinforce a word.

b. [Now you get what I will do. I want you to know what you must do in this process. I think giving you a work load makes you more actively involved and a better writer in the long run.]

103. Professor: Ok? Now, another thing that is going to be unusual is that I believe writing is process, and I believe it takes as long as it takes to get it right.

Richard Smith here is embarking on a paper that I think has the potential to be an article. Not just a class paper, not just a conference paper. And it actually, probably $^{\wedge}$ has the potential if he was in another field maybe this one to be a dissertation topic.

a. Turns around and walks closer to laptop as if going to sit at it but turns back around as if changed mind and needed to clarify some more about writing process she believes in first AND/OR to discuss the process by bringing in something about another student's paper. Goes back closer to the middle of the arrangement and starts using hands to reinforce the different types of papers the student's paper could be.

b. [I am trying to tell you that I will never give up on you because each person is different and I can see ahead and lead you to writing different types of papers if you agree to work with me.]

104. Professor: And we'll put this up and you'll see.

a. Points backwards to the screen as if she is segueing into the next part of the class by discussing the student's paper AND/OR as if to prepare students for how she will be changing something in the class soon.

b. [I want you to know up front that your work will be put up in front of others so get used to it right now.]

105. Professor: All right, so I believe writing is a process and all things that are a process, you learn by practicing those skills. Now any of you athletic? Basketball, soccer, soccer, like I know shit about soccer. Anyhow^, when you are little, most of us are older than death, and don't want to play soccer or basketball anymore, but when we are little, and we used to play these things, we practiced! And all athletes know that they have to practice these skills, and we drill it into ${ }^{\wedge}$ them. But we don't do the same things when it comes to writing, when it comes to reading. We talk about it other ways, we don't talk about these thing in terms of building skills.

a. She rolls her hands while further explaining the process of writing as it relates to practicing athletics as if all students have that background knowledge. Then she asks students about their life outside class as if to bring their experiences into the conversation AND/OR as if to show students she realizes they surely know more about soccer than she does AND/OR as if to provide a concrete base for understanding the writing process. 
b. AND/OR Then she contextualizes herself and the students in the same age category denoting none of them practice sports anymore as if assuming this must be true AND/OR as if making use of time to get to the point of practicing writing rather than continuing the vein of exploring similar experiences outside of class.

c. [In case you don't get it yet, I will use something we have probably all done at least once for background knowledge in explaining that writing is a process.]

106. Professor: Now, I don't think for the most part, the books you have to read, this term, for these classes are going to be difficult reads. In years past, I've used the Handbook of Adult Education and it was a difficult read, because the last version took a postmodern stance. And postmodernists believe that the only good writing is dense circuitous writing, on purpose! OK.

a. Makes a quick connection to the books the students have to read for this class and past classes as if everyone knows that reading and writing practice are related AND/OR as if it is important what book is chosen for the class to read AND/OR as if there is a connection between type of reading and type of writing $\mathrm{AND} / \mathrm{OR}$ as if there is a connection that relates to good writing and purpose and the postmodern stance somehow (OC: Do these students know what postmodern is already? Is this important somehow? Is the Professor stretching their thinking by going beyond what they might already know?) AND/OR as if it is important to know about others' purposes for writing.

b. [I want you to also know that writing is closely connected to reading. And I will throw in a new word in case you don't know it just to get your feet wet.]

107. Professor: So, and, but it takes practice sometimes, well it does, it takes practice to read academic literature, it takes practice to develop your reading skills, it's just practice, same with writing, so.

a. Reiterates what she means about connecting reading and writing practice with a few hand points and turns around to walk back to the front by the laptop as if she is done with that topic and intends to begin a new one.

b. [I think the practice makes perfect analogy is a good one.]

108. Professor: I also think some of you should get into peer groups possibly, join forces, send drafts back and forth, though I'm not going to organize that because you're always better off getting outside ()

a. Fiddles with the laptop and talks about peer groups as if needing a filler while she prepares the next slide AND/OR as if wants to say this before she forgets and this is a good time while she works on the next slide.

b. [I am not forcing you, but peer groups work well for you but also for me in the long run workwise.]

109. Professor: All right, we're going to start off talking about the components of a paper. I'm gonna walk through what I think are the components of a position paper which is what most of you will be writing in here from the way that I saw your purposes. Then I'm gonna go through some samples, I'm going to walk you 
through Lisa's paper, I'm gonna show you Miguel's paper from two years ago in this class, and uh, and then I'm gonna go through your purpose statements that you sent me, okay?

a. One hand points backwards up in the air to the screen while she tells the students what she is going to do in the next of the class, what kind of paper most of them will be writing based on what she received this week by email, and then what she will do in the final section of the class.

b. [I want to start talking about something else now, the parts to the paper and this is how I am going to do it. I do this so you can get used to the process looking at others' papers before your own go up. I think this is a less offensive way of leading up to seeing your own papers.]

110. Professor: Now, the other thing. I generally don't give breaks because I don't remember to give em, and if you really need a break, you've got to consult with Miguel, he's gonna have to work it out and he's gonna have to tell me to stop. Now, otherwise, I'm just going to go (.) until I'm done, [laugh], and then we go home ${ }^{\wedge}$. If you need to get up and go to the bathroom, they're down the hall. Or you can take the elevator or the stairs all the way down^ and go on the first floor. I don't know where they are on the second floor. I don't think I've been on the second floor, um.

a. Says now with emphasis as if to initiate a new topic or the new section of the class. She goes back to some housekeeping as if she forgot to say this in the beginning $\mathrm{AND} / \mathrm{OR}$ as if she remembers the students who came in late never heard about housekeeping policies in the class, hands still at elbow level. Then giggles as if it is humorous that $\mathrm{C}$ would stop her after hearing from students about needing breaks AND/OR as if apologizing for not stopping for breaks during the class. And then goes on in some detail about where restrooms are, pointing in different directions as if she wants students to know she cares to take the time to share these things for their future comfort, if needed.

b. [I want to confirm in case you missed it that we work straight through and so you know I care about your comfort AND/OR so you will not ever ask in the future, I will tell you again where the restrooms are located.]

111. Student: (undecipherable)

a. Student mumbles.

b. [I can't believe this that we don't have breaks! AND/OR I like that so we can go home early!]

112. Professor: Yeah, you can go out that door and down this hall, yeah, you're really concerned about the bathrooms?

a. She brings in some humor as if to ease students' fears about not being comfortable during class AND/OR as if to tell other students they are welcome to ask questions in this class. Looks directly at student who spoke and takes a sip out of her water bottle.

b. [I think humor eases the pain of students not getting what they think they need or want, like taking breaks AND/OR It feels right being humorous since most professors are so dry and aloof.] 
113. Students: [laugh]

a. Students laugh as if not sure.

b. [It feels out of character for a professor to talk so down to earth about going to the bathroom AND/OR It makes me uneasy to talk about the bathroom in a university setting.]

114. Student: I'm a nurse. You're worried about if we don't find them.

a. Student shares her perspective on why PROFESSOR gives so much attention to the logistics of the restrooms.

b. [I think I should bring back an academic perspective to this class in case we get off track here AND/OR I feel the need to show others that I am a nurse AND/OR I get the idea that it is appropriate in this class to turn the tables on the professor if I am doing it in a professional manner.]

115. Professor: So, <papers have parts $>$. Papers have parts, and the papers that you're going to do for me will have three parts. It will have an introduction^, a discussion section, and implications. The introduction ${ }^{\wedge}$ section contains some parts $^{\wedge}$.

a. Sits down for a split second and then stands up again, using two hands to emphasize that papers have parts. Then points to the screen with one hand from high to middle to low on the screen as if to show students where to look for the information she is sharing, continuously emphasizing the word parts.

b. [I must get back to academics here so let me show you the importance of the parts of the paper by emphasizing them carefully.]

116. Professor: Are you looking for a chair? We've got two over here. | And there are no other rooms in this place.

a. Another student walks in late and she stops talking about parts as if to make the new student feel comfortable immediately, waits a minute as if thinking what students are thinking about the crowded room and responds that there are no other rooms as if that is what she thinks they are wishing right now AND/OR as if she is concerned with their comfort level.

b. [It is always right to recognize someone who comes in the room so they know right off that I see them, they are included, and that I understand the cramped situation and we may not be comfortable, but I care.]

117. Professor: But she better be on time next time. [laugh], or she'll be sitting in the hall, [laugh] [laugh].

a. Comments to the late student about tardiness and what may happen next time as if trying to get a point across about being late in a humorous way. Sits back down in front of the laptop.

b. [But just in case, I want to show you that I would appreciate it if you would come early AND/OR I want to show you that if you would come earlier you would not be so cramped because you would have a wider choice of chairs to choose from but I can't run your life so I'll just throw in some humor so you might get my point that way.]

118. Professor: All right, now (.) a problem ${ }^{\wedge}$ statement. I sent you that article, or the chapter in the book from Merriam and Simpson, where they walk through a 
problem statement. Now, you're going to hear terms kind of bantied about, you might have already in your travels as students. Some people will talk about a thesis, ok? When you took uh, English, they probably talked about a thesis, um, what other ways do they talk about it? Well, that's good enough, we know that one.

a. Fixes slide and stands back up, moving hands out and in at elbow level explaining what she sent and why as if trying to make connections to experiences they may have had before as a student with this new term AND/OR stops at the English class example stating that we know that one as if she knows all students have heard about the word thesis in English class before.

b. [I am in my comfort zone now because I can talk about problem statements in my sleep. I like this part where I can show the students that they really do know about problem statements even though it is a new term for them AND/OR I don't have to emphasize words so much because I know this part so well and believe it so deeply AND/OR This part feels right because I can start bringing in students' outside experiences to get them into the topic of this class.]

119. Professor: But the thesis $\wedge$ and the problem statement is the beginning of a paper that grounds your paper in something. Ok? Some one of you wants to do something on obesity, ok? And, uh, I had a student last year that did something on obesity, I don't exactly remember how it went but it was on the educational ${ }^{\wedge}$ value of obesity programs that we have.

a. Contextualizes the new term problem statement with known term thesis to someone's paper in this class and past class as if it is important to connect past with present and future learning to make sense of a new project the students are embarking on in this class AND/OR as if to bring in a student in this class to raise community feeling that we are all in this together.

b. [This feels really great when I can bring in something a student just sent me and a paper a student did last term to make this real for the students in the room to get them into this right away AND/OR I really like to pull in past and present work from students because it makes what I am trying to show the students applicable to their situation and I think it makes them feel part of the class, my grander mission.] 
Pilot Study Transcripts

Stage Five A, Part 1- Reconstructive Data Analysis of Representative Classroom Observations (Initial Meaning Reconstruction: Low-Level Coding)

(Categories That Name Interactions)

$\begin{array}{ll}\text { Italicized lines } & \text { - General code categories that name interactions } \\ \text { Numbered lines } & \text { - Codes that give examples of category } \\ \text { Very objective } & \text { - Anyone in the room would agree } \\ \text { Slight objectivity } & \text { - Slight interpretation used } \\ \text { Medium objectivity - Most people in the room would probably agree }\end{array}$

Low-level Coding, Lines 1-119

General Code Category and Codes

Level of Objectivity

Ways of doing housekeeping as professor

1. Include students orally in the process

Very

2. Use laptop

Very

3. Blame system or institution for requirements

Very

4. Use of humor

Slight

5. As interruption of lecture portion of class

Very

Very

6. Through directives

Very

7. Through story

Very

Ways of including students in class

Slight

1. Learn names

Slight

2. Refer to student(s)

Slight

3. Tell story about past or present student

Slight

4. Ask questions

Very

5. Discuss students' class work

Very

6. Discuss students' histories, cultures, differences

Slight

7. Discuss students' outside experiences

Very

8. Relate students' outside experiences with lecture topic

Slight

9. Send them things via email

Very

10. Expect them to provide work samples each step of process

11. Share beliefs about students' potential openly

Slight

12. Use humor

Slight

13. Discuss her own history as student (empathy)

Medium

14. Learn something about student

Slight

15. Openly mention power level structure (teacher v. student)

Medium

16. Attempts to reduce power level structure

Medium

Medium

Ways of revealing her process

Very

Very

Very

Very

3. Talk about how she makes decisions

Very 
5. Talks about her own work Slight

6. Talks about why she does certain things Very

7. Talks about why she uses certain things Very

8. Talks about her own history in decision making Slight

9. Shows students the process she had used Very

10. Brings in her history with students Slight

11. Brings in her own and students' cultures, groups Medium

12. Compares her process to other faculty's Very

13. Compares her thinking to other faculty's Very

14. Relates students' outside life to classroom work Medium

15. Uses humor Medium

Ways reveal being out of the ordinary as a professor Slight

1. Act disorganized

2. Explicitly state the fact Very

3. Use humor Slight

4. Learn students' names Medium

5. Mention how things are done Slight

6. Use words not typically used in classrooms by professor Very

7. Talk about race, discrimination, culture, etc. Very

8. Talk about own work Slight

9. Talk about students' thinking Slight

10. Talk about beliefs $\quad$ Very

11. Talk about self as a person, human, former student Slight

12. Make fun of self $\quad$ Very

13. Make fun of students Very

14. Say something, take it back to make point Slight

15. Talk about never giving up on students' potential Very

16. Bring up histories have with students' work Slight

17. Encourage students to join in with humor Slight

18. Tell story about humanity, mistakes made, etc. Slight

19. Use new terms Medium

20. Discuss how make academic decisions based on prior class Slight

21. Explicitly connect past, present, future Slight

Ways of maintaining control, regaining attention, changing topic Slight

1. Use terms (so, now, all right, at any rate, anyhow, ok) Very

2. Specifically call on student for response Slight

3. Tell story about student Medium

4. Do housekeeping when lecture portion interrupted Slight

5. No official break Slight

6. Use humor Slight

7. Use hand movements Slight

8. Use eye contact Very

Ways of teaching writing Very

1. Use PPT slides as organizer Very

2. Use screen to provide examples of the process Very 
3. Expound on each "part" Very

4. Tell story (personal or student) with each "part" Very

5. State what she will be talking about Very

6. Discuss outside influences on each "part" Slight

7. Discuss why this is important in your life Slight

8. Discuss connections between writing, culture, history, etc. Slight

9. Include broader implications of writing Very

10. Talk about benefits of working together Slight

11. Make connections to reading Very

12. All time given to the teaching of writing Slight

13. Use humor Slight

Ways of conforming as a student Very

1. Walk in and sit down at table Very

2. Take out laptop or notebook and pen Very

3. Listen to professor Very

4. Raise hands unless told otherwise Medium

5. Laugh when professor tells joke Medium

6. Answer to roll call Very

7. Answer questions directly from professor Very

Ways of conforming to particular classroom norm as student Slight

1. Laugh at professor's sense of humor Slight

2. Reply to professor's sense of humor with sarcasm Slight

3. Agree with professor when asked Slight

Ways of bucking system as student Slight

1. Walk in late Slight

2. Play with IPOD during class Slight

3. Eat candy when no one else is eating Slight

Ways of bucking classroom norm as student Slight

1. Talk quietly to neighbor while professor talks Very

2. Act bored and pack up when ready to leave Very 
Pilot Study Transcripts

Stage Five A, Part 2- Reconstructive Data Analysis of Representative Classroom Observations (Initial Meaning Reconstruction: Explicit Meaning Reconstruction) (Possible Underlying Meanings)

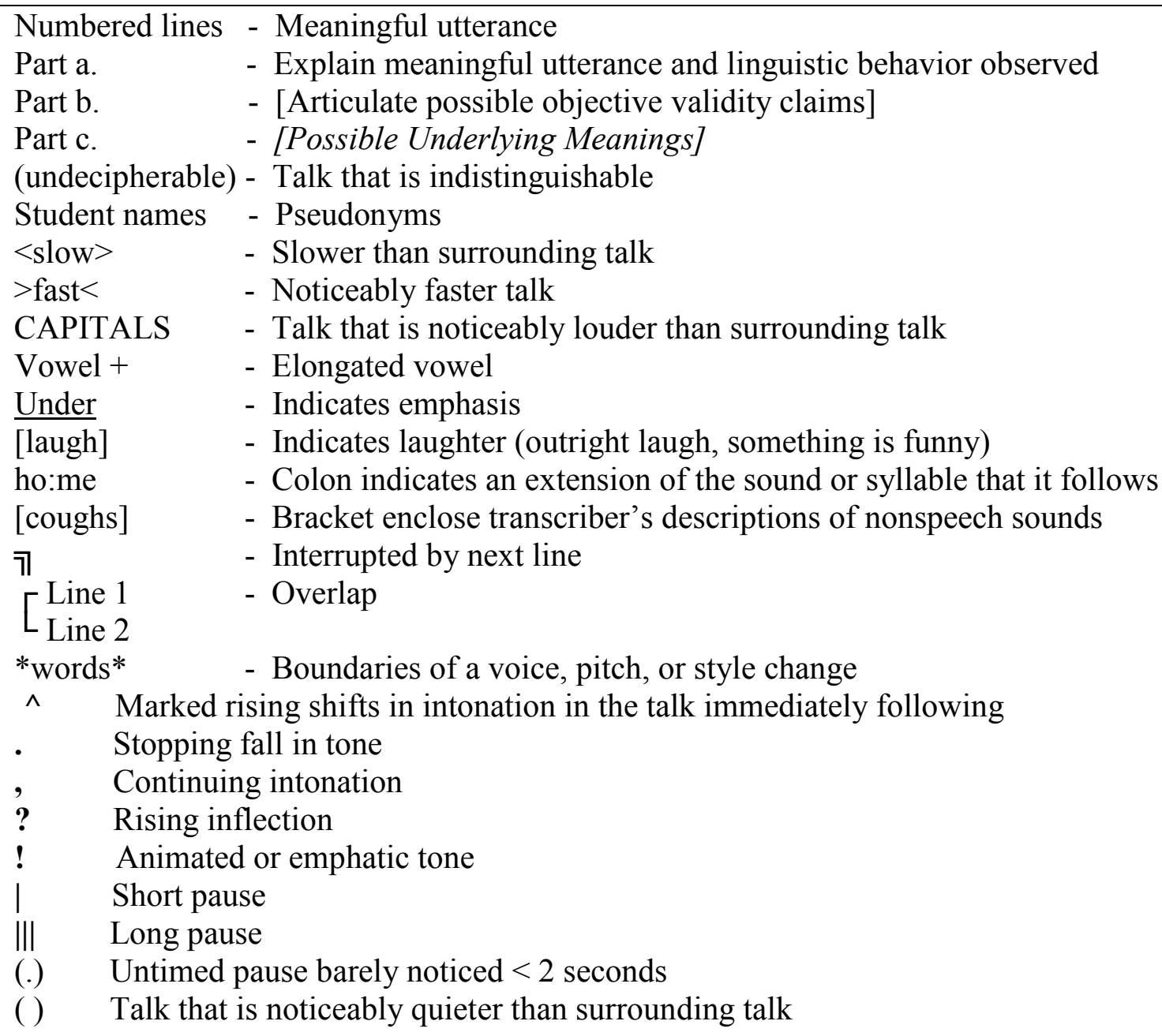

1. Students: [laugh]

a. Students are coming in and casually and quietly talking and laughing to whoever is near them as the professor fiddles with the laptop at the front of the class. A Power Point slide is showing on the screen. The long tables are situated in a rectangular shape so the students can sit on the outside and look towards each other. Several students have laptops. Most have notebooks to write in.

b. [We're having fun talking together in a classroom with other students before class officially starts AND/OR I need to find a seat before class starts so I don't get called on and potentially embarrassed.] 
c. [Students usually use laughter as a filler or as a way of hiding fear or tension about making a good grade in class even at this level, they really just want a grade AND/OR They want to make the least obvious entrance at the first of the semester because they do not know what to expect yet. Those who know the professor already are less apprehensive and quieter because they don't have to resort to nervous laughter.]

2. Students: (undecipherable)

a. Around 20 students are here now and either quiet or buzzing quietly to the person next to them. It is obvious who the professor is by the placement of the tables. The professor is setting up at the front table, the one right in front of the screen.

b. [It is acceptable to talk together quietly before the professor gets here AND/OR begins class.]

c. [Students are really just filling space with idle chit chat because they just want to get started so they can go home at this time of day after a long day's work, kids waiting etc.]

3. Professor: Uh. Who did? You know I left my notebook. Where's Jim?

a. Sitting down in front of laptop and looking around as if lost somebody or something.

b. [I always forget something AND/OR I can always ask Jim what I did with my notebook since he usually remembers what I do with stuff better than I do.]

c. [The professor probably realizes she forgot something or got sidetracked by something more interesting than taking roll AND/OR realizes some student knows about her notebook and would rather him look for it than her.]

4. Students [laugh]

a. Students all laughing at once as if someone just told a joke.

b. [This feels good when the professor asks for students to help AND/OR This professor did the same thing last time and I think that is funny AND/OR The professor loses things and it reminds me of myself AND/OR The professor does not match what I thought a professor does.]

c. [Students quickly think about how the professor gets ready to begin but usually don't dwell on it and just fill time with idle chit chat while waiting.]

5. Professor: Didn't he just? Who are you?

a. Point to a person as if that might be Jim.

b. [I thought I just saw Jim. To save face, I will ask someone if they are Jim to ease the embarrassment level. I usually have good luck with that tactic.]

c. [The professor really doesn't care if she doesn't recognize someone at this point but has a habit of pointing out students in the time of question AND/OR the professor really does care and hopes to find Jim really quickly now.]

6. Students: [laugh]

a. Students laughing at once again as if someone told another joke. 
b. [This may be a fun class since the professor cracks jokes AND/OR has a different sense of humor AND/OR I hope she does not point to me since I don't like to be called on even at this level.]

c. [Students really wonder when they will get started AND/OR Students wish class would be called so they can go home but make the best of it by having fun while they wait.]

7. Professor: Ohh, forget it. He's not here yet. It was me.

a. Throws up one hand in the air as if tossing something out.

b. [Oh, yeah, I remember where it is. I'll just admit my preoccupation out loud and get a laugh out of the students. AND/OR I like to make them feel at ease. They have had too many dry insensitive professors.]

c. [The professor usually figures out "it was her" instead of her students and she is glad to admit it AND/OR The professor just changes how things will work differently anytime something goes wrong.]

8. Students: [laugh]

a. Students all laugh together again as if someone told another joke.

b. [She's human, thank heavens. This may not be so hard after all. Being at the master's level may be something I can do after all. AND/OR Oh, no, she's a basket case. Can I endure this?]

c. [The first or second class students are very exuberant and think everything is funny AND/OR Students like to come in the room and get settled on the first few classes to make a good impression with their peers who don't know them yet. Later they get less exuberant like this.]

9. Professor: This is the hardest thing I do all year. Doris, were you here last time?

a. Checking things on the laptop as if it is required AND/OR being in charge of taking roll.

b. [I like to say things that students have to reconsider, like pretending something mundane is really hard. AND/OR I need to put this task forced upon me by the university into perspective. I hope the students caught that innuendo.]

c. [The professor really doesn't get into taking roll, it is not high up on the list of things to do that are important AND/OR DR does not put priority on tasks like taking roll since they are not important in the broader scheme of things so anything to do with it seem to get lost or misplaced making the task harder than it should be.]

10. Doris: Yes

a. Students are listening now as if they know the rules of school are the same as rules of university, that is, that when the teacher or professor begins checking roll, class begins and you become quiet. The class includes students from various cultures from looking around or listening to them answer the professor. Some are Black, some are Latino/a, a few are White with most appearing to be Latino/a and Black.

b. [I have been in school for so long that roll call is familiar to me.]

c. [Students want to be listed as present because it affects their grade so they quiet down and listen up for their name AND/OR Taking roll is a classic 
way of beginning class so students quiet down with no prompting other than the first name being called.]

11. Professor: Lola Lesser, does anybody know if she was here last two weeks ago?

a. Looks around as if she would know or see the person being called $\mathrm{AND} / \mathrm{OR}$ as if she knows the person is not there or would raise hand and be acknowledged.

b. [Where is she? I was hoping she would come to class. AND/OR I purposely look around and get people involved even in what seems like the smallest ways. This sets the tone for the class.]

c. [The professor wishes she could figure out a way to make this task go away or be easier and less of a headache. She wants to get on with important stuff.]

12. Professor: All right. Jose Ruiz?

a. Looks around briefly as if knows the person is there.

b. [This is boring AND/OR Why does the university require such mundane things from me? AND/OR Why don't I just let someone else do this task? I follow this rule to the $t$ in every class. I sure wouldn't do this if not required.]

c. [The professor feels she must do this task because she holds herself responsible for giving grades, getting to know students, and getting students involved and she intends to show this to the students AND/OR The professor just wants to hurry up.]

13. Professor: All right. Marissa Sanders?

a. Says all right as if to get attention of whole class. Looks up at student as if remembers her and she was there.

b. [I want to make sure I have the attention of everyone from the getgo.]

c. [The professor wants the students to be involved in this task AND/OR The professor wants control of the students' attention.]

14. Professor: Johnson I know was, ()

a. Did not look up as if she knows Johnson.

b. [I like knowing students so I can get this task over with AND/OR to let students see how I interact closely with students even at mundane levels.]

c. [The professor likes knowing people in the first couple of classes because that means they wanted to take the class because of her AND/OR that means she has a base upon which to build her community writing process/model.]

15. Professor: How about Natalia? [laugh]

a. Looks up briefly as if knows Carmen is not there but checking anyway and then giggles as if something is funny.

b. [Sometimes I tickle myself AND/OR I want to make the students feel like they can be comfortable.]

c. [The professor thinks it is funny that she can maintain attention just by taking roll AND/OR The professor laughs because she is not self confident and believes this will get the students more on her wavelength.]

16. Professor: All right. Niara? 
a. Looks up briefly as if looking for student.

b. [I may remember him if I just scan the room.]

c. [The professor knows a lot of students and remembers this name AND/OR The professor really does not know the student but just wants to finish this task.]

17. Niara: Yes

a. Niara answers that she is here today.

b. [I know how roll call goes.]

c. [Niara wants to fit in AND/OR Niara just rotely answers.]

18. Professor: Were you last?

a. Talks directly to the student as if to verify last week's attendance.

b. [It feels good looking at students' faces when I talk to them.]

c. [The professor feels the need to connect so asks about last week AND/OR The professor feels the need to do this task properly and it might help her

19. Niara: Yes fix her attendance sheet if she asks Niara about last week's attendance.]

a. Niara answers as if she knew the professor wanted to know about last week's attendance.

b. [Don't you remember? AND/OR Why don't you know me yet?]

c. [Niara is put out that the professor does not know her yet and doesn't remember her last week AND/OR Niara doesn't care either way but wants to be on the attendance sheet at present AND/OR Niara lies since the professor doesn't remember anyway thus getting by with a free attendance.]

20. Student: [cough]

21. Professor: Christy, you were too, Acacia was not because they lied to her, Alicia was not ${ }^{\wedge}$ Ginny was ${ }^{\wedge}$ Rachel was, you're Rachel right, Emily.

a. Speaks to Christy as if she remembers her. Then looks up as talks about Acacia at same time pushes a button on the laptop and brings that finger up into the air as she speaks. Each time makes big strokes as if to make sure the students see her take attendance. She looks at who she thinks is Rose to confirm and looks up to find Emily.

b. [I am getting tired of this so I feel like moving faster and acting out what I do so they know I am still taking attendance AND/OR I need to speed this up before I lose people over something unimportant in the scheme of things.]

c. [The professor really likes knowing why students are or are not in class so she finds out AND/OR The professor likes to buck the establishment and fits it in whenever she can.]

22. Emily: I was here

a. Answers as if she wants to clarify her attendance.

b. [I must make sure she knows I was here last time so I will use something other than a simple yes just to make sure.] 
c. [Emily thinks this is ridiculous AND/OR Emily wants to be recorded as here and is so glad the professor will try to be nosy about her whereabouts since she was in class. Emily feels lucky.]

23. Professor: You were here then. Are you here now?

a. Talks as if making connections between this and last week's attendance.

b. [It feels good when I can pull in historical construction even when taking roll.]

c. [The professor really enjoys thinking of insinuations that connect past, present, and future when possible AND/OR The professor likes to hear herself say words in a clever way.]

24. Professor: I think you were here when I called your name!

a. Converses with Emily as if her name is a very important topic.

b. [I like making people feel important. AND/OR She is taking over the power here so I will dominate the conversation.]

c. [The professor likes remembering why she does things AND/OR DR is having a good time playing on words.]

25. Students: (undecipherable)

a. Students mumble together.

b. [I see she really gets to know people AND/OR I wonder what she will say to me in the future? I better keep on my toes.]

c. [Students feel like the professor is with it AND/OR Students feel like the professor is nosy.]

26. Professor: $O k$, ok $\mid M i-a h$ ?

a. Stops and asks the student her name as if asking for correct pronunciation.

b. [What culture does this name come from? Better check pronunciation.]

c. [The professor really does want to know about this students' culture AND/OR The professor does not really care about the culture but want to make this task more interesting for the students.]

27. Mia: Me-ah.

a. Student says name as if to respectfully correct mispronunciation of name.

b. [It feels good when teachers ask me how to pronounce my name. It makes me feel important. AND/OR It is getting old having to say my name in every class year after year. I feel like an outcast.]

c. [Mia thinks it is good when professors act interested in her background AND/OR Mia is offended when professors act like she is from another country and holds it against them.]

28. Professor: $M e-a h$.

a. The professor repeats as if trying to say the name correctly.

b. [I need to repeat so I can remember. Years of this has shown me how important names are to people.]

c. [The professor assumes that all students want their cultures talked about AND/OR The professor does not really care what students want but she wants them to understand that culture is something that must be addressed.]

29. Mia: Yeah. 
a. Mia answers as if verifying the correct pronunciation.

b. [I'll just muffle my answer so we can just get off this topic.]

c. [Mia wishes she could crawl under the table AND/OR Mia doesn't care about the culture part but just wants to be counted present.]

30. Professor: I knew who you were ^ [laugh]!

a. With both hands as if directing an orchestra for a second AND/OR as if very happy she knew which student she was talking to.

b. [This feels good when I remember someone after only one class.]

c. [The professor really is happy when she gets to know new people.]

31. Mia: Yeah. That's a plus ${ }^{\wedge}$

a. Student agrees as if it was important.

b. [I guess she really means well, so I will go along.]

c. [Mia feels that the professor must really care so becomes more into the conversation AND/OR Mia sees that the professor really cares so she goes along with her.]

32. Professor: That's a plus! [laugh] I didn't have to ask ^ you. Ok, you were here last time too.

a. Reiterates the point as if this is an important part of the agenda for all to know.

b. [I love bringing in culture and community feel even during mundane tasks like taking roll.]

c. [The professor likes to make humor out of mundane topics.]

33. Professor: OK, I know June was, all right. NOW, is there somebody in the room right now that was here two weeks ago and here tonight that's not on my list ${ }^{\wedge}$ ? |ll

a. Points with one finger down for each time she said here as if to confirm she means right here in this room. Then spends a while finishing something on the laptop as students watch her.

b. [I'm ready to get done here so let me finalize the task right here!]

c. [The professor is happy this is drawing to a close AND/OR The professor thinks it works to use her hands to direct the class attention.]

34. Professor: Ok, how many of you received the things that I sent you today? | All right, uh, you will find that I-ah frequently will send you things the day of, actually, there is no rhyme or reason to my sending things.

a. She looks up as she asks the question, still sitting. Then she reconsiders what she asked as if the process of sending needed to be explained.

b. [I don't know if that came out right, what did I say? Let me rephrase that so students will think it was a funny comment AND/OR so that students will listen better thinking that I may be an oddball by letting out my thoughts. It feels good having students think that I am an oddball because most professors are so boring and closed.]

c. [The professor wants to know how her email system is working AND/OR The professor also likes to explicitly begin class with what the students will find that she does differently AND/OR The professor has learned from 
experience to begin class by drawing attention to her different way of doing things.]

35. Students: [laugh]

a. Muffled laugh as if smiling slightly out loud

b. [I may enjoy listening to you tell us how you think and do things, since most professors are so straight-laced and never let on that they really might think like you are about to expound on. I like this. AND/OR Oh, no, what have I gotten myself into? Surely, she has a rhyme or reason. I want to make an A in this class. Come on!]

c. [Students feel closer to professors when they realize they are human AND/OR Students are jolted out of their stupor and listen up to decide if this is all worth it when professors tell them something about themselves that is out of character.]

36. Professor: To tell you the truth ${ }^{\wedge}$,

a. Reconsidering again as she decided another way to explain her process of sending papers AND/OR as if reconsidering how to explain her process.

b. [I like coming at things from a different angle than anyone would expect. I want them to think about things so I'll just bring in how I do it.]

c. [The professor likes to get students' attention and then gain their approval AND/OR The professor likes to shock students.]

37. Professor: If one of you asks me a question, now the answer you ${ }^{\wedge}$ get from me may aggravate the shit out of me, and you notice that in my response.

a. She tells her process as if trying to get at the possible students' perspective when they receive a response from her.

b. [If I act like I think about them when I am reading their stuff and having a life at home, they will get the idea that I am not an oddball, but a person who they can talk to, relate to, and construct knowledge with.]

c. [The professor likes to shock students right off the bat so they will acclimate quicker to the norm of the classroom AND/OR The professor likes to shock the class right off so they will have a disorienting dilemma and transformative learning experience.]

38. Professor: But then ^ but then ^ I will decide it's not that dumb of a question [laugh] after all [laugh], it was my husband ${ }^{\wedge}$ that was really aggravating me, and I will try ${ }^{\wedge}$ to respond in a fuller message to the class because I think that it is something in fact that you don't understand as a group, ok^?

a. Stands up and starts using hands as if to reiterate the importance of the new way of explaining the process AND/OR showing students she rethinks her first response and reaction, acknowledging she realizes how students may feel when they get that first response and decides they all might need help, thus providing help on something they all might not understand.

b. [I can get my point across when I show them how I actually learn something by reconsidering what seems stupid when taken at face value, but upon rethinking it seems like a good question from someone else's 
perspective. I feel good when I show students that it is hard to be a critical thinker but I expect them to do the same thing sooner or later.]

c. [The professor feels good when she can pull shocked students back in to reality and show them she is human like them AND/OR The professor feels good when she can show students she really cares about them in a backhanded sort of way.]

39. Professor: <So you've got a message> about problem statements from me, right? And I took, I think I took two of my own papers?

a. Two hands going up in the air as she asks the first question, looking down at laptop as if to answer the second question she asks.

b. [I do lots of work here, did you at least retrieve the messages? AND/OR I like to ask them questions I already know, but sometimes I get sidetracked and maybe didn't even send what I meant to send so I'll put it in their court.]

c. [The professor feels it necessary to share her own writing with students as models because she missed out on that in her schooling AND/OR The professor wants students to see models of good writing.]

40. Students: (undecipherable)

a. Quiet buzzing of students' voices as if they don't want to be heard.

b. [I know the answer but am afraid to speak really loud.]

c. [Students say an answer under their breath when they want to be a part but do not want to be singled out in case their answer is wrong.]

41. Professor: What?

a. Looks at who asked the question as if to hear the question again.

b. [I wonder what you said but am not going to press the matter since I don't think it matters right now AND/OR since she looks not sure of herself.]

c. [The professor likes it when students start participating with comments.]

42. Professor: Yeah, and uh, I showed you what a problem statement with a purpose looked like from, I know from my CRT one and I don't remember what the other one was,

a. Quickly registers the question and begins using hands two up touching a point in the air and then lowers them a little to touch another point in the air to answer the question and then telling the students what was sent with one hand under chin as if she feels she has their attention now.

b. I'll just answer my own question since I think I remember what I sent, and it feels good to talk about my CRT paper. I like to banty terms around that I want them to hear for future reference anyway.]

c. [The professor likes to share her work AND/OR The professor shares her work first so students gets eased into the fact that their work will be shared in this open forum.]

43. Student: [cough]

44. Professor: but I think it was two of them,

a. Says this as if it were an afterthought.

b. [The second paper is not a priority right now in my mind.] 
c. [The professor likes to think out loud as a way of carrying on conversations with groups of students AND/OR as a way of making herself seem more human to the students.]

45. Professor: At any rate, uh $\mid$ all right, so, somebody asked me something and that's what I did,

a. Uses this phrase to change the topic some and using hands as if directing an orchestra in an up, down, and around fashion.

b. [I need to get back to the topic of this class or at least the topic of how I respond when someone asks me a question just in case I have had a busy day AND/OR in case someone doesn't understand my cultural behaviors.]

c. [The professor wants to openly summarize why she did what she did so students can get the gist of how she works.]

46. Professor: Now, today ^ I was preparing for class a little later than I would have liked because I distracted myself and did something else, and uh, and anyhow, so I sent these things late, >Now they're in your email ^ so you can go back and get them if you don't have them but it occurred to me that it might be useful for somebody to have ${ }^{\wedge}$ these handouts so you can print them out and write on them $<$, ok?

a. Using hands as if to direct an orchestra and talking more quickly as she goes along as if knowing what is coming next in the information being given.

b. [I have a life and want you to know that even so I will still do my work and will still provide you with all sorts of models for your own writing. I like to throw hints about writing on handouts and other things that I've learned along the way about the writing process AND/OR and other things that other professors could care less about sharing.]

c. [The professor likes to share how her life gets in the way sometimes like students' live do AND/OR The professor wants to share how she thinks about students in her preparations for class.]

47. Professor: So, that's why I sent them, you know, it's not required, it's just I was trying to be helpful. |

a. Two hand up together with the first word and then pointing to places in the air as if to make sure students see she is concluding the explanation.

b. [See, I really do care because I go beyond requirements of the university system. I want to help you become better writers.]

c. [The professor likes to remind students that the university requires things of her that are mundane but she goes beyond requirements when it is important for the students' success.]

48. Professor: All right, um |||

a. Starts to sit back down again, looks at laptop as if deciding where to go next.

b. [I need a second to remind myself what I want to talk about next.]

c. [The professor wants to sit down but decides that she needs to introduce the topic of tonight's academic portion standing up AND/OR The 
professor decides now is not the time to reduce power relations while introducing the academic portion of tonight's class.]

49. Professor: Now, I'm going to talk to you tonight about writing a research paper and, umm, I going to spend probably what some of you will consider an inordinate amount of time, trying to teach you how to write research papers. I do this for a variety of reasons. One, I believe very strongly about the notion that students and professionals have a voice, and the only reason we don't know what you know is because you don't know how to access the world of publication.

a. Stands back up, hands together at first, and casually walks into the middle of the oblong table arrangement with all students facing inward as if she is confident enough about what she is saying that there is no need to point to places in the air for emphasis AND/OR begins making two hand motions from elbow level in and out many times as she is talking as if she is sure of the meaning of what she is saying to the students (OC: Does she point in the air when she is searching for what comes next versus move hands at elbow level in and out when she deeply believes and knows what she is saying or going to say next?)

b. [I better preface how long I intend to talk about writing research papers because I know that students are not used to this and they need to know how important this is. It feels good to tell them what I believe about their potential because I have seen how students (including myself as a doc student) are treated and I am determined not to do have any student leave my class thinking they cannot write well or thinking that writing is not a process!]

c. [The professor feels it is right to introduce the topic and then pull in how she will do it differently with reasons for doing thing differently AND/OR The professor feels good when she can connect academic topic with voice and access.]

50. Professor: $O k$ ?

a. End of discussion.

b. [I feel like I made my point.]

c. [The professor likes to end a discussion with a word that commands attention briefly but effectively.]

51. What do you think, Johnny?

a. Looks at Johnny, hands elbow level together and still.

b. [I better lighten things up and bring in a student's remarks, a student I know well right now.]

c. [The professor enjoys asking students she knows what they think about how the class is going AND/OR The professor likes taking the attention off of herself when she is done with a topic or segment of discussion.]

52. Johnny: You are perfectly, perfectly right.

a. Sounds as if he halfway agrees but with tongue in cheek.

b. [I understand the professor and it is fun to be able to tease her and vice versa. My history with school, work, and society has been a disaster. She gives me hope. It's the least I can do at this point.] 
c. [Johnny really does agree with the professor AND/OR Johnny agrees and wants to say more but leaves it at that right now.]

53. Professor: [laugh] I like Johnny. [laugh]

a. Laughing, pointing with one hand toward Johnny.

b. [I know Johnny has had a hard time so I feel good when I let him show what he knows even if it is about me. Aren't I funny? Sometimes I tickle myself. I'm so glad I became a professor.]

c. [The professor enjoys making students feel like they are welcome.]

54. Professor: Anyhow, and uh, I think this because when I was a grad student, uh, in the 90s, look at my vitae, I'm very young, um, all four of my degrees come from the 1990s. Anyhow, um, when I was a grad student, I knew when I went to get my doctorate that I wanted to be a professor, that was what I was going to do, um, I figured it out but so, I didn't know what you needed to do as far as career ${ }^{\wedge}$ path to become a professor, so I started asking faculty^. You know, what does a student need to do as a student to become a faculty member later? And they said you need to publish.

a. Still standing, little bit closer to laptop, hands elbow level, slightly in and out as if truly believes and knows what she is saying, one hand up to confirm importance of four degrees, finger shaking at figured it out, point to three places on a ladder at career path, hands still low.

b. [I like bringing in my own history because I know how past, present, and future connect and maybe by doing so my students will begin to look at the world differently, at what they can do differently.]

c. [The professor wants the students to think it is good to have multiple degrees AND/OR be a faculty member AND/OR young with multiple degrees and faculty

55. OKa+y, [laugh], how do you do that ${ }^{\wedge}$ ? Well, no faculty members really tell you how to do that except for a few rare ones, like me^, and um, because most faculty assume that you go on and get a doctorate and somehow this magic of writing this dissertation which is really unlike any other kinds of writing, will magically make you understand the publication process. And it doesn't. It doesn't prepare you at all, uh, others ${ }^{\wedge}$ think that students have nothing more () to say, therefore, they shouldn't be encouraged |

a. Continues the same stance every now and then backing up a step and looking at laptop as if checking to see if she is getting closer to it, two hands sway left and right at somehow magically, then two hands pointing to places in front of her as if to stress unlike other kinds of writing AND/OR as if to stress that she disagrees with what other faculty think about writing and their students relationship to the magical process AND/OR hands higher than elbow level and pointing for emphasis when she emphasizes certain words when talking as if showing where she disagrees with other faculty.

b. [I learned that I needed telling but no one would do it and from that point in time I made a commitment to tell students how to do something like publishing papers explicitly. At this point, I can say I am a rare case at the 
cost of appearing a braggart because of how I have handled the class up to this point. They must see that I am trying to share what I know and have learned yet don't think myself a know it all.]

c. [The professor wants students to know she is rare as far a teaching writing explicitly and making the publication process less magical AND/OR It is good to be rare.]

56. Professor: [laugh]

a. Smile on face and hands in front of her swaying with upper body is as if she just got away with opening up a previously secret bit of information.

b. [I think I got away with that. That's funny to me. But I made my point.]

c. [The professor uses her humor to manage the classroom AND/OR The professor has fun teaching her class.]

57. It's getting more and more crowded, there's two seats up here. There's one here, this one's mine ||| Nobody wants to sit by me, Johnny.

a. Points to places to sit and then tells Jimmy no one wants to sit by her as if to ease student unfamiliarity with her and others AND/OR as if trying to get on the same level with students AND/OR trying to get students to enter into the conversation as people who know something and don't have to steer clear of the professor.

b. [I like to make people laugh and feel comfortable AND/OR like they can be humorous especially in a forced closed in setting like the university classroom.]

c. [The professor likes to make fun of herself to draw students in AND/OR to put everyone at ease.]

58. Johnny: [laugh]

a. Johnny laughs as if he thinks something is humorous.

b. [They will learn that sitting by you is okay as soon as they get to know you.]

c. [Johnny thinks the young students should see that he is accepting of the professor's humor. He has been ostracized before and does not want that for anyone else.]

59. Student: You're so ()

60. Professor: ||| All right, you know what, we are expecting some more. So, you're going to have to sit there, I hope you're ()

a. Stops to let students sit down and lets them take their time by talking to Miguel while they get comfortable.

b. [I really don't like when students come in late but don't want to belabor the point. Humor needed here.]

c. [The professor thinks that people usually come late for reasons outside of their control at this level. She knows students and who registered and they are not all here.]

61. Student: (undecipherable) [laugh]

a. Students laugh as a group as if to show that they feel comfortable and get the humor she presents.

b. [So that's we get if we come late?] 
c. [Students ask their neighbor how they will get the professor's feedback on their classwork with so many students in the class because they have been in too many crowded classrooms.]

62. Professor: And |||

a. Says as if biding for time.

b. [I need to get back on topic, but how?]

c. [The professor manages the class using words as placeholders.]

63. Actually, Miguel ^ was going to put the desks this way, but I thought this would be a better way. I think you might have a little more room sort of. If there's a fire drill though

a. Brings Miguel into the conversation as if to make students feel comfortable AND/OR as if to show she got the last say in arranging tables and then adds some humor as if to bring back the comfort level again for students.

b. [It works well to bring in a student and something they did in context to gain attention sometimes. I'll try it here.]

c. [Humor is a good way of gaining attention or changing the topic.]

64. Students: [laugh]

a. Students laugh as if to show they understood the humor of a crowded room and fire drill.

b. [It feels great to laugh at a professor's jokes.]

c. [Students hope that will never happen but they laugh because it is a thought that would not have occurred to them at this point.]

65. Professor: I think this row needs to go out this door so that that group can go out that door. Oh, yeah, the restrooms down this door. I

a. Pointing with two hands as if directing traffic, she continues the humor of the fire drill as if letting students know she heard their laughter AND/OR as if she wants to elevate ownership and comfort in the classroom and as if making a final input for student comfort she adds more about the restrooms and points to the door she is referencing (OC: does she point often because she thinks students are or should be looking at her or because of her cultural background of Italian or to elevate comfort level?)

b. [I like telling the whole story of what could happen. It's sort of like rethinking what is given at face value and coming up with something out of the norm.]

c. [The professor thinks it is good to bring in humor to assuage possibly uncomfortable situations. Too many people are in uncomfortable situations and she wants to alleviate that if she can.]

66. Professor: () Where was I in my sad tale?

a. Almost said as if an aside to get back to the topic of the class.

b. [I'm ready to begin talking about writing again.]

c. [The professor likes telling her story within the context of the lecture portion of the class. She has forgotten where she was for a split second so interjects a little more humor as she collects her thoughts.] 
67. Professor: Oh! The rest of you don't know anything worth getting published, therefore, nobody should teach you how. Now that's one reason. Another ${ }^{\wedge}$ reason is that if you come to graduate school, the assumption is you have a career $^{\wedge}$. It's my assumption that most of you work. Is there anybody here that doesn't have a regular job?

a. She starts in as if the new students knew what she was talking AND/OR didn't think about how they just got there (OC: I wonder if the new students thought SHE meant that they knew nothing and nobody should teach them. I wonder what they thought the reasons were that she is listing. I wonder if the students who had been there remembered where she left off from here.) Then she asks the class about their jobs as if to bring everyone into the conversation.

b. [I was talking about how professors don't think students know anything, so that's where I'll start. But I think differently, everyone has careers and that must mean they know something.]

c. [The professor asks about jobs to catch students' interest and contextualize the class in their lives, but at the same time forgets that the late students may think they don't know anything.]

68. Professor: $M e$, [laugh].

a. She answers her own question as she points with one hand in the air and laughs at her answer as if being a professor was not a regular job AND/OR as if trying to let students realize that any job could be considered not regular AND/OR as if trying to get students to think outside the box about their own job.

b. [What did I mean by regular, I'll bring myself into the conversation again and say that my job is not regular. It feels right when I laugh about myself in order to build the students' confidence.]

c. [Laughing at herself is part of her classroom management style which allows her to also make fun of students or talk about their work as examples of the process.]

69. Student: (undecipherable)

70. Professor: All right, but you're in the minority [cough] () but you are, and especially true in the fields that I represent, Adult Education and Human Resource Development.

a. Looks at the student who answered the question and then critiques the students' answer quickly as if to bring everyone back to the topic of the class papers, that is $\mathrm{AE} / \mathrm{HRD} \mathrm{AND} / \mathrm{OR}$ as if to introduce the topic of minorities.

b. [Was that an insult? I'd better clarify about minority here. I know I would bring it up sooner or later anyway.]

c. [The professor likes to represent AE/HRD and feels very connected and knowledgeable about the field.]

71. Professor: SO, if you have a job, how many of you never write a thing at work? 
a. Immediately emphasizes the word SO as if to not pursue the students' answer AND/OR as if to bring the topic back to writing. Then looks around for a second as if for nods or some response.

b. [It is important to relate this class to what students do in the real world, that is, work. I know the answer to this question already and so do they.]

c. [The professor changes the topic often or asks questions with little wait time because she has a lot to tell the students and needs to get on with the lecture portion.]

72. Professor: Yeah, most of you write at work. Now, how people perceive you at work has a lot to do with how you communicate. Not ${ }^{\wedge}$ the nature of your ideas... how you look, how you sound, whether they can pick up an accent in your writing.

a. Still standing at front of room near laptop. Sees she got a response and continues talking with one hand out at a little above elbow level on the topic of writing as if to introduce identity and communication and writing with work AND/OR as if trying to connect what she is saying to students through their work.

b. [It is also important to relate writing to culture and identity development.]

c. [The professor feels like it is her duty to interject culture and identity development because of her background with the topics. Discussion on these topics come very easy to her.]

73. Professor: I learned this in Columbus, Ohio, so it has nothing to do with a Spanish accent that I'm talking about. It has everything to do with a Black accent, or a Cleveland accent, ok, or West Virginia. Because even from different places in this country^, we write differently according to where we were brought up and how we talk. And generally^ one thing I can guarantee all of you, I write a whole hell of a lot better than I talk.

a. Holds one hand still by her neck, then begins explaining how different places write differently moving her hands up and down and then talks about her own writing keeping hands elbow level and still. Connects other cultures to her own as if bringing in connections so students can begin to understand writing as part of bigger picture AND/OR as if making connections to culture in general and writing in particular.

b. [It seems like the time to bring in the connection between culture and writing and language. I need to show them these are all related and that we are going to discuss them in this class.]

c. [The professor believes that talking about herself and cultures makes this class more meaningful for the students.]

74. Professor: [laugh], yeah, and in fact, you can probably read me and not think it was me because it is so good.

a. Brings in humor by talking about herself in ways that most people shy away from.

b. [It works well when I can insult myself in good humor. Students like that. It makes them feel comfortable, which I want in my classes.]

c. [The professor believes that all students can learn to write well even if they do not talk so well, but they must try anyway to communicate well.] 
75. Students: [laugh]

a. Some students laugh as if to say they got the humor and understand.

b. [I see, I can be my own person in here. AND/OR I see, writing is more than just words on the paper. AND/OR What is she talking about? I'm lost, I thought this was a research writing class.]

c. [Students tend to rely on their backgrounds and new topics are uptaken in various ways.]

76. Professor: No cussing nothing ^ [laugh]. The sentences are in the right order [laugh], uh, it's focused, organized, all of the things I'm not in person, [laugh].

a. Continues explaining the disconnect between her own writing and talking in person as if to show the importance for the class in their own thinking about writing AND/OR as if sharing with class that they can all learn to write well even if they don't talk well.

b. [I wonder if anyone gets it yet, the new students, that writing is a process connected to identity development and history and culture and that's why I talk about why I do stuff the way I do too.]

c. [The professor thinks it is okay and maybe even necessary to cuss in talk but not in writing.]

77. Professor: All right, SO, these are the reasons that I do this, I think that you've got something important to say. I think that you represent different groups, whether it be graduate students masters' level, graduate students at the doctoral level, different ethnic groups, different other kinds of groups, women, African American, Haitians, Jamaican, uh,

a. She discusses more about the connections between culture and writing and identity as if to say that everyone one in the class has all the pieces necessary for becoming a good writer AND/OR as if to take the spotlight off of her to the students AND/OR as if to share her mission of bringing in representation of disenfranchised groups even at this level.

b. [I think you are all important and I think we need to express out loud who and what we represent. I give you permission.]

c. [The professor is very knowledgeable about different groups and this is helpful when teaching writing to different groups.]

78. Student: [cough]

79. Professor: Other groups that in this country have been disenfranchised, and not [cough] allowed in.

a. She says the word disenfranchised in the context of writing as if to connect the two meanings for the students (OC: I wonder if anyone in the room has heard this word or knows its meaning?) AND/OR as if she assumes everyone knows this word and its meaning AND/OR as if she wants to share her mission with the students and invite them to join her on the mission and is doing this in the context of this class.

b. [I am ready to make my big plunge into racism. It feels good to say the word disenfranchised in this context when no one would expect it. Isn't this what it is all about? Accepting everyone 
c. [The professor knows a lot about disenfranchisement and believes most people in the room also know this word or should know it. She also thinks it connects to the writing process or doesn't care if it connects because it must be discussed so it can be addressed as a social justice matter.]

80. Professor: OK? So, those are some of the reasons that I'm doing this.

a. Concludes her reasons as if everyone remembers what reasons she is discussing even the ones who came in late (I wonder if they are lost?) $\mathrm{AND} / \mathrm{OR}$ as if she has already talked about the reasons in a previous class (I may have missed this in previous class. I know what she means because I have been her doctoral student for years. I wonder how long it took me to understand her mission like I do now as I listen to this class.)

b. [I hope they got the connection that writing and the reasons I conduct this class the way I do is all about inclusion and just practices.]

c. [The professor thinks it is good to conclude portions of the lecture periodically with a summary statement possibly to remind people where they are and where they are going.]

81. Professor: Now, my process is probably one most of you are not used to, and actually the nurses from summer from spring you didn't get a taste of this because we went on that giant escapade.

a. Hands mostly elbow level, but come out to reinforce a few words as she speaks. She refers back to the process she keeps talking about as if she realizes maybe some students are lost AND/OR as if she thinks maybe she should reiterate how most students are unfamiliar with her process $\mathrm{AND} / \mathrm{OR}$ as if she is bringing in her realization of the various ways people historically construct knowledge. Then she reminds nurses in the class who had her before of a project that got them waylaid from the process she is referring to here.

b. [I feel like reminding the class again that I know they are not used to my process and bringing in what happened in the past with some of the students in this class right now will reinforce context, history, and learning from what happens to us.]

c. [She almost got the nurses involved in her mission and wants to attempt involvement in this class too.]

82. Students: [laugh] [laugh]

a. Nurses laugh as if they remember.

b. [That was funny to think that the professor thought of our last class as an escapade. We are a community.]

c. [Other students wonder what is going on and whether they want to be a part or not. They wonder if class is more than just academics after all.]

83. Student: You had to bring that up, didn't you?

a. Student uses a bit of humor reminiscent of the professor's as if to connect to what the professor is saying here.

b. [I feel like I can be sarcastic in here.]

c. [The student wants to get on the good side of the professor as do most students and since there was a connection, she used the same humor.] 
84. Professor: [laugh]

a. Laughs as if to show she is enjoying the conversation and memories.

b. [I like students to feel like they can be sarcastic and reminisce with me and others in the class. I think it builds community.]

c. [The professor likes getting students involved and connected.]

85. Professor: I'm still living with it. We're still trying to do it.

a. Reminds students that there is much to get involved in in here and there is a history between her and her students.

b. [I'm trying to show you that I don't give up easily.]

c. [The professor feels good getting students to work with her. It also validates her mission.]

86. Student: It really $<\underline{\text { honed }}$ my lit search skills $>$.

a. The student spoke as if wanting others to know the importance of what they did in the class being referred to AND/OR as if to let others know to beware.

b. [I feel like I learned something at least and it was hard work.]

c. [The student feels very strongly about what happened but cannot elaborate here.]

87. Professor: It's ${ }^{\wedge}$ an important skill if you go on to get a doctorate. [laugh]

a. Says by framing face and smiling with two hands directly in front of her face.

b. [I believe in you so nothing we do is in vain.]

c. [The professor believes each student can get a doctorate if they do not give up. She did and she had everything against her.]

88. Professor: All right, so, I don't, I, one thing I learned, I'm never going to do that in that class again and it wasn't, it just wasn't the right place, but at any rate, because I probably will do such a stupid thing again, I know. [laugh]

a. Says all right and so as if to bring students back to the topic AND/OR continues on discussing the topic with the nurses anyway as if to historicize what happened in the context of this class referring to what was learned and how it will affect future actions.

b. [I probably should have learned to not venture out and go with the flow, but I know myself and I will do the same thing again if called for in context.]

c. [The professor thinks it is good to rethink things and learn from one's mistakes, because it is only human and students are human so they need to know she will rethink their mistakes too.]

89. Professor: All right, So, what I will do, is you'll send me drafts, and given my parameters timewise, I'll look at them and I'll give you feedback. I use track changes and I use comments. And I use short (.) to the point (.) sentences. And I have never learned Spanish, I don't know come from a polite culture. Those of you who haven't noticed that yet. I'm Italian, I'm the oldest, I have four brothers $^{\wedge}$, I was raised in a very male neighborhood^, in Cleveland, we're just like New Yorkers and Chicagoans, which means we're not very polite. [laugh] 
a. Holding hands together in front elbow level, periodically throwing out a hand slightly, going over the process of feedback integrating with her culture as if to provide reasons for how she gives feedback tied to her culture. Closes with a slight laugh looking quickly from one side to the other as if to get approval AND/OR as if to make everyone at ease.

b. [I'm trying to share with you how we do things in here, but don't expect me to be a typical professor that never lets you in on my own life. My cultural behaviors are learned from my own history and even though I am organized and work hard, I will still have cultural norms come out that you may not like or understand or be used to. So don't get offended is what I am trying to say. I hope you hear me.]

c. [The professor thinks she should tell students what to expect but to remind them that some things don't change, like culture.]

90. Professor: Nonetheless, Miguel is here to keep me in check, right? [laugh]

a. Points to Miguel as if to remind students they are included in this class

AND/OR to ease the comfort again between instructor/student power levels. And then laughs for a minute as if something is funny AND/OR as if it might be ironic that she must be kept in check.

b. [I am trying to show you how students become close to me over time and take over when I just don't get it. I give students permission.]

c. [The professor feels it is necessary to reduce power relations in various ways. One of them is to point out students who are examples of what she is talking about.]

91. Miguel: Yeah.

a. Answers in affirmative as if he knows what is expected AND/OR as if he wants to go along with the professor process or humor. (OC: Miguel has known the professor for a couple of years and understands where she is coming from.)

b. [I appreciate what she has done for me and how she gives me permission to think outside of the box.]

c. [Sometimes Miguel does not understand the humor and knows cultural humor is the last thing a person learns.]

92. Professor: Right? [laugh] [laugh]. Good luck to him^, huh^? [laugh]

a. Laughs as if tickled at the prospects of what she just said.

b. [I hope they got the humor in thinking Miguel can take over and tell me to do something. This is a good example of sharing the community of learners.]

c. [The professor thinks jokes about herself will reduce power relations between her and the students. She thinks of herself as a student too always learning.]

93. Professor: So, I just tell you this in way of explanation, because our cultures differ, and there are a lot of you in the room and there's only one of me,

a. Hand movements criss cross at differ and then open out and then hold up one finger to reinforce one of me as if students need reinforcement as they 
listen to her speak AND/OR as if students are still new to process and way of thinking DR has about writing and cultures.

b. [I want you to understand that we are all included here AND/OR I need you to know that I am cognizant of power level structures in university classroom settings.]

c. [The professor likes to explain things, especially about culture and power relations when they differ form the norm in society.]

94. Professor: Cuz he can't give you feedback on your writing. No offense, [laugh].

a. Points to Miguel and reminds students who cannot give feedback as if to reinforce her status AND/OR as if to reinforce Miguel's status.

b. [But I also want to remind you that I am leading the pack so to speak, I am the one teaching you how to write research papers even though some students have learned a lot.]

c. [The professor uses Miguel as an example of a student who has learned a lot but hasn't reached the final goal yet.]

95. Professor: Well, he can and he can't. I want to take that back, because Miguel has come a hell of a long way since I've met him. When ^did I meet ^you? Three years ago?

a. Takes back what she said as if rethinking how to say what she wants to say to the students AND/OR as if trying to take back an insult. Then turns directly full body to $\mathrm{C}$ and asks him when they met.

b. [Sometimes what I say sound like an insult so I need to clarify. And I will at the expense of sounding different. I just want you to know what I am thinking and how I believe in students.]

c. [The professor uses student examples as her style in making students feel comfortable, a part, or to understand her thinking.]

96. Miguel: 2006.

a. Miguel knew exactly when they met as if it coincided with a special event like when he began his degree.

b. [I remember the day we met for sure.]

c. [Miguel can remember the timing so well because it coincided with his arrival from South America to study in the states.]

97. Professor: Only two years ago? It seems like I've known you forever.

a. Starts turning back to face the class while states knowing him forever as if it were a bad or good thing AND/OR as if leading students to another thought.

b. [I want to share with you how will I come to know my students.]

c. [The professor likes to have a history with each one of her students.]

98. Professor: Anyhow, [laugh], all right, but Miguel has come a long way, because in the interview, the dissertation, the interview to get in to be a doc student, I didn't understand half of what he said. All I understood was a few references to Habermas, who else? Foucault, uh, [laugh].

a. Now she fills the class in on what she meant as if they need to understand the connection between language usage and culture and knowledge AND/OR as if students knew Habermas and Foucault (OC: Do AE/HRD 
masters students have knowedge of Habermas, Foucault?) AND/OR to try to take back an insult with an compliment on how smart $\mathrm{C}$ is since he knows about Habermas and Foucault enough to talk about them in conversation.

b. [I want you to connect intelligence with what people know not how they speak English.]

c. [The professor thinks it is good to know about critical thinkers since she professes to be one and asks students to do the same.]

99. Professor: Anyhow, so, well, you had only been here, what, a year at that point?

a. Faces Miguel again to ask him this question as if to reiterate how smart he is or the connection between language and culture and knowledge.

b. [I think he spoke pretty good English for only a year in the US.]

c. [The professor likes this student and admires what he has done.]

100. Miguel: Yeah.

101. Professor: And he did what I think is common for a lot of people that come here from another country to take-go to school and that is they sit silently in classes because they don't want to look stupid. And so you don't speak and you don't learn to speak it. He's forced to talk to me.

a. Hands held together elbow level, she talks about common behavior that she has seen for people who come here knowing another language better than English as if taking back the insult again to reiterate his intelligence AND/OR as if to share he cognizance of how students sit back and don't learn to speak English AND/OR as if to share how she relates to what people need to learn English better and also provides it for them, that is talking with them.

b. [I have seen lots of students in the same situation in a regular classroom, but with me I make people talk sooner or later by working closely with them on writing and other projects.]

c. [The professor has watched or studied about students from other countries who speak other languages and believes that they must be forced to speak to get better, like practicing writing to get better.]

102. Professor: So, anyhow, you've got the idea? I'm going to give you feedback within my time parameter. Now some of you may need to make appointments with me, may need to come in during my office hours. If you want help on your papers, though, don't come to me with nothing, come to me with a work plan, come to me with an outline, come to me with a draft of some sort, because I can't work from nothing^ and if you give me something concrete, I can give you something concrete back.

a. Still standing in front of class, hands mostly in front still elbow level and asking if they have the idea (OC: Idea of what? I wonder if students are staying with her?). She continues explaining the idea as if it is about talking to her so they can learn things AND/OR explaining the idea as if it is about the process of getting feedback in general AND/OR as if the idea is how she needs concrete evidence of their work before she can provide feedback. Hands up once to reinforce a word. 
b. [Now you get what I will do. I want you to know what you must do in this process. I think giving you a work load makes you more actively involved and a better writer in the long run.]

c. [The professor is busy and works hard, and she expects her students to work hard too. She will do anything to help you if you work hard too.]

103. Professor: Ok? Now, another thing that is going to be unusual is that I believe writing is process, and I believe it takes as long as it takes to get it right. Richard Smith here is embarking on a paper that I think has the potential to be an article. Not just a class paper, not just a conference paper. And it actually, probably $\wedge^{\wedge}$ has the potential if he was in another field maybe this one to be a dissertation topic.

a. Turns around and walks closer to laptop as if going to sit at it but turns back around as if changed mind and needed to clarify some more about writing process she believes in first AND/OR to discuss the process by bringing in something about another student's paper. Goes back closer to the middle of the arrangement and starts using hands to reinforce the different types of papers the student's paper could be.

b. [I am trying to tell you that I will never give up on you because each person is different and I can see ahead and lead you to writing different types of papers if you agree to work with me.]

c. [The professor wants to show students she knows a lot about types of papers and she keeps up on students' topics. She knows students by their topics.]

104. Professor: And we'll put this up and you'll see.

a. Points backwards to the screen as if she is segueing into the next part of the class by discussing the student's paper AND/OR as if to prepare students for how she will be changing something in the class soon.

b. [I want you to know up front that your work will be put up in front of others so get used to it right now.]

c. [The professor will also use students' writings as examples because she thinks this will bond the group and will allow students to build what they see from models into their own writing.]

105. Professor: All right, so I believe writing is a process and all things that are a process, you learn by practicing those skills. Now any of you athletic?

Basketball, soccer, soccer, like I know shit about soccer. Anyhow^, when you are little, most of us are older than death, and don't want to play soccer or basketball anymore, but when we are little, and we used to play these things, we practiced! And all athletes know that they have to practice these skills, and we drill it into ${ }^{\wedge}$ them. But we don't do the same things when it comes to writing, when it comes to reading. We talk about it other ways, we don't talk about these thing in terms of building skills.

a. She rolls her hands while further explaining the process of writing as it relates to practicing athletics as if all students have that background knowledge. Then she asks students about their life outside class as if to bring their experiences into the conversation AND/OR as if to show 
students she realizes they surely know more about soccer than she does AND/OR as if to provide a concrete base for understanding the writing process. AND/OR Then she contextualizes herself and the students in the same age category denoting none of them practice sports anymore as if assuming this must be true AND/OR as if making use of time to get to the point of practicing writing rather than continuing the vein of exploring similar experiences outside of class.

b. [In case you don't get it yet, I will use something we have probably all done at least once for background knowledge in explaining that writing is a process.]

c. [The professor feels good about connecting everyday life with academic processes.]

106. Professor: Now, I don't think for the most part, the books you have to read, this term, for these classes are going to be difficult reads. In years past, I've used the Handbook of Adult Education and it was a difficult read, because the last version took a postmodern stance. And postmodernists believe that the only good writing is dense circuitous writing, on purpose! OK.

a. Makes a quick connection to the books the students have to read for this class and past classes as if everyone knows that reading and writing practice are related AND/OR as if it is important what book is chosen for the class to read AND/OR as if there is a connection between type of reading and type of writing $\mathrm{AND} / \mathrm{OR}$ as if there is a connection that relates to good writing and purpose and the postmodern stance somehow (OC: Do these students know what postmodern is already? Is this important somehow? Is the professor stretching their thinking by going beyond what they might already know?) AND/OR as if it is important to know about others' purposes for writing.

b. [I want you to also know that writing is closely connected to reading. And I will throw in a new word in case you don't know it just to get your feet wet.]

c. [The professor believes in the connection between writing and reading and wants students to understand that connection also.]

107. Professor: So, and, but it takes practice sometimes, well it does, it takes practice to read academic literature, it takes practice to develop your reading skills, it's just practice, same with writing, so.

a. Reiterates what she means about connecting reading and writing practice with a few hand points and turns around to walk back to the front by the laptop as if she is done with that topic and intends to begin a new one.

b. [I think the practice makes perfect analogy is a good one.]

c. [The professor likes to reiterate something that is very important because sometimes students don't listen.]

108. Professor: I also think some of you should get into peer groups possibly, join forces, send drafts back and forth, though I'm not going to organize that because you're always better off getting outside () 
a. Fiddles with the laptop and talks about peer groups as if needing a filler while she prepares the next slide AND/OR as if wants to say this before she forgets and this is a good time while she works on the next slide.

b. [I am not forcing you, but peer groups work well for you but also for me in the long run workwise.]

c. [The professor likes to throw in ways for students to bond with the group and peer groups is one of her favorites. She has seen this work from her own days as a doctoral student desperate for feedback.]

109. Professor: All right, we're going to start off talking about the components of a paper. I'm gonna walk through what I think are the components of a position paper which is what most of you will be writing in here from the way that I saw your purposes. Then I'm gonna go through some samples, I'm going to walk you through Lisa's paper, I'm gonna show you Miguel's paper from two years ago in this class, and uh, and then I'm gonna go through your purpose statements that you sent me, okay?

a. One hand points backwards up in the air to the screen while she tells the students what she is going to do in the next of the class, what kind of paper most of them will be writing based on what she received this week by email, and then what she will do in the final section of the class.

b. [I want to start talking about something else now, the parts to the paper and this is how I am going to do it. I do this so you can get used to the process looking at others' papers before your own go up. I think this is a less offensive way of leading up to seeing your own papers.]

c. [The professor thinks it works well to talk about components or parts of papers.]

110. Professor: Now, the other thing. I generally don't give breaks because I don't remember to give em, and if you really need a break, you've got to consult with Miguel, he's gonna have to work it out and he's gonna have to tell me to stop. Now, otherwise, I'm just going to go (.) until I'm done, [laugh], and then we go home ${ }^{\wedge}$. If you need to get up and go to the bathroom, they're down the hall. Or you can take the elevator or the stairs all the way down^ and go on the first floor. I don't know where they are on the second floor. I don't think I've been on the second floor, um.

a. Says now with emphasis as if to initiate a new topic or the new section of the class. She goes back to some housekeeping as if she forgot to say this in the beginning AND/OR as if she remembers the students who came in late never heard about housekeeping policies in the class, hands still at elbow level. Then giggles as if it is humorous that $\mathrm{C}$ would stop her after hearing from students about needing breaks AND/OR as if apologizing for not stopping for breaks during the class. And then goes on in some detail about where restrooms are, pointing in different directions as if she wants students to know she cares to take the time to share these things for their future comfort, if needed. 
b. [I want to confirm in case you missed it that we work straight through and so you know I care about your comfort AND/OR so you will not ever ask in the future, I will tell you again where the restrooms are located.]

c. [The professor feels like she should remind students several times about taking no breaks formally since students sometimes don't listen the first time.]

111. Students: (undecipherable)

a. Students buzz quietly as if something needs to be commented on.

b. [I can't believe this that we don't have breaks! AND/OR I like that so we can go home early!]

c. [Students at this level feel like being asked to determine their own break is appropriate because, after all, this is graduate school.]

112. Professor: Yeah, you can go out that door and down this hall, yeah, you're really concerned about the bathrooms?

a. She brings in some humor as if to ease students' fears about not being comfortable during class AND/OR as if to tell other students they are welcome to ask questions in this class. Looks directly at student who spoke and takes a sip out of her water bottle.

b. [I think humor eases the pain of students not getting what they think they need or want, like taking breaks AND/OR It feels right being humorous since most professors are so dry and aloof.]

c. [The professor believes that humor can help manage squeamish or unpalatable information.]

113. Students: [laugh]

a. Students laugh as if something is funny.

b. [It feels out of character for a professor to talk so down to earth about going to the bathroom AND/OR It makes me uneasy to talk about the bathroom in a university setting.]

c. [Students at all levels enjoy being caught off guard in the classroom.]

114. Student: I'm a nurse. You're worried about if we don't find them.

a. Student shares her perspective on why DR gives so much attention to the logistics of the restrooms.

b. [I think I should bring back an academic perspective to this class in case we get off track here AND/OR I feel the need to show others that I am a nurse AND/OR I get the idea that it is appropriate in this class to turn the tables on the professor if I am doing it in a professional manner.]

c. [Student thinks being a nurse is an important job.]

115. Professor: So, <papers have parts>. Papers have parts, and the papers that you're going to do for me will have three parts. It will have an introduction^, a discussion section, and implications. The introduction ${ }^{\wedge}$ section contains some parts $^{\wedge}$.

a. Sits down for a split second and then stands up again, using two hands to emphasize that papers have parts. Then points to the screen with one hand from high to middle to low on the screen as if to show students where to 
look for the information she is sharing, continuously emphasizing the word parts.

b. [I must get back to academics here so let me show you the importance of the parts of the paper by emphasizing them carefully.]

c. [The professor remembers from past semesters that this was something that gave students trouble, so she divides the paper into parts to help them conceptualize what good writing is in this case.]

116. Professor: Are you looking for a chair? We've got two over here. | And there are no other rooms in this place.

a. Another student walks in late and she stops talking about parts as if to make the new student feel comfortable immediately, waits a minute as if thinking what students are thinking about the crowded room and responds that there are no other rooms as if that is what she thinks they are wishing right now AND/OR as if she is concerned with their comfort level.

b. [It is always right to recognize someone who comes in the room so they know right off that I see them, they are included, and that I understand the cramped situation and we may not be comfortable, but I care.]

c. [The professor thinks it is good to acknowledge students and that it is okay to bounce between academic and social issues. She believes students can handle the disruption at this level.]

117. Professor: But she better be on time next time. [laugh], or she'll be sitting in the hall, [laugh], [laugh].

a. Comments to the late student about tardiness and what may happen next time as if trying to get a point across about being late in a humorous way. Sits back down in front of the laptop.

b. [But just in case, I want to show you that I would appreciate it if you would come early AND/OR I want to show you that if you would come earlier you would not be so cramped because you would have a wider choice of chairs to choose from but I can't run your life so I'll just throw in some humor so you might get my point that way.]

c. [The professor likes to use student examples to make a point, even in social areas.]

118. Professor: All right, now (.) a problem ^ ${ }^{\wedge}$ statement. I sent you that article, or the chapter in the book from Merriam and Simpson, where they walk through a problem statement. Now, you're going to hear terms kind of bantied about, you might have already in your travels as students. Some people will talk about a thesis, ok? When you took uh, English, they probably talked about a thesis, um, what other ways do they talk about it? Well, that's good enough, we know that one.

a. Fixes slide and stands back up, moving hands out and in at elbow level explaining what she sent and why as if trying to make connections to experiences they may have had before as a student with this new term AND/OR stops at the English class example stating that we know that one as if she knows all students have heard about the word thesis in English class before. 
b. [I am in my comfort zone now because I can talk about problem statements in my sleep. I like this part where I can show the students that they really do know about problem statements even though it is a new term for them AND/OR I don't have to emphasize words so much because I know this part so well and believe it so deeply AND/OR This part feels right because I can start bringing in students' outside experiences to get them into the topic of this class.]

c. [The professor believes being a student is like traveling because she feels like she is also still learning.]

119. Professor: But the thesis^ and the problem statement is the beginning of a paper that grounds your paper in something. Ok? Some one of you wants to do something on obesity, ok? And, uh, I had a student last year that did something on obesity, I don't exactly remember how it went but it was on the educational ${ }^{\wedge}$ value of obesity programs that we have.

a. Contextualizes the new term problem statement with known term thesis to someone's paper in this class and past class as if it is important to connect past with present and future learning to make sense of a new project the students are embarking on in this class AND/OR as if to bring in a student in this class to raise community feeling that we are all in this together.

b. [This feels really great when I can bring in something a student just sent me and a paper a student did last term to make this real for the students in the room to get them into this right away AND/OR I really like to pull in past and present work from students because it makes what I am trying to show the students applicable to their situation and I think it makes them feel part of the class, my grander mission.]

c. [The professor remembers students' past papers and believes it is good to be able to talk about them with current students to show them all about connections.] 
Pilot Study Transcripts

Stage 5 B, Part 1-Reconstructive Data Analysis of Representative Classroom Observations (Pragmatic Horizon Analysis: Boundary-Making)

(Contextualization Cues, Interactional Units, Phases)

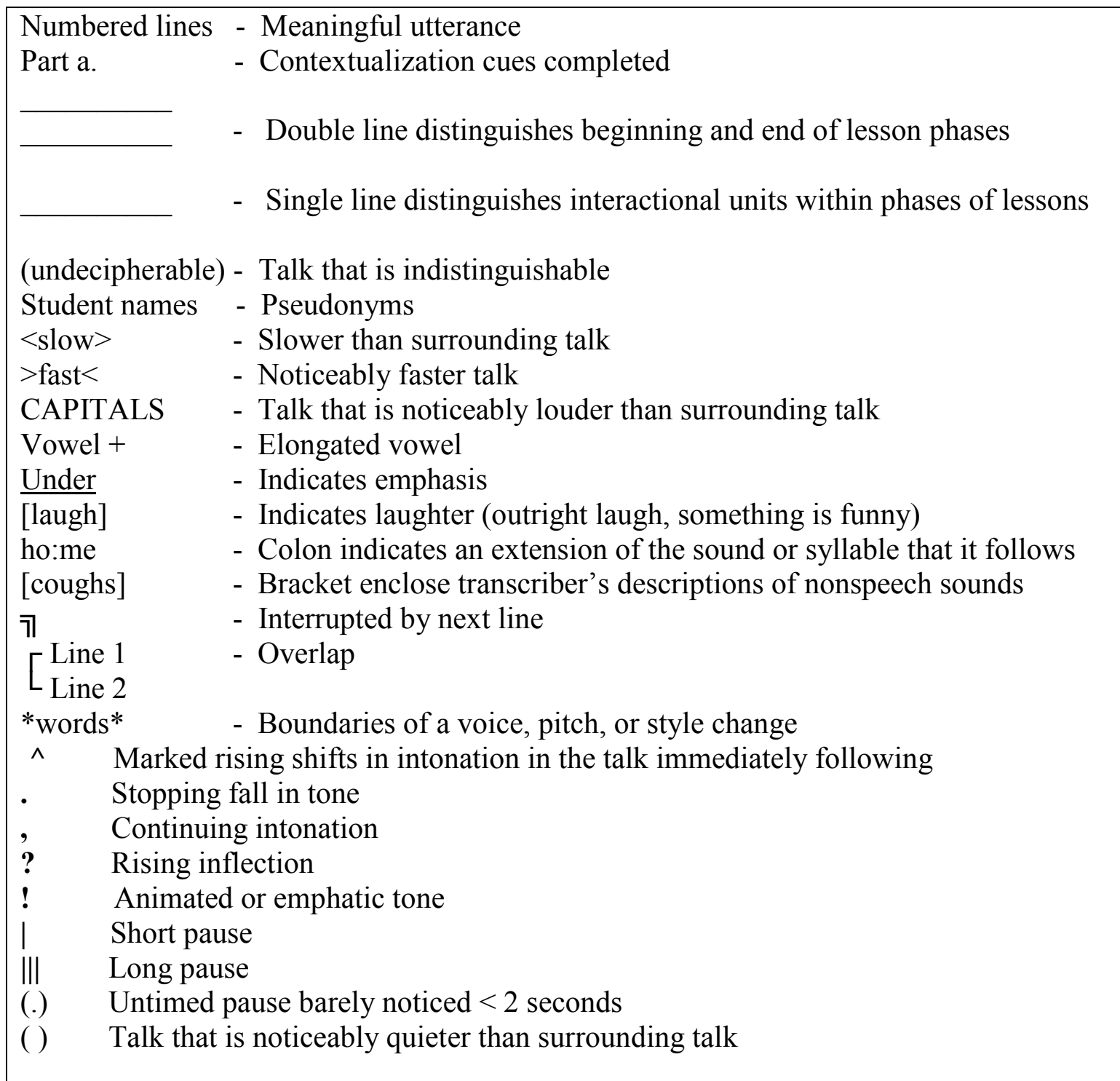




\section{PHASE 1, BEFORE CLASS OFFICIALLY BEGINS}

\section{INTERACTIONAL UNIT 1}

1. Students: [laugh]

a. Laughter [not signal to claim floor]

2. Students: (undecipherable)

\section{INTERACTIONAL UNIT 2}

3. Professor: Uhh. Who did? You know I left ^ my notebook, Where's Jim.

a. Uhh [indicates the beginning of a message unit and claims to speaking rights]; rising tone [signals question]; flat intonation pattern [maintains claim for speaking rights]

4. Students: [laugh]

a. Laughter [signals response to previous message unit but not claim to floor]

5. Professor: ^ Didn't he just, Who are you.

a. Shift in tone [indicates new message unit]; flat intonation of questions [maintains claim to floor]

6. Students: [laugh]

a. Laughter [no claim to floor]

7. Professor: Ohh ^,ok forget it. I'm not here yet. It was me (.).

a. Shift in tone and elongated ohh [signals new message unit and claim to speaking rights]; untimed pause [signals claim to maintain floor]

8. Students: [laugh]

a. Laughter [not a signal to claim the floor]

\section{INTERACTIONAL UNIT 3}

9. Professor: This is the hardest ${ }^{\wedge}$ thing I do all year, all right, Alex, Doris? Were you here last time?

a. Stress on This [signals beginning of new message unit]; rising intonation [signals a question]; continuous intonation [maintains claim for the floor and next turn-at-talk]

10. Doris: Yes,

a. Different speaker [signals new message unit but not claim for floor]

11. Professor: Lola Lesser? Does anybody know if she was here last, two weeks ago?

a. Stress on Lesser [indicates new message unit and maintains claim to floor; rising intonation signals question]

12. Professor: All right. Jose Ruiz? 
a. Shift in tone [signals beginning of message unit and claim to speaking rights]; rise in intonation [signals question]

13. Professor: All right, uh, Marissa Sanders?

a. Shift in tone [signals beginning of message unit]; rise in intonation [signals question]

14. Professor: Johnson I know ^ was, ()

a. Stress on Johnson [signals new message unit]; continuing intonation [maintains claim for next turn-at-talk]

15. Professor: $\underline{\text { How }}$ about Natalia? () [laugh]

a. Stress on Carmen [signals new message unit]; rise in intonation [indicates question]; laughter [does not signal claim to floor]

16. Professor: All right. Niara?

a. Stress on All [signals new message unit]; rise in intonation [indicates a question]

17. Niara: Yes

a. Different speaker [signals new message unit but not claim to floor]

18. Professor: Were you here last?

19. Niara: Yes

a. Different speaker and stress on were [indicates new message unit]; rising intonation [indicates a question]

a. Different speaker [indicates new message unit but not claim to floor]

20. Student: [cough]

\section{INTERACTIONAL UNIT 4}

21. Professor: Christy, you were too, Acacia was not because they lied to her, Alicia was not $^{\wedge}$ Ginny was ${ }^{\wedge}$ Ramon ${ }^{\wedge}$ was ${ }^{\wedge}$, you're Ramon right, Emily?

a. Different speaker and stress on Christy [signals a new message unit]; flat intonation [maintains claim for turn-at-talk]; rising intonation [indicates questions]

22. Emily: I was here,

a. Different speaker [indicates new message unit but not claim to floor]

23. Professor: ^ You were here then, Are you here now?

a. Different speaker and shift in tone [indicates new message unit]; rising intonation [signals question]

24. Professor: *^I think you were here when I called your ${ }^{\wedge}$ name!*

a. Pitch and style change [indicates new message unit]

25. Students: (undecipherable)

a. Undecipherable conversation [suggests that students interpret floor as open but no claim to floor]

26. Professor: $O k$, ok $\mid M i-a h$ ?

a. Different speaker and stress on $o k$ [indicates new message unit and claim for speaker's rights]; rising intonation [signals question]

27. Mia: Me-ah.

a. Different speaker and stress on $\mathrm{Me}$ - [indicates new message unit] 
28. DR Me-ah,

a. Different speaker and stress on $\mathrm{Me}$ - [indicates new message unit]; pause [indicates claim for floor and next turn-at-talk]

29. Mia: Yeah.

a. Different speaker [indicates new message unit]; fall in tone [indicates no claim for floor]

30. Professor: *I knew who you were ${ }^{\wedge *}[$ laugh]!

a. Different speaker and pitch and style change [indicates new message unit and claim for speaking rights]

31. Mia: Yeah, I guess ^ that's a plus.

a. Different speaker and stress on yeah [signals new message unit; stopping fall in tone [indicates no claim to next turn-at-talk]

32. Professor: *That's a plus! [laugh], I didn't have to ask ${ }^{\wedge}$ you* . $\mathrm{Ok}$, ^ you're Orlando, ok, you were here last time too?

a. Different speaker and stress on that's [signals a new message unit and claim to speaking rights]; shift in tone, pitch, and style [indicates speaker is maintaining floor]; rising intonation [indicates question]

33. Professor: $\underline{O k}$, I know June was, all right. NOW, is there somebody in the room right now that was here two weeks ago? and here tonight? that's not on my list ${ }^{\wedge}$ ? ||

a. Stress on Ok [signals a new message unit]; louder tone of NOW and continuous intonation with long pause [maintains the floor and indicates there is more to come]; rising intonation [indicates questions]

PHASE 2, INTRODUCTION TO HER PROCESS

\section{INTERACTIONAL UNIT 5}

34. Professor: Ok, ^ how many of you? received the things that I sent you today. | All right, uh, you will ^ find that I-ah ^frequently will send you things the day of, actually, there is no rhyme or reason to my sending things.

a. Shift in tone [indicates new message unit]; continuous intonation [maintains floor]

35. Students: [laugh]

a. Different speaker with laughter [does not claim floor but interprets pause as an open floor]

36. Professor: To tell you the truth,

a. Different speaker and continuous intonation [indicates new message unit and claim to next turn at talk]

37. Professor: If one of you asks me a question? now the answer you ^ get from me may aggravate the ${ }^{\wedge}$ shit out of me, and you ${ }^{\wedge}$ notice that in my response,

a. Continuous rising and falling intonation [indicates overlapping message unit and maintains claim to floor] 
38. Professor: But *then ${ }^{\wedge}$ but then ${ }^{\wedge}$ I will decide it's not that dumb of a question [laugh] after all [laugh], it was my husband ${ }^{\wedge}$ that was really aggravating me, * and I will try ^ to respond in a fuller message to the class, because I think that $<i t$ is something in fact that you don't understand as a group $>$, ok^?

a. Voice, pitch, and style change [indicates a new message unit and claim to maintain floor]; emphasis on try and class followed by slower than surrounding talk [indicates a change in thinking with new ideas presented to group as a whole]; rising intonation [indicates question and claim to next turn-at-talk]

\section{INTERACTIONAL UNIT 6}

39. Professor: <So you've got a message $>$ about ^ problem statements from me, right? And I took, I think I took two of my own papers?

a. Shift in speed of talk [indicates new message unit]; emphasis on problem and rising intonation [maintains floor]; pause and rising intonation [indicates there is more coming with a question]

40. Students: (undecipherable)

a. Different speaker undecipherable words [indicates new message unit interpreted as open floor but not claim to floor]

41. Professor: What?

a. Different speaker and rising tone [indicates new message unit with question]

42. Professor: Yeah, and uh, I showed you what a problem statement with a purpose looked like from, I know from my CRT one and I don't remember what the other one was,

a. Stress on yeah [indicates new message unit]; continuous flat intonation [indicates more to come and maintains floor and claims next turn-at-talk]

43. Student: [cough]

44. Professor: But I think it was two of them,

a. Slight stress on but [maintains floor]; flat intonation and pause [indicates more to come and maintains claim to next turn-at-talk]

45. Professor: At any rate, uh $\mid$ all right, so, somebody asked me something and that's what I did.

a. Use of at any rate and all right [indicates overlap from last message unit and maintains floor]; stress on that and falling tone [signals end of thought]

\section{INTERACTIONAL UNIT 7}

46. Professor: Now, ^ today, I was preparing for class, a little later than I would have liked because I distracted myself and did something else, and uh, and ${ }^{\wedge}$ anyhow, so I sent these things late, >Now they're in your email ^ so you can go back and get them if you don't have them but it occurred to me that it might be 
useful for somebody to have ${ }^{\wedge}$ these handouts so you can print them out and write on them $<$, ok?

a. Begin with now and stress on today [signals beginning of new message unit and claim to speaking rights]; faster yet mostly flat intonation [indicates maintaining floor and more to come]; rising intonation at end [indicates question and more to come]

47. Professor: So, that's why I sent them, you know, it's not required, it's just I was trying to be helpful. |

a. Slight stress on so [indicates new message unit]; pauses and continuous intonation [maintaining floor]

48. Professor: All right, um |||

a. Use of all right and um [indicates new message unit]

\section{PHASE 3, REASONS FOR HER PROCESS}

\section{INTERACTIONAL UNIT 8}

49. Professor: Now, ^ I'm going to talk to you tonight about writing a research paper and, umm, ^I going to spend ${ }^{\wedge}$ probably what some of you will consider an inordinate amount of time, trying to teach you how to write research papers. I do this for a variety of reasons. One, $<$ I believe very strongly in the notion $>$ that students and professionals have a voice, and the ^ only reason we don't know what you know is because you don't know <how to access the world of publication>.

a. Stress on now [indicates a new message unit]; stressing words and slowing down to enunciate certain phrases [indicates that this material is important and is a new way of thinking for the whole group to consider and maintains floor]

50. Professor: $O k$ ?

a. Rising intonation [suggests new message unit and question]

51. Professor: > What do you think, Johnny??

a. Faster talk [signals new message unit that breaks from previous idea]

52. Johnny: You are perfectly, perfectly right.

a. Different speaker [signals new message unit]

53. Professor: *[laugh] I like Johnny. [laugh]*

a. Different speaker and change in voice, pitch, style [indicates new message unit]; laughter [maintains claim to floor]

54. Professor: Anyhow, and uh, I think this, because when ${ }^{\wedge}$ I was a grad student, uh, in the 90s, >look at my vitae, I'm very young $<$, um, all four of my degrees come from the ^1990s. Anyhow, um, when^ I was a grad student, I knew when I went to get my doctorate that I wanted to be a professor, $>$ that was what I was going to do, $<$ um, $>$ I figured it out $<$ but so, $<$ I didn't know what you needed to do as far $a^{\wedge}{ }^{\wedge}$ career path to become a professor $>$, so I started asking faculty^. You know, 
what does a student need to $\underline{\text { do }}$ as a student to become a faculty member later? And $u$ h, they said $>$ you need to publish $<$.

a. Stress on anyhow [indicates new message unit and claim to speaking rights]; faster talk [indicates an aside]; slower talk and stressed words [maintains claim to floor with important maybe new material that needs to be thought about]

55. Professor: OK:ay, [laugh], how do you do that^? Well, no faculty members ${ }^{\wedge}$ really tell you how to do that except for a few rare ones, like me^, and um, because most faculty assume that you go on and get a doctorate and $<$ somehow this magic of writing this dissertation $>$ which is really unlike ${ }^{\wedge}$ any other kinds of writing, will magically make you understand the publication process. And it doesn't. It doesn't prepare you at ${ }^{\wedge}$ all, uh, others ${ }^{\wedge}$ think that students have nothing worthwhile to ${ }^{\wedge} \underline{\text { say }}$, therefore, they shouldn't be ${ }^{\wedge}$ encouraged $\mid$

a. Elongated syllable [indicates new message unit and claim to speaking rights]; laughter [maintains floor]; slower talk [indicates important material to think about]; stressed words and shifts in tone [maintains floor by emphasizing new way of thinking for whole group to think about]

56. Professor: [laugh]

a. Laughter [indicates new message unit and possible way to maintain floor or possible way to let what she said to sink in to whole group as new material]

\section{INTERACTIONAL UNIT 9}

57. Professor: ^It's getting more and more crowded, there's two seats up here. There's one ^here, this one's mine ||| Nobody wants to sit by me, Jimmy.

a. Shift in tone [signals new message unit]

58. Johnny: [laugh]

a. Different speaker and laughter [interprets an opening in floor]

59. Student: You're so ()

a. Different speaker [signals new message unit but no claim to floor]

60. Professor: ||| All right (.) You know what? We ${ }^{\wedge}$ are expecting some more. So, you're going to have to sit ${ }^{\wedge}$ there, I hope you're skinny.

a. New speaker [signals new message unit]; rising intonation [indicates question]; shifts in tone [indicate speaker is maintaining floor]

61. Student (undecipherable) [laugh]

a. Undecipherable talk and laugher [indicates interpret opening in floor and new message unit]

62. Professor: A:nd $\| \mid$

a. Elongated syllable [indicates speaker claiming floor]

63. Professor: ^Actually, Migule ${ }^{\wedge}$ was going to arrange it the other way, and I thought this would be a better way. $I^{\wedge}$ think you actually have a little bit more room sort of. If there's a ^fire drill though

a. Shift in tone [signals new message unit]; continuous intonation with few stressed words [signals more to come but maintains floor] 
64. Students: [laugh]

a. Laughter [not claim to floor]

65. Professor: I think ${ }^{\wedge}$ this row needs to go out this door so that that group can go out that door. Oh, yeah, > the restrooms are down this door $<$. I

a. Different speaker [signals new message unit]; stress of this row [indicates claim to speaking rights; repetitive stress of words and slow flat intonation [maintains claim to floor and next turn-at-talk]

\section{INTERACTIONAL UNIT 10}

66. Professor: () > Where was I in my sad tale? $<$

a. Faster talk [indicates new message unit and maintains claim to floor]; rising intonation [signals question and more to come]

67. Professor: Oh! The ^ rest of you don't ${ }^{\wedge}$ know anything worth getting published, therefore, ^nobody should teach you how. Now ${ }^{\wedge}$ that's one reason. Another ${ }^{\wedge}$ reason is, that if you come to graduate school, the assumption is you have a

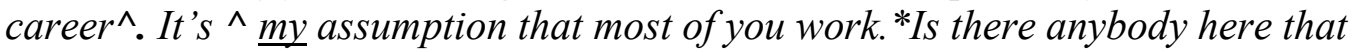
doesn't have a regular job?*

a. Stress on $o h$ [signals a new message unit and maintains claim to speaking rights]; continuous flow of intonation and stress of words [indicates more to come]; voice, pitch, style change and rising intonation at end [indicates question for whole group to think about]

68. Professor: $\underline{M e}$, [laugh].

a. Stress on $m e$ and laughter [maintains claim to floor]

69. Student: (undecipherable)

a. Undecipherable [signals new message unit interpret opening in floor]

70. Professor: All right, but you're in the minority [cough] I'm ^ not picking on you, but you are, and especially true in the fields that I represent, Adult Education and Human ${ }^{\wedge}$ Resource Development,

a. Stress on all right [signals new message unit]; pause [indicates more to come and claim speaking rights for next turn-at-talk]

71. Professor: So, if you have a $\wedge$ job, how many of you? never write a thing at work?

a. Stress on so [signals a new message unit and maintains floor]; rising intonation [indicates questions]

72. Professor: Yeah. Most of you write at work. Now, <how people perceive you at work $>$ has a lot to do with how you ${ }^{\wedge}$ communicate. $>$ Not ${ }^{\wedge}$ the nature of your ideas $<, \mid>$ how you look, how you sound, whether they can pick up an accent in your writing $<$,

a. Falling tone of yeah [signals new message unit and more to come]; slower talk [indicates important material to think about]; faster talk [indicates an aside or additional material that add something to the important material or maintaining floor]; pause at end [signals claim for next turn-at-talk]

73. Professor: $>$ I learned this in Columbus, Ohio, so it has nothing to do with a Spanish accent that I'm talking about $<$. It has ^everything to do with a Black accent, or a Cleveland accent, ok, or West Virginia. Because, even from different 
places in this ${ }^{\wedge}$ country, we write differently >according to where we were brought up and how we talk. $<$ And generally^ one thing I can guarantee all of you, $<$ I write a whole $h$ - of a lot better than I talk. $>$

a. Stress on I learned [signals new message unit]; slower talk [indicates new material to think about for the whole group and to maintain floor]; faster talk [indicates asides to add to new material]

74. Professor: [laugh], yeah, and in fact, you can probably read me and not think it was me because it is so good.

a. Stress and pause on yeah [signals new message unit and more to come]

75. Students: [laugh]

a. Laughter [does not make claim for floor]

76. Professor: ${ }^{*} N o{ }^{\wedge}$ cussing nothing* [laugh]. The sentences are in the right ${ }^{\wedge}$ order [laugh], uh, it's ^focused, ^organized, all of the things I'm not in person, [laugh].

a. Change in voice, pitch, style [signals new message unit]; laughter [maintains claim to floor]; repetitive stress on words [maintains floor and next turn-at-talk]

77. Professor: ^All right, so, these are the reasons that I do this, I think that you've got something important to ${ }^{\wedge}$ say. I think that you represent different groups, whether it be graduate students masters' level, graduate students at the doctoral level, different ethnic groups, different other kinds of groups, women, African American, Haitians, Jamaican, wh,

a. Shift in tone with all right and stress on so [signals new message unit and claims floor]; continuing intonation and stressing of words periodically [indicates more to come and maintaining floor]

78. Student: [cough]

79. Professor: Other groups that in ${ }^{\wedge}$ this country have been disenfranchised, and not [s cough] allowed in.

a. Stress on other groups [signals new message unit and maintains floor]; stopping fall in tone [indicates end of thought]

\section{PHASE 4, EXPLANATION OF HER PROCESS}

\section{INTERACTIONAL UNIT 11}

80. Professor: $\underline{O k}$ ? $\underline{\text { So }}$, those are some of the reasons that I'm doing this.

a. Stress on $o k$ and so [signal new message unit and maintaining floor]

81. Professor: Now, my process is probably one most of you are not used to, and $\wedge$ actually the nurses from summer, or from spring you didn't get a taste of this because we went on that giant escapade.

a. Stress on now and pause [indicates new message unit and more to come] 82. Students: [laugh] [laugh] 
a. Different speakers with laughter [indicate new message unit interpreted as opening in floor]

83. Student: You had to bring that up, didn't you?

a. Different speaker and stress of had [indicates new message unit and claim speaker's rights]

84. Professor: [laugh]

a. Different speaker and laughter [indicates new message unit but not claim for floor]

85. Professor: I'm still living with it, we're still trying to do it.

a. Stress on I'm and continuing intonation [indicates new message unit and claim of speaker's rights]

86. Student: It really $<\underline{\text { honed }}$ my lit search skills $>$.

a. Different speaker [signals new message unit]; slower talk and stress on honed [indicates claim of speaker's rights]

87. Professor: It's ${ }^{\wedge}$ an important skill if you go on to get a doctorate. [laugh]

a. Different speaker and stress of important [signals new message unit]; laughter [maintains floor for next turn-at-talk]

88. Professor: ^All right, so, I don't, I, one thing I learned, >I'm never going to do that in that class again< and it wasn't, it just wasn't the right place, but at any rate, > because I probably will do such a stupid thing again, I know $<$. laugh]

a. Shift in tone [signals new message unit]; faster talk [indicates asides to other material more important]; laughter [maintains claim to floor]

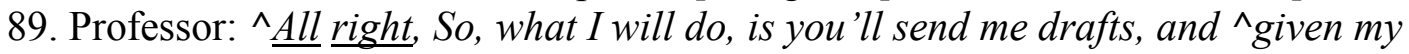
parameters ^timewise, I'll look at them, and I'll give you feedback. I use track^ changes and I use comments. And I use short (.) to the point (.) sentences. And I have never learned Spanish, I don't ^ come from a polite culture. Those of you who haven't noticed that yet. I'm ^Italian, I'm the oldest, I have four brothers ${ }^{\wedge}, I$ was raised in a very male neighborhood ${ }^{\wedge}$, in Cleveland, > we're just like New Yorkers and Chicagoans $<$, which means we're not very polite. [laugh]

a. Shift in tone and stress on all right [signals new message unit]; rising and falling intonation and untimed pauses [indicate more to come and maintains floor]

\section{INTERACTIONAL UNIT 12}

90. Professor: <Nonetheless, Miguel is here,> to keep me in check, right? [laugh]

a. Slower talk [signals new message unit]; rising intonation and laughter [indicates question to maintain floor and suggest rights to next turn-attalk]

91. Miguel: Yeah.

a. Different speaker [indicates new message unit but no claim to floor]

92. Professor: ^Right? [laugh]. [laugh]. *Good luck to him^, huh^^?*[laugh]

a. Different speaker [indicates new message unit]; laughter and change in voice, pitch, style maintains floor] 
93. Professor: So, I just tell you this in way of explanation, because our ${ }^{\wedge}$ cultures differ, and there are a lot of you in the ^ room and there's only one of me,

a. Stress on so [signals new message unit]; stress on differ [indicates important material]; continuing intonation [indicates more to come and maintains floor]

94. Professor: ^Cuz, he can't give you feedback on your writing. No offense, [laugh].

a. Shift in tone [signals new message unit]; laughter [maintains floor]

95. Professor: Well, he can and he can't. >I want to take that back, because Carlos has come a hell of a long way since I've met him. $<$ When ^did I meet ^you? Three years ago?

a. Stress on well [signals new message unit]; faster talk [indicates extra material; rising intonation [indicates questions]

96. Miguel: 2006.

a. Different speaker [indicates new message unit but not claim to floor]

97. Professor: Only two years ago? < It seems like I've known you forever.>

a. Different speaker [signals new message unit]; rising intonation and stress of words [indicates question and maintaining floor]

98. Professor: ^Anyhow, [laugh], all right, but ^ Miguel has come a long way, because in the interview, the dissertation, the interview to get in to be ${ }^{\wedge} \wedge \underline{d o c}$ student, I didn't understand ${ }^{\wedge}$ half of what he said. All I understood was a few references to ${ }^{\wedge}$ Habermas, who else? Foucault, uh, [laugh].

a. Shift in tone and use of laughter and stress of words [signals new message unit]; repetitive stress on words and laughter [maintains floor]

99. Professor: ^Anyhow, so, well, you had only been here, what, a year at that point?

a. Stress on anyhow and shift in tone [signals new message unit and maintains floor]

100. Miguel: Yeah.

a. Different speaker [signals new message unit but not claim for floor]

101. Professor: And ${ }^{\wedge} \underline{\text { he }}$ did what I think is <common for a lot of people that come here from another country to take-go to school $>\wedge$ and that is ${ }^{\wedge}$ they sit silently in classes because they don't want to look stupid. $>$ And so you don't speak and you don't learn to speak it. $<<H e$ 's forced to talk to me $>$.

a. Stress on he did and shift in tone [signals new message unit]; slower talk signals important material to think about]; faster talk [indicates aside material that adds to topic]

\section{INTERACTIONAL UNIT 13}

102. Professor: ^So, anyhow. You've got the idea? I'm going to give you $\wedge$ feedback within my time parameter. Now some of you may need to make appointments with ${ }^{\wedge}$ me, may need to come in during my office hours. <If you want help on your papers, though, $>$ don't come to me with nothing. Come to me with a work plan, come to me with an outline, come to me with a draft of some sort, because I can't work from nothing^ and if you give me something concrete, I can give you something concrete back. 
a. Shift in tone and falling tone at end of so anyhow [signals new message unit]; continuing flat intonation [indicates more to come and maintains floor]

103. Professor: ^ Ok? Now, ^ another thing that is going to be unusual, is that $\wedge$ I believe writing is process, and $\wedge^{\wedge} I$ believe it takes as long as it takes to get it right. Richard Smith here is embarking on a paper that I think has the potential to be an article. Not just a class paper, not just a conference paper. And it actually, probably ${ }^{\wedge}$ has the potential if he was in another field maybe this one to be a dissertation topic.

a. Rising intonation and shift in tone [signals new message unit]; continuing flat intonation and stress on periodic words [indicates more to come and maintains floor]

104. Professor: | And we'll put this up and and you'll see.

a. Pause [signals new message unit]; falling tone [indicates end of thought]

\section{INTERACTIONAL UNIT 14}

105. Professor: ^All right, so ${ }^{\wedge} \underline{\text { I believe writing is a process }}$ and $<$ all things that are a process, you learn by practicing those skills $>$. Now (.) any of you athletic? Basketball, soccer, soccer, like I know shit about soccer. Uh, ^ $\underline{\text { anyhow, }}$ when you are little, most of us are older than ^death, and don't want to play soccer or basketball anymore, but * when we are little, and we used to play these things*, we practiced! And all athletes know that they have to practice these skills, and we drill it into ${ }^{\wedge}$ them. But we don't do the same things when it comes to writing, when it comes to reading. We talk about it in other ways, we don't talk about these thing in terms of building skills.

a. Shift in tone and stress of I believe [signals new message unit]; slower talk [indicates new important material to think about]; continuing intonation [indicates more to come]; change in pitch, voice, style [indicates a new thought or way of thinking coming up]; falling tone at end [indicates claim for next turn-at-talk]

106. Professor: ${ }^{\wedge}$ Now, ${ }^{\wedge}{ }^{*} I$ don't think* for the most part, the books you have to read, this term, for these classes are going to be difficult reads. In years past, I've used the ${ }^{\wedge}$ Handbook of Adult Education and it was a difficult read, because the last version took a postmodern stance. And postmodern:ists believe that the only good writing is <dense circuitous writing >, on purpose! OK.

a. Shift in tone and stress on now [signals new message unit and claim to maintain speaking rights]; change in pitch [indicates new thought coming up]; continuous intonation [indicates more to come and maintain floor]; slower talk [indicates important material or new material to think about]

107. Professor: So, and, but it takes practice sometimes, $>$ well it does $<,<i t$ takes practice to read academic literature, it takes practice to develop your reading skills $>$, it's just practice, same with writing, so. 
a. Stress on so [signals new message unit]; stress on practice and slower talk [indicates important or new material]; faster talk [indicates aside material]; stress on $s o$ at end [indicates end of thought]

108. Professor: $I^{\wedge}$ also think some of you should get into *peer groups possibly*, join forces, send drafts back and forth, though I'm not going to organize that (.) because you're ^always better off getting outside ()

a. Shift in tone [signals new message unit]; change in voice, pitch, style [indicates new idea or thought for group to think about]; continuous intonation [maintains floor]

\section{PHASE 5, COMPONENTS OF PAPER}

\section{INTERACTIONAL UNIT 15}

109. Professor: ^All right, *we're going to start off* talking about the components of a paper. I'm gonna walk ^through what I think are the components of a ${ }^{\wedge}$ position paper which is what most of you will be writing in here from the way that I saw your purposes. And then I'm gonna go through some samples, I'm going to walk you through ^Leana's paper, I'm gonna show you ${ }^{\wedge}$ Carlos's paper from two years ago in this class, and uh, and then I'm gonna go through your purpose statements that you sent me, okay?

a. Shift in tone and change in voice, pitch, and style [signals new message unit and new thoughts coming up]; continuous intonation with repetitive shifts in tone [indicate maintaining floor and more to come]; rise in intonation [indicates question]

110. Professor: Now, the other thing. ${ }^{\wedge}<$ generally don't give breaks $>$ because I don't remember to give em, and if you really need a break, you've got to consult with ^Carlos, he's gonna have to work it ^out and he's gonna have to tell me to stop. Now, otherwise, I'm just going to ^go (.) until I'm done, [laugh], and then we go home ${ }^{\wedge}$. If you need to get ${ }^{\wedge}$ up and go to the ${ }^{\wedge}$ bathroom, they're down the hall. Or you can take the elevator or the stairs all the way down^ and go on the first floor. I don't know where they are on the second floor. I don't think I've been on the second floor, um.

a. Stress on now [signals new message unit]; slower talk [indicates important or new material to think about]; continous intonation and periodic shift in tone [indicates more to come and maintaining floor]

\section{INTERACTIONAL UNIT 16}

111. Students: (undecipherable)

a. Different speaker and undecipherable [signals new message unit interpreted as opening in floor] 
112. Professor: Yeah. You can go out that door and down this hall, yeah, *you're really concerned about the bathrooms?*

a. Falling tone of yeah [signals new message unit]; change in voice, pitch, and style [indicates change in thought]

113. Students [laugh]

a. Different speaker [signals new message unit]; laughter [interprets an opening in floor but no claim for floor]

114. Student: I'm a nurse. You're worried about if we don't find them.

a. Different speaker [signals new message unit]; stress on I'm a nurse [interprets opening in floor and claims speaker's rights for turn-at-talk]

\section{INTERACTIONAL UNIT 17}

115. Professor: $\underline{\text { So, }}$ <papers have parts $>$. Papers have parts, and the [signals new message unit papers that you're going to do for me will have three parts. It will have an ${ }^{\wedge}$ introduction, $a^{\wedge}{ }^{\wedge}$ discussion section, and implications. $<$ The introduction ${ }^{\wedge}$ section contains some parts ${ }^{\wedge}$.>

a. Different speaker [signals new message unit]; slower talk [indicates new and important information]; shifts in tone [indicate more to come and maintains floor]

116. Professor: Are you looking for a ^chair? We've got two up here. | And there are no other rooms in this place.

a. Rising intonation [indicates new message unit and question]; pause [indicates more coming]

117. Professor: *But she better be on time next time. * [laugh], or *she'll be sitting in the hall, * [laugh], [laugh].

a. Change in voice, pitch, and style [signal new message unit]; laughter [maintains claim for floor]

118. Professor: ^All right, | now (.) a problem ${ }^{\wedge}$ statement. I sent you that article from $u h$, or $>$ the chapter in the book from Merriam and Simpson $<$, where they walk through a problem statement. Now, you're going to hear terms kind of bantied about, you might have already in your travels as students. Some people will talk about a thesis, ok? When you took uh, English, they probably talked about a thesis, um, (what other ways do they talk about it?) Well, ^ that's good enough, we know that one.

a. Shift in tone, short pause, and pause [signal new message unit and more is coming]; , and stress on problem and statement [maintains claim for floor]; falling tones [indicate more to come]

119. Professor: But the thesis ${ }^{\wedge}$ and the problem statement is the ${ }^{\wedge}$ beginning of a paper <that grounds your paper in something $>$. Ok? Some one of you wants to do something on obesity, ok? And, uh, I had a student last year that did something on obesity, $>$ I don't exactly remember how it went $<$ but it was on the $<$ educational ${ }^{\wedge}$ value of obesity programs that we have $>$. 
a. Stress on thesis [signals new message unit]; slower talk [indicates something important to think about and maintains claim for floor]; faster talk [indicates extra material]

\section{INTERACTIONAL UNIT 18}


Pilot Study Transcripts

Stage 5 B, Part 2a/b-Reconstructive Data Analysis of Representative Classroom Observations (Pragmatic Horizon Analysis)

(Turntaking, Thematic Coherence)

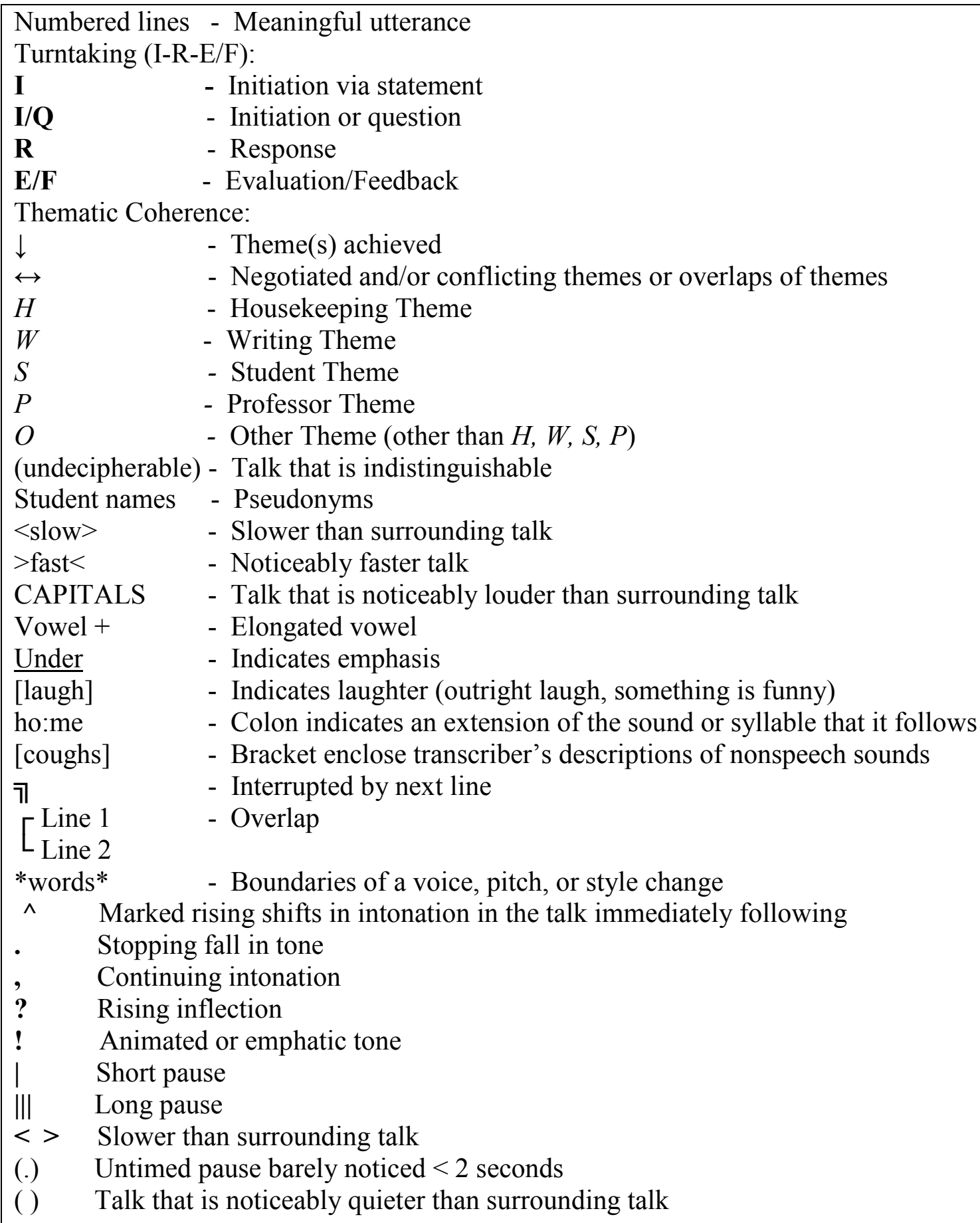


1. R Students: [laugh]

2. R Students: (undecipherable)

3. I/Q Professor: Uh. Who did? E/F You know I left my notebook. $\quad \downarrow \downarrow$ I/Q Where's Jim?

4. R Students: [laugh]

5. E/F Professor: Didn't he just? I/Q Who are you?

6. R Students: [laugh]

7. E/F Professor: Ohh, forget it. He's not here yet. It was me.

8. R Students: [laugh]

9. E/F Professor: This is the hardest thing I do all year.

$\mathbf{I} / \mathbf{Q}$ Doris, were you here last time?

10. R Doris: Yes

11. I/Q Professor: Lola Lesser, does anybody know if she was here last two weeks ago?

12. E/F Professor: All right. I/Q Jose Ruiz?

13. E/F Professor: All right. I/Q Marissa Sanders?

14. E/F Professor: Johnson I know was, ()

15. I/Q Professor: How about Natalia? [laugh]

16. E/F Professor: All right. I/Q Niara?

17. R Niara: Yes

18. I/Q Professor: Were you last?

19. R Niara: Yes

20. Student: [cough]

21. E/F Professor: Christy, you were too, Acacia was not because they lied to her, Alicia was not

${ }^{\wedge}$ Ginny was ^ Ramon was, you're Ramon right,

Emily.

22. R Emily: I was here

23. E/F Professor: You were here then. I/Q Are you here now? $\downarrow \downarrow \downarrow$

24. E/F Professor: I think you were here when I called your name!

25. R Student: (undecipherable)

26. E/F Professor: $O k$, ok $\mid \mathbf{I} / \mathbf{Q} M i-a h$ ?

27. R Mia: Me-ah.

28. E/F Professor: $M e-a h$.

29. R Mia: Yeah.

30. E/F Professor: I knew who you were ${ }^{\wedge}$ [laugh]!

31. R Mia. Yeah. That's a plus ${ }^{\wedge}$

32. E/F Professor: That's a plus! [laugh] I didn't have to ask

$\wedge$ you. Ok, you were here last time too.

33. E/F Professor: OK, I know June was, all right. I/Q NOW, is there somebody in the room right now that was here two weeks ago and here tonight that's not on my list ${ }^{\wedge}$ ? |II 34. I/Q Professor: Ok, how many of you received the things 
that I sent you today? | E/F All right, uh, you will find that I-ah frequently will send you things the day of, actually, there is no rhyme or reason to my sending things.

35. R Students: [laugh]

36. E/F Professor: To tell you the truth ${ }^{\wedge}$,

37. E/F Professor: If one of you asks me a question, now the answer you ${ }^{\wedge}$ get from me may aggravate the shit out of me, and you notice that in my response.

38. E/F Professor: But then ^ but then ^ I will decide it's not that dumb of a question [laugh] after all [laugh], it was my husband

${ }^{\wedge}$ that was really aggravating me, and I will try ${ }^{\wedge}$ to respond in a fuller message to the class because I think that it is something in fact that you don't understand as a group, ok^?

39. I/Q Professor: <So you've got a message> about problem statements from me, right? And I took, I think I took two of my own papers?

40. R Students: (undecipherable)

41. E/F Professor: What?

42. E/F Professor: Yeah, and uh, I showed you what a problem statement with a purpose looked like from, I know from my CRT one and I don't remember what the other one was, 43. Student: [cough]

44. E/F Professor: But I think it was two of them 45. E/F Professor: At any rate, uh | all right, so, somebody asked me something and that's what I did, 46. E/F Professor: Now, today ^ I was preparing for class a little later than I would have liked because I distracted myself and did something else, and uh, and anyhow, so I sent these things late, $>$ Now they're in your email ${ }^{\wedge}$ so you can go back and get them if you don't have them but it occurred to me that it might be useful for somebody to have ${ }^{\wedge}$ these handouts so you can print them out and write on them $<$, ok?

47. E/F Professor: So, that's why I sent them, you know, it's not required, it's just I was trying to be helpful.

48. E/F Professor: All right, um ||| 49. I Professor: Now, I'm going to talk to you tonight about writing a research paper and, umm, E/F I going to spend probably what some of you will consider an inordinate amount of time, trying to teach you how to write research papers. I do this for a variety of reasons. One, I believe very strongly about the notion that students 
and professionals have a voice, and the only reason we don't know what you know is because you don't know how to access the world of publication.

50. I/Q Professor: $O k$ ?

51. I/Q Professor: What do you think, Johnny?

52. R Johnny: You are perfectly, perfectly right.

53. E/F [laugh] I like Johnny. [laugh]

54. E/F Professor: Anyhow, and uh, I think this because when I

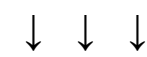

was a grad student, uh, in the 90s, look at my vitae,

I'm very young, um, all four of my degrees come from

the 1990s. Anyhow, um, when I was a grad student,

I knew when I went to get my doctorate that I wanted

to be a professor, that was what I was going to do, um,

I figured it out but so, I didn't know what you needed

to do as far as career ${ }^{\wedge}$ path to become a professor,

so I started asking faculty^. You know, what does a

student need to do as a student to become a faculty

member later? And they said you need to publish.

55. E/F Professor: $\mathrm{OKa}+y$, [laugh], how do you do that ${ }^{\wedge}$ ? Well, no

faculty members really tell you how to do that except

for a few rare ones, like me^, and um, because most

faculty assume that you go on and get a doctorate and

somehow this magic of writing this dissertation which

is really unlike any other kinds of writing, will magically

make you understand the publication process. And it

doesn't. It doesn't prepare you at all, uh, others

$\wedge$ think that students have nothing more () to say,

therefore, they shouldn't be encouraged |

56. E/F Professor: [laugh]

57. E/F Professor: It's getting more and more crowded, there's two seats up here. There's one here, this one's mine |||

Nobody wants to sit by me, Johnny.

58. R Johnny: [laugh]

59. E/F Student: You're so ()

60. E/F Professor: ||| All right, you know what, we are expecting

some more. So, you're going to have to sit there, I

hope you're ()

61. R Student: (undecipherable) [laugh]

62. E/F Professor: And |||

63. E/F Professor: Actually, Miguel ^ ${ }^{\wedge}$ was going to put the desks

this way, but I thought this would be a better way.

I think you might have a little more room sort of. If

there's a fire drill though 
64. R Students: [laugh]

65. E/F Professor: I think this row needs to go out this door so that that group can go out that door. Oh, yeah, the restrooms down this door. |

66. I/Q Professor: () Where was I in my sad tale?

67. E/F Professor: Oh! The rest of you don't know anything worth getting published, therefore, nobody should teach you how. Now that's one reason. Another ^ reason is that if you come to graduate school, the assumption is you have a career ${ }^{\wedge}$. It's my assumption that most of you work. I/Q Is there anybody here that doesn't have a regular job?

68. R Professor: $M e$, [laugh].

69. R Student: (undecipherable)

70. E/F Professor: All right, but you're in the minority [cough] ()

but you are, and especially true in the fields that I represent, Adult Education and Human Resource Development.

71. I/Q Professor: SO, if you have a job, how many of you never write a thing at work?

72. E/F Professor: Yeah, most of you write at work. Now, how people perceive you at work has a lot to do with how you communicate. Not $^{\wedge}$ the nature of your ideas... how you look, how you sound, whether they can pick up an accent in your writing.

73. E/F Professor: I learned this in Columbus, Ohio, so it has nothing to do with a Spanish accent that I'm talking about. It has everything to do with a Black accent, or a Cleveland accent, ok, or West Virginia. Because even from different places in this country^, we write differently according to where we were brought up and how we talk. And generally^ one thing I can guarantee all of you, I write a whole hell of a lot better than I talk.

74. E/F Professor: [laugh], yeah, and in fact, you can probably read me and not think it was me because it is so good.

75. R Students: [laugh]

76. E/F Professor: No cussing nothing ^ [laugh]. The sentences are in the right order [laugh], uh, it's focused, organized, all of the things I'm not in person, [laugh].

77. E/F Professor: All right, SO, these are the reasons that I do this, I think that you've got something important to say.

I think that you represent different groups, whether 


$$
H \quad W \quad S \quad P \quad O
$$

it be graduate students masters' level, graduate

students at the doctoral level, different ethnic groups,

different other kinds of groups, women, African

American, Haitians, Jamaican, uh,

78. Student: [cough]

79. E/F Professor: Other groups that in this country have

been disenfranchised, and not [s cough] allowed in.

80. I/Q Professor: OK? E/F So, those are some of the reasons

that I'm doing this.

81. E/F Professor: Now, my process is probably one most of you

are not used to, and actually the nurses from summer

from spring you didn't get a taste of this because

we went on that giant escapade.

82. R Students: [laugh] [laugh]

83. E/F Student: You had to bring that up, didn't you?

84. E/F Professor: [laugh]

85. E/F Professor: I'm still living with it. We're still trying to do it. $\downarrow \leftrightarrow \downarrow$

86. E/F Student: It really $<\underline{\text { honed }}$ my lit search skills $>$.

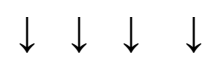

87. E/F Professor: It's $\wedge$ an important skill if you go on to get

a doctorate. [laugh]

88. E/F Professor: All right, so, I don't, I, one thing I learned,

I'm never going to do that in that class again and it wasn't,

it just wasn't the right place, but at any rate, because

I probably will do such a stupid thing again, I know. [laugh] $\downarrow \leftrightarrow \downarrow$

89. E/F Professor: All right, I So, what I will do, is you'll

send me drafts, and given my parameters timewise, I'll look

at them and I'll give you feedback. I use track changes

and I use comments. And I use short (.) to the point (.)

sentences. E/F And I have never learned Spanish, I don't

know come from a polite culture. Those of you who

haven't noticed that yet. I'm Italian, I'm the oldest,

I have four brothers ${ }^{\wedge}$, I was raised in a very male

neighborhood^, in Cleveland, we're just like New

Yorkers and Chicagoans, which means we're not very

polite. [laugh]

90. E/F Professor: Nonetheless, Miguel is here to keep me in check, right? [laugh]

91. R Miguel: Yeah.

92. I/Q Professor: Right? E/F [laugh] [laugh] Good luck to him^^, $h u h^{\wedge}$ ? [laugh]

93. E/F Professor: So, I just tell you this in way of explanation,

because our cultures differ, and there are a lot

of you in the room and there's only one of me,

94. E/F Professor: Cuz he can't give you feedback on your writing. 
No offense, [laugh].

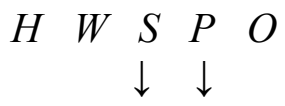

95. E/F Professor: Well, he can and he can't. I want to take

that back, because Miguel has come a hell of a long

way since I've met him. I/Q When ^did I meet ^you?

Three years ago?

96. R Miguel: 2006.

97. I/Q Professor: Only two years ago? E/F It seems like

I've known you forever.

98. E/F Professor: Anyhow, [laugh], all right, but Miguel has

come a long way, because in the interview, the

dissertation, the interview to get in to be a doc

student, I didn't understand half of what he said.

All I understood was a few references to Habermas,

who else? Foucault, uh, [laugh].

99. E/F Professor: Anyhow, so, well, I/Q you had only been here, what, a year at that point?

100. R Miguel: Yeah.

101. E/F Professor: And he did what I think is common

for a lot of people that come here from another

country to take-go to school and that is they sit

silently in classes because they don't want to look

stupid. And so you don't speak and you don't

learn to speak it. He's forced to talk to me.

102. I/Q Professor: So, anyhow, you've got the idea?

E/F I'm going to give you feedback within my time

parameter. Now some of you may need to make

appointments with me, may need to come in during

my office hours. If you want help on your papers,

though, don't come to me with nothing, come to me

with a work plan, come to me with an outline, come

to me with a draft of some sort, because I can't work

from nothing^ and if you give me something concrete,

I can give you something concrete back.

103. I/Q Professor: Ok? E/F Now, another thing that is

$\downarrow \downarrow \downarrow$

going to be unusual is that I believe writing is process, and

I believe it takes as long as it takes to get it right. Richard

Smith here is embarking on a paper that I think has the

potential to be an article. Not just a class paper,

not just a conference paper. And it actually, probably

$\wedge$ has the potential if he was in another field maybe

this one to be a dissertation topic.

104. E/F Professor: And we'll put this up and you'll see.

105. E/F Professor: All right, so I believe writing is a

process and all things that are a process, you learn 
by practicing those skills. I/Q Now any of you athletic?

E/F Basketball, soccer, soccer, like I know shit about soccer.

Anyhow^, when you are little, most of us are older than death, and don't want to play soccer or basketball

anymore, but when we are little, and we used to play

these things, we practiced! And all athletes know that

they have to practice these skills, and we drill it into

^ them. But we don't do the same things when it comes

to writing, when it comes to reading. We talk about

it other ways, we don't talk about these thing in terms

of building skills.

106. E/F Professor: Now, I don't think for the most part,

the books you have to read, this term, for these classes

are going to be difficult reads. In years past, I've used

the Handbook of Adult Education and it was a difficult

read, because the last version took a postmodern stance.

And postmodernists believe that the only good writing is dense circuitous writing, on purpose! OK.

107. E/F Professor: So, and, but it takes practice

sometimes, well it does, it takes practice to read academic

literature, it takes practice to develop your reading skills,

it's just practice, same with writing, so.

108. E/F Professor: I also think some of you should get into

peer groups possibly, join forces, send drafts back

and forth, though I'm not going to organize that

because you're always better off getting outside ()

109. I Professor: All right, we're going to start off talking

about the components of a paper. E/F I'm gonna walk

through what I think are the components of a position

paper which is what most of you will be writing in here

from the way that I saw your purposes. Then I'm

gonna go through some samples, I'm going to walk

you through Lisa's paper, I'm gonna show you

Miguel's paper from two years ago in this class, and

uh, and then I'm gonna go through your purpose

statements that you sent me, I/Q okay?

110. E/F Professor: Now, the other thing. I generally

don't give breaks because I don't remember to give em, and

if you really need a break, you've got to consult with

Miguel, he's gonna have to work it out and he's gonna

have to tell me to stop. Now, otherwise, I'm just going

to go (.) until I'm done, [laugh], and then we go home ${ }^{\wedge}$.

If you need to get up and go to the bathroom, they're

down the hall. Or you can take the elevator or the 
stairs all the way down^ ${ }^{\wedge}$ and go on the first floor.

I don't know where they are on the second floor.

I don't think I've been on the second floor, um.

111. R Student: (undecipherable)

112. E/F Professor: Yeah, you can go out that door

and down this hall, yeah, you're really concerned

about the bathrooms?

113. R Students: [laugh]

114. E/F Student: I'm a nurse. You're worried about

if we don't find them.

115. E/F Professor: So, <papers have parts >. Papers

have parts, and the papers that you're going to do for me

will have three parts. It will have an introduction ${ }^{\wedge}, a$

discussion section, and implications. The introduction

$\wedge$ section contains some parts ${ }^{\wedge}$.

116. I/Q Professor: Are you looking for a chair?

$\mathbf{E} / \mathbf{F}$ We've got two over here. | And there are no other rooms in this place.

117. E/F Professor: But she better be on time next time. [laugh], or she'll be sitting in the hall, [laugh], [laugh].

118. E/F Professor: All right, now (.) a problem ${ }^{\wedge}$

statement. I sent you that article, or the chapter in the book

from Merriam and Simpson, where they walk through a

problem statement. Now, you're going to hear terms

kind of bantied about, you might have already in your

travels as students. Some people will talk about a thesis,

ok? When you took uh, English, they probably talked

about a thesis, um, what other ways do they talk

about it? Well, that's good enough, we know that one.

119. E/F Professor: But the thesis^ and the problem

statement is the beginning of a paper that grounds your

paper in something. Ok? Some one of you wants to do

something on obesity, ok? And, uh, I had a student last

year that did something on obesity, I don't exactly

remember how it went but it was on the educational

$\wedge$ value of obesity programs that we have. 
Pilot Study Transcripts

Stage 5 B, Part 2c-

Reconstructive Data Analysis of Representative Classroom Observations

(Pragmatic Horizon Analysis)

(Intertextuality)

\begin{tabular}{|c|c|}
\hline \multicolumn{2}{|c|}{ Numbered lines - Meaningful utterance } \\
\hline \multicolumn{2}{|c|}{$\begin{array}{l}\text { Intertextuality: } \\
I P\end{array}$} \\
\hline \multirow{2}{*}{$\begin{array}{l}I P \\
I A\end{array}$} & - Intertextuality Proposed \\
\hline & - Intertextuality Acknowledged (superficial knowledge of existence) \\
\hline$I R$ & - Intertextuality Recognized (underlying familiarity and understanding) \\
\hline$S C$ & - Social Consequence \\
\hline NT & - New Topic \\
\hline $\mathrm{R}$ & - Request \\
\hline $\mathrm{C}$ & - Confirmation \\
\hline $\mathrm{X}$ & - Occurrence \\
\hline \multicolumn{2}{|c|}{ Turntaking (I-R-E/F): } \\
\hline I & - Initiation via statement \\
\hline $\mathbf{I} / \mathbf{Q}$ & - Initiation via question \\
\hline $\mathbf{R}$ & - Response \\
\hline $\begin{array}{l}\mathbf{E} / \mathbf{F} \\
\text { (undecipherable }\end{array}$ & - Evaluation/Feedback \\
\hline \multirow{2}{*}{$\begin{array}{l}\text { (undecipherable) } \\
\text { Student names }\end{array}$} & - Talk that is indistinguishable \\
\hline & - Pseudonyms \\
\hline$<$ slow $>$ & - Slower than surrounding talk \\
\hline$>$ fast $<$ & - Noticeably faster talk \\
\hline CAPITALS & - Talk that is noticeably louder than surrounding talk \\
\hline Vowel + & - Elongated vowel \\
\hline Under & - Indicates emphasis \\
\hline [laugh] & - Indicates laughter (outright laugh, something is funny) \\
\hline \multirow{2}{*}{$\begin{array}{l}\text { ho:me } \\
\text { [coughs] }\end{array}$} & - Colon indicates an extension of the sound or syllable that it follows \\
\hline & - Bracket enclose transcriber's descriptions of nonspeech sounds \\
\hline \multirow{2}{*}{7} & - Interrupted by next line \\
\hline & - Overlap \\
\hline \multicolumn{2}{|l|}{ Line 2} \\
\hline \multicolumn{2}{|r|}{ - Boundaries of a voice, pitch, or style change } \\
\hline \multirow{2}{*}{\multicolumn{2}{|c|}{$\begin{array}{l}\text { Marked rising shifts in intonation in the talk immediately following } \\
\text {. Stopping fall in tone }\end{array}$}} \\
\hline & \\
\hline \multicolumn{2}{|c|}{ Continuing intonation } \\
\hline \multicolumn{2}{|c|}{ ? $\quad$ Rising inflection } \\
\hline \multicolumn{2}{|c|}{ ! Animated or emphatic tone } \\
\hline \multicolumn{2}{|c|}{ I Short pause } \\
\hline Long pau & \\
\hline Slower th & an surrounding talk \\
\hline Untimed 1 & pause barely noticed $<2$ seconds \\
\hline Talk that & is noticeably quieter than surrounding talk \\
\hline
\end{tabular}


1. R Students: [laugh]

$\begin{array}{llll}I P & I A & I R & S C \\ & \mathrm{X} & & \end{array}$

2. R Students: (undecipherable)

NT

3. I/Q Professor: Uh. Who did? E/F You know I left

my notebook. I/Q Where's Jim?

4. R Students: [laugh]

5. E/F Professor: Didn't he just? I/Q Who are you? $\quad \mathrm{X} \quad \mathrm{R}$

6. R Students: [laugh]

7. E/F Professor: Ohh, forget it. He's not here yet.

It was me.

8. R Students: [laugh]

9. E/F Professor: This is the hardest thing I do all year.

$\mathbf{I} / \mathbf{Q}$ Doris, were you here last time?

10. R Doris: Yes

11. I/Q Professor: Lola Lesser, does anybody know if she was here last two weeks ago?

12. E/F Professor: All right. I/Q Jose Ruiz?

$\mathrm{X} \quad \mathrm{R}$

$X \quad R$

13. E/F Professor: All right. I/Q Marissa Sanders? $\quad \mathrm{X} \quad \mathrm{R}$

14. E/F Professor: Johnson I know was, ()

15. I/Q Professor: How about Natalia? [laugh]

16. E/F Professor: All right. I/Q Niara?

$\mathrm{X}$

$\mathrm{X} \quad \mathrm{R}$

$\mathrm{X} \quad \mathrm{R}$

17. R Niara: Yes

18. I/Q Professor: Were you last?

$\mathrm{C}$

19. R Niara: Yes

$\mathrm{X} \quad \mathrm{R}$

$\mathrm{C}$

20. Student: [cough]

21. E/F Professor: Christy, you were too, Acacia was $\quad \mathrm{X} \quad \mathrm{R}$

$\mathrm{R}$ not because they lied to her, Alicia was not

${ }^{\wedge}$ Ginny was ${ }^{\wedge}$ Ramon was, you're Ramon right,

Emily.

22. R Emily: I was here

23. E/F Professor: You were here then. I/Q Are you here now?

24. E/F Professor: I think you were here when I called your name!

25. R Student: (undecipherable)

$\mathrm{X} \quad \mathrm{C}$

26. E/F Professor: $O k, o k \mid \mathbf{I} / \mathbf{Q} M i-a h$ ?

NT

27. R Mia: Me-ah.

$\mathrm{X} \quad \mathrm{R} \quad \mathrm{C}$

28. E/F Professor: $M e-a h$.

$\mathrm{C}$

29. R Mia: Yeah.

30. E/F Professor: I knew who you were ^ [laugh]!

31. R Mia: Yeah. That's a plus ${ }^{\wedge}$

32. E/F Professor: That's a plus! [laugh] I didn't have to ask

^ you. Ok, you were here last time too.

33. E/F Professor: OK, I know Judy was, all right. 
$\mathbf{I} / \mathbf{Q} N O W$, is there somebody in the room right now

IP IA IR SC

that was here two weeks ago and here tonight that's not on my list ${ }^{\wedge}$ ? |||

NT R R

34. I/Q Professor: Ok, how many of you received the things that I sent you today? | E/F All right, uh, you will find that I-ah frequently will send you things the day of, actually, there is no rhyme or reason to my sending things.

35. R Students: [laugh]

36. E/F Professor: To tell you the truth ${ }^{\wedge}$,

37. E/F Professor: If one of you asks me a question,

$\begin{array}{lll}\mathrm{X} & \mathrm{R} & \mathrm{X}\end{array}$

now the answer you ${ }^{\wedge}$ get from me may aggravate the shit out of me, and you notice that in my response.

38. E/F Professor: But then ^ but then ${ }^{\wedge}$ I will decide X it's not that dumb of a question [laugh] after all [laugh],

${ }^{\wedge}$ it was my husband that was really aggravating me, and I will try ${ }^{\wedge}$ to respond in a fuller message to the class because I think that it is something in fact that you don't understand as a group, ok^?

39. I/Q Professor: $<$ So you've got a message $>$ about $\quad \mathrm{X} \quad \mathrm{R} \quad \mathrm{C}$ problem statements from me, right? And I took, I think I took two of my own papers?

40. R Students: (undecipherable) NT

41. E/F Professor: What? X

42. E/F Professor: Yeah, and uh, I showed you what a X problem statement with a purpose looked like from, I know from my CRT one and I don't remember what the other one was,

43. Student: [cough]

44. E/F Professor: But I think it was two of them $\quad \mathrm{X}$

45. E/F Professor: At any rate, uh | all right, so, $\quad \mathrm{X}$

$\mathrm{R} \quad \mathrm{C}$

somebody asked me something and that's what I did, 46. E/F Professor: Now, today ^ I was preparing for class a little later than I would have liked because I distracted myself and did something else, and uh, and anyhow, so I sent these things late, >Now they're in your email ^ so you can go back and get them if you don't have them but it occurred to me that it might be useful for somebody to have ${ }^{\wedge}$ these handouts so you can print them out and write on them $<$, ok?

47. E/F Professor: So, that's why I sent them, you know, it's not required, it's just I was trying to be helpful. | X 48. E/F Professor: All right, um |||

49. I Professor: Now, I'm going to talk to you tonight about 
IP IA IR SC

writing a research paper and, umm, I going to spend probably what some of you will consider an inordinate amount of time, trying to teach you how to write research papers. I do this for a variety of reasons. One, I believe very strongly about the notion that students $\mathrm{X}$ and professionals have a voice, and the only reason we don't know what you know is because you don't know how to access the world of publication.

50. I/Q Professor: $O k$ ?

51. I/Q Professor: What do you think, Johnny?

$\mathrm{R}$

52. R Johnny: You are perfectly, perfectly right.

$\mathrm{R}$

53. E/F Professor: [laugh] I like Johnny. [laugh]

54. E/F Professor: Anyhow, and uh, I think this because when I was a grad student, uh, in the 90s, look at my vitae, I'm very young, um, all four of my degrees come from the 1990s. Anyhow, um, when I was a grad student, I knew when I went to get my doctorate that I wanted to be a professor, that was what I was going to do, um, I figured it out but so, I didn't know what you needed to do as far as career ${ }^{\wedge}$ path to become a professor, so I started asking faculty^. You know, what does a student need to do as a student to become a faculty member later? And they said you need to publish.

55. E/F Professor: $\mathrm{OKa}+\mathrm{y}$, [laugh], how do you do that ${ }^{\wedge}$ ?X Well, no faculty members really tell you how to do that except for a few rare ones, like $\mathrm{me}^{\wedge}$, and um, because most faculty assume that you go on and get a doctorate and somehow this magic of writing this dissertation which is really unlike any other kinds of writing, will magically make you understand the publication process. And it doesn't. It doesn't prepare you at all, uh, others $\wedge$ think that students have nothing more () to say, therefore, they shouldn't be encouraged |

56. E/F Professor: [laugh]

$\mathrm{R}$

57. E/F Professor: It's getting more and more crowded, there's two seats up here. There's one here, this one's mine || Nobody wants to sit by me, Johnny. NT 58. R Johnny: [laugh]

59. E/F Student: You're so ()

60. E/F Professor: || All right, you know what, we are NT expecting some more. So, you're going to have to sit there, I hope you're ()

61. R Student: (undecipherable) [laugh]

62. E/F Professor: And $\| \mid$ 
63. E/F Professor: Actually, Miguel ${ }^{\wedge}$ was going to put

IP IA IR SC

the desks this way, but I thought this would be a better way. I think you might have a little more room sort of. If there's a fire drill though

64. R Students: [Laugh]

65. E/F Professor: I think this row needs to go out this door so that that group can go out that door. Oh, yeah, the restrooms down this door. |

$\mathrm{C}$

66. I/Q Professor: () Where was I in my sad tale?

67. E/F Professor: Oh! The rest of you don't know

anything worth getting published, therefore, nobody

should teach you how. Now that's one reason.

Another ${ }^{\wedge}$ reason is that if you come to graduate

school, the assumption is you have a career^. It's

my assumption that most of you work. I/Q Is there

anybody here that doesn't have a regular job?

68. R Professor: $M e$, [laugh].

69. R Student: (undecipherable)

70. E/F Professor: All right, but you're in the

minority [cough] ()but you are, and especially

true in the fields that I represent, Adult Education

and Human Resource Development.

71. I/Q Professor: SO, if you have a job, how many

of you never write a thing at work?

X R

72. E/F Professor: Yeah, most of you write at work.

Now, how people perceive you at work has a lot to do with how you communicate. Not ${ }^{\wedge}$ the nature of your ideas... how you look, how you sound, whether they can pick up an accent in your writing.

73. E/F Professor: I learned this in Columbus, Ohio, so it has nothing to do with a Spanish accent that I'm talking about. It has everything to do with a Black accent, or a Cleveland accent, ok, or West Virginia. Because even from different places in this country^, we write differently according to where we were brought up and how we talk. And generally^ one thing I can guarantee all of you, I write a whole hell of a lot better than I talk.

74. E/F Professor: [laugh], yeah, and in fact, you can probably read me and not think it was me because it is so good.

75. R Students: [laugh]

X R

$\mathrm{C}$

76. E/F Professor: No cussing nothing ^ [laugh]. The X sentences are in the right order [laugh], uh, it's focused, 
IP IA IR SC

organized, all of the things I'm not in person, [laugh].

77. E/F Professor: All right, SO, these are the reasons

that I do this, I think that you've got something

important to say. I think that you represent different

groups, whether it be graduate students masters'

level, graduate students at the doctoral level, different,

ethnic groups different other kinds of groups, women,

African American, Haitians, Jamaican, uh,

78. Student: [cough]

79. E/F Professor: Other groups that in this country

have been disenfranchised, and not [cough] allowed in. X $\mathrm{R}$

80. I/Q Professor: OK? E/F So, those are some of the reasons that I'm doing this.

81. E/F Professor: Now, my process is probably one most of you are not used to, and actually the nurses

from summer from spring you didn't get a taste of

this because we went on that giant escapade.

82. R Students: [laugh] [laugh]

83. E/F Student: You had to bring that up, didn't you?

84. E/F Professor: [laugh]

85. E/F Professor: I'm still living with it. We're still

trying to do it

86. E/F Student: It really $<\underline{\text { honed }}$ my lit search skills $>$.

NT $\quad$ R

$\mathrm{C}$

87. E/F Professor: It's ^ an important skill if you go

on to get a doctorate. [laugh]

88. E/F Professor: All right, so, I don't, I, one thing I

learned, I'm never going to do that in that class again

and it wasn't, it just wasn't the right place, but at

any rate, because I probably will do such a stupid

thing again, I know. [laugh]

C

89. E/F Professor: All right, I So, what I will do, is

you'll send me drafts, and given my parameters

timewise, I'll look at them and I'll give you feedback.

I use track changes and I use comments. And I use

short (.) to the point (.) sentences. $\mathbf{E} / \mathbf{F}$ And I have

never learned Spanish, I don't know come from a

polite culture. Those of you who haven't noticed

that yet. I'm Italian, I'm the oldest, I have four

brothers $^{\wedge}$, I was raised in a very male

neighborhood^, in Cleveland, we're just like

New Yorkers and Chicagoans, which means

we're not very polite. [laugh]

X R

$\mathrm{C}$

90. E.F Professor: Nonetheless, Miguel is here to keep me in check, right? [laugh]

C 
91. R Miguel: Yeah.

IP IA IR SC

92. I/Q Professor: Right? E/F [laugh] [laugh].

$\mathrm{C}$

Good luck to him^, huh^? [laugh]

93. E/F Professor: So, I just tell you this in way of

explanation, because our cultures differ, and there

are a lot of you in the room and there's only one of

me,

94. E/F Professor: Cuz he can't give you feedback on

your writing. No offense, [laugh].

$\mathrm{X} \quad \mathrm{C}$

95. E/F Professor: Well, he can and he can't. I want

to take that back, because Miguel has come a hell

of a long way since I've met him. I/Q When ^did I $\quad$ NT $\quad \mathrm{R}$

meet ^you? Three years ago?

96. R Miguel: 2006.

97. I/Q Professor: Only two years ago? E/F It seems

like I've known you forever.

98. E/F Professor: Anyhow, [laugh], all right, but Miguel

has come a long way, because in the interview, the

dissertation, the interview to get in to be a doc

student, I didn't understand half of what he said.

All I understood was a few references to Habermas, who else? Foucault, uh, [laugh].

$\mathrm{X} \quad \mathrm{C}$

99. E/F Professor: Anyhow, so, well, I/Q you had only been here what, a year at that point?

100. R Miguel: Yeah.

101. I/Q Professor: And he did what I think

is common for a lot of people that come here from

another country to take-go to school and that is they

sit silently in classes because they don't want to look

stupid. And so you don't speak and you don't

learn to speak it. He's forced to talk to me.

$\mathrm{X}$

C

102. I/Q Professor: So, anyhow, you've got

the idea? $\mathbf{E} / \mathbf{F}$ I'm going to give you feedback within my

time parameter. Now some of you may need to make

appointments with me, may need to come in during

my office hours. If you want help on your papers,

though, don't come to me with nothing, come to me

with a work plan, come to me with an outline, come

to me with a draft of some sort, because I can't work

from nothing^ and if you give me something concrete,

I can give you something concrete back.

$\begin{array}{lll}\mathrm{X} & \mathrm{R} & \mathrm{C}\end{array}$

103. I/Q Professor: Ok? E/F Now, another

thing that is going to be unusual is that I believe

writing is process, and I believe it takes as long as it 
IP IA IR SC

takes to get it right. Richard Smith here is embarking

on a paper that I think has the potential to be an

article. Not just a class paper, not just a conference

paper. And it actually, probably ^ has the potential

if he was in another field maybe this one to be a dissertation topic.

$\mathrm{X}$

$\mathrm{C}$

104. E/F Professor: And we'll put this up and you'll see.

105.

E/F Professor: All right, so I believe

$\mathrm{X}$

C

writing is a process and all things that are a process,

you learn by practicing those skills. $\mathbf{I} / \mathbf{Q}$ Now any of you

athletic? E/F Basketball, soccer, soccer, like I know shit

about soccer. Anyhow^, when you are little, most of

us are older than death, and don't want to play soccer

or basketball anymore, but when we are little, and

we used to play these things, we practiced! And all

athletes know that they have to practice these skills,

and we drill it into ${ }^{\wedge}$ them. But we don't do the same

things when it comes to writing, when it comes to

reading. We talk about it other ways, we don't talk

about these thing in terms of building skills.

106. E/F Professor: Now, I don't think for the

most part, the books you have to read, this term, for

these classes are going to be difficult reads. In years

past, I've used the Handbook of Adult Education

and it was a difficult read, because the last version

took a postmodern stance. And postmodernists

believe that the only good writing is dense circuitous

writing, on purpose! OK.

$\mathrm{X} \quad \mathrm{R}$

$\mathrm{C}$

107. E/F Professor: So, and, but it takes practice

sometimes, well it does, it takes practice to read

academic literature, it takes practice to develop your

reading skills, it's just practice, same with writing, so. $\mathrm{X}$

$\mathrm{C}$

108. E/F Professor: I also think some of you

should get into peer groups possibly, join forces, send

drafts back and forth, though I'm not going to organize $\mathrm{X}$

that because you're always better off getting outside ()

109. I Professor: All right, we're going to start

off talking about the components of a paper. E/F I'm gonna

walk through what I think are the components of a position

paper which is what most of you will be writing in here

from the way that I saw your purposes. Then I'm

gonna go through some samples, I'm going to walk

you through Lisa's paper, I'm gonna show you 
Miguel's paper from two years ago in this class, and uh, and then I'm gonna go through your purpose

statements that you sent me, $\mathbf{I} / \mathbf{Q}$ okay?

110. E/F Professor: Now, the other thing. I generally don't give breaks because I don't remember to give em, and if you really need a break, you've got to consult with Miguel, he's gonna have to work it out and he's gonna have to tell me to stop. Now, otherwise, I'm just going to go (.) until I'm done, [laugh], and then we go home ${ }^{\wedge}$. If you need to get up and go to the bathroom, they're down the hall. Or you can take the elevator or the stairs all the way down^ and go on the first floor. I don't know where they are on the second floor. I don't think I've been on the second floor, um.

111. R Student: (undecipherable)

112. E/F Professor: Yeah, you can go out that door and down this hall, yeah, you're really concerned about the bathrooms?

113. R Students: [laugh]

114. E/F Student: I'm a nurse. You're worried about if we don't find them.

115. $\quad$ E/F Professor: So, <papers have parts $>$. Papers have parts, and the papers that you're going to do for me will have three parts. It will have an introduction $^{\wedge}$, a discussion section, and implications. The introduction ${ }^{\wedge}$ section contains some parts^. 116. I/Q Professor: Are you looking for a chair? E/F We've got two over here. | And there are no other rooms in this place.

117. E/F Professor: But she better be on time next time. [laugh], or she'll be sitting in the hall, [laugh], [laugh].

$\mathrm{R}$

$\mathrm{C}$

C

$\mathrm{C}$

$\mathrm{C}$

$\mathrm{C}$

118. E/F Professor: All right, now (.) a problem $\wedge$ statement. I sent you that article, or the chapter in the book from Merriam and Simpson, where they walk through a problem statement. Now, you're going to hear terms kind of bantied about, you might have already in your travels as students. Some people will talk about a thesis, ok? When you took uh, English, they probably talked about a thesis, um, what other ways do they talk about it? Well, that's good enough, we know that one. 119. E/F Professor: But the thesis ${ }^{\wedge}$ and the problem statement is the beginning of a paper that grounds your paper in something. Ok? Some one of 
IP IA IR SC

you wants to do something on obesity, ok? And, uh,

I had a student last year that did something on obesity,

I don't exactly remember how it went but it was on the

educational $\wedge$ value of obesity programs that we have. $\mathrm{X}$

$\mathrm{R}$

C 
Pilot Study Transcripts

Stage 5 C - Reconstructive Data Analysis of Representative Classroom Observations

(Validity Reconstructions)

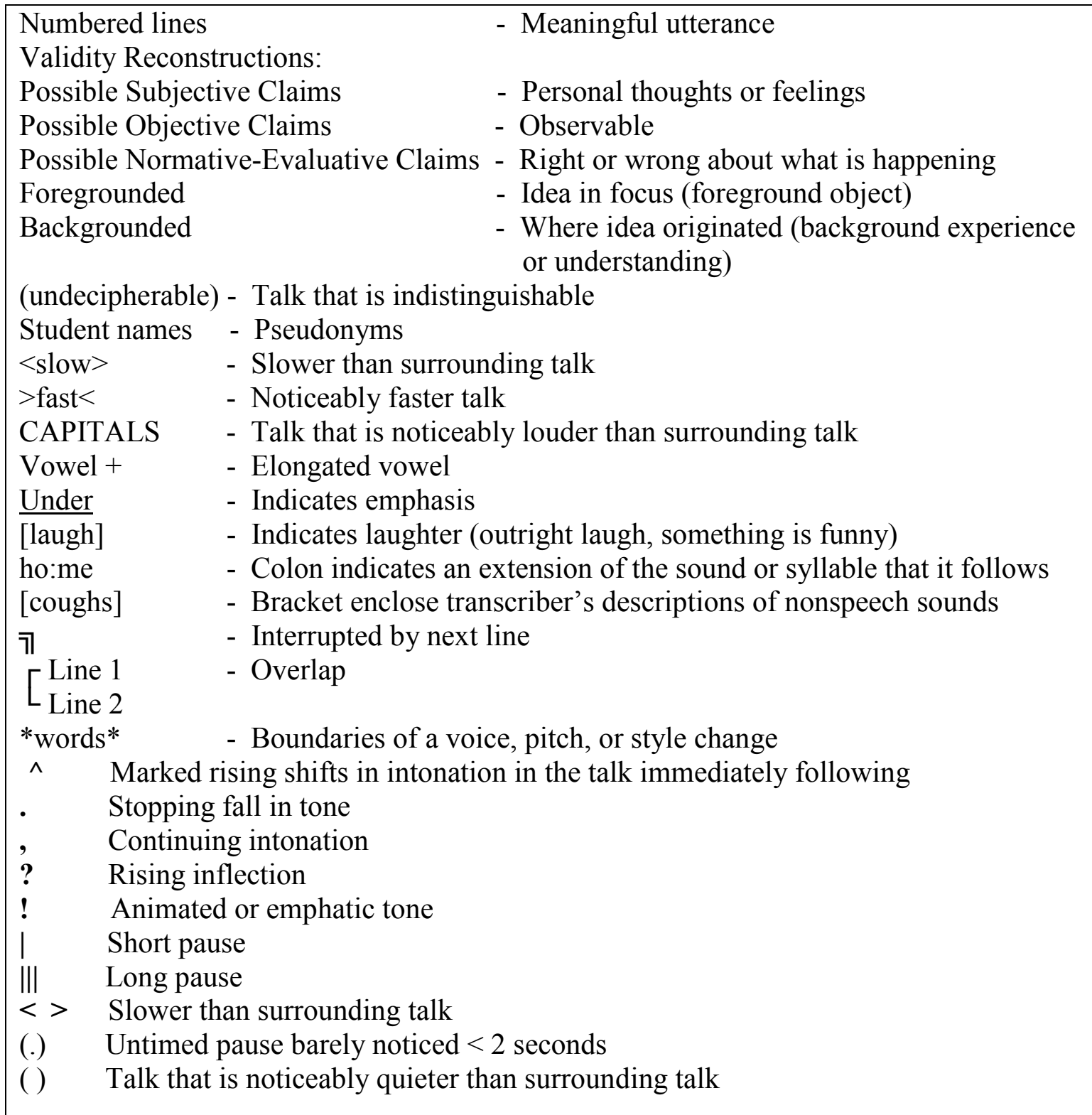

1. Students: [laugh]

2. Student: (undecipherable)

Possible subjective claims

Foregrounded, Immediate

I think something is funny. I like to talk to friends before class starts.

Less Foregrounded, Less Immediate 
I am nervous about this class, because I am a terrible writer, so it helps to laugh. I am a good student so I can relax (identity claim).

Backgrounded, Remote

I want to buck the establishment which says sit quietly in classrooms.

Possible objective claims

Very Foregrounded, Very Immediate

The professor is here and most of them like us to enjoy being here.

Highly Backgrounded, Remote

This is a university and students get on the bad side of professors if they don't act happily involved from the getgo. Laughing is a way to resist "be quiet" rules of university classrooms.

\section{Possible normative-evaluative claims}

Quite Foregrounded, Quite Immediate

It is okay to relax and laugh a little before class at the university level.

Less Foregrounded, Less Immediate

Laughter is accepted by most professors before class.

Backgrounded, Remote

Professors are responsible for calling class to order.

3. Professor: Uh. Who did? You know I left my notebook. Where's Jim?

\section{Possible subjective claims}

Foregrounded, Immediate

I want help finding something.

Less Foregrounded, Less Immediate

I am disorganized (identity claim). I have too much going on so let me get somebody to help.

Backgrounded, Remote

I depend on Joe to help me. Students are too distant at first so I want to wake them up.

Possible objective claims

Very Foregrounded, Very Immediate

I need to get class started. I want the students to get involved immediately.

Highly Backgrounded, Remote

This is a university and I am expected to be organized. Professors get a bad rap if they are not ready for class to start.

\section{Possible normative-evaluative claims}

Quite Foregrounded, Quite Immediate

It is right for professors to have students helping them with housekeeping tasks.

Less Foregrounded, Less Immediate

It is not good for me to leave my stuff at home. I will blame my student helper. It is good to show students that I know names and will call on them.

Backgrounded

Professors want students to think they are on top of things. Students are easier on the professor when they know the student helper made the mistake.

4. Students: [laugh]

5. Professor: Didn't he just? Who are you?

6. Students: [laugh] 
7. Professor: Ohh, forget it. He's not here yet. It was me.

\section{Possible subjective claims}

\section{Foregrounded Immediate}

I give up. I made the mistake and I can't blame the student.

Less Foregrounded, Less Immediate

I want to seem human. I want students to feel comfortable making mistakes.

Backgrounded

I can't ever remember everything so I'll put that on the back burner.

\section{Possible objective claims}

Very Foregrounded, Very Immediate

The students are watching me to see what is important in this class.

Highly Backgrounded, Remote

This is a university class and it is time to start housekeeping.

Possible normative-evaluative claims

Quite Foregrounded, Quite Immediate

It is right for the professor to take responsibility.

Less Foregrounded, Less Immediate

The professor knows best. It is good for students to feel like they can joke around.

Backgrounded, Remote

Professors get good ratings if they are more human. The professor is ultimately responsible for everything in the class. Students should be part of classroom routines.

8. Students: [laugh]

9. Professor: This is the hardest thing I do all year. Doris, were you here last time?

10. Doris: Yes

11. Professor: Lola Lesser, does anybody know if she was here last two weeks ago?

12. Professor: All right. Jose Ruiz?

13. Professor: All right. Marissa Sanders?

14. Professor: Johnson I know was, ()

15. How about Natalia? [laugh]

16. All right. Niara?

17. Niara: Yes

18. Professor: Were you last?

19. Niara: Yes

20. Student: [cough]

21. Professor: Christy, you were too, Acadia was not because they lied to her, Alicia was not ${ }^{\wedge}$ Ginny was ${ }^{\wedge}$ Ramon was, you're Ramon right, Emily.

\section{Possible subjective claims}

Foregrounded Immediate

I want to check roll correctly. I want to fix up last week's roll call. I want to involve students in this task.

Less Foregrounded, Less Immediate

I am a critical theorist professor (identity claim) when I talk about what is wrong with the university system.

Backgrounded

I like to stir up trouble and get students thinking out of the box right off the bat. 


\section{Possible objective claims}

Very Foregrounded, Very Immediate

A student's absence is not always their fault. University staff can make mistakes.

Highly Backgrounded, Remote

The university system does not care about its students.

\section{Possible normative-evaluative claims}

Quite Foregrounded, Quite Immediate

It is right for the professor to learn names and count attendance properly. I care about each student.

Less Foregrounded, Less Immediate

It is good to involve students in the university setting.

Backgrounded, Remote

Students need to know that the university system makes mistakes. It is right to let the students know that the university system is uncaring.

22. Emily: I was here

23. Professor: You were here then. Are you here now?

24. Professor: I think you were here when I called your name!

25. Student: (undecipherable)

26. Professor: $O k, o k \mid M i-a h$ ?

27. Mia: Me-ah.

28. Professor: $M e-a h$.

29. Mia: Yeah.

30. Professor: I knew who you were ${ }^{\wedge}$ [laugh]!

31. Mia: Yeah. That's a plus ^

32. Professor: That's a plus! [laugh] I didn't have to ask ^ you. Ok, you were here last time too.

\section{Possible subjective claims}

Foregrounded Immediate

I want to ask about my students. I like going beyond my required duties as a professor.

Less Foregrounded, Less Immediate

I want to learn names. I want students to realize that I care about them and their

whereabouts.

Backgrounded

I am a good professor and caring person.

Possible objective claims

Very Foregrounded, Very Immediate

The students will learn about more than writing in here. University professors usually do not go beyond their required duties. University professors do not usually know or remember their students especially in the first couple of classes.

Highly Backgrounded, Remote

Names are a hint about people's cultures.

Possible normative-evaluative claims

Quite Foregrounded, Quite Immediate 
It is a positive when professors learn students' names. It is a positive when professors remember names after the first class.

Less Foregrounded, Less Immediate

It is the professor's responsibility to learn names even in the university setting. Students like to be more than a number. It is good when students talk in class.

Backgrounded, Remote

It is good for the professor to spend time now getting to know students' names. We can get more done later if we feel like a community and know each other's names.

33. Professor: OK, I know June was, all right. NOW, is there somebody in the room right now that was here two weeks ago and here tonight that's not on my list ${ }^{\wedge}$ ? III

34. Professor: Ok, how many of you received the things that I sent you today? | All right, uh, you will find that I-ah frequently will send you things the day of, actually, there is no rhyme or reason to my sending things.

\section{Possible subjective claims}

Foregrounded Immediate

I want you to receive things by email from me. I want you to get to know how I think and do things.

Less Foregrounded, Less Immediate

I want you to know that I do things differently. I want you to all feel included before we get started.

Backgrounded

I want to make sure my system and process worked. I want to give myself an out if something in the process does not work well.

\section{Possible objective claims}

Very Foregrounded, Very Immediate

This is a university classroom and there must be a system of collecting and distributing assignments. This classroom will have a different type of system than you are used to as students.

\section{Highly Backgrounded, Remote}

I care about you and want to explain my process of assigning work and including you in the class happenings related to learning.

\section{Possible normative-evaluative claims}

Quite Foregrounded, Quite Immediate

It is right for the professor to explain the class process. It is right to use email. It is right to check roll.

Less Foregrounded, Less Immediate

The professor can make changes in the typical process of assigning work and handling relationships with students.

Backgrounded, Remote

Professors can be different and get by with it. It is right for the professor to be run things differently in a university classroom.

35. Students: [laugh]

36. Professor: To tell you the truth ${ }^{\wedge}$, 
37. If one of you asks me a question, now the answer you ^ get from me may aggravate the shit out of me, and you notice that in my response.

38. But then ^ but then ${ }^{\wedge} I$ will decide it's not that dumb of a question [laugh] after all [laugh], it was my husband ${ }^{\wedge}$ that was really aggravating $m e$, and I will try ${ }^{\wedge}$ to respond in a fuller message to the class because I think that it is something in fact that you don't understand as a group, $\mathrm{ok}^{\wedge}$ ?

\section{Possible subjective claims}

\section{Foregrounded Immediate}

I want you to understand that I react and then reconsider what I just did. I want you to know I have a life that sometimes interferes with my thinking.

Less Foregrounded, Less Immediate

I want you to know that I care about my students in the long run (identity claim). I care about what students learn and I want to explain to them what they don't understand. Backgrounded I can cuss and get by with it. I do my work at home.

\section{Possible objective claims}

Very Foregrounded, Very Immediate

University students want to learn something. University students ask questions because they don't understand something.

Highly Backgrounded, Remote

Professors who listen to questions from students will realize that many have the same question. Professors need to answer questions if they follow system rules.

\section{Possible normative-evaluative claims}

Quite Foregrounded, Quite Immediate

It is right for the professor to answer questions. Caring professors will answer questions. Less Foregrounded, Less Immediate

The professor who is good will read and answer students' questions. Good professors will not let personal issues override classroom learning.

Backgrounded, Remote

It is okay for professors to work at home as long as they can separate personal and academic issues. It is okay for professors to work at home as long as students are not slighted. Good professors care about their students and take time to answer their questions.

39. Professor: <So you've got a message > about problem statements from me, right? And I took, I think I took two of my own papers?

40. Student: (undecipherable)

41. Professor: What?

42. Professor: Yeah, and uh, I showed you what a problem statement with a purpose looked like from, I know from my CRT one and I don't remember what the other one was,

43. Student: [cough]

44. Professor: But I think it was two of them,

45. Professor: At any rate, uh | all right, so, somebody asked me something and that's what I did, 
46. Professor: Now, today ^ I was preparing for class a little later than I would have liked because I distracted myself and did something else, and uh, and anyhow, so I sent these things late, >Now they're in your email ^ so you can go back and get them if you don't have them but it occurred to me that it might be useful for somebody to have ${ }^{\wedge}$ these handouts so you can print them out and write on them $<$, ok?

47. Professor: So, that's why I sent them, you know, it's not required, it 's just I was trying to be helpful. |

48. Professor: All right, um ||

49. Professor: Now, I'm going to talk to you tonight about writing a research paper and, umm, I going to spend probably what some of you will consider an inordinate amount of time, trying to teach you how to write research papers. I do this for a variety of reasons. One, I believe very strongly about the notion that students and professionals have a voice, and the only reason we don't know what you know is because you don't know how to access the world of publication.

\section{Possible subjective claims}

\section{Foregrounded Immediate}

I want to make connections now between you, me, writing, the process and why. I want you to buy in to the process we will be using. I want to explain why I do things like this. I believe in students' potential.

Less Foregrounded, Less Immediate

I am a caring professor (identity claim) because I go beyond requirements. I teach how to write and connect the process to what I believe about students. I like to throw out new terms like problem statement and CRT.

\section{Backgrounded}

I want to start slipping in critical theory and other connections to the process we will use both in writing and in the way the course plays out.

\section{Possible objective claims}

Very Foregrounded, Very Immediate

Papers are a big part of this class since this is a writing for publication course. Students are important in this class. Students have a voice that is usually overlooked.

Highly Backgrounded, Remote

Most students have been in classes that do not highlight their voice. Most university classes do not spend a lot of time on how to write research papers. Most university professors do not believe in students' potential to become good writers.

\section{Possible normative-evaluative claims}

\section{Quite Foregrounded, Quite Immediate}

It is right for the professor to believe in students' potential. It is right to give students voice. Professors are responsible for believing in students and teaching them to write. Less Foregrounded, Less Immediate

The professor who gives students voice cares about them. Most professors do not care and do not teach. It is good to share papers with students. It is good to share the professor's and other students' own writing with students as models for their future writing. Backgrounded, Remote 
It is right for professors to let students in on the inner workings of the university system.

50. Professor: $O k$ ?

51. Professor: What do you think, Johnny?

52. Johnny: You are perfectly, perfectly right.

53. Professor: [laugh] I like Johnny. [laugh]

54. Professor: Anyhow, and uh, I think this because when I was a grad student, uh, in the 90s, look at my vitae, I'm very young, um, all four of my degrees come from the 1990s. Anyhow, um, when I was a grad student, I knew when I went to get my doctorate that I wanted to be a professor, that was what I was going to do, um, I figured it out but so, I didn't know what you needed to do as far as career ${ }^{\wedge}$ path to become a professor, so I started asking faculty^. You know, what does a student need to do as a student to become a faculty member later? And they said you need to publish.

55. Professor: OKa+y, [laugh], how do you do that^? Well, no faculty members really tell you how to do that except for a few rare ones, like me^, and um, because most faculty assume that you go on and get a doctorate and somehow this magic of writing this dissertation which is really unlike any other kinds of writing, will magically make you understand the publication process. And it doesn't. It doesn't prepare you at all, uh, others ${ }^{\wedge}$ think that students have nothing more () to say, therefore, they shouldn't be encouraged |

\section{Possible subjective claims}

Foregrounded Immediate

I want to share my background as a student with you. I want you to know that I am not like most faculty.

Less Foregrounded, Less Immediate

I want you to start believing in yourself as a writer.

Backgrounded

I want to teach you more than just how to write papers. I want to open up the secret world of academia for you.

\section{Possible objective claims}

Very Foregrounded, Very Immediate

The students who already know me can add to the conversation. Professors talk about academics and/or their personal lives in class.

Highly Backgrounded, Remote

Professors can talk about what is wrong at the university level to get their point across.

\section{Possible normative-evaluative claims}

Quite Foregrounded, Quite Immediate

It is right to explain to students how the process of this class originated. It is bad when faculty keep secrets from their students about career paths or writing papers.

Less Foregrounded, Less Immediate

It was not right when faculty do not believe in students' potential or teach them exactly how to become a faculty member. Faculty should encourage and prepare their students. Backgrounded, Remote

Professors should share connections with their students to reduce power relations in the classroom. 
56. Professor: [laugh]

57. Professor: It's getting more and more crowded, there's two seats up here. There's one here, this one's mine ||| Nobody wants to sit by me, Johnny.

58. Johnny: [laugh]

59. Student: You're so ()

60. Professor: ||| All right, you know what, we are expecting some more. So, you're going to have to sit there, I hope you're ()

61. Student (undecipherable) [laugh]

62. Professor: And |||

63. Professor: Actually, Miguel ^ was going to put the desks this way, but I thought this would be a better way. I think you might have a little more room sort of. If there's a fire drill though

64. Students: [laugh]

65. Professor: I think this row needs to go out this door so that that group can go out that door. Oh, yeah, the restrooms down this door.

66. Professor: () Where was I in my sad tale?

67. Professor: Oh! The rest of you don't know anything worth getting published, therefore, nobody should teach you how. Now that's one reason. Another ${ }^{\wedge}$ reason is that if you come to graduate school, the assumption is you have a

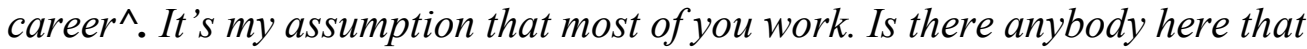
doesn't have a regular job?

68. Professor: $M e$, [laugh].

69. Student: (undecipherable)

70. Professor: All right, but you're in the minority [cough] () but you are, and especially true in the fields that I represent, Adult Education and Human Resource Development.

71. Professor: SO, if you have a job, how many of you never write a thing at work?

72. Professor: Yeah, most of you write at work. Now, how people perceive you at work has a lot to do with how you communicate. Not ${ }^{\wedge}$ the nature of your ideas... how you look, how you sound, whether they can pick up an accent in your writing.

73. Professor: I learned this in Columbus, Ohio, so it has nothing to do with a Spanish accent that I'm talking about. It has everything to do with a Black accent, or a Cleveland accent, ok, or West Virginia. Because even from different places in this country^, we write differently according to where we were brought up and how we talk. And generally^ one thing I can guarantee all of you, I write a whole h- of a lot better than I talk.

74. Professor: [laugh], yeah, and in fact, you can probably read me and not think it was me because it is so good.

75. Students: [laugh]

76. Professor: No cussing nothing ^ [laugh]. The sentences are in the right order [laugh], uh, it's focused, organized, all of the things I'm not in person, [laugh].

77. Professor: All right, SO, these are the reasons that I do this, I think that you've got something important to say. I think that you represent different groups, whether it be graduate students masters' level, graduate students at the doctoral 
level, different ethnic groups, different other kinds of groups, women, African American, Haitians, Jamaican, uh,

78. Student: [cough]

79. Professor: Other groups that in this country have been disenfranchised, and not [cough] allowed in.

80. Professor: OK? So, those are some of the reasons that I'm doing this.

\section{Possible subjective claims}

Foregrounded Immediate

I want to bring culture to your attention. I want to tell you how I write well but do not

appear so when you hear me talk. I want to share with you what I believe about including students of all walks of life.

Less Foregrounded, Less Immediate

I have had experience with different groups (identity claim).

Backgrounded

I want to teach you the connections between writing, difference, and caring about others.

Possible objective claims

Very Foregrounded, Very Immediate

Most graduate students work and write at work. The common assumption of how people look or sound equals their writing ability is not accepted by this professor.

Highly Backgrounded, Remote

This class will be more than about writing. This class will make connections between personal and academic things.

\section{Possible normative-evaluative claims}

Quite Foregrounded, Quite Immediate

It is okay to talk about cultures, different groups, and why they matter in this class. It is okay to write and talk differently.

Less Foregrounded, Less Immediate

It was okay to talk inappropriately and be disorganized while simultaneously being a good writer.

Backgrounded, Remote

It is okay to question our thinking. It is okay to bring up hard to discuss topics in this university classroom.

81. Professor: Now, my process is probably one most of you are not used to, and actually the nurses from summer from spring you didn't get a taste of this because we went on that giant escapade.

82. Students: [laugh] [laugh]

83. Student: You had to bring that up, didn't you?

84. Professor: [laugh]

85. Professor: I'm still living with it. We're still trying to do it.

86. Student: It really $<$ honed my lit search skills $>$.

87. Professor: It's ${ }^{\wedge}$ an important skill if you go on to get a doctorate. [laugh]

88. Professor: All right, so, I don't, I, one thing I learned, I'm never going to do that in that class again and it wasn't, it just wasn't the right place, but at any rate, because I probably will do such a stupid thing again, I know. [laugh] 
89. Professor: All right, So, what I will do, is you'll send me drafts, and given my parameters timewise, I'll look at them and I'll give you feedback. I use track changes and I use comments. And I use short (.) to the point (.) sentences. And I have never learned Spanish, I don't know come from a polite culture. Those of you who haven't noticed that yet. I'm Italian, I'm the oldest, I have four brothers $^{\wedge}$, I was raised in a very male neighborhood ${ }^{\wedge}$, in Cleveland, we're just like New Yorkers and Chicagoans, which means we're not very polite. [laugh]

\section{Possible subjective claims}

Foregrounded Immediate

I want to include students in the conversation and get them to think about their history. I want to teach students about my process of feedback on their papers. I want to remind students about the importance of culture and how that connects to writing.

Less Foregrounded, Less Immediate

I am interested in my students.

Backgrounded

I am a good professor because I learn from my mistakes or admit my mistakes (identity claim).

\section{Possible objective claims}

Very Foregrounded, Very Immediate

Students can be humorous or sarcastic in this classroom. The professor says she is not polite and relates that to her culture.

Highly Backgrounded, Remote

Cultural behaviors will be understood in this class. This is an inclusive class.

\section{Possible normative-evaluative claims}

Quite Foregrounded, Quite Immediate

It is okay to banter humor and sarcasm back and forth with the professor in this class.

Less Foregrounded, Less Immediate

It was important to be tolerant of different processes and cultural behaviors in this class.

Backgrounded, Remote

An inclusive classroom teaches more than just academic material.

90. Professor: Nonetheless, Miguel is here to keep me in check, right? [laugh]

91. Miguel: Yeah.

92. Professor: Right? [laugh]. [laugh]. Good luck to him^^, huh^? [laugh]

93. Professor: So, I just tell you this in way of explanation, because our cultures differ, and there are a lot of you in the room and there's only one of me,

94. Professor: Cuz he can't give you feedback on your writing. No offense, [laugh].

95. Professor: Well, he can and he can't. I want to take that back, because Miguel has come a hell of a long way since I've met him. When ^did I meet ^you? Three years ago?

96. Miguel: 2006.

97. Professor: Only two years ago? It seems like I've known you forever.

98. Professor: Anyhow, [laugh], all right, but Miguel has come a long way, because in the interview, the dissertation, the interview to get in to be a doc student, I didn't understand half of what he said. All I understood was a few references to Habermas, who else? Foucault, uh, [laugh]. 
99. Professor: Anyhow, so, well, you had only been here, what, a year at that point?

100. Miguel: Yeah.

101. Professor: And he did what I think is common for a lot of people that come here from another country to take-go to school and that is they sit silently in classes because they don't want to look stupid. And so you don't speak and you don't learn to speak it. He's forced to talk to me.

102. Professor: So, anyhow, you've got the idea? I'm going to give you feedback within my time parameter. Now some of you may need to make appointments with me, may need to come in during my office hours. If you want help on your papers, though, don't come to me with nothing, come to me with a work plan, come to me with an outline, come to me with a draft of some sort, because I can't work from nothing^ and if you give me something concrete, I can give you something concrete back.

103. Professor: Ok? Now, another thing that is going to be unusual is that I believe writing is process, and I believe it takes as long as it takes to get it right. Richard Smith here is embarking on a paper that I think has the potential to be an article. Not just a class paper, not just a conference paper. And it actually, probably ^ has the potential if he was in another field maybe this one to be a dissertation topic.

104. Professor: And we'll put this up and you'll see.

105. Professor: All right, so I believe writing is a process and all things that are a process, you learn by practicing those skills. Now any of you athletic?

Basketball, soccer, soccer, like I know shit about soccer. Anyhow^, when you are little, most of us are older than death, and don't want to play soccer or basketball anymore, but when we are little, and we used to play these things, we practiced! And all athletes know that they have to practice these skills, and we drill it into ${ }^{\wedge}$ them. But we don't do the same things when it comes to writing, when it comes to reading. We talk about it other ways, we don't talk about these thing in terms of building skills.

106. Professor: Now, I don't think for the most part, the books you have to read, this term, for these classes are going to be difficult reads. In years past, I've used the Handbook of Adult Education and it was a difficult read, because the last version took a postmodern stance. And postmodernists believe that the only good writing is dense circuitous writing, on purpose! OK.

107. Professor: So, and, but it takes practice sometimes, well it does, it takes practice to read academic literature, it takes practice to develop your reading skills, it's just practice, same with writing, so.

108. Professor: I also think some of you should get into peer groups possibly, join forces, send drafts back and forth, though I'm not going to organize that because you're always better off getting outside ()

109. Professor: All right, we're going to start off talking about the components of a paper. I'm gonna walk through what I think are the components of a position paper which is what most of you will be writing in here from the way that I saw your purposes. Then I'm gonna go through some samples, I'm going to walk you 
through Lisa's paper, I'm gonna show you Migulel's paper from two years ago in this class, and uh, and then I'm gonna go through your purpose statements that you sent me, okay?

\section{Possible subjective claims}

Foregrounded Immediate

I am trying to teach you that we are all in this together and that I am not the only one in the room who knows something. We will all work in here.

Less Foregrounded, Less Immediate

I care about working with my students. I will work as hard as my students. Everyone in here will learn to write and more as long as they don't give up. I will not give up.

Backgrounded

I will provide multiple ways for you to learn to write and become tolerant too.

\section{Possible objective claims}

Very Foregrounded, Very Immediate

Students know a lot and can teach others what they know. The professor relinquishes power by giving students the rights to form their own study groups.

Highly Backgrounded, Remote

This is an inclusive class in which everyone knows something. Academics can be learned better when outside influences are related to the topic.

\section{Possible normative-evaluative claims}

Quite Foregrounded, Quite Immediate

It is right to require students to work as hard as the professor. It is right to talk about other students' work and use as models. It is right to make academic decisions (about what to learn, what to read, what to write, the process we will use) based on my past experiences as a student or professor.

Less Foregrounded, Less Immediate

It is good when students can learn academics via everyday examples. It is good when students can learn from each other. It is good to share what I have learned in academic settings with my students.

Backgrounded, Remote

People who work together and share intellectual knowledge will gain a greater understanding of themselves and others which ultimately brings us closer as a society.

110. Professor: Now, the other thing. I generally don't give breaks because I don't remember to give em, and if you really need a break, you've got to consult with Miguel, he's gonna have to work it out and he's gonna have to tell me to stop. Now, otherwise, I'm just going to go (.) until I'm done, [laugh], and then we go home ${ }^{\wedge}$. If you need to get up and go to the bathroom, they're down the hall. Or you can take the elevator or the stairs all the way down^ and go on the first floor. I don't know where they are on the second floor. I don't think I've been on the second floor, um.

111. Student: (undecipherable)

112. Professor: Yeah, you can go out that door and down this hall, yeah, you're really concerned about the bathrooms?

113. Students: [laugh]

114. Student: I'm a nurse. You're worried about if we don't find them. 
115. Professor: So, <papers have parts $>$. Papers have parts, and the papers that you're going to do for me will have three parts. It will have an introduction ${ }^{\wedge}$, a discussion section, and implications. The introduction ${ }^{\wedge}$ section contains some parts $^{\wedge}$.

116. Professor: Are you looking for a chair? We've got two over here. | And there are no other rooms in this place.

117. Professor: But she better be on time next time. [laugh], or she'll be sitting in the hall, [laugh], [laugh].

118. Professor: All right, now (.) a problem ${ }^{\wedge}$ statement. I sent you that article, or the chapter in the book from Merriam and Simpson, where they walk through a problem statement. Now, you're going to hear terms kind of bantied about, you might have already in your travels as students. Some people will talk about a thesis, ok? When you took uh, English, they probably talked about a thesis, um, what other ways do they talk about it? Well, that's good enough, we know that one.

119. Professor: But the thesis^ and the problem statement is the beginning of a paper that grounds your paper in something. Ok? Some one of you wants to do something on obesity, ok? And, uh, I had a student last year that did something on obesity, I don't exactly remember how it went but it was on the educational ${ }^{\wedge}$ value of obesity programs that we have. 
Pilot Study Transcripts

Stage Six A-Building Primary Record from Interviews

(Listen to and Transcribe Meaningful Utterances)

$\begin{array}{ll}\text { Numbered lines } & \text { - Meaningful utterance } \\ \text { (undecipherable) - Talk that is indistinguishable } \\ \text { [laugh] } & \text { - Indicates laughter (outright laugh, something is funny) } \\ \text { Student names } & \text { - Pseudonyms } \\ \text { CAPITALS } & \text { - Talk that is noticeably louder than surrounding talk }\end{array}$

1. [showing videorecording]

2. Miguel: [stopped videorecording at line 49 of classroom observations transcript] Okay. That come up to my mind when when was explain why she sent emails us. um. I have think a lot about this only this class not other class because the professor.

3. Miguel: My reflection is now watching on the tv and up to my mind, it is, um professors have different styles to run a class, to run a coursework, and um, and in this case, for example I have a different style than her.

4. Miguel: I don't go through emails that much that, though, but I don't know and I have told her that people get confused doing that. Umm. But she like that like that style and she really trusts on that style. Specifically, it is when I reply on email, I don't reply to everyone even because even though the information that is inside can benefit someone, uh, I think that the confusion getting so many emails is bigger than the benefit of having that information, the specific information. But what I like to do is just reply person by person.

5. Researcher: Instead of reply to all, is that what you are saying?

6. Miguel: All of them.

7. Researcher: $O k$.

8. Miguel: But umm, but in this case, the professor doesn't like that way,

9. Researcher: [laugh]

10. Miguel: She likes to reply to everyone.

11. Miguel: But I have had and this is part of the reflection, I have had many mentors in my life. Professors, other professors, umm, and I have realized after a while that they were right in the way that they were doing something. So my reflection now it is, um, professor is sort of a dilemma, you know. Professor's style, umm receives a lot of criticism because of you never know, you are younger, you don't have that experience and you don't know if the professor is right in what he is doing.

12. Miguel: In this specific case, I really don't agree. I really think that people is getting confused with her style. And I'm just (undecipherable) an example with something, I think that the the style is not working, but I don't know. That person, she is the professor, she has the (undecipherable), and you never

13. Researcher: You're basing it only on this class but just any class you have had with her or taught with her? 
14. Miguel: It is in all the classes and um, yeah, and um,

15. Researcher: Because this one just started, it's only like the second one

16. Miguel: Yes, it was like the second one or the, yeah, the second one. Yes, but um, yes, and I am I have been thinking about, yes, I will say that her channel to communicate with people is through reading, through emails.

17. Researcher: $O k$.

18. Miguel: Other professor that I am working with (undecipherable) they have a totally different style which is phone calls, drop by in their office, and um.

19. Researcher: ... Johnny, (undecipherable) anything? I heard your name somewhere, did you hear your name? It's so quiet, maybe we should shut (undecipherable) thanks uh

20. Johnny: Well, uh, uh, I will say that uh, that I agree [laugh]

21. Researcher: About with what Miguel - ?

22. Johnny: No, no. I agree with her with her with email.

23. Researcher: Oh, ok, you agree with her email.

24. Johnny: Because uh, many many of the students uh, I think that I got the feeling from many courses that they still thinking in the development of the course as an undergraduate. So when they get over here to the graduate school, Miguel is almost a doctor, you have to put a lot of uh, of getting organized, and uh, not all of them have the I see I got the feeling from (undecipherable) don't got their own discipline to do the readings for example, or do the research, uh, this type of program is for so many different backgrounds, especially we are in Adult Education but uh, (undecipherable) courses

25. Researcher: [laugh]

26. Johnny: And the other part, uh, HRD. It's one of the things that like I say, they talk a different language and bring it back a set of rules, syllabus

27. Researcher: She provides a syllabus, you're saying?

28. Johnny: Yes, the only thing with her is that the syllabus has to be more clear. (undecipherable) writer (undecipherable) many people (undecipherable) $50 \%$ but uh, good to get to know,

29. Johnny: And about the email, to everybody, uh, like I say, our way Latin Americans, that's [laugh] something new to begin with

30. Researcher: $O h ! O k$.

31. Johnny: And here, um, many people, uh, get a little uncomfortable when everybody knows what you're writing

32. Researcher: Reply to all?

33. Johnny: Reply to all, but uh, I think that that put you even to know what you're writing or don't write,

34. Researcher: [laugh]

35. Johnny: Or ask or don't ask, they got the advantages and the disadvantages, I think it is a good tool to get the people in the in the good track. That's that's about it,

36. Johnny: Oh, that Miguel, he's too impatient, (indecipherable) settle [laugh]

37. Researcher: Good, good, anything else?

38. [continue showing videorecording of classroom observations transcript] 
39. Miguel: [stops videorecording at line 55] I have co, comment [laugh]

40. Researcher: Okay [laugh]

41. Miguel: We're never going to finish on time.

42. Researcher: Oh, let's make sure we don't make y'all late to class [laugh]

43. Johnny: No, no we still have time.

44. Researcher: Okay, yeah, I know when we are going to stop, I'm not sure how

45. Miguel: I'm going to be

46. Johnny: [laugh]

47. Miguel: Finally, there's uh, her agenda in this part of the class (undecipherable) and I think that less than 5\% of the class (undecipherable) numbers so I start thinking in who agenda is the class and how you can you know, align your agenda with the classes.

48. Miguel: We never talk with the students about their goals about why they are here and uh, we were talking about that. She knows many of them and probably she is at some point aligned with that agenda but I, I'm not sure.

49. Miguel: So that is first is ah, she bring the agenda.

50. Miguel: She (undecipherable) the comment is, she brought up the 4 degrees and uh, I'm not that old, former young, you know? It's good to be young, and it's good to have many degrees.

51. Johnny: Well, sorry the, about the writing, uh, of course, in one part I agree with Miguel I surely uh base his course in students to open up their eyes like myself, I bring all my life to write, but uh nobody cares or give me a chance to write so I feel uh comfortable, I just write for the papers whenever I don't like what they write,

52. Johnny: But uh, right now I see the young professionals more worry as about how much money they gonna make.

53. Johnny: I really follow up on develop their fields.

54. Johnny: So, what I get from the university some boards, and everything else, all scholars first of all, are getting old, they are reading the same thing, in the syllabus they got a book book or field that we read today I saw about two or three quotes from the same one from the other book.

55. Researcher: So this was in another class?

56. Johnny: No, no, book for, ah, people are famous in this thing (undecipherable)

57. Researcher: Oh, I see, I see

58. Johnny: So, it's good, it's good, and uh, really, I appreciate uh, what she's do but uh, I hope that she uh check the everybody's files, the office and more or less see uh

59. Researcher: You're talking about the -

60. Johnny: Who's going to be interested in that, however, my opinion.

61. Johnny: Here in the states, pro-professional that doesn't know how to communicate is is in bad shape, because here they want to speak, they want to write, they want to get up and say openly what they feel and what they like and what they don't like is they want to (undecipherable) It's like that in our society now. Everybody is doing the things for us. And look the mess that we are in today 
because the young people have to do something about and to write is one of the things,

62. Johnny: But he can no fix the problem that comes directly come from middle and high school, so, that's something that the university should get uh, do with them with that respect.

63. Researcher: What do you mean when you said -

64. Johnny: Well, as in relation to what Miguel said, you know, our class, you see the faces, probably he sees them better [laugh] from his corner and uh, you talk to the people and they are in in different planet when they (undecipherable)

65. Researcher: But they, what they, like he said, their goals, is that what you are talking about?

66. Johnny: No, uh, like I say, they got different interests, uh, before you put them

67. Researcher: Ok, I think I understand.

68. Johnny: To (undecipherable) or get a, or become a good writer, you know, WRITE or think about,

69. Researcher: Something,

70. Johnny: However, however, maybe those people never have any, a person talk to them like she does, so I believe that that's the merit Professor, because I don't think, uh, I haven't got in touch in my life, and it's very long one somebody that uh, push so much that the people learn to spread themself. But I say this, this is very important, very important.

71. Johnny: [stopped videorecording at line 110] [cough] I've got a comment. When she's gonna start explaining how to write a paper, uh, in undergraduate work, we used to take English (undecipherable), English, right, and there are other teachers who don't write well your report but uh, we work in MLA help we present and we come to the graduate and you gotta change to the APA [laugh]

72. Researcher: $O h$, [laugh] the MLA, the APA, right,

73. Johnny: Oh, eh, some other people that'll that one, but uh, but actually I guess it's too much improvisation, actually don't learn how to write in undergrad

74. Researcher: Oh, in undergrad?

75. Johnny: With exception, for example, somebody can go to the law school, or have a good family, and plus the, diction (undecipherable) for your talk, that comes out from the family, [laugh] a good family background and you read well and uh, because you read and report knowledge better so you can take (undecipherable).

76. Johnny: Some of the teachers that really know you through what you write, they get the image of the student, uh, you know but uh you can write they probably think that you are a dummy. (undecipherable) Label, level is uh, the advantage is the classes are small, get the chance to know.

77. Johnny: But uh, like I said I wish there was some force like uh, the job is doing here, (undecipherable) group, uh, (undecipherable) they are doing some good job,

78. Johnny: They want, want, they have to learn to write they don't want to be just one more, make the money, and that's it, but (undecipherable) some paralegal and do the job

79. Researcher: I had one question. She calls on you a lot in class. What's happening when she does that, I guess, I mean, I don't know what you're thinking, 
80. Miguel: I think that in the in the rational part, (undecipherable) but the yes, when she (undecipherable) again, but I think that it's okay, there's nothing wrong with that in all your,

81. Researcher: I didn't know what you were thinking, but I did notice she called on you a lot and,

82. Miguel: Also, I think it is part of her style

83. Researcher: So, as a support of what she is saying or as an example? Oh, ok.

84. Miguel: It's it's yes, she what I think is is part of selling, you know, (undecipherable) that connect part of buy in in the, always those agenda are aligned with, though she's selling that, you know, (undecipherable) I view to try to get support, with the ultra thing that some time there is a trick there, and she's (undecipherable) eyeing that,

85. Miguel: Oh, I don't think that it is a rational thing, but behind that, um, I mean, not easy to follow,

86. Miguel: To me, not that true that everyone is worker, you know, everyone can do something, everyone can write, yeah, everyone can write, but to publish, you have to overcome very well established.

87. Miguel: And um, 50\% of people that buy the agenda that she (undecipherable) time with what they want is when she is supporting with simple examples, exceptions, that I think for most of the people I will do say waste of waste of time (undecipherable)

88. Researcher: She calls on you too.

89. Johnny: Yeah, and I, like I say, we are speculating too but, uh, being what she is, I guess, uh, program

90. Miguel: Program Leader

91. Johnny: For College of Education. One, patience on how that our university publishes and uh, I don't know the story behind the, the I don't know the university uh, publish, (undecipherable) years later,

92. Johnny: Uh, like I say, maybe that from ten people may be interested

93. Miguel: Yeah, I know,

94. Johnny: But I guess the idea of her is getting involved and seeing if, you know, we hit somebody because uh,

95. Johnny: Professor must be conscious that many of the people there are waiting for 9:05 or whatever

96. Researcher: It is over,

97. Johnny: That one other thing is the way the course and everybody to do the reading and the

98. Miguel: Yeah, I- I rise that question that possible because in this university system public research university so she has to make people publish and she has to press to do

99. Miguel: And perhaps she's most probably she's conscious that other people is waiting, but I wonder if if she checked the agenda on that less people would want to be waiting in the class?

100. Researcher: That's a good question, 
101. Miguel: And I wonder if that is possible because there is a system here playing a role

102. Researcher: System, that's what you [to Johnny] brought up too.

103. Johnny: Yeah, but, uh, at least she's the head on our school

104. Researcher: [laugh]

105. Johnny: In our program, you know, and I guess, but uh, she's doing it, she's worry about later on, the, that any university (undecipherable) UCLA or Cal State, and California (undecipherable) State (undecipherable), they worry, they call you, see where you are, what you have been done, doing, uh, they just wrote they published a 50 years book,

106. $\quad$ Researcher: Uh-huh, what people are doing

107. Johnny: People and they got

108. Miguel: They include you

109. Johnny: Yeah! [laugh]

110. Researcher: [laugh] Huh,

111. Johnny: So they are happy, this is my graduate, over here, you know, you don't, you, uh, say, for example, you know,

112. Johnny: Even that maybe writing, punctuating, (undecipherable), there is one part of of getting the feeling getting confidence self reward.

113. Johnny: And I say it once, I say it a thousand times, right now, if you're professional, and you have to speak in public, you won't know where because somebody will do it for you.

114. Johnny: And uh, that that that is or you're in and you're know is not really interested we have to push with different angles. What I see is that probably another institution within the university get involved along with her. For example, the, what they call it, the graduate association (undecipherable) resume out to you help prepare the kids for that, another one for who want to be teachers but the other things people involved people don't learn in the books learn in the class. That is the Academy of Teaching you people have in the university but nobody knows, nobody knows is the other part, and I don't know if the K-12 part with Dr. L or Dr. L or what ever the name, what she's doing around that part. Cuz, uh, it's even worse, they say that we need teachers but the teachers get out they don't get a job.

115. Researcher: Get your teacher [laugh]

116. Miguel: What are the other numbers?

117. Researcher: Do you want to watch the last 2 minutes? Don't want you to get in trouble.

118. Johnny: Where, so little done, oh 2 minutes you said it's 20.

119. [continue showing videorecording of classroom observations]

120. Researcher: She's talking about the obesity remember,

121. Johnny: [stopped videorecording at line 119 of classroom observations transcript] No, what happened is, uh do you have any oth-whatever you have written there do you have uh? 
122. Researcher: Well, my my goal here was to just have, I could see if you are thinking in any way like I am thinking to merge it with my analysis. And one of my questions was, that's one of my big questions, one of the other ones, laughter.

123. Johnny: Her what?

124. Researcher: Her laughter. When I did the, when I did this, the script, laugh laugh laugh laugh laugh laugh was all (undecipherable) out. Have you ever thought about that? [laugh]

125. Johnny: But she have uh,

126. Professor: Because many profe-

127. Johnny: They have their own way to say things, [laugh] and uh maybe the style of whatever, Miguel, Miguel know her, but uh,

128. Johnny: But we can call an education the classroom management uh,

129. Johnny: Talking here with adults many already have (undecipherable) and though,

130. Johnny: Yeah, that's selling more than the idea than regular undergraduate school, that's the difference between regular level and graduate level because it's the selling of the class management, that you perform, the way that you have to perform,

131. Johnny: But uh, one is to (undecipherable) to focus on what uh, (undecipherable) you want (undecipherable) now the other may be testing how much you know about what she think that you know (undecipherable)

132. Johnny: With the silent (undecipherable) for, I guess she get a little nervous and then he get on him or get on me whatever, that she's more, you know, that had more uh, more understanding to get an answer maybe, but uh, that that's her class and her style. That's her class and her style and uh,

133. Johnny: I would say that uh, people who come over here, I see it, (undecipherable) read this and, in my opinion, they won't be able to read that. [laugh]

134. Miguel: What I think about that, it is, uh, well, the, bathroom (undecipherable) especially hard for me because I don't (undecipherable) half of her jokes

135. Johnny: [laugh]

136. Miguel: And so there are very cultural and very

137. Researcher: Ok, that's a that's a cultural thing which is another thing I know (undecipherable) ways (undecipherable) that I never thought of that

138. Miguel: It is uh, it is uh, I don't, it's uh, I just don't understand the joke, I feel one of

139. Researcher: You don't catch the, you don't catch what's funny

140. Miguel: One, yeah, one, I think one of the harder things to get from a culture is the

141. Johnny: Well, uh, one one of the things uh talking about culture, uh, I think from what I got the chance to (undecipherable) Here's a very sensitive woman (undecipherable) the effect will have a hundred years (undecipherable) So that is one thing that (undecipherable) talking about (undecipherable) 
142. Johnny: But also the style and uh, what she's trying to get to the other ones because she mention and cuz she knows a lot, (undecipherable)

143. Johnny: One of the things was the doctoral student is writing a paper about very whatever, (undecipherable) the other and what to teach, like uh, one of the things uh, talking about is social justice. Social justice is uh 40 years so many fields - with adult education. That that part I didn't know until the readings that we have in (undecipherable) I'm really surprised

144. Researcher: What's the name of this course?

145. Johnny: Huh?

146. Researcher: What's the name of this course?

147. Miguel: Individual learning

148. Researcher: What?

149. Miguel: Individual learning

150. Researcher: Oh, my goodness, I thought it was writing for publication or something, I don't know.

151. Johnny: No, no.

152. Researcher: Oh, really?

153. Johnny: So,

154. Miguel: Yeah, there's, yeah,

155. Researcher: Oh, [laugh]

156. Johnny: It's, it's, and uh, well, he [nod toward Miguel] read already all the articles and books, I don't, so many of the things that I mention to you is because I had the chance to (undecipherable) take a part today. They call it, uh, (undecipherable) -cal justice

157. Miguel: (undecipherable) Mental justice

158. Johnny: (undecipherable) Mental justice

159. Miguel: I'm going to just

160. Researcher: Tell her we are coming. We can stop, Miguel, because she has allowed us to do this, so,

161. Miguel: Well, she knows where we are too

162. Johnny: That environmental justice, to tell you the true, I

163. Miguel: I have my last comment about the laughing stuff

164. Researcher: [laugh]

165. Miguel: For your records

166. Johnny: [laugh]

167. Researcher: I hope it's recorded [laugh] or I'll have to get y'all again [laugh]

168. Miguel: It is her, I think she, one of her abilities is to manage, her moods, um, very good, I mean, I mean, she can change moods drastically

169. Researcher: Um-huh

170. Miguel: From being very upset and pissed off to be very, you know,

171. Johnny: (undecipherable)

172. Miguel: Happy and and laughing

173. Johnny: (undecipherable) Sometimes she get mad [laugh]

174. Researcher: [laugh] [accidentally touches microphone] Oops [laugh] 
175. Miguel: So, uh, I (undecipherable) she use uh, she use the laughing style for, or, it's part of her style, it's part of her style. She can say very, you know, tough,

176. Johnny: Very strong

177. Miguel: Very strong comments. She can make very strong comments. And uh, and then, she, uh, she change the moods of the room, you know, (undecipherable)

178. Johnny: Well, uh, one one of the things, uh, that I may say at this time and I got a little uh problem with her right in the second class last last quarter is because she is very very direct, $u$,

179. Johnny: In a woman and as a teacher, that's good, at this level,

180. Johnny: But you can no be that way with everybody because [laugh] you don't know the backgrounds.

181. Johnny: And then it's different by that in the (undecipherable) I will say the environment so diversified that we have

182. Miguel: I thinking if if (undecipherable) have the skill to laugh about herself and about situations, that defect, or that, you know, problem that she has that she doesn't differentiate (undecipherable) whom she's talking and um, abilities to open spaces would be really bad

183. Johnny: Yeah [laugh]

184. Researcher: [laugh]

185. Miguel: So I think it is because she can change her mood to use the sense of humor to (undecipherable) in rooms and balance, at some point, you know, she can balance her style which I think I agree sometimes (undecipherable) and work with somebody

186. Researcher: That's a good point cuz it was throughout just all you know, so that's a theme, you know, that's a good part of having (undecipherable)

187. Miguel: Yeah, she's very extreme, she is very extreme, you know (undecipherable)

188. Researcher: [laugh]

189. Johnny: [laugh] But but she she know what she's doing, she know, we know

190. Researcher: [laugh]

191. Miguel: Well, I hope so [laugh] I hope so she know [starts walking toward door]

192. Researcher: I thank y'all so much, I hope this worked, oh, my gosh, I'll come get my stuff. 
Pilot Study Transcripts

Stage Seven A- Reconstructive Data Analysis of Interviews

(Initial Meaning Reconstruction: Low-Level Coding)

(Categories That Name Interactions)

Italicized headings - General code categories that name interactions
Numbered lines $\quad$ - Codes that give examples of category
Very objective $\quad$ - Anyone in the room would agree
Slight objectivity
Medium objectivity - Most people in the room would probably agree

Low-level Coding of Student Interview

General Code Category and Codes

Ways of talking about professor's style

1. Different styles among professors or teaching assistants

2. Hers is to send emails

3. Hers is to reply to all

4. Different styles than students

5. Confusion of emails is bigger than benefit

6. People have reasons for their styles

7. People trust their own styles

8. Same style in all of her classes

9. Communication channel through reading and emails

10. Calls on students as part of her style to provide examples and get new students to buy in to her agenda

11. Emails cause confusion

12. Maybe the professor is right even if observer or student thinks otherwise

13. Style not working

14. 50\% of students get confused with her style

15. Talk different language

16. Syllabus not clear enough

17. Reply to all uncomfortable for some people

18. Email something new to some cultures (Latin Americans)

19. Reply to all may be a way to get people on the right track

20. Advantages and disadvantages with reply to all

21. Syllabus has books that repeat things and quotes

22. Syllabus needs updating

23. Need to check students' files and interests and include in course agenda

25. If want to get students to become involved and become better writers, then find out their interests and goals first

26. If checked students' goals, would they still be waiting
Level of Objectivity

Very

Very

Very

Very

Very

Very

Medium

Very

Medium

Very

Medium

Very

Medium

Very

Very

Medium

Medium

Very

Very

Very

Very

Medium

Medium

Very

Medium 
for class to end?

Medium

27. Calls on students to fill in silence

Medium

28. Calls on students to sell classroom management

Very

29. Calls on students to test what students know

Medium

30. Influences students down the long run

Slight

31 . Very direct

32. Can't be direct with everybody

Very

Very

33. Makes strong comments

34. Can't open spaces if don't differentiate uptake from

Very students

Medium

35. Very extreme

Very

Ways of talking about students' perceptions of course

1. Wonder if professor is right in their style

Very

2. Undergraduate mindset

3. Not disciplined yet to do readings

Medium

Medium

Very

4. From different backgrounds

Very

5. Talk different language

Medium

6. Different set of rules as in syllabus

Medium

7. Need to be careful what you write since reply to all everyone sees

8. Course opens up students' eyes

Very

Very

9. Somebody cares

10. Somebody gives students a chance to write

Very

11. More worried about making money than writing

Very

12. Not following up getting better in fields

Very

13. It is important for students to be interested in course

Medium

14. It is important for students to be included in the agenda

Very

15. Most don't understand readings

Very

Very

16. Students' faces tell they are waiting for class to end

17. Everyone can work and write but not everyone can publish

18. Professor can get mad

Very

Very

Very

Ways of talking about professor's agenda Very

1. Less than 5\% students understand her agenda Very

2. Not aligned with classes

Very

3. Knows many students but may not align with agenda

Very

4. Never talk about students' agendas

Very

5. Multiple degrees is good

Very

6. Being young is good

Very

7. Merit in that she pushes people and talks to them about what they can do

Very

8. Selling agenda through student examples

Very

9. $50 \%$ of students will jump on writing agenda and try 
to publish Very

10. $50 \%$ of students will be disappointed or not join agenda Very

11. Simple examples are discussed so students will join writing agenda

12. Works with other K-12 professor on writing agenda

13. To teach social justice

14. To teach what she knows which is a lot

15. To provide mental justice

16. Is probably conscious of students waiting for 9:05

Medium

Very

Very

Very

Medium

Very

Ways of talking about institutional and societal influences Medium

1. Dilemma being a professor Very

2. Professors criticized by students, especially younger ones Very

3. Authors using same quotes (gets old)

Medium

4. People need to know how to communicate to get ahead in society

5. Writing is one of the ways to get ahead

6. Must be well established to become published

7. University needs to become involved in solving the mess we are in

Medium

Medium

Very

Very

Very

9. Graduate and undergraduate different and students have to relearn things such as APA style

10. Don't learn to write well in undergraduate

Very

Medium

11. Family background sets the stage for who has already learned, who learns well, who can communicate, who is ahead and so on

12. Teachers judge students by how they write and communicate

13. Dummy classes - one advantage is they are smaller

14. Bad writers are put in dummy classes

15. Program leader must push publication

16. System plays a role

Very

17. Program leader's worries about publishing affects agenda

18. University areas should work together

19. Working together at university level would help with professor's agenda

20. As woman, good to be direct and strong at this level

21. She knows what she is doing

22. Education is classroom management

23. Self confidence booster when write better

Medium

Medium

Meduim

Very

Very

Very

Very

Very

Very

Very

Very

Medium

Ways of talking about laughter V Very

1. Her own style Very

2. Classroom management Very 
3. Selling agenda at graduate level, differ from undergrad

Medium

4. Cultural humor hard to understand

Very

5. Manage moods very well

Very

6. Part of her style

Very

7. Changes mood of room

Very

8. Backgrounds accept differently

Very

9. Diversified environment must be considered

Very

1. [showing videorecording from classroom observations]

2. Miguel: [stopped videorecording at line 49 of classroom observations transcript] Okay. That come up to my mind when when was explain why she sent emails us. um. I have think a lot about this only this class not other class because the professor.

3. Miguel: My reflection is now watching on the tv and up to my mind, it is, um professors have different styles to run a class, to run a coursework, and um, and in this case, for example I have a different style than her.

4. Miguel: I don't go through emails that much that, though, but I don't know and I have told her that people get confused doing that. Umm. But she like that like that style and she really trusts on that style. Specifically, it is when I reply on email, I don't reply to everyone even because even though the information that is inside can benefit someone, uh, I think that the confusion getting so many emails is bigger than the benefit of having that information, the specific information. But what I like to do is just reply person by person.

5. Researcher: Instead of reply to all, is that what you are saying?

6. Miguel: All of them.

7. Researcher: $O k$.

8. Miguel: But umm, but in this case, the professor doesn't like that way,

9. Researcher: [laugh]

10. Miguel: She likes to reply to everyone.

11. Miguel: But I have had and this is part of the reflection, I have had many mentors in my life. Professors, other professors, umm, and I have realized after a while that they were right in the way that they were doing something. So my reflection now it is, um, professor is sort of a dilemma, you know. Professor's style, umm receives a lot of criticism because of you never know, you are younger, you don't have that experience and you don't know if the professor is right in what he is doing.

12. Miguel: In this specific case, I really don't agree. I really think that people is getting confused with her style. And I'm just (undecipherable) an example with 
something, I think that the the style is not working, but I don't know. That person, she is the professor, she has the (undecipherable), and you never

13. Researcher: You're basing it only on this class but just any class you have had with her or taught with her?

14. Miguel: It is in all the classes and um, yeah, and um,

15. Researcher: Because this one just started, it's only like the second one

16. Miguel: Yes, it was like the second one or the, yeah, the second one. Yes, but um, yes, and I am I have been thinking about, yes, I will say that her channel to communicate with people is through reading, through emails.

17. Researcher: $O k$.

18. Miguel: Other professor that I am working with (undecipherable) they have a totally different style which is phone calls, drop by in their office, and um.

19. Researcher: ... Johnny, (undecipherable) anything? I heard your name somewhere, did you hear your name? It's so quiet, maybe we should shut (undecipherable) thanks uh

20. Johnny: Well, uh, uh, I will say that uh, that I agree [laugh]

21. Researcher: About with what Miguel - ?

22. Johnny: No, no. I agree with her with her with email.

23. Researcher: Oh, ok, you agree with her email.

24. Johnny: Because uh, many many of the students uh, I think that I got the feeling from many courses that they still thinking in the development of the course as an undergraduate. So when they get over here to the graduate school, Miguel is almost a doctor, you have to put a lot of uh, of getting organized, and uh, not all of them have the I see I got the feeling from (undecipherable) don't got their own discipline to do the readings for example, or do the research, uh, this type of program is for so many different backgrounds, especially we are in Adult Education but uh, (undecipherable) courses

25. Researcher: [laugh]

26. Johnny: And the other part, uh, HRD. It's one of the things that like I say, they talk a different language and bring it back a set of rules, syllabus

27. Researcher: She provides a syllabus, you're saying?

28. Johnny: Yes, the only thing with her is that the syllabus has to be more clear. (undecipherable) writer (undecipherable) many people (undecipherable) 50\% but uh, good to get to know,

29. Johnny: And about the email, to everybody, uh, like I say, our way Latin Americans, that's [laugh] something new to begin with

30. Researcher: $O h$ ! Ok.

31. Johnny: And here, um, many people, uh, get a little uncomfortable when everybody knows what you're writing

32. Researcher: Reply to all?

33. Johnny: Reply to all, but uh, I think that that put you even to know what you're writing or don't write,

34. Researcher: [laugh] 
35. Johnny: Or ask or don't ask, they got the advantages and the disadvantages, I think it is a good tool to get the people in the in the good track. That's that's about it,

36. Johnny: Oh, that Miguel, he's too impatient, (indecipherable) settle [laugh]

37. Researcher: Good, good, anything else?

38. [continue showing videorecording of classroom observations transcript]

39. Miguel: [stops videorecording at line 55] I have co, comment [laugh]

40. Researcher: Okay [laugh]

41. Miguel: We're never going to finish on time.

42. Researcher: Oh, let's make sure we don't make y'all late to class [laugh]

43. Johnny: No, no we still have time.

44. Researcher: Okay, yeah, I know when we are going to stop, I'm not sure how

45. Miguel: I'm going to be

46. Johnny: [laugh]

47. Miguel: Finally, there's uh, her agenda in this part of the class (undecipherable) and I think that less than 5\% of the class (undecipherable) numbers so I start thinking in who agenda is the class and how you can you know, align your agenda with the classes.

48. Miguel: We never talk with the students about their goals about why they are here and uh, we were talking about that. She knows many of them and probably she is at some point aligned with that agenda but I, I'm not sure.

49. Miguel: So that is first is ah, she bring the agenda.

50. Miguel: She (undecipherable) the comment is, she brought up the 4 degrees and uh, I'm not that old, former young, you know? It's good to be young, and it's good to have many degrees.

51. Johnny: Well, sorry the, about the writing, uh, of course, in one part I agree with Miguel I surely uh base his course in students to open up their eyes like myself, I bring all my life to write, but uh nobody cares or give me a chance to write so I feel uncomfortable, I just write for the papers whenever I don't like what they write,

52. Johnny: But uh, right now I see the young professionals more worry as about how much money they gonna make.

53. Johnny: Not really follow up on develop their fields.

54. Johnny: So, what I get from the university some boards, and everything else, all scholars first of all, are getting old, they are reading the same thing, in the syllabus they got a book book or field that we read today I saw about two or three quotes from the same one from the other book.

55. Researcher: So this was in another class?

56. Johnny: No, no, book for, ah, people are famous in this thing (undecipherable)

57. Researcher: Oh, I see, I see

58. Johnny: So, it's good, it's good, and uh, really, I appreciate uh, what she's do but uh, I hope that she uh check the everybody's files, the office and more or less see uh

59. Researcher: You're talking about the -

60. Johnny: Who's going to be interested in that, however, my opinion. 
61. Johnny: Here in the states, pro-professional that doesn't know how to communicate is is in bad shape, because here they want to speak, they want to write, they want to get up and say openly what they feel and what they like and what they don't like is they want to (undecipherable) It's like that in our society now. Everybody is doing the things for us. And look the mess that we are in today because the young people have to do something about and to write is one of the things,

62. Johnny: But he can no fix the problem that comes directly come from middle and high school, so, that's something that the university should get uh, do with them with that respect.

63. Researcher: What do you mean when you said -

64. Johnny: Well, as in relation to what Miguel said, you know, our class, you see the faces, probably he sees them better [laugh] from his corner and uh, you talk to the people and they are in in different planet when they (undecipherable)

65. Researcher: But they, what they, like he said, their goals, is that what you are talking about?

66. Johnny: No, uh, like I say, they got different interests, uh, before you put them

67. Researcher: Ok, I think I understand.

68. Johnny: To (undecipherable) or get a, or become a good writer, you know, WRITE or think about,

69. Researcher: Something,

70. Johnny: However, however, maybe those people never have any, a person talk to them like she does, so I believe that that's the merit Professor, because I don't think, uh, I haven't got in touch in my life, and it's very long one somebody that uh, push so much that the people learn to spread themself. But I say this, this is very important, very important.

71. Johnny: [stopped videorecording at line 110] [cough] I've got a comment. When she's gonna start explaining how to write a paper, uh, in undergraduate work, we used to take English (undecipherable), English, right, and there are other teachers who don't write well your report but uh, we work in MLA help we present and we come to the graduate and you gotta change to the APA [laugh]

72. Researcher: $O h$, [laugh] the MLA, the APA, right,

73. Johnny: Oh, eh, some other people that'll that one, but uh, but actually I guess it's too much improvisation, actually don't learn how to write in undergrad

74. Researcher: Oh, in undergrad?

75. Johnny: With exception, for example, somebody can go to the law school, or have a good family, and plus the, diction (undecipherable) for your talk, that comes out from the family, [laugh] a good family background and you read well and uh, because you read and report knowledge better so you can take (undecipherable).

76. Johnny: Some of the teachers that really know you through what you write, they get the image of the student, uh, you know but uh you can write they probably think that you are a dummy. (undecipherable) Label, level is uh, the advantage is the classes are small, get the chance to know.

77. Johnny: But uh, like I said I wish there was some force like uh, the job is doing here, (undecipherable) group, uh, (undecipherable) they are doing some good job, 
78. Johnny: They want, want, they have to learn to write they don't want to be just one more, make the money, and that's it, but (undecipherable) some paralegal and do the job

79. Researcher: I had one question. She calls on you a lot in class. What's happening when she does that, I guess, I mean, I don't know what you're thinking,

80. Miguel: I think that in the in the rational part, (undecipherable) but the yes, when she (undecipherable) again, but I think that it's okay, there's nothing wrong with that in all your,

81. Researcher: I didn't know what you were thinking, but I did notice she called on you a lot and,

82. Miguel: Also, I think it is part of her style

83. Researcher: So, as a support of what she is saying or as an example? Oh, ok.

84. Miguel: It's it's yes, she what I think is is part of selling, you know, (undecipherable) that connect part of buy in in the, always those agenda are aligned with, though she's selling that, you know, (undecipherable) I view to try to get support, with the ultra thing that some time there is a trick there, and she's (undecipherable) eyeing that,

85. Miguel: Oh, I don't think that it is a rational thing, but behind that, um, I mean, not easy to follow,

86. Miguel: To me, not that true that everyone is worker, you know, everyone can do something, everyone can write, yeah, everyone can write, but to publish, you have to overcome very well established.

87. Miguel: And um, 50\% of people that buy the agenda that she (undecipherable) time with what they want is when she is supporting with simple examples, exceptions, that I think for most of the people I will do say waste of waste of time (undecipherable)

88. Researcher: She calls on you too.

89. Johnny: Yeah, and I, like I say, we are speculating too but, uh, being what she is, I guess, uh, program

90. Miguel: Program Leader

91. Johnny: For College of Education. One, patience on how that our university publishes and uh, I don't know the story behind the, the I don't know the university uh, publish, (undecipherable) years later,

92. Johnny: Uh, like I say, maybe that from ten people may be interested

93. Miguel: Yeah, I know,

94. Johnny: But I guess the idea of her is getting involved and seeing if, you know, we hit somebody because uh,

95. Johnny: Professor must be conscious that many of the people there are waiting for 9:05 or whatever

96. Researcher: It is over,

97. Johnny: That one other thing is the way the course and everybody to do the reading and the

98. Miguel: Yeah, I- I rise that question that possible because in this university system public research university so she has to make people publish and she has to press to do 
99. Miguel: And perhaps she's most probably she's conscious that other people is waiting, but I wonder if if she checked the agenda on that less people would want to be waiting in the class?

100. Researcher: That's a good question,

101. Miguel: And I wonder if that is possible because there is a system here playing a role

102. Researcher: System, that's what you [to Johnny] brought up too.

103. Johnny: Yeah, but, uh, at least she's the head on our school

104. Researcher: [laugh]

105. Johnny: In our program, you know, and I guess, but uh, she's doing it, she's worry about later on, the, that any university (undecipherable) UCLA or Cal State, and California (undecipherable) State (undecipherable), they worry, they call you, see where you are, what you have been done, doing, uh, they just wrote they published a 50 years book,

106. Researcher: Uh-huh, what people are doing

107. Johnny: People and they got

108. Miguel: They include you

109. Johnny: Yeah! [laugh]

110. Researcher: [laugh] Huh,

111. Johnny: So they are happy, this is my graduate, over here, you know, you don't, you, uh, say, for example, you know,

112. Johnny: Even that maybe writing, punctuating, (undecipherable), there is one part of of getting the feeling getting confidence self reward.

113. Johnny: And I say it once, I say it a thousand times, right now, if you're professional, and you have to speak in public, you won't know where because somebody will do it for you.

114. Johnny: And uh, that that that is or you're in and you're know is not really interested we have to push with different angles. What I see is that probably another institution within the university get involved along with her. For example, the, what they call it, the graduate association (undecipherable) resume out to you help prepare the kids for that, another one for who want to be teachers but the other things people involved people don't learn in the books learn in the class. That is the Academy of Teaching you people have in the university but nobody knows, nobody knows is the other part, and I don't know if the K-12 part with Dr. L or Dr. L or what ever the name, what she's doing around that part. Cuz, uh, it's even worse, they say that we need teachers but the teachers get out they don't get a job.

115. Researcher: Get your teacher [laugh]

116. Miguel: What are the other numbers?

117. Researcher: Do you want to watch the last 2 minutes? Don't want you to get in trouble.

118. Johnny: Where, so little done, oh 2 minutes you said it's 20.

119. [continue showing videorecording of classroom observations]

120. Researcher: She's talking about the obesity remember, 
121. Johnny: [stopped videorecording at line 119 of classroom observations transcript] No, what happened is, uh do you have any oth-whatever you have written there do you have uh?

122. Researcher: Well, my my goal here was to just have, I could see if you are thinking in any way like I am thinking to merge it with my analysis. And one of my questions was, that's one of my big questions, one of the other ones, laughter.

123. Johnny: Her what?

124. Researcher: Her laughter. When I did the, when I did this, the script, laugh laugh laugh laugh laugh laugh was all (undecipherable) out. Have you ever thought about that? [laugh]

125. Johnny: But she have uh,

126. Professor: Because many profe-

127. Johnny: They have their own way to say things, [laugh] and uh maybe the style of whatever, Miguel, Miguel know her, but uh,

128. Johnny: But we can call an education the classroom management uh,

129. Johnny: Talking here with adults many already have (undecipherable) and though,

130. Johnny: Yeah, that's selling more than the idea than regular undergraduate school, that's the difference between regular level and graduate level because it's the selling of the class management, that you perform, the way that you have to perform,

131. Johnny: But uh, one is to (undecipherable) to focus on what uh, (undecipherable) you want (undecipherable) now the other may be testing how much you know about what she think that you know (undecipherable)

132. Johnny: With the silent (undecipherable) for, I guess she get a little nervous and then he get on him or get on me whatever, that she's more, you know, that had more uh, more understanding to get an answer maybe, but uh, that that's her class and her style. That's her class and her style and uh,

133. Johnny: I would say that uh, people who come over here, I see it, (undecipherable) read this and, in my opinion, they won't be able to read that. [laugh]

134. Miguel: What I think about that, it is, uh, well, the, bathroom (undecipherable) especially hard for me because I don't (undecipherable) half of her jokes

135. Johnny: [laugh]

136. Miguel: And so there are very cultural and very

137. Researcher: Ok, that's a that's a cultural thing which is another thing I know (undecipherable) ways (undecipherable) that I never thought of that

138. Miguel: It is uh, it is uh, I don't, it's uh, I just don't understand the joke, I feel one of

139. Researcher: You don't catch the, you don't catch what's funny

140. Miguel: One, yeah, one, I think one of the harder things to get from a culture is the

141. Johnny: Well, uh, one one of the things uh talking about culture, uh, I think from what I got the chance to (undecipherable) Here's a very sensitive 
woman (undecipherable) the effect will have a hundred years (undecipherable) So that is one thing that (undecipherable) talking about (undecipherable)

142. Johnny: But also the style and uh, what she's trying to get to the other ones because she mention and cuz she knows a lot, (undecipherable)

143. Johnny: One of the things was the doctoral student is writing a paper about very whatever, (undecipherable) the other and what to teach, like uh, one of the things uh, talking about is social justice. Social justice is uh 40 years so many fields - with adult education. That that part I didn't know until the readings that we have in (undecipherable) I'm really surprised

144. Researcher: What's the name of this course?

145. Johnny: Huh?

146. Researcher: What's the name of this course?

147. Miguel: Individual learning

148. Researcher: What?

149. Miguel: Individual learning

150. Researcher: Oh, my goodness, I thought it was writing for publication or something, I don't know.

151. Johnny: No, no.

152. Researcher: Oh, really?

153. Johnny: So,

154. Miguel: Yeah, there's, yeah,

155. Researcher: Oh, [laugh]

156. Johnny: It's, it's, and uh, well, he [nod toward Miguel] read already all the articles and books, I don't, so many of the things that I mention to you is because I had the chance to (undecipherable) take a part today. They call it, uh, (undecipherable) -cal justice

157. Miguel: (undecipherable) Mental justice

158. Johnny: (undecipherable) Mental justice

159. Miguel: I'm going to just

160. Researcher: Tell her we are coming. We can stop, Miguel, because she has allowed us to do this, so,

161. Miguel: Well, she knows where we are too

162. Johnny: That environmental justice, to tell you the true, I

163. Miguel: I have my last comment about the laughing stuff

164. Researcher: [laugh]

165. Miguel: For your records

166. Johnny: [laugh]

167. Researcher: I hope it's recorded [laugh] or I'll have to get y'all again [laugh]

168. Miguel: It is her, I think she, one of her abilities is to manage, her moods, um, very good, I mean, I mean, she can change moods drastically

169. Researcher: Um-huh

170. Miguel: From being very upset and pissed off to be very, you know,

171. Johnny: (undecipherable)

172. Miguel: Happy and and laughing 
173. Johnny: (undecipherable) Sometimes she get mad [laugh]

174. Researcher: [laugh] [accidentally touches microphone] Oops [laugh]

175. Miguel: So, uh, I (undecipherable) she use uh, she use the laughing style for, or, it's part of her style, it's part of her style. She can say very, you know, tough,

176. Johnny: Very strong

177. Miguel: Very strong comments. She can make very strong comments. And uh, and then, she, uh, she change the moods of the room, you know, (undecipherable)

178. Johnny: Well, uh, one one of the things, uh, that I may say at this time and I got a little uh problem with her right in the second class last last quarter is because she is very very direct, uh,

179. Johnny: In a woman and as a teacher, that's good, at this level,

180. Johnny: But you can no be that way with everybody because [laugh] you don't know the backgrounds.

181. Johnny: And then it's different by that in the (undecipherable) I will say the environment so diversified that we have

182. Miguel: I thinking if if (undecipherable) have the skill to laugh about herself and about situations, that defect, or that, you know, problem that she has that she doesn't differentiate (undecipherable) whom she's talking and um, abilities to open spaces would be really bad

183. Johnny: Yeah [laugh]

184. Researcher: [laugh]

185. Miguel: So I think it is because she can change her mood to use the sense of humor to (undecipherable) in rooms and balance, at some point, you know, she can balance her style which I think I agree sometimes (undecipherable) and work with somebody

186. Researcher: That's a good point cuz it was throughout just all you know, so that's a theme, you know, that's a good part of having (undecipherable)

187. Miguel: Yeah, she's very extreme, she is very extreme, you know (undecipherable)

188. Researcher: [laugh]

189. Johnny: [laugh] But but she she know what she's doing, she know, we know

190. Researcher: [laugh]

191. Miguel: Well, I hope so [laugh] I hope so she know [starts walking toward door]

192. Researcher: I thank y'all so much, I hope this worked, oh, my gosh, I'll come get my stuff. 
Pilot Study Transcripts

Stage Eight A-High-level Coding

(Supported by Matches between Interview Statements

and Exemplary Pragmatic Horizon Analyses)

\begin{tabular}{|ll|}
\hline Italicized headings & - General code categories that name interactions \\
Numbered lines & - Codes that give examples of category \\
PHA & - Exemplary pragmatic horizon analysis match to support code \\
SI & - Student interview statement match to support code \\
PI & - Professor interview statements match to support code \\
Italicized codes & - Meaningful utterance quoted from interview \\
H & - High level of abstraction (much interpretation involved) \\
VH & - Very high level of abstraction (much higher interpretation) \\
T & - Transcript \\
$\#$ A & - Stage number \\
L & - Line number \\
Student names & - Pseudonyms \\
Turntaking: & \\
R & - Response \\
I & - Initiation \\
I/Q & - Initiation via Question \\
I, Q & - Initiation and a Question \\
E/F & - Evaluation/Feedback \\
Intertextuality: & \\
$I P$ & - Intertextuality Proposed \\
$I A$ & - Intertextuality Acknowledged (superficial knowledge of existence) \\
$I R$ & - Intertextuality Recognized (underlying familiarity and understanding) \\
$S C$ & - Social Consequence \\
NT & - New Topic \\
R & - Request \\
C & - Confirmation \\
X & - Occurrence \\
& \\
\hline
\end{tabular}

High-level Coding

General Code Category and Codes/Level of Abstraction

Ways professor reproduces dominant ideology

1. PHA/VH - T5B1, L33: Conducts required tasks

2. PHA/VH - T5B1, L102: Predominant teacher talk/lecture

3. PHA/VH - T5B1, L49: Lesson phases guided by Power Point slides

4. PHA/H - T5B2ab, L71: Uses traditional I-R-E/F sequence

5. PHA/VH - T5B2ab, L50: Asks questions with known answer and provides short wait time for response 
6. PHA/VH - T5B2ab, L68: Answers own questions

7. PHA/VH - T5B2c, L72L72: Confirms own recognition of intertextuality

8. PHA/H - T5B2c, L70: Represents AE/HRD field in university

9. SI/H - T7A, L 105, Johnny: In our program, you know, and I but uh, she's doing it, she's worry about later on, the, that any university (undecipherable) UCLA or Cal State, and California(undecipherable) State (undecipherable), they worry, they call you, see where you are, what you have been done, doing, uh, they just wrote they published a 50 years book,

10. SI/H - T7A, L, 98, Miguel: Yeah, I- I rise that question that possible because in this university system public research university so she has to make people publish and she has to press to do

Ways students reproduce dominant ideology (good student identity)

1. PHA/VH - T5B1, L10: Answer professor by remote

2. $\mathrm{PHA} / \mathrm{VH}$ - T5B2ab, L100: Wait for end of class, respond if asked

3. SI/H - T7A, L95, Johnny: Professor must be conscious that many of the people there are waiting for 9:05 or whatever

4. PHA/VH - T5B2ab, L36-51: Listen to professor for long stretches

5. SI/H - T7A, L64, Johnny: Well, as in relation to what Miguel said, you know, our class, you see the faces, probably he sees them better [laugh] from his corner and uh, you talk to the people and they are in in different planet when they (undecipherable)

Ways professor resists dominant ideology

1. Teaches about social justice

a. PHA/H - T5B2c, L72: Teaches ways of gaining social access

b. PHA/H - T5B2c, L55: Encourages students to use voice to gain access

c. PHA/VH - T5B2c, L105: Professor uses intertextualities (connections) to confirm social consequences of teaching

d. SI/H - T7A, L143, Johnny: One of the things was the doctoral student is writing a paper about very whatever, (undecipherable) the other and what to teach, like uh, one of the things uh, talking about is social justice. Social justice is uh 40 years so many fields - with adult education. That that part I didn't know until the readings that we have in (undecipherable) I'm really surprised

e. PHA/H - T5, L77: Encourages students to push themselves into uncharted academic and social areas they might not otherwise traverse

f. SI/VH - T7A, L156, Johnny: It's, it's, and uh, well, he [nod toward Miguel] read already all the articles and books, I don't, so many of the things that I mention to you is because I had the chance to (undecipherable) take a part today. They call it, uh, (undecipherable) -cal justice; Miguel: (undecipherable) Mental justice; Johnny: (undecipherable) Mental justice 
g. PHA/H - T5B2ac, L77, 79: Different groups are represented in this class, some of which have been historically disenfranchised, and she will address this issues

h. SI/H - T7A, L76, Johnny: Some of the teachers that really know you through what you write, they get the image of the student, uh, you know but uh you can write they probably think that you are a dummy. (undecipherable) Label, level is uh, the advantage is the classes are small, get the chance to know.

2. Cares about students, whereas system and some other faculty may not

a. PHA/H - T5B1, L9: Wants to know students by name and interests

b. PHA/VH - T5B1, L21: Will help students and not lie to them

c. SI/VH - T7A, L114, Johnny: And uh, that that that is or you're in and you're know is not really interested we have to push with different angles. What I see is that probably another institution within the university get involved along with her. For example, the, what they call it, the graduate association (undecipherable) resume out to you help prepare the kids for that, another one for who want to be teachers but the other things people involved people don't learn in the books learn in the class. That is the Academy of Teaching you people have in the university but nobody knows, nobody knows is the other part, and I don't know if the K-12 part with Dr. $L$ or Dr. L or what ever the name, what she's doing around that part. Cuz, uh, it's even worse, they say that we need teachers but the teachers get out they don't get a job.

d. PHA/H - T5B2c, L55: Teaches students access to world of publication so can further career

e. PHA/H - T5B2c, L38: Explains how she answers students fully

f. PHA/H - T5B2ab, L47: Explains how she is helpful

3. Produces own style of communication

a. PHA/H - T5B2c, L46: Uses email to provide feedback to students

b. SI/H - T7A, L16, 18: Miguel: Yes, it was like the second one [class of the semester] or the, yeah, the second one. Yes, but um, yes, and I am I have been thinking about, yes, I will say that her channel to communicate with people is through reading, through emails .... Other professor that I am working with (undecipherable) they have a totally different style which is phone calls, drop by in their office, and um.

c. $\mathrm{PHA} / \mathrm{H}-\mathrm{T} 5 \mathrm{~B} 2 \mathrm{c}, \mathrm{L} 88,110$ : Direct in communication

d. SI/H - T7A, L178, Johnny: Well, uh, one one of the things, uh, that I may say at this time and I got a little uh problem with her right in the second class last last quarter is because she is very very direct, $u$ h,

e. PHA/VH - T5B2ab, L98: Uses students' experiences as examples for whole class

f. SI/VH - T7A, L132, Johnny: With the silent (undecipherable) for, I guess she get a little nervous and then he get on him or get on me whatever, that she's more, you know, that had more uh, more understanding to get an 
answer maybe, but uh, that that's her class and her style. That's her class and her style and $u$,

g. PHA/VH - T5B2ab, L103: Uses students' work as examples for whole class

h. SI/VH - T7A, L82, Miguel: Also, I think it [calling on students] is part of her style

4. Uses humor

i. PHA/H - T5B2ab, L42: Uses her own work as examples for whole class

a. PHA/VH - T5B2ab, L92: Laughs at herself and situation

b. SI/VH - T7A, L182, Miguel: I thinking if if (undecipherable) have the skill to laugh about herself and about situations, that defect, or that, you know, problem that she has that she doesn't differentiate (undecipherable) whom she's talking and um, abilities to open spaces would be really bad

c. PHA/VH - T5B1, L68: Uses laughter (humor) to manage classroom

d. SI/H - T7A, L27, 28, Johnny: They have their own way to say things, [laugh] and uh maybe the style of whatever, Miguel, Miguel know her, but uh, but we can call an education the classroom management uh,

e. PHA/H - T5B1, L9: Incorporates humor into required tasks

f. SI/VH - T7A, L185: Miguel: So I think it is because she can change her mood to use the sense of humor to (undecipherable) in rooms and balance, at some point, you know, she can balance her style which I think I agree sometimes (undecipherable) and work with somebody. Yeah, she's very extreme, she is very extreme, you know (undecipherable)

5. Pushes own agenda

a. PHA/H - T5B1, L49: Explains the reasons for her process

b. SI/H - T7A, L49, Miguel: So that is first is ah, she bring the agenda.

c. PHA/H - T5B1, L55: Explains why her process is better than other faculty's

d. SI/H - T7A, L2: Okay. That come up to my mind when when was explain why she sent emails us. um. I have think a lot about this only this class not other class because the professor.

e. PHA/VH - T5B2c, L54: Explains the long term social consequences of her process

f. SI/VH - T7A, L50, Miguel: She (undecipherable) the comment is, she brought up the 4 degrees and uh, I'm not that old, former young, you know? It's good to be young, and it's good to have many degrees.

g. PHA/VH - T5B2c, L81: Presents multiple new topics and intertextualities (connections) at once to make her points

h. PHA/H - T5B2ab, L115: Explains paper assignments

6. Teaches about culture

a. PHA/H - T5B2ab, L72, 73: Uses to explain that how people communicate is how others view us

b. PHA/H - T5B2ac, L77, 79: Uses to explain that different groups have been historically disenfranchised

c. PHA/H - T5B2ac, L88: Uses to explain her direct way of communicating 
d. PHA/H - T5B2ab, L93: Uses to explain differences in professor versus student in relation to feedback

e. PHA/H - T5B2ab, L101: Uses to explain access for students new to U.S. university

Ways students resist professor's ideology

1. Communication style

a. SI/H - T7A, L4, Miguel: I don't go through emails that much that, though, but I don't know and I have told her that people get confused doing that. Umm. But she like that like that style and she really trusts on that style. Specifically, it is when I reply on email, I don't reply to everyone even because even though the information that is inside can benefit someone, uh, I think that the confusion getting so many emails is bigger than the benefit of having that information, the specific information. But what I like to do is just reply person by person.

b. SI/H - T7A, L29-33, Johnny: And about the email, to everybody, uh, like I say, our way Latin Americans, that's [laugh] something new to begin with. And here, um, many people, uh, get a little uncomfortable when everybody knows what you're writing .... Reply to all, but uh, I think that that put you even to know what you're writing or don't write,

c. PHA/VH - T5B1, L34-35: Students did not acknowledge getting emails

d. SI/H - T7A, L28, Johnny: Yes, the only thing with her is that the syllabus has to be more clear (undecipherable) writer (undecipherable) many people (undecipherable) 50\% but uh, good to get to know,

e. SI/VH - T7A, L130, Johnny: Yeah, that's selling more than the idea than regular undergraduate school, that's the difference between regular level and graduate level because it's the selling of the class management, that you perform, the way that you have to perform,

f. SI/H - T7A, L142: Johnny: But also the style and uh, what she's trying to get to the other ones [get students who are lost or not interested] because she mention and cuz she knows a lot, (undecipherable)

2. Cultural uptake and interests of students

a. SI/H - T7A, L64-68: Johnny: Well, as in relation to what Miguel said, you know, our class, you see the faces, probably he sees them better [laugh] from his corner and uh, you talk to the people and they are in in different planet when they (undecipherable) .... No, uh, like I say, they got different interests, uh, before you put them to (undecipherable) or get a, or become a good writer, you know, WRITE or think about,

b. SI/H - T7A, L24, Johnny: Because uh, many many of the students uh, I think that I got the feeling from many courses that they still thinking in the development of the course as an undergraduate. So when they get over here to the graduate school, Miguel is almost a doctor, you have to put a lot of uh, of getting organized, and uh, not all of them have the I see I got the feeling from (undecipherable) don't got their own discipline to do the readings for example, or do the research, uh, this type of program is for so 
many different backgrounds, especially we are in Adult Education but uh, (undecipherable) courses

c. SI/H - T7A, L52, Johnny: But uh, right now I see the young professionals more worry as about how much money they gonna make. Not really follow up on develop their fields.

d. PHA/VH - T5B2c, L1-119: Student rarely provides an input

e. SI/H - T7A, L132, Johnny: With the silent (undecipherable) for, I guess she get a little nervous and then he get on him or get on me whatever, that she's more, you know, that had more uh, more understanding to get an answer maybe, but uh, that that's her class and her style. That's her class and her style and $u$,

f. SI/H - T7A, L182: Miguel: I thinking if if (undecipherable) have the skill to laugh about herself and about situations, that defect, or that, you know, problem that she has that she doesn't differentiate (undecipherable) whom she's talking and um, abilities to open spaces would be really bad

g. SI/VH - T7A, L141, Johnny: Well, uh, one one of the things uh talking about culture, uh, I think from what I got the chance to (undecipherable) Here's a very sensitive woman (undecipherable) the effect will have a hundred years (undecipherable) So that is one thing that (undecipherable) talking about (undecipherable).

3. Professor's agenda

a. SI/H - T7A, L84: Miguel: It's it's yes, she what I think is is part of selling, you know, (undecipherable) that connect part of buy in in the, always those agenda are aligned with, though she's selling that, you know, (undecipherable) I view to try to get support, with the ultra thing that some time there is a trick there, and she's (undecipherable) eyeing that, oh, I don't think that it is a rational thing, but behind that, um, I mean, not easy to follow,

b. SI/VH - T7A, L86: Miguel: To me, not that true that everyone is worker, you know, everyone can do something, everyone can write, yeah, everyone can write, but to publish, you have to overcome very well established.

c. SI/H - T7A, L87: Miguel: And um, 50\% of people that buy the agenda that she (undecipherable) time with what they want is when she is supporting with simple examples, exceptions, that I think for most of the people I will do say waste of waste of time (undecipherable)

d. SI/H - T7A, L47: Finally, there's uh, her agenda in this part of the class (undecipherable) and I think that less than 5\% of the class (undecipherable) numbers so I start thinking in who agenda is the class and how you can you know, align your agenda with the classes.

e. SI/H - students' interests not integrated into class

f. SI/H - T7A, L48, 49, Miguel: We never talk with the students about their goals about why they are here and uh, we were talking about that. She knows many of them and probably she is at some point aligned with that agenda but I, I'm not sure. So that is first is ah, she bring the agenda. 
g. SI/VH - T7A, L146: Researcher: What's the name of this course? Researcher: Oh, my goodness, I thought it was writing for publication or something, I don't know.

h. SI/H - T7A, L26, Johnny: And the other part, uh, HRD. It's one of the things that like I say, they talk a different language and bring it back a set of rules, syllabus

4. Professor's humor

a. SI/H - T7A, L134-140, Miguel: What I think about that, it is, uh, well, the, bathroom (undecipherable) especially hard for me because I don't (undecipherable) half of her jokes. And so there are very cultural and very .... It is uh, it is uh, I don't, it's uh, I just don't understand the joke, I feel one of .... One, yeah, one, I think one of the harder things to get from a culture is the

b. SI/H - T7A, L182, Miguel: I thinking if if (undecipherable) have the skill to laugh about herself and about situations, that defect, or that, you know, problem that she has that she doesn't differentiate (undecipherable) whom she's talking and um, abilities to open spaces would be really bad

c. SI/H - T7A, L189, Johnny: [laugh] But but she she know what she's doing, she know, we know

d. PHA/VH - T5B2c, L110-112: Humor used to maintain floor

5. Professor's dilemma

a. SI/H - T7A, L10-11, Miguel: But I have had and this is part of the reflection, I have had many mentors in my life. Professors, other professors, umm, and I have realized after a while that they were right in the way that they were doing something. So my reflection now it is, um, professor is sort of a dilemma, you know. Professor's style, umm receives a lot of criticism because of you never know, you are younger, you don't have that experience and you don't know if the professor is right in what he is doing. In this specific case, I really don't agree. I really think that people is getting confused with her style. And I'm just (undecipherable) an example with something, I think that the the style is not working, but I don't know. That person, she is the professor, she has the (undecipherable), and you never

b. SI/H - T7A, L99-101, Miguel: And perhaps she's most probably she's conscious that other people is waiting [for class to end], but I wonder if if she checked the agenda on that less people would want to be waiting in the class? And I wonder if that is possible because there is a system here playing a role

c. SI/H - T7A, L90-94, Miguel: Program Leader; Johnny: For College of Education. One, patience on how that our university publishes and uh, I don't know the story behind the, the I don't know the university uh, publish, (undecipherable) years later, Uh, like I say, maybe that from ten people may be interested; Miguel: Yeah, I know; Johnny: But I guess the idea of her is getting involved and seeing if, you know, we hit somebody because uh, 
d. SI/H - T7A, L95, Johnny: Professor must be conscious that many of the people there are waiting for 9:05 or whatever

Ways students reproduce professor's ideology

1. SI/H - T7A, L51, open up students' eyes, Johnny: Well, sorry the, about the writing, uh, of course, in one part I agree with Miguel I surely uh base his course in students to open up their eyes like myself, I bring all my life to write, but uh nobody cares or give me a chance to write so I feel uncomfortable, I just write for the papers whenever I don't like what they write,

2. SI/H - T7A, L70, talks to students and pushes them, Johnny: However, however, maybe those people never have any, a person talk to them like she does, so I believe that that's the merit Professor, because I don't think, uh, I haven't got in touch in my life, and it's very long one somebody that uh, push so much that the people learn to spread themself. But I say this, this is very important, very important.

3. SI/H - T7A, L112, writing builds self-confidence, Johnny: Even that maybe writing, punctuating, (undecipherable), there is one part of of getting the feeling getting confidence self reward.

4. SI/H - T7A, L113, good writing provides success in public and career, Johnny: And I say it once, I say it a thousand times, right now, if you're professional, and you have to speak in public, you won't know where because somebody will do it for you.

5. PHA/VH - T5B2ab, L83, use same humor, Student: You had to bring that up, didn't you? 


\section{APPENDIX G}

Summary of Data Collection and Analysis Stages

Stage Components Data Source

Stage One-

Data Collection for the Primary Record

$\begin{array}{ll}\text { Passive Classroom Observations } & \text { Video-recorded Observations } \\ & \text { Primary Record Notes } \\ & \text { Field Journal Notes } \\ & \text { Contact Summary Sheets }\end{array}$

Stage Two-

Building a Primary Record from Classroom Observations

Determine Representative Segment Video-recorded Observations

Stage Three-

Dialogical Data Generation

$\begin{array}{ll}\text { Interviews with Students } & \text { Participants } \\ \text { Interviews with Teachers } & \text { Representative Video Segments } \\ & \text { Field Journal Notes } \\ & \text { Contact Summary Sheet }\end{array}$

Stage Four-

Building a Primary Record from Representative Video Segments

Transcribe Meaningful Utterances Representative Video Segments

Analyze Meaningful Utterances Primary Record Transcripts 
and Linguistic Behavior Observed

Analyze Possible Objective Validity Claims

Stage Five-

Reconstructive Data Analysis of Representative Classroom Interactions

Initial Meaning Reconstruction Primary Record Transcripts

Low-level coding

Explicit initial meaning reconstruction

Pragmatic Horizon Analysis (CDA)

Contextualisation cues

Boundary-making

Turn-taking patterns

Thematic coherence

Intertextuality

Validity Reconstruction

Subjective validity claims

Objective validity claims

Normative-evaluative validity claims

Stage Six-

Building a Primary Record from Interviews

Transcribe participant interviews Digitally-recorded Interviews

Stage Seven-

Reconstructive Data Analysis of Interviews

Initial Meaning Reconstruction Interview Transcripts 


\section{Low-level coding}

Stage Eight-

High-level Coding

Abstract Analysis

Match for Support

Thematic Analysis

Comparative Analysis
Primary Record Transcripts

Interview Statements

Exemplary Pragmatic Horizon

Analyses

Stage Nine - Final Reconstructive Data Analysis

Interactive Power Analysis

Pragmatic Horizon Analyses

Validity Claims

Validity Reconstructions

Interactive Power Analysis

Raw Codes from each classroom

Interactive Power Analysis

Findings from all classrooms 


\section{APPENDIX H}

\section{Part 1: Field Journal Notes for Classroom Three Observations 2/02/09}

Today when I walked in, Mr. Frederek said that he expected the same 4 kids and this class was unruly since it was after lunch. I set up and then one by one they came in. Once they all got there, it was the same thing. Three of them were disrespectful, cursing, sarcastic etc. One was totally silent. All were Latino. He ignored much of the sassy talking but every once in a while he said LOUDLY for them to stop it, not to bother the quiet guy, and to leave each other alone. But one time, someone said "What is that word slut?" (when reading fault). That was it, he got security and kicked him out. It makes me wonder whether and why he decides to ignore and not this time. I believe it has to do something with when it hurts someone else.

The lessons go in a pattern. First, he lectures about something. Then he gives them a worksheet, then they watch a film, them he lectures some more and and answers questions about the worksheets (more than one or until time runs out). So he stands there while they do (or don't do and instead talk etc) the worksheets. They were asked today to put the \# of earthquakes in each state. He gave them or opened their books to a page where they found a map to use.

Lots of side conversations came up today. One guy left several times w/ a pass. One guy left once w/out a pass. Mr. Frederek ignored him. Report cards were passed out, and they discussed what they would tell their parent and why they got certain grades.

The lesson had nothing to do w/ the kids in any way. It was as if it were snapped out of the air as it floated by one day. No one says hello when they come in. No one acts 
like they care at all about each other. The teacher is nice but is there to get paid. He does not like the job, it appears to me. He may be afraid of the guys or fear losing control.

This is a banking/transmission style classroom to the hilt. When the film comes on, no one watches or if they do it is for one second. They talk and go to the bathroom the teacher does not know how to get the kids engaged or invested. It is a "this is what I'm after" style of class. It's as if knowledge is one thing and the answers can be guessed until right. For example, he asked them a question and the guys guessed 10.9...no...

10.11...no...what's between those (seeking the answer 10.10).

\section{Part 2: Contact Summary Sheet for Observations and Interviews}
Contact Type: Videotaping day2
Site:
$\underline{\text { Classroom \#3 }}$

Observation

Date:

- $02 / 02 / 09$

Group/Individual Videotape Feedback Interview

1. What were the main concerns during this contact?

The students continued to be disrespectful, foul in language, and sarcastic to the teacher. The only humor in the room was among the 3 ringleaders. One student was totally silent. The teacher ignored many of the comments, but one in particular got one kid kicked out. But he returned soon.

2. Summarize the information received or not received on each target question for this contact.

Question

\section{$\underline{\text { Information }}$}

1. Learning processes were those of transmission/banking education to the t. $\mathrm{He}$ presented material, gave worksheets, waited for them to put answers down. The questions on the worksheets were those like putting a number for how many 
earthquakes in certain states. The teacher on the third sheet said we still have time and this one is easy. But in the end, he took it back up. Suspension practices were those of ignoring and once kicking out someone for saying something that may have been hurtful to a girl or something to that effect. Learning was a guessing game to get the right answer. Grades come from worksheets.

2. The teacher explained that the kids were unruly in this class because they just got back from lunch and were riled up. He says very little really, but that he is not used to this school. For one, when kids were absent he never knows where they are. In the Catholic and other school in Sweden he always knew where kids were.

3. Cycles of social identity reproduction include being in the cursing club, knowing certain topics like music and being in charge of the streets, speaking Spanish as a way of being in this club they are in - the against the teacher club. The academic identity reproduction was one of trying to get the least out of it that would let them pass the day. Academics meant what grade you made, not what you learned.

4. Today they were worried about why the white guy (they called him) didn't talk. They tried to get him to talk, but the teacher says leave him alone, he doesn't like that. They talk about themselves as being a group of sorts, such as when one guy wants his backpack from another room, one other guy said to the teacher to let him go, let him do this and that and then he tells the guy to go on home after he gets what he needs. One guy went to get it himself after the friend failed at getting it. He was back in a second and said he ruled the school when the teacher saw him coming, he gave it to him quick. 
5. I haven't seen all the classrooms yet, but the boys in this class are rude and think they run the school and classroom for sure. The other classrooms have silent boys and boys that quietly follow directions and slyly try to get away w/ things like going to another website during class.

3. What else was interesting, informative, or important during this contact?

The fact that the students blatantly talked using foul language and the teacher did not seem to have a clue as to how to raise them up into nicer young men. He is not there for that, he is there to give the some facts and have them regurgitate them back.

4. What are some new or remaining target questions for the next contact?

Will he kick someone out again and why? I think he was one who rarely used suspension, but inside the class it is mayhem.

5. What are additional concerns after this contact?

What they are learning is good for nothing for them. The content is not related to anything that would get the kids to critically think about what they are doing and move them forward into moral growth and literacy. 


\section{APPENDIX I}

\section{Lightly-Structured Videorecording Feedback Interview Protocol}

Introduction: Hello, we are going to have an (do something different in the next part of our) interview session together. I will use this digital recorder to record what we say. I will place it between us so everything we say will be heard clearly. You may say anything you wish, nothing you say will leave this room. You will not be penalized for anything you say. You will be anonymous when I write up my research report (discuss what anonymous means). In fact, your ideas may help change schools for the better. Your thoughts will assist me so I can compare what you think was happening and why to what I think was happening and why. You may also stop the interview at any time.

I have brought a 20-minute segment for you to watch from the videorecordings that were taken in your classroom a few days ago. I have my own thoughts about what is happening and why in this segment. But I would like to hear your version, or your story, of what was happening and why. While we watch the segment, feel free to stop the videorecording and talk about something that is happening and why. You can also talk about what you were thinking, feeling, or doing and why.

I may also stop the videorecording at certain parts where I have questions and tell you what I think was going on and why in that part. I would like for us to compare my version and yours so I can understand better what is happening and why during class. Do you have any questions?

Concluding Ideas: Do you have anything else to add?

Closure: Thank you very much for your opinions and ideas! 


\section{APPENDIX J \\ Lightly-Structured Depth Interview Protocol}

\section{Introduction:}

Hello, we are going to have an interview session together. I will use this digital recorder to record what we say. I will place it between us so everything we say will be heard clearly. You may say anything you wish, nothing you say will leave this room. You will not be penalized for anything you say. You will be anonymous when I write up my research report. You may also stop the interview at any time. Do you have any questions?

\section{Open Questions:}

Please tell me how you became a teacher. What is your educational background?

How did you come to this school?

What is an ordinary teaching day like for you?

What is an ordinary day like for your students?

Can you tell me more?

\section{Follow-up life part probing questions related to literacy:}

Please tell me about your classroom. What are things used for? What do people do?

\section{Classroom One, Follow-up society part probing questions related to literacy:}

I am going to name some things I have seen or heard talked about in your classroom.

Please tell me about each one. Who uses it? How is it used? Why is it used?

-Positive Behavioral Support (PBS)

-PBS Chart

-Report Cards (grades)

-Culinary Arts supplies, apparel, kitchen 
-Passes

-Vocabulary

-Journals

-Computer

-Glossary

-Recipe Cards

-Point Sheet

-White Board

-Wall Posters

-Labels

-Referral forms

-Tests

Classroom Two, Follow-up society part probing questions related to literacy:

I am going to name some things I have seen or heard talked about in your classroom.

Please tell me about each one. Who uses it? How is it used? Why is it used?

-Positive Behavioral Support (PBS)

-PBS Chart

-Report Cards (grades)

-Passes

-Textbooks

-Worksheets

-Magazines

-Books 
-Point Sheet

-White Board

-Wall Posters

-Computers

-Referral forms

-Tests

Classroom Three, Follow-up society part probing questions related to literacy:

I am going to name some things I have seen or heard talked about in your classroom.

Please tell me about each one. Who uses it? How is it used? Why is it used?

-Positive Behavioral Support (PBS)

-PBS Chart

-Report Cards (grades)

-Passes

-Textbooks

-Worksheets

-Overhead

-Point Sheet

-White Board

-Wall Posters

-Computer

-Screen

-Referral forms

-Tests 
-Science supplies and kits

-Science lab

Classroom Four, Follow-up society part probing questions related to literacy:

I am going to name some things I have seen or heard talked about in your classroom.

Please tell me about each one. Who uses it? How is it used? Why is it used?

-Positive Behavioral Support (PBS)

-PBS Chart

-Report Cards (grades)

-Passes

-Textbooks

-Worksheets

-Folders

-Books

-TV

-Overhead

-Point Sheet

-White Board

-Wall Posters

-Computer

-Referral forms

-Tests

-Suspension list (Why are students are on the list?) 
-Attendance list (What is the ethnicity of your students? Why are students here? How does your current list differ from the list you gave me at the beginning of this study? -Did I leave anything out?

May I take a picture of each item when we finish the interview?

\section{Concluding Ideas:}

Do you have anything else to add?

Closure: Thank you very much for your opinions and ideas! 


\section{APPENDIX K}

Classroom Three Transcripts

Stage Four A-Building Primary Record from Representative Classroom Observations (Transcribe Meaningful Utterances)

Stage Four B - Building Primary Record from Representative Classroom Observations (Explain Meaningful Utterances and Linguistic Behavior Observed)

Stage Four C-Building Primary Record from Representative Classroom Observations (Articulate Possible Objective Validity Claims)

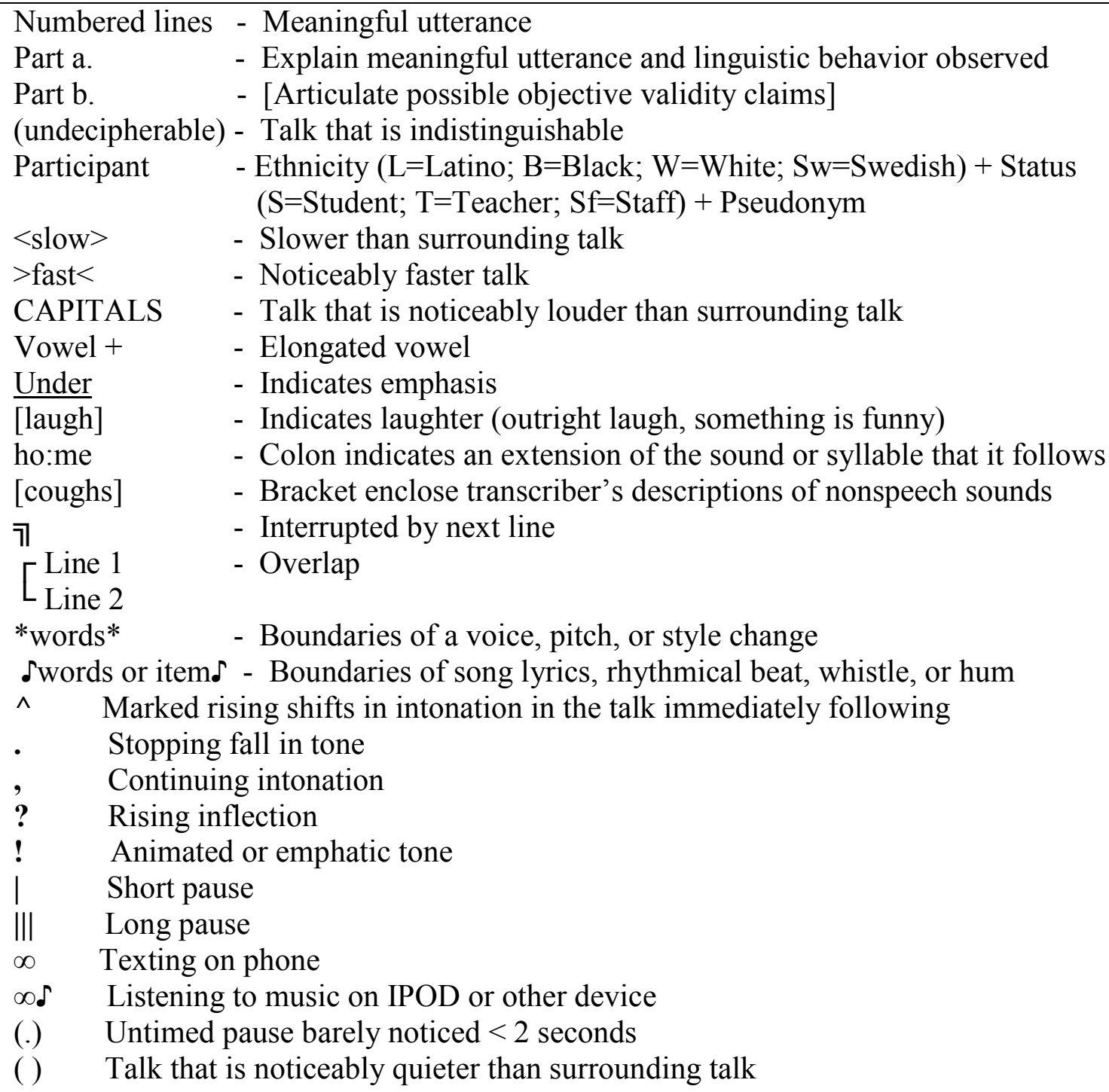

165. Sw-T-Frederek: LEAVE HIM ALONE! THAT'S ENOUGH!

a. Loudly yells at the two boys who are egging Antonio on as if he is not going to let this happen. 
b. [Okay, I have to resort to yelling again because they just don't get it, man, $\mathrm{AND} / \mathrm{OR}$ this is hard.]

166. L-S-Carlos: Hey, what does it say there? Sluts? Like sluts?

a. Smirks, then looks at board ready to take notes asking the questions as if he were totally serious.

b. [He writes horribly, the only word that I know that comes close to that is sluts. Let me ask him and find out.]

167. Sw-T-Frederek: you're out of here.

a. Abruptly walks to the door as if he is going to call security to come get

Carlos and as if his serious demeanor meant nothing serious.

b. [That was the last straw, he is gone!]

Transcribed verbatim meaningful utterances by the speakers, Mr. Frederek and Carlos, are entered in italics in Lines 165-167. The linguistic behaviors observed are nonverbal actions and interactions that describe how the words were spoken. For example in Line 165, Mr. Frederek spoke with a loud voice as if he was not going to let this happen. As if is used to recognize that this observation is only one perspective to which others may disagree. After ignoring the students' taunting behaviors for a long time, Mr. Frederek resorted to using a loud voice to get the others to stop bothering Antonio (i.e., truth claim or assertion). He is exasperated at the effort this takes. Carlos decided to shape up and start copying off the board. He could not read one word and the only guess he could conjure up was sluts. He smirked at Mr. Frederek's illegible writing. However, Mr. Frederek viewed Carlos's attempts as troublemaking rather than a serious effort. Translating truth claims into validity claims entails describing the linguistic behaviors Mr. Frederek and Carlos exhibit. What Mr. Frederek and Carlos may be explicitly or implicitly thinking as they interacted is bracketed as possible validity claims. 


\section{APPENDIX L}

\section{Transcription Key}

(.) Untimed pause barely noticed $<.2$ seconds

$>$ fast $<\quad$ Noticeably faster talk

$<$ slow $\quad$ Slower than surrounding talk

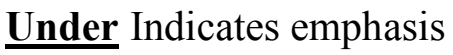

.,?! $\quad$ Punctuation used to mark speech delivery, not grammar. A period indicates a stopping fall in tone; a comma means continuing intonation; a question mark means rising inflection; an exclamation point means animated or emphatic tone

[laugh] Indicates laughter (outright laugh, something is funny)

CAPITALS Talk that is noticeably louder than surrounding talk

ho:me Colon indicates an extension of the sound or syllable that it follows

.hh Audible inbreath

hh Audible outbreath (often with laughter)

$\wedge \quad$ Marked rising shifts in intonation in the talk immediately following

[coughs] bracket enclose transcriber's descriptions of nonspeech sounds

[bell rings] other features of talk

( ) Talk that is noticeably quieter than surrounding talk

(undecipherable) uneasy or agreeable mumbling, or talking to themselves

$\begin{array}{ll}\mid & \text { Short pause } \\ || & \text { Long pause } \\ 7 & \text { Interrupted by next line } \\ {\left[\begin{array}{ll}\text { Line 1 } \\ \text { Line 2 }\end{array}\right.} & \text { Overlap }\end{array}$


Vowel $+\quad$ Elongated vowel

*words* Boundaries of a voice, pitch, or style change

Italics Verbal behavior (meaningful utterances, i.e., talk)

OC: $\quad$ Observer comments

Student Unidentified student speaking

Students Many students speaking at once

(Berkenkotter \& Thein, 2005, p. 205; Bloome, Carter, Christian, Otto, \& Shuart-Faris, 2005, p. 245) 


\section{APPENDIX M \\ Conversational Functions \\ (utterances may serve different functions as noted by AND/OR in analyses or validated by dialogical data)}

Acknowledgment

Acknowledging an argument

Agreeing

Allocating a turn

Allocating turn/testing an argument

Asserting a statement of fact, contesting a challenge

Assigning turn and setting up a response

Beginning a new argument

Challenging

Challenging the application of an illustration

Challenging argument

Challenging a statement of fact

Checking that interlocutors are paying attention

Clarifying

Clarifying the argument

Confirming the argument

Connecting parts of an argument — as in "therefore"

Connecting to next phase of argument

Connecting one part of an argument to another

Connecting the supposition to the text and to previous response

Contesting extension of argument to a new premise

Contesting statement of fact

Defining

Defining a linguistic form

Designating turn

Disagreeing $\mathrm{w} /$ the premise

Elaboration

Elaboration of claim

Elaborating argument

Ending a previous interaction/initiating a new interaction

Establishing a common premise

Establishing a common referent

Exploring the challenge in line ---

Explaining

Informing

Initiating a narrative

Initiating a new topic

Illustrating a counterargument

Locating argument $\mathrm{w} /$ students 
Maintaining floor/establishing a norm for turn at talk as bounded by the boundaries of an argument of "making my point"

Managing turn-taking

Naming interlocutors

Opening up a turn at talk

Providing turn space

Question

Repeating a topic

Repetition of inference

Rephrasing argument

Requesting validation of the argument

Response/Acknowledgment

Restating

Restating argument

Rephrasing an argument

Request for grounding of argument

Restarting the argument

Shifting to a new interactional structure

Side comment

Statement

Statement if the form of a question

Statement in the form of a rhetorical question

Statement of fact/establishing the experiential basis for an argument

Stating an argument

Stating a premise

Telling a story

Turn holder

Validating locating argument w/ students 


\section{APPENDIX N}

\section{Argument Elements}

Attempting to make a claim

Backing

Challenging assumption implicit in the warrant

Challenging claim and backing

Claim

Contesting the argument in lines ---

Contesting the warrant

Contrastive claims

Contrastive grounding

Defining

Defining and backing for a rebuttal

Defining claim

Defining terms

Evaluating the warrant

Grounding

Qualifier

Rejection of particular backing, refuting a potential rebuttal, establishing a warrant

Request for grounding

Seeking backing

Shifting the argument

Transforming contesting in line ---- to a claim

Validating a claim

Warrant

Warrant and rationale

Warrant for claim in line ----

Warrant for rebuttal 


\section{APPENDIX O}

Classroom Three Transcripts

Stage Five A, Part 1- Reconstructive Data Analysis of Representative Classroom Observations (Initial Meaning Reconstruction: Low-Level Coding)

(Categories That Name Interactions)

$\begin{array}{ll}\text { Italicized lines } & \text { - General code categories that name interactions } \\ \text { Numbered lines } & \text { - Codes that give examples of category } \\ \text { Very objective } & \text { - Anyone in the room would agree } \\ \text { Slight objectivity } & \text { - Slight interpretation used } \\ \text { Medium objectivity - Most people in the room would probably agree }\end{array}$

Ways of using/allowing language in class as teacher

Slight

1. Ignore many underlying meanings

Slight

2. Allows sarcastic language

Very

3. Allows argument until gets disruptive

4. Talks very loud when addressing disruptive behavior after multiple attempts to stop it

Very

Ways of dealing with interruptions as teacher

Very

1. Respond to inappropriate talk when it may hurt another student

2. Ignore inaudible talk

3. Allows yelling to take its course as long as hurt to another student not imminent

4. Allows to build up to crescendo before responds

Slight

Very

Very

Slight

Medium

Ways of bucking classroom norm/maintaining control as student

1. Push teacher to the limit

Slight

2. Talk about outside of school things during class

Slight

3. Whisper if necessary to communicate

Medium

4. Ignore teacher when get involved talking about out

of school issues

5. Egg peers on to maintain image

Slight

6. Talk about outside of school interests for the

majority of class as long as can get by with it

Very

Slight

Very

This is a sampling of low-level coding from Classroom Three Transcripts that apply to what happened in Lines 165-167. Both category heading codes are slightly objective since slight interpretation was used in their construction. Low-level codes are open to multiple access which means anyone in the room who was listening would probably 
agree that it happened. Medium objectivity of low-level codes (e.g., Allows to build up to crescendo before responds) means some interpretation was used to determine the code. Slightly objective low-level codes (e.g., Ignore many underlying meanings) involve slight interpretation. Low-level codes with slight and medium levels of objectivity will be supported through horizon analysis and validity reconstruction procedures. 
c. [The teacher understands that Antonio is being made fun of but gives no indication that he knows the details AND/OR uses his loud voice to call off the sarcasm.]

166. L-S-Carlos: Hey, what does it say there? Sluts? Like sluts?

a. Smirks, then looks at board ready to take notes asking the questions as if he were totally serious.

b. [He writes horribly, the only word that I know that comes close to that is sluts. Let me ask him and find out.]

c. [Carlos misread the teacher's patience level AND/OR likes getting the teacher very angry and knows how to do it AND/OR waited too late to be honestly be interested and blurted out a word he knows that is similar to the one on the board.]

167. Sw-T-Frederek: you're out of here.

a. Abruptly walks to the door as if he is going to call security to come get Carlos and as if his serious demeanor meant nothing serious.

b. [That was the last straw, he is gone!]

c. [The teacher is in the heat of the moment and misread Carlos's sincerity AND/OR realizes Carlos will never stop since he has been doing this for a long time now AND/OR and needs to rid the class of Carlos or it will go downhill from here on out.]

This is a sample transcription with inferences of possible underlying meanings related to Lines 165-167. A possible underlying meaning of the loud voice that came out of nowhere is that Mr. Frederek may be pretending to not know what the guys are bothering Antonio about and/or it usually calls off their sarcasm for the moment. Carlos may have misread the teacher's patience limit and/or likes to and knows how to get the teacher angry. On the other hand, Carlos may truly be interested and simply blurted out an inappropriate word. It is possible that Mr. Frederek either misjudged Carlos's sincere efforts to straighten up and/or realized that Carlos will not stop at this point. Mr. Frederek also may think that if he does not remove Carlos right now, the class will fall apart. Science and literacy learning was not totally disrupted. However, it was limited due to the long period of time that Mr. Frederek ignored the continual bothering of Antonio before he used his loud voice. Carlos's attempt to return to the topic of Science may have been 
an opportunity to draw him back in but Mr. Frederek chose to remove him. Later, one of the students asked Mr. Frederek why he kicked Carlos out. This question insinuated that it was not clear why Mr. Frederek chose that moment to lose his patience and/or the possibility that this was uncommon. 


\section{APPENDIX Q}

Classroom Three Transcripts

Stage 5 B, Part 1-Reconstructive Data Analysis of Representative Classroom

Observations (Pragmatic Horizon Analysis: Boundary-Making)

(Contextualization Cues, Interactional Units, Phases)

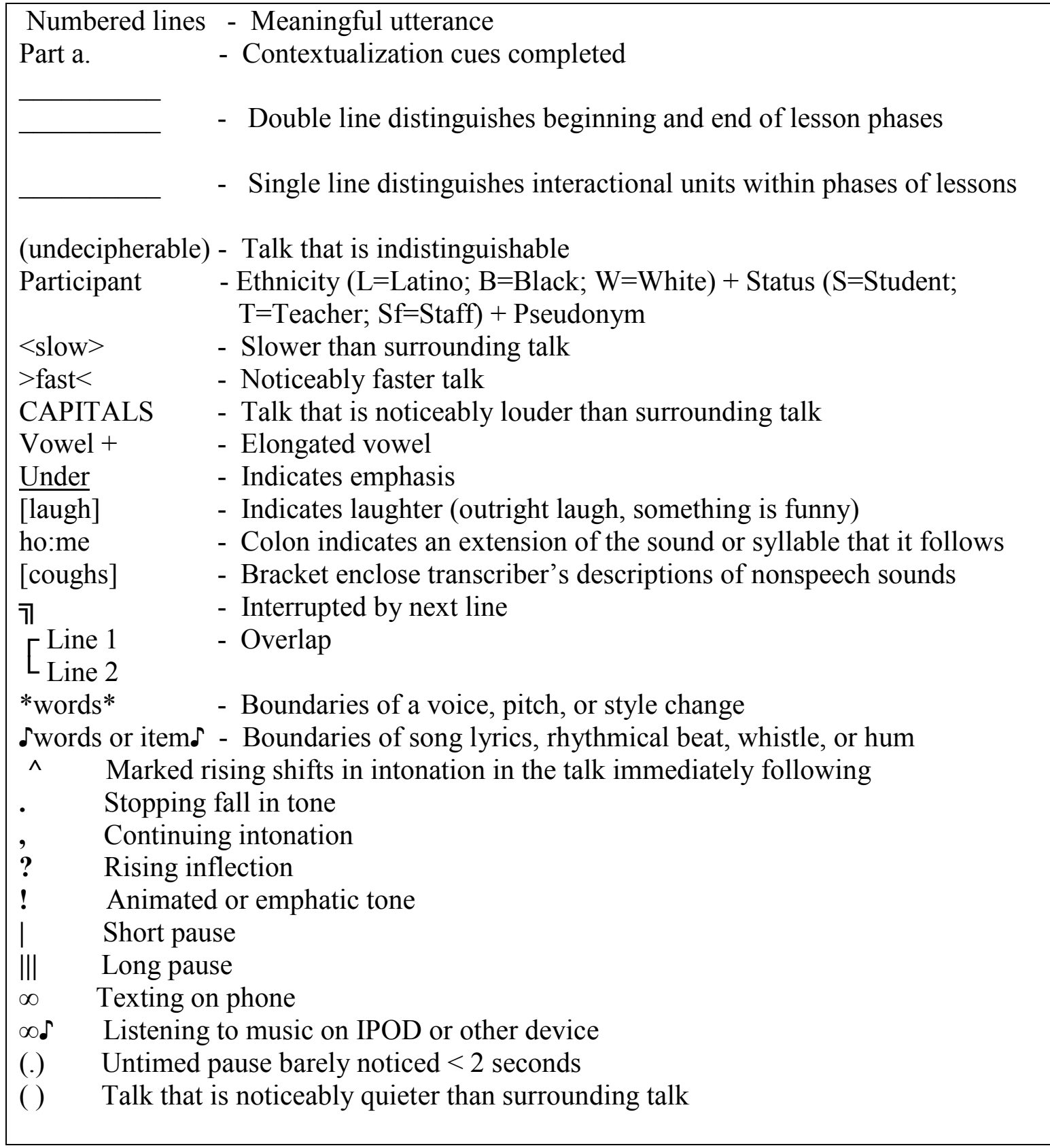




\section{PHASE 17, TEACHER ATTEMPTS TO STOP STUDENT TALK WITH LOUD AND EMPHATIC DIRECTIVES AND THREAT OF KICKING STUDENT OUT OF CLASS}

\section{INTERACTIONAL UNIT 38}

165. Sw-T-Frederek: LEAVE HIM ALONE! THAT'S ENOUGH!

a. Different speaker [indicates new message unit]; talk that is noticeably louder than surrounding talk with emphatic tone and continuous intonation [indicates claim for floor but not next turn-at-talk]

166. L-S-Carlos: (smirks, then looks at board ready to take notes) Hey, what does it say there? Sluts? Like sluts?

a. Different speaker [indicates new message unit]; continuous intonation [indicates claim for floor but not next turn-at-talk]; rising inflection [signals question]

167. Sw-T-Frederek: (abruptly walks to the door) you're out of here.

a. Different speaker [indicates new message unit]; continuous intonation with stopping fall in tone [indicates claim for floor but not next turn-at-talk]

\section{PHASE 18, STUDENT TALK SIMULTANEOUSLY STALLS TEACHER ATTEMPT TO KICK HIM OUT OF CLASS WITH LOUD AND EMPHATIC DIRECTIVES AND INTRODUCTION OF EARTHQUAKES}

\section{INTERACTIONAL UNIT 39}

168. L-S-Carlos: YO, BUT WHAT DOES IT SA+Y? YOU DON'T KNOW HOW TO WRITE, DOG! (holds left arm straight out toward the walking teacher) | HEY! (quickly takes off jacket and grabs yellow uniform shirt, glances at camera) (I gotta put on my shirt.) What does it say there? WHAT DOES IT SAY THERE?!

a. Different speaker [indicates new message unit]; talk that is noticeably louder than surrounding talk with emphasis on yo and pattern of emphatic tones and continuous intonation [indicates claim for floor but not next turn-attalk]; rising inflection [signals question]

This is a sample transcript of contextualization cues completed (if cues were missed in previous transcripts), boundary-making, interactional units, and phases of lessons. By this stage, contextualization cues throughout the transcripts are finalized. In Line 166, the 
question mark is noted with the transcription symbol that indicates not only a question begin asked by how it is asked with the rising inflection. Transcription symbols also show the boundaries of a change in voice, pitch, or style. For example, Mr. Frederek spoke much louder in Line 165 than in Line 167 as noted with capital letters when he voiced, "LEAVE HIM ALONE!" The explanation mark indicates an animated or emphatic tone.

This sample transcript also identifies/interprets message unit boundaries. Lines 165-167 denoted a new message unit with a different speaker. Line 168 has been included in this sample to denote a new message unit and claim to speaking rights, identifiable by stress on the first word, noted by underlining "YO." He elongates the vowel in "SA+Y" to animate his voice tone. Each new speaker uses continuous intonation patterns to claim floor and maintain the floor, but not to claim next turn-attalk. This pattern may mean that they expect a back-and-forth word volley.

This sample transcript also indicates interactional units. The beginning of INTERACTIONAL UNIT 38 is proposed with a new speaker, Mr. Frederek, stating, "LEAVE HIM ALONE!" A line is drawn above this message unit to denote the beginning of the interactional unit. Closure of INTERACTIONAL UNIT 38 is dictated by Mr. Frederek, denying Carlos's attempt to seem interested in copying a word. A line is drawn below this message unit to denote closure of INTERACTIONAL UNIT 38. Two lesson phases are also indicated. The boundary for PHASE 17, TEACHER ATTEMPTS TO STOP STUDENT TALK WITH LOUD AND EMPHATIC DIRECTIVES AND THREAT OF KICKING STUDENT OUT OF CLASS, was initiated in Line 165 when the teacher said, "LEAVE HIM ALONE!” PHASE 18, STUDENT TALK 
SIMULTANEOUSLY STALLS TEACHER ATTEMPT TO KICK HIM OUT OF CLASS WITH LOUD AND EMPHATIC DIRECTIVES AND INTRODUCTION OF EARTHQUAKES, was introduced in Line 168 when Carlos yelled, "YO!" indicating his disagreement to Mr. Frederek's decision for him to leave the classorom. The full transcript would include the conclusion of this lesson. 


\section{APPENDIX R}

Classroom Three Transcripts

Stage 5 B, Part 2a/b-Reconstructive Data Analysis of Representative Classroom Observations (Pragmatic Horizon Analysis)

(Turntaking, Thematic Coherence)

\begin{tabular}{|c|c|}
\hline \multirow{2}{*}{\multicolumn{2}{|c|}{$\begin{array}{l}\text { Numbered lines - Meaningful utterance } \\
\text { Turntaking (I-R-E/F): }\end{array}$}} \\
\hline & \\
\hline I & - Initiation via statement \\
\hline $\mathbf{I} / \mathbf{Q}$ & - Initiation or question \\
\hline $\mathbf{R}$ & - Response \\
\hline $\mathbf{E} / \mathbf{F}$ & - Evaluation/Feedback \\
\hline \multicolumn{2}{|c|}{ Thematic Coherence: } \\
\hline$\downarrow$ & - Theme(s) achieved \\
\hline$\leftrightarrow$ & - Negotiated and/or conflicting themes or overlaps of themes \\
\hline$E$ & - Earthquake Theme \\
\hline$S$ & - Student Outside of School Theme (or off the E topic) Theme \\
\hline Si & - Silence \\
\hline$H$ & - Housekeeping (pencils, folders) \\
\hline$R$ & (undecipherable) - Talk that is indistinguishable \\
\hline Participant & $\begin{array}{l}\text { - Ethnicity }(\mathrm{L}=\text { Latino; } \mathrm{B}=\mathrm{Black} ; \mathrm{W}=\mathrm{White})+\text { Status }(\mathrm{S}=\text { Student; } \\
\mathrm{T}=\text { Teacher; } \mathrm{Sf}=\text { Staff })+ \text { Pseudonym }\end{array}$ \\
\hline$<$ slow $>$ & - Slower than surrounding talk \\
\hline$>$ fast $<$ & - Noticeably faster talk \\
\hline CAPITALS & - Talk that is noticeably louder than surrounding talk \\
\hline Vowel + & - Elongated vowel \\
\hline Under & - Indicates emphasis \\
\hline$\overline{[\text { laugh }]}$ & - Indicates laughter (outright laugh, something is funny) \\
\hline \multirow{2}{*}{ ho:me } & - Colon indicates an extension of the sound or syllable that it follows \\
\hline & - Bracket enclose transcriber’s descriptions of nonspeech sounds \\
\hline \multirow{2}{*}{ 근 1} & - Interrupted by next line \\
\hline & - Overlap \\
\hline \multicolumn{2}{|l|}{ Line 2} \\
\hline \multicolumn{2}{|r|}{ Boundaries of a voice, pitch, or style change } \\
\hline \multicolumn{2}{|r|}{ Boundaries of song lyrics, rhythmical beat, whistle, or hum } \\
\hline \multicolumn{2}{|c|}{$\wedge \quad$ Marked rising shifts in intonation in the talk immediately following } \\
\hline \multicolumn{2}{|c|}{ - Stopping fall in tone } \\
\hline \multicolumn{2}{|c|}{, Continuing intonation } \\
\hline \multicolumn{2}{|c|}{ ? $\quad$ Rising inflection } \\
\hline ! Anim & d or emphatic tone \\
\hline Short & use \\
\hline Long & \\
\hline$\infty \quad$ Textin & n phone \\
\hline
\end{tabular}



$\infty$ Listening to music on IPOD or other device
(.) Untimed pause barely noticed $<2$ seconds
( ) Talk that is noticeably quieter than surrounding talk

E S Si $\quad$ S R

165. FSw-T-Frederek: LEAVE HIM ALONE!

THAT'S ENOUGH!

166. I/QL-S-Carlos: (smirks, then looks at board ready to take notes) Hey, what does it say there? Sluts? Like sluts?

167. RSw-T-Frederek: (abruptly walks to

the door) you're out of here.

This is a sample transcript with turntaking, including I-R-E/F sequences, and thematic coherence indicated. In Line 165, Mr. Frederek initiates the interactional unit with feedback to students' previous taunting. In Line 166, Carlos initiates with a question to try to change the subject. Mr. Frederek responds in Line 167 with, "you're out of here." This was not a typical I-R-E/F sequence of participation between the teacher and students during the lesson. Rather, this sequence begins with feedback to disruptive behavior. The student is the one initiating a question, but the teacher responds again to behavior. The Science literacy learning in this sequence is missing. This sample transcription also indicates the analysis of thematic coherence. A reprimand theme interrupts student behavior that is off the topic of earthquakes in Line 165. Carlos attempts to achieve thematic coherence between an assumed theme of earthquakes and his off the topic of earthquakes behavior. However, Mr. Frederek does not accept the theme Carlos proposes. Instead, he returns to the reprimand of Carlos's off the topic behavior.

At this point, there is a standstill and no thematic coherence was achieved. If Mr. Frederek had taken up Carlos's proposal to begin an earthquake theme and continue to 
ignore his behavior of bothering others, thematic coherence may have been achieved. However, Mr. Frederek denied Carlos's proposal and Carlos was removed for a breather. While this scene was occurring, other students waited silently, watching. Science literacy learning theme was momentarily at a standstill. After Carlos left, however, Mr. Frederek immediately and calmly picked up the topic of earthquakes with the remaining students. Even though Jack asked why he kicked Carlos out, Mr. Frederek answered but continued the earthquake theme fairly successfully. Students consented to both why Jack got kicked out and to the proposed earthquake theme by listening. At first, as noted in field journal notes, the research implied this as a non-caring attitude about Carlos. However, after the teacher and student interviews, it was evident that this was an isolated incident, rarely occurred, and Mr. Frederek was a caring teacher based on how he treated students over time. Overall, thematic coherence was achieved, even during proposed theme interruptions, by Mr. Frederek's decision to retain Science language in the midst of interruptions based on students' behavioral history in his class. Science and students' cultural interests were part of the ongoing (i.e., temporal) classroom context and linguistic (i.e., pragmatic) behaviors that were exhibited and negotiated. 


\section{APPENDIX S}

Classroom Three Transcripts

Stage 5 B, Part 2c-Reconstructive Data Analysis of Representative Classroom Observations (Pragmatic Horizon Analysis: Intertextuality)

\begin{tabular}{|c|c|}
\hline \multicolumn{2}{|c|}{ Numbered lines - Meaningful utterance } \\
\hline Intertextuality: & \\
\hline IP & - Intertextuality Proposed \\
\hline$I A$ & - Intertextuality Acknowledged (superficial knowledge of existence) \\
\hline$I R$ & - Intertextuality Recognized (underlying familiarity and understanding) \\
\hline$S C$ & - Social Consequence \\
\hline NT & - New Topic \\
\hline $\mathrm{R}$ & - Request \\
\hline $\mathrm{C}$ & - Confirmation \\
\hline $\mathrm{X}$ & - Occurrence \\
\hline \multicolumn{2}{|c|}{ Turntaking (I-R-E/F): } \\
\hline I & - Initiation via statement \\
\hline $\mathbf{I} / \mathbf{Q}$ & - Initiation or question \\
\hline $\mathbf{R}$ & - Response \\
\hline $\mathbf{E} / \mathbf{F}$ & (undecipherable) - Talk that is indistinguishable \\
\hline Participant & $\begin{array}{l}\text { - Ethnicity }(\mathrm{L}=\mathrm{Latino} ; \mathrm{B}=\mathrm{Black} ; \mathrm{W}=\text { White })+\text { Status }(\mathrm{S}=\text { Student; } \\
\mathrm{T}=\text { Teacher; } \mathrm{Sf}=\text { Staff })+ \text { Pseudonym }\end{array}$ \\
\hline$<$ slow $>$ & - Slower than surrounding talk \\
\hline$>$ fast $<$ & - Noticeably faster talk \\
\hline CAPITALS & - Talk that is noticeably louder than surrounding talk \\
\hline Vowel + & - Elongated vowel \\
\hline$\underline{\text { Under }}$ & - Indicates emphasis \\
\hline$\overline{\text { [laugh }]}$ & - Indicates laughter (outright laugh, something is funny) \\
\hline & - Colon indicates an extension of the sound or syllable that it follows \\
\hline [coughs] & - Bracket enclose transcriber's descriptions of nonspeech sounds \\
\hline \multirow{2}{*}{ 그 Line 1} & - Interrupted by next line \\
\hline & - Overlap \\
\hline \multicolumn{2}{|l|}{$[$ Line 2} \\
\hline \multicolumn{2}{|c|}{ *words* - Boundaries of a voice, pitch, or style change } \\
\hline \multicolumn{2}{|c|}{ Swords or item $\delta$ - Boundaries of song lyrics, rhythmical beat, whistle, or hum } \\
\hline \multicolumn{2}{|c|}{$\wedge \quad$ Marked rising shifts in intonation in the talk immediately following } \\
\hline Stopping $\mathrm{f}$ & fall in tone \\
\hline Continuing & g intonation \\
\hline Rising infl & flection \\
\hline Animated & or emphatic tone \\
\hline Short paus & \\
\hline Long paus & \\
\hline Texting on & phone \\
\hline
\end{tabular}



$\infty$ Listening to music on IPOD or other device
(.) Untimed pause barely noticed $<2$ seconds
( ) Talk that is noticeably quieter than surrounding talk

165. Sw-T-Frederek: LEAVE HIM ALONE!

THAT'S ENOUGH!

166. L-S-Carlos: (smirks, then looks at

board ready to take notes) Hey, what does it say

there? Sluts? Like sluts?

167. Sw-T-Frederek: (abruptly walks to

the door) you're out of here.

168. L-S-Carlos: $\underline{Y O}, B U T$ WHAT DOES

IT SA+Y? YOU DON'T KNOW HOW TO WRITE,

IP IA IR SC

$D O G$ ! (holds left arm straight out toward the

walking teacher) | HEY! (quickly takes off jacket

and grabs yellow uniform shirt, glances at camera)

NT $\quad X \quad X$

NT R

(I gotta put on my shirt.) What does it say there?

WHAT DOES IT SAY THERE?!

This is a sample transcription with intertextualities indicated. In Line 165, Mr. Frederek proposes intertextuality by calling for a stop to the ongoing taunting of each other, simultaneously assuming the students will recognize the proposal and understand the underlying social consequence of the situation. However, Carlos's response was a new proposal of what appeared to be a superficial request for information related to the Science topic at hand. However, Mr. Frederek does not accept Carlos's proposal but, instead, confirms the social consequence of being removed from the classroom with "you're out of here."

To acknowledge an intertextuality means the person admits that it exists at a surface level; to recognize an intertextuality means the person understands and can distinguish the intertextuality at a deeper level and can use it for some purpose. Carlos confirmed the social consequence of his actions and simultaneously requested 
acknowledgment and recognition of intertextuality from Mr. Frederek by asking what does say again and excusing his misunderstanding with Mr. Frederek's bad handwriting. He continued by requesting another level of recognition and acknowledgment of intertextuality and of the social consequence of not wearing his uniform shirt. He grabbed his yellow shirt and tried to quickly put it back on as he yelled. He knew that the security guard would come in, find that he did not have on his uniform shirt as required by administration, and turn him for that infraction as well.

This sample illustrates heteroglossic (Bakhtin, 1981) intertextualities that existed between how long Mr. Frederek would ignore bothering behavior, why he finally lost his patience when the word fault on the board got misconstrued inappropriately, why uniform rules were not enforced inside the classroom, background history of students' behavior, and how and why Science literacy learning took place in this classroom. The researcher interpreted the teacher's and students' possible beliefs to conduct analysis of intertextualities based on an understanding of the teacher's and his students' beliefs from patterns observed in their verbal and nonverbal behaviors over a period of time. For 40 years, research has consistently documented the link between teachers' perceptions and expectations of students and decision making in the classroom (Noguera, 2003; Rist, 1970). Research has also shown how teachers rely on their backgrounds, beliefs, and experiences for their teaching practices (Cochran-Smith \& Zeichner, 2005; DarlingHammond \& Bransford, 2005). 


\section{APPENDIX T}

Classroom Three Transcripts

Stage 5 C-Reconstructive Data Analysis of Representative Classroom Observations (Validity Reconstructions)

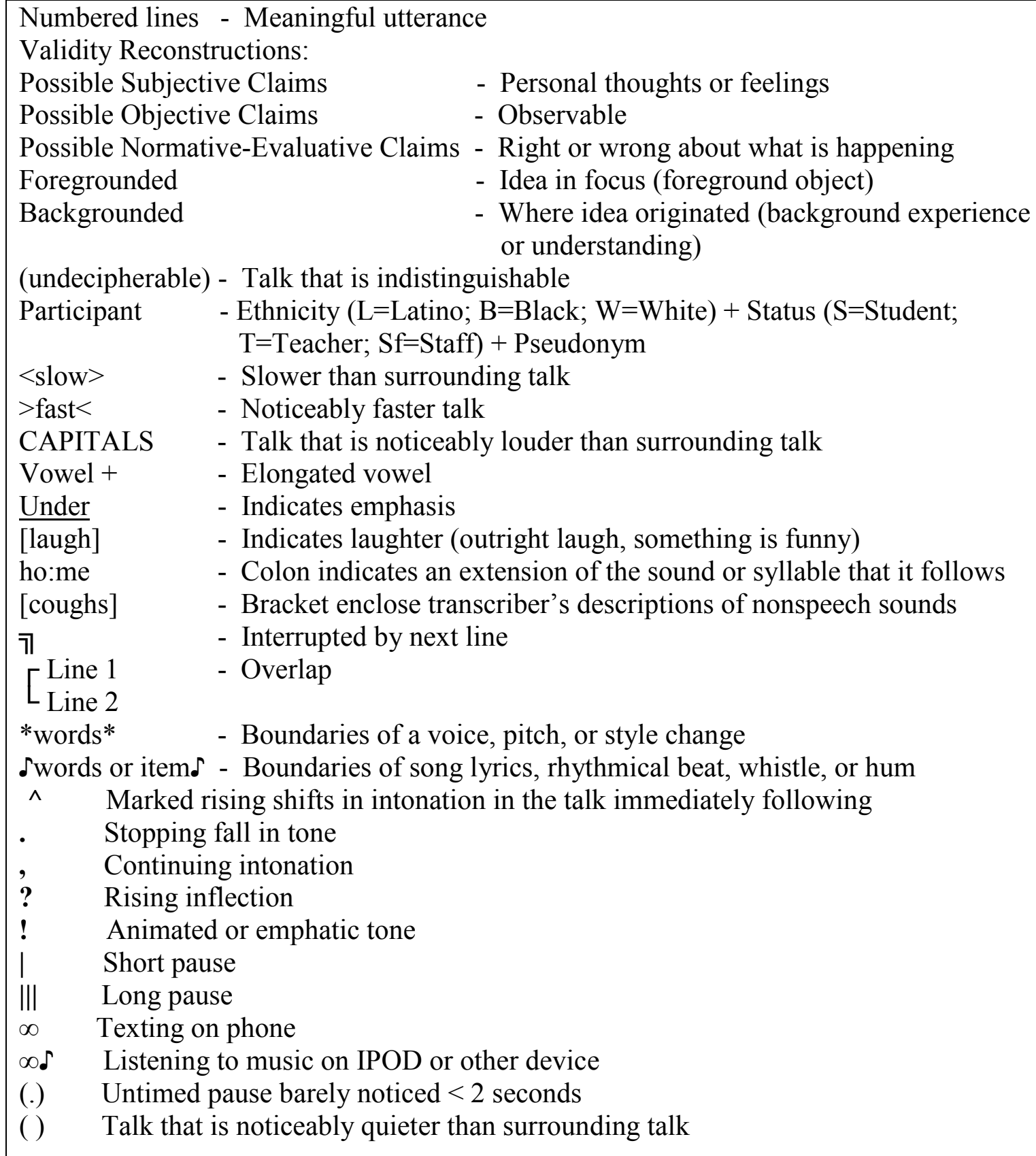


133. L-S-Jack: What happened to the board? It's not erased properly.

134. Sw-T-Frederek: I know, it's dirty.

135. L-S-Jack: What?

136. Sw-T-Frederek: it's ok, ok, so we have these different $<$ fault lines, $>$ these plates, and when they intersect, we have fault lines

137. L-S-Carlos: $\infty \delta$ (turns it on loudly)

138. Sw-T-Frederek: Carlos.

139. L-S-Carlos: $\infty \delta$ (continues to listen)

140. Sw -T-Frederek: Carlos. Carlos.

141. L-S-Carlos: $\infty$ S (looks up) it's not on, it's not on.

142. Sw-T-Frederek: Stop it!

143. L-S-Jack: (briefly turns around and looks and turns back to the front, chewing straw)

144. Students: (look forward, expressionless)

145. L-S-Jack: (points to something on his arm looking at Antonio)

146. L-S-Antonio: (nods to Jack) (undecipherable)

147. Sw-T-Frederek: We have faults.

148. L-S-Carlos: $\infty \delta$ (puts head on desk)

149. L-S-Jack: (mouths something to Antonio, scrunches mouth and leans head back a little)

150. Sw-T-Frederek: Where you have these faults, you have earth:quakes. (walks over to put textbook on $2^{\text {nd }}$ table where Julio sits) You're going to draw this first put today's date (points to board with textbook in hand) the $2^{\text {nd }}$ of February, uh, your book, the page. (looks over to Carlos) Carlos. Carlos. Turn it off now.

151. L-S-Carlos: $\infty \delta$ (rhythmic move of arm, reaches in pocket to turn it off, smiles)

152. L-S-Jack: (rubs eyes, mimics rhythmic move of arms)

153. L-S-Carlos: Damn, dog, I wanted to listen to my ipod.

154. Sw-T-Frederek: (holding two textbooks up to demonstrate) These plates

155. L-S-Jack: (turns around to smile at Carlos)

156. L-S-Carlos: I'm gonna listen to him (points hand toward the teacher)

157. Sw-T-Frederek: (abruptly stacks two books and walks to $4^{\text {th }}$ table, leans over to get notebook off the table, turns around and leans over $1^{\text {st }}$ table, leaves notebook there)

158. L-S-Antonio: (looks at Jack and Carlos expressionless)

159. L-S-Carlos: ${ }^{\wedge}$ Come on, hoss. [laugh]

160. L-S-Jack: [laugh]

161. Sw-T-Frederek: don't worry about it (faces students again)

162. L-S-Jack: (looks at Antonio) that was straight, chillin' mack

163. L-S-Carlos: He's Mr. T right now. [laugh]

164. L-S-Carlos: [laugh]

165. Sw-T-Frederek: LEAVE HIM ALONE! THAT'S ENOUGH!

\section{Possible subjective claims}

Foregrounded, Immediate 
I love to teach science when I finally get a chance in this alternative education school. Science knowledge is in books and teachers deliver that knowledge. However, it is fun to change things around a bit and deliver in several different ways. We like science but just can't help but interrupt to evaluate what someone even the teacher is doing for fun to get the approval of our peers (identity claim).

Less Foregrounded, Less Immediate

We do things like listen to our ipod, text, or stare into space to pass the time in school. I feel like a good teacher when the students are quiet and listening to me lecture (identity claim).

Backgrounded, Remote

I am a good teacher if I reprimand students who break the rules or make fun of others.

Possible objective claims

Quite Foregrounded, Quite Immediate

The teacher is delivering knowledge and the students are considering accepting it, but their other interests take over.

Highly Backgrounded, Remote

Students in alternative schools often disregard what the teacher is teaching with disruptive behavior or other behavior that shows they are not engaged.

\section{Possible normative-evaluative claims}

Quite Foregrounded, Quite Immediate

It is not acceptable to listen to the ipod when teachers lecture. It is acceptable when the teacher calls students' names for doing this or breaking other rules.

Less Foregrounded, Less Immediate

Calling students' names is the first step in appropriate disciplining of misbehavior.

Backgrounded, Remote

Teachers are responsible for making students listen. Students in alt ed always try to get away with things.

166. L-S-Carlos: (smirks, then looks at board ready to take notes) Hey, what does it say there? Sluts? Like sluts?

167. Sw-T-Frederek: (abruptly walks to the door) you're out of here.

168. L-S-Carlos: YO, BUT WHAT DOES IT SA+Y? YOU DON'T KNOW HOW

TO WRITE, DOG! (holds left arm straight out toward the walking teacher) |

$H E Y$ ! (quickly takes off jacket and grabs yellow uniform shirt, glances at camera)

(I gotta put on my shirt.) What does it say there? WHAT DOES IT SAY THERE?!

169. Sw-T-Frederek: (talking to security at the door)

170. L-S-Carlos: What the fuck? (putting on shirt) III

171. Students: (wait silently facing forward)

172. L-S-Jack: (undecipherable)

173. L-S-Carlos: Yo, what does it say there? (left arm moving once from right to left)

174. Sw-T-Frederek: (talking to security at the door)

175. L-S-Jack: (looks back briefly to Carlos)

176. L-S-Carlos: Yo, what does it say there? (left arm moving straight out) |

177. Sw-T-Frederek: Fault, fault. 
178. L-S-Carlos: That's fault? Are you serious? (holds pencil between both hands in front of him propped on the table, then points with one finger to the word on the board) Faults?

179. Sw-T-Frederek: Faults, you know, you are causing these problems, just be quiet.

180. L-S-Carlos: I'm just 7

181. Sw-T-Frederek: If you don't understand what it says, write it down, but be quiet!

182. L-S-Jack: (looks back briefly to Carlos and laughs)

183. L-S-Carlos: Oh, my God, how can I write it down if I don't know it says?

184. Sw-T-Frederek: YOU'RE OUT FROM HERE!

185. L-S-Carlos: Go', dog, fuck (holds head low and shakes his head)

186. Sw-T-Frederek: Yeah, you have these fault lines (door opens)

187. Sw-T-Frederek: I need Carlos out.

188. B-Sf-Jones: Let's go.

189. Sw-T-Frederek: Go now. ^ $\underline{\text { Go now. }}$

190. L-S-Carlos: (writing, looks up briefly) Hold on.

191. B-Sf-Jones: Let's go now.

192. L-S-Carlos: (writes) hold on, so it's faults? ||| (calmly and slowly stands up, pulls up pants, fixes shirt, gets jacket off chair, fixes it, looks for arm holes, turns it right side out)

193. L-S-Jack: (looks back and forth from Carlos and security guard)

194. B-Sf-Jones: You can put it on outside, man, come on.

195. L-S-Carlos: (undecipherable) (puts on jacket, gets backpack and leaves)

196. L-S-Jack: What's gonna be the difference?

197. Students: (sit facing forward, expressionless)

198. Outside: (undecipherable)

199. Sw-T-Frederek: $o k$.

200. L-S-Jack: Why'd you kick him out?

201. Sw-T-Frederek: Why? You know,

202. L-S-Jack: Why do you look at me with that face? (points once with right arm, smiling) [laugh]

203. L-S-Antonio: [laugh] (knees up against table)

204. L-S-Julio: $\infty$

205. Sw-T-Frederek: You too are the next

206. L-S-Jack: Why?

207. Sw-T-Frederek: Well, it's complication (undecipherable)

208. L-S-Jack: (waves right hand and shakes head no, smiling) I'll say it is.

209. Sw-T-Frederek: Jack, I will call your father, (holding two textbooks to demonstrate)

210. L-S-Jack: naw, naw, naw, naw, naw, naw (moves paper on table)

\section{Possible subjective claims}

Foregrounded, Immediate

I like to wait until the last minute to get down to business, but I misjudged the teacher's patience this time. I will take a lot of things but not cursing or downgrading women. 
Less Foregrounded, Less Immediate

This is the last straw for this kid. Even if I was serious which I was this time, the teacher has it out for me.

Backgrounded, Remote

Alt ed students push the limits of authority and that is why they are here. Alt ed teachers always give too many reminders and end up sending kids to CSI.

\section{Possible objective claims}

\section{Quite Foregrounded, Quite Immediate}

The teacher who yells ends up calling CSI. Students yell when they see inequities in class. Students are silent when they do not want to be involved in getting kicked out.

Highly Backgrounded, Remote

The teacher has the power and the students do not. Students are not asked their opinion about anything from academics to behavior to life.

\section{Possible normative-evaluative claims}

Quite Foregrounded, Quite Immediate

It is okay to use one's power to maintain control as the teacher in alt ed classes.

Less Foregrounded, Less Immediate

It is not right to tease or belittle anyone in class if the teacher is not with it in the humor department.

Backgrounded, Remote

Principals have power over the teacher which can work both ways either against or for the teacher and usually against the student. Society thinks alt ed kids are bad.

This is a sample transcription of validity reconstructions which are possible reasons the teacher and the students could give to defend their actions. A larger portion of the entire transcript was provided in order to more clearly illustrate validity reconstruction. Possible subjective reasons are derived from analyzing what each participant's personal thought or feelings could be in the particular situation, taking into condsideration what happened beforehand. Possible subjective reasons also incorporate what the teacher and the students may consider what it means to produce or reproduce a "good student" or "good teacher" identity. For example, the immediate idea in focus (e.g., Foregrounded, Immediate) for the first possible subjective claim analyzed for the teacher in the sample provided is based on how the teacher teaches Science and seems to love it. Since Mr. Frederek used the textbook for all aspects of his lesson, albeit presented in various ways, 
one possible reason he does so is because he thinks Science knowledge comes from the book. The possible subjective reasons are written as if Mr. Frederek is speaking (i.e., "I love to teach Science ... . Science knowledge is in books ...”). In the same scene, the Backgrounded, Remote (i.e., where the idea originated in his background experiences) possible subjective reason could be "I am a good teacher if I reprimand students who break the rules or make fun of others."

Possible objective reasons deal with what is observed in the setting. For example, a Quite Foregrounded, Quite Immediate possible objective claim noted by the researcher and written as if she is talking is, "The teacher is delivering knowledge and the students are considering accepting it, but their other interests take over." Possible objective claims are also analyzed according to previous occurrences observed. On the other hand, possible normative-evaluative reasons involve analyzing what may be right or wrong about what is happening. Normative-evaluative claims are written in third person as if someone is telling someone else what they think. To analyze normative-evaluative, the researcher incorporates the cultural, social, and political context of the setting knowing that possible claims are never final. These claims are possibilities gleaned from the researcher's perspectives gained in her own life experiences with others and from what she has learned about others' perspectives while participating in the study. For example, a possible normative-evaluative claim that is Quite Foregrounded, Quite Immediate in this classroom scene is that "It is not acceptable to listen to the ipod when teachers lecture. It is acceptable when the teacher calls students' names for doing this or breaking other rules." A Less Foregrounded, Less Immediate normative-evaluative claim is that "Calling students' names is the first step in appropriate disciplining of misbehavior." Finally, a 
Backgrounded, Remote normative-evaluative claim may be that "Teachers are responsible for making students listen. Students in alternative education always try to get away with things." Such claims make an effort to analyze what is considered right or wrong in this setting based on the broader context as well. Often, what is considered right and wrong may take for granted what is good or not good for the teacher or students to do. These taken-for-granted notions have implications that are taken up later in analyses. Validity reconstruction gives a more precise idea about what is happening in the classrooms. For example, setting negotiations are begun sporadically and/or stopped midstream by the the students due to possible fears of being caught by an authority figure in the act of not being a good student or not following the rules (e.g, Carlos grabbing his uniform shirt to put it on before the security guard comes). Mr. Frederek was possibly swayed by the dominant ideologies about school (e.g., using textbooks), but he prioritized the business of reducing categorizations and hierarchies that may involve students getting bothered and hurt by others over infractions as not wearing a uniform shirt in class. Mr. Frederek's attempted to protect students in the classroom may be thwarted by interruptions and other conflicts in the classroom. However, he still managed to maintain patience and control in order to teach Science. Students also managed to maintain their particular identities and learn Science at the same time. Interactive power relations are implicated throughout validity reconstructions. 


\section{APPENDIX U \\ Classroom Three Transcripts \\ Stage Seven A- Reconstructive Data Analysis of Student Interviews \\ (Initial Meaning Reconstruction: Low-Level Coding) \\ (Categories That Name Interactions)}

Italicized headings - General code categories that name interactions
Numbered lines

General Code Category and Codes

Level of Objectivity

Ways of talking about student behavior

Afraid to go to principal

Knows when he may get called in by the principal

Acting up makes classroom fun

Student won't talk because peers bother him too much

Some students bother others when they are quiet

Are quiet in new school until other student bothers them

to the point of anger and then start acting up

It builds up on the teacher until he kicks them out

Get in worse trouble if break one rule while getting

caught breaking another

Ways of talking about teacher actions

Makes funny faces when gets mad

Only gets mad for something really bad like putting hole in wall

Teacher is good

Patient

Good teachers do not just kick kids out

Calling home straightens student up

Does not write referrals much

Can't understand handwriting

Good teacher, knows his stuff

Searches for answer if he doesn't know

Gets funny faces when he doesn't know something

Goes around to each student who is not doing anything and

helps them start working

Always scratching his ear

Observes teacher actions for clues as to what is going on
Very

Medium

Slight

Medium

Very

Very

Very

Very

Very

Very

Very

Very

Very

Very

Slight

Very

Medium

Very

Very

Very

Very

Medium

Very

Slight 
Teacher threatens to call father to tone student down

Very

Can't understand with Swedish accent

Very

Frustrating when can't understand

especially when trying to copy correctly and ask him what it says Very

Frustrating when answers questions by beating around the bush Very

Voice suddenly changes pitch and loudness and is funny Very

This transcript offers a sample of low-level coding from Classroom Three Student

Interview Transcripts that apply to what happened in Lines 165-167. All category

heading codes are very objective since no interpretation was used in their construction.

Low-level codes are open to multiple access which means anyone in the room who was listening would probably agree that it happened. Medium objectivity of low-level codes (e.g., Acting up makes classroom fun) means some interpretation was used to determine the code. Slightly objective low-level codes (e.g., Good teachers do not just kick kids out) involve slight interpretation. Low-level codes with slight and medium levels of objectivity will be supported through horizon analysis and validity reconstruction procedures. The student interviews illuminated how in tune they were to teachers' actions in the classroom. Also, students were able to understand why teachers made certain decisions even though they often disagreed with their reasoning. Overall, students wanted to have a good time in the classroom, they wanted to learn, and they thought teachers who could provide both were good teachers. 


\section{APPENDIX V \\ Classroom Three Transcripts \\ Stage Seven B - Reconstructive Data Analysis of Teacher Interviews \\ (Initial Meaning Reconstruction: Low-Level Coding) \\ (Categories That Name Interactions)}

Italicized headings - General code categories that name interactions
Numbered lines

General Code Category and Codes

Level of Objectivity

Ways of talking about referrals

Very

Don't give often (2-3 per week is unusual)

Very

Referrals must be written if student is to stay longer than

15 minutes in CSI

Very

Students who get referrals can't go back to home school

Very

Too harsh punishment

Very

Should be something in between

Doesn't want to reduce students' chances for going back to

Very

their home school

Slight

Referrals are political

Very

Lots of referrals on school's record makes it look bad downtown Very

They don't encourage referrals

Very

Doesn't know what happens to referrals once they leave his hand Very

Not in control of process from writing referral to outcome Slight

Referral may not be executed even if he writes it Very

Teachers do not make decisions that affect student's placements Medium

Ways of talking about himself as a teacher Very

Ignores some infractions $\quad$ Very

Ignores uniform rule $\quad$ Very

Knows what some kids do outside of school Very

Protects students who he think will get hurt by other students Very

May support wrong person in conflicts because

can't know who started it

Being in this environment often lose good judgment

Very

Slight

talk to administration about persistent issues to protect student Very

Thinks he can solve problems with students

when knows them well

Very

King of patience means he gives too many breaks

Very

Can't send students out all the time, have to find balance

Very 
Don't notice little things like student changing uniform shirt Very

So much takes place he doesn't notice little things Very

Wants to keep students in class as much as

possible so picks battles Very

Issue has to be big enough to kick student out of class Very

Leaving class often causes more problems than staying Very

Teachers have to juggle decisions between

short-long term outcomes Very

History of behavior used to make referral decisions Very

Rules have to be bent when dealing with kids Slight

If someone is not working, will encourage them (open text) Very

Choose which disruptive behavior gets his attention Very

Some behaviors are more irritating when the room is quiet Very

Doesn't remember things that were going on in the tape Very

When issue ends well, he forgets about it Very

Watching self on DVD good for self/staff development Very

So many things happen every day every class period Very

Teaching here is hard on teachers Slight

Ways of talking about student behavior Very

Boxing give respect to student Very

Thinks students pick on each in other classes and it

stems over to his class Very

Some students are the instigators Very

Some students know right from wrong Very

Some parents will steer student in right direction if he calls them Very

Language is an issue when teacher doesn't speak Spanish Very

Notices that students' relationships change constantly Very

Teachers can't possibly know what started conflicts

between students Very

Tend to get used to constant problems and overlook them Very

Conflicts and issues constantly changing Very

Students make fun of his accent and writing all the time Very

Students who are troublemakers 24-7 get kicked out more often Very

Students who are not constantly troublemakers get kicked

out less often because it may cause more problems later Very

Security in halls watch students Very

Inappropriate language from one kid reaps different

outcomes than from another kid depending on level

of troublemaking

Very

Students act differently depending on time of day Medium

Dynamics change according to students in class Very 
This transcript offers a sample of low-level coding from Classroom Three Teacher Interview Transcripts that apply to what happened in Lines 165-167. All category heading codes are very objective since no interpretation was used in their construction. Low-level codes are open to multiple access which means anyone in the room who was listening would probably agree that it happened. Medium objectivity of low-level codes (e.g., Studens act differently depending on time of day) means some interpretation was used to determine the code. Slightly objective low-level codes (e.g., Teaching here is hard on teachers) involve slight interpretation. Low-level codes with slight and medium levels of objectivity will be supported through horizon analysis and validity reconstruction procedures. Teacher interviews illuminated their implicit theories when taken into consideration with their actions in the classroom. For example, Mr. Frederek seemed to ignore students' behaviors for long periods of time as he taught Science. He explained during the interview his understanding of the political nature of writing referrals and as a result, tried to find a balance between keeping students in the classroom and dealing with disruptive behavior himself by teaching Science in enjoyable ways. $\mathrm{He}$ also knew what students thought about his inevitable patience, for example, even though he did not seem aware of it to an observer. 


\section{APPENDIX W}

Classroom Three Transcripts

Stage Eight A-High-level Coding

(Supported by Matches between Interview Statements and Exemplary Pragmatic Horizon Analyses)

\begin{tabular}{|ll|}
\hline Italicized headings & - General code categories that name interactions \\
Numbered lines & - Codes that give examples of category \\
PHA & - Exemplary pragmatic horizon analysis match to support code \\
SI & - Student interview statement match to support code \\
TI & - Teacher interview statements match to support code \\
Italicized codes & - Meaningful utterance quoted from interview \\
H & - High level of abstraction (much interpretation involved) \\
VH & - Very high level of abstraction (much higher interpretation) \\
T & - Transcript \\
$\#$ & - Stage number \\
L & - Line number \\
Student names & - Pseudonyms \\
Turntaking: & \\
R & - Response \\
I & - Initiation \\
I/Q & - Initiation via Question \\
I, Q & - Initiation and a Question \\
E/F & - Evaluation/Feedback \\
Intertextuality: & - Intertextuality Proposed \\
$I P$ & - Intertextuality Acknowledged (superficial knowledge of existence) \\
$I A$ & - Intertextuality Recognized (underlying familiarity and understanding) \\
$I R$ & - Social Consequence \\
$S C$ & - New Topic \\
NT & - Request \\
R & - Confirmation \\
C & - Occurrence \\
X & \\
\hline
\end{tabular}

Ways students resist dominant ideology regarding classroom interactions (agentic student identity)

1. PHA/H - T5B1, L2: Curse openly in class.

2. $\mathrm{PHA} / \mathrm{H}-\mathrm{T} 5 \mathrm{~B} 1, \mathrm{~L} 8,64,183$ : Yell in class.

3. PHA/H - T5B1, L15: Say nothing in class.

4. PHA/H - T5B1, L26, 144-146: Whisper when discussing outside of school issues.

5. PHA/H - T5B1, L31-35, 62: Talk in a way that upholds image with peers.

6. PHA/H - T5B1, L44-47, 65-67, 84: Say things to egg each other on.

7. $\mathrm{PHA} / \mathrm{H}-\mathrm{T} 5 \mathrm{~B} 1, \mathrm{~L} 86,97$ : Argue with teacher. 
8. $\mathrm{PHA} / \mathrm{H}-\mathrm{T} 5 \mathrm{~B} 1, \mathrm{~L} 90$ : Bring in outside issues into class to maintain image.

9. $\mathrm{PHA} / \mathrm{H}-\mathrm{T} 5 \mathrm{~B} 1, \mathrm{~L} 30$ : Talk to teacher as if he is a peer.

10. PHA/H - T5B1, L131, 137: Listen to ipod during class.

11. PHA/H - T5B1, L, 348-365, 411-422: Become actively involved in own curriculum/ practices during class.

12. PHA/H - T5B2ab, L126, 321: Evaluation commonly used to talk to teacher.

13. PHA/H - T5B2ab, L48, 52, 66: Evaluation and feedback commonly used to talk to other students.

14. PHA/H - T5B2ab, L203-204, 261: Responses to teacher initiations are often nonverbal.

15. PHA/H - T5B2c, L54-72: New topics are constantly being introduced.

a. SI/VH - T6A, L15-40, L-S-Antonio: We're talking about the fight that um, remember when he said he punched John, and then, Scorpion, John?

Naw, naw, it was not a fight, he was just making it up, you know, play, we was just playing. No, Carlos said he punched John, but he was playing. He didn't say it to like, he said it serious but he didn't do it. He was just making that up. and then I got up. [and Jack told him to] stop fucking around with him. Oh, you [Jack] said ghetto or something like that. And then Carlos was like, oohh. [Jack keeps saying] no, he's talking to me. [LS-Jack:] I'm just carrying on.

b. SI/VH - T6A, L43-48, L-S-Jack: and then you go *oh, no, I don't want to be here no more. Ohohohhh!* L-S-Antonio: but they got me mad, both of them

c. SI/VH - T6A, L55-62, L-S-Jack: [repeat say it again] for him to get mad. We were playing around. He does get mad. L-S-Antonio: it's funny when he gets mad. Yeah, he like, his face is funny.

d. SI/VH - T6A, L97-112, L-S-Antonio: he [L-S-Julio] wouldn't talk. L-SJack: he won't ever say anything. He only says something once in a while to his other friends, but like. When he's with his other friends, he'll talk, but only a little bit. L-S-Antonio: yeah, but Carlos is bothering him too much. That's why he doesn't talk, cuz anything that he says- L-S-Jack: ever since he came into the school, I don't know, somebody started bothering him. L-S-Antonio: yeah. L-S-Jack: so he's quiet because Carlos was talking to him so he can talk and be friendly but he don't want to and Carlos from there on said oh, I'm going to bother you and bother you and bother you until you start talking. L-S-Antonio: so that happened to me, that's what Carlos did to me. Yeah, at the beginning of the year, I used to come in and try to do my work and then go to sleep. And they used to throw paper bombs at me and then one day I just got mad, threw one back and started cursing $a$ lot and then whatever.

e. TI/VH - T6B, L224-237, Sw-T-Frederek: for some reason they're they're you know, that's, but they have had a fairly good relationship, you know before, the month before but there's always something coming up but I don't know what's been going on, I know that he he was in boxing before and then he stopped I think you know, you get a lot of respect if you attend 
a boxing match, but if you stop you're like outside. Yeah, they have talked about it before. But uh, yeah, no I remember they came to me earlier in the day and there was some issue in another class. I don't know if you remember, I told you about this yeah, there was an issue because I moved I don't know but, I don't know what this issue is really, you know, they start, he has some friends too in school and there might be another class that he's with them and he's they're kind of picking on one another so and then another class group and he doesn't have anybody that you know

f. TI/VH - T6B, L250, Sw-T-Frederek: yeah, these students are so uh, they have this conflict one second you know and then the next minute it's completely different, like it never existed, so

g. TI/VH - T6B, L262, Sw-T-Frederek: I don't know, they sometimes make fun of my accent. [laugh] they talk about my writing all the time.

h. TI/VH - T6B, L264-288, Sw-T-Frederek: [to only talking to security for Antonio but sending Carlos to security] because he wants, because I think I go outside to get some personnel and then he uh, things like this I don't notice. So what he did was, yeah, I think I did talk to somebody outside the door, and yeah, then I changed my mind. Because I wanted to have him in class, that issue was not big enough. I think the issue was not big enough. I think that it was not big enough for him to leave, I think that would cause more problems, but him and I think he's he's um, he's a troublemaker 247 and he will try to get you know, he will try to create you know, I think there was something on the board that he said that he couldn't read it but then he translated it to something inappropriate, yeah, because it's just going to get worse and worse with him, it's there's not too much you can do when he gets started. Well, they are supposed to receive a pass. The watch the hallways and stuff like that. I thought that maybe there was something that that had led up to (undecipherable) asked him why. I remember it was something on the board and he translated it to something inappropriate and I knew that it was just going to continue and continue and continue and I think it was maybe, yeah, and also because he had just bothered him, but Carlos you know, he's a good student too but some days and this is the worst period of the day because it's after lunch and they are all (undecipherable) up

i. TI/VH - T6B, L290-292, Sw-T-Frederek: but it's interesting that just one person can change the whole dynamics in the classroom and uh, the interactions. Antonio is not really doing anything right now. Antonio, he is the one that sits over there and he was really a good student when he first came here. He did everything. To be in this school for a long-for a longer period of time is not good. You shouldn't be, the purpose is to be sent back to your normal school. I think 18 weeks [time requirement] but it used to be 9 weeks which is much better, in 9 weeks you can keep up, you know, concentration, but after 18 weeks, it's uh, so many bad bad influences for a lot of kids. 
This transcript offers a sample of high-level coding, supported by matches between interview and primary record analyses. One high-level category heading code,

Ways students resist dominant ideology regarding classroom interactions (agentic

student identity) is abstracted from multiple exemplary pragmatic horizon analysis matches to support the code. It is considered a high level, or abstract, code due to its taken-for-granted nature in classrooms. Then, exemplary pragmatic horizon analyses that represent examples of the code are listed below it. For example, one way students resist dominant ideology regarding classroom interactions is to say things to egg each on. Several matches from student interviews and the teacher interview using a very high level of abstraction (much higher interpretation) to support this code and the exemplary pragmatic horizon analyses are then added to the list. For example, student interview selections discuss how the students egg each other on and probably should not; the teacher interview selections discuss how he deals with students who egg each other on. All entries support each other in the implied message that students resist dominant ideology in class and teachers deal with it in their own way. 


\section{APPENDIX X}

\section{Interactive Power Analysis}

First, list types of power employed or claimed by participants and analyze common forms. Second, determine cultural milieu or cultural themes drawn upon by participants and its distribution. Third, ask why (need understanding of cultural milieu to answer).

1. Before class, tacit agreement is to either sit in chairs and wait for teacher or curse and walk around before sitting down. Julio is silent, apparently by choice.

Antonio sits and waits. Jack and Carlos are most verbal and animated at this point, accepting classroom norm with some reservations and using agency to communicate with each other. Frederek at door directing students or protecting classroom from intruders with normative claims used like come on.

2. Frederek - uses nonverbal and verbal normative power claims (as teacher claiming authority by walking around continually among and in front of students - got to do work - not much eye contact providing social distance but speaking quietly to certain ones that need his protection from the others to gain solidarity with them; teachers should protect students who are getting bothered in the school where students bother other students that's why they are here in the first place obscure the contractual point sheet modes of power by foregrounding normative power claims as protector) as he passes out folders and says got to do (students come to school to do work in folders), ignoring conversation (until louder) between Jack, Antonio, and Carlos, seemingly an accepted classroom norm to converse (referencing counterculture of fighting) using cursing/wait for teacher to start (often gives candy later on after settled down in classwork and leaving each other alone in behavior, tacit agreement among students and teacher)

a. Jack - accepts claim talks and waits

b. Carlos - accepts claim talks and waits

c. Antonio - accepts claim talks and waits, stands up once angry, sits back down

d. Julio - accepts claim waits

3. Frederek - uses normative claim with watch your language (underlying rules to be enforced at expense of bothering others - his definition of setting - he steps in, not to acknowledge his authority being challenged but to protect other students sort of like interactively established contract with students they know he will protect them and he wants them to all stay there under these terms, but challenge him too far and he will use underlying CSI option for few minutes, trying to not write referrals until very necessary, even when his authority is challenged by being made fun of his language and writing, he ignores and continues to administer normative power claims of leave him alone until he has to resort to coercive power claims of calling the student's home if he doesn't tone down) to Antonio but when realizes his error in who cursed, redirects his call to Jack - finally gains consent of students and then will administer contract with candy as reward for consenting to his terms of not bothering anyone else. He gives many opportunities 
to consent to his authoritative claim to do the work and let him teach the material and usually gets consent - he enforces school rules to protect students in classroom from being bothered, so that he can teach science and so that the students have a good time, he rewards them with candy to solidify the deal (contract) they agree upon in the end. The students construct an acceptable identity by talking about what they like and letting him teach at the same time. If they get out of hand making fun of people, the teacher will call them on it, they are allowed to make fun of him because his authority is not challenged anyway it seems. He does not want it to get too far, so he ignores until someone is being bothered other than him. When he teaches Science, he provides information in pictorial ways and it gains the students' attention, he changes mode every 15 minutes or so, drawing, overhead, etc. and then when well on their way, will pass out candy. He answers questions if they ask, but does not initiate the questions to students.

a. Jack - rejects claim with sarcasm to retain identity and agency

b. Carlos - rejects claim with sarcasm to retain identity and agency, then changes subject and returns to conversation with Jack and Antonio

c. Antonio - rejects claim by yelling back at Frederek that he is unfair in his judgment call, retaining identity and agency

d. Julio - silent the entire time, Frederek only noticing him if gets bothered or lacks supplies

4. Frederek - interactively established contract with have pencils (tacit contract in school that they will work/have supplies and he will give points) but resorts to normative claim as teacher when guys continue to bother Antonio with leave each other alone

a. Jack - appears to accept first claim but rejects second claim with sarcasm to Antonio

b. Carlos - accepts pencil but rejects second claim with sarcasm to Antonio

c. Antonio - rejects first and second claim with cursing to get guys off his back, sarcastic retort and then gets up and heads for door threatening to leave (agency to take care of the situation by himself since the teacher did not do it)

5. Frederek - accepts interactively established contract from Antonio and tries to get him to stay with you have been in this class and walking by him (I'll protect you in here and not write referral if you will stay) and with Carlos with you are the one who should be leaving (tacit you broke the classroom norms here) but interjects normative claims with Jack and Carlos with loud be quiet leave him alone and sit down to Antonio (Frederek allows some claims to be made by students either by ignoring their references to cultural differences unless it bothers another student, or by letting them talk quietly about community processes like fighting while he passes out folders or by challenging how the class is run when the teacher is not protecting someone like he tacitly claims to be able to do or by challenging his coercive attempts to send a student to CSI as last straw even though the question may be legitimate about sluts, it came at the end of historically disruptive period of time by the same student or by using coercive 
power claim to call home if he knows the student will ultimately stop disrupting when he hears this, a good student with a good family he'll do that, otherwise if the student has shown he is not good and has no support at home or makes bad choices and doesn't seem to care about them, he'll kick him out ultimately. Frederek makes decisions based on what is happening in classroom with little reference to school rules. Bottom line, he wants kids to like science and class and he doesn't want to ruin their chances by writing referrals)

a. Antonio - opens interactively established contract with teacher telling him to call counselor to change schedule, rejects normative claims to sit down

b. Jack - accepts claim to leave Antonio alone doesn't want to get into trouble

c. Carlos - accepts claim to leave Antonio alone and tries to get him to stay fearing repercussions for the guys; then uses coercive power with Antonio dropping his rank in the peer group (references peer group from latino culture as attempt to identify them separately from school)

6. Frederek - uses coercive power with talking to CSI staff about Antonio

a. Antonio - accepts claim and comes in with agency of choice demeanor

b. Jack - accept claim with laughter and sarcasm

c. Carlos - accept claim with laughter and sarcasm

7. Frederek - uses normative claim with ok and leave each other alone now (most common claim), don't talk to him, that's enough, don't look at him, Jack's name

a. Antonio - gets upset again

b. Jack - accepts claim with all right, I'm ready, but looks around quietly

c. Carlos - opens notebook accepts claim

8. Frederek - uses coercive power claim to be quiet or he'll call Jack's home (second common claim)

a. Jack accepts claim with all right all right

b. Others - accept claim

9. Frederek - uses normative claim to begin lesson and to tell Carlos to turn off IPOD with Carlos and stop it, tries to begin lecture, then to Carlos again with Carlos, turn it off, then says don't worry about it to Antonio (interactively established contract that he protects students) followed by loud leave him alone that's enough (normative claim)

a. Jack - rejects claim with sarcasm about how long they have been working on earthquakes, saying Frederek deserves an award for patience, laughing, saying board is dirty, mouthing to Carlos, then laughs as what Carlos says, then accepts Antonio's stare as straight, chillin', mack

b. Carlos - rejects claim by pulling up hood and listening to IPOD, turns it up loud, rejects claim by continuing to listen, mouth words to Antonio, and appears to accept second normative claim to turn it off but with reservation to maintain identity and agency that he wanted to listen to it but tells Jack that he is going to listen to Frederek, but immediately eggs Antonio on

c. Antonio - accepts claim but also nods to Jack, stares at guys, guys accept at straight, chillin, mack 
10. Frederek - uses coercive power (used when the history of a situation has gone on too long and someone gets hurt or demeaned even if not in the room like in this case with the word sluts for faults - ignores much of what goes on but finally has to step in and remove student - not obvious to students though that this is why he got kicked out all they heard was the one word, but they also know that the teacher is patient and finally cracks like most people - they try him until he gives a reaction) by saying you're out of here and going to get CSI staff for Carlos, never accepts challenge to authority but waits for security using normative claims as tells Carlos what the word is, that he causes the problems, to be quiet, to write it down even when he doesn't understand it but be quiet! And when Carlos continues to argue, he says loud you're out from here. To security, I need Carlos out (normative claim)

a. Carlos - appears to accept normative claim to leave Antonio alone with what does that say (to words to copy on board) sluts? But rejects coercive power by reclaiming his position of wanting to copy but couldn't read the word an innocent mistake he really thought faults was sluts, some cursing, more asking, disbelief, and questioning how he can copy if he doesn't know the word - never accepts normative claim and obligingly accepts coercive claim but with agency and identity in tact by not giving in without a fuss, taking a while to write the word and put on his jacket.

b. Jack - rejects claim with question, why did you kick him out?

c. Julio - texting silently (has been part of period)

d. Antonio - laughs, accept claim but with reservation

11. Frederek - uses coercive power (he enforces his own rules by calling home rather than kick him out) to Jack's question, and interjects he says you may be next then uses interactively established contract proposal with will call home and then uses normative claim for lecture time but answers Jack's question about report cards

a. Jack - accepts claim to call father by waving hands to say don't call him with naw naw naw and asks questions about earthquake but questions him when he breaks a pencil as an example to maintain identity and agency, then writes

b. Antonio - accepts claim

c. Julio - rejects claim, texting, begins writing soon

12. Frederek - uses unstated normative claim to lecture - but answers Jack's question about report cards, and enters into Jack's proposal for interactively established contract, rejects proposal from Jack about report card, ignores proposal to go get bookbag continues to lecture, ignores pencil tapping during overhead lecture, confirms what's in box (goggles)

a. Jack - almost immediately asks when they get their report cards, argues that he will copy if Frederek will let him look quickly; then asks Antonio about his bookbag followed by Frederek if he can go get it, then begins pencil tapping incessantly during lecture, then asks what's in box, goes to restroom in classroom, returns to pencil tap while Antonio and Frederek talk 
b. Carlos returns (CSI time limit w/out referral, admin rule) accepting normative rule to listen to lecture but with quiet insult to Frederek to maintain identity and agency, begin writing, tapping pencil, head on chin)

c. Julio - looking at overhead, accepts claim

d. Antonio - looking at floor, accepts claim, asks several lecture related questions and Frederek answers

13. Frederek - uses unstated normative claim (as teacher - students do work at school and teachers teach) to pass out seatwork, handout, map, then stated normative

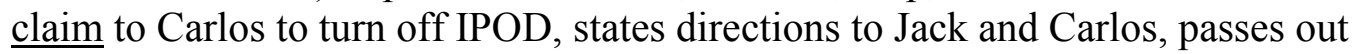
maps, handouts, and starbursts, walks around

a. Jack - accepts claim but with sarcastic remark (cultural humor referenced and music culture and identity - references the way Frederek talks with his accent, making fun of it, positions the student peer group as latino versus Swedish - counterculture that is interested in gang activities and status, during academic conversations know a lot and voice what they know to position themselves as intelligent, but trouble with law got them here, not in same status as Black kids; family important don't want them to call home to bother them with school stuff supposed to mind teacher; ) then talks about what he knows, questions Frederek's gulf of mexico comment, then asks Carlos what lyrics on bookbag mean, hums them while listen to Frederek's directions, then changes subject to starbursts while working on handout, looks through magazine, maintains agency and identity, teases Antonio

b. Carlos - partially accepts claim with hand on elbow holding up head appearing to accept claim and asks question, hums while listening to IPOD and accepts claim to turn off, tells Jack what lyrics mean, asks Frederek about directions, curses class doesn't get it, maintaining agency and identity, throws paper on floor, says Frederek should pass out week instead

c. Antonio - accepts claim still looking at fiddling with overhead, sleeping demeanor, throws starbursts, maintaining agency and identity, scrunches eyes at Jack for teasing and puts head on table again

d. Julio - writing, accepts claim

This stage is the process of critically reflecting on what has been discovered thus

far in the research study. Having gone through the other stages, interactive power analysis is akin to theoretical notes. It is conducted away from other materials other than key definitions jotted down for referral during the process, the researcher's memory and feelings flow forth. It is a hermeneutical, reiteritative, and thought-provoking process in which the researcher's understandings flow together as the three steps are conducted. 
First, cultural power was viewed as distortions, and tacit normative-evaluative claims were recognized and reflected on as unequal power relations of participants caught up in unconscious or semiconscious interactions. To spot the distortions, the researcher must recognize the claim to universality to the participants' world referenced by each normative-evaluative claim considered. Failure of claims to represent the mutual interest of the whole group was considered cultural power at play. Cultural power was discovered through hermeneutic position taking, or inferencing, when the plea or reference to universality was spotted as a sham. 
VITA

\section{DEBRA MAYES PANE}

October 3, 1950

1972

1993

1993-1995, 2004-2008

1995-2004, 2007-2008
Born, Hereford, Texas

Bachelor of Arts in Teaching

Sam Houston State University, Huntsville, Texas

Master of Science, K-12 Reading

Florida International University, Miami, Florida

Adjunct, Graduate Teaching Assistant Instructor, Reading Camp Director

Florida International University, Miami, Florida

Language Arts Teacher, P/T Executive Director TROY Community Academy Miami-Dade County Public Schools, Florida, Educational Alternative Outreach Program

Dissertation Evidence Acquisition Fellowship Recipient \& Dissertation Year Fellowship Recipient Florida International University Graduate School

\section{PUBLICATIONS AND PRESENTATIONS}

Pane, D. M. (in press). Viewing classroom discipline as negotiable social interaction: A communities of practice perspective. Teaching and Teacher Education: An International Journal of Research and Studies (2009), doi:10:1016/j.tate.2009.05.002

Pane, D. M. (in press). [Review of the book Critiquing Praxis]. New Horizons in Adult Education and Human Resource Development, xx(x), xx-xx

Pane, D. M., \& Diaz, R. (in press). [Review of the book Interactive qualitative analysis: A systems method for qualitative research]. New Horizons in Adult Education and Human Resource Development, $x \mathrm{x}(\mathrm{x}), \mathrm{xx}-\mathrm{xx}$

Pane, D. M., \& Salmon, A. (in press). The experience of isolation in alternative education: A heuristic research study. Western Journal of Black Studies [Special Issue: Literacy as a Social Divide]

Pane, D. M. (2009). Third space: Blended teaching and learning. Journal of the Research Center for Educational Technology [Special Issue], 5, Art. 8, http://www.rcetj.org 
Pane, D. M., \& Rocco, T. S. (2009). Critical Microethnography: The Search for Emancipatory Methods. Forum: Qualitative Social Research (FQS), 10(2), Art. 7, http://www.qualitative-research.net/index.php/fqs/issue/view/31

Pane, D. (2006). Pre-service teacher education for diversity. In A. Heron-Hruby (Ed.), American Reading Forum Online Yearbook, 26, http://www.americanreadingforum.org/06_yearbook/html/arf_06_pane.htm

Nackoney, C., \& Pane, D. (2006). [Review of the book Auto/Biography and Auto/Ethnography: Praxis of Research Method]. New Horizons in Adult Education and Human Resource Development, 20(3), 47-50

Pane, D. M. (2009). Reducing the discipline gap among African American students: Learning in classroom communities of practice. In M. Plakhotnik, S. Nielson, \& D. M. Pane (Eds.), Proceedings of The Eighth Annual College of Education Research Conference (pp. 92-97). Miami, FL: Florida International University. (Lorraine R. Gay Award for Excellence in Research/Scholarship - Best Graduate Student Paper) (College of Education Graduate Student Network Award - Best Student Conference Paper)

Pane, D. M. (October, 2009). Critical microethnographic method. Qualitative Inquiry in the Caribbean Conference 2009, School of Education University of the West Indies, Mona, Jamaica.

Pane, D. M. (March, 2007). Finding third space in pre-service teacher education. Presented at the 2007 Annual Florida International University Graduate Student Association Scholarly Forum, Miami, Florida.

Pane, D. (April, 2006). Transformative teaching and learning in at-risk schools. Paper presented at the American Educational Research Association (AERA) Division K Graduate Student Seminar, San Francisco, California.

Pane, D. (March, 2006). New teacher education for diversity. Paper presented at the 2006 Florida International University Annual Graduate Student Association Scholarly Forum, Miami, Florida. (Awarded $2^{\text {nd }}$ Place) 